\title{
The Meteorological Aspects of Siting Large Wind Turbines
}

T. R. Hiester

W. T. Pennell

January1981

Prepared for the U.S. Department of Energy under Contract DE-AC06-76RLO 1830

Pacific Northwest Laboratory Operated for the U.S. Department of Energy by Battelle Memorial Institute 


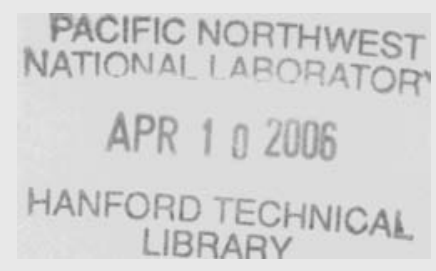

$\mathrm{NOTICE}$

This report was prepared as an account of work sponsored by the United States Government. Neither the United States nor the Department of Energy, nor any of their employees, nor any of their contractors, subcontractors, or their employees, makes any warranty, express or implied, or assumes any legal liability or responsibility for the accuracy, completeness or usefulness of any information, apparatus, product or process disclosed, or represents that its use would not infringe privately owned rights.

The views, opinions and conclusions contained in this report are those of the contractor and do not necessarily represent those ot the United Siates Government or the United States Department of Energy.

\author{
PACIFIC NORTHWEST LABORATORY \\ operated by \\ BATTELLE \\ for the \\ UNITED STATES DEPARTMENT OF ENERGY \\ Under Contract DE-AC06-76RLO 1830
}

\author{
Printed in the United States ot America \\ Available from \\ National Technical Information Service \\ united States Department or Commerce \\ 5285 Port Royal Road \\ Springfield. Virginia 22151
}

Price: Printed Copy S

*: Microfiche $\mathbf{5 3 . 0 0}$

NTIS

'Pages Selling Price

$\begin{array}{ll}001-025 & \$ 4.00 \\ 026-050 & \$ 4.50 \\ 051-075 & \$ 5.25 \\ 076-100 & \$ 6.00 \\ 101-125 & \$ 6.50 \\ 126-150 & \$ 7.25 \\ 151-175 & \$ 8.00 \\ 176-200 & \$ 9.00 \\ 201-225 & \$ 9.25 \\ 226-250 & \$ 9.50 \\ 251-275 & \$ 10.75 \\ 276-300 & \$ 11.00\end{array}$


THE METEOROLOGICAL ASPECTS OF SITING LARGE WIND TURBINES

\author{
T. R. Hiester \\ W. T. Pennell
}

January 1981

Prepared for the U.S. Department of Energy under Contract DE-AC06-76RLO 1830

Pacific Northwest Laboratory Richland, Washington 99352 


\section{ACKNOWFDGEMENTS}

This document, which was supported by the U.S. Department of Energy under Contract DE-AC06-76RLO 1830, synthesizes the work of others. For those whose works have appeared in the open literature, the source is acknowledged by citations in the text. However, for those who provided supportive words and concepts, the authors would like to acknowledge them specifically and to take responsibility for any misrepresentation, reinterpretation or elaboration of their contributions. These individuals include: M. M. Orgi1l, C. H. Huang, R. L. Drake, J. V. Ramsdell, and D. S. Renné of the Pacific Northwest Laboratory; E. W. Hewson, J. E. Wade, R. W. Baker, and R. L. Whitney of Oregon State University; S. N. Walker and T. G. Zambrano of AeroVironment, Inc., Pasadena, California; K. M. Barnett of New Mexico State University; W. Frost of FMG Associates, Inc., Tullahoma, Tennessee; and R. W. Marrs and D. R. Gaylord of the University of Wyoming.

The authors would also like to thank Debbie Atkin, Judy Childs, and Rosemary Ellis for their never-ending effort to see this document typed. 


\section{PREFACE}

Writing a report on site selection for large wind turbines poses a special problem, because the potential users of the report represent various professional backgrounds, and the problem of site selection encompasses many disciplines. As a result, precisely what should be included and in what detail becomes a central concern.

In this document, the meteorological aspects of the site selection problem are addressed, and other areas, such as machine design and wind energy economic evaluation, are only mentioned when they touch on meteorological siting concerns. However, we felt that a basic look at the relationship of nonmeteorological and meteorological aspects of the problem, upon which our suggested solutions are based, was necessary. All or part of this introductory material, which is presented in Chapter 1, may be familiar to those already working with this interdisciplinary problem. For those wishing only to determine the scope and intent of a meteorological site selection program, the basic meteorological problem, along with techniques and strategies that may be used to solve it, are addressed in Chapter 2.

The amount of detail required in discussing the technical issues involved in the remaining chapters and appendices varies substantially. For example, the discussion of numerical modeling is designed to acquaint the reader with the approaches and theories used in numerical modeling. As a result, it requires that the reader have a sufficient mathematical background to assess the models discussed and determine their applicability to a site selection program. At the same time, the discussion of biological and geomorphological indicators requires that the reader be versed in general engineering and science. Therefore, we have attempted to summarize in a readily understandable manner the conclusions and recommendations in each chapter.

We believe that this presentation will enable potential users to assess the meteorological aspects of the siting problem, to determine ways to solve the problem, and to integrate these solutions into the overall site selection program. 


\section{SUMARY}

This report, which focuses on the meteorological aspects of siting large wind turbines (turbines with a rated output exceeding $100 \mathrm{~kW}$ ), has four main goals. The first is to outline the elements of a siting strategy that will identify the most favorable wind energy sites in a region and that will provide sufficient wind data to make responsible economic evaluations of the site wind resource possible. The second is to critique and summarize siting techniques that were studied in the Department of Energy (DOE) Wind Energy Program. The third goal is to educate utility technical personnel, engineering consultants, and meteorological consultants (who may have not yet undertaken wind energy consulting) on meteorological phenomena relevant to wind turbine siting in order to enhance dialogues between these groups. The fourth goal is to minimize the chances of failure of early siting programs due to insufficient understanding of wind behavior.

The problems of wind turbine siting are briefly outlined in Chapter 1. The main meteorological problem in siting is variability of the wind resource. The spatial variability of the wind resource makes it difficult to identify potential wind turbine cluster sites. The temporal variability makes it difficult to know the economic value of the wind resource, which is dominated by the conventional fuels that wind energy intermittently replaces. The magnitude and diurnal variation of the wind resource experienced by a large wind turbine cannot be accurately predicted from near-surface measurements. This requires, once a potential site is identified, that measurements from a tall tower be made to responsibly assess wind energy value.

Strategies for solving the meteorological problems are outlined in Chapter 2. Depending upon the specific problem, wind prospecting begins by screening the utilities' region of interest to identify potential candidate sites. The regional wind energy atlases, prepared for DOE by the Pacific Northwest Laboratory (PNL) and its subcontractors (Appendix 1) are the starting point for identifying areas with an economically viable wind resource. In addition, the wind prospector can use numerical models (Chapter 3 ); consideration of airflow interaction with topography (Chapter 5 and Appendix 2); measurement (Chapter 10); 
and studies of wind effects on tree deformation (Chapter 6), on geological features (Chapter 7), and on use of land by people (Chapter 8). Once candidate sites are identified they are instrumented near hub height (about $200 \mathrm{ft}$ ) for detailed sampling of wind speed and direction (about 1 minute averages, Chapter 2 and Chapter 10). If the site still looks economically viable, a cluster of wind turbines is designed (Chapter 9), possibly with the aid of wind tunnel models (Chapter 4).

Following the outline of siting strategies in Chapter 2, specific technical discussions follow in the remaining chapters. The major conclusions of these technical discussions are summarized below.

Numerical models provide an objective method for interpolating wind resource characteristics between data stations. Models may be used to identify good resource areas within a larger region or used to estimate climatological statistics at a given site. Simple models, in which conservation of mass is the only physical constraint, are useful where topography is the major influence on airflow. A hierarchy of models based on the conservation of momentum and thermodynamic energy have been developed that should be capable of indicating where topographical and thermodynamic effects enhance the resource. However, these complex models are often difficult to use. Modeling is not a standalone siting tool but should be used in concert with other techniques. The sensitivity of model results to input data and assumptions should be tested for every region where they are applied.

Physical models (wind tunnels, towing tanks) are superior to numerical models when small areas of land (cluster size) are being modeled for stability conditions that are nearly neutral. Neutral stability exists when vertical motions of air are neither enhanced nor suppressed by effects of the temperature structure of the atmosphere. As the modeled region or height of terrain features become larger and the thermal stratification departs from neutrality, physical modeling becomes less able to model all of the important aspects of the real atmosphere. The best uses for physical modeling are for cluster site analysis and development of generic understanding of flow over specific terrain shapes. 
Estimating topographic effects on wind flow (such as the acceleration of wind over a ridge) is the oldest technique of wind energy site assessment, and is still valuable today. Little quantitative information exists so an experienced boundary layer meteorologist should be consulted. Topographical indicators of wind potential assist in understanding flow over terrain, knowing where to measure, and in interpreting measurement and modeling program results.

Wind-deformed trees are examples of biological indicators of wind. Biological indicators are very useful in screening fairly large areas for evidence of persistent winds. They may also be useful when investigating flow around specific topographical features. It is often possible to estimate the annual mean wind speed to within \pm 20 percent from trees. However, biological indicators provide only qualitative information.

The uncertainty of quantitative information derived from geomorphological indicators (such as wind-formed sand dunes) is large, but the techniques can be applied quickly. Therefore, this technique is recommended only if the geological information can be obtained at little additional cost, e.g., as part of site-screening visits for other purposes.

No specific method exists for using social and cultural indicators of wind power potential. The wind prospector should remain alert, however, to certain clues, such as previous uses of wind power in an area, location of snow fences, and evidence of turbulence or high wind damage.

Designing a cluster of wind turbines requires knowledge of how closely wind turbines can be spaced and of how the cluster's geometric design affects overall performance. Cluster design is based on our understanding of how the wakes created by individual machines evolve and interact and of how these wakes affect the performance of downstream machines. Results of numerical modeling studies, wind tunnel simulations, and a limited number of experiments in which the wakes of full-scale machines were observed are used to develop wind turbine spacing guidelines. These studies indicate that at spacings greater than 10 rotor diameters, the effect of the wake of one turbine on a downstream machine will be negligible. At a spacing of 7 to 8 diameters, 
there could be some reduction in the output of downwind machines, particularly at night when the intensity of atmospheric turbulence is low. At a spacing of less than 5 diameters, there is evidence that reductions in the output of downwind machines would be significant. Considerations of the effects of turbine wakes on cluster performance are most critical in the prevailing wind directions. In nonprevailing wind directions, wind turbines could be spaced very close together in order to reduce land requirements.

Wind measurements are the only sure way to assess the resource, and only then when the correct instruments are used properly to measure the appropriate quantities. Conventional cup and vane anemometry is adequate for all siting work, but reliability is a prime consideration. Numerous recording system options are discussed. Near-surface (10-m) measurement has some limited value in the early stages of site selection, as do short-term measurements to detect turbulence and flow separation zones using kite anemometers and tethered balloons. Ultimately a tall tower is required for site evaluation, because techniques to extrapolate 10-m measurements to hub height for a large wind turbine do not work well, especially over nonflat terrain. A rigorous program of instrument maintenance and calibration should be implemented to assure that quality decisions will be made based on measurements. 


\section{CONTENTS}

ACKNOWEDGEMENTS

ii

PREFACE

SUMARY

1.0 INTRODUCTION

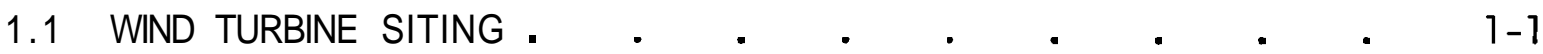

1.2 THE WIND RESOURCE $\cdot$.

1.3 WIND ENERGY MACHINES $\cdot$. $\quad . \quad \cdot \quad \cdot$.

1.3.1 Converting Wind Energy to Electrical or
Mechanical Energy.

1.3.2 Computing Machine Output . . . . . . 1-21

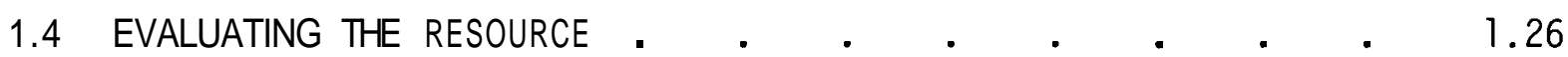

1.5 CONCLUSIONS . . . . . . . . . . . . . 1-30

2.0 STRATEGIES FOR WIND TURBINE SITING . . . . . .

2.1 THE NATURE OF THE PROBLEM • $2-1$

2.2 BASIC APPROACHES TO SITING . . . $2-2$

2.3 TECHNIQUES FOR ESTIMATING WIND RESOURCES . . . . $2-3$

2.3.1 Numerical Modeling . . . . 2-3

2.3.2 Physical Modeling . . . . . 2-6

2.3.3 Topographical Indicators . . . . . . 2-6

2.3.4 Biological Indicators . . . . . 2 . 2-6

2.3.5 Geomorphological Indicators . . . . . 2-6

2.3.6 Social and Cultural Indicators . . . . . 2-7

2.4 A. SITE-SELECTION STRATEGY . . • . $2-7$

2.4.1 Analyzing the Region of Interest. . . . . 2-10

2.4.2 Evaluating Candidate Resource Areas . . . . 2-10

2.4.3 Screening Potential Candidate Sites . . . . 2-13

2.4.4 Evaluating Candidate Sites . . . . . 2-14

2.4.5 Developing a Site. . . . 2-19 
2.5 CONCLUSIONS AND RECOMMENDATIONS . . . . . . . 2-24

2.5.1 Data Requirements for Siting . . . . . $2-25$

2.5.2 Sampling Periods . . . . . . . • $2-27$

3.0 NUMERICAL MODELING .

3.1 GOVERNING EQUATIONS . . . . . . . . . . . .

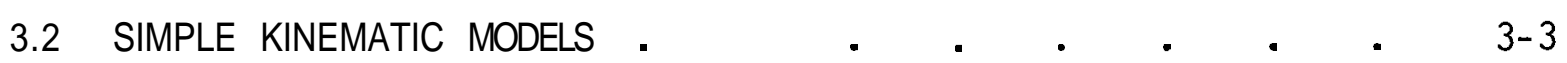

3.2.1 Mass Consistent Objective Analysis of Wind . . . 3-4
Fields.

3.2.2 Data Requirements and Initialization . . . . . 3-7

3.2.3 Verification Results . . . . $3-9$

3.2.4 Conclusions and Recommendations . . . . . . 3-26

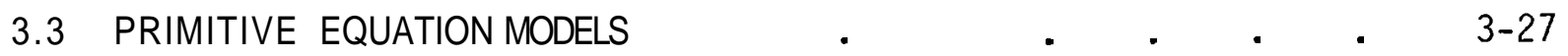

3.3.1 Boussinesq Equations . $\quad$. $\quad$. $\quad$. $3-27$

3.3.2 Anelastic Equations . . . . . . . . . $3-31$

3.3.3 Nonhydrostatic Models . . . . . . . . 3-32

3.3.4 Closure Assumption . . . . . . 3-32

3.3.5 Data Requirement and Initialization . . 3 . 36

3.3.6 Application and Results . . . . 3-37

3.3.7 Conclusions and Recommendations . . . 3- . 39

3.4 CONCLUSIONS AND RECOMMENDATIONS . . . . . . . . . $3-50$

4.0 PHYSICAL MODELING . $\quad$. $\quad$. $\quad . \quad 4-1$

4.1 THEORETICAL FOUNDATIONS AND LIMITATIONS . $\quad$. . . . $4-2$

4.1.1 Achieving Dynamic Similarity . . . . . 4-4

4.1.2 Achieving Similarity in the Boundary Conditions . . 4-9

4.2 VERIFICATION RESULTS . . . 4 4-17

4.3 CONCLUSIONS AND RECOMMENDATIONS . . . . . . 4-24

5.0 TOPOGRAPHICAL INDICATORS OF WIND PONER POTENTIAL .

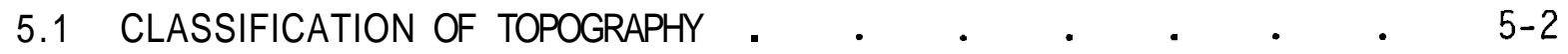

5.2 ROW OVER FLAT TERRAIN . . . . . . . . . . . $5-3$

5.2.1 Homogeneous Flat or Gently Sloping Terrain . . . 5-5

5.2.2 Homogeneous Flat Terrain with Obstacles . . . 5-9 
5.2.3 Flat Terrain with Roughness Changes

5.2.4 Flat Terrain with Horizontal Temperature Gradients

5.3 SMALL-SCALE TERRAIN . $\quad \cdot \quad \cdot \quad \cdot \quad \cdot \quad \cdot \quad \cdot \quad \cdot \quad \cdot 5-27$

5.3.1 Elevated Feature . . . . . . . . . . $5-28$

5.3.2 Flow Over Depressions . . . . . . . . . $5-47$

5.4 ROW OVER LARGE-SCALE TERRAIN FEATURES . • . • . • 5-49

5.4.1 Flow Over Large-Scale Elevated Features . . . 5-51

5.4.2 Large-Scale Depressions . . . . . . $5-62$

5.5 SUMMARY AND RECOMMENDATIONS .

6.0 BIOLOGICAL INDICATORS OF WIND PONR POTENTIAL . . . . . 6-1

6.1 TREES AS INDICATORS OF MEAN WIND SPEED . . . . • . • 6-1

6.1.1 Description of the Indices of Wind Deformation . . 6-2

6.1.2 Mean Wind Speed and Tree Deformation . . . . 6-7

6.2 INDICATORS OF PREVAILING WIND DIRECTION . . . . . . . $6-9$

6.2.1 Winter Wind Flagging . . . . . . . . . 6-14

6.2.2 Growing Season Wind Flagging . . . . . 6-14

6.2.3 Flagging By Winds During Both Growing . . . . 6-14

6.3 INDICATORS OF SEVERE WIND OR ICE DAMAGE . . . . . . 6-15

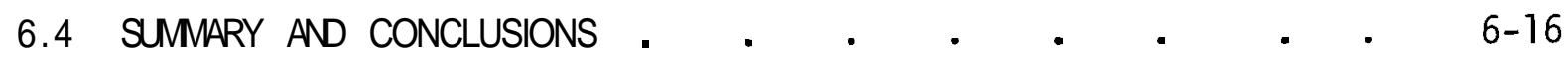

7.0 GEOMORPHOLOGICAL INDICATORS OF WIND POWER POTENTIAL .

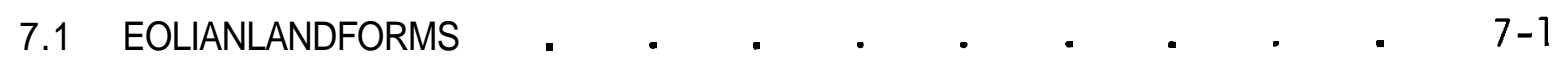

7.2 ESTIMATING WIND SPEEDS .

7.2.1 Sand Size Distribution and Wind Speed . . . . 7-4

7.2.2 Dune Migration and Wind Speeds . . . . . 7-12

7.2.3 Wind Speed and Other Eolian Activities . . . 7-16

7.3 EOLIAN INDICATORS $\propto F$ WIND DIRECTION . $\quad . \quad \cdot \quad \cdot \quad$ 7-17

7.4 CONCLUSIONS AND RECOMMENDATIONS .

8.0 SOCIAL AND CULTURAL INDICATORS OF WIND POWER POTENTIAL . 
9.0 WIND TURBINE WAKES AND CLUSTER DESIGN

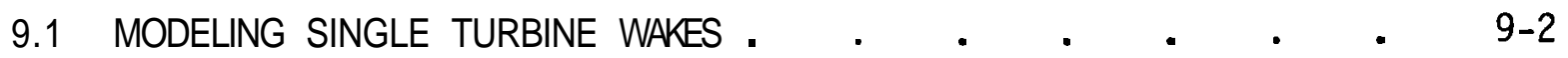

9.1.1 Numerical Modeling of Wind Turbine Wakes . 9-3

9.1.2 Physical Modeling of Wind Turbine Wakes . 9-5

9.1.3 General Behavior of Wind Turbine Wakes . . . 9-10

9.2 WAKE INTERACTIONS AND CLUSTER PERFORMANCE . . • • • $\quad$ 9-24

9.2.1 Approximate Numerical Models . . • . . . 9-24

9.2.2 Cluster Design Using Lissaman's Model . . . . 9 9-25

9.2.3 Cluster Design Using Physical Modeling . . • 9-28

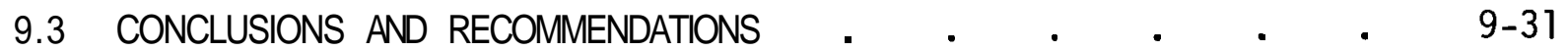

9.3.1 Numerical and Physical Modeling Approaches
to Cluster Evaluation. . . . 9-31

9.3.2 General Spacing Guidelines for Large . . . . . 9-32
Wind Turbines . . . 9

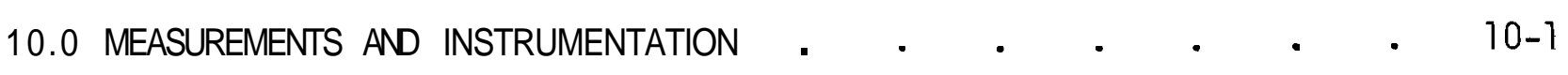

10.1 CONCEPTS OF A MEASUREMENT SYSTEM . . . . . . . $10-1$

10.2 WIND SENSING SYSTEMS $. \quad . \quad . \quad . \quad . \quad . \quad . \quad . \quad 10-4$

10.2.1 Cup Anemometers . . . . . . . . . 10-5

10.2.2 Wind Vanes . . . . . . . . . . 10-7

10.2.3 Propeller Anemometers . . . . . . . 10-9

10.2.4 Tethered Balloon Systems . . . . . . 10-10

10.2.5 Kites and Kite Anemometers . . . . . . 10-11

10.2.6 Remote Sensing Systems . . . . . 10-13

10.2.7 Specialty Sensors . . . . . . . 10-17

10.2.8 Conclusion . . . . . . . . . . $10-19$

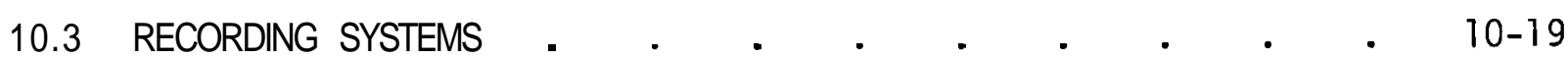

10.3.1 Class I Systems . . . . . . . . 10-21

10.3.2 Class II Systems . . . . . . . . . $10-22$

10.3.3 Class III Systems . . . . . . . . $10-24$

10.3.4 Class IV Systems . . . . . . . . 10-28

10.3.5 Remote Access or Telemetry Data Link. . . 10-34

10.4 ICE DETECTORS . . . . . . . . . . 10-34 
10.5 SATELLITE AND AIRCRAFT IMAGERY OF THE EARTH . . . . 10-35

10.6 INSTRUMENT DEPLOYMENT AND SITING STAGES . . . .

10.6.1 Analysis of Region of Interest . . . . . 10-39

10.6.2 Candidate Resource Area Evaluation . . . . 10-39

10.6.3 Potential Candidate Site Screening . . . . 10-46

10.6.4 Candidate Site Evaluation and Site Development . 10-47

10.7 QUALITY ASSURANCE $\quad . \quad$. . . . . . . . 10-51

10.8 SUMARY . . . . . . . . . . $10-52$

APPENDIX 1: REGIONAL WIND RESOURCE ASSESSMENT . . . . A.1-1

APPENDIX 2: SONE BACKGROUND METEOROLOGY PERTINENT TO

SITING LARGE WECS .

APPENDIX 3: VARIABILITY OF THE WIND RESOURCE . . . . . . A.3-1

$\begin{array}{ll}\text { APPENDIX 4: } & \text { ESTIMATING AVERAGE WIND SPEEDS RROM } \\ & \text { AVERAGE SAND TRANSPORT RATES }\end{array}$

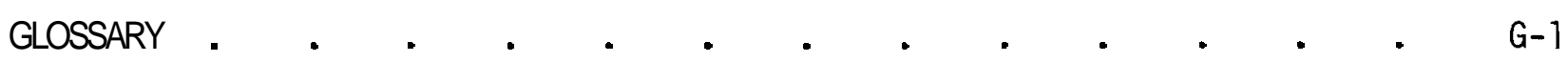




\section{FIGURES}

1.1 Surface Winds of the General Circulation . 1 . 1-6

1.2 Annual Average Wind Power at $50 \mathrm{~m}$ Above Well-Exposed

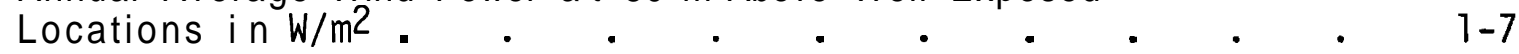

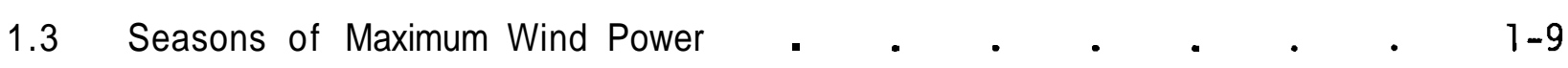

1.4 Seven Years of Monthly Mean Wind Speeds for Butte

1.5 Annual Average Diurnal Variation at Three Elevations . . . 1-12

1.6 Monthly Averaged Diurnal Wind Speed Cycle for Great

1.7 Some Types and Configurations of WECS . . . . . . . . . 1-15

1.8 Power Coefficients for Some Typical Wind Turbines . . . . 1-18

1.9 Forces and Wind Velocity at a Blade Element $\quad$. 1-18

1.10 Power Coefficient of a Typical Large Wind Turbine . . . 1-20

1.11 Example of Power Output Curve for a Typical Large $1-21$

1.12 Comparison of Measured and Predicted Power - Mod-OA,

1.13 Interaction of Wind Turbine Power Characteristics and Wind Statistics to Produce the Wind Turbine Power Frequency Distribution.

1.14 Example of Percent Downtime as a Function of Mean
Wind Speed and Cutout Speed .. . . . . .

1.15 Percent Time Running at Rated as a Function of Cutout,
Rated and Mean Wind Speeds . . . . . . . $1-25$

1.16 Estimate of Expected Average Power Output for Wind Turbines as a Function of Cutout, Rated and Mean Wind Speeds and Rated Power Output . $1-25$

1.17 Cost of Energy and Annual Energy Production Versus
Site Annual Mean Wind Speed for a Hypothetical Machine 
FIGURES (contd)

2.1 Applicability of Siting Techniques to the Steps of

the Wind Prospecting Approach . . 2-4

2.2 Applicability of Site-Screening Techniques to the

Problem of Evaluating a Predetermined Site . . . 2-5

2.3 Flow Chart Summary of Utility Strategy for Siting
Large Wind Turbines

3.1 Perspective of the Terrain on Oahu, Hawaii, Looking 3-10

Southwestward .

3.2 Wind Speed Contours at $15 \mathrm{~m}$ Above the Surface for

Idealized Tradewind Flow over Oahu . . 3-11

3.3 Oahu Topographic Schematic with Data Network Locations . . 3-12

3.4 Comparison of Calculated to Observed Wind Speed for 24 Oahu Simulation Events (Simulation I) . 3-14

3.5 Comparison of Calculated to Observed Wind Speed for 24 Oahu Simulation Events (Simulation II) . 3-14

3.6 Comparison of Calculated to Observed Wind Speed for 24 Oahu Simulation Events (Simulation III). 3-15

3.7 Comparison of Calculated to Observed Mean Wind Speed

Based Upon 24 Oahu Simulation Events (Simulation I). 3-15

3.8 Comparison of Calculated to Observed Mean Wind Speed

Based Upon 24 Oahu Simulation Events (Simulation II) . 3-16

3.9 Comparison of Calculated to Observed Mean Wind Speed

Comparison of Observed and Predicted Seasonal Mean Wind
Speeds for Mt Tom, Massachusetts

3.11 Comparison of Observed and Predicted Diurnal Cycles for
Each Season a t Mt. Tom, Massachusetts.

3.12 Hourly Average Power Density over Chesapeake Bay,
Wintertime Conditions. . . . . . . 3-40

3.13 Hourly Average Power Density over Chesapeake Bay,
Wintertime Conditions. . . . . . . 3-41

3.14 Daily Average, Layer Mean Power Density over Chesapeake
Bay, Wintertime Conditions. . . . . 3-42 
FIGURES (contd)

3.15 Hourly Average Power Density over Chesapeake Bay, Summertime Conditions . $. \quad . \quad . \quad . \quad . \quad . \quad$.

3.16 Hourly Average Power Density over Chesapeake Bay,

3.17 Daily Average, Layer-Mean Power Density over Chesapeake

Bay, Summertime Conditions . . . . . . . 3-45

3.18 Hourly Average Power Density over South Texas Coast,

3.19 Hourly Average Power Density over South Texas Coast, Summertime Conditions . . . . . . . . . 3-47

3.20 Daily Average, Layer-Mean Power Density over South Texas Coast, Summertime Conditions . . . . . . 3-48

4.1 Potential Temperature Profile from the Air Mass Transformation

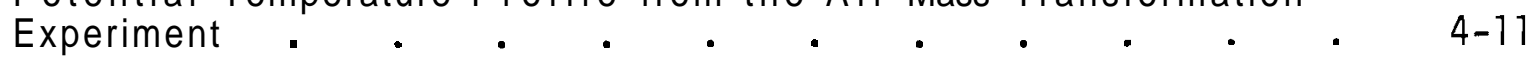

4.2 Profile of the $U$ and $V$ Component from the Air Mass

Transformation Experiment . . . . . . 4-12

4.3 Measurements of the Longitudinal and Vertical Components of Turbulence Intensity $. \quad . \quad . \quad . \quad . \quad . \quad .443$

4.4 Scatter Diagrams of the Rakaia Gorge Field Measurements Versus Wind Tunnel Simulation Measurements .

4.5 Annual Mean Wind Speed, $\mathrm{m} / \mathrm{sec}$, over Oahu . . . . . 4-21

4.6 Contour Plot of Relative Wind Speeds . . . . . . 4-22

Scatter Diagram of Kahuku Point Field Measurements
Versus Wind Tunnel Simulations . . . . . . 4-24

5.1 Wind Speed at Three Levels as a Function of Roughness
Length. . . . . . . . 5-6

5.2 The Configuration of Geostrophic Wind and Terrain Slope that Results in the Maximum Ageostrophic Wind at the Top of the Nocturnal Boundary Layer Shortly After Sunset .

5.3 Flow Over an Obstacle That Produces Horseshoe Vortices and Roof Corner Vortices 
FIGURES (contd)

5.4 Lateral Profiles of Mean Velocity Deficit and Turbulence

Excess in the Wake of a Building at $0^{\circ}$. . . .

5.5 Lateral Profiles of Mean Velocity Deficit and

Turbulence Intensity Excess in the Wake of

a Building at $47^{\circ}$.

5.6 Decay of the Maximum Mean Velocity Difference Behind

the Block Building with Downwind Distance for Two

Wind Directions . . . . . . . . . . 5-15

5.7 Lateral Profiles of Mean Velocity Deficit and Turbulence

Intensity Excess Measured with a Hot-Film Anemometer.

5.8 Vertical Profiles of Velocity Deficit on the Hemisphere

Wake Center1ine. . . . . . . . . . 5-17

5.9 Comparison of the Decay of the Centerline Velocity Excess

in the Wakes of Hemispheres in the Smooth- and Rough-Wall

Boundary Layers

5.10 Streamlines of the Thermal Wind and of the Wind for

Onshore Flow from Cool Ocean to Hot Land

5.11 Cross Section of a Well Developed Afternoon Sea Breeze . 5-26

5.12 Hypothetical Wind Speed Profiles Over a Small Two-

Dimensional Range . . . . . . . . . . 5-29

5.13 Comparison of Model Predicted and Measured Wind Speeds . 5-33

5.14a The Effect of Upwind Slope on the Speed-up Factor . 5-36

5.14b The Effect of Downwind Slope on the Speed-up Factor . 5-36

5.15 Criterion for Flow Separation over Two-Dimensional Hills . 5-38

5.16 Contours of Longitudinal Turbulence Intensity with

Superimposed Streamlines . . . . . . . . 5-39

5.17 Contours of the Speedup of Upstream Winds and Contours of the Turbulence Intensity for Flow over a Cliff.

5.18 Contours of the Speedup of Upstream Winds and Contours of the Turbulence Intensity for Flow over an Escarpment with 1:1 Slope 
FIGURES (contd)

5.19 Contours of the Speedup of Upstream Winds and Contours of the Turbulence Intensity for Flow over an Escarpment with 1:2 slope

5.20 Contours of the Speedup of Upstream Winds and Contours of the Turbulence Intensity for Flow over an Escarpment with 1:4 Slope . . . . . . . . . . 5-44

5.21 Windspeed $v_{m}$ Versus $v_{f}$. . . . . . . . . . . 5-54

5.22 Gravity Waves Forced by Flow Over a Ridge . . . 5-57

5.23 A Schematic of the Wind Pattern and Velocity Profile through a Mountain Pass . . . . . . . . 5-63

5.24 The Daily Sequence of Mountain and Valley Winds . . . 5-65

5.25 Possible WECS Sites in Sloping Valleys and Canyons where $\quad 5-66$

5.26 Possible WECS Sites Where Prevailing Winds are Channeled by Valleys . . . . . . . . . . . . 5-67

6.1 Distribution of Trees in the United States Being Calibrated for Use as Indicators of Wind Power Potential

6.2 The Griggs-Putnam Index of Wind Deformation . . 6-4

6.3 The Barsch Rating of Wind Deformation . . . . . . 6-5

6.4 The Technique for Computing the Deformation Ratio Index
for Conifers and Hemispherically Crowned Trees . 6-7

6.5 Core Samples from Windward and Leeward Side of the Tree, Mounted and Cross-Dated

6.6 The Relationship Between the Griggs-Putnam Index for Ponderosa Pine and Mean Annual Wind Speed.

6.7 Guide to Wind Deformation Indices for Pinus

6.8 Guide to Wind Deformation Indices for Quercus . . 6-13

7.1 Diagram Showing Plan and Profile Views of Simple Dunes . . 7-3

7.2 Threshold Velocity as a Function of Sand Grain Diameter and Sand Density. 
FIGURES (contd)

7.3 Structure of Three Wind Profiles over a Dune . . . . 7-7

7.4 Grain Size Distribution of a Typical Sample of the Wyoming Ferris Dune Field . . • . . . . . 7-9

7.5 Setting Velocities in $\mathrm{cm} / \mathrm{sec}$ of Quartz Spheres in

7.6 Assuming a Rayleigh Distribution of Wind Speeds, Equation (7.11) Shows That the Average Sand Transport

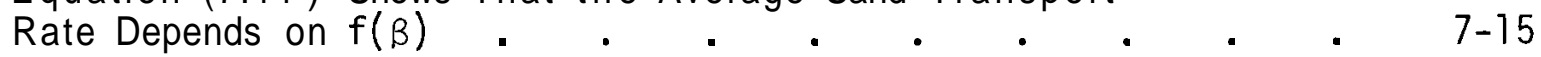

9.1 The Structure of the Wake Assumed in Lissaman's Model 9-3

9.2 Wake Centerline Velocity Deficits Behind a 0.2 m Rotating Model of a Darrieus Rotor and Two Tea-Strainer Models of Different Diameter

9.3 Available Power Ratios Behind a $0.2 \mathrm{~m}$ Rotating Model of a Darrieus Rotor and Two Tea-Strainer Models of Different Diameters

9.4 Wake Centerline Deficits Behind a Model Wind Turbine and a Full-Scale 5.3-m Darrieus Wind Turbine.

9.5 Predicted Centerline Velocity Deficits Using Lissaman's Model for Various Values of the Turbulence Intensity.

9.6 For Low Levels of Ambient Turbulence: Wake Velocity Deficits Behind a $0.25-\mathrm{m}$ Horizontal-Axis Model Wind Turbine at Various Downstream Distances

9.7 For Decaying Grid-Generated Turbulence: Wake Velocity Deficits Behind a $0.25-\mathrm{m}$ Horizontal-Axis Model Wind Turbine at Various Downstream Distances

9.8 For Turbulence in a Wind Tunnel Boundary Layer $0.6 \mathrm{~m}$ Deep: Wake Velocity Deficits Behind a $0.25-\mathrm{m}$ HorizontalAxis Model Wind Turbine at Various Downstream Distances

9.9 Lateral Velocity Profíles in Low Ambient Turbulence Conditions

9.10 Lateral Velocity Profiles in Decaying Grid-Generated Turbulence 
FIGURES (contd)

9.11 For Low Ambient Turbulence Intensity: Profiles of Turbulence Intensity Measured at Lateral Positions

9.12 For Decaying Grid-Generated Turbulence: Profiles of Turbulence Intensity Measured at Lateral Positions.

9.13 For Turbulence in a Wind Tunnel Boundary Layer 0.6 m Deep: Profiles of Turbulence Intensity Measured at Lateral Positions

9.14 Cluster Efficiency as a Function of Wind Turbine Spacing.

9.15 Cluster Efficiency as a Function of Wind Direction.

10.1 Wind Speed Frequency Distribution from Kingsley, Nebraska.

10.2 Wind Speed Frequency Distribution from San Gorgonio, California.

10.3 Diurnal Variation of Wind Speed by Season for Boone, North Carol ina

10.4 Wind Speed Persistence in Speed Class . . . 10-29

10.5 Probability of Difference Between 50-Second Averages of Wind Direction Exceeding $10^{\circ}$ as a Function of Comparison Interval

10.6 Frequency Distribution of Parallel and Normal Shear Components 


\section{TABLES}

1.1 Variation of the Yearly Estimates of the Correlation Coefficient of Monthly Mean Wind Speeds Between

Whitehall and Butte, Montana

3.1 Oahu Data Base Surface Observation Stations . . . . . 3-13

3.2 Station Wind Speed Results Correlation Quantities . 3-17

3.3 Station Wind Direction Results Correlation Quantities 3-18

3.4 Comparison of Simulated and Measured Wind Speeds i $\mathrm{n} \mathrm{m} / \mathrm{sec}$. $3-24$

3.5 Data Supplied for Primitive Equation Model Verification Exercise on Oahu . . . . . . . . . . . 3-37

5.1 Wake Behavior of Variously Shaped Buildings and Shelter Belts . . . . . . . . . . . . 5-21

5.2 Frenkiel's WECS Site Suitability Based on Slope of Hill and Wind Gradient in Lowest 40 Meters . . . . . . 5-46

6.1 Statistical Results and Error Analysis for the Calibration of the Indices G (Griggs-Putnam Index), B (Barsch Index), D (Deformation Ratio), and C (Compression Ratio) With Mean Annual Wind Speed.

7.1 Wind Speeds Inferred from Eolian Sand Size Distribution

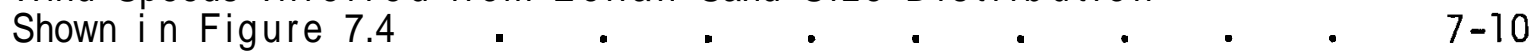

9.1 Recommended Values of Power Law Coefficient and Turbulence Intensity for the Lissaman Wind Turbine Wake Model.

9.2 A Procedure for Cluster Design and Performance Evaluation Based on the Lissaman Wake Model. . . . . . 9-27

10.1 Statistical Summary of Comparison Between Kite Anemometer and BAO Tower

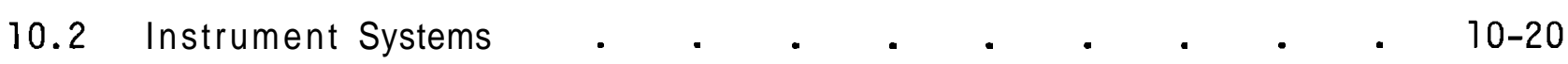

10.3 Summary of Remote Sensing Products Useful for Wind Energy
Site Selection

10.4 Arc Over Which Wind Speed and Wind Direction Measurements are Accurate 


\section{TABLES (contd)}

10.5 Summary of Measurements and Instrumentation for Wind Prospecting Strategy 


\subsection{INIRODUCTION}

Abundant and inexhaustible wind can be a valuable source of electrical energy in a power network. For this resource to be utilized, an efficient wind energy conversion system (WECS) or a cluster of WECS ${ }^{(a)}$ must be erected upon sites that have an adequate wind resource. To determine the adequacy of the resource, the magnitude and characteristics of the wind at a site, as well as the economic constraints specific to the site, must be assessed. Once an optimal site has been determined, how effectively the wind resource is exploited depends on the WECS characteristics. Undoubtedly, utilities will decide to install a WECS or a cluster of WECS based on analysis of the wind resource and the cost of exploiting it, and on consideration of the impacts of adding WECS on utility operations. Such analyses require estimates of the energy generation characteristics of the WECS that may be compared to estimates for other potential sites or other forms of power generation.

An effective WECS siting program should provide the necessary information for selecting, evaluating, and ultimately committing to sites. To help utilities(b) identify and evaluate sites, this document presents strategies and techniques that may be used by those involved in selecting potential WECS sites. The meteorological issues are the principal concern. This introductory chapter begins by discussing the general problems of wind turbine siting and by pointing out the meteorological problems for which this document provides solutions. The ways that the solutions to the meteorological problems are shaped by wind resource characteristics, by wind machine characteristics, and by wind energy economic assessment procedures are also discussed briefly.

\subsection{WIND TURBINE SITING}

One of the principal differences between the siting of wind turbines and the siting of conventional power plants is that the performance of WECS is

(a) The acronym WECS is used throughout this report to denote singular or plural as required by the context.

(b) References made to utilities in this report may also refer to third party entrepreneurs or meteorological and engineering consultants. 
governed by their locations. The sensitivity of the performance of a cluster of wind turbines to its location adds new considerations to the process of siting generation facilities. The problems of power system planning for conventional systems must sti11 be addressed. Such problems include:

- load forecasting

- generation system reliability

- generation system cost

- power-flow

- transmission system reliability

- land use, availability, and acquisition

- environmental and socioeconomic impact assessments

- site engineering and construction

- site security and safety.

In addition to these traditional problems, wind turbine siting requires further unique considerations in the following areas:

- meteorological issues

- site selection

- site evaluation

- site development

- site operations

- assessment of potential hazards

- WECS hardware

- choice of WECS

- operating with WECS

- performance prediction

- WECS economic evaluation

- value and cost assessments

- value uncertainty

- yet-to-be articulated issues.

The considerations added to the power plant siting process by wind turbine siting requirements are not independent of the aforementioned dimensions of conventional power plant siting, For example, acquisition of land is a problem 
in either case. Any procedure for siting wind turbines must blend in with conventional procedures as much as possible. (a) A study to consider and meld al1 aspects of wind turbine siting is currently underway (De Meo 1979) and reports by Park et al. (1978), JBF Scientific Corporation (1979), and Marsh (1979) discuss many aspects of wind turbine siting.

This report focuses on a portion of the wind turbine siting problem, namely the meteorological aspects of siting large wind turbines. The meteorological siting problem, as discussed in this report, may be broken into three broad and somewhat overlapping phases. The first is that of site selection. The problem in this phase is to locate sites where the wind blows hard enough and frequently enough that there is a good likelihood that the site will be of sufficient economic value, or at least be of superior value to other sites in its neighborhood. The solution to this problem requires an understanding of the wind resource, its origin, spatial and temporal distribution, and its interaction with topography. Most of this report is devoted to achieving this understanding.

Once candidate sites have been identified, the problem becomes one of evaluating the potential of the site. Appropriate measurements must be made at a site to enable planners to make this evaluation. To determine what data are needed requires understanding of wind turbine operating characteristics, WECS economic evaluation procedures, and wind turbine/utility operations. Sections 1.3 and 1.4 provide brief discussions on wind turbine characteristics and WECS economics from which site evaluation data collection strategies, presented in Chapter 2, will be formulated. Whether or not those data are completely adequate for certain utility operations and reliability assessment procedures has not yet been articulated.

(a) Although this document does not specifically address the nonmeteorological siting issues, the strategies presented here do attempt to anticipate the timing and nonmeteorological data requirements (neither of which are fully articulated yet for many issues) of a fully integrated siting program. Thus, it is assumed that general land use and availability questions would be addressed before pinpointing candidate sites, and that preliminary engineering and construction feasibility at an identified candidate site would be assessed before embarking upon an elaborate meteorological measurement program. 
WES cluster development problems include determining locations and arrangements for the wind turbines, assessing the meteorological characteristics that affect WECS operations and service life, and addressing wind behavior that affects power output characteristics of the cluster and WES interaction within the cluster. WECS hardware performance characteristics and electrical engineering concerns once again impact the meteorological data collection strategies. However, it is not yet clear what peculiar data requirements there might be, if any, to deal with those concerns. The treatment of wind turbine clustering in this report, therefore, deals primarily with the depletion of the wind resource in the wakes of wind turbines and does not address electrical interactions in a cluster. If actual experience in future cluster operations makes certain siting data requirements apparent that are not met by the recommendations made here, appropriate adjustments to these recommendations will be made.

The routine operation of wind turbine clusters in a power network may require, or at least may be enhanced by, a knowledge of wind characteristics that affect the day-to-day operation of WECS in a utility mix of equipment. Research into the impacts of wind speed forecasts and the occurrence of wind characteristics or wind events on utility operations is an active research area in the Federal Wind Energy Program (Wegley 1979). Whether or not wind data collected during the siting process will be of use in later operations is not yet clear, so no specific recommendations are made here.

\subsection{THE WN RESOURCE}

If the atmosphere and oceans are considered as an engine powered by the sun, then wind and weather are its products. However, the heating of the earth and the subsequent energy transfers are very uneven. In the tropics, the earth's surface faces the sun directly, but in polar regions the sun's rays are tangential, which reduces the radiant energy received per unit area. Similarly, continents, oceans, mountains, deserts, ice fields, rivers, and forests cause strong local differences in energy transfer. The spatial variations in heat transfer create variations in the pressure field that cause 
air to move in response to forces directed from high-to-low pressure. In the vertical direction, the pressure gradient force is usually cancelled by the downward gravitational force; therefore, the winds blow predominantly in the horizontal plane in response to the horizontal pressure gradients. The atmosphere then strives to mix the different temperature and pressure air masses distributed across the earth's surface. This mixing process creates the 1 billion megawatts (MW) of kinetic energy available in the winds. Understanding and predicting wind systems that accomplish this mixing and redistribution of energy are the goals of meteorology.

The processes involved in the mixing and redistribution of energy are complex and occur over a broad range of time and space scales. In addition to the pressure gradient and gravitational forces, the inertia of the air, the earth's rotation, and friction with the earth's surface (manifested by turbulence within a few kilometers $(\mathrm{km})$ of the ground) affect winds. The influence of each of these forces on wind systems differs depending on the scale of motion considered. One wind system, called the general circulation, involves wind patterns that cover the entire planet, Large-scale, prevailing nearsurface wind directions of the general circulation are shown in Figure 1.1. This figure is an oversimplification because it does not reflect the effects continents have on the wind distribution. Upper atmosphere components of the general circulation include mid-latitude jet streams. The mid-lati tude westerly jet streams migrate southward over the United States in the winter and have a great influence on mid-latitude cyclonic storms (which are the next smaller scale wind system) because the jet stream moves and steers these storms. Since storms provide a large portion of the wind power in some areas, knowing jet stream behavior helps significantly in interpreting a season or year of field measurements. For example, in the winter of 1976-1977 the jet stream continually steered storms from the Pacific Ocean north of the U.S. West Coast, resulting in a West Coast drought and lower than normal wind speeds, but consistently steered severe storms out of Canada into the U.S. Northeast. This activity is believed to have been related to a sea-surface temperature anomaly in the Pacific Ocean. Smaller scale systems than the jet streams and 


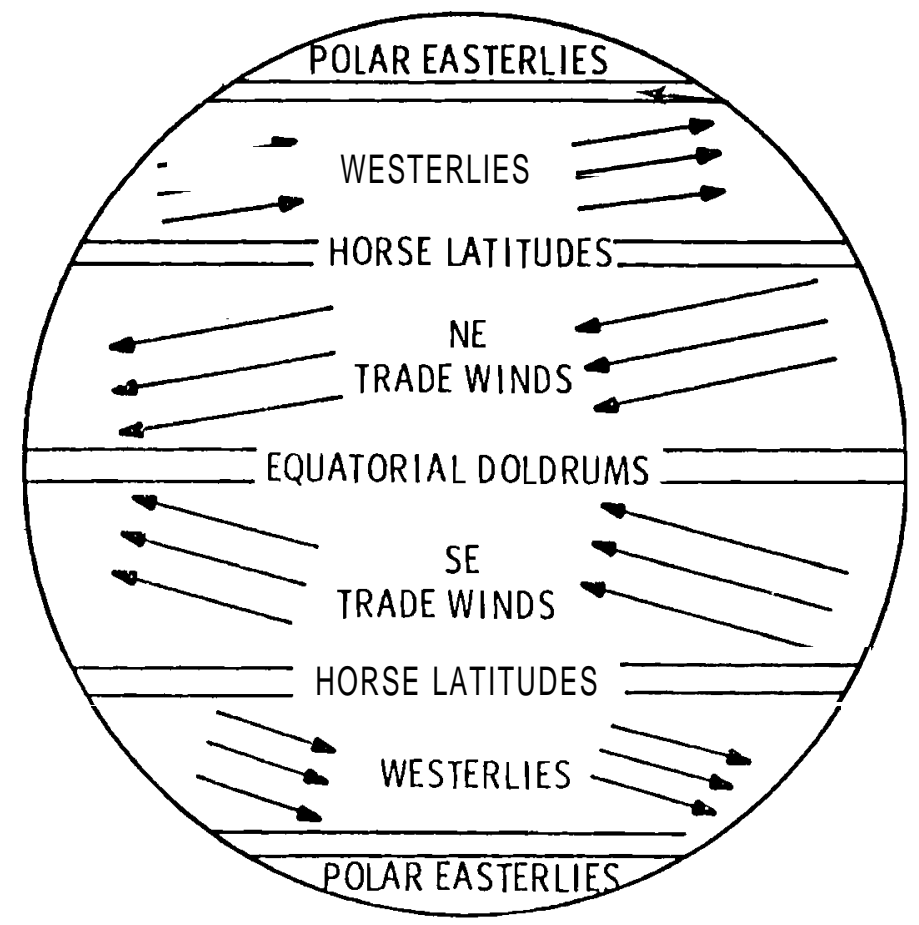

FIGURE 1_. I - Surface Winds of the General Circulation

cyclonic storms are the mesoscale wind systems. Mesoscale effects on wind flow include topographical and thermal influences on flows relevant to WECS siting (discussed in Chapter 5) and thunderstorms. At the smallest scale are the microscale motions, which include turbulence.

Each of the above circulation patterns affects the winds, and thus the viability of the wind energy resource, at a given location.

Quantifying the wind resource over very large areas is an aid to locating specific exploitable regions. An analysis of the wind resource in the United States by Elliott (1977) focused on the 48 contiguous states (see Figure 1.2). The map in Figure 1.2 gives an estimate of the annual average wind power in $\mathrm{W} / \mathrm{m}^{2}$ that is available for extraction. The values reflect the influence of mountains, bodies of water, and large plains. However, topographical enhancement or reduction of winds may considerably modify these estimates (see Chapter 5). In mountainous regions (indicated by $M$ on the map), the estimates reflect the lower limits expected for the typical we11-exposed sites. 


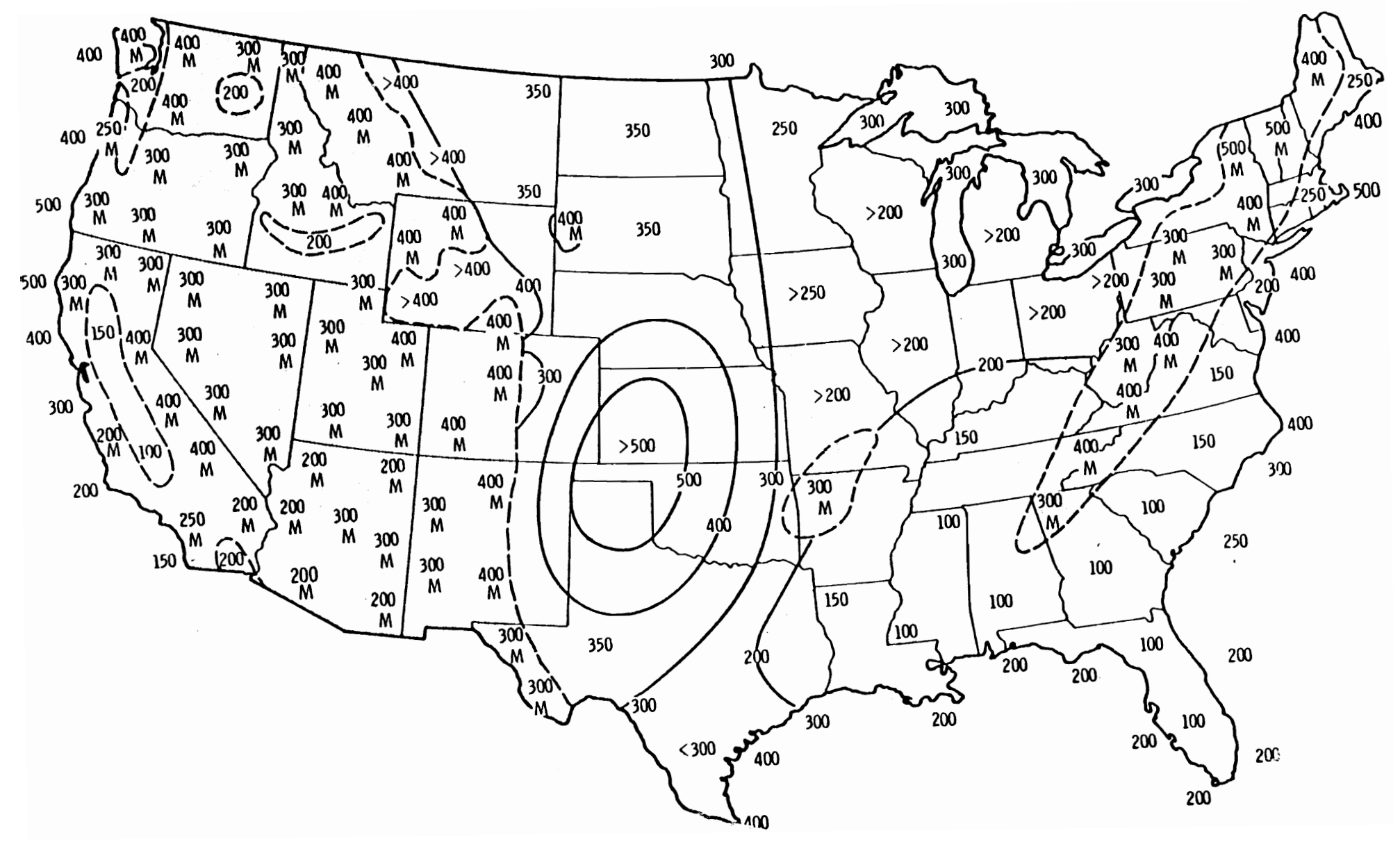

FIGURE 1.2 Annual Average Wind Power at $50 \mathrm{~m}$ Above Well-Exposed Locations in $\mathrm{W} / \mathrm{m}^{2}$. The "M" refers to mountains or ridge locations (E11iott 1977). 
In certain nonmountainous regions, the mean annual wind power is high $\left(\geq 400 \mathrm{~W} / \mathrm{m}^{2}\right)$. High wind power also appears over the central and southern Great Plains, offshore and exposed coastal sites of the Northeast and Northwest, and in parts of Wyoming, Montana and the south Texas coast. A11 mountainous regions, except possibly some areas of the Southwest, are expected to have sites with considerable wind power. However, over most of the Southeast, wind power is quite low $\left(100 \mathrm{w} / \mathrm{m}^{2}\right)$.

Seasonal patterns of maximum wind power are similar to annual patterns (see Figure 1.3). Over the eastern one-third of the nation, maximum wind power occurs during the winter and early spring. A spring maximum occurs over the Great Plains, the north-central states, the Texas coast, most nonmountainous regions in the West (e.g., the basins and broad valleys), and offshore areas of central and southern Cal ifornia. Winter maxima occur over all mountainous regions, except for some areas in the lower Southwest where the spring wind power appears about equally as large. A summer maximum of wind power is shown to occur only on the offshore areas of southern Oregon and northern California.

The maps in Figures 1.2 and 1.3 are rather coarse; much important wind data is filtered out of these diagrams. For example, these maps do not contain the detailed structure that would be produced by air flowing over individual mountain ranges, large river systems, large bays, and expanses of rolling hills. In addition, the temporal information given is too coarse to help much in the siting of WECS.

A more detailed evaluation of the U.S. wind power potential is being conducted for 12 regions within the U.S. and its territories. (See Appendix 1 for examples of the products of the Northwest regional wind resource assessment). From these regional analyses, which are to be completed in 1981, a new national synthesis will be created to update that of Elliott (1977).

Even after the regional assessments are completed, the principal problem in wind turbine siting will continue: the wind resource is extremely variable. Annual average wind speed, turbulence levels, seasonal wind patterns and diurnal changes in the wind can alter drastically over relatively short distances 


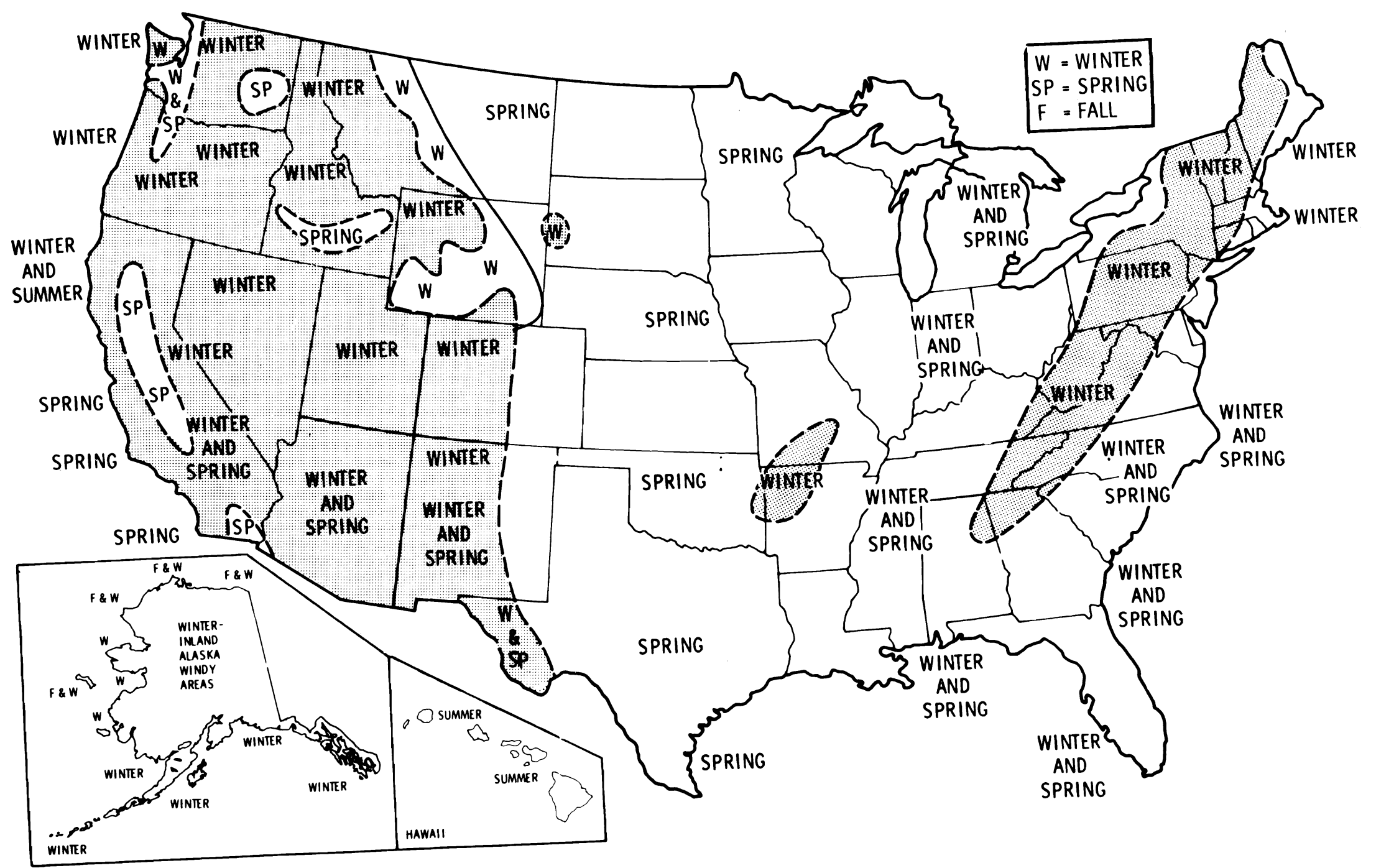

FIGURE 1.3. Seasons of Maximum Wind Power (Elliott 1977) 
and elevations. These wind characteristics can also vary significantly from year to year, which can make interpreting the wind climatology at a site from short-term measurenients difficult. An extended discussion of wind variability is given in Appendix 3, from which the following examples have been excerpted.

- Horizontal and Interannual Variability

To illustrate how certain wind characteristics may vary with horizontal separation, two stations in Montana have been chosen. Butte and Whitehall are both located in the mountains of southwestern Montana. Whitehall is only 17 miles away from Butte, but is separated from Butte by a ridge. Whitehall is near the mouth of a long, northeasterly sloping valley. In these valleys the highest winds are observed in the winter, when strong winds at higher elevations are from the southwest along the valley axis.

Figure 1.4 illustrates the behavior of monthly averaged wind speeds at Butte and Whitehall. The average wind speeds at Whitehall were expected to be higher than those at Butte because of the orientation of the valleys. This assumption was correct, as the horizontal lines representing the 7 -year averages show.

Figure 1.4 also shows that the wind characteristics vary from year to year as well as over distances. For example, Whitehall shows peaks in winter wind speeds, but Butte shows no consistency from year to year in its correlation with Whitehall. The lack of consistent correlation from year to year is also shown in Table 1.1. As seen in this example, measurements made at one location may not apply to other nearby locations, particularly in regions of significant topography. Furthermore, changes from year to year imply that some wind characteristics, such as seasonal variations about the annual average wind speed, may require more than one year of measurements to determine the wind climatology. Close cooperation between meteorologists and power system planners is required to define the length of measurement program required in a given region. 


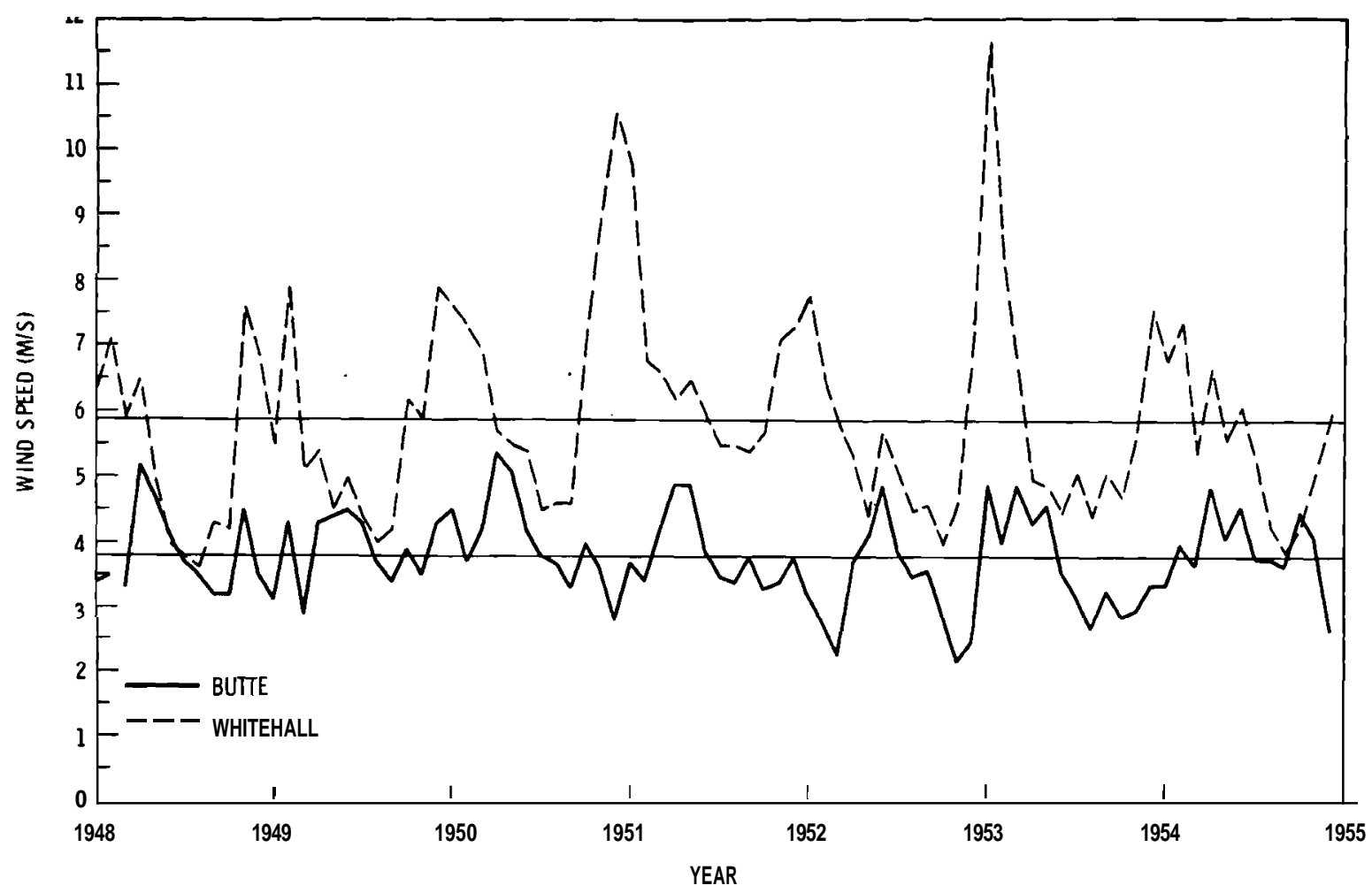

FIGURE 1.4. Seven Yèars of Monthly Mean Wind Speeds for Butte and Whitehall, Montana. Horizontal Tines represent 7-year means.

TABLE 1.1. Variation of the Yearly Estimates of the Correlation Coefficient of Monthly Mean Wind Speeds Between Whitehall and Butte Montana. The correlation coefficient over 84 months is $\mathrm{r}=0.12$.

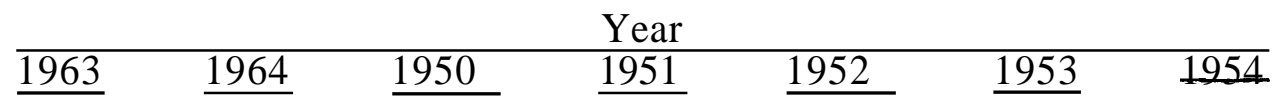

$\begin{array}{llllllll}\begin{array}{l}\text { Correlation } \\ \text { Coefficient }\end{array} & 0.27 & 0.23 & -0.38 & 0.01 & -0.20 & 0.54 & 0.03\end{array}$

- Vertical Wnd Variability

Figure 1.5 shows the annual average diurnal wind variation at three levels taken from a year's data collected from a tall meteorological tower in central Oklahoma (Crawford and Hudson 1973). The levels correspond to: the height of typical surface wind measurements, the hub height of a multi-megawatt large wind turbine, and the top of the rotor disk of a large wind turbine. 


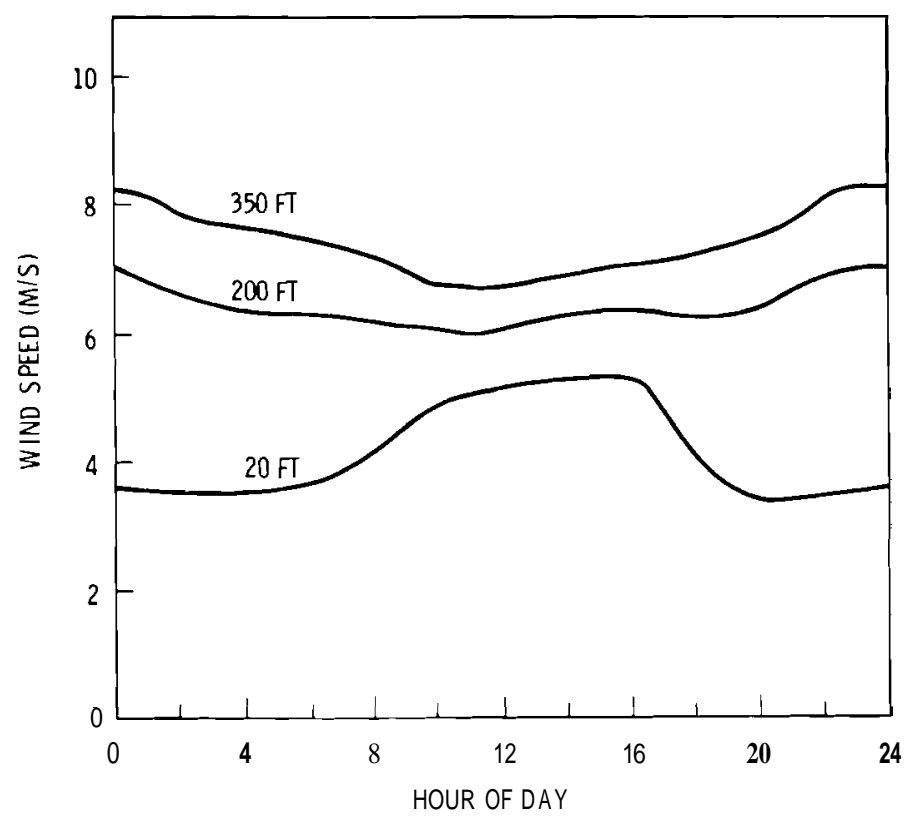

FIGURE 1.5. Annual Average Diurnal Variation at Three Elevations. Results are based on a single year of measurement at a site near Oklahoma City, Oklahoma. Elevations correspond to a typical surface measurement height; hub height for a large, horizontal axis wind turbine; and the top of the rotor disk of such a turbine.

Figure 1.5 illustrates how greatly diurnal behavior can change over vertical distances comparable to the size of large wind turbines. A wind turbine located at a site with wind behavior as depicted in Figure 1.5 would, on an annual average, produce more energy at night than during the day. This is dramatically different than what would have been expected from simple extrapolations of surface data. Since extrapolation of near-surface winds to higher levels cannot be done with sufficient accuracy (see Appendix 2), i t is clear that at some point in the siting process direct measurements at levels comparable to the height of the wind turbine will be required.

- Temporal and Interannual Variabi lity

Figure 1.6 is an example of the year-to-year differences in diurnal wind behavior in the month of July at Great Falls International Airport, Montana. In this figure, the monthly averaged diurnal cycles for three consecutive 


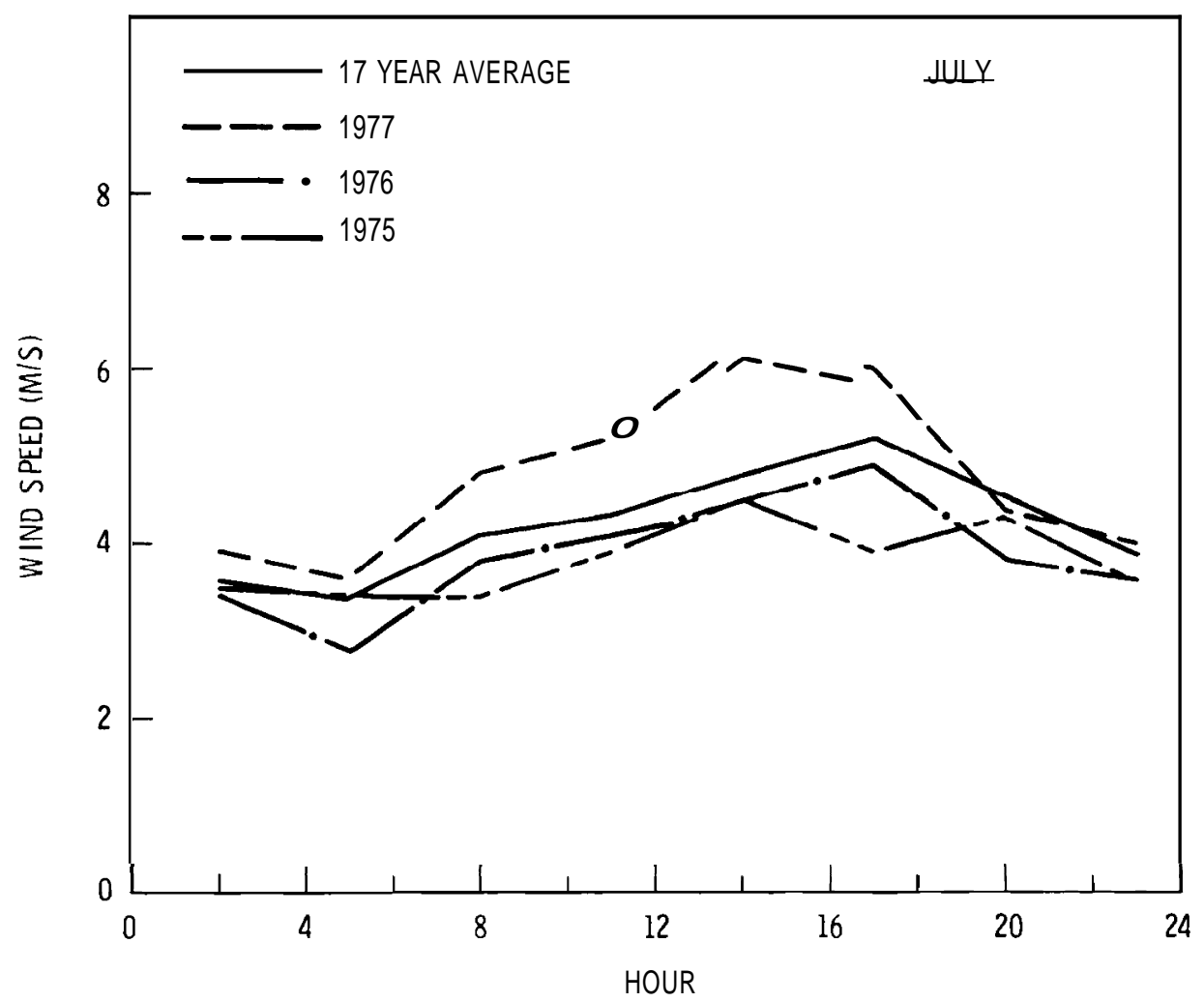

FIGURE 1.6. Monthly Averaged Diurnal Wind Speed Cycle for Great Fal1s, Montana. Average diurnal cycle for 1 month in each season for 3 consecutive years are compared with a 17-year mean.

years are compared to the long-term July diurnal cycle. The figure shows that year-to-year differences can be significant. Although gross features of the diurnal cycle can be established with a single year of data, more detailed characteristics (such as the amplitude of the diurnal modulation and the timeof-day of maximum winds) cannot.

Discovering potential sites and confidently assessing the long-term behavior of wind energy resources at those sites are the principal meteorological problems of wind turbine siting. To do this effectively requires thorough understanding of the variability of the wind resource. Once a potential site has been identified, wind machines capable of efficient conversion of wind energy at the proposed site must be identified and considered in terms of the site wind characteristics. Wind machines, their response to the variable 
wind resource, and the implications of their response for wind data collection strategies are the subjects of the following section.

\subsection{WIND ENGGY MACHINES}

Throughout history people have converted the kinetic energy of the wind into more usable forms of energy. With the exception of sail power for boats and ships, most of these conversions have been to mechanical energy transmitted via the torque on a rotating shaft. Most wind energy machines then involve some form of rotor to serve as the direct link between moving air and a rotating shaft. Rotors, obviously, rotate about an axis, either horizontally or vertically. Figure 1.7 shows six types of WECS. These types do not represent every developed or proposed WECS, nor are they all suited to driving megawatt-scale generators. However, these machines illustrate various design concepts.

Part A of Figure 1.7 is the familiar water pumper, still used extensively in parts of the United States. It faces into the wind guided by a tail that acts like a weather vane tail. In high winds the angle between the tail and the rotor may be intentionally reduced from its normal operating position. Still, the tail lines up with the wind so less frontal area of the rotor disk is exposed to the wind, thus reducing the power captured and the wind loading on the machine. The solidity, which is the ratio of blade area to the area swept by the blades, is high. High solidity results in a high torque when the rotor starts from rest. Diameters of these machines are up to about $5 \mathrm{~m}$.

Figure 1.76 shows a two-bladed horizontal-axis machine with a generator located within the nacelle atop the tower. Each blade resembles a wing in shape and may be as long as $20 \mathrm{~m}$ to $50 \mathrm{~m}$. Electrical capacities of the wind turbine generators would correspondingly run from $0.1 \mathrm{MW}$ to a few $\mathrm{MN}$ for these blade sizes. The blades are positioned on the downwind side of the tower. This downwind position is a naturally stable configuration, which helps keep the blades downwind of the tower as the wind direction changes. Motors and brakes are used to change and hold the orientation. All wind machines experience changes in wind speed with height, usually caused by friction retarding 


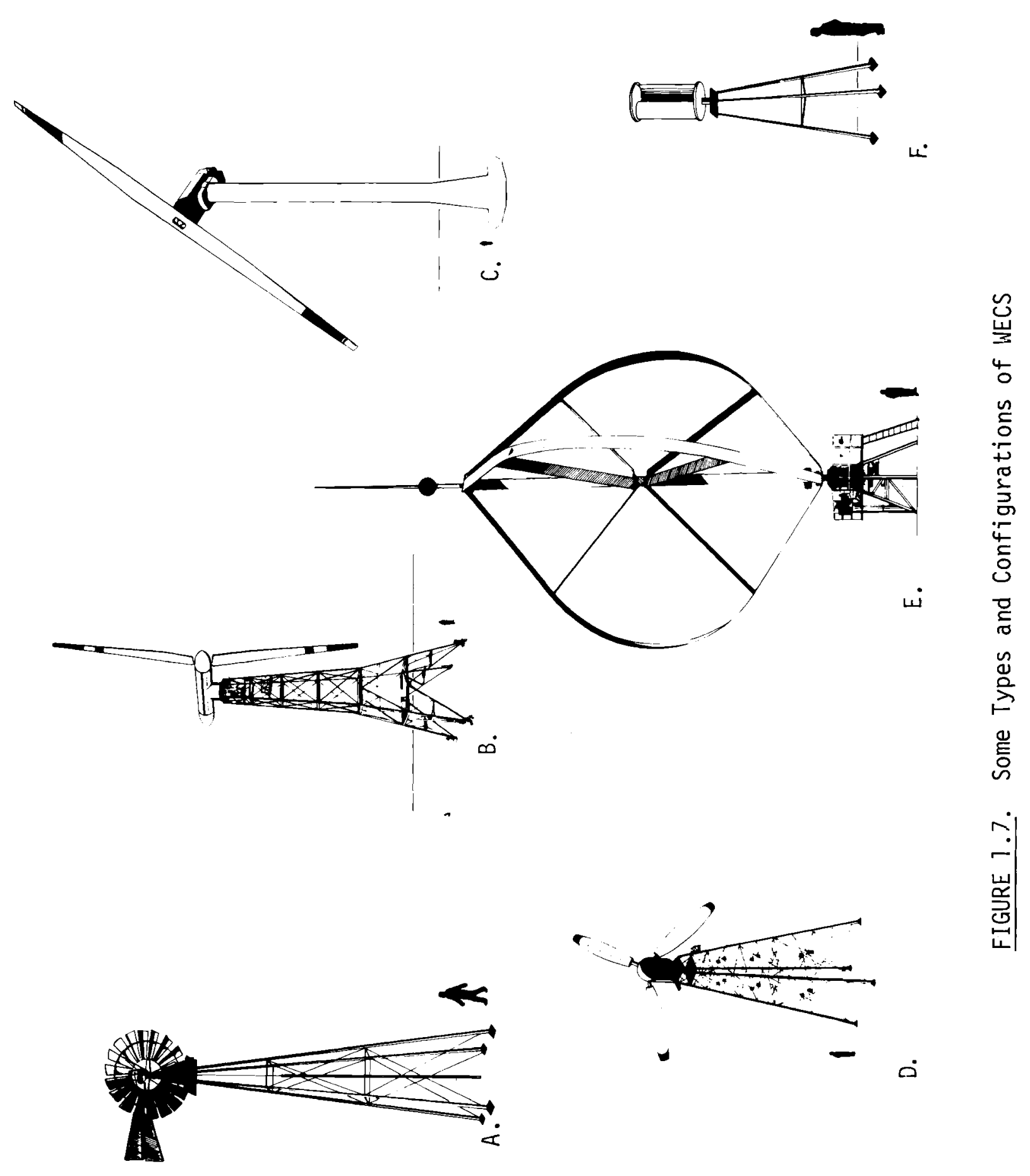


the near-surface winds. This causes cyclic stresses to be put on each blade as it passes from the high-wind portion of its rotation into the low-wind portion. These cyclic stresses are accentuated when the machine is in the downwind configuration because of the "wind shadow" of the tower. The loads on the blades are transmitted to the tower. The tower shown is a "stiff" tower, i.e., the natural frequency of the tower is higher than the frequency of the cyclic loads on it.

A two-bladed wind machine may also be designed to operate in the upwind configuration shown in Figure 1.7C. This design avoids tower shadow problems. The cyclic stresses caused by the change in wind with height are also reduced by allowing the blades to "teeter" on the main hub axle. The example shown has a "soft" tower, which means the natural frequency of the tower is less than the frequency of the cyclic stresses on it during normal operation. A soft tower is less expensive but as the blades begin turning the system must pass through the natural or resonant frequency.

Figure 1.70 shows a three-blade upwind-configuration machine. In this machine, the driving or wing-shaped portion of the blade does not begin right at the hub but at some distance from the hub. Because the forcing near the hub is weak, little energy is lost by this adaptation. The shafts upon which the blades are mounted may be rotated to control the pitch of the "wing" with respect to the airflow. This positioning may also occur on all or portions of the blades of machines $B, C$, or $D$.

Two types of vertical-axis machines are shown in Figure 1.7. Part E is the Darrieus rotor and part $F$ the Savonius. An advantage of vertical-axis machines is that they do not have to be turned into the wind as the wind direction varies, and they are influenced less by the change of the wind with height. The generator is easily mounted at ground level with a vertical-axis rotor. Darrieus blades are wing-shaped in cross section, whereas the Savonius rotor is an S shape capped at upper and lower ends. The blades on the Darrieus move through the air much faster than do the Savonius blades. The slower, heavier Savonius derives its rotation mostly from drag on the rotor rather than lift on a wing and therefore behaves more like the pumper of Figure 1.7A. 
The amount of energy in the wind that can be extracted and converted to mechanical or electrical energy depends upon a complex chain of actions. This chain begins as the wind interacts with the rotor, which converts the wind energy to mechanical energy. The mechanical energy could then drive a generator that produces electricity or perhaps drive a pump. The way that this conversion of wind energy occurs is discussed below.

\subsubsection{Converting Wind Energy to Electrical or Mechanical Energy}

The ratio of the power captured and delivered as mechanical or electrical power to the power in the wind impinging on the wind machine is the power coefficient $\left(C_{P}\right)$. A similar power coefficient exists for the rotor, which is the ratio of the power delivered to the rotating shaft by the rotor to the power in the wind. The behavior of the rotor power coefficient as a function of tip speed ratio (the ratio of blade tip speed to wind speed) is illustrated for several types of rotors in Figure 1.8. In this figure, the differences in efficiencies and tip speeds of the two-blade wind machines and the Savonius or the pumper are apparent. The higher solidity in the Savonius and the pumper gives them a higher starting torque. The higher starting torque and the lower tip-speed ratio make these types of machines ideal for low-speed uses, such as pumping and milling.

The behavior of the rotor power coefficient for horizontal axis wind turbines can be understood by examining the forces on the blade that make the rotor rotate (see Figure 1.9). The wind velocity $\left(V_{W}\right)$ strikes the blade element, but since the blade is moving at speed $V_{B}$, the blade sees a relative wind vector $\left(V_{R}\right)$. The angle of attack (a) is the angle between $V_{R}$ and the chord of the blade. The pitch angle $(\beta)$ is the angle between the plane of rotation of the blade element and the chord of the blade. There is a $1 \mathrm{ift}$ force (L) caused by the aerodynamics of the blade and a drag force (D). The resultant force $(F)$ has a component in the direction of rotation that sustains blade speed $V_{B}$. The useful power transferred to the blade depends upon the lift and drag characteristics of the airfoil, $V_{R}$, and $a$. Because the blade rotates, $V_{B}$ increases with distance from the hub. For a given wind velocity 


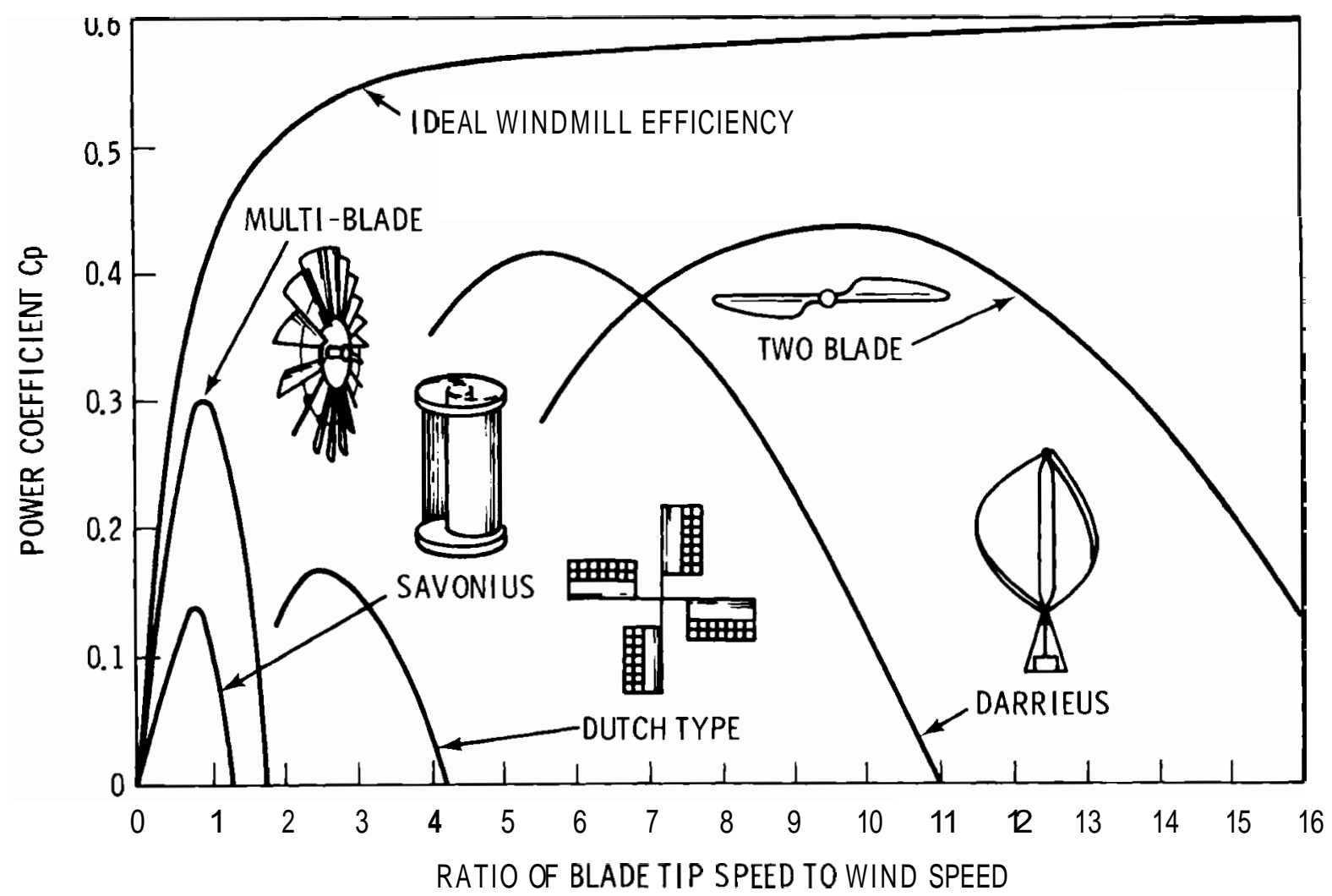

FIGURE 1.8. Power Coefficients for Some Typical Wind Turbines

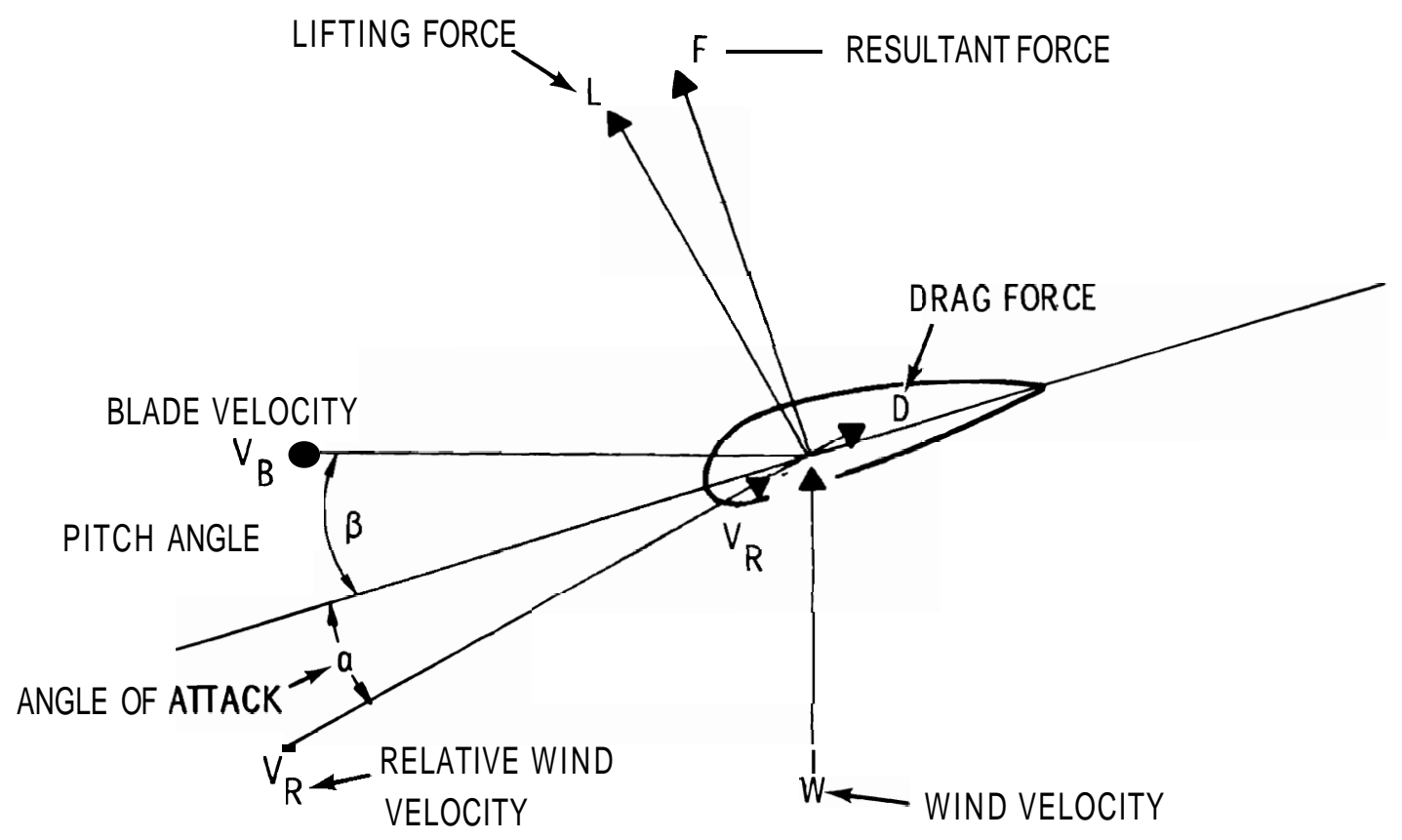

FIGURE 1.9. Forces and Wind Velocity at a Blade Element 
$V_{W}$, the angle between $V_{W}$ and $V_{R}$ is a function of the radius also. To keep all segments of the blade at peak efficiency at wind velocity $V_{W}$, the blade must twist from a high angle to the plane of rotation at its root to a small angle at its tip. Since the blade roots contribute little to the power extracted, some designs such as that in Figure 1.7D trade away this power for the less expensive nontwisted blade. As the wind speed increases, the rotation rate increases, the tip speed ratio increases, and the power coefficient increases until a maximum rotor efficiency is achieved. Then as the wind speed increases, the angle of attack becomes too large and the efficiency declines until some point where the blade stalls and lift ceases.

Optimum rotor efficiency as a function of wind speed requires that an efficient angle of attack be preserved. This can be achieved in different ways. If the rotation rate of the rotor is variable such that the tip speed ratio is nearly constant, optimum efficiency results. This is frequently the strategy of systems using induction generators that do not require a constant generator shaft rotation rate. However, most large wind turbines using synchronous generators require that the generator shaft turn at a constant rate. For those systems, gear shifting between the rotor and the generator at discrete intervals can maintain the tip speed ratio in a desired optimum range. Still another approach is to adjust the pitch of a portion or all of each blade.

Operation of large wind turbines is usually controlled by a microprocessor that initiates turbine maneuvers based in part on the state of the wind, usually sampled at the nacelle. The strategy for sampling and processing the wind data is programmed into the wind turbine microprocessor, and is a result of energy-capture optimization studies of the designers. At very low wind speeds, there is not enough power in the wind to power the system so the turbine remains on standby. When the winds exceed a specified cut-in wind speed for a sufficient length of time, the control system initiates startup procedures, and some minutes later the turbine feeds power into the grid. If the wind speed or power output drops below some specified level the turbine is shut down again. As the wind speed increases, the various mechanisms discussed 
above are employed to enhance efficient energy capture. At the rated wind speed the power output reaches the rated power of the generator. At wind speeds above rated, pitch control or flaps on the blade are used to reduce rotor efficiency by spilling excess power from the machine such that the power output remains at the rated output of the generator. If the wind direction changes for a specified amount of time and by a given amount (which may be a function of wind speed) the control system may initiate a reorientation of the turbine. If the wind direction changes by a large amount or if the wind speed exceeds a cut-out speed, the control system will order the machine to shut down.

When the efficiencies of all elements of the power train are combined with the rotor efficiency, a power coefficient versus windspeed curve may be drawn (see Figure 1.10). If this $C_{P}$ is multiplied by the power in the wind at a given wind speed (a density of air is assumed and the area of the rotor disk is used) a power output curve as seen in Figure 1.11 results.

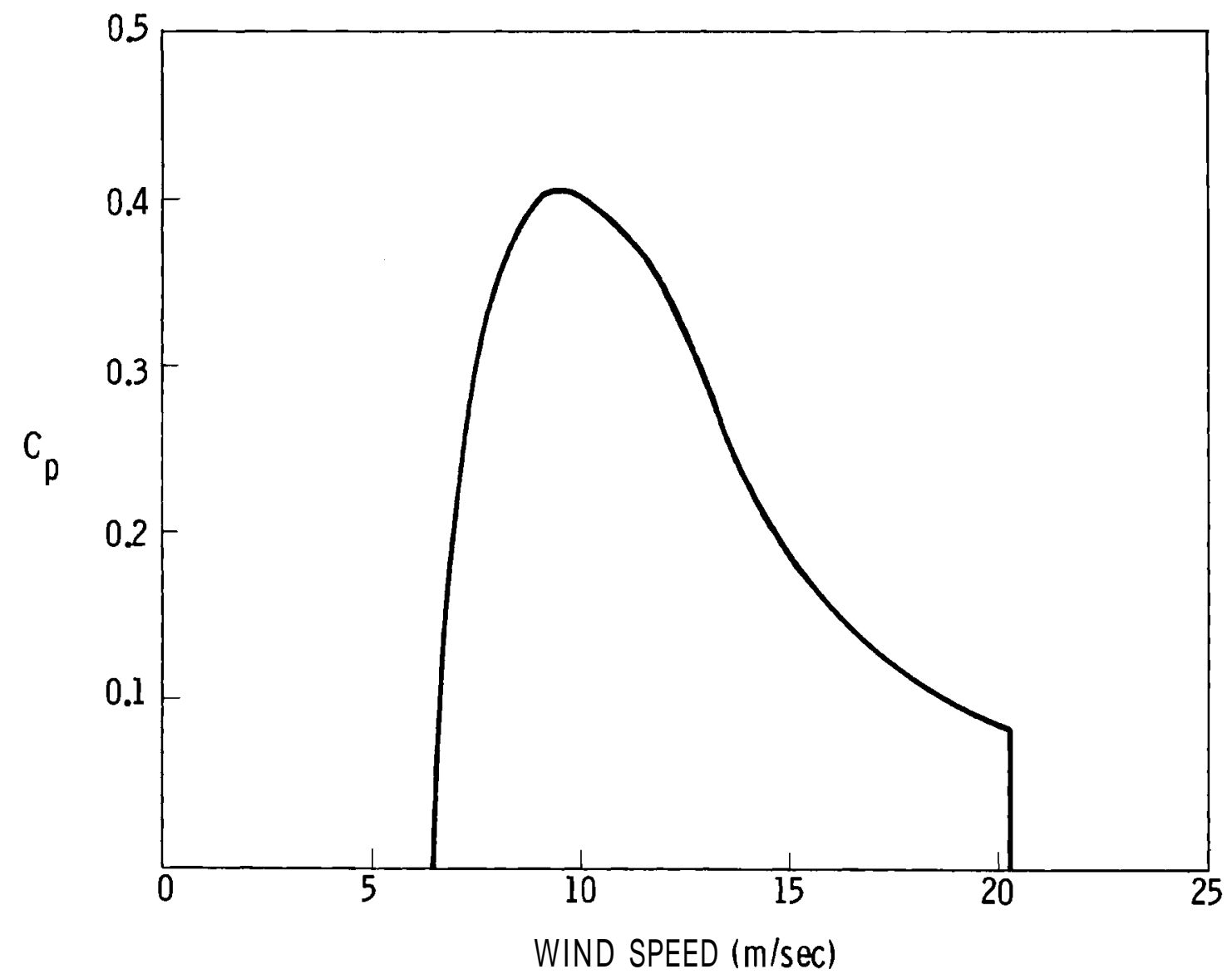

FIGURE 1.10. Power Coefficient of a Typical Large Wind Turbine 


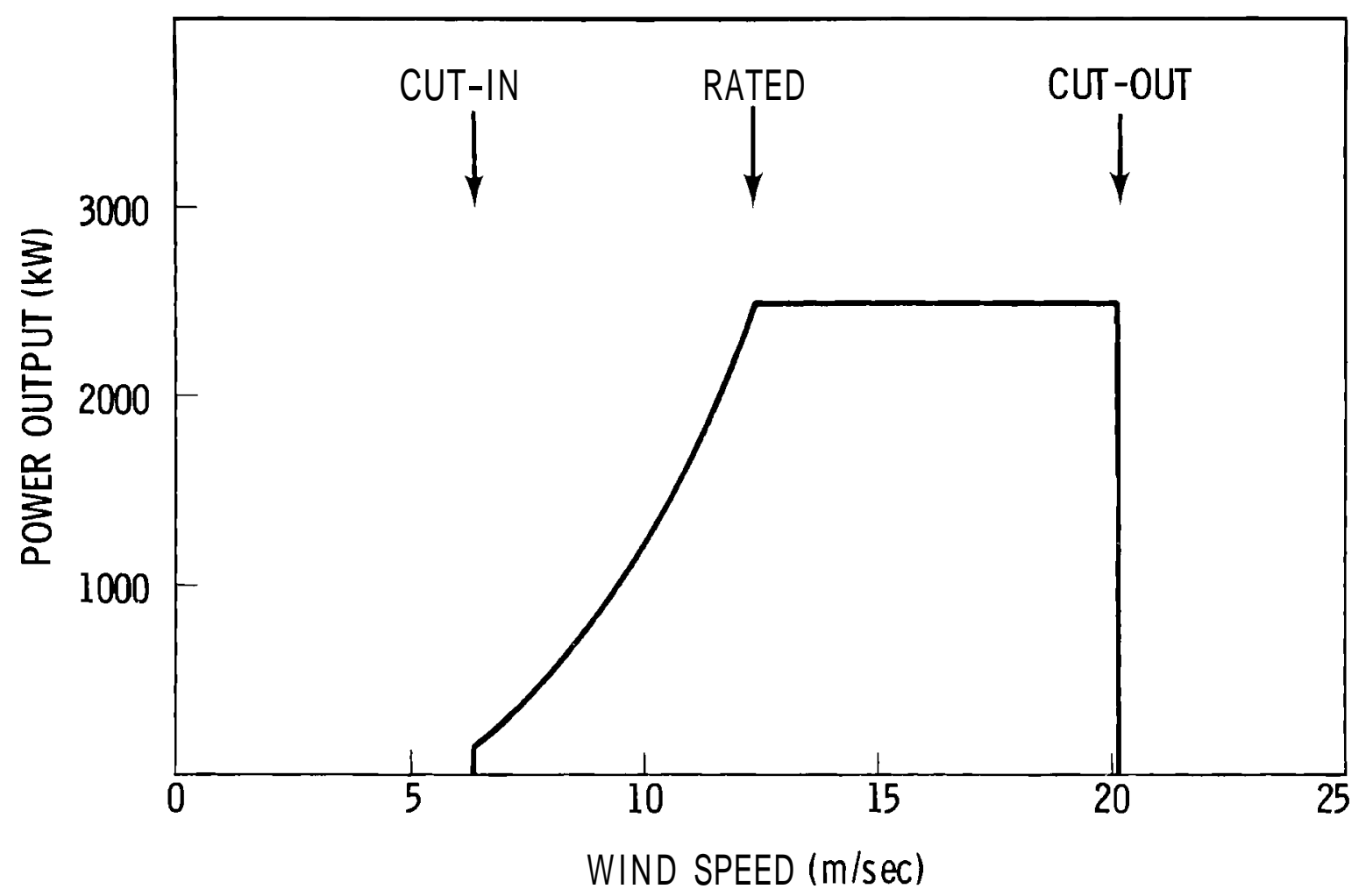

FIGURE 1.11. Example of Power Output Curve for a Typical Large Wind Turbine. Wind speeds refer to hub height.

\subsubsection{Computing Machine Output}

A fundamental objective of a wind turbine siting program is to collect data that enables a utility to predict the future power and energy output characteristics of machines to be erected at the site. Power output characteristics are most simply estimated using power output curves like the one in Figure 1.11.

Hub-height wind speeds, frequently sampled (e.g., 2-minute averages), can be used to predict power output when the wind turbine is synchronized with the grid. The usefulness of the power curve to model large wind turbine performance in this way has been reasonably well verified in the field as Figure 1.12 shows (Glasgow and Robbins 1979). The scatter around each centroid of points could result from a number of causes such as: yaw error (misalignment of turbine and wind); horizontal wind speed variations between meteorological 


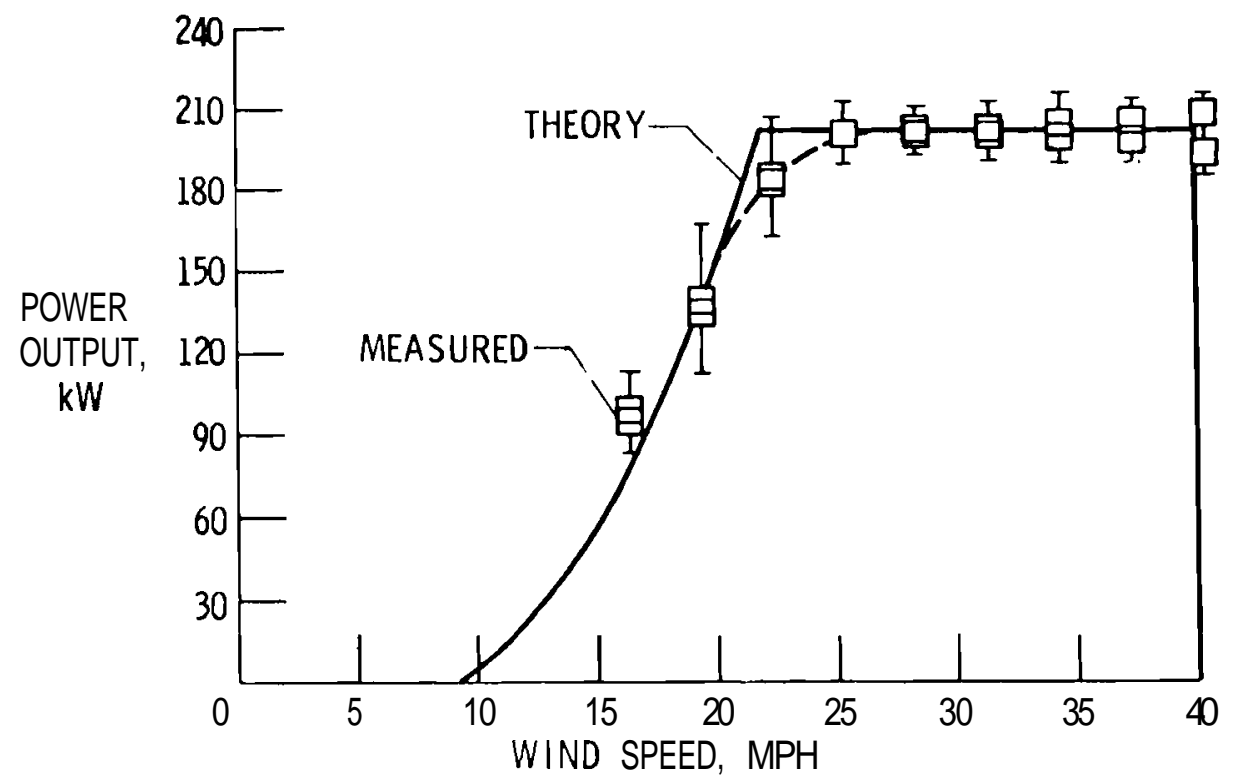

FIGURE 1.12. Comparison of Measured and Predicted Power, Mod-OA, 200-kW Wind Turbine, Clayton, New Mexico (Glasgow and Robbins 1979)

tower and turbine; and wind shear (variation of wind speed with height). However, the accuracy to which wind speeds averaged over other intervals of time, e.g., hourly, can model the power output of large wind turbines is not known. The behavior of machines with pitch control, yawing mechanisms, gear shifting, and programmed startup and shutdown procedures is complex. Data capable of simulating the control procedures of a large wind turbine are a logical upper limit to the sophistication of data collection necessary to achieve hourly energy production estimates required for wind turbine energy resource economic evaluation (see Section 1.4). The data collection procedures are discussed in Chapter 2. (a) Simpler estimates can, of course, be made with hourly averaged data or wind speed probability distributions, but with some presumed and undetermined loss of accuracy.

(a) As discussed in Section 1.1, this report deals only with meteorological data requirements for energy planning purposes and does not deal with additional data that may or may not be required for studying wind turbine impacts on utility system operations. 
Qiff (1977) demonstrated a method for simply estimating the long-term average extractable power (expressed in $\mathrm{kWh} / \mathrm{yr}$ ) by multiplying the poweroutput curve by the measured wind speed probability distribution and integrating the resulting curve over all wind speeds (see Figure 1.13). The area under the third curve represents the average power output, a number which may be used to compare with other sites or machines. Cliff found that if the mean wind speed is $>4.5 \mathrm{~m} / \mathrm{sec}$, this technique can be used by assuming a Rayleigh distribution $^{(a)}$ and will provide reasonable estimates of the average extractable power even though the actual distribution may differ substantially from a Rayleigh distribution. This result occurs because of the filtering effect the wind turbine power curve has upon the wind speed probability distribution. of course, a serious siting investigation will measure the distribution directly, at least. Although these methods were applied to long-term power output, the method could also be applied to the seasonal or diurnal variations of mean wind speed to obtain approximate averages of the temporal variation of power output characteristics.

Cliff (1977) generated a number of useful simplified curves for making quick and basic estimates of wind turbine performance characteristics. A Rayleigh distribution was assumed and a simplified wind turbine performance curve was used, which was similar to that in Figure 1.11 but with a linear ramp between cut-in and rated wind speeds. The shaded area of the distribution in Figure 1.13 represents the fraction of time the machine will be down or not operating because of winds below cut-in or above cut-out speed. Figure 1.14 shows the percentage of downtime versus annual mean wind speed with various machine characteristics. Similarly, Figure 1.15 shows the percentage of time a machine is running at its rated output. Figure 1.16 illustrates the average

(a) The Rayleigh distribution is an analytical expression of a probability density function of wind speed. It seems to f it many observed wind speed distributions reasonably well, although there are exceptions. The advantage of using the Rayleigh distribution is that it is completely specified by one parameter, the long-term average wind speed. Therefore, if only the average wind speed is known or measured, a reasonable estimate of the probabilities of occurrence of wind speeds around the mean can be made (see Chapter 10). 


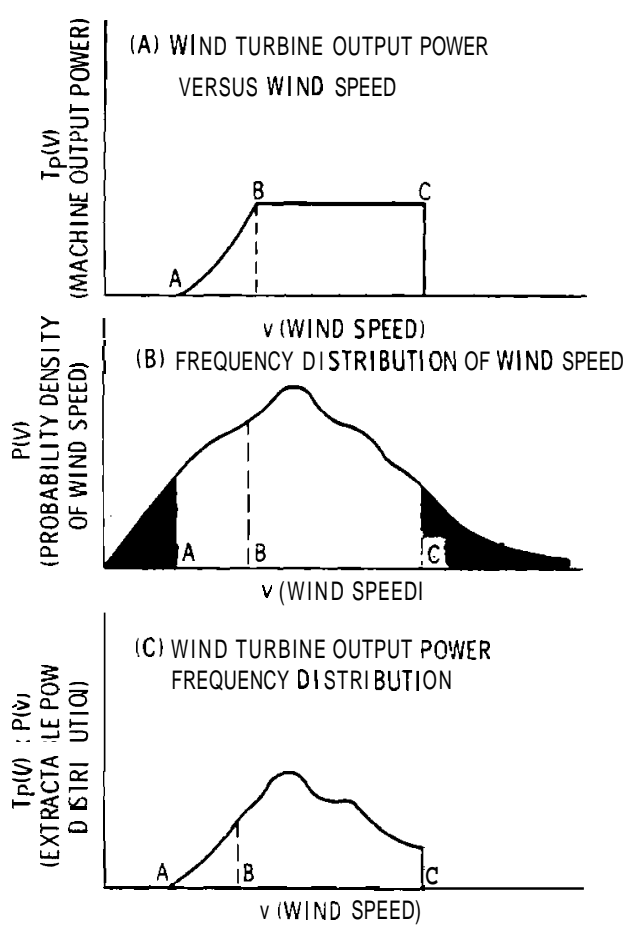

FIGURE 1.13. Interaction of Wind Turbine Characteristics and Wind Statistics to Produce the Wind Turbine Power Frequency Distribution

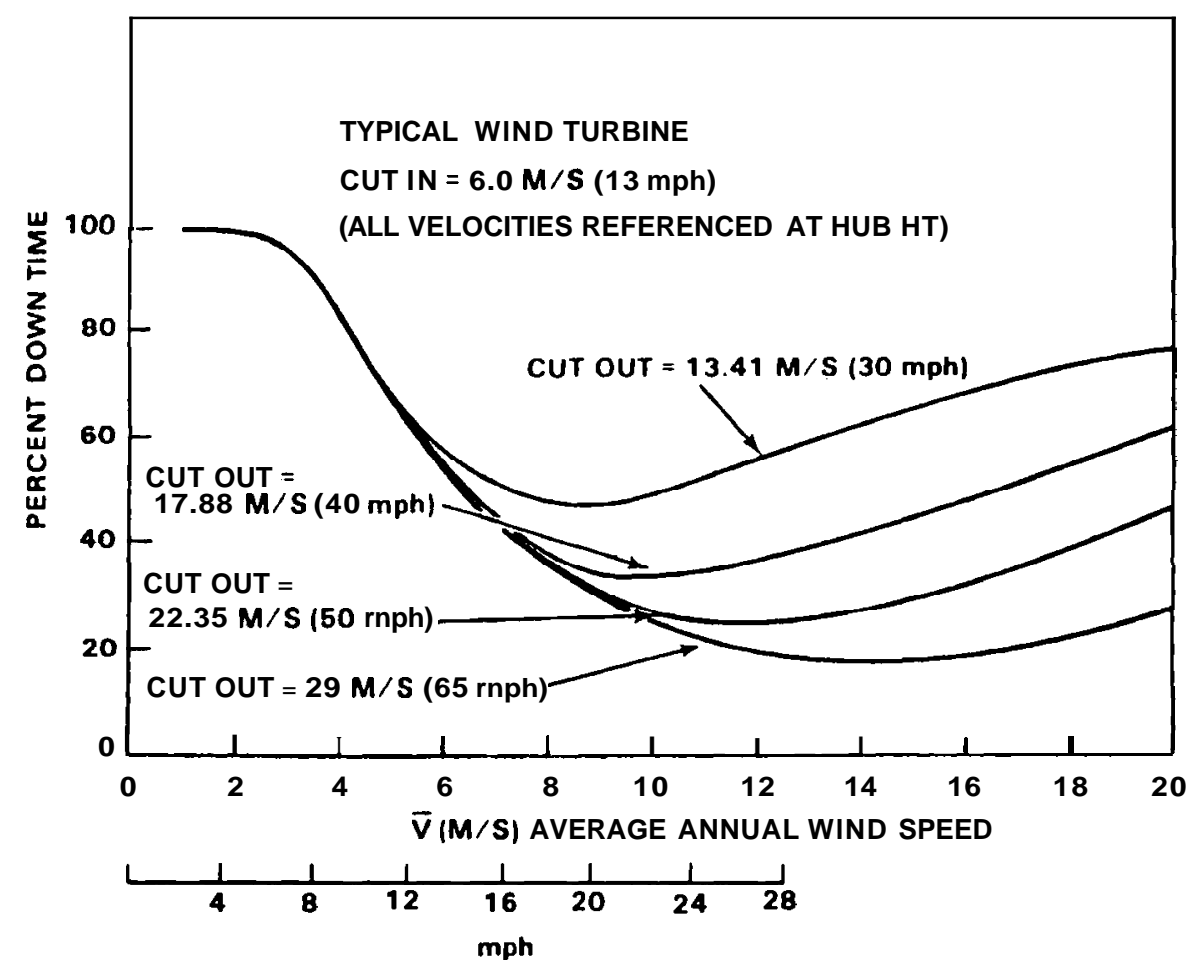

FIGURE 1.14. Example of Percent Downtime as a Function of Mean Wind Speed and Cutout Speed 


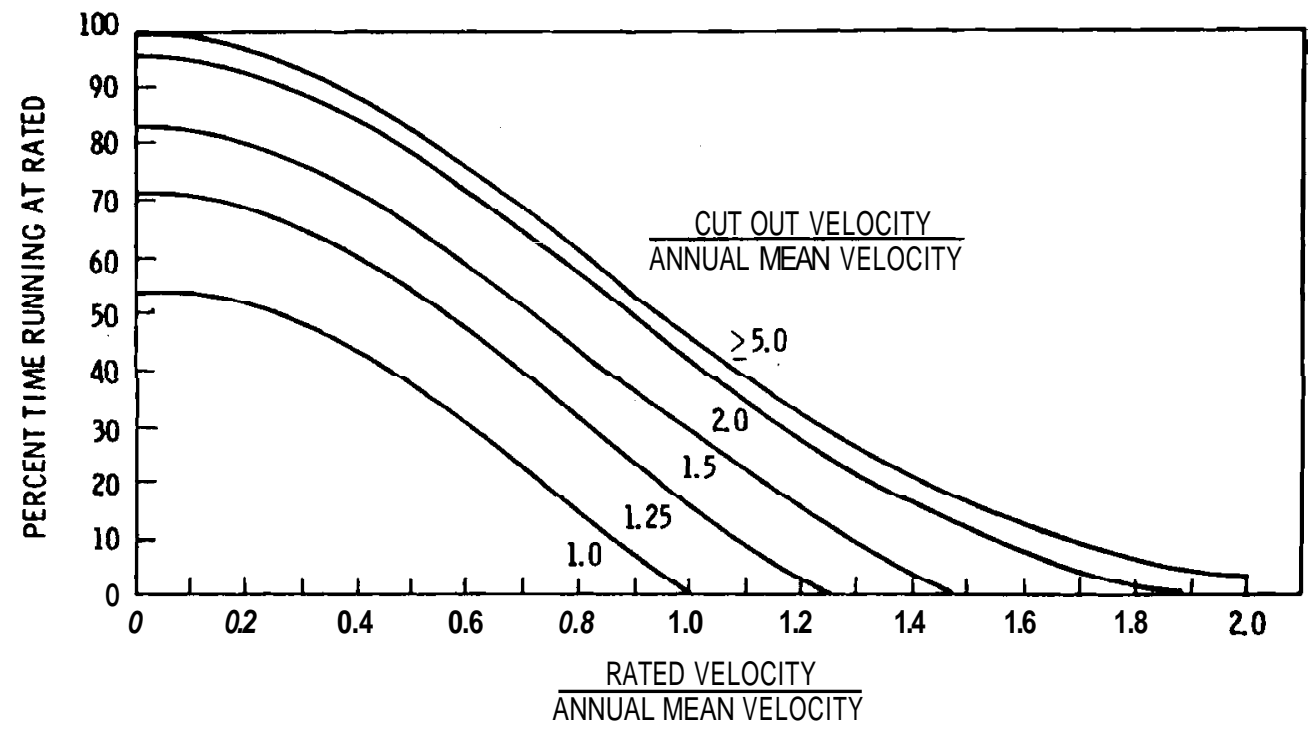

FIGURE 1.15. Percent Time Running at Rated as a Function of Cutout, Rated and Mean Wind Speeds

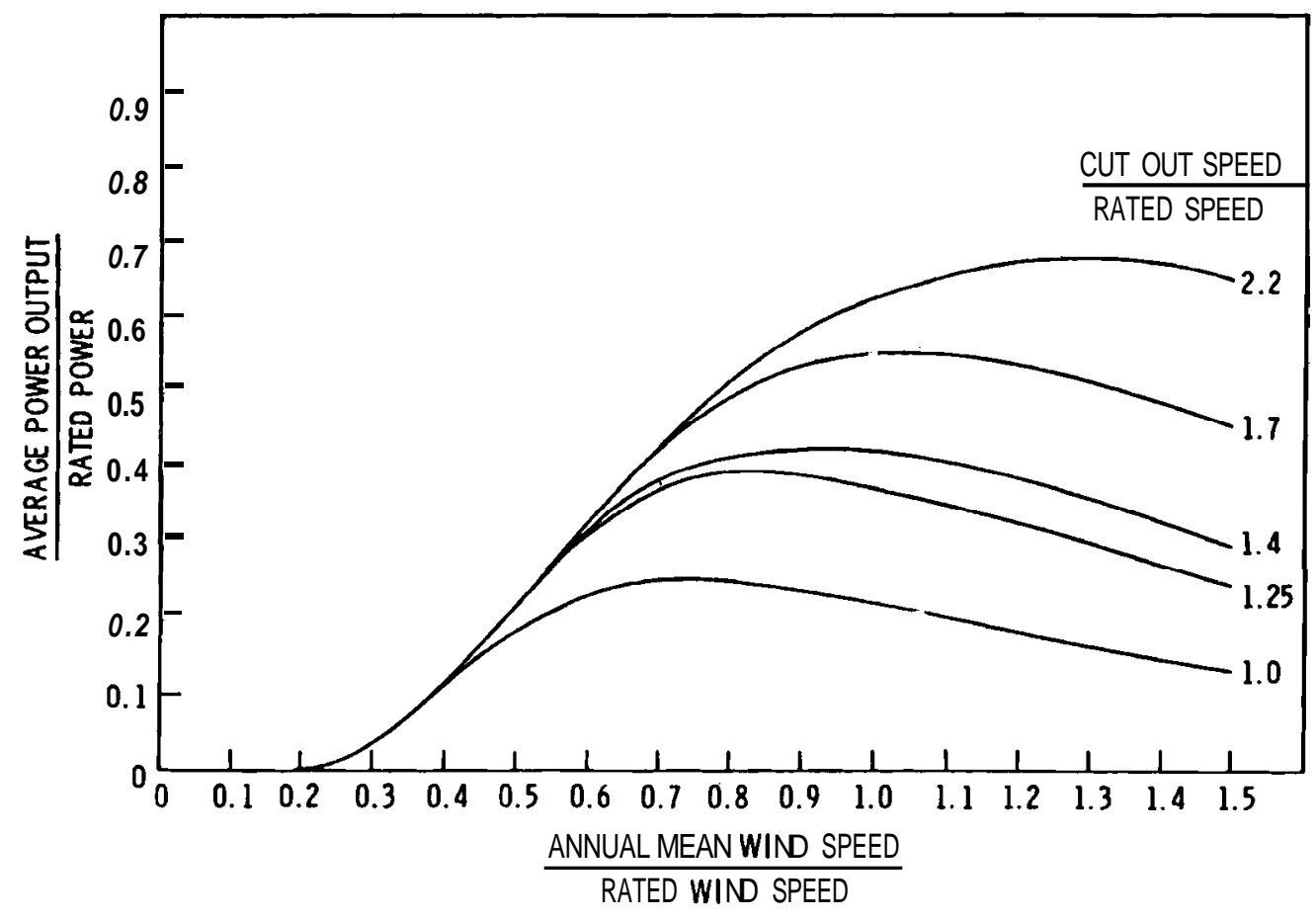

FIGURE 1.16. Estimate of Expected Average Power Output for Wind Turbines as a Function of Cutout, Rated and Mean Wind Speeds and Rated Power Output. 
power output versus mean wind speed for various machine characteristics. A $100 \%$ availability was assumed so that if an actual availability factor of $90 \%$ (10\% downtime due to scheduled or unscheduled shutdowns caused by other than insufficient or excessive winds) is anticipated, the results in these figures should be adjusted accordingly.

These techniques are the tools for making basic estimates of the energy production characteristics for a specified machine and the wind characteristics of a particular site. However, the decision to install wind turbines at a site depends on the analysis of the value and cost of the energy produced.

\subsection{EVALUATING THE RESOURCE}

Generic models have been developed to analyze the value and cost of energy produced by a cluster of WECS if the existing planning procedures used by utilities are slightly modified [see JBF Scientific Corp. (1979) and Marsh (1979).] The Electric Power Research Institute (EPRI) also discusses standard methods of evaluating electrical generation alternatives (EPRI 1979). Once the value of wind energy (VOE) is computed, it is compared with the cost of wind energy produced (COE). If the VOE is predicted to be greater than the COE at a given site for a given WECS cluster, then it would be economically beneficial to develop the site. If the COE exceeds the VOE, utility system costs would be higher if the cluster were developed than if conventional alternatives were chosen. In this latter case, the wind turbine cluster could only be developed if other considerations (e.g., societal values or institutional mandate) dictated that the consequent electricity rate increases would be preferable to the conventional option.

To determine the value of wind energy, utility system production cost estimates are obtained for a base case without wind energy, and for a modified case where wind-generated electricity is used to satisfy a portion of the load before the modified production cost estimates are made. Typically, hourly estimates of wind turbine or wind turbine cluster energy production are used for the basic wind energy data. As discussed in Section 1.3.2, accurate 
hourly wind turbine energy production estimates may require wind data more frequently than hourly to satisfactorily simulate hourly energy production. The generation mix of conventional equipment assumed for the modified case may be the same as the base case; if so the wind turbines are used as fuel savers only. Or, the generation mix may be optimized to account for capacity credit awardable to WECS. However, the issue of capacity credit for wind turbines is sti11 highly controversial. The stream of conventional system production costs in both cases are computed and expressed as a present value in the analysis year. The difference in the levelized production costs of the two cases is the savings obtained by adding WECS to the generation mix of the utility. These savings result from fuel savings for the conventional equipment and to a lesser extent from the reduction in fixed costs for maintaining a reserve margin for utility system reliability using conventional equipment (JBF Scientific Corp. 1979). The production cost savings are the value of the wind energy to a utility and may be expressed in $\$, \$ / k W$, or $\$ / k W h$. The VOE therefore represents the maximum amount of money a utility could afford to spend on WECS over the life of the machine without increasing system production costs.

Seasonal and diurnal variations of the wind significantly affect the value of wind energy, since these factors determine the types of fuel and equipment that the WECS will displace. Utilities will attempt to operate the equipment that is least expensive to run (low incremental costs) to satisfy base loads and only run the more expensive equipment (high incremental costs) as it is needed for peak loads. If the load has a nocturnal minimum a larger fraction of the utility's nocturnal load might be served by a low-fuel-cost but high-capital-cost system such as nuclear; during the daytime peak load the high-fuel-cost peaking equipment would be operating. In this instance, wind energy from a site with a daytime maximum would be of more value than from a site with a nighttime maximum, since energy produced at the first site would displace more high-fuel-cost equipment than the second site. It is even conceivable that, at significant penetrations of WECS not all of the windgenerated electricity available at minimum load conditions could be fully utilized. For example, a nighttime load could be met by nuclear (which is not 
allowed to load follow) and combustion turbines (for load following and maintaining reliability). In this circumstance the wind energy cannot displace nuclear, nor can it be allowed to displace much of the fossil fuel load following plants since they are required for maintaining reliability. Therefore, wind characteristics must be matched to both load and utility operational characteristics to be of maximum value.

The cost of energy is the present value of the stream of costs associated with purchasing, siting, installing, owning, operating, and maintaining a WECS. It is therefore a minimum levelized price that must be charged for energy consumed to satisfy revenue requirements. Like the VOE, the predicted COE from a proposed cluster of WECS could be expressed in $\$, \$ / \mathrm{kW}$, or $\$ / \mathrm{kWh}$.

One method (EPRI 1979) to estimate the COE uses the following equation:

$$
C O E=\frac{I C \cdot F C R+L F \cdot A O M}{A E P}
$$

where

$$
\begin{aligned}
& \mathrm{COE}=\text { the cost of energy expressed in } \phi / \mathrm{kWh} \\
& I C=\text { initial system cost including purchase of the system, site } \\
& F C R=\text { levelized fixed charge rate including the return on capital, }
\end{aligned}
$$

The first term in the numerator of the COE equation describes the levelized fixed costs. The EPRI Technical Assessment Guide discusses in detail the factors that contribute to the FCR. For systems with 30 -year lifetimes in 
investor-owned utilities the FCR is roughly 18\%; for municipal utilities it can be less. The second term is the levelized annual 0\&M costs. The choice of the LF, which is in the neighborhood of 2, is also discussed in the EPRI report. A third term, the levelized fuel costs, would appear for thermal plants. This term would only appear for wind systems if the utility had to pay royalties on the energy produced at a site to the local landowner.

The site with the greatest positive difference between the VOE and the COE would be the most economically viable. To increase the VOE, a site with the best temporal match between power produced and load should be selected, subject to any operational constraints. To decrease the COE, a site with higher mean annual energy production should be selected (see Figure 1.17).

Exactly how much time should be spent on locating a site that would satisfy both of these criteria would depend on a utility's needs. For example, a remote utility that generates its power with diesels alone will have a smaller range of incremental costs than most larger utilities. Therefore, the VOE would be high and fairly independent of the temporal distribution of the wind resource. The focus of attention in a meteorological prospecting program, in this case, is locating sites with maximum annual energy production. Wind prospecting for utilities with a broad range of incremental costs and large temporal variations in load will require more consideration of the temporal distribution of the wind resource. As Figure 1.5 shows, this can translate into requiring good definition of the vertical distribution of wind at a site.

At present, the tradeoffs between siting efforts and improved definition of the temporal distribution of the wind resource are not well understood since studies on the sensitivity of the VOE to wind characteristics are yet to be done. However, examining the sensitivity of the COE is straightforward, as indicated in Figure 1.17. For example, a utility has an option of two sites, each with different projected site development costs (initial costs) and different values of the mean annual energy production. Depending on the utility's goals, the total initial cost of a WECS cluster may be less at the higher wind speed site, especially if a specified amount of wind-generated 
ANNUAL ENERGY PRODUCTION $\left(\mathrm{kWh} \times 10^{\mathrm{o}}\right)$

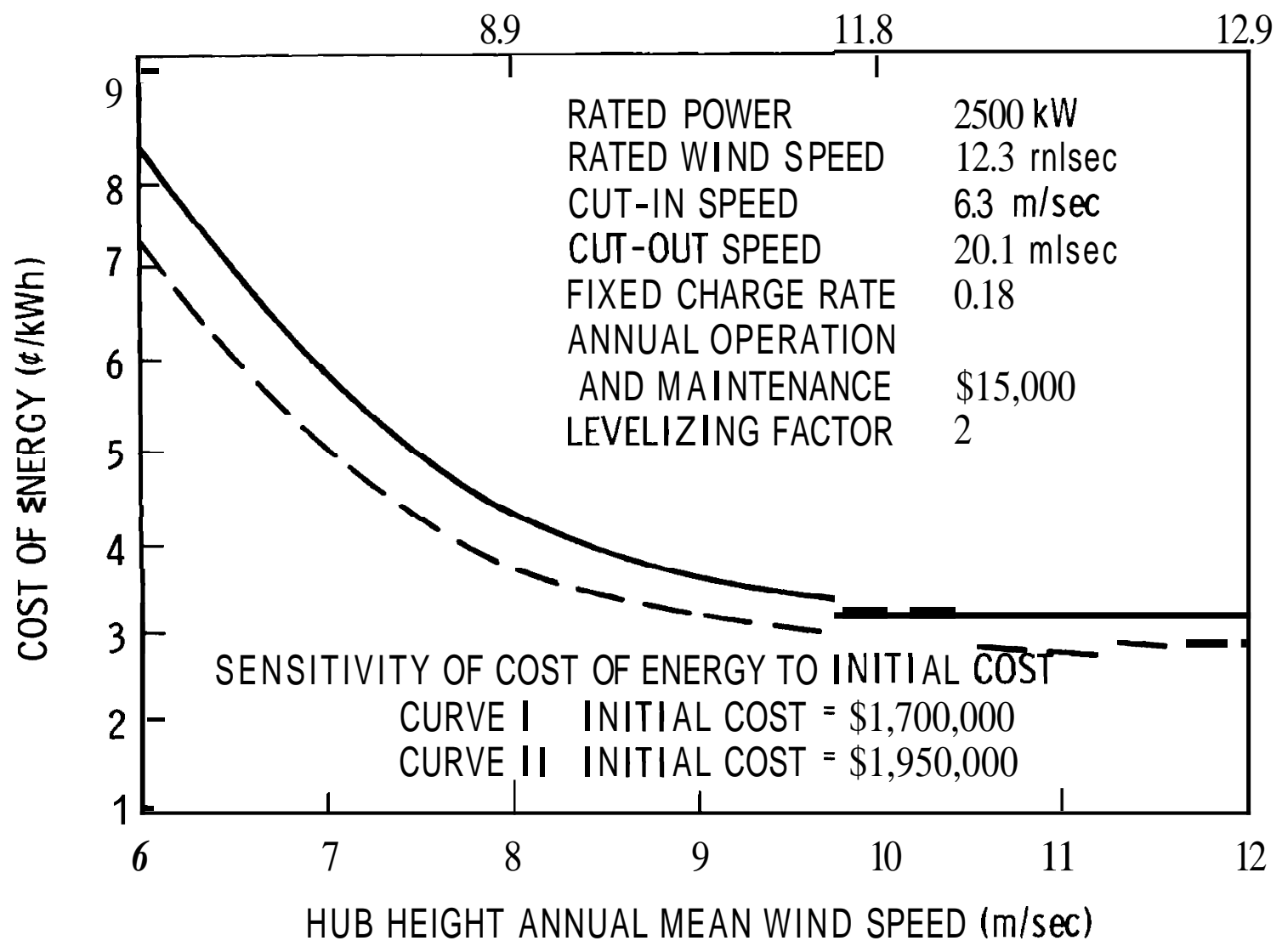

FIGURE 1.17. Cost of Energy and Annual Energy Production Versus Site Annual Mean Wind Speed for a Hypothetical Machine, Assuming Rayleigh Distribution and $100 \%$ Availability

energy is needed, since fewer machines will be required to produce the same amount of energy at the higher wind speed site. In this instance, efforts to find a better site, i.e., one with higher mean wind speeds, would result in reductions both in the initial capital investment and the cost of energy per kWh.

\subsection{CONCLUSIONS}

This report addresses the meteorological aspects of the multifaceted problem of large wind turbine siting. The data needs of utility resource planners are the primary focus of the siting strategies developed. The variability of the wind resource, the characteristics of wind energy machines, and 
the procedures of economic evaluation of wind energy systems dictate what meteorological data are required. There may or may not be additional sitespecific meteorological data requirements to analyze the integration of wind systems into the operations of the utility system. However, those data requirements have not yet been articulated so this report does not deal with the utility operational aspects of wind turbine siting.

To help utilities select sites that best fit their needs, siting strategies and techniques such as those presented in this document may be used in full or in part by those involved in selecting potential WECS sites. The overall strategies and approaches discussed in Chapter 2 provide the framework for site selection. The individual techniques described in Chapter 3 through 8 support the overall strategies. However, once the cluster site or sites are selected, the arrangement of machines in the cluster for maximum cluster energy output must be considered (see Chapter 9 for machine wakes and cluster design). In Chapter 10, the uses and types of instrumentation needed for making meteorological measurements are explained. Through careful consideration of the information presented here, a plan for selecting sites can be designed that fits the individual utility's needs. 


\section{REFERENCES}

Betz, A. 1928. "Windmills in the Light of Modern Research." Tech. Memo. No. 474, U.S. National Advisory Committee for Aeronautics, Washington, DC.

Cliff, W. C. 1977. The Effect of Generalized Wind Characteristics on Annual Power Estimated from Wind Turbine Generators. PNL-2436, Pacific Northwest Laboratory, Richland, Washington.

Electric Power Research Institute (EPRI). 1979. Technical Assessment Guide. EPRI PS-1201-SR, Electric Power Research Institute, Palo Alto, California.

Elliott, D. L. 1977. Synthesis of National Wind Energy Assessments.

BNWL-2220, WIND-5, Pacific Northwest Laboratory, Richland, Washington.

Glasgow, J. C. and W. H. Robbins. 1979. "Utility Operational Experience on the NASA/DOE MOD-OA $200 \mathrm{~kW}$ Wind Turbine." In Proceedings of the Workshop on Economic and Operational Requirements and Status of Large Scale Wind Systems. EPRI ER-1110-SR (DOE CONF. \#790352), Altas Corp., Santa Cruz, California.

JBF Scientific. 1979. Wind Energy Systems Application to Regional Utilities. Vol. 1, JBF Scientific, Wilmington, Massachusetts.

Marsh, W. D. 1979. Requirements Assessment of Wind Power Plants in Electric Utility Systems. EPRI ER-978-SY, General Electric Company, Schenectady, New York.

Park, J. and D. Schwind. 1978. Wind Power for Farms, Homes, and Small Industry. RFP-2841/1270/78/4, National Technical Information Service, Springfield, Virginia.

Wegley, H. L. 1979. "Overview of Wind Characteristics for Operations." In Proceedings of the Conference and Workshop on Wind Energy characteristics and Wind Energy Siting 1979, American Meteorological Society, Boston, Massachusetts. 


\subsection{STRATEGIES FOR WIND TURBINE SITING}

\subsection{THE NATURE OF THE PROBLEM}

One of the principal differences between siting wind turbine generators and siting conventional power plants is that the performance of a wind turbine generator (the total energy produced by the machine over a given period as well as the temporal behavior of this energy production) is completely governed by the turbine's location. This sensitivity to location opens a new dimension to the process of siting generating facilities; it makes the site-selection process for wind turbine generators even more critical than the site-selection process for conventional power plants.

The sensitivity of wind turbine performance to location arises, of course, because the fuel source (the wind) varies with location. The spatial variability of the wind depends on the local topography, the complexity of the meteorology within a region, the season of the year, and the time of day. While most people are aware that the strength of the wind does vary over short distances, few are aware of how large this variability can be. Section 1.2 and Appendix 3 discuss the problem of resource variability in some detail. These discussions conclude that significant variations in both the magnitude and the temporal behavior of the wind can occur over fairly short distances. This includes short distances in the vertical, as well, for as illustrated in Appendix 3 , the behavior of the wind near the surface may not be representative of the wind blowing through the rotor disk of a large wind turbine.

The complexity of wind behavior means that wind turbine performance cannot be predicted accurately without wind measurements at the site. These measurements will have to be at heights comparable to the hub heights of the machines under consideration, and, therefore, they represent a significant expense. The purpose of a wind turbine siting program should be to pinpoint potential wind turbine sites as rapidly as possible and to pick potential sites that have a high probability of being feasible. If this approach is followed, the number of locations where expensive on-site measurements are required will be minimized. 


\subsection{BASIC APPROACHES TO SITING}

There are two approaches to siting wind turbine generators: "wind prospecting" and evaluating a predetermined site. In the first approach, a large area (the region of interest) is screened for places that experience suitably high wind speeds with sufficient frequency to make wind-generated electricity economically attractive. Once these places are found, the detailed wind measurements needed to determine economic value are made. The steps involved in wind prospecting are:

Wind Prospecting

1. Analyze Region of Interest. A large region, perhaps $200,000 \mathrm{~km}^{2}$, is screened for candidate resource areas $\left(210,000 \mathrm{~km}^{2}\right)$ that appear attractive.

2. Evaluate Candidate Resource Areas. A candidate resource area is screened for potential candidate sites that experience usable winds and satisfy pertinent land use and accessibility criteria.

3. Screen Potential Candidate Sites. The potential candidate sites are reviewed for candidate sites.

4. Evaluate Candidate Site. Wind data are collected at the candidate sites and the sites are evaluated.

5. Develop Site. A site is chosen and the best locations for individual machines are identified.

The second approach, evaluating a predetermined site, is followed when the potential candidate sites are already known. For example, a utility may own or have access to one or several parcels of land with apparent wind energy potential. In this case, the siting procedure is:

Evaluating a Predetermined Site

1. Establish Feasibility. A preliminary evaluation is made of the wind energy potential.

2. Evaluate Site. Representative wind data are collected.

3. Develop Site. The best locations are identified for individual machines. 


\section{TECHNIQUES FOR ESTIMATING WIND RESOURCES}

Because the wind resource shows so much spatial variability and because the cost of on-site measurements can be large, many utilities are interested in techniques for estimating the wind energy potential of a possible site without having to make on-site wind measurements. Quite a few techniques for doing this either exist or have been proposed. These techniques can be used screen candidate resource areas for sites with high potential or to estimate wind energy characteristics at a specific location. Among these techniques are:

- numerical modeling of flow over terrain

- physical modeling of flow over terrain

- topographical indicators of wind energy potential

- biological indicators of wind energy potential

- geomorphological indicators of wind energy potential

- social and cultural indicators of wind energy potential.

The intelligent use of these siting techniques requires a thorough understanding of their strengths and weaknesses. Chapters 3 through 8 provide detailed discussions of each of these techniques, and Figures 2.1 and 2.2 show where each technique can be applied in the siting process. The following discussion describes each technique briefly. The discussion should assist the reader in understanding how each would be applied in the site-selection strategy outlined in the next section.

\subsubsection{Numerical Model ing}

Numerical models provide an objective method for estimating the effects of terrain on airflow and for interpolating wind data from locations where there are wind observations to locations where there are none. The models find solutions to equations that describe the complex interactions between the atmosphere and the earth's surface. The accuracy achieved in these solutions depends on the accuracy and density of the data and the amount of realism inherent in the mathematical relationships that make up the model (see Chapter 3). 


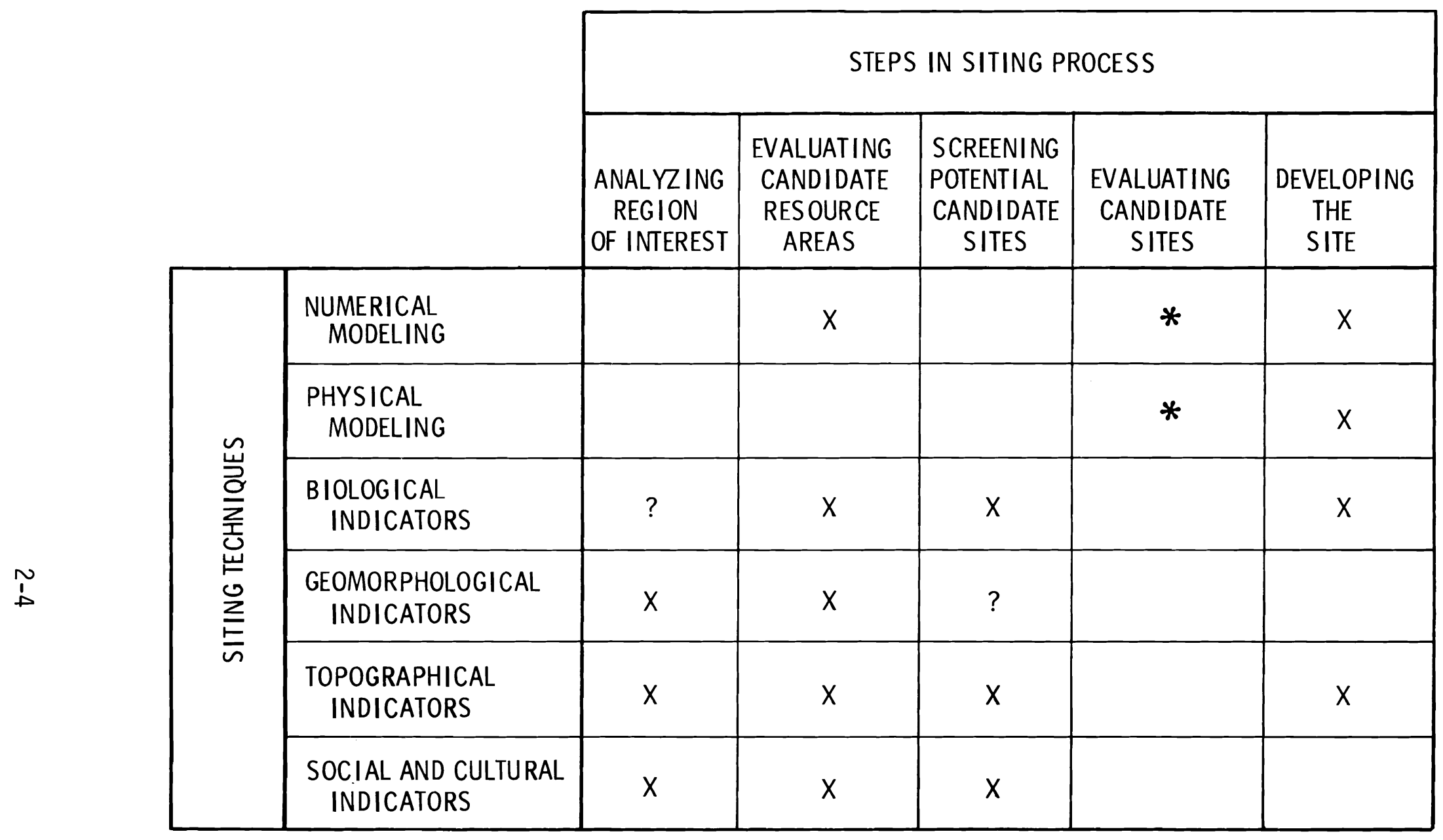

$X$-TECHNIQUES ARE APPLICABLE

?-TECHNIQUES HAVE LIMITED APPLICABILITY

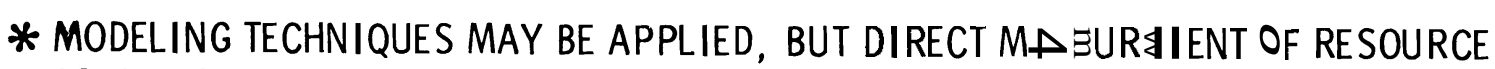
IS NECESSARY FOR FIRM VALUE ESTIMATES

FIGURE 2.1. Applicability of Siting echniques to the steps of the Wind Prospecting Approach 


\begin{tabular}{|c|c|c|c|c|}
\hline & & \multicolumn{3}{|c|}{ STEPS IN SITING PROCESS } \\
\hline & & $\begin{array}{l}\text { ESTABLISHING } \\
\text { FEAS IBILITY }\end{array}$ & $\begin{array}{c}\text { EVALUATING } \\
\text { THE } \\
\text { SITE }\end{array}$ & $\begin{array}{c}\text { DEVELOPING } \\
\text { THE } \\
\text { SITE }\end{array}$ \\
\hline \multirow{6}{*}{ 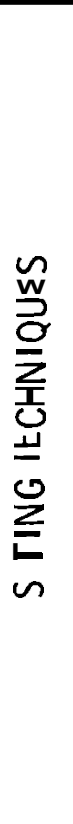 } & $\begin{array}{l}\text { NUMERICAL } \\
\text { MODELING }\end{array}$ & $\mathrm{x}$ & * & $\mathrm{x}$ \\
\hline & $\begin{array}{l}\text { PHYSICAL } \\
\text { MODELING }\end{array}$ & & * & $x$ \\
\hline & $\begin{array}{l}\text { BIOLOGICAL } \\
\text { INDICATORS }\end{array}$ & $x$ & & $\mathrm{x}$ \\
\hline & $\begin{array}{l}\text { GEOMORPHPLOGICAL } \\
\text { INDICATORS }\end{array}$ & $\mathrm{x}$ & & \\
\hline & $\begin{array}{l}\text { TOPOGRAPHICAL } \\
\text { INDICATORS }\end{array}$ & $x$ & & $\mathrm{X}$ \\
\hline & $\begin{array}{l}\text { SOCIAL AND CULTURAL } \\
\text { INDICATORS }\end{array}$ & $x$ & & \\
\hline
\end{tabular}

\section{$X$-TECHNIQUES ARE APPLICABLE}

* MODELING TECHNIQUES MAY BE APPLICABLE, BUT DIRECT MEASUREMENT OF RESOURCE IS NECESSARY FOR FIRM VALUE ESTIMATES

FIGURE 2.2. Applicability of Site-Screening Techniques to the Problem of Evaluating a Predetermined Site. The applicability of numerical modeling to the feasibility step depends on the size of the area under examination and on the complexity of the terrain. 


\subsubsection{Physical Model ing}

Flow over terrain may also be modeled by placing a scale model of the terrain in a suitably designed wind tunnel. This approach can yield useful information as long as the modeled region is no more than a few tens of kilometers in size, and the portion of the atmosphere to be studied is the lowest few hundred meters. Wind tunnels are limited in their ability to simulate all aspects of flow over terrain; however, their ability to simulate the effects of small-scale terrain features on nearly neutral flow are superior to currently available numerical models. Hence, physical model ing could be quite valuable when the exact layout of a cluster of machines is being planned (see Chapter 4).

\subsubsection{Topographical Indicators}

Historically, wind machines have been sited by applying empirical guidelines describing the general effects of terrain or surface obstacles on the wind. An excellent example of the application of this technique to the problem of site screening is the British site-screening program of the late 1940s and early 1950s described by Golding (1955). Topographical guidelines are either based on a physical understanding of how topography affects flow or on experience gained through observation. An understanding of these guidelines is also invaluable in interpreting the results of numerical and physical model ing studies or of measurement programs (see Chapter 5).

\subsubsection{Biological Indicators}

The shapes of certain species of trees are good indicators of high winds. Trees are particularly useful in regions where large, local variations in wind speed can be expected or in regions where wind data are sparse. The qualitative relationship between tree shape and wind speed has been recognized for years [Putnam (1948) used biological indicators in looking for wind turbine sites during the 1930s]. Recently, more quantitative work has been done on the relationship between tree shape and wind shape (Wade and Hewson 1979, see Chapter 6).

\subsubsection{Geomorphological Indicators}

Certain features of the land surface, such as sand dunes, are a direct consequence of wind action. These features are called eolian and can be 
detected from satellite or aircraft photographs. These features are useful in locating potential areas of strong winds in arid regions. Since eolian landforms can be caused by occasionally strong winds over a period of years, their existence is not a guarantee of persistently strong winds. This technique must then be combined with a good understanding of wind climatology if it is used (see Chapter 7).

\subsubsection{Social and Cultural Indicators}

Indirect information about the wind can be found by examining land use patterns and by questioning people who live or work in areas that are expected to be windy. For example, people dislike living in areas with persistent winds, so areas with high wind energy potential seldom coincide with large population centers. Agricultural use of the land is also an indicator: windy areas are more likely to be used for grazing than for row crops. Other indicators could be the locations of snow fences along the highways or the locations of frequent wind damage to power lines (see Chapter 8 ).

\subsection{A SITE-SELECTION STRATEGY}

The site-selection strategy described in this section is for a utility considering the introduction of several wind turbine generators into its mix of generating equipment. The utility is assumed to be looking for areas where perhaps 5 to 50 megawatt-size machines can be placed in reasonable proximity, and the scope of its search covers a large geographical area. The utility is also assumed to be looking for several of these machine clusters that could be developed sequentially. Since the decision as to where and when to develop clusters is an economic one, the strategy concentrates on documenting the meteorological factors that affect economic viability. Other issues such as land use constraints, accessibility, proximity to existing transmission corridors, environmental impact and public acceptance are clearly important, but they are beyond the scope of this report and are only mentioned in general terms. The strategy is for wind prospecting. The parallel strategy for evaluating a predetermined site is not described since it is an abridgment of the one for prospecting. 
Figure 2.3 is a flow chart outlining a decision-making process for developing a system of wind turbine clusters. The remainder of this chapter is devoted to describing the steps of the strategy in more detail and outlining the wind data requirements for each step. The strategy provides a framework in which to organize a site selection program rather than a detailed, stepby-step procedure. The details of a site-selection program are dictated by the unique problems of each utility since the relative importance of issues such as land use constraints, accessibility, public acceptance, environmental constraints and so forth will vary. Local topography and meteorology will have a deciding influence on which of the techniques for estimating wind resources should be used in locating potential sites. None of the the techniques described in Section 2.2 are foolproof and all require considerable knowledge and experience to apply correctly. Because of this, it is strongly recommended that an experienced boundary-layer meteorologist be included in a site-selection team. Most large utilities will have this expertise on their staff; otherwise, consultants should be employed. At present, the relevant meteorological expertise is most likely to be found in firms that have specialized in air pollution problems in complex terrain.

Because a cluster of megawatt-size wind turbines represents a reasonably large capital investment, the siting strategy outlined in Figure 2.3 is thorough. The wind characteristics at a site not only govern the energy output of a cluster but they also can affect the service life of the equipment and both the scheduled and unscheduled maintenance costs. It is assumed, therefore, that wind characteristics affecting turbine output, service life and operating costs will have to be documented before a utility will commit funds to wind turbine installation. (a)

Nevertheless, it is important that the level of effort involved in siting match the size of the problem. If, for example, a utility is considering the

(a) Since wind turbine clusters will probably be developed a few machines at a time, documentation of site characteristics may not have to be as thorough as if the entire cluster were installed at once. Operating experience with the first few machines will be more convincing of the potential of a site than years of data collection. 


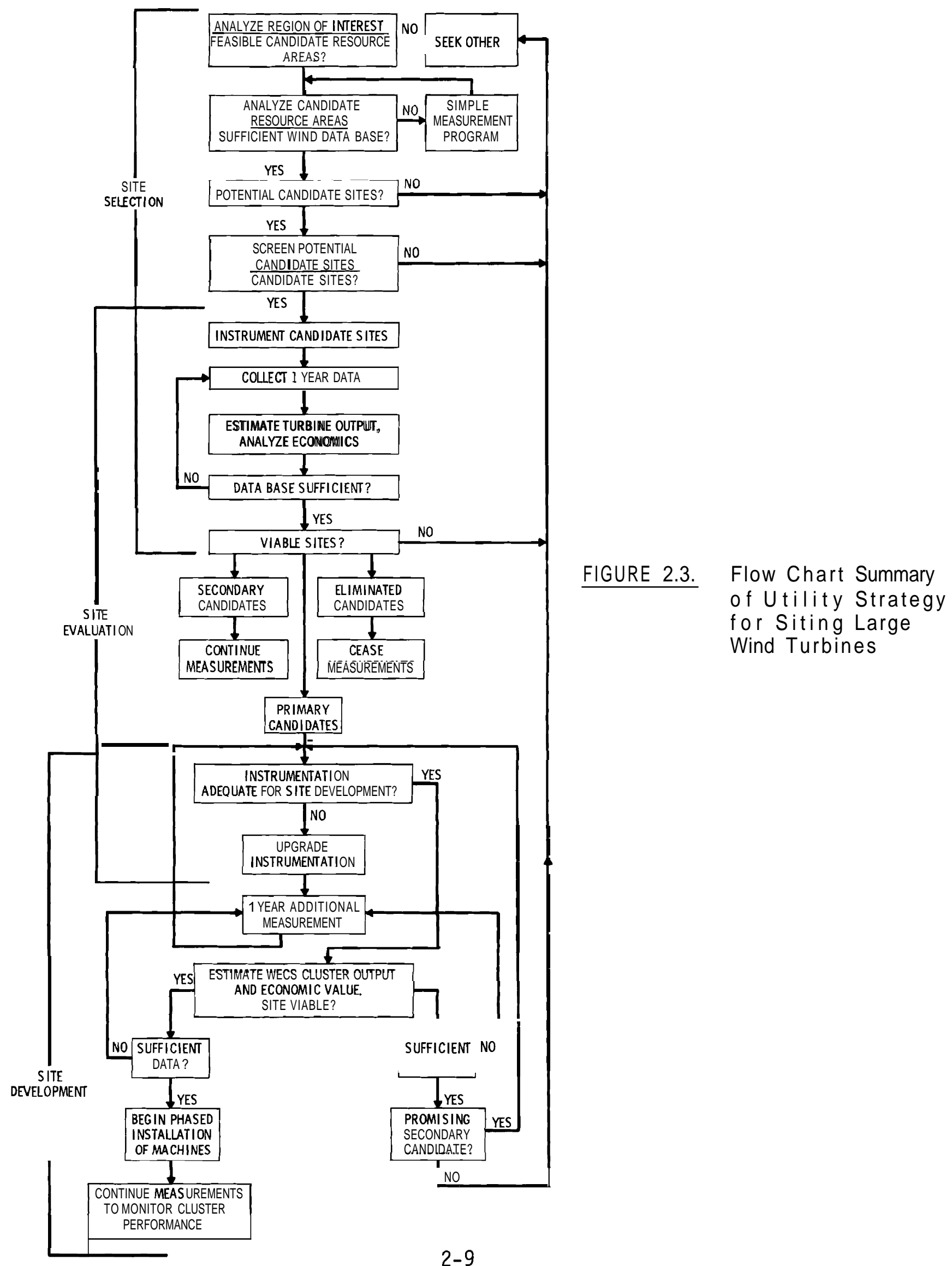


installation ot one or two test machines to enable the utility to gain experience with wind turbine equipment, the siting strategy described below is far too involved. A test site must have sufficient wind energy potential to exercise the machine, but detailed understanding of how the machine will perform at the site is unnecessary.

\subsubsection{Analyzing the Region of Interest}

In this step, potential high wind areas are identified and then evaluated on the basis of criteria such as: how seasonal variations in wind speed match seasonal variations in the load, the probability of finding clusters of a minimum useful size, land use restrictions, location of roads and transmission lines, accessibility, and meteorological and other hazards. A key data source for this step is the regional wind resource assessments prepared for DOE by PNL. These assessments, which are described in Appendix 1, provide information on wind characteristics with a resolution of about 30 kilometers. Combining this information with any additional wind information the utility may have, wind resource analysts will be able to identify those areas within the region of interest that have the greatest potential.

A particular concern both in large area analysis and in later siting decisions should be terrain. Locating WECS clusters in flat areas could result in lower costs for siting evaluations and operation, since wind characteristics tend to be more benign over gentle terrain. In more rugged terrain, the horizontal variability in the wind resource is greater, and the chance of encountering large wind shear and turbulence is also larger. Both possibilities result in higher costs for site evaluation and in the possibility of reduced service life and increased maintenance costs.

\subsubsection{Evaluating Candidate Resource Areas}

If wind power is feasible, an analysis of the region of interest will have identified several attractive areas. If necessary, these areas should be divided into sections on the order of $10,000 \mathrm{~km}^{2}$ in order to facilitate further analysis. Within the areas, called candidate resource areas, the wind energy potential will vary considerably. One area ma contain several potential 
sites. To identify potential candidate sites within a candidate resource area, the areas should be screened for sites with promising wind energy (see Figure 2.1 for techniques that could be used). In areas void of data, a supplemental wind measurement program may be required.

The amount of wind data required to identify potential candidate sites depends upon the terrain and the meteorological complexity of the area. If the topography of an area is complicated and the seasonal and diurnal variations in the meteorology are large, at least 1 year's surface data are required for an analysis of the general flow characteristics of a candidate resource area. (a) In conducting such an analysis the surface data should come from locations that will provide the most information on the air masses flowing through the area; i.e., data should be available from the major valleys parallel to the prevailing flow as well as the major passes and major ridges perpendicular to the prevailing flow.

The time required to complete this phase of the site selection process depends on the number of candidate resource areas and on how well the wind resource within the area is understood. If the wind resource is poorly documented, supplementary measurements may be needed. This will require time to plan the program, deploy the instruments, collect the data, analyze it and interpret the results. For a data collection period of 1 year, about 18 months to 2 years could be required to complete the process from planning to interpretation.

Numerical models could be useful in the analysis of candidate resource areas. Numerical models require input data representing mean flow conditions over the candidate resource area. The averaging times for input data should be short compared to the time scale of the changing pressure patterns that drive the flow but long compared to the turbulent fluctuations that are irrelevant to the general flow patterns through the region. Data requirements for a fairly simple numerical simulation would be:

(a) Here, analysis means an estimate of the flow at all points in a given region subject to the constraints of the physical laws governing the atmosphere. 
- synchronous 1-hr averages of surface wind speed and direction at key locations

- representative vertical profiles of wind speed, direction, and temperature.

In analyzing the wind resource of a candidate resource area with a numerical model, a small group of simulations that presumably represent a cross section of the climatology are performed. Of course, which days will be representative of the climatology cannot be known until a considerable amount of data have been collected and scrutinized. More detail on how numerical models can be applied to site screening and site evaluation is given in Chapter 3.

A program of analysis as rigorous as the one just described could provide a great deal of information about the wind energy potential of a candidate resource area, even if the anemometers providing the input data were not located in what the analysis showed to be high wind areas. However, a less rigorous approach may also be taken. In this approach, supplemental data are collected at what are presumed to be the windiest locations (see Chapter 5 for techniques to estimate these locations) and the goal is simply to document this resource. Numerical models could be used to help site anemometers for wind resource documentation by running the model for intelligent guesses of the prevailing flow patterns. An example of how this approach can be used to understand the prevailing wind speed patterns over an area is given in Section 3.2.3. A model could also be used to test how sensitive the location of high wind areas are to the accuracy of the input data. If the location of high wind areas and the wind speeds at those areas are very sensitive to the input guesses, one should take this as an indication that locating potential candidate sites may prove difficult and that a more thorough documentation of the wind climatology may be required.

The goal in this phase of site selection is to pinpoint potential candidate sites with high wind energy potential. If supplemental measurements are made, their purpose is either to drive a numerical model of flow over the candidate resource area or to document wind energy potential at locations that are 
assumed to have it. The sampling period will be 1 year, and cost constraints will probably limit anemometer heights to less than $30 \mathrm{~m}$. The height limitation makes it impossible to determine in detail the diurnal wind cycle at elevations comparable to the hub height of a multi-megawatt horizontal-axis wind turbine (see Appendix 3). Likewise, collecting wind data for only a single year will eliminate the possibility of determining the long-term seasonal modulation of wind energy potential (again, see Appendix 3). However, the gross wind power potential on an annual average basis can probably be estimated reasonably well by a single year of wind measurement (Corotis 1980).

The equipment required for wind measurements at this stage of the siteselection process depends on the purpose of the data collection. Data for driving numerical wind flow models must be in a time-series format with all data loggers time-synchronized. Data for mesoscale wind documentation can be i $n$ a summarized format (i.e., a wind rose or joint probability distribution of wind speed and direction). Chapter 10 gives more detailed information on wind sensors and data-logging equipment.

\subsubsection{Screening Potential Candidate Sites}

After a number of potential candidate sites have been identified, they must be screened through site visits. During these visits, the surrounding terrain is examined and any small-scale terrain features or obstacles that could affect wind characteristics at the site are noted. Factors that could affect the cost of developing the site are also examined. Site screening is not a time-consuming process and, depending on the number of sites, can be completed in a few weeks to months.

Valuable information on wind characteristics at the potential candidate site can be obtained by examining the vegetation and by measuring the wind profile with such inexpensive systems as wind-sensing kites or instrumented tethered balloons. Profile measurements made during site visits would be used primarily to identify obvious potential hazards, such as flow separation and the turbulence and high wind shear that accompany them. One cannot expect to obtain a reliable wind profile climatology through such a small set of observations, however. This type of information requires continuous observation over long time periods. 


\section{Evaluating Candidate Sites}

The objective of this step is to determine how wind machines will perform at each site and to ascertain the site or combination of sites that will result in power output characteristics best matching the needs of the utility. The type of wind data needed at this stage of the site-selection process depends on the number of sites to be evaluated, the size of the wind turbine cluster, and the size and complexity of the utility system that the machines will be serving. If a large number of sites remain after the potential candidate sites have been screened, and if the wind resources of these sites are sti1l uncertain, a preliminary site evaluation program may be necessary. The program would be similar to the wind resource documentation program described in Section 2.4.2 and would be accomplished by installing inexpensive wind measuring equipment at the sites. Equipment such as wind-run anemometers, mechanical weather stations or simple, rugged anemometers coupled to smart data loggers would be satisfactory. The anemometers would be mounted on short masts (10 $\mathrm{m}$ high, for example), and the measurements would be used simply to verify the existence of a usable wind resource.

Once the number of candidate sites is reasonable, the actual process of candidate site evaluation begins. As indicated in Figure 2.3, the objective of candidate site evaluation is to identify the site or sites most attractive for the installation of wind turbine generators. Since this decision will probably be based on economics, an understanding is required of how wind turbines placed at the various sites will affect energy production costs for the entire utility.

The impact of wind turbine generators on the energy production costs of utilities has been the subject of several recent studies (General Electric Company 1979, JBF Scientific Corporation 1979, Van Kuiken et a1. 1980). The General Electric and JBF studies showed, for example, that the economic value of wind turbines (that is, the price a utility can afford to pay for a machine) depends on the modifications wind-generated electricity make to the utility's load ${ }^{(a)}$ and on the utility's mix of generating equipment. If energy production

(a) When evaluating the worth of wind energy it is convenient to use the wind energy to satisfy a portion of the load, thereby creating a "modified load" that must be satisfied by conventional equipment, subject to operational constraints (see Section 1.4). 
costs vary widely for the various units in the mix, the way in which the output of a cluster satisfies that load on an hour-by-hour basis (or less) will be important. On the other hand, if production costs are fairly uniform for the various units in the mix (as they might be for a very small utility), the total energy produced over a given period might be the most important factor influencing economic value and not details on how turbine or cluster output modify the utility's load.

Determining the impact of wind characteristics on economic value requires a model to transform wind data into information on energy production. The appropriate model to use in estimating energy production will depend on the response characteristics of the machine, the behavior of the wind through the rotor disk and the averaging time that is chosen for the wind data. At present, there are no thoroughly verified methods for simulating the day-to-day energy production of large wind turbines given wind data from a site. The reason for this is that data on wind turbine performance at sites with detailed wind measurements are only being obtained now.

Traditionally, wind turbine energy production has been modeled by combining hub-height wind speeds with the steady-state performance characteristic of the machine (see Section 1.3.2). (a) In the traditional approach, an average hub-height wind speed is computed for a given time interval and the average power output for that interval is assumed to be given by the steady-state performance characteristic. The average power output that actually occurs may differ from the estimate for several reasons: the wind at hub height may not have been representative of flow through the rotor disk of the turbine; the machine may not have been on-line and synchronized with the utility system during the entire time interval; and if the machine is a horizontal axis type, there could have been significant yaw errors (and consequent reductions in net flow through the rotor disk) between the wind direction and the axis of the machine.

(a) The steady-state performance characteristic of a wind turbine is the relationship between wind speed and power output (Figure 1.11) that would exist if the machine were operating in a uniform, steady flow such as exists in a wind tunnel. 
Another approach to modeling wind turbine energy production is to attempt a more detailed simulation of machine operation by combining wind data (speed and direction) with a detailed simulation of the machine's startup, shutdown and synchronization procedures as well as its yawing behavior. Accomplishing this type of simulation requires wind data with fairly short time resolution (1 to 2 min averages, or less). Average power output for these short intervals is computed by first determining whether the machine is synchronized, computing the average component of wind velocity normal to the plane of the rotor disk and then computing average power output for the time interval from the steadystate performance characteristic. Miller (1980) provides a more detailed description of this approach to energy production modeling.

Utilities, however, need to know the accuracies of the different approaches to energy production modeling so they can choose the one most appropriate to their needs. The method that will be used to model individual turbine and cluster output must be known before planning a site measurement program because the energy production model will be one factor determining the wind data to be collected. Although there is not a great deal of information on how well the various energy production models perform, there is enough information available for some educated guesses to be made. Analysis of data from the MOD-OA and MOD-1 ${ }^{(a)}$ machines at Clayton, New Mexico, and Boone, North Carolina, has shown that when the machine is synchronized for the entire time interval, there is good agreement between the average power output predicted by the steady-state performance characteristic and the average power output actually delivered to the utility system (Robbins and Baldwin 1980; also, see Figure 1.12). (b) The studies at Clayton also suggest that when the traditional approach to modeling energy production is used to estimate total energy production for a time period of a month to a year, the actual energy production will be overestimated by approximately 10 to 25 percent. (c) The results of recent research

(a) The MOD-OA, MOD-1 and MOD-2 machines are operated by the National Aeronautics and Space Administration (NASA) and the Department of Energy (DOE).

(b) Hub-height wind data were used. Also, considerably more data have been analyzed at Clayton than at Boone.

(c) The magnitude of the overestimate depends on the gustiness of the wind and the responsiveness of the machine. 
also suggest that in modeling energy production, the averaging time for wind data should be less than 1 hour. For the traditional method, 5 to 10 minutes is probably reasonable.

Presumably, a detailed simulation of wind turbine operation of the type described by Mller (1980) would result in more accurate estimates of energy production than the traditional approach. However, this hypothesis has not been tested by data from an actual turbine installation. Nevertheless, Miller has compared the results of traditional simulations of energy production with results from a detailed simulation of the NASA/DOE MOD2 wind turbine using wind data from several DOE candidate sites. (a) In these comparisons, Miller found that energy production estimates by the traditional method were 10 to 20 percent higher than the detailed simulations.

In addition to depending on the method used to estimate the energy production at a site, the required wind data could also depend on the machine size. The results obtained at Clayton suggest that energy production from intermediatesize machines (rotor diameters less than $40-50 \mathrm{~m}$ ) can be estimated with wind data from a single height (hub height) above the surface. Larger machines, however, could require wind measurements at several levels above the ground in order to represent the mean flow through the rotor disk. This possibility arises because the amount that the wind may vary across a rotor disk increases as the blade length increases. How accurately wind data from a single level will estimate energy production for a machine having a rotor diameter as large as $100 \mathrm{~m}$ will not be known until performance tests are conducted on these large machines.

The wind data collected in the site evaluation stage will be a compromise between the desire to obtain the best possible simulation of machine (and cluster) performance and the need to control costs. Wind data sufficient for a detailed simulation of machine operation are recommended. The cost differences between collecting data for a detailed simulation and collecting data for a traditional simulation are small. The minimum data requirement for a detailed simulation are 1 - to 2 -min averages of the two horizontal components of wind

(a) M1ler (1980) and private communication. 
velocity at or near hub height $(a)$ for the machine or machines under consideration. For machines more than $50 \mathrm{~m}$ in diameter, some thought should be given to collecting data at additional levels (such as $\pm 2 / 3$ of the rotor radius) if test results for larger machines show that this could improve estimates of energy production significantly.

A full simulation of machine operation should produce not only the best estimate of energy production but also valuable information on machine behavior at the various candidate sites. Miller's simulations of large wind turbines have shown that the number of startup, shutdown and yawing operations can vary greatly from site to site ${ }^{(b)}$ The importance of these maneuvers is that they place significant loads on blades and other structural elements of a machine (Neustadter 1979). An excessive number of these operations could have an effect on maintenance costs and, perhaps, on the service life of a machine. Estimates of the annual number of startup, shutdown and yawing operations for the various machines under consideration could be another parameter to use in ranking candidate sites.

Wind measurements should be made at enough locations to document typical wind behavior over the site. The required number of locations will be a matter of judgment, and it will depend on the size of the site and the complexity of the terrain in and around the site. In reasonably flat terrain, a single measurement location should suffice.

In order to compute energy production, information on air temperature is needed in order to compute the air density at a site. Machine performance data are given for a standard air density; thus, the performance characteristic for a given machine must be corrected for any significant density difference. Air density variations due to air pressure fluctuations about the mean barometric pressure amount to no more than \pm 2 percent, but density variations due to temperature changes can amount to about 20 percent at typical sites in the northern U.S. The error in estimating air density can be reduced by about one-half if the monthly average temperatures are known at the site. A more

\footnotetext{
(a) A constratnt to consider is that the Federal Aviation Administration (FAA) requires reliable beacon lights on towers over $200 \mathrm{ft}$. Bringing an ac power line to a remote site is an expense that must be weighed.

(b) Private communication.
} 
accurate determination of air density requires on-site data with sufficient time resolution to resolve daily changes in the diurnal temperature pattern.

The data processing equipment used in eval uating candidate sites must produce time series of wind speed, wind direction and temperature (if this parameter is measured). The equipment must sample the wind and temperature transducers, compute averages of the two horizontal components of wind direction and record these data, along with the time, on tape. Data can be recorded at each tower or transmitted to a central recording location. Recording the data at a central location provides obvious advantages in managing the data and in maintaining system reliability; however, it requires large initial expenses. The reader is again referred to Chapter 10 for additional information on instrumentation systems.

The length of time spent in the site evaluation phase depends on the behavior of the wind at the various candidate sites and on the characteristics of the utility. If a decision can be based on the levelized cost of energy, a single year of data collection should be sufficient. After a year of data collection at the various sites, a reasonable estimate of annual average energy production will be possible. It will also be possible to make reasonable estimates of the uncertainty in this estimate of annual average energy production. If, on the other hand, the economic value of wind-generated electricity is a strong function of how the turbines modify the utility's load on an hourly or subhourly basis, more than 1 year of data collection could be required, since it is necessary to know how the several wind characteristics that affect load modifications might vary from year-to-year. ${ }^{(a)}$ In any case, a decision on how long to measure will rest on the differentials between the cost of wind-generated electricity and the utility's energy production costs as wel1 as on the sensitivity of the economic value of wind turbines to uncertainties in wind characteristics.

\subsubsection{Developing a Si te}

At the completion of the site evaluation stage, some sites will have emerged as viable candidates for the installation of machines and some will

(a) See Appendix 3 for a discussion of the interannual variability of key characteristics such as the diurnal and seasonal modulations of wind speed. 
have been eliminated. This process of elimination is achieved by considering the economic value of the energy generated at each site, the cost of developing each site, the suitability of each site for trouble-free wind turbine operation, and the acceptability of each site to the public. At this point, a site could be chosen as the prime candidate for a wind turbine cluster even though a complete understanding of the nature of the wind resource at the site may not have been obtained. Once the primary site is chosen, the remaining viable sites become secondary candidates and data collection at those sites could continue. At the primary site, the process of site development would begin. The purpose of this step is to:

- locate each machine in the cluster

- verify the energy production characteristics of the cluster

- determine the effects of cluster output on the dynamics of the utility system

- ensure that the character of the wind resource is compatible with troublefree operation of the turbines.

Such an intensive evaluation requires detailed knowledge of the flow through a section of atmosphere having a vertical dimension of 100 to $150 \mathrm{~m}$ and horizontal dimensions equal to the size of the cluster. Thus, the first step is to decide whether existing instrumentation on the site is sufficient to provide the information needed. The amount of instrumentation required to characterize the flow through a cluster will depend on the size of the machines, their number, the size of the cluster area, and the nature of the terrain in and around the site. The decision on whether to upgrade the instrumentation will again require examination of the trade-offs between the need for detailed documentation of the wind resource and the need to keep costs reasonable.

Techniques that could be used to determine whether to upgrade the existing instrumentation system include subjective interpretation of how the flow varies over the site (see Chapter 5 ) and, if the complexity of the problem warrants, numerical or physical modeling (Chapters 3 and 4 ). If the decision 
is to upgrade the instrumentation system, numerical and physical modeling can be very helpful in planning the layout of the new system, particularly for sites in hilly areas.

If the decision is to upgrade the instrumentation system, the adequacy of the new system layout should be evaluated after a year of operation. The same analysis techniques would be used in this evaluation as were used in the initial layout. The evaluation could indicate a need to redesign the layout, particularly for cluster sites in complicated terrain. If a new layout is required, the instrumentation are redeployed and the cycle repeated. During each year of measurement the interannual variability of key wind characteristics is analyzed (or the interannual variability of the economic value computations is examined) and a decision made on when to de-emphasize measuring and to start installing machines. The length of the measurement period will depend, as discussed in Section 2.4.4, on both the nature of the wind resource and the nature of the utility.

There are several reasons for upgrading instrumentation systems; one of the major ones is to improve estimates of energy production by the cluster. In the site evaluation stage, energy production estimates will be made with the minimum instrumentation necessary. One must now judge whether this minimum is sufficient. If energy production estimates in the site evaluation stage were based on hub-height data, one should consider whether these estimates would be improved significantly by better documentation of the flow through the rotor disk. This decision will obviously be influenced by the size of the machines under consideration and the probability of experiencing significant wind shear at the site. Another consideration would be the necessity of increasing the number of locations where wind measurements are made within the cluster. This decision will be influenced by cluster size and terrain complexity. In order to improve energy production estimates, upgrading the instrumentation system would only involve increasing the number of levels and the number of locations where wind measurements are made. Increasing the sampling rates over that used in site evaluation would not be required.

Another reason for upgrading instrumentation would be to evaluate the possible influences of minute-to-minute fluctuations in cluster output on the dynamics of the utility system (Zaininger 1980). Modeling fluctuations in 
cluster output on the time scale of minutes could require documentation of variations in wind behavior around the cluster. Modeling the minute-to-minute behavior of a cluster also requires an algorithm to transform fluctuations in wind speed and direction into fluctuations in electrical output. At the present time, there are no verified algorithms for this purpose; hence, the exact data requirements for this type of simulation are also unknown. In any respect, one should determine whether fluctuations in the output of a turbine cluster would have any significant impact on the utility system before upgrading the instrumentation system, and gathering the data needed to simulate these fluctuations. A gross analysis of the importance of the problem can be accomplished through a parametric analysis of the dynamic behavior of the system (Zaininger 1980).

Some of the wind characteristics most important in affecting the operation of wind turbines at a site include wind direction variability and the behavior of the wind speed in the neighborhood of a machine's cut-in and cut-out speeds. As discussed in the previous section, these characteristics govern the number of startup, shutdown and yawing operations which could, in turn, affect operation and maintenance costs as well as service life of the equipment. Data for estimating the average number of startup, shutdown and yawing operations per year will have been collected in the site evaluation stage of site selection-if data for a full simulation of machine operation have been collected. In the site development stage, it must be determined whether this data is representative of conditions over the entire site.

Other wind characteristics that could affect the successful operation of wind generators at a site are frequent, extreme wind gusts and turbulence. (a) Sufficient information for judging whether a site is subject to an unreasonable number of extreme wind events will have been collected during the site evaluatiol stage.

(a) In this context, wind gusts are defined as events occurring at scales much larger than the size of the rotor disk. As "seen" by a machine, a gust is a sudden increase in wind speed that is uniform across the rotor disk. Turbulence, on the other hand, is composed of wind events occurring at scales comparable to the size of the rotor disk. 
High levels of turbulence can usually be avoided by not siting wind turbines near obstacles or near topographic features known to promote separation (see Chapter 5). In addition, some information on turbulence levels at hub height will have been collected during the site evaluation phase and would be used to avoid sites with unacceptably large turbulence levels. Hub-height data, however, do not always give a clear impression of conditions over the entire rotor disk, and generic guidelines are known to have exceptions. Hence, these procedures may not always guarantee that a site is free of turbulence-related problems.

There has not been sufficient experience with large wind turbine generators to establish how important turbulence might be as a factor in siting. The scales of turbulence important to wind turbines are on the order of the size of the rotor disk. Since wind speed and direction vary across the rotor disk and since the wind vector at any point in space varies on a time scale of seconds, the blades of a wind turbine will experience fluctuating, differential loads. Many persons associated with the design of large wind turbines believe that turbulence-related loads are not significant when compared with other design loads. The argument is that the machines will be sufficiently robust to be unaffected by turbulence at any practical site; thus, collecting turbulence data would be an unnecessary expense. However, the magnitude of the fluctuating wind shears a machine will experience depends on the size of the rotor disk, and there has been little experience with really large machines. It is also known that turbulence near the earth's surface varies with time of day, the season of the year and the nature of the surrounding terrain. But, there is little detailed understanding of turbulence behavior in the lowest 100 to $150 \mathrm{~m}$ of the atmosphere except over smooth ground.

If turbulence is measured at a site, the purpose would be to document those aspects that could affect operation and maintenance costs of wind turbines or their service life. Proper documentation of the relevant characteristics requires representative measurements of the flow across the rotor disk of the machine in question. This requires a meteorological tower of about the same height as the top of the rotor disk. The tower should be instrumented at a 
minimum of three levels with anemometers sufficiently responsive to resolve the important scales of motion (a distance constant of about $5 \mathrm{~m}$ is acceptable; see Chapter 10). The data should be collected and displayed in such a way that a manufacturer could interpret the data's probable impact on performance and service life.

One way of cataloging turbulence information would be joint probability distributions of the changes in wind speed and wind direction across the rotor disk as a function of mean hub-height wind speed (or wind speed ranges). These distributions should be formed with 5- to 10-sec average data, so that the changes will be representative of eddies completely engulfing the rotor. Much of the data could be processed on site by smart data loggers in order to reduce the amount of data to be archived.

Once sufficient data have been collected to understand the distribution of the wind resource over the site, the individual machines in the cluster can be located. Final location of each machine will require consideration of the effects of turbine wakes on the performance of individual machines in the cluster and on the performance of the cluster as a whole. Chapter 9 discusses the question of wind turbine wakes in some detail and suggests a procedure for designing the layout of a cluster and predicting its performance once the design is completed.

\subsection{CONCLUSIONS AND RECOMMENDATIONS}

There are, basically, two approaches to siting wind turbines--wind prospecting and evaluating a predetermined site. The steps involved in each of the two approaches are:

Wind Prospecting

1. Analyze Region of Interest

2. Evaluate Candidate Resource Areas

3. Screen Potential Candidate Sites

4. Evaluate Candidate Sites

5. Develop Site 


\section{Evaluating a Predetermined Site}

1. Establish Feasibility

2. Evaluate Site

3. Develop Site.

A number of techniques have been identified that can be applied to the various steps in each siting approach. Not all techniques are applicable to each step in the two approaches. Figures 2.1 and 2.2 indicate the applicability of various siting techniques to the different steps in prospecting and evaluating a predetermined site. An intelligent siting strategy entails applying appropriate siting techniques to the relevant steps in the siting process in a logical and economical fashion.

To illustrate the siting process, a strategy was proposed for wind energy prospecting. The decision process involved in the strategy is illustrated in Figure 2.3. A strategy for evaluating a predetermined site was not described in detail since that approach is simply an abridgment of the prospecting process.

\subsubsection{Data Requirements For Siting}

Wind data requirements are an item of particular interest in wind turbine siting. They change with each step in the siting process and vary according to which siting techniques are being applied. A summary of wind data requirements for each step in the wind prospecting approach are:

2.5.1.1 Analyzing the Region of Interest. Analysis on this geographical scale is accomplished with existing wind information. Wind resource assessments have been prepared for each state in the United States. These should provide an adequate data base for this stage of the site selection process.

2.5.1.2 Evaluating Candidate Resource Areas. Wind data requirements for this stage depend on whether the goal is to carry out a rigorous analysis of the entire candidate resource area using a numerical model or simply to document the wind resource at what are believed, a priori, to be the windiest locations within the region. The latter option, though simpler, entails more risk of error. Typical data requirements for a model-based analysis are: 
- synchronous 1-hour averages of surface wind speed and direction at key locations in region; i.e., at major valleys parallel to prevailing flow, major passes, major ridges perpendicular to prevailing flow

- representative vertical profiles of wind speed, direction, and temperature.

If a rigorous analysis of the flow is not performed, data requirements are less stringent. Useful screening information can be obtained with wind-run anemometers. However, summarized information on hourly wind speed and direction is more useful.

2.5.1.3 Screening Potential Candidate Sites. In screening a large number of potential candidate sites, wind profile measurements from inexpensive systems such as tethered balloons or kites are recommended. This data is used to look for large wind shear or high turbulence levels.

2.5.1.4 Evaluating Candidate Sites. If a large number of sites remain after the potential candidate sites have been screened, a preliminary site evaluation program may be necessary. The objective would be to verify the existence of a usable wind resource, and the program would use inexpensive equipment with anemometers mounted on short masts. Wind run anemometers, mechanical weather stations or simple, rugged anemometers combined with smart data loggers would be suitable hardware.

The objective of the full site evaluation program is to collect the wind data needed for determining the economic value of wind-generated electricity at each candidate site. The data requirements are 1 to 2 -min averages of the two horizontal components of the wind vector at hub height for the machines i $n$ question. Some knowledge of the temperature behavior at the site is needed in order to determine air density. It is possible that collecting data at several levels across the rotor disk could improve energy production estimates for very large machines. However, analysis of wind and performance data from several experimental installations will be needed before it can be determined if wind data collection at more than one level is economically justified.

2.5.1 .5 Developing a Site. This stage of the siting process requires sufficient knowledge of the flow over the site that the location of each 
machine can be determined. For large sites where the local terrain relief(a) is on the order of the size of the machines, measurements at several locations could be required. Numerical and physical modeling of the flow over the site can be useful in determining if this is the case. If wind data have been collected at an insufficient number of locations, an upgrading of the measurement system is necessary. However, the type of data collected (e.g., 1- to 2-min averages at hub height of the two horizontal components of the wind vector) would be the same as in the site evaluation stage.

Another goal in this stage of siting is to obtain final assurance that there is no wind behavior, unique to the site, that could adversely affect wind turbine operations or turbine service life. The wind characteristics most likely to fall in this category are an excessive number of extreme gust events or excessively high turbulence. Sites experiencing an excessive number of extreme gusts should be eliminated during the candidate site evaluation process. Documenting turbulence characteristics at a site would only be considered for very large machines, since it is unlikely that machines having rotor disks less than $50 \mathrm{~m}$ in diameter would experience significantly large, fluctuating wind shears. If turbulence is documented, a tall tower, about as high as the turbines in question, is required. The tower should be instrumented a three levels with reasonably responsive anemometers (about a $5 \mathrm{~m}$ distance constant). One anemometer should be at hub height and the other two should be near the top and bottom of the rotor disk. The data must be processed so that the results could be interpreted by manufacturers in terms of probable effects on operation and maintenance and service life.

\subsubsection{Length of Measurement Period}

In a siting program, wind measurement has two objectives: to determine whether wind turbines installed at a particular site will be an economically viable investment and to ensure that turbines installed at a particular site will be operationally successful. Wind data should be collected for as long as it takes to resolve these issues. Determining the economic value of windgenerated electricity at a particular site will undoubtedly be the factor that governs the length of the measurement program.

(a) The typical elevation differences between low points and high points. 
If a decision to install wind turbines can be based on the net energy produced over a given period, a single year of data collection should be sufficient. With a year's data, a reasonable estimate of average annual energy production can be made, and it is fairly easy to make reasonable estimates of the uncertainty in this estimate.

If the economic value of wind-generated electricity is a strong function of how the turbines modify the utility's load, more than a year's data collection could be required. It depends on how the wind characteristics that affect load modification (such as, the diurnal and seasonal modulation of the wind) vary from year-to-year. A decision on how long to measure would rest on the differentials among the cost of wind-generated electricity, the utility's production costs and on the sensitivity of economic value to these uncertainties. The decision on when to start installing machines would depend on each utility's assessments of the costs and the risks. 


\section{REFERENCES}

Corotis, R. B. 1980. Handbook for the Application of Statistical Techniques to Wind Characteristics at Potential Wind Energy Conversion Sites. DOE/ET/20283-3, Available from National Technical Information Service, Springfield, Virginia.

General Electric Company. 1979. Reauirements Assessment of Wind Power Plants in Electric Utility Systems. EPRI ER-978-SY (3 volumes), Electric Power Research Institute, Palo Alto, California.

Golding, E. W. 1955. The Generation of Electricity by Wind Power. John Wiley \& Sons, New York.

JBF Scientific Corporation. 1979. Wind Energy Systems Application to Regional Utilities. U.S. DOE Contract EX-76-C-01-2438, JBF Scientific Corporation, Wilmington, Massachusetts.

Miller, A. H. 1980. "Implications of the Effects of Wind Characteristics on the Operation of Large Wind Turbines." in Proceedings of the 15th Intersociet, Energy Conversion Engineering Conference, American Institute of Aeronautics and Astronautics, New York.

Neustadter, H. E. 1979. "The Use of Wind Data With an Operational Wind Turbine in a Research and Development Environment." In Proceedings of the Conference and Workshop on Wind Energy Characteristics and Wind Energy Siting 1979, American Meteorological Society, Boston, Massachusetts.

Putnam, P. C. 1948. Power From the Wind. Van Nostrand, New York.

Robbins, W. H. and D. H. Baldwin. 1980. "Large Wind Turbines: A Utility Option for Generation of Electricity." 1980 Solar Program Review Meeting, Electric Power Research Institute, Palo Alto, California.

Van Kuiken, J. C., W. A. Buehring, C. C. Huber, and K. A. Hub. 1980. Reliability, Energy, and Cost Effects of Wind-Powered Generation Integrated With a Conventional Generating System. ANL/AA-17, Argonne National Laboratory, Argonne, Illinois.

Wade, J. E. and E. W. Hewson. 1979. "Trees as a Local Climatic Wind Indicator." J. Appl. Meteor. 18:1182-1187.

Zaininger, H. W. 1980. Wind Power Generation Dynamic Impacts on Electric Utility Systems. EPRI AP-1614, TPS 79-775, Electric Power Research Institute, Pa1o Alto, California. 


\subsection{NUMERICAL MODELING}

Because of the spatial variability of the wind resource, wind data from fixed observing stations can only be used to evaluate the wind energy potential at nearby sites. In some cases, wind data from well-exposed stations in flat, homogenous terrain may be representative of conditions over a large area. In other cases, wind data from one location may be unrepresentative of conditions 100 meters away. Clearly, techniques are needed to gauge the effect of terrain on wind characteristics and to estimate critical wind characteristics at locations remote from the nearest observing station.

Numerical models provide an objective method for estimating the effects of terrain on airflow and for interpolating wind data from locations where there are observations to locations where there are none. To do this, the models find solutions to equations that describe the complex interactions between the atmosphere and the earth's surface. The accuracy achieved in these solutions depends on the accuracy and the density of the input data, as well as the amount of realism inherent in the mathematical relationships that make up the model.

Numerical models can be applied to the siting process in three ways: to screen mesoscale areas (dimensions on the order of $10,000 \mathrm{~km}^{2}$ ) for subregions that experience the highest winds, to estimate wind characteristics at specific predetermined sites, and to understand how the flow over a cluster site might affect the placement of each machine. This chapter reviews two basic types of models that have been proposed for use in site screening and site evaluation: kinematic and primitive equations. The results of experiments to verify these models are also reviewed, and a critique of the models' capabilities is given. However, before the models are presented, equations governing the dynamics of the atmosphere are discussed.

\subsection{GOVERNING EQUATIONS}

The dynamics of the atmosphere can be described by the equations of motion for a viscous, compressible Newtonian fluid in a uniform gravitational field. When these equations are written in a coordinate system fixed with respect to 
the earth's surface (i.e., a rotating or noninertial coordinate system), the equations of motion are:

$$
\begin{gathered}
\frac{\partial u_{i}}{\partial t}+u_{j} \frac{\partial u_{i}}{\partial x_{j}}=-\frac{1}{\rho} \frac{\partial P}{\partial x_{i}}-g \delta_{3 i}-2 \varepsilon_{i j k} \Omega_{j} u_{k} \\
+\frac{1}{\rho} \frac{\partial}{\partial x_{k}}\left(\mu \frac{\partial u_{i}}{\partial x_{i}}-\frac{2}{3} \mu \delta_{i k} \frac{\partial u_{j}}{\partial x_{j}}\right)
\end{gathered}
$$

The terms on the left of Equation (3.1) represent the acceleration of an arbitrary fluid element and the terms on the right describe the forces, per unit mass, acting on the element. The first term on the right-hand side of Equation (3.1) is the pressure force, the second term represents the force caused by gravitational acceleration, ${ }^{(a)}$ the third term is the Coriolis force, and the last term represents viscous forces.

The equations of motion have been written in the Einstein summation convention. Thus, a term with a single subscript or index (e.g., $u_{i}$ ) represents the ith component of a vector quantity. If an index is repeated within a given term, the term is summed over all values the index can assume, for example:

$$
u_{j} \frac{\partial u_{i}}{\partial x_{j}}=u_{1} \frac{\partial u_{i}}{\partial x_{1}}+u_{2} \frac{\partial u_{i}}{\partial x_{2}}+u_{3} \frac{\partial u_{i}}{\partial x_{3}}
$$

The terms $\delta_{i j}$ and $\varepsilon_{i j k}$ are the Kronecker delta and the alternating unit tensor, respectively. The Kronecker delta, $\delta_{i j}$, is zero if $i \neq j$ and unity if $i=j$. The alternating unit tensor, $\varepsilon_{i j k}$, is zero if at least two of the three indices are the same, +1 if $i, j$ and $k$ form a cyclic permutation, and -1 if $i, j, k$ are in a noncyclic order. Note that $\varepsilon_{i j k} \Omega_{j} u_{k} \equiv \vec{\Omega} \times \vec{u} .(b)$

In addition to the equations of motion, expressions representing the conservation of mass, energy (or, as written below, entropy), and water vapor are

(a) Actually, g is the effective gravitational acceleration, which is less than actual gravity because of the effects of the centrifugal acceleration of the earth's rotation.

(b) See the glossary for the definition of terms not explained in the text. 
needed to describe the complete atmospheric system. These equations are, respectively:

$$
\begin{gathered}
\frac{\partial \rho}{\partial t}+\frac{\partial}{\partial x_{i}}\left(\rho u_{i}\right)=0 \\
\frac{\partial \theta}{\partial t}+u_{i} \frac{\partial \theta}{\partial x_{i}}=\frac{\Theta}{\rho C_{p}} \frac{\partial}{\partial x_{i}}\left(\frac{k_{T}}{T} \frac{\partial T}{\partial x_{i}}\right)+\dot{I}+S_{\Theta}
\end{gathered}
$$

and

$$
\frac{\partial q}{\partial t}+u_{i} \frac{\partial q}{\partial x_{i}}=\frac{\partial}{\partial x_{i}}\left(k_{q} \frac{\partial q}{\partial x_{i}}\right)
$$

In Equation (3.3), $\theta=T\left(P_{0} / P\right)^{R / c_{p}}$ is a measure of entropy (called the potential temperature) and $I$ is the production of entropy as a result of viscous dissipation and other irreversible processes. The term $S_{\Theta}$ represents the rate of entropy production caused by internal heat sources or heat sinks. Equations (3.1) through (3.4) have been written assuming that the water content of the atmosphere is always in the vapor phase. Thus, $S_{\Theta}$ results from the absorption or emission of thermal radiation by the atmosphere.

\subsection{SIMPLE KINEMATIC MODELS}

Obtaining numerical solutions to Equations (3.1) through (3.4) is a formidable problem and many attempts have been made to find simplified approaches. These approaches try to simplify the equations of motion while still retaining most of the essential physics contained in the full equations. This section describes a simplified approach that has received a great deal of attention as a possible siting tool.

Meteorologists have long been faced with the problem of producing weather maps from data collected at widely scattered, and unevenly spaced, observing stations. Historically, these maps were produced by hand, and the interpolation of meteorological quantities between stations was done subjectively. With the advent of digital computers, much effort has been spent on automating this process. 
Sasaki (1958) proposed one of the early schemes for producing objectively analyzed wind fields. His approach was modified for use in producing wind fields over topography by Sherman (1978). Sherman's method starts with all available observations of the wind vector within the region of interest. An initial guess of the wind field is then made at every point in a three-dimensional grid that covers this region. The initial guess is obtained by an arbitrary, ad hocinterpolation scheme. This initial guess of the wind field is then adjusted until the equation of continuity is satified for every grid element. At grid points closest to the earth's surface, the wind vector must be parallel to the earth's surface. Because the approach outlined above produces wind fields satisfying the equation of mass continuity, models of this type are often called mass consistent.

\subsubsection{Mass-Consistent Objective Analysis of Wind Fields}

A mass-consistent objective analysis scheme can be formulated as a problem in the calculus of variations. For example, if $u_{i 0}$ is the initial guess of the ith velocity component at a given point and $u_{i}$ is the final, modified value, the sum of the squares of the differences between $u_{i 0}$ and $u_{i}$ at a given point is:

$$
\alpha_{i}^{2}\left(u_{i}-u_{i 0}\right)^{2}
$$

where $\alpha_{i}^{2}$ are specified weighting factors. (The physical significance of these weighting factors will be explained below.) Given the quantity (3.5), a functional, $\mathrm{H}$, is defined by integrating expression (3.5) over the entire volume of interest, i.e.,

$$
H=\int_{V} \alpha_{i}^{2}\left(u_{i}-u_{i 0}\right)^{2} d V
$$

The modified velocity field, $u_{i}$, must satisfy the equation of continuity (Equation 3.2), which can be rewritten as:

$$
\frac{\partial u_{i}}{\partial x_{i}}+\frac{1}{\rho}\left(\frac{\partial \rho}{\partial t}+u_{i} \frac{\partial \rho}{\partial x_{i}}\right)=0
$$


Since the terms in parentheses are generally very small in most flows (see Section 3.3.1), the equation of continuity is approximately

$$
\frac{\partial u_{i}}{\partial x_{i}}=0
$$

The initial guess of the wind field, $u_{i 0}$, is obtained by interpolating actual wind observations over the volume of interest. Therefore, our initial guess of the field is assumed to contain valuable information on the true wind structure. The smallest adjustments possible that will result in a final wind field that satisfies continuity should be made to the initial field. In other words, Equation (3.6) should be minimized subject to the constraint that

$$
\int_{V} \frac{\partial u_{i}}{\partial x_{i}} d V=0
$$

This problem can be solved by Lagrange's method of undetermined multipliers (Mathews and Walker 1964). By this technique, minimizing Equation (3.6) subject to the constraint given in Equation (3.9) is equivalent to minimizing the functional

$$
\begin{gathered}
I(u, v, w, \lambda)=\iiint\left\{\alpha_{1}^{2}\left[\left(u-u_{0}\right)^{2}+\left(v-v_{0}\right)^{2}\right]\right. \\
\left.+\alpha_{3}^{2}\left(w-w_{0}\right)^{2}+A \frac{\partial u_{i}}{\partial x_{i}}\right\} d x d y d z
\end{gathered}
$$

where

- $x, y, z$ are cartesian coordinates fixed with respect to the earth's surface

- $u, v, w$ are the modified velocity components in the $x, y, z$ directions, respectively

- $u_{0}, v_{0}, w_{0}$ are the corresponding initital values

- $\lambda(x, y, z)$ is the Lagrangemultiplier. In Equation (3.10), $\alpha_{1}$ and $\alpha_{2}$ have been set equal. 
The weighting factors $\alpha_{1}$ and $\alpha_{3}$ govern the size of the wind field adjustments needed to minimize Equation (3.10). The size of the adjustment will be inversely proportional to the magnitude of the weighting factor. In principle, the weighting factors can be made a function of their position relative to the measurement locations. If, for instance, they are made large near the locations of actual wind measurements, the adjustment to the initial wind field will be small. Reducing the size of the weighting factors as the distance from the nearest measurement location increases will cause the largest adjustments to be made at the grid locations where the initial guess is most uncertain.

The most important role of the weighting factors, however, is to simulate the effects of thermal stratification. Except very near the earth's surface, the atmosphere is always stably stratified; thus, buoyancy forces will resist vertical motion. When encountering a large isolated topographical feature, the air is more likely to flow around the object than over it. As a result of stable stratification, the mean magnitude of vertical motions in the atmosphere (averaged over several minutes, for example) are on the order of $10^{-2}$ to $10^{-3} \mathrm{~m} / \mathrm{sec}$, except very near abrupt topographical features or within cumulus clouds. Horizontal motions, however, are on the order of meters per second. As the functional defined in Equation (3.10) is minimized, the vertical component should not change by more than $10^{-2} \mathrm{~m} / \mathrm{sec}$, whereas the horizontal components may change as much as several meters per second. If the magnitude of $\alpha_{1}$ is on the order of unity, $\alpha_{3}$ should be a factor of 100 larger in order to achieve the desired result.

Equation (3.10) can be minimized if the following equations are solved:

$$
\begin{gathered}
2 \alpha_{1}^{2}\left(u-u_{0}\right)-\frac{\partial \lambda}{\partial x}=0 \\
2 \alpha_{1}^{2}\left(v-v_{0}\right)-\frac{\partial \lambda}{\partial y}=0 \\
2 \alpha_{3}^{2}\left(w-w_{0}\right)-\frac{\partial \lambda}{\partial z}=0 \\
\frac{\partial u}{\partial x}+\frac{\partial v}{\partial y}+\frac{\partial w}{\partial z}=0
\end{gathered}
$$


subject to the boundary conditions,

$$
\lambda \delta(\vec{V} \cdot \hat{n})=0
$$

where $\delta()$ is the variation of the quantity in parentheses (Lass 1957) and $\hat{n}$ is a unit vector normal to the boundary surface. Setting $\lambda \equiv 0$ at the boundaries is appropriate for "open" or "flow-through" boundaries, while making $\delta(\vec{V} \cdot \hat{n}) \equiv 0$ allows no adjustment to the velocity component normal to the boundary surface. As can be seen from Equations (3.11) through (3.13), the latter boundary condition is equivalent to

$$
\hat{n} \cdot \nabla \lambda=0 \text {. }
$$

\subsubsection{Data Requirements and Initialization}

Before a mass-consistent objective analysis scheme can create an adjusted wind field for the area in question, an initial guess must be supplied at every grid point in the domain of the model. To make an initial guess, information on topography and the flow patterns within the area is needed. Information on topography must be at a resolution compatible with the grid size of the model. In most applications, data on flow patterns will be limited to a few observations of surface wind speed and direction. Sometimes, information on wind structure above the surface is available from a nearby rawinsonde station.

When only surface wind information is available, one possible procedure for initializing the model is to create a wind profile, or sounding, at every point having a surface wind observation. A common way to do this is by an ad hoc extrapolation scheme, such as

$$
\begin{aligned}
& S(z)=S\left(z_{0}\right)\left(\frac{z}{z_{0}}\right)^{p}, z \leq z_{b 1} \\
& S(z)=S\left(z_{b 1}\right), z_{b l} \leq z \leq z_{\max }
\end{aligned}
$$


where

- $S(z)$ is the wind speed at a height $z$ above the surface

- $z_{b l}$ is an arbitrarily chosen boundary layer depth

- $z_{\max }$ is the top of the model domain

- $p$ is frequently chosen as 0.14.

In using Equation (3.17) as an extrapolation method, the wind direction is usually held constant. After a vertical sounding is produced over each wind observation station, the data are interpolated horizontally to fill the remaining grid points.

If a rawinsonde sounding is available, it can be used to aid in the vertical extrapolation of surface wind data. One possible approach assumes that in addition to the sounding, both wind speed and direction are available at each of the points having surface wind data. If $R$ is the elevation (relative to sea level) of the rawinsonde station and $P$ the elevation of the station that has only surface observations, then for $P>R, z_{b}=H$, where $H$ is an arbitrary height above the surface (on the order of $100 \mathrm{~m}$ ). For $P<R, z_{b}=$ $\mathrm{R}-\mathrm{P}+\mathrm{H}$. Above $\mathrm{z}_{\mathrm{b}}$, the wind speed and direction are adjusted to the sounding by a linear interpolation scheme. This scheme is

$$
q(z)=q_{u a}(z)+\left[q\left(z_{b 1}\right)-q_{u a}\left(z_{b l}\right)\right] \frac{\left(z_{\max }-z\right)}{\left(z_{\max }-z_{b}\right)}
$$

where $q(z)$ is the quantity (speed or direction) at a height $z$ above the surface and $q_{u a}(z)$ is the quantity at $z$ according to the sounding.

Once a wind profile is produced above each observing station, the remaining points are again filled by a horizontal interpolation scheme, such as setting the wind components at the remaining points equal to a weighted average of all of the input values. A $1 / r^{2}$ weighting factor is frequently used where

$$
r^{2}=\left(x-x_{s}\right)^{2}+\left(y-y_{s}\right)^{2}
$$

and $x_{s}, y_{s}$ are the coordinates of each input station. This field provides the $u_{0}, v_{0}$, and $w_{0}$ values in Equations (3.11) through (3.13). 


\section{$\underline{\text { Verification Results }}$}

Since an objective analysis scheme based on the calculus of variations minimizes adjustments to the initial guess of the wind field, the accuracy and representativeness of the input data are crucial. However, estimates of how wel1 the model may perform in a given application can only be made subjectively by evaluating the performance of the model on similar problems and under similar conditions. Recently, two studies (Traci et a1. 1979; Endlich et al. 1980) were conducted to evaluate the ability of mass-consistent numerical models to simulate airflow over complex topography and to predict certain wind characteristics at potential wind turbine sites.

In the first of these two studies, a mass-consistent model was applied to areas where the network of surface wind measurements was reasonably dense. A subset of the surface wind observations was used to initialize the model and observed winds were then compared with simulated winds at locations not used in the initialization. Comparisons were made for a number of cases and for varying numbers of input data stations. The island of Oahu in Hawaii, the most populated of the Hawaiian Islands, was used as the site in this study. The island is small (approximately $1570 \mathrm{~km}^{2}$ in area) but rugged (see Figure 3.1). Its principal topographic features are ridges that lie along the eastern and western edges of the island and run north to south. The average elevation of the windward (eastern) ridge is about $600 \mathrm{~m}$. The leeward ridge is slightly higher. The maximum elevation on the island is $1225 \mathrm{~m}$.

Because it is situated in the northern portion of the northeastern tradewinds, Oahu is embedded in one of the steadiest, most homogeneous wind regimes on earth. It is also small enough that the effects of diurnal heating of the island on the flow patterns should be small in comparison to the effects of

topography. (a) The lateral boundary conditions of a model describing flow over Oahu should be fairly steady and reasonably well defined. Therefore, Oahu should provide a straightforward test of a model's ability to simulate the effect of topography on airflow.

(a) This is true, at least, for the scales of motion resolvable by models such as those described in this chapter. 


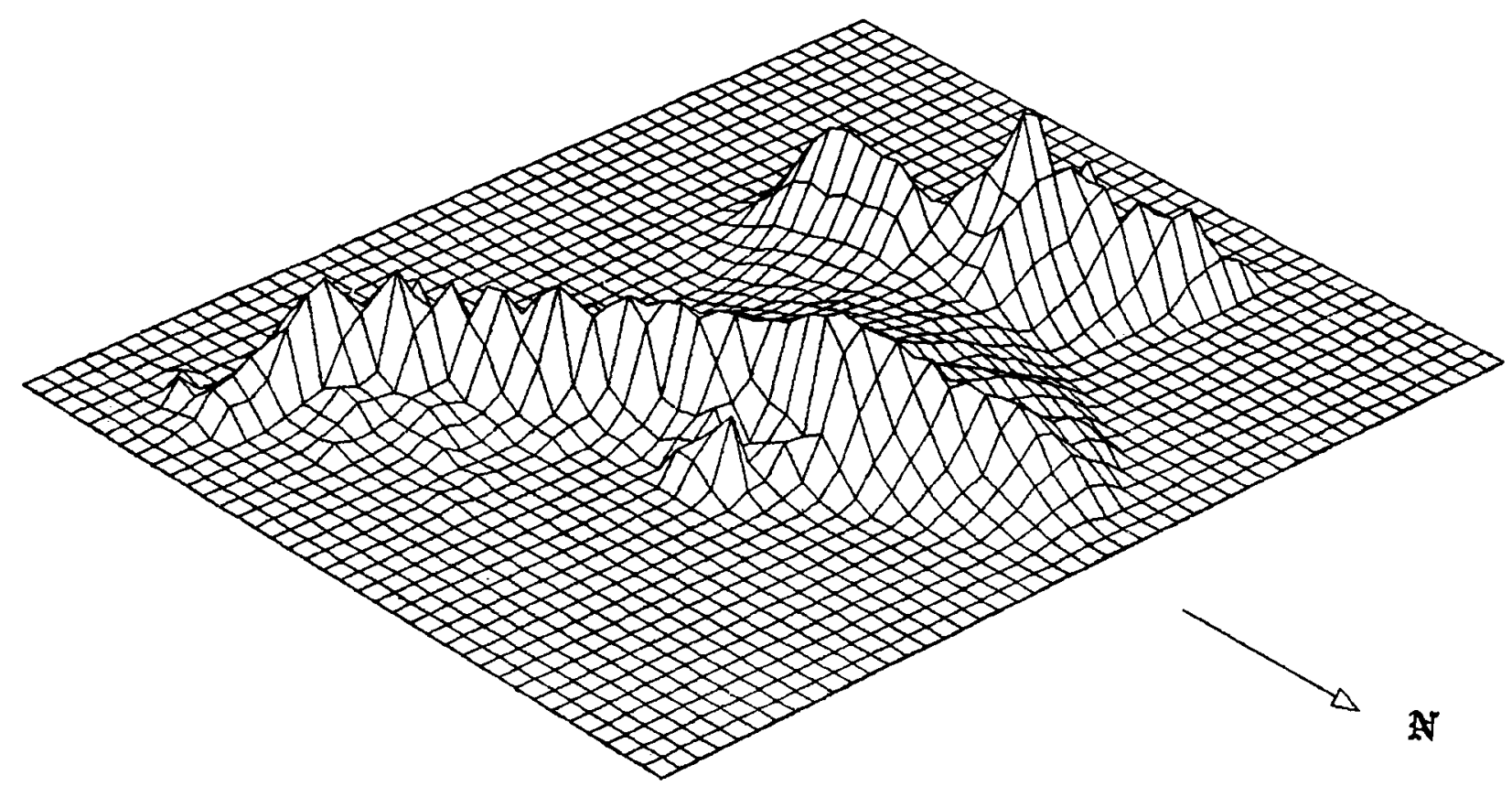

FIGURE 3.1. Perspective of the Terrain on Oahu, Hawaii, Looking Southwestward

First, the ability of a mass-consistent model, called NOABL, to simulate general features of the flow over Oahu was investigated (Traci et al. 1979). (a) Since NOABL is written in a nonorthogonal, terrain-conformal coordinate system, the form of the equations solved by the model differs from Equations (3.11) through (3.14) [see Traci et al. (1978), and Phillips (1979) for a complete description of the model].

In simulating the general features of tradewind flow over Oahu, NOABL was run with a grid having a resolution of $1600 \mathrm{~m}$ in the horizontal. The size of the modeled domain was $77 \mathrm{~km}$ in the east-west direction, $53 \mathrm{~km}$ in the northsouth direction, and $2 \mathrm{~km}$ in the vertical. The model was initialized by assuming a uniform wind direction of $065^{\circ}$ (wind from the north has a direction of $0^{\circ}$ ). A uniform wind speed of $10 \mathrm{~m} / \mathrm{sec}$ was assumed at $200 \mathrm{~m}$ above the surface. The wind speed followed a $1 / 7$ power law (see Equation 3.17a) below the 200-m level and was constant above it. The results of this simulation are shown in Figure 3.2. The figure shows a definite influence of the terrain on

(a) The model was developed for DOE by Science Applications, Inc. of San Diego, CA. 


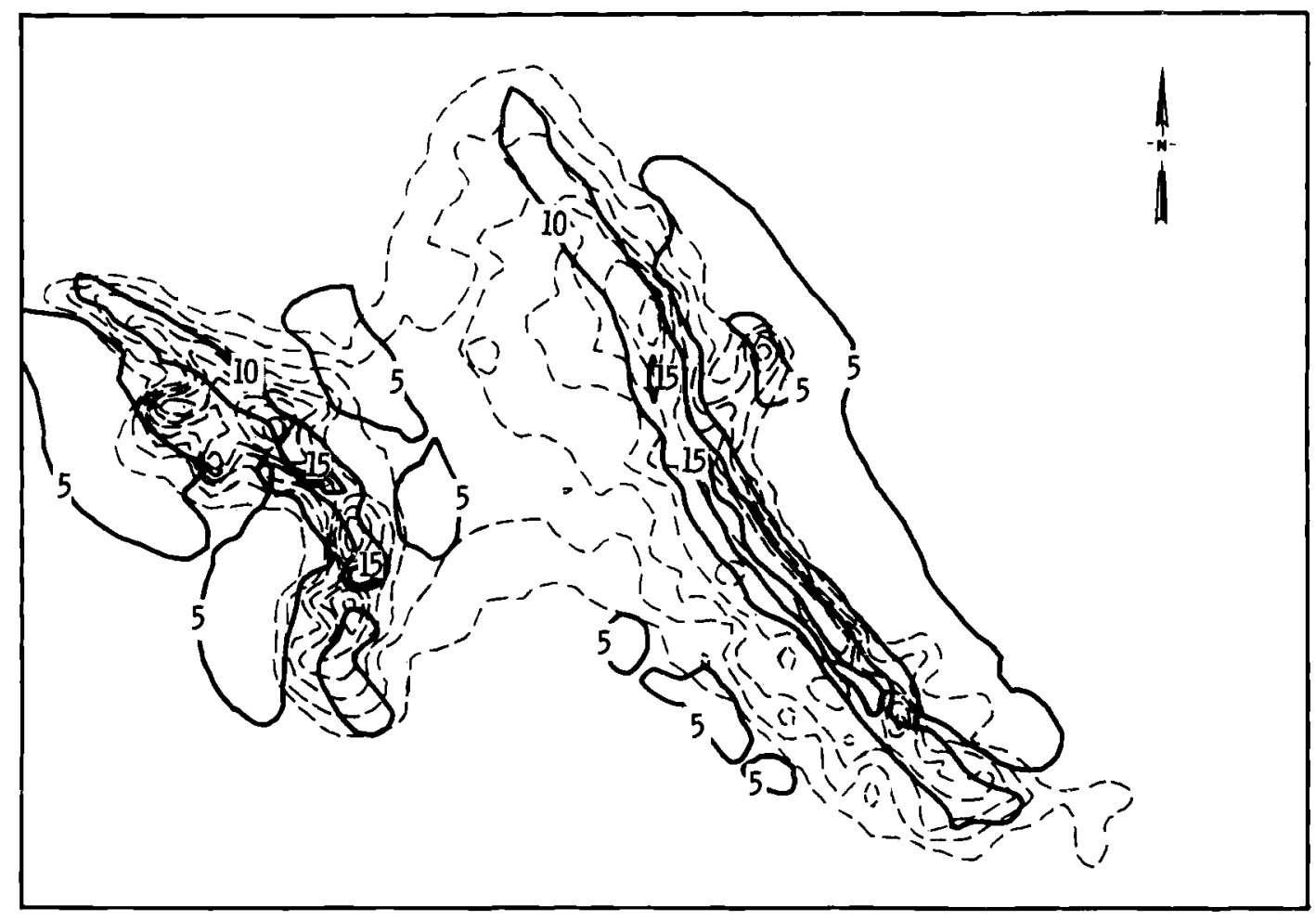

FIGURE 3.2. Wind Speed Contours at $15 \mathrm{~m}$ Above the Surface for Idealized Tradewind Flow Over Oahu. The free stream wind speed at $200 \mathrm{~m}$ was $10 \mathrm{~m} / \mathrm{s}$ and the wind direction was $65^{\circ}$.

the flow. Over the mountain ridges and around the points, wind speeds are enhanced; in the central valley and on the east side of the windward mountain range, wind speeds are much lower. These features correspond very well to observations of the surface wind pattern.

Since the hypothetical tradewind case indicated that gross features of flow over the island could be simulated with very crude input, a more quantitative comparison between model simulations and observations was undertaken. In this step, data was used from the Oahu surface wind network (Shinn et al. 1979). The data set consists of hourly averaged surface wind observations at as many as 21 locations on Oahu (see Figure 3.3). Measurements were made for a 24-month period starting in August 1976 and ending in July 1978. In addition to the surface wind observations, rawinsonde soundings were available twice a day from Lihue on the neighboring island of Kauai. 


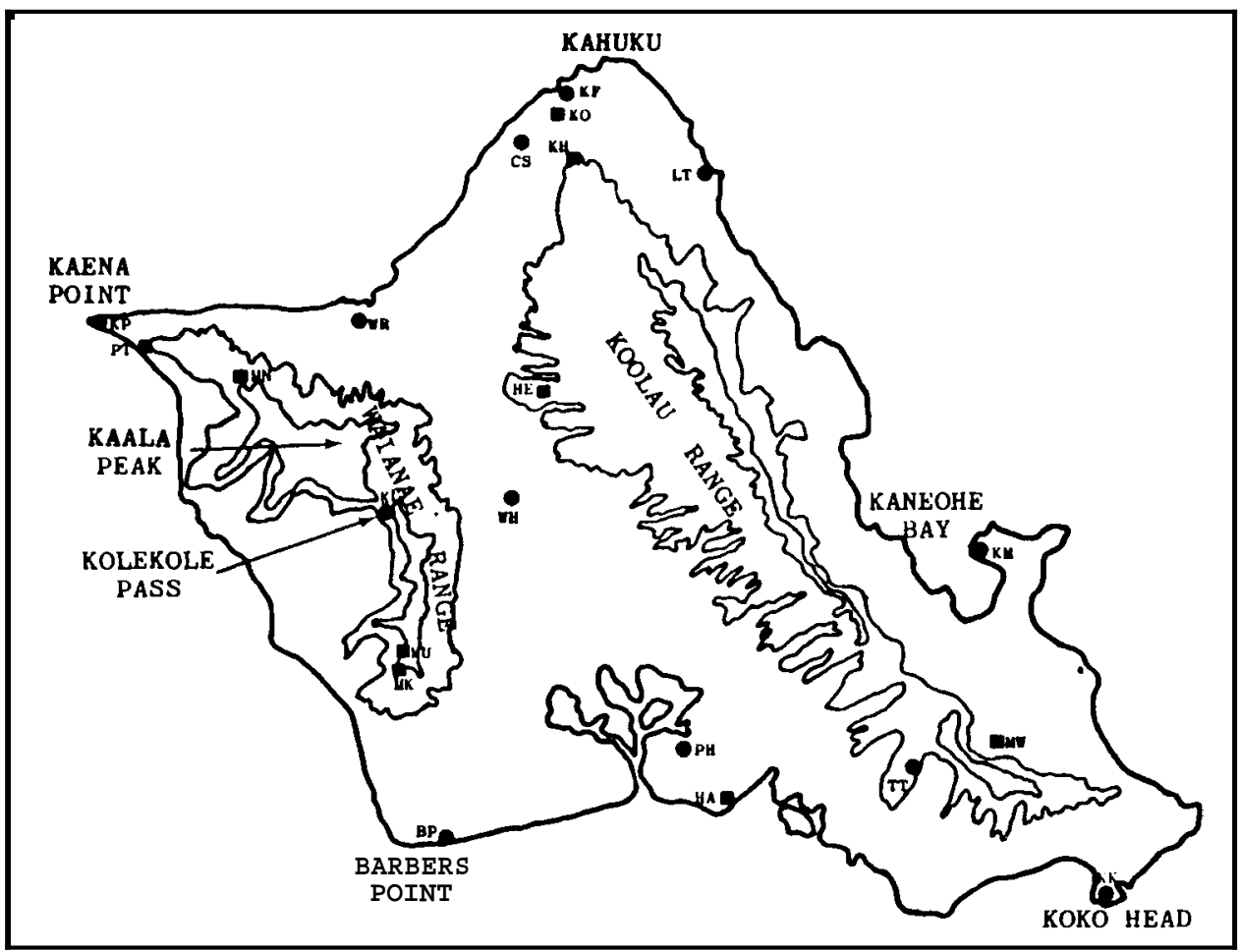

FIGURE 3.3. Oahu Topographic Schematic with Data Network Locations

From the first 12 months of measurements, 12 days (one for each month) were chosen as representative of conditions for the entire year. (a) NOABL was then used to produce simulated wind fields at two times on each of the 12 days. These times, 0200 and 1400 local time, corresponded to the times of the Lihue sounding. Three sets of 24 wind field simulations were made with differing amounts of input data: one set used up to six surface stations plus the Lihue sounding, another used as many as three surface stations plus the sounding, and the third was initialized with data from only the sounding. The stations used for the three cases are indicated in Table 3.1. The initial guess of the wind field was made using the procedure for input sets with rawinsonde soundings that was described in Section 3.2.2 [see Traci et al. (1978) for more details].

Figures 3.4 through 3.9 compare simulated wind speeds with those observed at stations not used to initialize the model. The simulated speeds are from the 15-m level, which is the level in the model closest to the level of the surface stations. The first three figures (3.4 through 3.6) compare all 24

(a) Private communication from C. S. Sherman, Lawrence Livermore Laboratory. 
TABLE 3.1. Oahu Data Base Surface Observation Stations

\begin{tabular}{|c|c|c|c|c|c|}
\hline & \multirow[b]{2}{*}{ Code } & \multicolumn{2}{|c|}{$\begin{array}{c}\text { UIM Coordinates } \\
(\mathrm{km})\end{array}$} & \multirow{2}{*}{$\begin{array}{l}\text { Ground } \\
\text { Level } \\
(\mathrm{m} \text { ASL) }\end{array}$} & \multirow{2}{*}{$\begin{array}{c}\text { Instrument } \\
\text { Height } \\
\text { (m AGL) }\end{array}$} \\
\hline & & North & East & & \\
\hline \multicolumn{6}{|l|}{ LLL Stations } \\
\hline Kahuku H i 11 & $\mathrm{KH}$ & 2396.68 & 603.17 & 300 & 10 \\
\hline Laie & LT & 2394.83 & 610.74 & 3 & 26 \\
\hline Mokuleia & MN & 2383.18 & 583.06 & 609 & 15 \\
\hline Waialua & WR & 2386.51 & 590.52 & 5.5 & 15 \\
\hline \multicolumn{6}{|l|}{ Permanent Stations } \\
\hline Barbers Point NAS & BP & 2355.98 & 595.48 & 3 & 3 \\
\hline Comsat Corp.-Paumalu & CS & 2396.98 & 599.84 & 137 & 15 \\
\hline Honolulu Airport-NWS & HA & 2358.27 & 612.50 & 2 & 7.6 \\
\hline Wheeler AFB & WH & 2375.90 & 599.12 & 250 & 3.7 \\
\hline Kaneohe MCAS & KM & 2373.02 & 626.40 & 2 & 4.5 \\
\hline Kaena Point-SAMTEC & $\mathrm{KP}$ & 2386.01 & 575.65 & 270 & 7.6 \\
\hline Pear 1 Harbor-FWC & $\mathrm{PH}$ & 2361.63 & 609.30 & 3 & 11 \\
\hline Mauna Kapu-USN & MK & 2366.58 & 592.88 & 782 & 23.2 \\
\hline \multicolumn{6}{|l|}{ Univ. of Hawaii Stations } \\
\hline Kahuku Field & KF & 2399.30 & 602.62 & 12 & 15 \\
\hline Kahuku Opana & KO & 2398.80 & 602.20 & 162 & 14 \\
\hline Koko Head & KK & 2352.57 & 634.36 & 195 & 11 \\
\hline Maunawili & MW & 2361.86 & 627.54 & 125 & 9 \\
\hline Mauna Kapu & MU & 2367.14 & 593.18 & 825 & 12 \\
\hline Helemano & $\mathbf{H E}$ & 2382.15 & 601.15 & 336 & 14 \\
\hline Tantalus & TT & 2360.20 & 622.80 & 596 & 21 \\
\hline Kolekole Pass & $\mathrm{KL}$ & 2375.28 & 591.60 & 527 & 29 \\
\hline Kaena Point Tower & PT & 2384.93 & 577.54 & 385 & 55 \\
\hline
\end{tabular}

simulations. The last three (3.7 through 3.9) compare means of the 24 simulations at each observing station. These figures show that considerable differences between simulated wind speeds and the observed hourly averages can exist at any given point. This is not surprising, since many of the physical processes affecting flow over terrain are not simulated in a mass-consistent model. Spatial resolution is also a factor in explaining these differences, since the wind speed simulations produced by a model represent an average over 


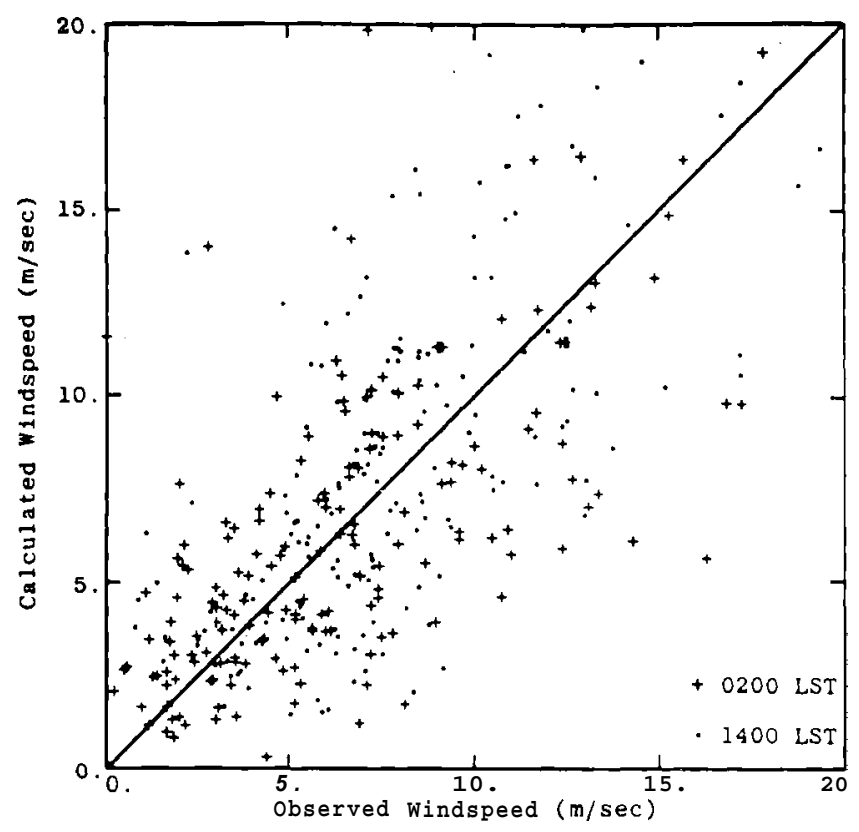

FIGURE 3.4. Comparison of Calculated to Observed Wind Speed for 24 Oahu Simulation Events (Simulation I: 6 surface stations plus upper air record. Source: Traci e t al. 1978).

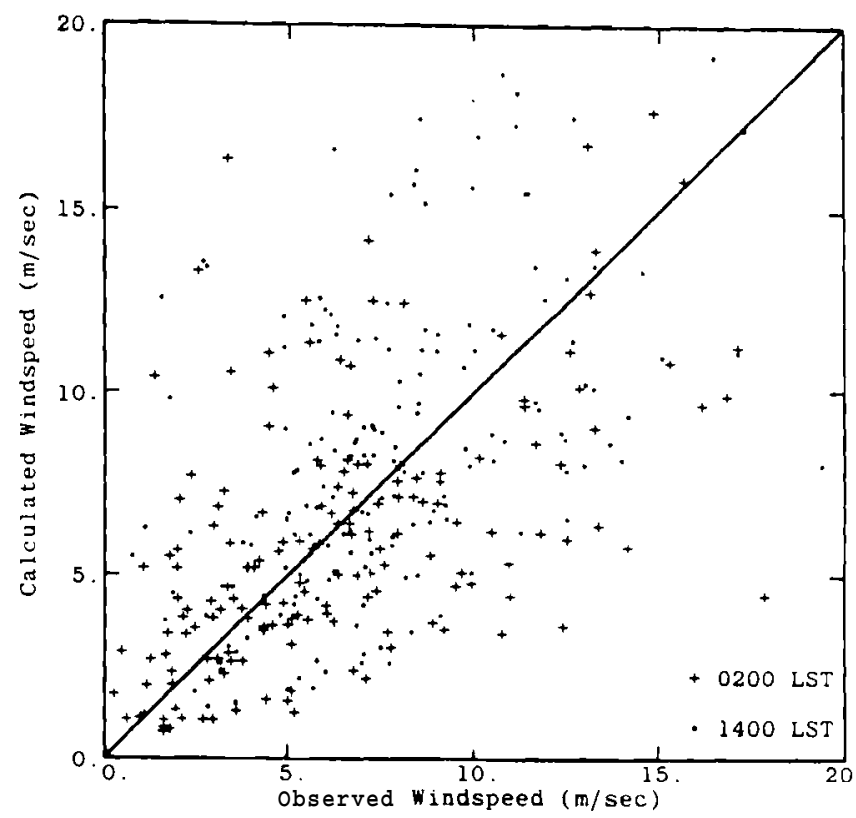

FIGURE 3.5. Comparison of Calculated to Observed Wind Speed for 24 Oahu Simulation Events (Simulation II: 3 surface stations plus upper air record. Source: Traci et al. 1978). 


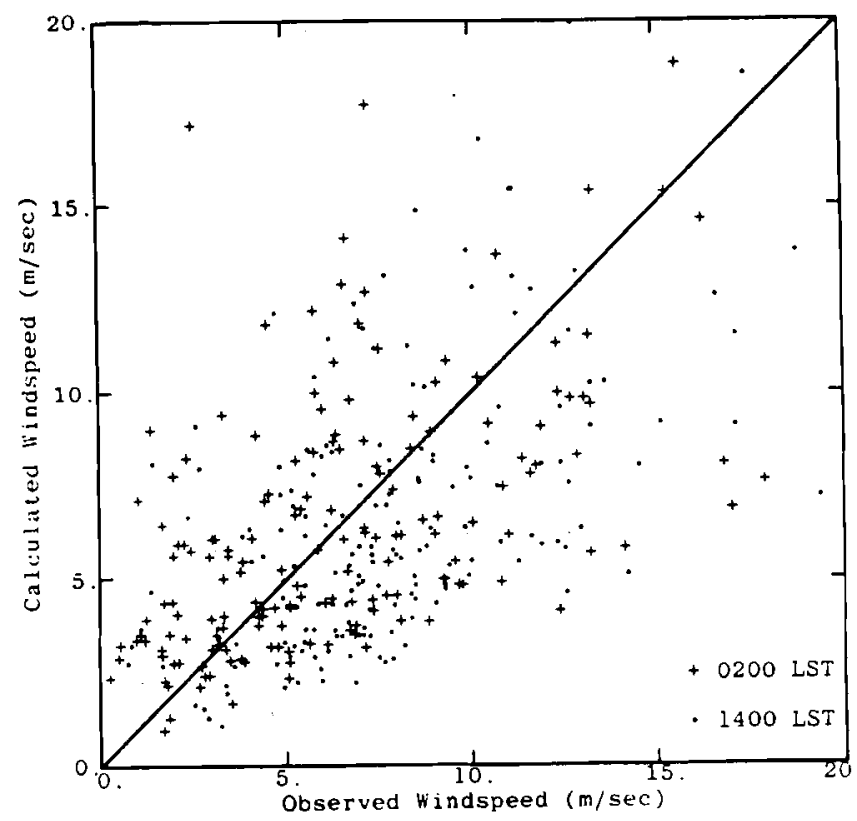

FIGURE 3.6. Comparison of Calculated to Observed Wind Speed for 24 Oahu Simulation Events (Simulation III: upper air record only. Source: Traci et al. 1978).

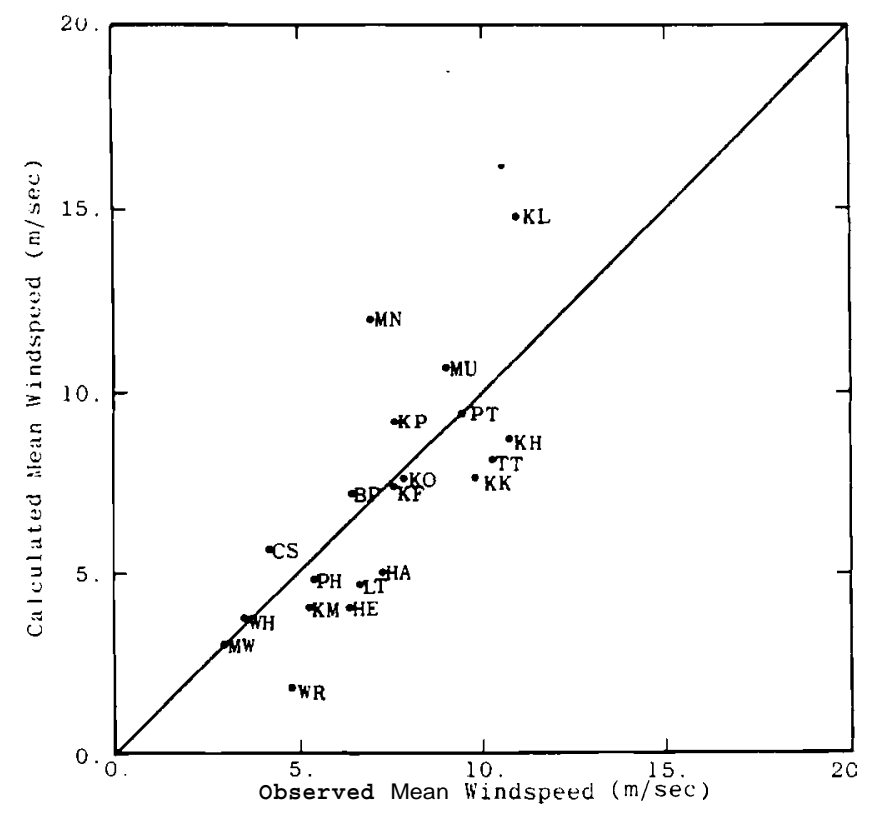

FIGURE 3.7. Comparison of Calculated to Observed Wind Speed Based Upon 24 Oahu Simulation Events (Simulation I: 6 surface stations plus upper air record. Source: Traci et al. 1978). 


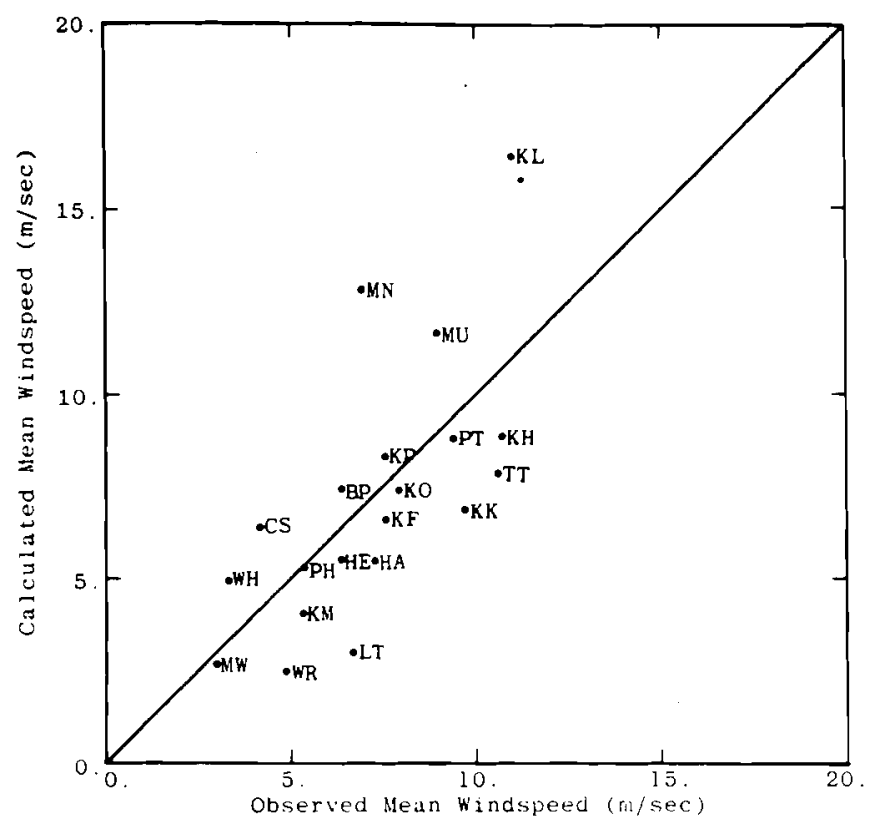

FIGURE 3.8. Comparison of Calculated to Observed Wind Speed Based Upon 24 Oahu Simulation Events (Simulation II: 3 surface stations plus upper air record. Source: Traci et al. 1978).

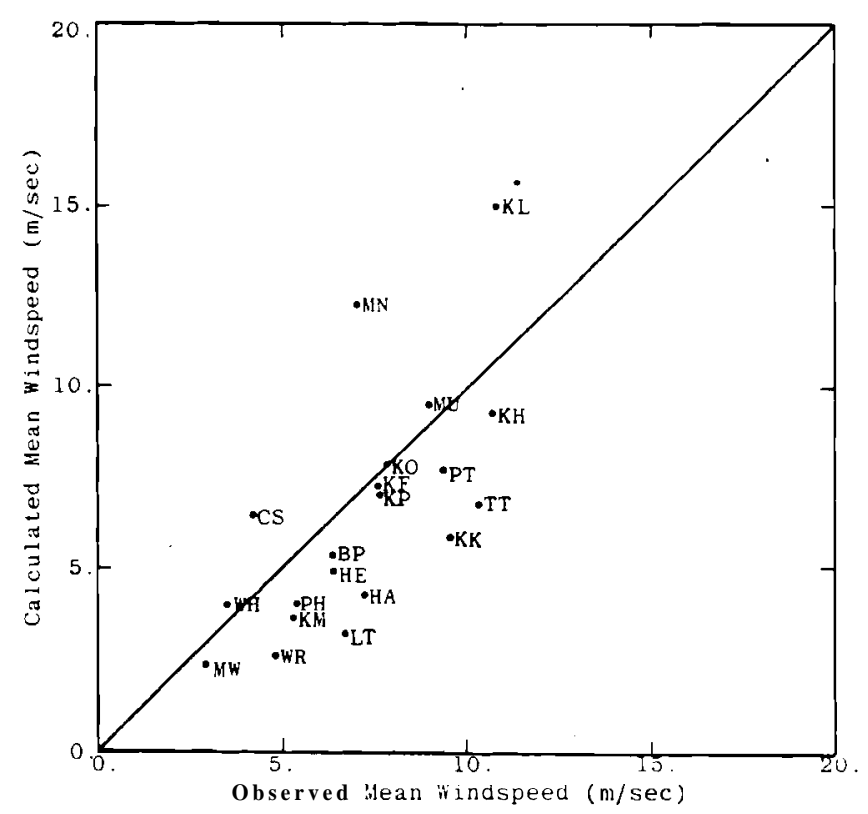

FIGURE 3.9. Comparison of Calculated to Observed Wind Speed Based Upon 24 Oahu Simulation Events (Simulation III: upper a ir record only. Source: Traci et al. 1978). 
the grid cell. For the simulations depicted in Figures 3.4 and 3.9, the horizontal dimensions of these cells are $1600 \mathrm{~m}$ The wind observations with which these simulations are compared, on the other hand, are essentially representative of points and are affected by features not resolvable by the model grid.

The last three figures show that the wind speed distribution over the island is represented reasonably well by the mass-consistent model--at least on the average. That is, the stations that were the windiest in the simulations tended to be the windiest of the observed stations (see Tables 3.2 and 3.3).

TABLE 3.2. Station Wind Speed Results Correlation Quantities: 24 Event Oahu Verification Matrix, Oahu Wind Data Network (Source: Traci et al. 1978)

\begin{tabular}{|c|c|c|c|c|c|c|}
\hline \multirow{2}{*}{$\begin{array}{l}\text { Simulation Description } \\
\text { (Events used in Analysis) }\end{array}$} & \multicolumn{2}{|c|}{$\begin{array}{c}\text { Least Syuares Linear Fit } \\
\text { Puranteters }\end{array}$} & \multirow{2}{*}{$\begin{array}{l}\text { Joul Nean Square } \\
\text { Error } \\
\text { HUSE, o } \\
\text { (m/sec) }\end{array}$} & \multirow{2}{*}{$\begin{array}{l}\text { Mein bias } \\
(\mathrm{m} / \mathrm{sec})\end{array}$} & \multirow{2}{*}{$\begin{array}{c}\text { Mean } \\
\text { Deviation } \\
\bar{d}_{\mathrm{q}}, \mathrm{m} / \mathrm{sec}\end{array}$} & \multirow{2}{*}{$\begin{array}{c}\text { Linear } \\
\text { Currellation } \\
\text { Coefticient } \\
\text { r }\end{array}$} \\
\hline & $\mathbf{a}_{0}$ & $\mathbf{a}_{1}$ & & & & \\
\hline $\begin{array}{l}\text { Simulation I : } 6 \text { surface \& upper a1r } \\
\text { - } 0200 \text { events } \\
\text { - } 1400 \text { events } \\
\text { - all } 24 \text { sets }\end{array}$ & $\begin{array}{l}2.3 \\
1.9 \\
2.1\end{array}$ & $\begin{array}{l}0.63 \\
0.79 \\
0.72\end{array}$ & $\begin{array}{l}3.3 \\
3.4 \\
3.4\end{array}$ & $\begin{array}{r}-0.01 \\
0.34 \\
0.17\end{array}$ & $\begin{array}{l}2.4 \\
2.7 \\
2.5\end{array}$ & $\begin{array}{l}0.63 \\
0.64 \\
0.65\end{array}$ \\
\hline $\begin{array}{l}\text { Simulation } 11: 3 \text { surface \& upper a2r } \\
\text { - } 0200 \text { events } \\
\text { - } 1400 \text { evepts. } \\
\text { - all } 24 \text { events }\end{array}$ & $\begin{array}{l}3.4 \\
3.2 \\
3.2\end{array}$ & $\begin{array}{l}.43 \\
.69 \\
.59\end{array}$ & $\begin{array}{l}4.2 \\
4.1 \\
4.1\end{array}$ & $\begin{array}{r}-0.11 \\
0.78 \\
0.33\end{array}$ & $\begin{array}{l}2.8 \\
3.0 \\
2.9\end{array}$ & $\begin{array}{l}0.43 \\
0.53 \\
0.51\end{array}$ \\
\hline $\begin{array}{l}\text { Simulation } 111 \text { : upper air only } \\
\text { - } 0200 \text { events } \\
\text { - } 1400 \text { events } \\
\text { - all } 24 \text { events }\end{array}$ & $\begin{array}{l}3.5 \\
2.4 \\
3.1\end{array}$ & $\begin{array}{l}.48 \\
.53 \\
.48\end{array}$ & $\begin{array}{l}3.7 \\
3.6 \\
3.6\end{array}$ & $\begin{array}{r}0.24 \\
-1.27 \\
-0.51\end{array}$ & $\begin{array}{l}2.8 \\
2.9 \\
2.0\end{array}$ & $\begin{array}{l}0.51 \\
0.54 \\
0.52\end{array}$ \\
\hline
\end{tabular}

$$
\begin{aligned}
& \mathrm{q}_{\mathrm{calc}}=\mathrm{a}_{\mathrm{O}}+\mathrm{a}_{1} \mathrm{q}_{\mathrm{obs}} \\
& \overline{\mathrm{b}}_{\mathrm{q}}=\frac{1}{\mathrm{~N}} \sum\left(\mathrm{q}_{\mathrm{calc}}-\mathrm{q}_{\mathrm{obs}}\right)=\text { mean bias } \\
& \overline{\mathrm{d}}_{\mathrm{q}}=\frac{1}{\mathrm{~N}} \sum\left|\mathrm{q}_{\mathrm{calc}}-\mathrm{q}_{\mathrm{Obs}}\right|=\text { mean deviation } \\
& \mathrm{o}_{\mathrm{q}}=\mathrm{RMSE}=\left\{\frac{1}{\mathrm{~N}} \sum\left(\mathrm{q}_{\mathrm{calc}}-\mathrm{q}_{\mathrm{Obs}}\right)^{2}\right\}^{\frac{1}{2}}=\text { root mean square error }
\end{aligned}
$$


TABLE 3.3. Station Wind Direction Results Correlation Quantities: 24 Event Oahu Verification Matrix, Oahu Wind Data Network (Source: Traci et al. 1978)

\begin{tabular}{|c|c|c|c|}
\hline $\begin{array}{l}\text { Simulation Description } \\
\text { (Events used in Analysis) }\end{array}$ & $\begin{array}{l}\text { Hoot Meun Square } \\
\text { Error } \\
\text { RuSE. O } \\
\text { (degrees) }\end{array}$ & $\begin{array}{l}\text { Mean Hais } \\
\bar{t}_{1,} \\
\text { (degrees) }\end{array}$ & $\begin{array}{l}\text { Mean Deviation } \\
\bar{d}_{f}(\text { degrees) }\end{array}$ \\
\hline $\begin{array}{l}\text { Simulation I : } 6 \text { surface \& upper a } \mathbf{r} \\
-\quad 0200 \text { events } \\
\text { - } 1400 \text { events } \\
\text { all } 24 \text { events }\end{array}$ & $\begin{array}{l}51.3 \\
37.0 \\
49.2\end{array}$ & $\begin{array}{l}-4.4 \\
-10.9 \\
-7.7\end{array}$ & $\begin{array}{l}35 . \\
29 . \\
32 .\end{array}$ \\
\hline $\begin{array}{l}\text { Simulation } \mathrm{II}^{8}: 3 \text { surface } \& \text { upper air } \\
\text { - } 0200 \text { events } \\
\text { - } 1400 \text { events } \\
\text { - a } 1124 \text { events }\end{array}$ & $\begin{array}{l}51.3 \\
50.3 \\
50.8\end{array}$ & $\begin{array}{l}-8.7 \\
-12.6 \\
-10.6\end{array}$ & $\begin{array}{l}36 . \\
33 . \\
35 .\end{array}$ \\
\hline $\begin{array}{l}\text { Simulation III: upper air only } \\
\text { - } 0200 \text { events } \\
\text { - } 1400 \text { events } \\
\text { - al1 24 events }\end{array}$ & $\begin{array}{l}52.9 \\
50.8 \\
51.9\end{array}$ & $\begin{array}{l}-6.2 \\
-8.5 \\
-7.1\end{array}$ & $\begin{array}{l}39 . \\
35 . \\
37\end{array}$ \\
\hline
\end{tabular}

Tables 3.2 and 3.3 list the root-mean-square error, mean deviation, correlation coefficient and other parameters for the three cases. The mean deviations between simulated and observed wind speed and direction are about $3 \mathrm{~m} / \mathrm{sec}$ and $30^{\circ}$, respectively. The statistics indicate that less than half of the variance in the wind speed at a given point (proportional to the correlation coefficient squared) is due to the gross effects of large-scale topography as modeled by NOABL.

The figures and the model also illustrate that the number of stations used in initializing the model in this study had only a small effect on the accuracy of the adjusted wind field, This result should not be taken as a general conclusion, since situations where small-scale phenomena, such as sea breeze, have an important effect on wind structure will not be so insensitive to the amount of input data.

A model that simulates individual wind fields is only a partial solution to the siting problem. Equally important is how many model runs are needed to 
provide the desired results. For screening purposes, all that may be needed are maps of annual or seasonal mean wind speeds over an area. These maps could then be used to pinpoint the regions in which further wind prospecting would be concentrated. If modeling is to be used to evalute the wind potential at a specific site, climatological estimates of more detailed wind characteristics, such as the wind speed probability density function or the diurnal behavior of the wind, may be required. One way of producing either maps of wind potential or site-specific estimates of critical wind characteristics would be to obtain numerical simulations for all input data available.

Assuming that hourly wind data were available, the model would be run for each hour and hourly estimates of wind speed and direction would be provided at every grid point within the model's domain for as long a period as thought necessary. The desired products would be obtained by analyzing all of the individual simulations. This approach is obviously impractical. Even using a model as simple as NOABL would require large amounts of computer time. The only practical solution is to run the model on a small subset of the available input data.

Analyzing the available input data subjectively and classifying them into a small number of representative cases would provide such a subset. The model would be run on these cases, and the results weighted according to the frequency at which the cases are observed. This procedure is more easily described than performed, since the criteria for subjectively classifying wind fields are not obvious.

Walton et a1. (1980) describe a procedure for classifying wind fields that makes use of principal components (or eigenvector) analysis. The procedure can be outlined as follows: Assume that over the region of interest surface wind observations are available at $\mathrm{N}$ locations. A t each of these $\mathrm{N}$ locations, a total of $M$ simultaneous observations have been made. Each individual observation of the wind vector can be represented by a complex number

$$
s=s e^{i \theta}
$$

where $s$ is the wind speed and $\theta$ is the wind direction. The entire set of wind observations can be expressed as a $N$ by $M$ matrix $S$ where $N<M$. The elements of $S$ are 


$$
s_{\mathrm{km}}=\mathrm{s}_{\mathrm{km}} \mathrm{e}^{\mathrm{i} \theta_{\mathrm{km}}}
$$

where $k$ denotes the location of the observation and $m$ denotes the time. Hardy and Walton (1978) showed that at any time m,

$$
S_{m}=\sum_{j=1}^{N} C_{j m} \hat{E}_{j}
$$

where $S_{m}$, the mth column of the matrix $S$, represents the wind observations at a11 N locations at time $m$ In Equation (3.22), $\hat{E}_{j}$ is the $j$ th eigenvector defined by the set of $N \cdot M$ observations and $C_{j m}$ are a set of expansion coefficients that size and rotate the elements of $\hat{E}_{j}$. The eigenvectors simply represent an efficient coordinate system for representing the wind vector observations.

As seen in Equation (3.22), the magnitude of $C_{j m}$ determines the relative importance of each eigenvector in representing the set of wind observations. In most applications of principal components analysis, the relative importance of the various eigenvectors varies considerably, and the observations can be represented by a small subset of the $\mathrm{N}$ eigenvectors. In addition, the magnitudes of the expansion coefficients of the most significant eigenvector (the primary expansion coefficients) are frequently much larger than the expansion coefficients of the second most significant eigenvector. This property is the key to the wind classification procedure described by Walton et al. (1980).

The first step in the process is to compare the temporal behavior of the primary expansion coefficients for each day in a period of interest (like a month) with the temporal behavior on every other day. Two days are said to be similar if their primary exparision coefficients are the same within prescribed error limits. As many types of days can be defined as are needed. Once the day-types are defined and each day classified, a typical day can be computed for each day-type (Knox and Walton 1978) or an actual day within a given type can be chosen as being representative of that type. The success of this procedure rests on the assumption that day-types will be easy to define and that individual days will be easy to classify. 
Once each day in the period of interest is assigned to a type and after a typical day has been selected to represent each type, the mass-consistent model MATHEW (Sherman 1978) is run for each representative day (Walton et al. 1980). Annual wind statistics are then derived at any grid point by computing a weighted sum (weighted by frequency of occurrence) of model solutions for each typical day.

Endlich et al. (1980) have developed another approach that minimizes the amount of subjectivity involved in analyzing the input data. The approach can be used either in screening mesoscale areas for potential sites or in estimating wind characteristics at pre-selected locations (see Endlich et al. 1980 for details on application). The method is based on a mass-consistent model similar to NOABL. (a) If Equations (3.11) through (3.14) are combined by substituting Equations (3.11) through (3.13) into Equation (3.14) an equation of the form

$$
\nabla^{2} \lambda=F(x, y, z)
$$

is obtained. This equation shows that the problem of finding the adjusted wind field is linear, and solutions can be found by linearly combining any number of component solutions.

The component solutions can be obtained from the eigenvectors defined by the wind observations. However, the way the wind observations are treated in the approach of Endlich et al. (1980) results in a different type of eigenvector than the method of Wat ton et a1. (1980). In this approach (see SRI International 1979), the wind vector at any location $k$ is represented by the two horizontal components $u_{k}$ and $v_{k}$. The observations are expressed by a $2 \mathrm{~N}$ by $\mathrm{M}$ matrix, $\mathrm{S}$, where $2 \mathrm{~N}<\mathrm{M}$ and the $\mathrm{mth}$ column of $\mathrm{S}$ is

(a) The model, called COMPLEX, differs from NOABL in that certain terms related to terrain slope are neglected for the sake of reducing computation time. The neglected terms are important in regions of very large slope. 


$$
S_{\mathrm{m}}=\left[\begin{array}{c}
\mathrm{s}_{1 \mathrm{~m}} \\
\cdot \\
\cdot \\
\mathrm{s}_{\mathrm{km}} \\
\cdot \\
\mathrm{s}_{(2 N) \mathrm{m}}
\end{array}\right]=\left[\begin{array}{c}
u_{1 \mathrm{~m}} \\
v_{1 \mathrm{~m}} \\
\cdot \\
\cdot \\
u_{n m} \\
v_{n m}
\end{array}\right]
$$

The wind observations at any time $m$ are expressed

$$
S_{m}=\sum_{j=1}^{2 N} a_{j m} \hat{E}_{j}+\bar{s}
$$

where $\overline{\mathrm{s}}$ is a vector formed from the time means of the $u$, $v$ wind components at each observing station and $\hat{E}_{j}$ is the $j$ th eigenvector.

Since the eigenvectors are orthogonal $\left(\hat{E}_{p} \cdot \hat{E}_{q}=\delta_{p q}\right)$, the coefficients $a_{j m}$ are given by

$$
a_{j m}=\sum_{k=1}^{2 N}\left(s_{k m}-\bar{s}_{k}\right) e_{k}
$$

where $e_{k j}$ are the elements of the $j t h$ eigenvector. The eigenvectors, $\hat{E}_{j}$, are eigenvectors of the covariance matrix whose elements are defined

$$
C 0_{i j}=\sum_{m=1}^{M}\left(s_{i m}-\bar{s}_{i}\right)\left(s_{j m}-\bar{s}_{j}\right)
$$

Once the eigenvectors are found, the mass-consistent model is only run $2 \mathrm{~N}+1$ times--once for each of the $2 \mathrm{~N}$ eigenvectors and once using the time mean data as input. (a) Thus, at any point $r$ in the model domain,

(a) Frequently, the observed fields can be represented with a small subset of the eigenvectors. In this case, the mode1 would be run using the mean data and the most significant eigenvectors as input. 


$$
\left[\begin{array}{l}
u_{r m} \\
v_{r m}
\end{array}\right]=\left[\begin{array}{l}
\bar{u}_{r} \\
\bar{v}_{r}
\end{array}\right]+\sum_{j=1}^{2 N} a_{j m}\left[\begin{array}{l}
u_{r j} \\
v_{r j}
\end{array}\right]
$$

where $u_{r j}$ and $v_{r j}$ are the adjusted $u, v$ components at $r$. These components are obtained when the $\mathrm{jth}$ eigenvector is used as input to the model. The components $\bar{u}_{r}, \bar{v}_{r}$ are the adjusted components obtained when the time means are used as input. Using Equation (3.28), estimates of the time series are obtained at every point of interest and any desired wind characteristics are computed.

One particular problem with this approach, however, is obtaining initial fields (see Section 3.2.2) when the mass-consistent model is run on the eigenvectors. Since the eigenvectors are a mathematical artifice and do not represent any physically realizable flow, it is not certain how eigenvectors obtained from surface wind observations should be vertically extrapolated. One possible method is given by Endlich et al. (1980). This is less of a concern in the approach used by Waton et al. (1980) since the eigenvector analysis is used to identify specific days to be used as model input.

To test the accuracy of the procedures described above, evaluation programs were conducted in which certain wind characteristics were computed.(a) Wa ton et a . (1980) compute annual average estimates of mean and median wind speeds and Weibull distribution fits to the predictions using principal components analysis and MATHEW. The verification was derived from seven stations on Oahu, beginning ir August 1976 and ending in July 1977. Table 3.4 compares the predicted and observed mean wind speeds for that period.

Seven of DOE's candidate wind turbine sites were used in the evaluation study of Endlich et al. (1980). Among the computed wind characteristics were the annual mean wind speed, the annual mean diurnal cycle and seasonal means of wind speed. The numerical model used 12 months of data from nearby National Weather Service stations for input. The actual wind characteristics at each site were obtained from one year's data gathered during the candidate site evaluation program (Renné and Sandusky 1979). For the majority of the

(a) The evaluation program tests both the eigenvectors approach to representing the initial data as well as the mass-consistent windfield code. 
TABLE 3.4. Comparison of Simulated and Measured Wind Speeds in $\mathrm{m} / \mathrm{sec}$ for Seven Oahu, Hawaii Stations (see Table 3.1). Average from August 1976 through July 1977 (Walton et al. 1980).

\begin{tabular}{|c|c|c|c|}
\hline Site & Measured & Simulated & Difference \\
\hline MN & 5.4 & 6.5 & +1.1 \\
\hline $\mathrm{KH}$ & 9.1 & 7.2 & -1.9 \\
\hline$H A$ & 5.9 & 4.3 & -1.6 \\
\hline MW & 2.7 & 3.7 & +1.0 \\
\hline MU & 7.2 & 6.4 & -0.8 \\
\hline TT & 9.3 & 8.9 & -0.4 \\
\hline K3 & 6.4 & 7.0 & +0.6 \\
\hline
\end{tabular}

simulations the 12-month period used in running the numerical model did not coincide with the 12 months of wind measurements at the site.

Table 3.5 compares computed and observed annual mean wind speed at seven candidate sites. Four of the sites (Boone, San Gorgonio, Block Island and Clayton) were used to "tune" the procedure. The tuned procedure was then used to simulate the wind characteristics at the other three locations. As the table shows, the procedure predicted the annual mean wind speeds very well (although more tests are needed before one could say that the success shown in Table 3.5 is typical).

TABLE 3.5. Comparison of Simulated and Measured Wind Speeds i $\mathrm{n} \mathrm{m} / \mathrm{sec}$ (Annual Averages for 1977) (Endlich et al. 1980)

\begin{tabular}{|c|c|c|}
\hline Site & Measured & Simulated \\
\hline $\begin{array}{l}\text { Boone, NC } \\
\text { San Gorgonio, CA } \\
\text { Block Island, RI } \\
\text { Clayton, NM } \\
\text { Ludington, MI } \\
\text { Holyoke, MA } \\
\text { Huron, SD }\end{array}$ & $\begin{array}{l}8.0 \\
8.0 \\
7.5 \\
7.6 \\
7.9 \\
7.3 \\
6.9\end{array}$ & $\begin{array}{l}8.1 \\
7.2 \\
7.0 \\
5.8 \\
7.2 \\
7.4 \\
6.7\end{array}$ \\
\hline
\end{tabular}

Figures 3.10 and 3.11 show the seasonal and diurnal wind speed distributions predicted for Holyoke, Massachusetts. The Holyoke site is on the top of Mt. Tom, a high hill in western Massachusetts. The agreement between the 
simulated and observed wind characteristics is typical of the three untuned sites. The seasonal wind speed distribution is predicted fairly well. The diurnal distributions, however, are not as close.

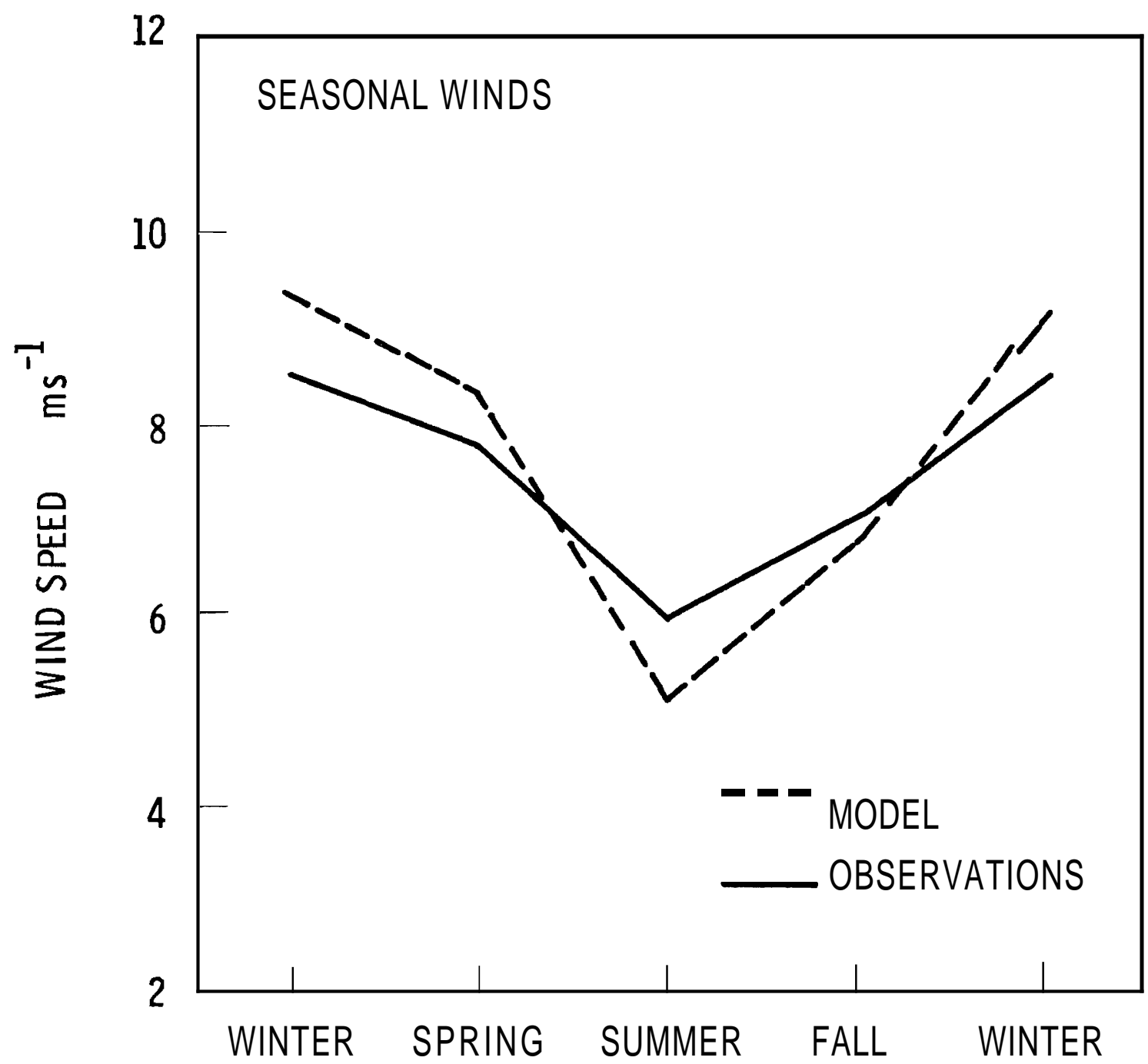

FIGURE 3.10. Comparison of Observed and Predicted Seasonal Mean Wind Speeds for Mt. Tom, Massachusetts. Predictions are based on an eigenvector analysis procedure coupled with a mass consistent model (Endlich et al. 1980). 


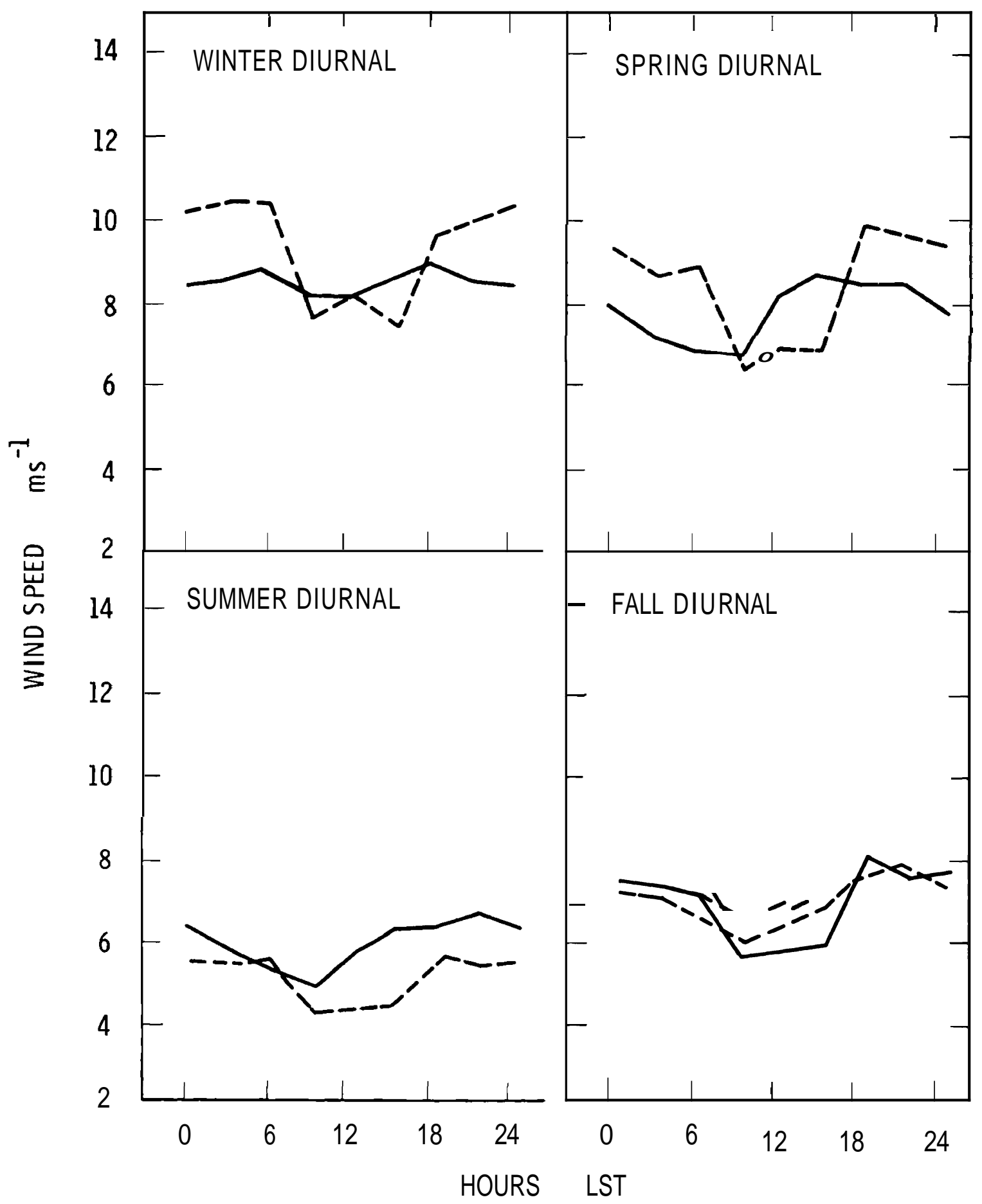

FIGURE 3.11. Comparison of Observed and Predicted Diurnal Cycles for Each Season at Mt. Tom, Massachusetts. Predictions are based on an eigenvector analysis procedure coupled with a mass consistent model (Endlich et a1. 1980). 
Successful1y representing the diurnal cycle is a fundamental problem with the objective analysis approach to wind field simulation. Mudh of the information on wind structure, particularly the temporal modulation of wind speed and direction, is contained in the input data. This is obvious from Equations (3.25) and (3.28). The coefficients $a_{j m}$ are defined by the input data; the temporal behavior of the adjusted wind field at any point in the model domain will reflect the temporal behavior of that data set. Therefore, a mass-consistent objective analysis model can generate a diurnal cycle, for example, at any point that is different from the cycle inherent in the input data only when there is a consistent diurnal modification of wind direction. If this is the case, there could be some locations where topographic modification of the wind field was so sensitive to wind direction that a considerable diurnal modification of the wind characteristics could occur. This, however, is not the usual mechanism responsible for the diurnal wind cycle (see Appendix 2).

\subsubsection{Concl usions and Recommendations}

Verification studies show that kinematic models, such as the mass-consistent objective analysis model discussed in this chapter, describe the general features of flow over terrain fairly well, if the gross effect of topography on mass conservation is the dominant mechanism forcing the flow. Less skill has been demonstrated in predicting detailed wind characteristics at specific sites, especially when the observed characteristics differ substantially from the input data. Therefore, successful use of these models depends on good input data.

Wind data from observation stations must be dense enough to give a general idea of flow patterns through the modeled region, and the data used must be representative of the turbine sites in the region. These models generally reproduce the same temporal characteristics as the input data. If nontopographic effects (such as sea breeze circulation, drainage flows in valleys, roughness changes, etc.) on a scale smaller than the modeled region are thought to be important, the input data must be dense enough to resolve these effects expl icitly. 
Mass-consistent models should be used only if the model's grid is capable of resolving the important topographical features and only if terrain relief on the scale resolvable by the model is greater than 200 to $300 \mathrm{~m}$. Otherwise, there will be little adjustment in the initial guess of the wind field. For island applications, utilization of a mass-consistent model on islands less than 2000 to $3000 \mathrm{~km}^{2}$ in area should be valid. Such islands are small enough that sea-breeze circulations are less important than topographic forcing. For applications on continents or islands larger than this, the importance of small-scale thermally driven circulations or small-scale surface roughness changes on the wind structure will have to be judged subjectively. The importance of many of these small-scale features could be seasonally dependent.

\subsection{PRIMITIVE EQUATION MODELS}

To achieve economy in the use of computer resources, mass-consistent objective analysis models ignore much of the physics governing the flow of fluids. Recently, models have been built that attempt to mimic the atmosphere as closely as possible, by solving the fundamental (or primitive) equations that describe atmospheric dynamics.

Actually, the fundamental equations of motion, shown in Equations (3.1) through (3.4) are not solved in a primitive equation model, since these equations describe the entire spectrum of atmospheric motions (including scales of motion unimportant to the problem of flow over terrain). To make the modeling problem more tractable, the fundamental equations of motion are usually simplified. Such simplifications, however, limit the generality of resulting models and may even change the character of certain types of atmospheric motion. These changes in character can be so significant that certain motions are no longer realistically simulated. Thus, in order to interpret the results of primitive equation models properly, the effects of the various assumptions on the fidelity of model simulations must be understood.

\subsubsection{Boussinesq Equations}

One set of equations, frequently used to describe atmospheric motion, is the so-called Boussinesq equations. In deriving these equations, the thermodynamic variables $P, T$, and $\rho$ are expressed as the sum of a reference state, 
denoted by the subscript "o", and a deviation from the reference state, denoted by the superscript "I":

$$
\begin{aligned}
& P=P_{0}+P^{\prime} \\
& T=T_{0}+T^{\prime} \\
& \rho=\rho_{0}+\rho^{\prime}
\end{aligned}
$$

The reference states have the property

$$
\nabla_{2} P_{0}=\nabla_{2} T_{0}=\nabla_{2} P_{0}=0
$$

where $\nabla_{2} \equiv\left(\hat{i} \frac{\partial}{\partial x}+\hat{\mathbf{j}} \frac{\partial}{\partial y}\right)$ and $\hat{\mathbf{i}}, \hat{\mathbf{j}}$ are unit normal vectors in the $\mathrm{x}$ and $\mathrm{y}$ directions, respectively. The Boussinesq equations then follow from substituting Equation (3.29) into Equations (3.1) through (3.4) and applying the following restrictions [see Dutton and Fichtl (1969) or Busch (1973) for details]:

1. The dynamic viscosity $(\mu)$ the thermal conductivity $\left(k_{T}\right)$, and diffusion coefficient $\left(\mathrm{k}_{\mathrm{q}}\right)$ are constant.

2. The ratios $\left|P^{\prime} / P_{0}\right|<<1,\left|T^{\prime} / T_{0}\right|<<1$, and $\left|\rho^{\prime} / \rho_{0}\right|<<1$.

3. The reference states $P_{0}$ and $T_{0}$ are defined such that

$$
\frac{\partial P_{0}}{\partial z}=-\rho_{0} g
$$

and

$$
\frac{\partial T_{0}}{\partial z}=-\frac{g}{c_{p}}
$$

4. The heat generated by viscous effects is negligible. 
5. Vertical scales of motion are small compared to the scale height

$$
\left|\frac{1}{\rho_{0}} \frac{\partial \rho_{0}}{\partial z}\right|^{-1}
$$

(at sea level, this scale height is on the order of $10^{4} \mathrm{~m}$ ).

Given these restrictions, the equations of continuity, momentum, entropy, and water vapor mass fraction are

$$
\begin{gathered}
\frac{\partial u_{i}}{\partial x_{i}}=0 \\
\frac{\partial u_{i}}{\partial t}+u_{j} \frac{a u_{i}}{\partial x_{j}}=-\frac{1}{\rho_{0}} \frac{\partial p^{\prime}}{\partial x_{i}}-g \frac{\rho^{\prime}}{\rho_{0}} \delta_{3 i}+v \frac{a^{2} u_{i}}{a x_{j} x_{j}}-2 \varepsilon_{i j k \Omega_{j} u_{k}} \\
\frac{\partial \theta}{\partial t}+u_{i} \frac{\partial \theta}{\partial x_{i}}=\theta_{0} k \frac{\partial}{\partial x_{i}}\left[\frac{1}{T}\left(\frac{\partial T^{\prime}}{\partial x_{i}}-\frac{g}{c_{p}} \delta_{3 i}\right)\right]+S_{\Theta} \\
\frac{\partial q}{\partial t}+u_{i} \frac{\partial q}{\partial x_{i}}=k_{q} \frac{\partial^{2} q}{\partial x_{i} \partial x_{i}}
\end{gathered}
$$

where $\theta=\theta-\theta_{0}, \frac{\partial \theta_{0}}{a t}+u_{i} \frac{\partial \theta_{0}}{\partial x_{i}} \equiv 0, k=k_{T} /\left(\rho c_{P}\right)$ and irreversibility, $I$, has been neglected.

An additional assumption that is usually made in contructing models is the hydrostatic assumption, i.e.,

$$
\frac{\partial P^{\prime}}{\partial z}+g \rho^{\prime}=0
$$

This equation replaces the momentum equation for the vertical velocity, $u_{3}$, since the terms 


$$
v \frac{\partial^{2} u_{3}}{\partial x_{j} \partial x_{j}}
$$

and

$$
2\left(\varepsilon_{3 j k} \Omega_{j} u_{k}\right)
$$

are of negligible magnitude.

The Boussinesq equations eliminate sound waves as possible solutions (Holton 1972). When time-dependent equations are solved by finite difference methods, the maximum time step is governed by the maximum rate at which disturbances can propagate through the modeled domain. If the time step is larger than allowed, computational instability will result, and the solution wi11 blow up. Acoustic waves are the most rapid disturbances that can move through a model. Acoustic waves are also unimportant to the problem of modeling flow over terrain. If they are eliminated, longer time steps can be taken, which reduce model running time. Thus, the absence of sound waves is a very desirable attribute.

The hydrostatic Boussinesq equations do not, however, represent all scales of internal gravity waves accurately. When the atmosphere is perturbed, as it is when flowing over uneven terrain, waves, with gravity as the restoring force, are excited in the atmosphere. These waves affect local pressure gradients and therefore influence surface winds. Although the hydrostatic approximation is valid for long wavelength gravity waves on the order of $50 \mathrm{~km}$ or greater, its representation of the small-scale gravity waves that could be forced by abrupt topographic features is not accurate. For example, there is a high frequency cutoff for gravity waves in the atmosphere that depends on the local vertical temperature gradient. For a given stable temperature lapse rate, gravity waves will not be observed with frequencies above a given value. Since temperature lapse rate is not constant with height in the real atmosphere, waves within a certain range of wavelengths can become trapped at various levels within the atmosphere. The range of wavelengths that can become 
trapped and the level or levels at which trapping can occur depend on how the temperature lapse rate varies with height (Tapp and White 1976). This trapping or ducting phenomenon is responsible for the occurrence of the standing lee waves commonly observed in mountainous areas. However, in a hydrostatic model, there is no high frequency cutoff and hence, no trapping or ducting of wave energy. Hydrostatic models are not capable of reproducing such nuances of gravity wave behavior.

Since gravity wave behavior can significantly affect wind behavior near the surface, it is important that this behavior be properly modeled. (a) According to Tapp and White (1976), the hydrostatic approximation is definitely invalid if the ratio of the vertical to the horizontal length scales of motions in the atmosphere equals or exceeds unity. In fact, nonhydrostatic effects could still be significant if the ratio of these scales is less than one. As a rule of thumb, the hydrostatic assumption becomes questionable for stably stratified flow over topography when the relief of the dominant terrain features in the modeled domain approaches something like a $1-\mathrm{km}$ elevation change in a distance of $2 \mathrm{~km}$.

\subsubsection{Anelastic Equations}

A model does not have to be hydrostatic to be free of pesky sound waves. Sound waves can be eliminated from the equations of motion without significantly affecting the ability to model nonhydrostatic motions. The so-called anelastic assumption does this by making the same assumptions as were made in the derivation of the Boussinesq equations except for one assumption (Ogura and Phillips 1962). In deriving the anelastic set of equations, the assumption that vertical scales of motion are small compared to the scale height

$$
\left|\frac{1}{\rho_{0}} \frac{\partial \rho_{0}}{\partial z}\right|^{-1}
$$

is relaxed (Dutton and Fichtl 1969). When the vertical scale restriction is lifted, the continuity equation is

(a) The characteristics of gravity waves forced by terrain depend on the behavior of the wind profile through the atmosphere, as well as the vertical temperature structure (Klemp and Lilly 1975). Both temperature and wind behavior can change from day to day and, indeed, from hour to hour. This variability is one reason for the complexity of surface wind behavior over rugged terrain. 


$$
\frac{\partial \rho_{0}}{\partial t}+\frac{\partial}{\partial x_{i}}\left(\rho_{0} u_{i}\right)=0
$$

The anelastic assumption is $\partial \rho_{0} / \partial t=0$.

Ogura and Phillips (1962) showed that the anelastic equations do not allow wave solutions for frequencies greater than the frequencies of gravity waves. In other words, density fluctuations on the time scale of sound waves are filtered out and density fluctuations on the time scale of gravity waves are not. Thus, a set of equations is achieved that is free of acoustic waves but is realistic in its treatment of small-scale gravity waves. The disadvan tage of the anelastic equation set is its greater complexity.

\subsubsection{Nonhydrostatic Models}

Recently, Tapp and White (1976) and Carpenter (1979) have developed a nonhydrostatic model that uses the fully compressible continuity equation (see Equation 3.2). In this model, the restrictions that acoustic waves place on the allowable time step are circumvented by the way terms involving the vertical and horizontal propagation of sound waves are treated in the finite difference equations. According to Tapp and White (1976), the resulting finite difference equations are stable for time steps as long as those typically used in a hydrostatic model.

\subsubsection{Closure Assumption}

Models that start with the same basic set of equations are not always equal in the results they produce, since the solutions depend on:

- the initial conditions assumed

- how the boundary conditions are treated (see Section 3.3.5)

- how details such as thermal radiation, clouds, precipitation, turbulent transport are handled

- which numerical procedures are used to integrate the equations.

The numerical procedures used to obtain solutions can have a profound effect on the results, and the literature abounds with discussions of the advantages 
and disadvantages of various techniques. A discussion of numerics is clearly beyond the scope of this chapter; however, the reader should be aware of their importance in explaining differences between models.

Thermal radiation affects the behavior of winds in the atmosphere, particularly at night. However, radiation heat transfer is too complicated to be realistically treated in a model designed primarily to simulate wind behavior. Therefore, radiation heating or cooling (term $S_{\Theta}$ in Equation 3.33) is parameterized by various simplified schemes that vary from model to model.

Clouds and condensation are important, though, because cloud cover affects the transport of heat by thermal radiation and condensation affects (as an internal heat source) atmospheric motions. The primitive equation models discussed in this chapter neglect the effects of cloud formation on atmospheric dynamics. However, the effect of cloud cover on thermal radiation can be very important. Several of the radiation parameterization schemes used in primitive equation modeling include this effect.

The way turbulence transport is treated in a model affects the behavior of the simulated wind fields. All current mesoscale models made use of the Reynolds assumptions in treating turbulence. In this approach, each variable is split into a mean (denoted by a "-") and a fluctuating part (denoted by a "I"):

$$
\begin{aligned}
& u_{i}=\bar{u}_{i}+u^{\prime}{ }_{i}, \bar{u}_{i}^{\prime}=0 \\
& \theta=\bar{\theta}+\theta^{\prime}, \bar{\theta}^{\prime}=0 \\
& P^{\prime}=\bar{p}+p^{\prime}, \bar{p}^{\prime}=0 \\
& q=\bar{q}+q^{\prime}, \bar{q}^{\prime}=0
\end{aligned}
$$

where fluctuations in potential temperature and thermodynamic temperature are assumed equal. 
For a time-dependent, nonhomogenous flow, there is some question as to what is meant by a "mean." In modeling flow over terrain, two time scales are important. One is the time scale of the turbulence $\left(\tau_{t}\right)$ :

$$
\tau_{t}=\frac{H}{w_{*}}
$$

where $\mathrm{H}$ is the depth of the atmospheric boundary layer and $\mathrm{w}_{*}$ is a velocity scale characteristic of turbulent motions in the boundary layer. The other time scale, $\tau_{P}$, represents the rate at which the large-scale pressure field changes

$$
\tau_{p}=\left|\frac{1}{P_{0}} \frac{\partial p^{\prime}}{\partial t}\right|^{-1}
$$

As long as $\tau_{P} \gg \tau_{t}$, a mean can be defined, e.g.,

$$
\bar{u}_{i}=\frac{1}{2 \tau_{m}} \int_{t-\tau_{m}}^{t+\tau_{m}} d t
$$

if $\tau_{t} \ll \tau_{m} \ll \tau_{P}$.

Substituting Equation (3.37) into the hydrostatic Boussinesq equations and neglecting the horizontal gradients of turbulence quantities, the equations become

$$
\begin{gathered}
\frac{\partial \bar{u}}{\partial x}+\frac{\partial \bar{v}}{\partial y}+\frac{\partial \bar{w}}{\partial z}=0 \\
\frac{\partial \bar{u}}{\partial t}+\bar{u} \frac{\partial \bar{u}}{\partial x}+\bar{v} \frac{\partial \bar{u}}{\partial y}+\bar{w} \frac{\partial \bar{u}}{\partial z}=-\frac{1}{\rho_{0}} \frac{\partial \bar{p}}{\partial x}+f \bar{v}-\frac{\partial\left(\overline{u^{\prime} w^{\prime}}\right)}{\partial z} \\
\frac{\partial \bar{v}}{\partial t}+\bar{u} \frac{\partial \bar{v}}{\partial x}+\bar{v} \frac{\partial \bar{v}}{\partial y}+\bar{w} \frac{\partial \bar{v}}{\partial z}=-\frac{1}{\rho_{0}} \frac{\partial \bar{P}}{\partial y}-f \bar{u}-\frac{\partial\left(\bar{v}^{\prime} w^{\prime}\right)}{\partial z}
\end{gathered}
$$




$$
\begin{aligned}
& \frac{\partial \bar{\theta}}{\partial t}+\bar{u} \frac{\partial \bar{\theta}}{\partial x}+\bar{v} \frac{\partial \bar{\theta}}{\partial y}+\bar{w} \frac{\partial \bar{\theta}}{\partial z}=-\frac{\partial\left(\overline{w^{\prime} \theta^{\prime}}\right)}{\partial z}+S_{\theta} \\
& \frac{\partial \bar{q}}{\partial t}+\bar{u} \frac{\partial \bar{q}}{\partial x}+\bar{v} \frac{\partial \bar{q}}{\partial y}+\bar{w} \frac{\partial \bar{q}}{\partial z}=-\frac{\partial\left(\overline{w^{\prime} q^{\prime}}\right)}{\partial z}
\end{aligned}
$$

where all terms involving molecular transport are neglected and $f$ is the Coriolis parameter, $f=2 \Omega_{r} \sin \phi\left(\Omega_{r}=\right.$ the earth's rotational speed and $\phi=$ latitude).

To close the set of equations, some relationship is needed between the turbulent transport terms and the mean field quantities. The oldest and most widely used relationship is

$$
\overline{w^{\top} r^{\top}}=-K_{r} \frac{\partial r}{\partial z}
$$

where $\bar{r}$ and $r^{\prime}$ are the mean and fluctuating portions of an arbitrary quantity, $r$. Equation (3.46) defines a turbulent transport coefficient, $K_{r}$, analogous to the molecular transport coefficient. Unlike the molecular transport coefficient, $K_{r}$ is a "property" of the flow and not the fluid; it can also have a positive or negative sign; $(\mathrm{a})$ for, the sign of $\bar{W}^{\top} r^{\top}$ is not necessarily determined by the sign of $\partial \bar{r} / \partial z$. O'Brien (1970) gives a formulation of the turbulent transport coefficient for momentum that is frequently used.

Another closure scheme is to relate the turbulent transport to certain turbulence quantities such as the turbulence kinetic energy, $e=\frac{1}{2} \bar{u}_{i}^{\prime} u^{\prime}{ }_{j}$, or the variances, ( $\overline{\theta^{\top} 2}$ or $\overline{q^{\top} 2}$ ) of scalar quantities (Mellor and Yamada 1974). A third approach is to derive the equations relating the turbulent transports to the mean and fluctuating fields and attempt to solve for the transport terms directly (Donaldson 1973). Introducing new equations involving turbulence quantities introduces new unknowns into the equation set. The new unknowns must be related to previously defined quantities (i.e., closed); thus, the above approaches are often called second-order closure schemes. Second-order schemes obviously lead to a more complicated model. Proponents

(a) Although negative transport coefficients are sometimes found when Equation (3.45) is applied to observations, $k_{r}$ cannot be negative in a numerical model. 
of second-order closure argue that this approach is more rigorous and results in turbulent transports that are related to the structure of the flow field in a more real istic manner. However, many of the assumptions made in closing the second-order equations or in simplifying some of the terms in these equations are of unknown accuracy or of questionable validity.

N matter which technique is used to represent turbulent transports all have been developed and tested for horizontally homogenous, level terrain. $\mathrm{How}$ accurately they apply to situations where local terrain relief is not small compared to the boundary layer depth is not known.

\subsubsection{Data Requirements and Initialization}

Primitive equation models require more data than objective analysis models. The data supplied must be sufficient to allow the initial values of every variable to be specified at each grid point in the model. The data must also define how these variables evolve at the model's boundaries. Conditions at the lateral and upper boundaries must be prescribed that are consistent with the large-scale meteorology. Frequently, existing data are insufficient to define boundary conditions and reasonable assumptions must be made. The whole issue of boundary conditions and how finite difference equations should be formulated in the neighborhood of boundaries is, like numerics, an issue of extreme importance. Errors in the way boundaries are treated in a model can propagate throughout the domain. (See Klemp and Lilly 1978 for a discussion of the effects of the upper boundary conditions on wind field simulations over rugged topography.)

When large-scale meteorology is fairly steady, conditions at the earth's surface control the behavior of the near-surface wind. The surface conditions that are important include fixed conditions (such as surface roughness or surface albedo) and variable conditions (such as temperature, or heat flux, and moisture mass fraction, or evaporation rate). Surface and moisture conditions can either be specified (if known) or computed from soil, heat, and moisture budgets at each time step.

The exact procedures for initializing a primitive equation model and the types of information needed at the surface depend on the particular model 
being used and data availability in the region under investigation. To indicate the amount of data required to run a primitive equation model, Table 3.6 lists data supplied to primitive equation modelers who were simulating airflow over Oahu, Hawaii, as part of a DOE model verification exercise. The most critical data needs are the wind, temperature, humidity and horizontal pressure gradients at the start of integration and information on how these quantities change with time at the lateral boundaries. Reasonable assumptions can be made for the other items in Table 3.6 if they are not available.

TABLE 3.6. Data Supplied For Primitive Equation Model Verification Exercise on Oahu

\begin{tabular}{|c|c|}
\hline Data Type & Data \\
\hline Topography & $\begin{array}{l}\text { Average terrain heights in } \\
1600 \mathrm{~m} \times 1600 \mathrm{~m} \text { grid box for } \\
48 \times 38 \text { box grid }\end{array}$ \\
\hline Roughness Length & For each grid box \\
\hline $\begin{array}{l}\text { Incident Solar } \\
\text { Radiation }\end{array}$ & Computed \\
\hline Vegetation Type & 5 categories \\
\hline Soil Type & $\begin{array}{l}\text { U.S.D.A classification - significant } \\
\text { local types }\end{array}$ \\
\hline Aibedo & $\begin{array}{l}\text { Determined from vegetation and soi } 1 \\
\text { type }\end{array}$ \\
\hline $\begin{array}{l}\text { Sea Surface } \\
\text { Temperature }\end{array}$ & Single value determined from satellite \\
\hline $\begin{array}{l}\text { Radiosonde } \\
\text { Soundings }\end{array}$ & $\begin{array}{l}\text { (Winds, temperature, humidity) at the } \\
\text { standard observing times }\end{array}$ \\
\hline Surface & $\begin{array}{l}850 \text { and } 700 \mathrm{mb} \text { analyses at the standard } \\
\text { observing times }\end{array}$ \\
\hline
\end{tabular}

\subsubsection{Applications and Results}

Unti1 recently, mesoscale primitive equation models were used primarily as research tools in meteorology. A good portion of this research has concentrated on the study of sea breeze circulations (e.g., Pielke 1974, and Tapp and White 1979). Early studies concentrated on problems where the topography 
was negligible; however, more recent work has been directed to problems where the topography is important (Mahrer and Pielke 1976, Nickerson and Magaziner 1976, Carpenter 1979). Verification of these studies has largely been qualitative, especially with respect to wind simulations, because: 1) the major objective of many of these studies was to explain the large-scale features of mesoscale meteorology in a fairly qualitative way and 2) the available data were seldom adequate for a rigorous verification to be performed.

Pielke and Mahrer (1978) used a hydrostatic primitive equation model to simulate surface winds and vertical motions at the top of the boundary layer for a summer day in south Florida. The day chosen for verification was one in which the large-scale meteorological situation was simple and steady. Under these conditions, afternoon weather was usually dominated by the buildup of large cumulus clouds and by the rain showers they produced. The effects of clouds and showers on atmospheric flow patterns were not simulated by the model as it did not allow for condensation.

Comparing model simulations with available wind and radar data (the latter indicating the locations of rain showers) showed the model simulating the general features of flow over the south Florida peninsula very well. The model did best in predicting the initial locations of cumulus clouds and rain showers. The locations where showers were first observed were close to the areas of maximum vertical motion, as predicted by the model. The model's performance in simulating details in the surface wind field was not as good. Much of the problem was probably due to the effects of shower activity on surface winds. In regions well away from these showers, the agreement between simulated and observed wind speed and direction was much better. (a)

Recently, the University of Virginia mesoscale model was applied to the problem of screening coastal sites for wind power potential (Garstang et al. 1979). As with all modeling studies, a critical question is the number of model runs that are required to simulate the wind climatology. A primitive

(a) Without access to the original data, quantitative comparisons of wind speed and direction in the shower-free areas are impossible. A quick comparison of figures in Pielke and Mahrer (1978) shows afternoon winds from the east to southeast at about $5 \mathrm{~m} / \mathrm{sec}$ occurring along the showerfree eastern coast in both the simulations and the observations. 
equation model is costly to run and, since it produces so much information, costly to analyze. In the study of coastal zone wind energy, Garstang et al. analyzed the climatology of seven geographical regions along the Atlantic and Gulf coasts. The large-scale meteorological situation was divided into six broad categories. The frequency of occurrence of each meteorological category was determined for all seven regions as a function of season. In the approach, model runs were only made for the nieteorological categories significant to the wind energy potential.

Figures 3.12 through 3.20 are maps of wind power flux (watts $/ \mathrm{m}^{2}$ ) exemplifying some of the products a primitive equation model can produce (Garstang et al. 1979). Two geographical areas are shown. One is the Chesapeake Bay area; the other is along the south Texas coast near Brownsville. The Chesapeake Bay area is one of very complex shoreline, while the south Texas shore has much simpler geography. Another difference is that the large-scale flow for the Chesapeake Bay area is offshore in both cases, but the large-scale flow for the south Texas case is onshore.

The two Chesapeake Bay cases are for both a typical winter day and a summer day. The large-scale fields used to initialize the model were taken from the analyses of Garstang et al. (1979). Large-scale meteorological conditions were held constant for the length of the simulations. Surface conditions, however, were allowed to respond to the soil heat and moisture budgets. Model runs were always started at sunrise.

Figures 3.12 and 3.13 show maps of average wind power flux for the hours 1400 to 1500 LST and 2200 to 2300 LST on the typical winter day. Figure 3.14 shows a map of daily averaged wind power flux for a typical winter day.

Figures 3.15 through 3.17 show these same features for a typical summer day. The figures indicate significant niovements in the centers of maximum wind power flux, both with time of day and with season. The figures also indicate significant changes in diurnal modulation with season.

Figures 3.18 through 3.20 show the same type of information as the previous figures but for the area near Brownsville. One of the most interesting 


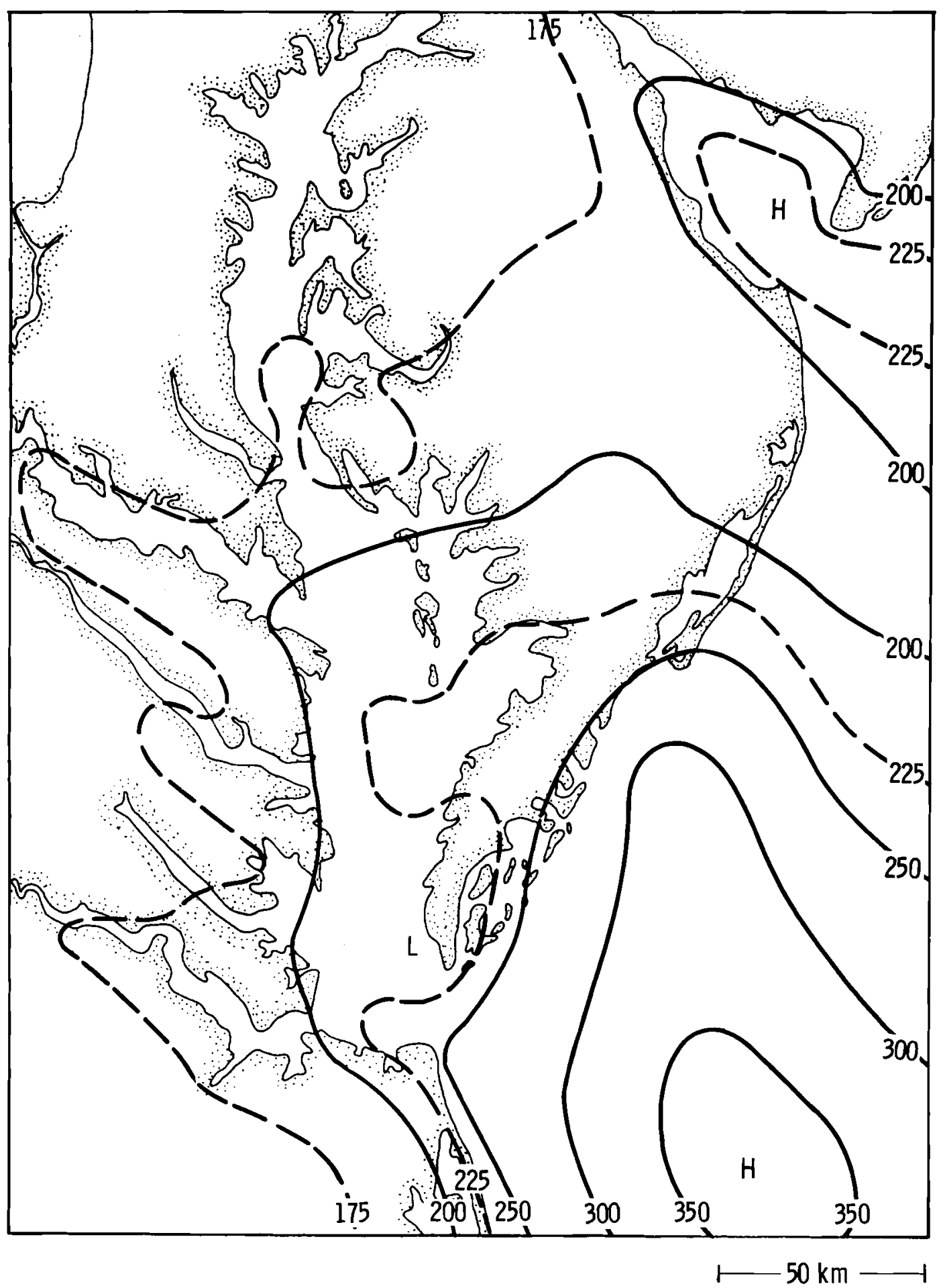

FIGURE 3.12. Hourly Average Power Density (watts $/ \mathrm{m}^{2}$ ) Over Chesapeake Bay, Wintertime Conditions, at $50 \mathrm{~m}$ Above the Surface for the Hour 1400 to 1500 Local Standard Time (LST). Six sets of predicted meteorological variables, at 10 -min intervals, were used to determine the average (Garstang et al. 1979). 


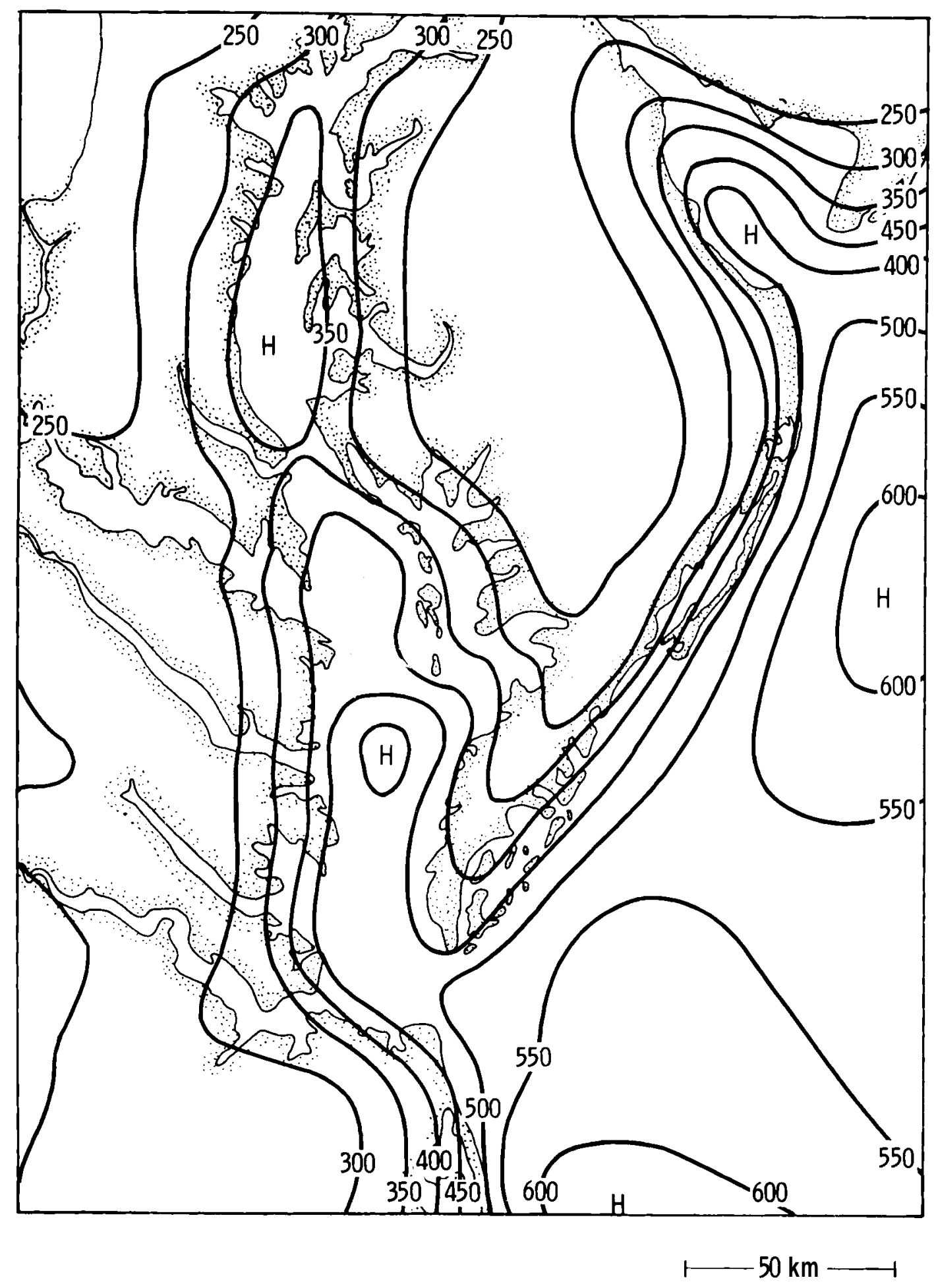

FIGURE 3.13. Hourly Average Power Density (watts $/ \mathrm{m}^{2}$ ) Over Chesapeake Bay, Wintertime Conditions, at $50 \mathrm{~m}$ Above the Surface for the Hour 2200 to 2300 Local Standard Time (LST). Six sets of predicted meteorological variables, at 10-min intervals, were used to determine the average (Garstang et al. 1979). 


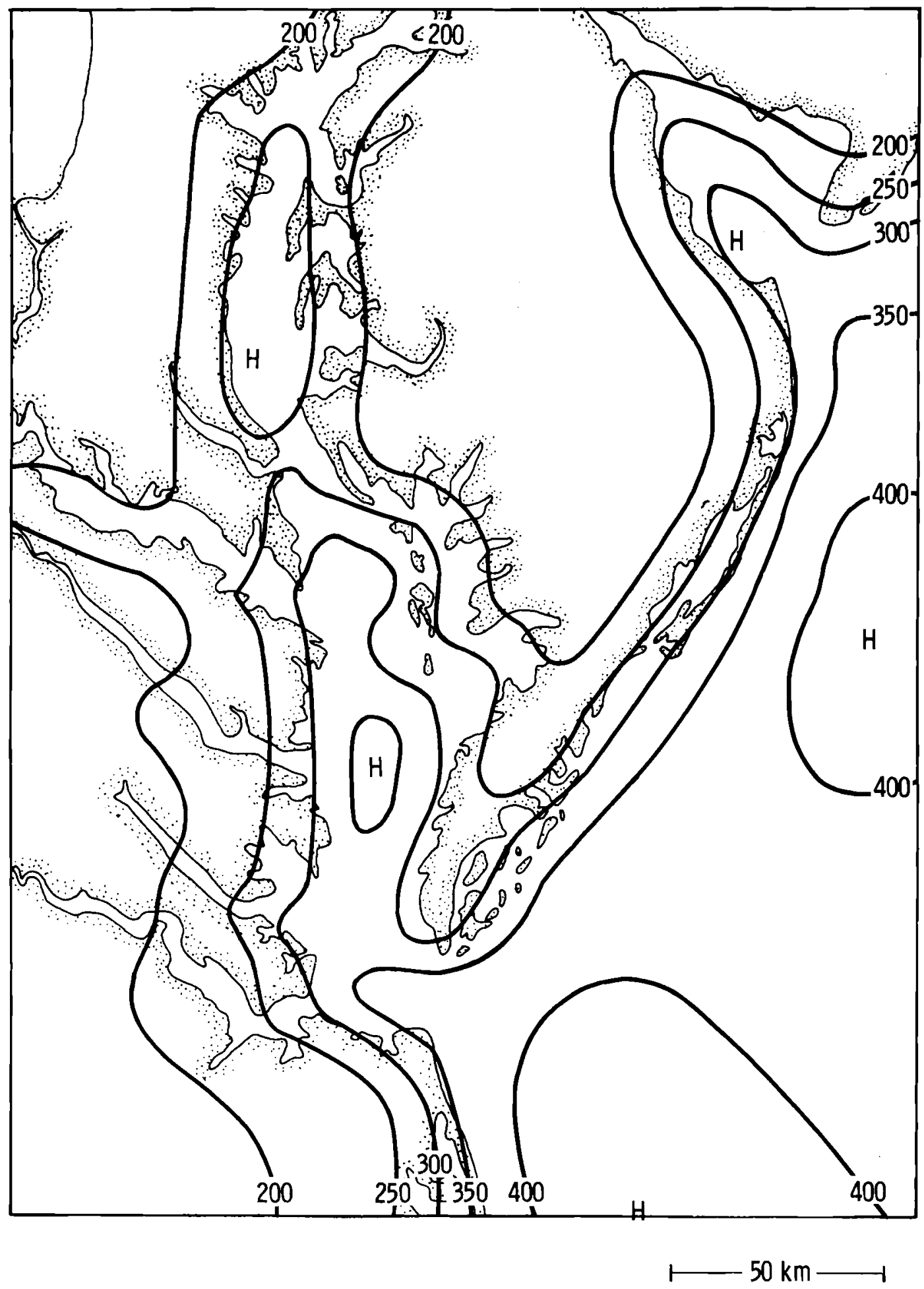

FIGURE 3.14. Daily Average, Layer-Mean Power Density (watts $/ \mathrm{m}^{<}$) Over Chesapeake Bay, Wintertime Conditions, for the Lowest $100 \mathrm{~m}$ of the Atmosphere. Heights above the surface and weightings (in parentheses) used in the layer averaging are: $10 \mathrm{~m}(0.20), 30 \mathrm{~m}(0.20), 50 \mathrm{~m}(0.35)$, $100 \mathrm{~m}(0.25)$ (Garstang et a1. 1979). 


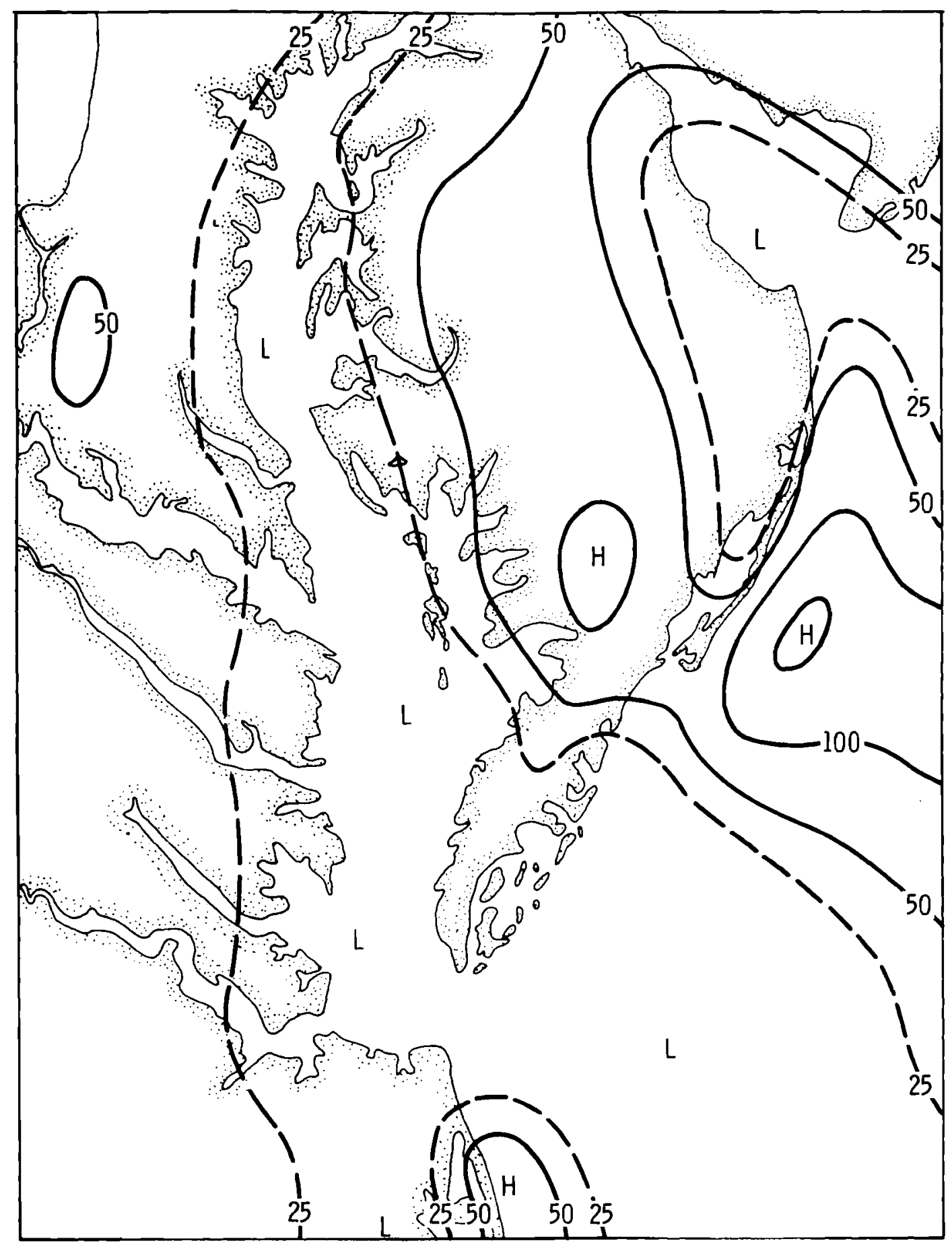

$\vdash-50 \mathrm{~km} \longrightarrow$

FIGURE 3.15. hourly Average Power Density (watts $/ \mathrm{m}^{2}$ ) Over Chesapeake Bay, Summertime Conditions, at $50 \mathrm{~m}$ Above the Surface for the Hour 1400 to 1500 Local Standard Time (LST). Six sets of predicted meteorological variables, at 10 -min intervals, were used to determine the average (Garstang et al. 1979). 


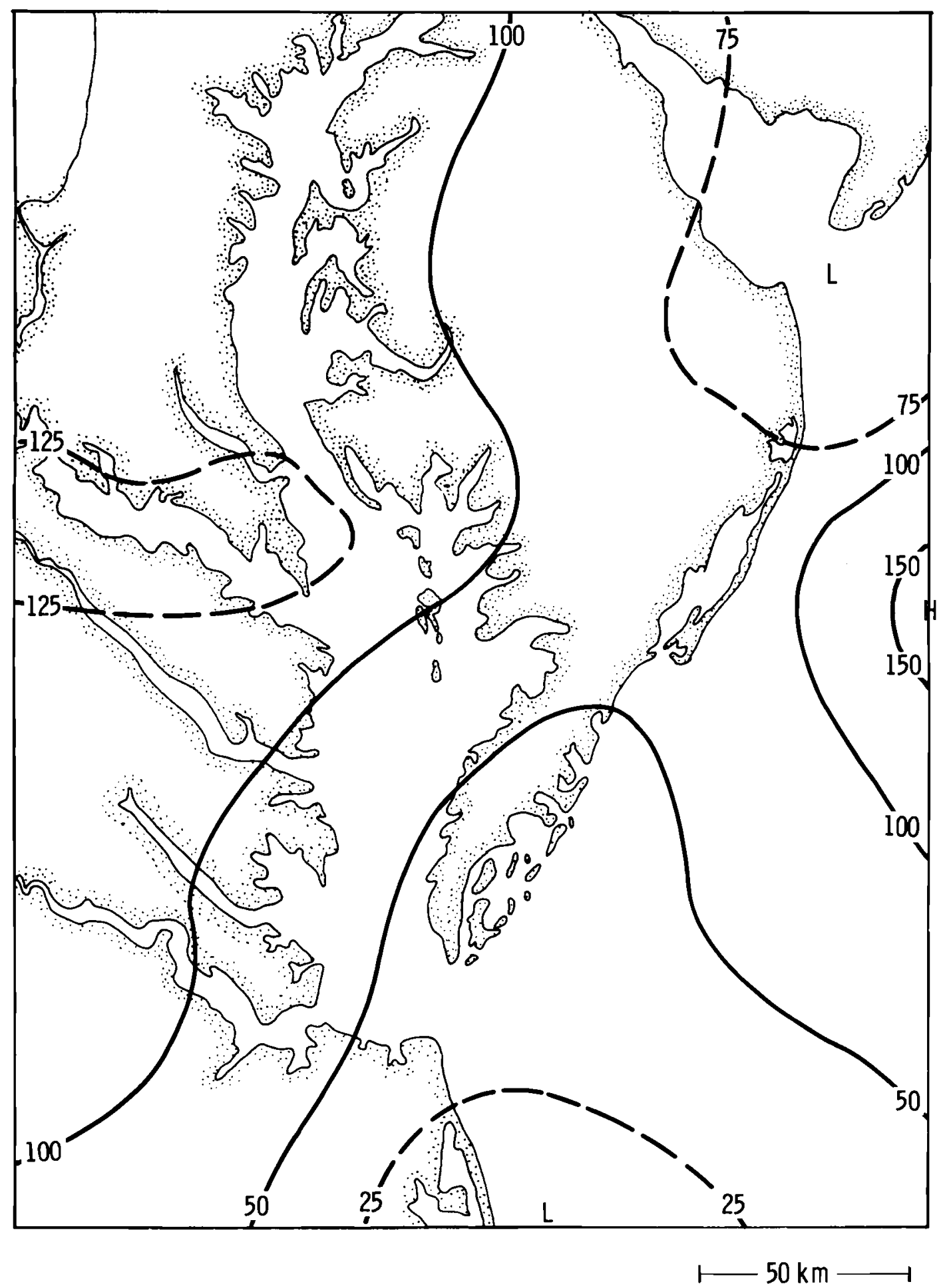

FIGURE 3.16. Hourly Average Power Density (watts $/ \mathrm{m}^{2}$ ) Over Chesapeake Bay, Summertime Conditions, at $50 \mathrm{~m}$ Above the Surface for the Hour 2200 to 2300 Local Standard Time (LST). Six sets of predicted meteorological variables, at 10-min intervals, were used to determine the average (Garstang et al. 1979). 


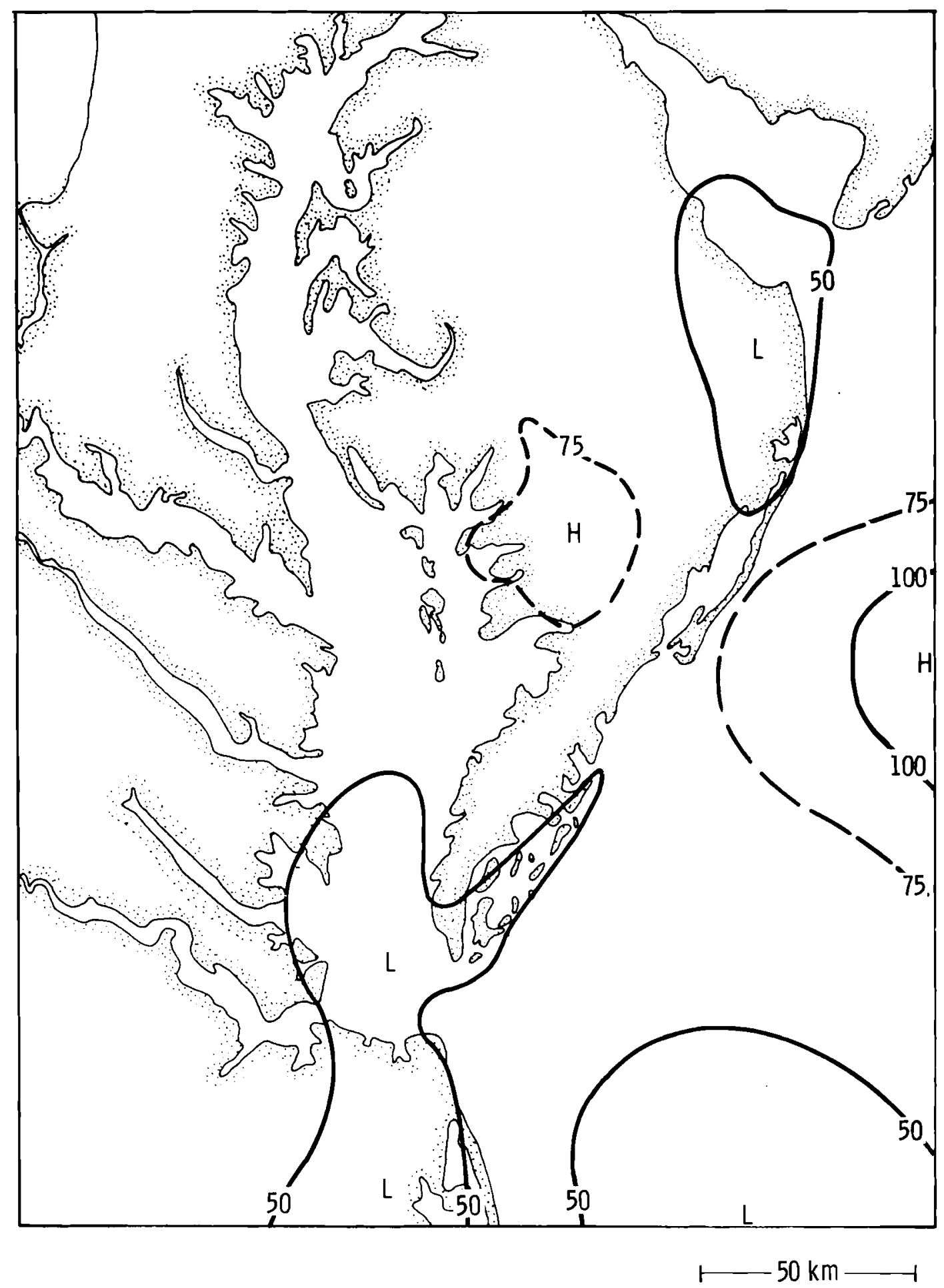

FIGURE 3.17. Daily Average, Layer-Mean Power Density (watts $/ \mathrm{m}^{2}$ ) Over Chesapeake Bay, Summertime Conditions, for the Lowest $100 \mathrm{~m}$ of the Atmosphere. Heights above the surface and weightings (in parentheses) used in the layer averaging are: $10 \mathrm{~m}(0.20), 30 \mathrm{~m}(0.20), 50 \mathrm{~m}(0.35)$, $100 \mathrm{~m}(0.25)$ (Garstang et al. 1979). 


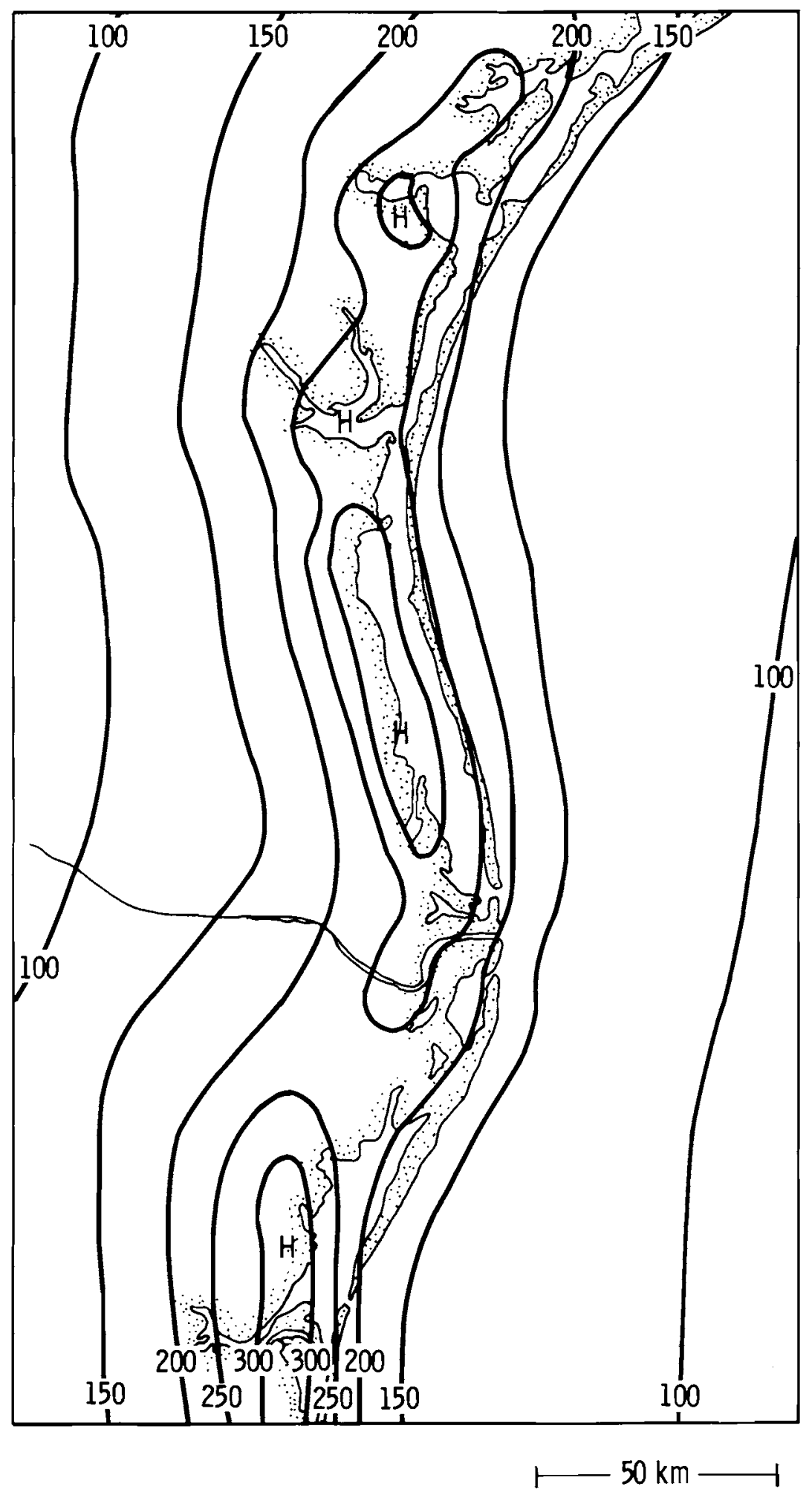

FIGURE 3.18. Hourly Average Power Density (watts $/ \mathrm{m}^{2}$ ) Over South Texas Coast, Summertime Conditions, at $50 \mathrm{~m}$ Above the Surface for the Hour 1400 to 1500 Local Standard Time (LST). Six sets of predicted meteorological variables, at 10-min intervals, were used to determine the average (Garstang et a1. 1979). 


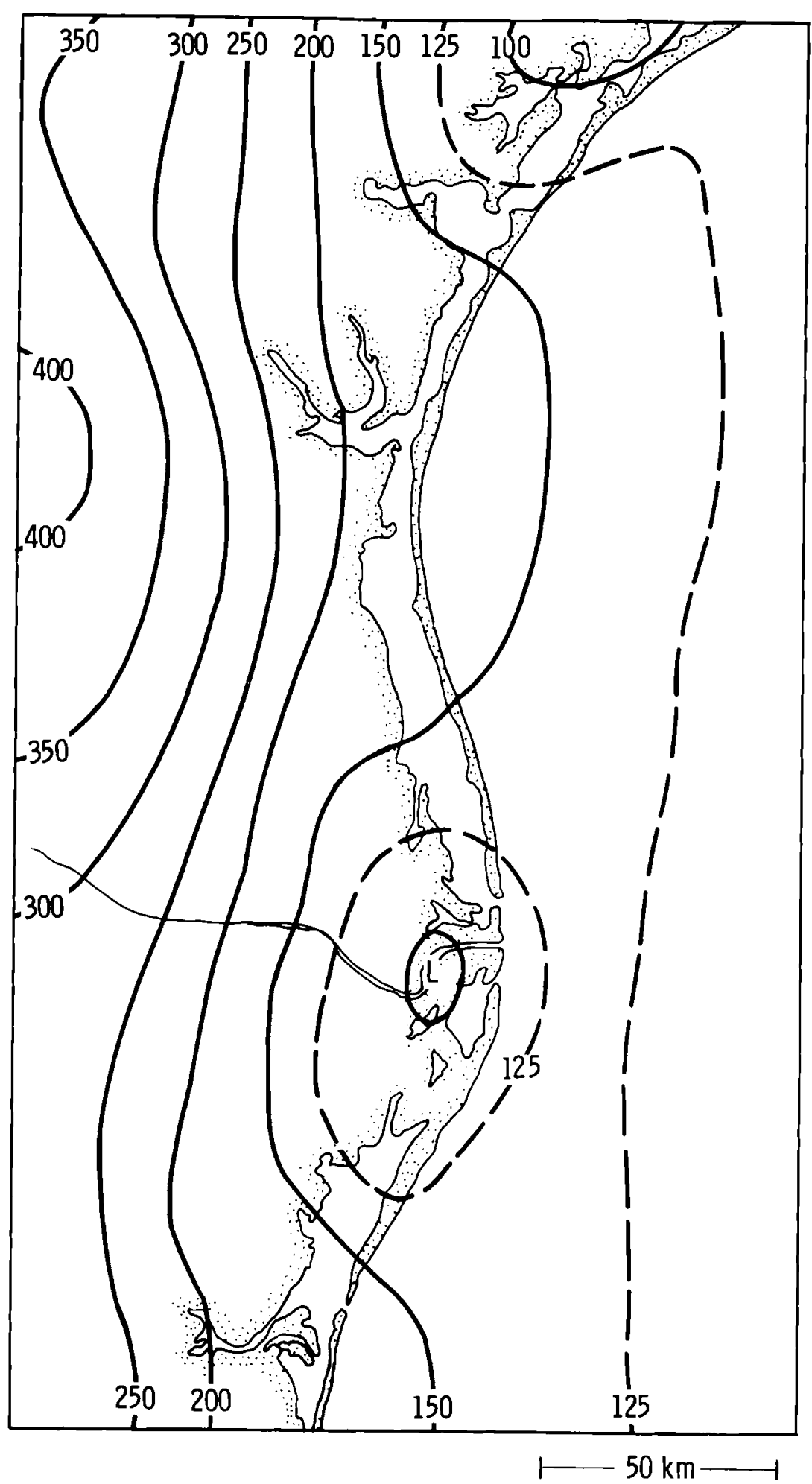

FIGURE 3.19. Hourly Average Power Density (watts $/ \mathrm{m}^{2}$ ) Over South Texas Coast, Summertime Conditions, at $50 \mathrm{~m}$ Above the Surface for the Hour 2200 to 2300 Local Standard Time (LST). Six sets of predicted meteorological variables, at 10-min intervals, were used to determine the average (Garstang et al. 1979). 


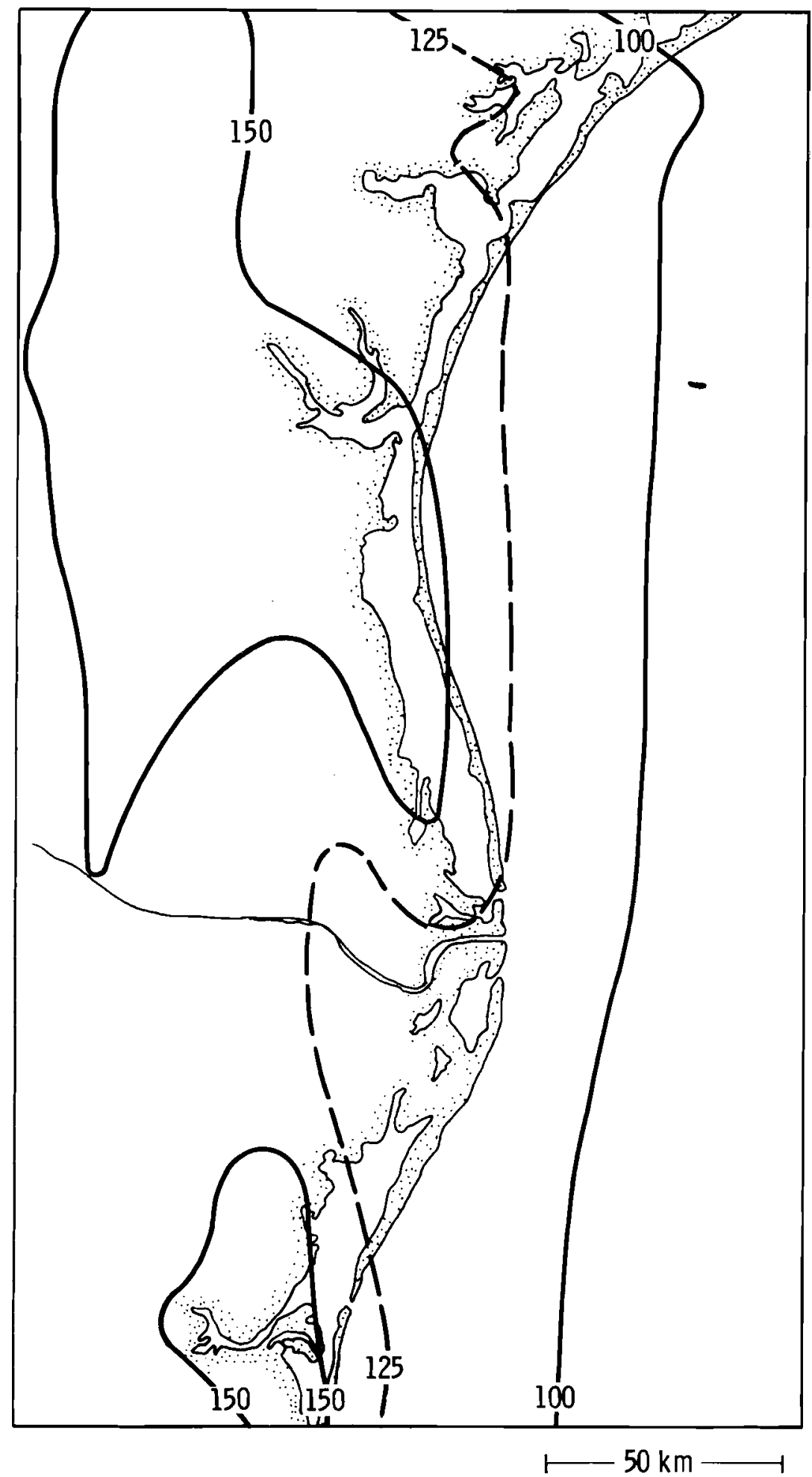

FIGURE 3.20. Daily Average, Layer-Mean Power Density (watts $/ \mathrm{m}^{2}$ ) Over South Texas Coast, Summertime Conditions, for the Lowest $100 \mathrm{~m}$ of the Atmosphere. Heights above the surface and weightings (in parentheses) used in the layer averaging are: $10 \mathrm{~m}(0.20), 30 \mathrm{~m}(0.20), 50 \mathrm{~m}(0.35)$, $100 \mathrm{~m} \mathrm{(0.25)} \mathrm{(Garstang} \mathrm{et} \mathrm{a} \mathrm{1.} \mathrm{1979).}$ 
results shown in the Figures 3.18 through 3.20 is how the available wind power can change along a linear coastline. These variations show that even for simple geographies preferred locations could exist for wind turbines. These locations would be where the sea breeze interacts sufficiently with the largescale flow to have a significant effect on wind speed.

\subsubsection{Conclusions and Recommendations}

Primitive equation models contain sufficient descriptions of the dynamics and thermodynamics of atmospheric flow that they can account for the effects that changes in thermal stratification, in surface temperature distribution, and in surface roughness can have on the near-surface wind field. Primitive equation models, therefore, should be useful in understanding wind flow patterns over areas where changes of the type described above are important. Since these models can be run on domains covering a wide range of scales, they can be used either to screen large areas for potential cluster sites or to understand complexities in the flow over a single site and assist in machine placement.

However, primitive equation models require large computers, consume large blocks of computer time, and require more tinkering by the user. Because of their complexity, the models can only be run a few times--corresponding to the most significant (or typical) meteorological events that affect the wind power potential of a region. A small number of runs mandates one to be cautious about the climatological significance of the results.

In an attempt to find a compromise between the realism of a threedimensional, time-dependent primitive equation model and the speed of the kinematic schemes, layer-averaged primitive equation models have been developed (Danard 1977, Venkatesh and Danard 1977, Lavoie 1972). The layer-averaged models are two-dimensional. In these models, the primitive equations are satisfied on the average over a layer with a thickness on the order of the boundary layer thickness. Surface boundary conditions are similar to other models but heuristic assumptions must be made about conditions at the top of the layer. Models of this type have been used to study sea and lake breezes. 
One-level models should be fast enough to be competitive with kinematic objective analysis schemes. They should do a better job than objective analysis methods in areas where differences in surface temperature have important effects on the wind (this has not been thoroughly tested, however). Problems arise, however, with upper boundary conditions. These conditions have an important influence on the resulting wind patterns and their accuracy under a wide range of situations is unknown. One-dimensional primitive equations are also non-linear. Hence, they cannot be substituted for a mass-consistent model in the eigenvector approach to generating wind time series at a remote site (see Section 3.3.3). Solutions to nonlinear equations cannot be obtained by combining coniponent solutions.

\subsection{CONCLUSIONS AND RECOMMENDATIONS}

The primary problem with all numerical models is a lack of verification. There have not been enough experiments in which model simulations have been compared with good field measurements. This will remain the case for a long time, since there are not very many good data sets for experiments of this type. The expense of gathering good verification data is large, and the task of gathering enough data to allow the flow over a complex piece of topography to be deduced unequivocally from the data alone is impossible. The only alternative is good judgment. The person analyzing model results should accept or reject them on the basis of how well he feels the model simulates the important physical processes controlling the flow.

Three-dimensional primitive equation models should be capable of simulating the wind patterns produced either when a large-scale flow interacts with the sea breeze circulation or from changes in surface roughness. Less is known of the ability of primitive equation models to handle rugged topography and the effects of surface temperature variations in sloping terrain (which can cause drainage winds, etc.) The usefulness of primitive equation models is in locating areas where local circulations, in addition to topographic 
profile, enhance wind power potential. (a) The existence of these enhanced regions would have to be verified by on-site measurements. Simple kinematic models should be used only in situations where the primary effects of topography are in blocking or channeling the flow or in situations where the input data set is dense enough to define the general flow conditions over the area.

Since conclusions based on modeling (and particularly primitive equation modeling) are based on a small number of simulations, caution is advised. When applying the models, studies should be conducted to determine how sensitive the location of important wind features might be to relatively small changes in the initial or boundary conditions. Results of such studies could indicate the likelihood of actually finding the enhanced areas. One should also be cautious in attaching too much significance to the magnitude of wind speed. predicted by a model. The patterns predicted by the model are probably more accurate than their magnitudes. Finally, a user should not take model predictions of the boundary layer wind profile or the behavior of the diurnal cycle too seriously. The methods used in existing models to simulate these characteristics of flow over terrain are simply too crude to warrant this level of confidence.

(a) As discussed above, existing primitive equation models are not capable of simulating the effects of cumulus cloud activity on the wind field. However, even in the case where the local topography creates preferred locations for cumulus formation, the exact location and behavior of this activity is too random to affect the wind climatology in a way important to wind energy potential. 


\section{REFERENCES}

Busch, N. E. 1973. "On the Mechanics of Atmospheric Turbulence." Chapter 1 in Workshop on Micrometeorology, D. A. Haugen (ed.). Science Press for the American Meteorological Society, Ephrata, Pennsylvania.

Carpenter, K. M. 1979. "An Experimental Forecast Using a Non-Hydrostatic Mesoscale Model." Q. J.R. Meteorol. Soc. 105:629-655.

Danard, M. B. 1977. "A Simple Model for Mesoscale Effects of Topography on Surface Winds." Mon. Wea Rev. 105:572-581.

Donaldson, C. duP. 1973. "Construction of a Dynamic Model of the Production of Atmospheric Turbulence and the Dispersal of Atmospheric Pollutants." Chapter 8 in Workshop on Micrometeorology, D. A. Haugen (ed.). Science Press for the American Meteorological Society, Ephrata, Pennsylvania.

Dutton, J. A. and G. H. Fichtl. 1969. "Approximate Equations of Motion for Gases and Liquids." J. Atmos. Sci. 26:241-254.

Endlich, R. M., F. L. Ludwig, C. M. Bhumralkar and M. A. Estoque. 1980. A Practical Method for Estimating Wind Characteristics at Potential Wind-Energy Conversion Sites. SRI Project 8349, SRI International, Men1o Park, California.

Garstang, M., S. Nnaji, R. A. Pielke, J. Gusdorf, C. Lindsey and J. W. Snow. 1979. Coastal Zone Wind Energy Part 1. RL0/2344-77/78/79. Available from National Technical Information Service, Springfield, Virginia.

Hardy, D. M. and J. J. Walton. 1978. "Principal Components Analysis of Vector Wind Measurements." J. Appl. Meteor. 17:1153-1162.

Holton, J. R. 1972. An Introduction to Dynamic Meteorology. Academic Press, New York, pp. $120-158$.

Klemp, J. B. and D. K. Lilly. 1975. "The Dynamics of Wave Induced Downslope Winds." J. Atmos. Sci. 32:320-339.

Klemp, J. B. and D. K. Lilly. 1978. "Numerical Simulation of Hydrostatic Mountain Waves." J. Atmos. Sci. 35:78-107.

Knox, J. B. and J. J. Walton. 1979. "A Computational Site Screening Methodology for Wind Energy." Presented at the UNITAR Conference on Long Term Energy Resources, Montreal, Canada, November 26-December 7, 1979.

Lass, H. 1957. Elements of Pure and Applied Mathematics. McGraw-Hill, New York, New York, pp. 288-294.

Lavoie, R. L. 1972. "A Mesoscale Model of Lake Effect Storms." J. Atmos. Sci. 29:1025-1040. 
Mahrer, Y. and R. A. Pielke. 1976. "Numerical Simulation of the Airflow Over Barbados." Mon. Wea Rev. 104:1392-1402.

Mathews, J. and R. L. Walker. 1964. Mathematical Methods of Physics.

Benjamin Cummings, Men1o Park, California, pp. 322-341.

Mellor, G. L. and T. Yamada. 1974. "A Hierarchy of Turbulence Closure Models for Planetary Boundary Layers." J. Atmos. Sci. 31:1791-1806.

Nickerson, E. C. and E. L. Magaziner. 1976. A Three-Dimensional Simulation of Winds and Non-Precipitating Orographic Clouds Over Hawaii. NOAA Technical Report, ERL-377-APCL 39, National Oceanic and Atmospheric Administration, Washington, DC.

O'Brien, J. J. 1970. "A Note on the Vertical Structure of the Eddy Exchange Coefficient in the Planetary Boundary Layer." J. Atmos. Sci. 27:1213-1215.

Ogura, Y. and N. A. Phillips. 1962. "Scale Analysis of Deep and Shallow Convection i $n$ the Atmosphere." J. Atmos. Sci. 19:173-179.

Phillips, G. T. 1979. A Preliminary Users Guide for the NOABL Objective Analysis Code. DOE/ET/20280/78-1. Available from National Technical Information Service, Springfield, Virginia.

Pielke, R. A. 1974. "A Three-Dimensional Model of the Sea Breezes Over South Florida." Mon. Wea. Rev. 102:115-139.

Pielke, R. A. and Y. Mahrer. 1978. "Verification Analysis of the University of Virginia Three-Dimensional Mesoscale Model Prediction over South Florida for July 1973." Mon. Wea. Rev. 106:1568-1589.

Renné, D. S. and W. F. Sandusky. 1979. "DOE Candidate Site Meteorological Measurement Program." Presented at the International Energy Agency Expert Meeting, Boone, North Carolina.

Sasaki, Y. 1958. "An Objective Analysis Based on the Variation Method." J. Meteor. Soc. of Japan 36:1-12.

Sherman, C. A. 1978. "A Mass Consistent Model for Wind Fields Over Complex Terrain." J. Appl. Meteor. 17:312-319.

Shinn, J. H., C. A. Sherman, J. J. Walton, K. L. Hill, B. R. Clegg and D. Whisler. 1979. Oahu Surface Wind Networks: Summary of Data August 1976 Through July 1978. UCID-18232, Lawrence Livermore Laboratory, Liverniore, California.

SRI International. 1979. Estimation of Wind Characteristics at Potential Wind Energy Conversion Sites. PNL-3074, Pacific Northwest Laboratory, Richland, Washington. 
Tapp, M. C. and P. W. White. 1976. "A Non-Hydrostatic Mesoscale Model." Q. J. R. Meteor. Soc. 102:277-296.

Traci, R. M., G. T. Phillips and P. C. Patnaik. 1978. Developing a Site Selection Methodology for Wind Energy Conversion Systems. DOE/ET/20280-3. Available from National Technical Information Service, Springfield, Virginia.

Traci, R. M., G. T. Phillips and K. C. Rock. 1979. Wind Energy Siting Methodology Windfield Model Verification Program, I. Oahu, Hawaii Data Set. DOE/ET/20280-80/1. Available from National Technical Information Service, Springfield, Virginia.

Traci., R. M.. D. C. Wvatt. P. C. Patnaik and G. T. Phillios, 1980. Wind Energy Siting Methodology Windfield Model verification' Program, I $\bar{I}$. Nevada Test Site Data Set. D0E/ET/20280-80/2. Available from National Technical Information Service, Springfield, Virginia.

Venkatesh S. and M. B. Danard. 1977. "A Model for Computing Small-Scale Wind Variations over a Water Surface." Boundary Layer Meteor. 14:35-57.

Walton, J. J., C. A. Sherman and J. B. Knox. 1980. Wind Power Site Screening Methodology Final Report. UCRL-52938, Lawrence Livermore Laboratory, Livermore, California. 


\subsection{PHYSICAL MODELING}

Physical modeling involves placing a scaled model of an object into a wind tunnel, water tunnel (also known as a flume), or a towing tank in order to determine how the object interacts with a fluid flowing over it. Physical modeling has a very long history of use in aerodynamics, naval engineering, and structural design. In more recent years, it has been used to model atmospheric flows in air pollution studies (Cermak 1975). The primary reason for turning to physical modeling is that current numerical models are still incapable of accurately simulating many problems in fluid mechanics. A scaled model in a flow facility represents a physical analogue to the real problem in nature; and if the study is constrained to consider only those problems that can be properly posed in a flow facility, physical modeling can yield results far more accurate than numerical models (also see Snyder 1979).

Since the models used in a physical modeling study are orders of magnitude smaller than the real object, it is not obvious that the results obtained will be transferable to nature. We will show in the next section that physical modeling results are transferable as long as certain constraints are met. These constraints limit the ability of physical modeling to simulate all of the physical variables that can affect flow over terrain. Flow over terrain can be physically modeled with reasonable accuracy, as long as the modeled region is no more than a few tens of kilometers in size, the portion of the atmosphere to be studied is the lowest few hundred meters, and the boundary layer is nearly neutral to unstable. (a) The ability of physical modeling to simulate flow over small-scale terrain features in nearly neutral flow is superior to currently available numerical models. Physical modeling, therefore, can be very useful in the final stages of wind turbine siting. It can be used in determining the placement of individual machines in a WECS cluster (see Chapter 9) or in planning the layout of wind monitoring equipment within a small area. Physical modeling of atmospheric flows does require large, specialized facilities, however. Riley and Delisi (1977) as well as Hunt and Fernholz (1975) provide listings of institutions that have proper facilities for modeling atmospheric flows.

(a) The last constraint is very important and is discussed in more detail in the next section. 


\subsection{THEORETICAL FOUNDATIONS AND LIMITATIONS}

The theoretical foundation of physical modeling is the principle of similarity. The principle states that if certain dynamic and kinematic constraints are met, flow over a dimensionally similar model will be identical to flow over the full-size object -- as long as boundary conditions are also the same. The dynamic constraints can be found by analyzing the equations that describe fluid flow. A thorough analysis of similarity constraints on the modeling of atmospheric flows has been made by numerous authors (e.g., Snyder 1972 and 1979; Meroney et a1. 1978a). This section will review the highlights of Snyder's analysis and stress those issues important to wind energy applications of physical modeling.

We assume that atmospheric motions in a dry atmosphere can be represented by the Boussinesq equations (see Section 3.3.1). These equations are

$$
\begin{gathered}
\frac{\partial u_{i}}{\partial t}+u_{j} \frac{\partial u_{i}}{\partial x_{j}}=-\frac{1}{\rho_{0}} \frac{\partial p^{\prime}}{\partial x_{i}}+\frac{g}{T_{0}} T^{\prime} \delta_{3 i}-2 \varepsilon_{i j k} \Omega_{j} u_{k}+v \frac{\partial^{2} u_{i}}{\partial x_{j} \partial x_{j}} \\
\frac{1}{\theta_{0}}\left[\frac{\partial \theta}{\partial t}+u_{i} \frac{\partial \theta}{\partial x_{i}}\right]=k \frac{\partial}{\partial x_{i}}\left[\frac{1}{T}\left(\frac{\partial T^{\prime}}{\partial x_{i}}-\gamma \delta_{3 i}\right)\right] \\
\frac{\partial u_{i}}{\partial x_{i}}=0
\end{gathered}
$$

where $\gamma \equiv g / c_{P}$ is the adiabatic lapse rate.

These equations can be made dimensionless through the use of certain scaling parameters that are defined by the boundary conditions of the problem. These scaling parameters are a length-scale characteristic of the problem (L), a characteristic velocity scale $U_{r}$, a density scale $\rho_{r}$, a temperature difference $T_{r}^{\prime}$ and an angular velocity $\Omega_{r}$. With these scaling parameters, we define the dimensionless variables

$$
\hat{x}_{i}=\frac{x_{i}}{L} \quad \hat{u}_{i}=\frac{u_{i}}{U_{r}}
$$




$$
\begin{array}{ll}
\hat{t}=\frac{U_{r}}{L} t & \hat{\rho}=\frac{\rho_{0}}{\rho_{r}} \\
\hat{p}=\frac{p^{\prime}}{\rho_{r} U_{r}^{2}} & \hat{T}^{\prime}=\frac{T^{\prime}}{T_{r}^{\prime}} \\
\hat{\Omega}_{j}=\frac{\Omega_{j}}{\Omega_{r}} & \hat{\theta}=\frac{\theta}{\theta_{0}}
\end{array}
$$

With these definitions, the dimensionless equations are

$$
\begin{gathered}
\frac{\partial \hat{u}_{i}}{\partial \hat{t}}+\hat{u}_{j} \frac{\partial \hat{u}_{i}}{\partial \hat{x}_{j}}=-\frac{1}{\hat{\rho}} \frac{\partial \hat{p}}{\partial \hat{x}_{i}}+\frac{1}{F r^{2}} \hat{T}^{\prime} \delta_{3 i}+\frac{2}{R o} \varepsilon_{i j k} \hat{\Omega}_{j} \hat{u}_{k}+\frac{1}{R e} \frac{\partial^{2} \hat{u}_{j}}{\partial \hat{x}_{j} \partial \hat{x}_{j}} \\
\frac{\partial \hat{\theta}}{\partial \hat{t}}+\hat{u}_{i} \frac{\partial \hat{\theta}}{\partial \hat{x}_{i}}=\frac{1}{P e} \frac{\partial}{\partial \hat{x}_{i}}\left[\frac{1}{\hat{T}}\left(\frac{\partial \hat{T}^{\prime}}{\partial \hat{x}_{i}}-\gamma \delta_{3 i}\right)\right] \\
\frac{\partial \hat{u}_{i}}{\partial \hat{x}_{i}}=0
\end{gathered}
$$

where

$F r \equiv \frac{U_{r}}{\left(g L T_{r}^{\prime} / T_{0}\right)^{1 / 2}}$ is the Froude number

Ro $\frac{U_{r}}{L \Omega_{r}}$ is the Rossby number

Re $\equiv \frac{U_{r} L}{V}$ is the Reynolds number

and

Pe $\frac{U_{r} L}{K}$ is the Peclet number. 


\subsubsection{Achieving Dynamic Similarity}

If Equations (4.4), (4.5) and (4.6) are an adequate mathematical model of atmospheric flow, we can form the following conclusion: flow over a properly scaled model of a section of terrain will be similar to flow over the actual terrain, provided each of the coefficients $\mathrm{Fr}$, Ro, Re, and Pe have the same values in the modeled flow as in the atmosphere and provided the dimensionless boundary conditions are also identical. (a)

Making the dimensionless coefficients in the modeled flow equal to those in the actual flow is frequently impossible, however. This is a common problem in physical modeling and its resolution requires some judgment on the part of the modeler. The solution is to first determine which of the dimensionless coefficients are most important in governing the dynamics of the problem at hand. The important coefficients are matched and the lesser ones are allowed to assume whatever values they will. The validity of results obtained under these conditions depends upon the validity of these assumptions. A modeler must be careful not to push his simulations too far beyond the range in which his similarity assumptions apply.

In modeling atmospheric flows, it is impossible to match Rossby, Reynolds, and Peclet numbers with the values they have in the atmosphere. The scaling parameter, $\Omega_{r}$, will be the same in the laboratory as in the flow to be modeled. Thus, if the scale factor for the model is to be $1: 1000$, fluid velocities in the simulations will also have to be reduced by a factor of 1000 to achieve the same Rossby number. Assuming that such small speeds could be achieved, reductions in fluid velocity of this magnitude would make matching the Reynolds and Peclet numbers possible only if $\mathrm{v}$ and $\mathrm{K}$ can be reduced accordingly. Such reductions, however, are out of the question. The resolution of this dilemma is to decide which is more important in properly simulating the dynamics of the problem at hand -- matching the Rossby number or matching the Reynolds and Peclet numbers?

(a) A properly scaled model has the same scaling factor in a11 three dimensions; that is, the vertical dimension, for instance, is not exaggerated with respect to the horizontal ones. 
The magnitude of the Rossby number can vary widely in atmospheric flow, depending upon the scale of the motion involved. In order to determine the scale atmospheric motions must reach before the effect becomes appreciable, consider the following argument: in order for the effect to be significant, it must have the same order of magnitude as the pressure force driving the flow; that is,

Now

$$
\left|\frac{1}{\rho_{0}} \frac{\partial p^{\prime}}{\partial x_{i}}\right| \simeq\left|\varepsilon_{i j k} \Omega_{j} u_{k}\right|
$$

$$
\left|\frac{1}{\rho_{0}} \frac{\partial p^{\prime}}{\partial x_{i}}\right| \simeq \frac{u_{r}^{2}}{L}
$$

and

$$
\left|\varepsilon_{i j k} \Omega_{j} u_{k}\right| \simeq U_{r} \Omega_{r}
$$

therefore,

$$
L \simeq \frac{U_{r}}{\Omega_{r}} .
$$

The rotational velocity of the earth (i.e., $\Omega$ ) is about $10^{-4} \mathrm{rad} / \mathrm{sec}$. If $U_{r}$ is about $10 \mathrm{~m} / \mathrm{sec}$, the scale at which the Coriolis effect becomes comparable to the pressure force is about $100 \mathrm{~km}$.

The problem can also be considered from the point of view of the time scales defined by the flow. Assume that we are considering flow over a section of terrain having a dimension of $L$. The time required for air to flow over the section is

$$
\tau_{f} \simeq \frac{L}{U_{r}}
$$

If the section of terrain is small enough, the air will flow across it before there can be any observable effect caused by the earth's rotation. In other words, $\tau_{f}$ will be small compared to a time scale set by the earth's rotation: 


$$
\tau_{r}=\frac{1}{\Omega_{r}} .
$$

The two time scales will be comparable if

$$
L E \frac{U_{r}}{G}
$$

which is the same result obtained previously.

If the area to be modeled is no larger than a few tens of kilometers, the effects of the earth's rotation on flow over the area will be negligible. Thus the Rossby number of the physical model need not be the same as in the actual flow. This does not mean, however, that the Coriolis effects on the boundary conditions can be completely neglected. Near the surface of the earth, the Coriolis force is observed through its effect on wind direction. In the northern hemisphere, the Coriolis effect will cause the wind to rotate in a clockwise direction as the distance above the surface increases. The wind profile above a section of terrain will show changes in both magnitude and direction. This rotation of the wind vector with height must be replicated in the boundary conditions of a physical model if the principle of similarity is to be strictly obeyed.

Maintaining strict similarity in the case of the Reynolds and Peclet numbers is also a problem in the physical modeling of atmospheric flows. For example, wind tunnels are the facilities most frequently used in physical modeling studies. Hence, the properties $v$ and $k$ in the wind tunnel are those of the atmosphere. If the scale reduction is a factor of 1000 , the wind speed would have to be increased by the same factor to achieve similarity with respect to those terms in Equations (4.4) and (4.5) multiplied by the Reynolds and Peclet numbers. Besides the impossibility of achieving such astronomical speeds in a wind tunnel, increasing wind speeds by this factor would mean that the wind tunnel flow would no longer be incompressible and that the effects of viscous heating in the entropy Equation (4.5) could not be neglected. In other words, the hypersonic flow in the wind tunnel would no longer be similar to flow in the atmosphere. 
Fortunately, however, the situation is saved by the principle of Reynoldsnumber independence. According to this principle, the Reynolds-number sensitivity of most features of fluid flow decreases as the Reynolds number increases. Above a certain Reynolds number, therefore, the behavior of the flow "sufficiently far from any boundaries" will not change significantly as the Reynolds number is increased. The same conclusion holds with respect to the Peclet number and the behavior of the temperature field. The arguments backing this principle are mainly heuristic, but they are based on a considerable amount of experimental evidence.

The principle of Reynolds-number independence is somewhat vague, however; there is little guidance as to the magnitude of the "certain" Reynolds number or as to how far from a boundary we must go before the flow is Reynolds-number insensitive. As to the first issue, a flow will probably be Reynolds-number independent if the Reynolds number is about two orders of magnitude larger than the critical Reynolds number and if the ratio of the scale of the dominant turbulent eddies to the dominant terrain features is the same in the model as in the actual flow. The critical Reynolds number in this case is the maximum Reynolds number at which laminar flow can be observed. Meroney et a1. (1978a) suggest that for nearly neutral flows the Reynolds number, based on the boundary layer depth, should exceed $10^{4}$. However, the critical Reynolds number is a function of thermal stratification (see Coantic 1978), and a flow that ma be Reynolds-number independent under neutral or convectively unstable stratification could be Reynolds-number dependent under stable conditions. With respect to the second restriction, that comparisons between physical models and the real atmosphere should be restricted to regions "sufficiently far" from the boundaries, "sufficiently far" is probably a distance considerably larger than the size of the roughness elements.

The last of the dimensionless constants to be considered is the Froude number. The Froude number is a measure of the role of buoyancy in fluid motions and cannot be neglected in the simulation of atmospheric flow (see Section 3.2). Since the exact value of the reference temperature $T_{r}^{\prime}$ is arbitrary, the Froude number defined by Equation (4.4) can be rewritten as 


$$
F r^{2}=\frac{u_{r}^{2}}{g L^{2}(\Gamma+\gamma) / T_{0}}
$$

where

$$
\Gamma=\frac{\partial T}{\partial z}
$$

is the atmospheric lapse rate and

$$
\gamma=-\frac{\partial T_{0}}{\partial z}=\frac{g}{c_{p}}
$$

is the lapse rate for a neutrally stratified, dry atmosphere. The square of the Froude number is related to the Richardson number

$$
R i=\frac{g \partial \theta / \partial z}{T_{0}\left[(\partial u / \partial z)^{2}+(\partial v / \partial z)^{2}\right]}
$$

since

$$
\frac{\partial \theta}{\partial z}=\Gamma+\gamma
$$

and $(\partial u / \partial z)^{2}+(\partial v / \partial z)^{2}$ is assumed to be related to $U_{r}{ }^{2} / L^{2}$.

Temperature profiles in the atmosphere are frequently complex; thus, the Froude number or the Richardson number can vary greatly with height. On a typical summer afternoon, for example, the temperature lapse rate in the atmosphere can be unstable near the surface, nearly neutral through much of the boundary layer, and then stable above the boundary layer. Thus, the Froude number at a given level in the atmosphere can be real or imaginary and can change in value by several orders of magnitude. Since the Froude number is such a strong function of height and has such an influence on the character of the flow, it is important that its behavior as a function of $\hat{x}_{i}$ be the same 
in simulations as in the actual flow. This is assured if the initial and boundary conditions in a physical simulation are strictly similar.

In reality, however, dynamic similarity with respect to the Froude number can only be maintained over a limited range of stabilities. Equation (4.7) shows that a scale reduction of $1: 1000$ requires increasing the lapse rate difference $(\Gamma+y)$ by $10^{6}$ if the wind speed is held constant. For flows having nearly neutral stratification, the wind speeds in a physical modeling facility can probably be varied by a considerable amount (from $\sim 1 \mathrm{~m} / \mathrm{sec}$ to $\sim 10 \mathrm{~m} / \mathrm{sec}$ ) and still achieve Reynolds number independence. Nevertheless, even if the wind speed in a model were made an order of magnitude smaller than the atmospheric flow, a scale reduction of 1:1000 would require an increase in $(\Gamma+y)$ of 10,000 . (a) This makes the simulation of highly stratified flows difficult, particularly in wind tunnels. Very few wind tunnel facilities, for instance, are even capable of simulating stratified flows. Those that are capable can only simulate fairly simple conditions such as a constant, mildly stable lapse rate (a problem in simulating lateral boundary conditions; see below). The best facilities for maintaining Froude number similarity under stable conditions are towing tanks. These facilities are capable of simulating large and fairly complex stratifications (Riley and Del isi 1977).

Because of the experimental difficulties in simulating stratified flow, most physical modeling studies are conducted in wind tunnels under neutrally stratified conditions and no surface heat flux. In the strictest sense, these simulations only apply to atmospheric flows that also have no surface heat flux and are neutrally stratified over the entire depth of the atmosphere--conditions that are never observed. In a practical sense, however, restrictions on the validity of neutrally stratified simulations are not nearly so severe. This point wi 11 be discussed in more detail in the next section.

\subsubsection{Achieving Similarity in the Boundary Conditions}

As Snyder (1972) points out, similarity requires that the dimensionless velocity, temperature, pressure, and density fields of the modeled flow and

(a) The adiabatic lapse rate, y, is $9.8 \times 10^{-3} \mathrm{~K} / \mathrm{m}$; thus, to model an isothermal layer, $\Gamma=0,(\Gamma+\gamma)$ must be $98 \mathrm{~K} / \mathrm{m}$ for a scale reduction of $1: 1000$. 
the atmospheric flow be identical at the beginning of a simulation. These fields must also be the same for all subsequent times at the boundaries. This restriction means that the mean fields, as well as all turbulence characteristics, must be matched to the relevant atmospheric conditions. Again, achieving exact similarity in the boundary conditions is impossible. Most modelers attempt to match the means, the variances, and the surface distribution of heat and momentum flux and assume this degree of similarity to be sufficient.

Physical modeling studies are usually attempted on non-time-dependent flows only. In this situation, matching initial conditions is equivalent to ensuring that the flow field approaching the section of modeled terrain is representative of the pertinent atmospheric conditions. Realistic simulation of the wind characteristics in the flow approaching modeled terrain is an area of particular concern to physical modelers. It is generally accepted that proper simulation of boundary layer characteristics is most important. At a minimum, the wind profile in the boundary layer should be realistic and the boundary layer depth should show the proper scale relationship to terrain features.

Figures 4.1 and 4.2 illustrate profiles of potential temperature and the two horizontal components of wind velocity measured over the open ocean after the passage of a strong cold front. Because the sea-surface temperature was $10^{\circ}$ to $12^{\circ} \mathrm{C}$ warmer than the air, turbulent mixing in the boundary layer was vigorous. This mixing is due to the large upward heat flux and the large production of turbulent energy by buoyancy forces (see Appendix 2). The figures illustrate that complicated wind profiles can occur over the simplest of terrain and since strong winds at many locations are associated with the meteorological conditions that exist during and after frontal passages, it would seem useful to discuss the dynamics of this situation in a little more detail. The strong wind shears illustrated in Figure 4.2 are the result of the strong horizontal temperature gradients experienced after the passage of fronts. With large horizontal temperature gradients, the pressure gradient is a function of altitude, This varying pressure gradient can result in strong wind shears at any level in the atmosphere (see Holton 1972, Chapter 3; and Appendix 2).

(a) The situation in the neighborhood of cold fronts is also complicated by the fact that the pressure forces can be changing rapidly with time. 
Boundary layer mixing greatly reduces the magnitude of the wind shear. The figures illustrate this point very well. Within the boundary layer, the wind profile behaves reasonably well--at least in the sense that the wind direction remains relatively constant. If the boundary layer wind speed profile shown in Figure 4.2 could be approximated in the physical modeling facility, flow within the boundary layer could be simulated. Failure to match the complicated wind and temperature structure above the boundary layer would be unimportant as long as local terrain relief (and the size of the wind turbine) were small compared to the boundary layer depth (a point discussed in more detail below).

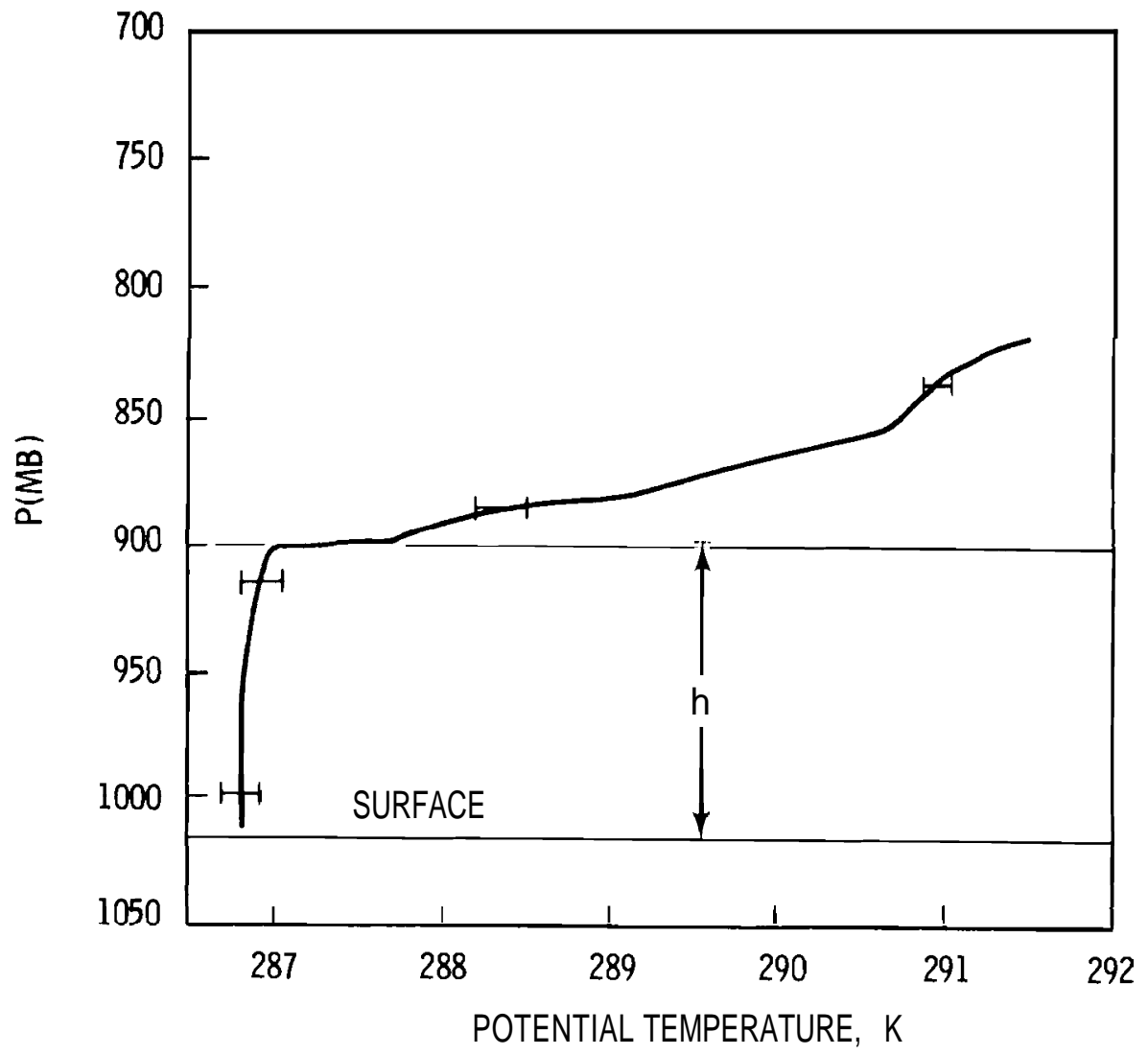

FIGURE 4.1. Potential Temperature Profile from the Air Mass Transformation Experiment (AMTEX) in the East China Sea $\left(25^{\circ} \mathrm{N}, 125^{\circ} 30^{\prime} \mathrm{E}\right)$ at 0300 GMT, 18 February 1975, Following the Passage of a Cold Front. Error bars indicate the amount of scatter in the measurements (after Pennel 1 et a1. 1979). 

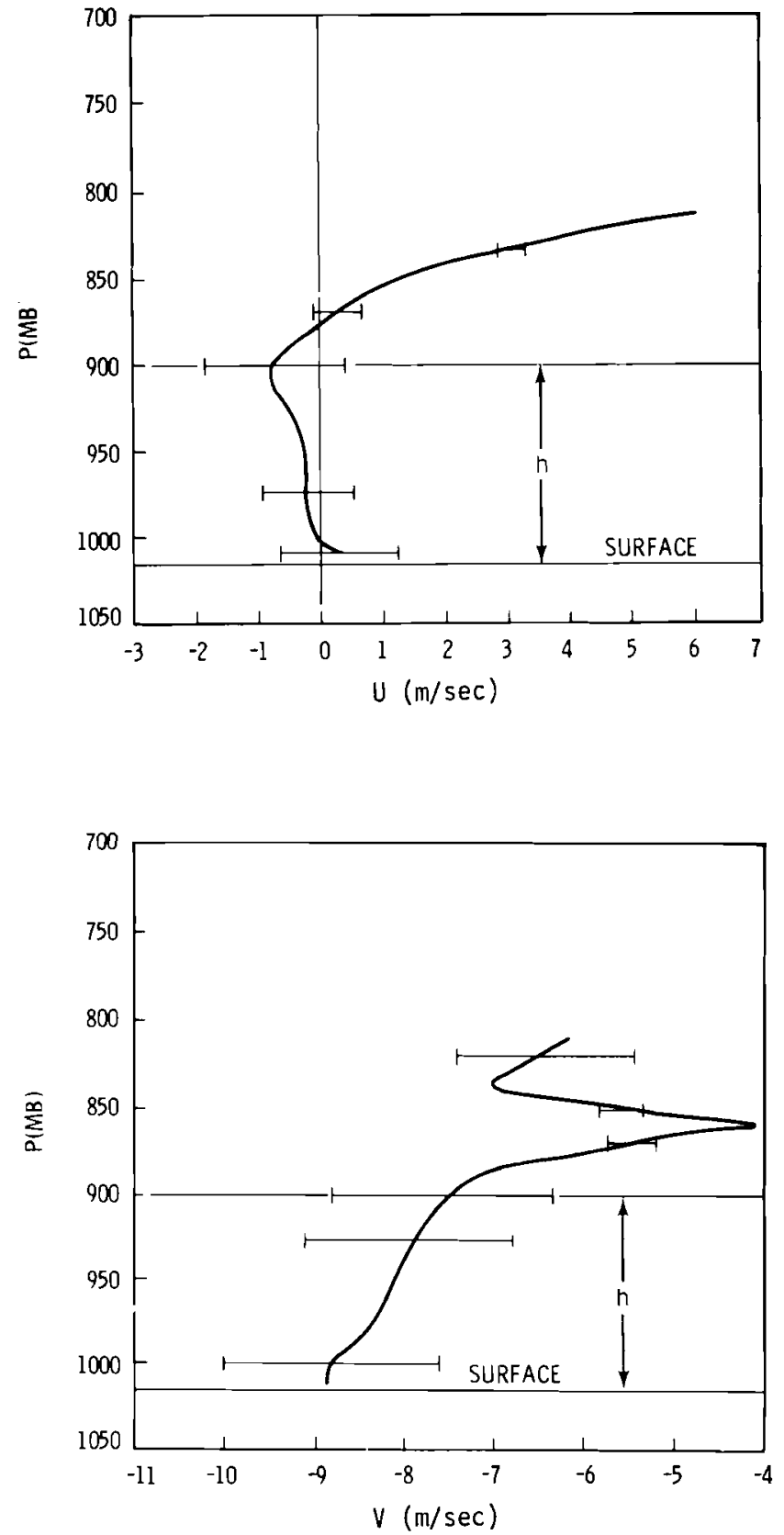

FIGURE 4.2. Profile of the $u$ (,Eastward) Component and v (Northward) Component of the Wind From the Air Mass Transformation Experiment (AMTEX) in the East China Sea $\left(25^{\circ} \mathrm{N}, 125^{\circ} \cdot 30 \mathrm{E}\right)$ at 0300 GMT, 18 February 1975, Following the Passage of a Cold Front. Error bars indicate the amount of scatter in the measurements (after Pennell et a1. 1979). 
Obtaining a boundary layer thick enough to simulate the typical daytime boundary layer is not a particular problem in a wind or water tunnel. It can be accomplished by placing large roughness elements on the floor of the tunnel upstream of the modeled terrain. When the surface is roughened in this way, the boundary layer will grow much more rapidly than it would over a smooth floor. Even with a boundary layer of proper thickness, its turbulence characteristics can differ from the characteristics of the atmospheric boundary layer. Figure 4.3 compares turbulence measurements in the neutrally stratified boundary layer over a wind tunnel floor (Meroney et a1. 1977) with measurements in an atmospheric boundary layer over the open ocean (Pennell and LeMone 1974). The atmospheric measurements were made as close to neutral conditions as is likely to be observed in the atmosphere. The figures show fairly good agreement in the lower $40 \%$ of the boundary layer. In the upper portion of the boundary layer, the discrepancies between the wind tunnel and atmospheric boundary layers are large. In the atmospheric boundary layer, there is a very small positive heat flux between the sea surface and the air. The boundary layer, in other words, is convectively unstable. This slight positive heat flux is enough to result in a significant transport of turbulence energy (mechanically generated near the surface) by buoyancy to the outer portion of the boundary layer. This mechanism of turbulence transport is missing in the strictly neutral boundary layer. The point at which the behavior of turbulence in the atmosphere shows significant differences from the behavior of a wind tunnel flow moves toward the surface as the atmospheric boundary layer becomes more unstable (see Appendix 2). Sometimes, an attempt is made to increase turbulence levels in the outer portion of wind tunnel boundary layers through the use of turbulence-producing grids or vortex generators (Meroney et al. 1977). However, these procedures produce turbulence that has considerably different properties than the turbulence in an atmospheric boundary layer. (a)

The failure of a neutrally stratified flow facility to produce a boundary layer that completely mimics the atmosphere is probably not significant for

(a) A more realistic simulation of the turbulence i $n$ an unstable atmospheric boundary layer can be achieved by heating the wind tunnel floor. If wind speeds in the tunnel are not too high (on the order of $1 \mathrm{~m} / \mathrm{sec}$ ), the heating rates required to achieve Froude number (or Richardson number) similarity for unstable boundary layer flows are not excessive. 


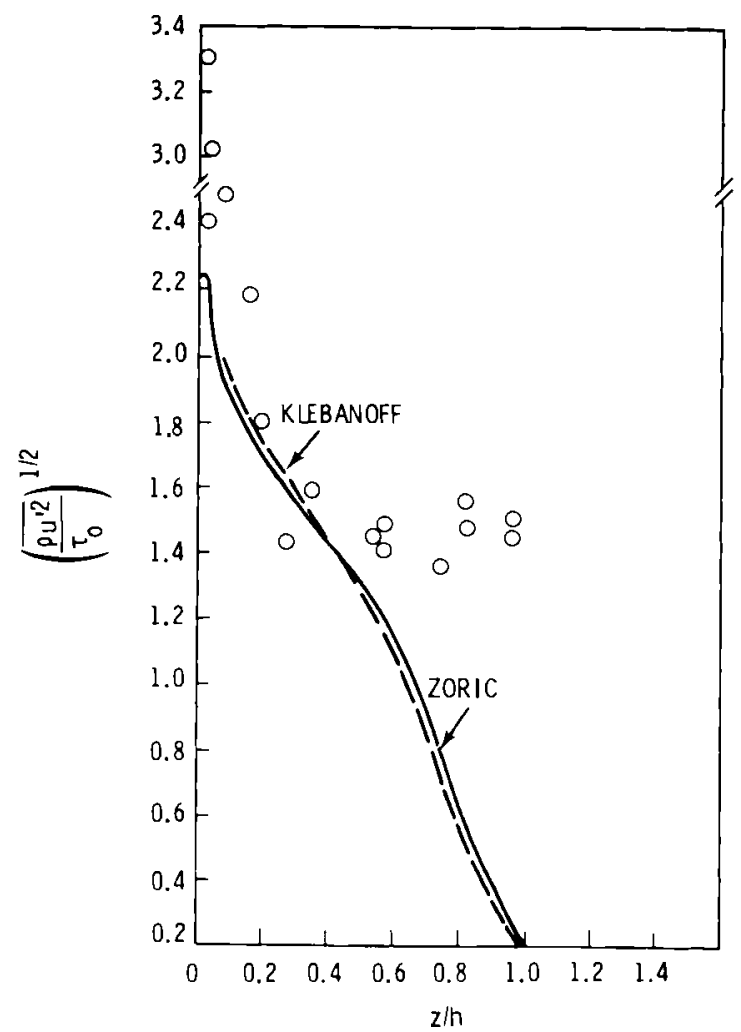

(a)

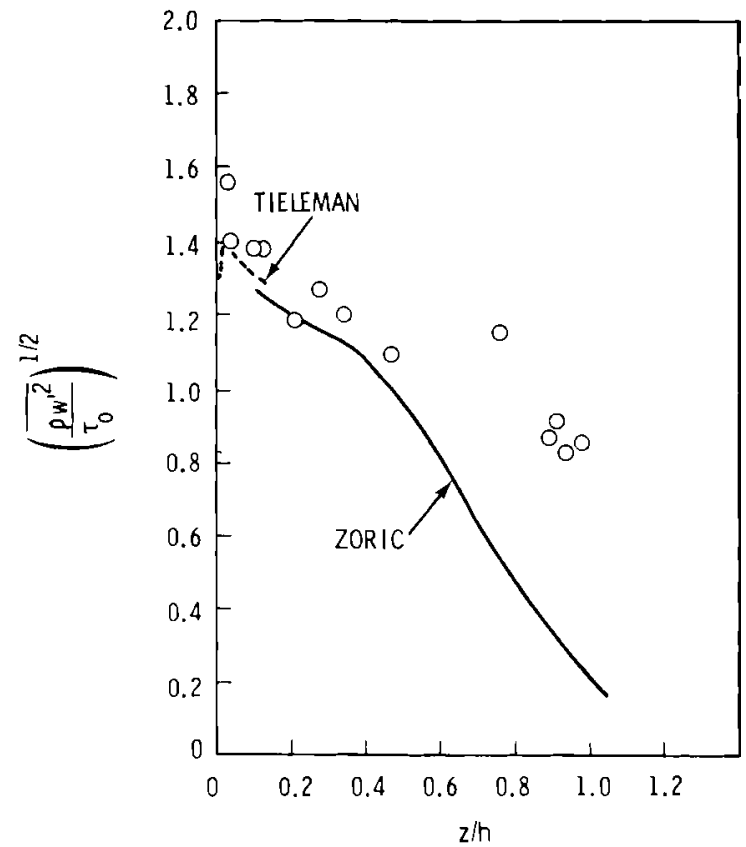

(b)

FIGURE 4.3. Measurements of the Longitudinal (a) and Vertical (b) Coniponents of Turbulence Intensity. Comparison is between measurements in a neutrally stratified boundary layer over a wind tunnel floor (Zoric 1968, Klebanoff 1954, and Tieleman 1967 as cited by Meroney et al. 1977) and measurements in nearly neutral atmospheric boundary layer over open ocean (Pennell and LeMone 1974). 
many situations of importance in wind energy siting. If winds near the earth's surface are moderate to strong and if there is positive heat transfer from the earth's surface to the atmosphere, a fairly deep atmospheric boundary layer will develop. The depth of this boundary layer can range from several hundred to several thousand meters. Because of strong turbulent mixing, the temperature lapse rate in the boundary layer will be nearly neutral (Figure 4.1), except for a negligibly thick layer near the surface that will be unstably stratified. Such a boundary layer is unstable, not neutral, even though the lapse rate over the bulk of the boundary layer is adiabatic. As discussed above, a major difference between the unstable atmospheric boundary layer and the neutral boundary layer in a wind or water tunnel is in the structure of the turbulence. Flow in the convectively unstable boundary layer can be modeled by a neutral flow in a wind tunnel if the scale of the terrain is much less than the depth of the boundary layer. Under this condition, differences in the flows due to the differences in turbulence structure should be secondary.

There are additional reasons for restricting the simulation of flow in an unstable atmospheric boundary by a neutral wind tunnel boundary to small terrain features. Unstable atmospheric boundary layers are always capped by stably stratified air as in Figure 4.1. If terrain relief is not smaller than the boundary layer depth, interactions between the terrain and this stable air can affect the flow significantly. In addition, if the terrain features are large enough, temperature variations over the surface of the terrain can generate secondary flows that can also affect flow near the surface (see Section 5.4.1).

On sunny days, the conditions described above generally hold over land surfaces. This is not the case under many other situations. Over land surfaces, boundary layer thickness and the vigor of the turbulent mixing process show considerable seasonal and diurnal variation. At night, for instance, boundary layer thicknesses can shrink considerably, reaching depths of $100 \mathrm{~m}$ or less. Thermal stratification and wind profiles near the surface can become very complicated and the wind and temperature fields can show considerable horizontal variabi 1ity on a very small scale (Lenschow et al. 1979). Physical modeling facilities are simply not capable of handling situations where there are large, 
spatial variations of wind speed, wind direction, and temperature on scales comparable to the size of the modeled region.

Assuming that a satisfactory representation of an atmospheric boundary layer can be achieved in the modeling facility, the last requirement for a successful simulation of atmospheric flow is to obtain similarity in the distribution of surface heat and momentum fluxes. Usually, physical modeling studies are restricted to neutral conditions because, as was pointed out in the previous section, it is very difficult to maintain both Reynolds and Froude number similarity when there are large reductions in scale. Simulating neutral conditions makes specification of the surface heat flux simple. It is zero everywhere.

Similarity in the surface distribution of momentum flux is achieved by producing a surface roughness distribution over the model that represents the surface roughness distribution of the actual terrain. One way of doing this is to follow the Jensen criterion (Snyder 1972). According to this rule, the roughness length of the model is scaled such that

$$
z_{0, m}=\left(\frac{L_{m}}{L_{f}}\right) z_{0, f}
$$

The Jensen criterion, however, frequently conflicts with the need to maintain a surface that is aerodynamically rough.

As was discussed in the previous section, one of the ironclad laws of physical modeling is that the Reynolds number of the model must be large enough that the flow is Reynolds-number insensitive. Numerous experiments have shown (Schlichting 1960) that certain quantities in a turbulent flow that depend on the properties of the flow near the surface (such as the drag coefficient) remain Reynolds-number dependent as long as the surface is smooth If the surface is roughened, a Reynolds number will be reached where the drag coefficient, for example, no longer changes. When this limit is reached, the flow is said to be aerodynamically rough. The consensus of experimental studies cited by Schlichting (1960) is that a flow will be aerodynamically rough if 


$$
\frac{u_{*} z_{0}}{v}>2.5
$$

Keeping conditions over the model aerodynamically rough helps ensure that the flow will be Reynolds-number independent. Therefore, when Equation (4.9) conflicts with the Jensen criterion, this equation can be used to set the minimum roughness length on the model. The surfaces with larger roughness are then scaled accordingly.

The criterion expressed in Equation (4.9) also sets a lower limit on the resolution of the model results applied to the atmosphere. The roughness length $\left(z_{0}\right)$ is about an order of magnitude smaller than the physical dimensions of the roughness element; therefore all objects that are physically represented in the model must be larger than about $10 z_{0}$. This does not turn out to be a serious restriction, however. For a typical wind tunnel,

$$
0.05 \mathrm{~m} / \mathrm{sec}<u_{\star}<1 \mathrm{~m} / \mathrm{sec}
$$

and $v=1.5 \times 10^{-5} \mathrm{~m}^{2} / \mathrm{sec}$. Substituting these quantities into Equation (4.9) gives a minimum roughness length of $4 \times 10^{-5} \mathrm{~m}$ to $8 \times 10^{-4} \mathrm{~m}$. For a scaling ratio of 1000:1, this gives a resolution limit of about 5 to $10 \mathrm{~m}$ in the fullscale flow.

\subsection{VERIFICATION RESULTS}

Studies comparing simulations of flow over topography obtained by physical modeling with field measurements are as rare as similar studies on numerical simulations. The reasons are the same as discussed in Section 3.3.4-the extreme difficulty in obtaining good field observations. Recently, two studies were conducted that compared the ability of large wind tunnels to simulate flow over two different types of terrain. In one study (Meroney et al. 1978b), simulations of flow in the region of the Rakaia Gorge, New Zealand, were compared with field measurements. In the second study (Chien at al. 1979), the same was done for the Kahuku Point area of Oahu, Hawaii. In both of the studies the thermal stratification of the simulation was neutral. 
In the Rakaia Gorge study a section of terrain $6.1 \mathrm{~km}$ wide and $18.3 \mathrm{~km}$ long, centered over the gorge, was modeled at a scale of 1:5000. This area is in a region thought to have great wind energy potential. Great care was exercised in reproducing the contour of the terrain and in representing the surface roughness distribution. Meroney et al. (1978b) provide extensive details on how this was accomplished.

Flow simulations were compared with field measurements on two separate days. The field measurements consisted of two sets of 15-min average winds taken with calibrated instruments at an elevation of $10 \mathrm{~m}$. Measurements were made at a total of 27 previously selected sites. The total elapsed time was approximately 6 hours. During the time mobile measurements were made, continuous wind observations were taken at two fixed locations. Three measurement teams were used. Two teams covered either side of the gorge while the third team traveled up the gorge by boat. On each of the two days chosen for measurement, winds were moderate to strong, winds were from the same general direction, and skies were overcast. These conditions suggest that the atmospheric boundary layer was more or less neutral (see Appendix 2), at least near the surface.

Comparisons of simulated and observed wind speeds for each of the two days are presented in Figure 4.4. The figure compares "10-m" wind speeds that have been normalized by the wind speed at the top of the boundary layer, Since actual wind speeds can change considerably over a 6 -hr period, a method had to be devised to account for the time lapses between the 27 mobile wind measurements. To accomplish this the ratio of a 15-min average wind at a mobile site to the corresponding 15-min average at one of the fixed sites was assumed to be a function of location but not of time. Accordingly, each of the 27 wind measurements could be adjusted to a standard reference time. Local noon was chosen as the reference, and the adjustment was made by the simple algorithm

$$
u_{m}\left(t_{0}\right)=u_{m}(t) \frac{u_{s}\left(t_{0}\right)}{u_{s}(t)}
$$


(a)
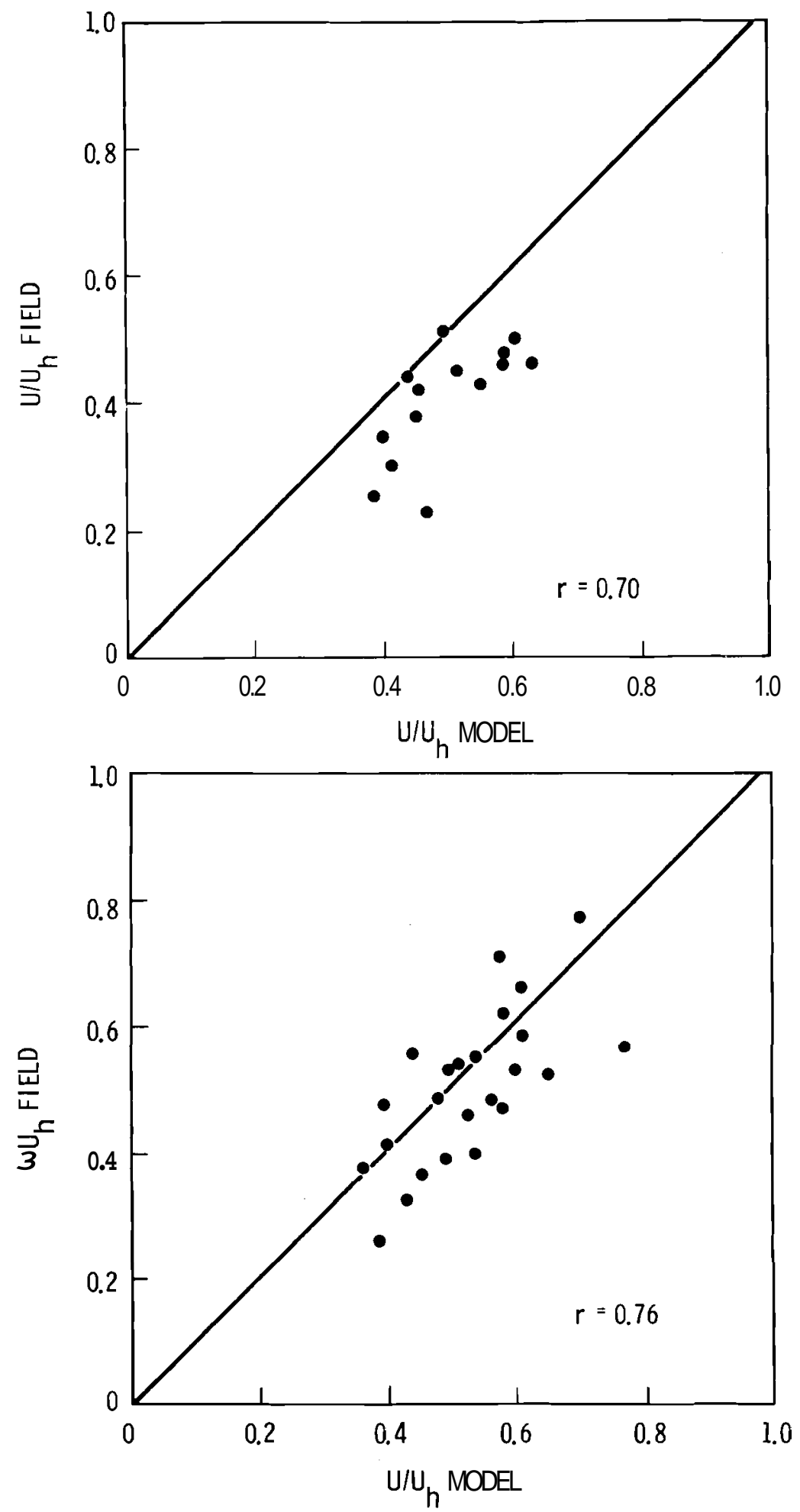

FIGURE 4.4. Scatter Diagrams of Rakaia Gorge Field Measurements Versus Wind Tunnel Simulation Measurements for 25 November 1977 (a) and 28 December 1977 (b) (Meroney et al. 1978b). U/Uh is the wind speed at $10 \mathrm{~m}$ normalized by the free stream wind speed. 
where $t_{0}$ is the reference time' and the subscripts $m$ and stand for the mobile stations and the fixed station, respectively. The free stream velocity $u_{h}$ was measured in the wind tunnel model. Then, the following relationship was assumed for the field measurements.

$$
\left[\frac{u_{s}\left(t_{0}\right)}{u_{h}\left(t_{0}\right)}\right]_{\text {field }}=\left[\frac{u_{s}}{u_{h}}\right]_{\text {model }}
$$

Figure 4.4 shows good agreement between the simulations and the observations for these two cases. The physical model not only shows skill in simulating the mean wind speed distribution but also demonstrates good performance in ranking the sites according to wind speed. However, the limitations of these comparisons should also be noted. These comparisons represent spot checks under carefully selected conditions, not an exhaustive verification. The validity of the comparisons is also limited by the assumptions made in the analysis of the field measurements.

In the second experiment, flow over a 1:3840 scale model of the Kahuku Point area was studied in a large, neutrally stratified wind tunnel (Chien et al. 1979). The area included in the model is indicated in Figure 4.5. The model was carefully constructed in order to faithfully represent the topography and the surface roughness distribution. Flow over the model surface was mapped for three prevailing wind directions: $045^{\circ}, 067^{\circ}$, and $090^{\circ}$. Figure 4.6 is an example of the wind speed distribution at a simulated height of $50 \mathrm{~m}$ for a prevailing wind direction of $067^{\circ}$.

Chien et a1. (1979) compared these simulations with field measurements obtained by a research team from the University of Hawaii. Measurements were conducted at a number of sites in the Kahuku Point area from August 8, 1978 to October 10, 1978. The measurements were made from vans equipped with $30-\mathrm{ft}(\sim 9-\mathrm{m})$ towers. Six-minute average values of wind speed and direction were made from the tops of the towers. At any one site, continuous measurements were made for at least 24 hours. To account for the fact that simultaneous measurements were not taken, all wind speeds were normalized by the wind speed at a fixed reference station. 


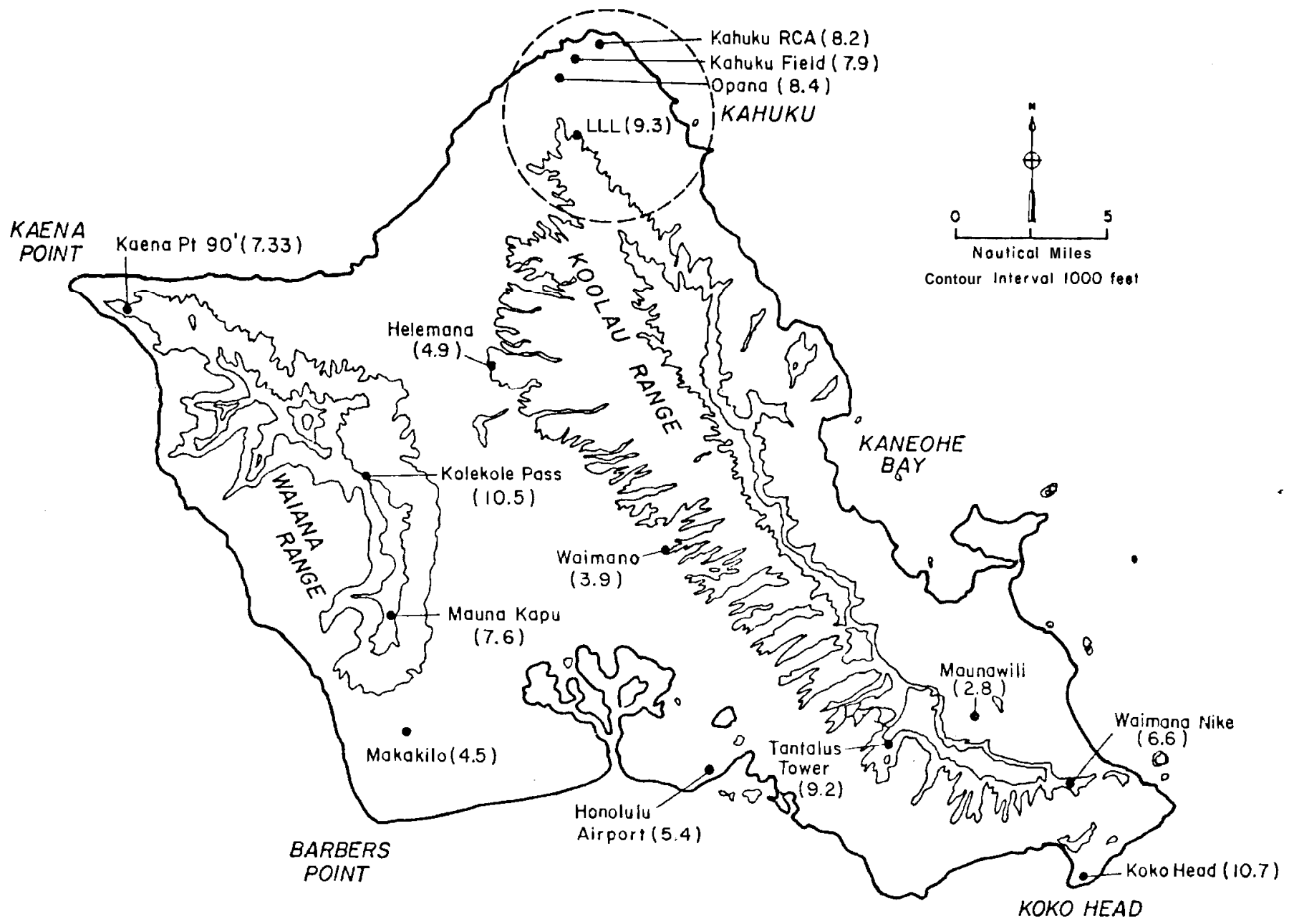

FIGURE 4.5. Annual Mean Wind Speed, $\mathrm{m} / \mathrm{sec}$, at Sites Over Oahu and the Area (circled) Covered in the Wind Tunnel Simulation. (Chien et al. 1979) 


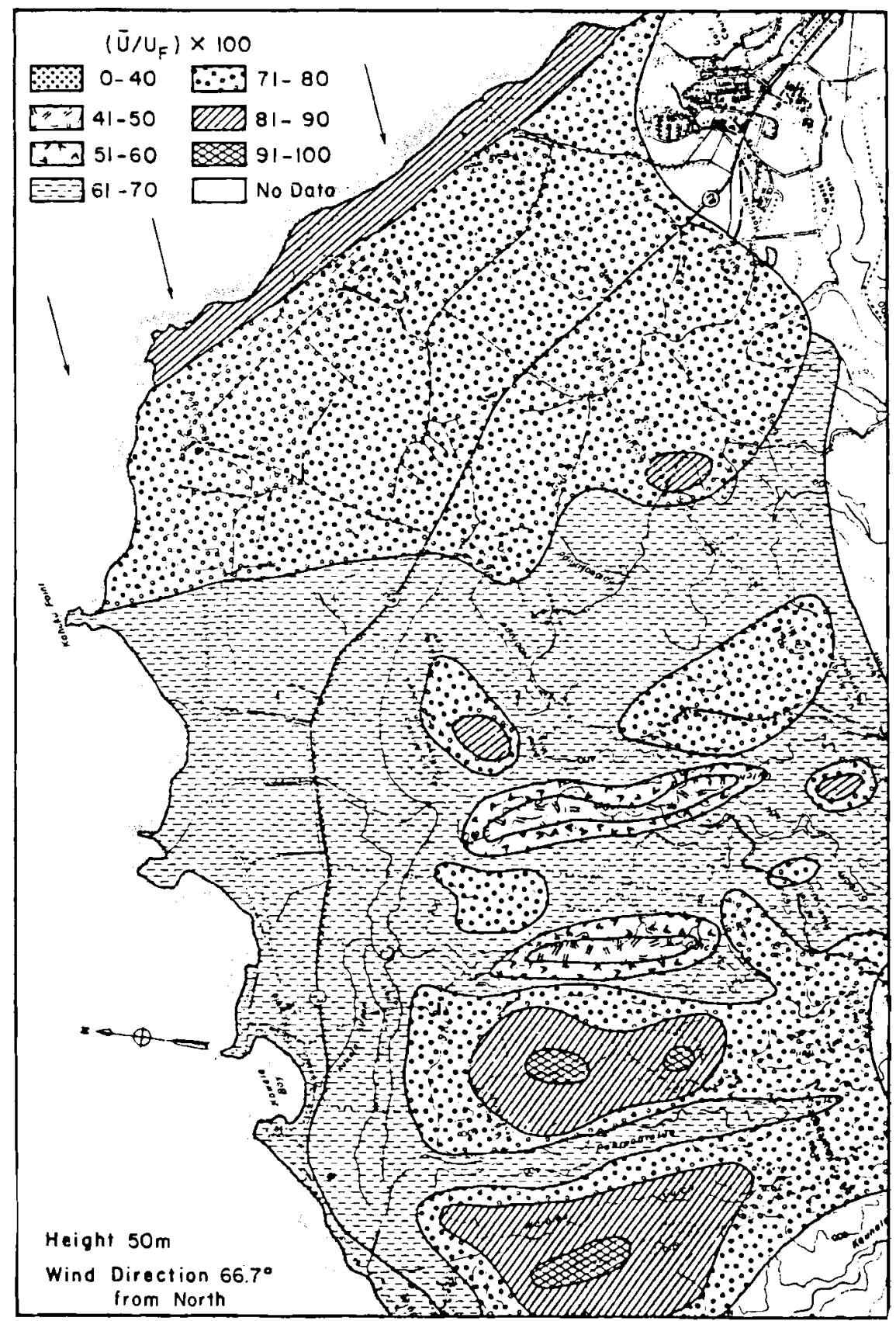

FIGURE 4.6. Contour Plot of Relative Wind Speeds, i.e., the Ratio of Simulated Wind Speed to Measured Simulated Free Stream Wind Speed in \%, Over the Kahuku Point Model at Equivalent 50-m Height for Wind Direction of $067^{\circ}$ (Chien et al. 1979) 
Comparisons between the wind tunnel simulations and the field measurements were made in order to gauge the accuracy of the simulations. Average normalized wind speed and average wind direction are tabulated for each of the field sites in Chien et al. (1979). The tabulation also includes normalized wind speeds at a simulated elevation of $10 \mathrm{~m}$ for the three prevailing wind directions studied in the wind tunnel. Normalized wind speed estimates are presented at every point corresponding to the location of a field measurement. Using those tabulated results we made a comparison of simulated to observed wind speeds. A field measurement was compared with it s corresponding wind tunnel value if the average wind direction at the field site was within $\pm 10^{\circ}$ of one of the three wind directions simulated in the wind tunnel. The field data that did not meet this condition were not used in our comparison. The field measurement was compared, of course, with the wind tunnel simulation having the wind direction closet to the actual flow. Chien et al. (1979) also compared laboratory simulations with field measurements, but the method they used was somewhat different.

There is an implicit assumption in our comparisons that the average wind direction at a field site equals the free stream wind direction of the time period in question. (a) This may not be the case. Therefore, field measurements in regions where the wind tunnel simulations showed significant terrain effects on wind direction were rejected. Field measurements were also rejected from sites where the anemometer exposure was thought to be poor (Chien et al. 1979).

Figure 4.7 shows the results of these comparisons. Agreement between simulations and observations in the Kahuku Point experiment is poorer than in the Rakaia Gorge study. The reasons for this lie in the way the field data were collected for these two studies. In the Rakaia Gorge experiment, data were collected under meteorological conditions close to the conditions that exist in a wind tunnel. At Kahuku Point, a broader range of conditions was sampled. Chien et al. (1979) note that on several days there was evidence of either a sea-breeze influence on the flow or an anabatic flow

(a) A better method of determining the free stream wind direction would have been to use a well-exposed reference station. We did not have access to data that would have allowed us to do this. 
(see Section 5.4.1) up the slope of the Koolau Ridge. If those days were not included in the comparisons, the performance of the simulations vis-a-vis the field measurements would be greatly improved. However, a comparison that eliminated troublesome meteorological conditions would not be a proper indication of the model's validity. We assume that a person using physical modeling to understand flow over a region is interested in the model's ability to produce information of climatological significance. In this case the comparisons must be made against the full range of conditions affecting flow over Kahuku Point, not just a selected subset of these conditions.

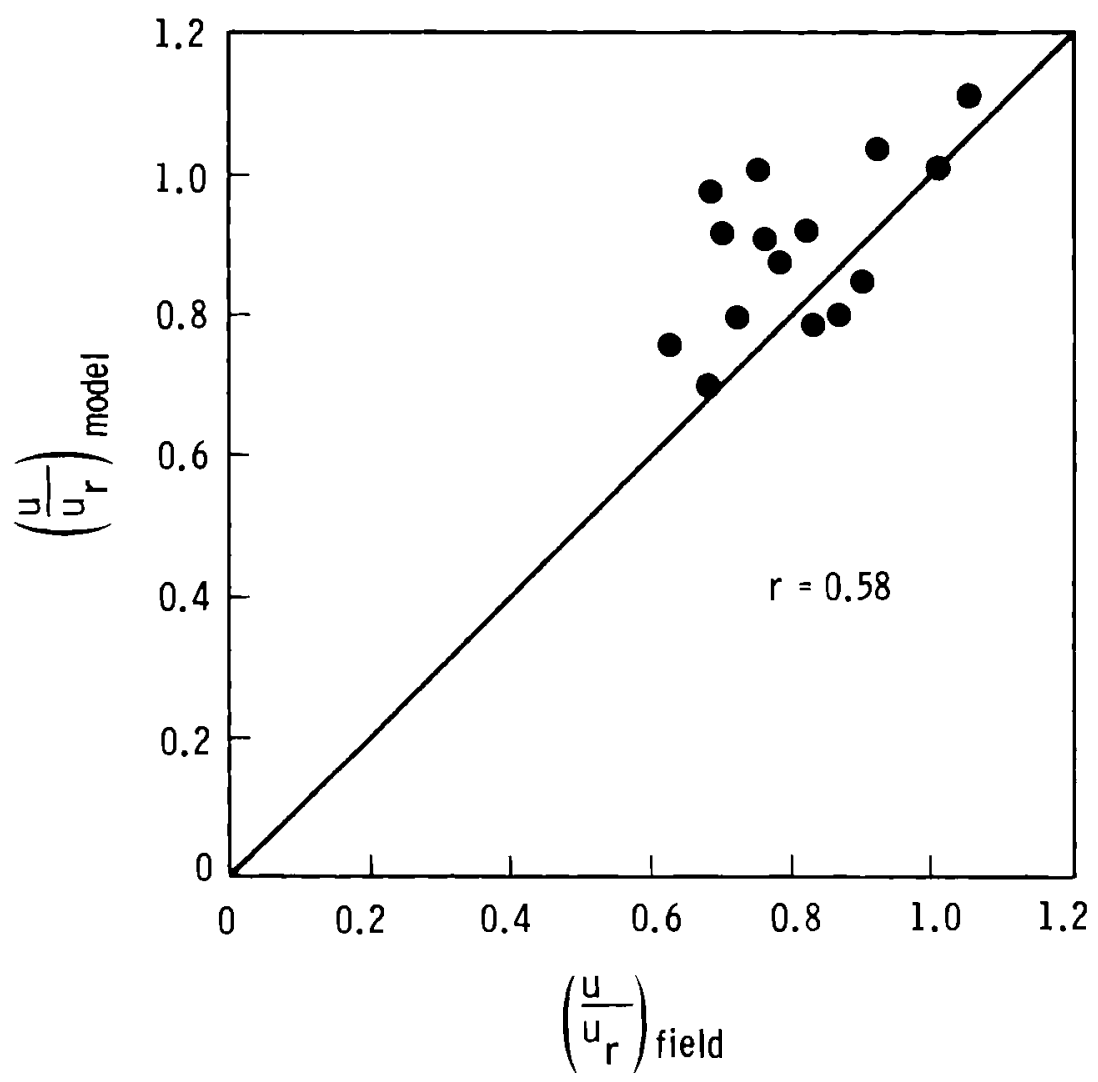

FIGURE 4.7. Scatter Diagram of Kahuku Point Field Measurements Versus Wind Tunnel Simulations. $u / u_{r}$ is the $10-m$ wind speed normalized by the wind speed at a reference station.

\subsection{CONCLUSIONS AND RECOMMENDATIONS}

The theoretical justification for physical modeling is the similarity principle. This principle states that the flow over a geometrically similar 
model will be analogous to flow over the actual object if the two flows are dynamically and kinematically simi lar and if the initial and boundary conditions are similar. True similarity rarely exists when there is a large reduction in size between the object and the model. A lack of complete similarity is certainly the case when atmospheric flows are modeled; however, it also occurs in more traditional applications of physical modeling, such as the modeling of ships or of aircraft. Useful results can still be obtained in these cases, as long as the neglected similarity requirement has a small influence on the resulting flow.

Analysis of the equations of motion shows that the key requirements for modeling atmospheric flow over regions smaller than a few tens of kilometers are a large Reynolds number, a Froude number (or Richardson number) identical to the actual flow, and identical initial and boundary conditions. How satisfactorily a physical model satisfies these requirements is largely a function of stability. The similarity constraints are met most easily for slightly to moderately unstable flows when local terrain relief is small compared to the depth of the boundary layer. (a) Under these conditions, flow in the boundary layer should be fairly homogeneous and the wind profiles simple with little turning of the wind vector with height. This situation results in fairly simple boundary conditions and the atmospheric flow can probably be represented by a neutral boundary layer in a wind or water tunnel.

The physical modeling of highly stable flows over complex terrain is much more difficult. As the Froude number becomes smaller (or the Richardson number larger), it becomes increasingly difficult to maintain both Reynolds number and Froude number similarity. The boundary conditions also become more complex. Variations in surface temperature over the terrain become important factors in driving the flow. There may also be large horizontal and vertical variations in the wind and temperature fields due to weak or nonexistent turbulent mixing. The complexity of the flow field makes the matching of lateral (or approach) boundary conditions especially difficult. Fortunately, however, highly stable

(a) These conditions are representative of partly cloudy to sunny days with moderate to high wind speeds. 
flows are associated with fairly low wind speeds, and modeling this stability range is probably not important in wind energy applications.

Maintaining similarity in the boundary conditions and the Froude number is the principal challenge in modeling neutral to slightly stable flows. Under moderate-to-strong wind speed conditions, this stability range is most 1ikely to be observed at night. For a given wind speed, stably stratified (or even neutral) boundary layers are less turbulent than convectively unstable ones. Less vigorous mixing means that these boundary layers will be more shallow than the unstable boundary layer and that wind and temperature fields will show more spatial and temporal variation. Boundary layer depths will probably range from 100 to $500 \mathrm{~m}$; thus local terrain relief may not be small compared to this depth. When the terrain relief is comparable to the depth of the boundary layer, the structure of the wind and temperature fields above the boundary layer can influence flow near the surface. All of these effects make it very difficult to represent the wind and temperature fields approaching the modeled terrain in a realistic manner. As the ratios of boundary layer depth to local terrain relief become larger and the Froude numbers become smaller, the accuracy of neutral simulations in the wind tunnel become increasingly uncertain. However, it is impossible to judge a priori how dissimilar a neutral simulation, or even stable simulation using approximate boundary conditions, may be from the actual flow.

Even considering these limitations, physical modeling (and, in particular, wind tunnel modeling) can be a useful tool in siting. Under neutral to mildly unstable conditions, wind tunnel modeling should give reasonably accurate information on the effects of terrain profile and roughness on wind speed distributions near the surface, on the wind profile and on turbulence (including the location of zones of probable flow separation). These simulations should be superior to the capabilities of currently available numerical models even when a neutrally stratified flow is used to represent the actual conditions. The accuracy of physical models of stable flow over terrain is more uncertain. For stable flows, it is very difficult to model the approach boundary conditions since these boundary conditions can be complex and since small changes in 
their structure can affect the flow significantly. These boundary conditions will also seldom be known with any precision. However, very similar problems exist in the numerical modeling of stable flows over terrain, as well.

Finally, there is one problem common to all modeling techniques: how representative are a few model runs to typical conditions over the site? The wind climatology of a real section of terrain is made up of a series of highly complicated, ever-changing flows. When flow over real terrain is modeled, an attempt is made to reproduce the actual wind climatology with a very small number of steady state flows obtained by using highly simplified boundary conditions. These simulations of the real world can provide useful guidance, but their basic limitations must always be kept in mind. 


\section{REFERENCES}

Cermak, J.E. 1975. "Applications of Fluid Mechanics to Wind Engineering." J. Fluid Engineering. 97: (1)1.

Chien, H.C., R.N. Meroney and V.A. Sandborn. 1979. Sites for Wind Power Installations: Physical Modeling of the Wind Field Over Kahuku Point, Oahu, Hawaii. DOE/ET/20292. Available from National Technical Information Service, Springfield, Virginia.

Coantic, M.F. 1978. An Introduction to Turbulence in Geophysics and Air-Sea Interactions. AGARD-AG-232. Available from National Aeronautics and Space Administration, Langley Field, Virginia.

Holton, J.R. 1972. An Introduction to Dynamic Meteorology. Academic Press, New York.

Hunt, J.C.R. and H. Fernholz. 1975. "Wind-Tunnel Simulation of the Atmospheric Boundary Layer: A Report on Euromech 50." J. Fluid Mech. 70:543-559.

Klebanoff, P.S. 1954. Characteristics of Turbulence in a Boundary Layer with Zero Pressure Gradient. NACA TN 3178, National Aeronautics and Space Administration, Washington, DC.

Lenschow, D.H., B.B. Stankov and L. Mahrt. 1979. "The Rapid Morning Boundary-Layer Transition." J. of Atmos. Sci. 36:2108-2124.

Meroney, R.N., V.A. Sandborn, R.J.B. Bowmeester and M. Rider. 1977. Sites For Wind Power Installations: Wind Tunnel Simulation of the Influence of Two Dimensional Ridges on Wind Speed and Turbulence. RLO/2438-77/1. Available from National Technical Information Service, Springfield, Virginia.

Meroney, R.N., V.A. Sandborn, R.J.B. Bowmeester, H.C. Chien and M. Rider. 1978a. Sites for Wind Power Installations: Physical Modeling of the Influence of Hills, Ridges and Complex Terrain on Wind Speed and Turbulence. RL0/2438-77/3. Avai 1able from National Technical Information Service, Springfield, Virginia.

Meroney, R.J., A.J. Bowen, D. Lindley and J. Pearce. 1978b. Wind Characteristics Over Complex Terrain: Laboratory Simulation and Field Measurements at Rakaia Gorge, New Zealand. RL0/2438-77/2. Available from National Technical Information Service, Springfield, Virginia.

Pennell, W.T., and M.A. LeMone. 1974. "An Experimental Study of Turbulence Structure in the Fair-Weather Tradewind Boundary Layer." J. Atmos. Sci. 31:1308-1323.

Pennell, W.T., D. H. Lenschow and M.A. LeMone. 1979. "Some Aspects of Turbulence in the Atmospheric Boundary Layer." Studies in Heat Transfer. J.P. Hartnet, T.F. Irvine, Jr., E. Pfender and E.M. Sparrow (eds). McGraw-Hill, New York, New York. 
Riley, J.J. and D.P. Delisi. 1977. Survey of Laboratory Modeling of Plume Dynamics. EPRI EA-323, Project 677-2, Electric Power Research Institute, Palo Alto, California.

Snyder, W.H. 1972. "Similarity Criteria for the Application of Fluid Models to the Study of Air Pollution Meteorology." Boundary-Layer Meteorology. $\underline{3}: 113-134$.

Snyder, W.H. 1979. Guideline for Fluid Modeling of Atmospheric Diffusion. EPA-45014-79-016, Environmental Protection Agency, Research Triangle Park, North Carolina.

Schlichting, H. 1960. Boundary Layer Theory. McGraw-Hi11, New York.

Tieleman, H.W. 1967. "Viscous Region of Turbulent Boundary Layer."

Ph.D. dissertation. Report No. CER67-68HWT21, Colorado State University, Ft. Collins, Colorado.

Zoric, D.L. 1968. "Approach of Turbulent Boundary Layer to Similarity." Ph.D. dissertation. Report CER68-69DLZ9, Colorado State University, Ft. Collins, Colorado. 


\subsection{TOPOGRAPHICAL INDICATORS OF WIND PONR POTENTIAL}

Topographical indicators of wind power potential are guidelines developed from empirical, experimental, theoretical, and experiential understanding of air flow interaction with terrain. The development of topographical indicators represents the integration of all that is known about mesoscale and microscale meteorology. This chapter is a brief review of the topographical indicators that may be used in siting large wind turbines.

Properly used, topographical indicators are the most cost-effective technique for short cutting the siting process. In the past, interpretations of flow over terrain have been applied to siting wind machines without supporting measurements. It was sufficient in past rural applications to know that the wind would be stronger here than there. Optimal siting of large wind turbines requires more information: Exactly how strong is it here? At what times is it strong? Is it too turbulent? In this context the use of topographical indicators is still a valuable technique, but the issues of when and how to use them, and how far to trust them, are complex.

Topographical guidelines are used alone when there is no other choice or when the cost of an error is minimal. In conjunction with measurements or modeling, the topographical guidelines become powerful supplements.

In the candidate resource area evaluation stage of wind prospecting, one uses topographical guidelines to determine locations for anemometers for confirming measurements throughout an area. These anemometers could easily be relocated if the incoming data indicated that the anemometer's siting was not optimum.

At the potential candidate site screening stage, topographical indicators can distinguish a primary from a secondary potential candidate site. For example, of two candidate cluster sites with roughly equal wind power potential, topographical indicators might suggest that one site experiences higher levels of turbulence than the other. This suggestion might result from the shapes of upwind topographic protrusions. 
In all but the simplest cases the information presented here is qualitative. Generic flow guidelines have used information from theory, from numerical simulations, from physical simulations of flow over scale model terrain, and from actual measurements of flow around full-scale features. Succinct generalizations drawn from these studies may sound thorough; however, reliance upon them must be tempered. Information in this chapter should therefore be used as a guideline to:

- understand flow-terrain interactions

- indicate where to make measurements

- interpret measurements already made.

These topics are discussed for several types of topographical indicators. The types of topography are identified in the next section.

\subsection{CLASSIFICATION OF TOPOGRAPHY}

Topographical features may be natural or man-made. Meaningful classifications of these features are difficult, arbitrary, and often indefinite. In addition, the structure of the atmospheric flow in the planetary boundary layer (PBL) itself, i.e., roughly the first kilometer of the atmosphere where direct heat and momentum exchanges with the earth's surface occur (Appendix 2), affects the terrain and flow interaction. These interactions between the terrain features and atmospheric flow features provide the framework for classifications used here.

Topography may be classified as one of the following three major groups:

- flat terrain

- small-scale terrain

- large-scale terrain.

The distinction between large and small scale is drawn from the response of the PBL to the interaction with the feature. For example, consider a small hill of which the height is but a small fraction of the boundary layer depth. The flow accelerates over the hill but, in the Lagrangian sense, is past the hill before turbulence near the surface can communicate the interaction to the rest of the PBL. The statistical properties of the flow downwind of the hill are indistinguishable from those upwind of the hill. For a large hill that 
extends through a significant portion of the PBL or even above the height of the PBL of the surrounding terrain, the structure of the entire boundary layer and the atmosphere above is modified by the interaction of flow and hill. Said in another way, small-scale terrain features are those that one could hope to model as small elements in a neutrally stratified wind tunnel experiment (Chapter 4), whereas all else is probably considered large scale.

The distinction between large and small scale therefore depends in a rough way on the relation between the boundary layer height to the range of terrain heights. A given terrain feature won't necessarily be always large or always small. For example, in a region of strong daytime heating and nighttime cooling, a 400-m hill by day may be considered a 400-m mountain at night because of the diurnal oscillation in PBL height and stability.

In addition to scale, other classifying features are based on the sense of elevation change, i.e., elevated features versus depressions. A further classification is based on whether or not the terrain features have a long axis that can be exploited in order to describe the flow in a two-dimensional cross section, e.g., ridge versus hill or valley versus basin.

The remainder of this chapter discusses terrain features categorized by:

- scale of features compared to PBL height: flat, small scale or large scale

- type of feature: elevated or depressed

- existence or nonexistence of a simplifying axis; e.g., ridge or hill.

\subsection{FLOW OVER FLAT TERRAIN}

Flow over flat terrain has been studied extensively with theory, measurements, and wind tunnel simulations. Real terrain is never truly flat; there are always small-scale irregularities or very gentle large-scale slopes. The choice of topics that can be discussed reflects the predominance of theoretical and wind tunnel work. This section covers flow over: 
- homogenous flat or gently sloping terrain

- homogenous flat terrain with obstacles

- flat terrain with roughness changes

- flat terrain with horizontal temperature gradients.

Because no terrain is truly flat, there must be usable criteria for defining flatness. Terrain that is not actually flat can be treated as flat if the departure from flatness has no perceptible effect on the wind and turbulence characteristics in the region between the ground and the top of a wind machine's rotor disk. There are two ways that nonflat terrain can influence the wind characteristics of that layer: 1) if small-scale terrain irregularities disturb the near-surface flow so that a universal surface layer wind profile ( $\log$ law, see Appendix 2) is not established or 2) if the influence affects the entire boundary layer as an external force but still allows universal wind profiles to be established near the surface. With respect to small-scale terrain irregularities, the terrain may be considered flat if (Frost 1979):

- elevation differences between the wind turbine site and the surrounding terrain are not greater than $50 \mathrm{~m}$ within $10 \mathrm{~km}$ of the site

- no hill has an aspect ratio (height divided by diameter) greater than $1 / 50$ within $5 \mathrm{~km}$ upstream and $1 \mathrm{~km}$ downstream of the site

- the difference between the elevation of the base of the rotor disk and the lowest elevation within $5 \mathrm{~km}$ upstream is greater than three times the maximum elevation difference within the same distance.

This definition provides a rough guideline for deciding whether theories developed for flat terrain are applicable. However, this is only a rough guideline. With stably stratified flow, the boundary layer may never reach steady state downstream of a small disturbance, whereas under unstable conditions the mixing may be so thorough as to erase the flow's memory of the disturbance much more quickly than the above guidelines suggest. As will be seen in the section on homogeneous flat terrain, the land is never flat enough to have no influence on PBL structure. 


\subsubsection{Homogeneous Fl at or Gently Sl oping Terrain}

Homogeneous terrain implies that the ground is flat and that the surface and air temperature distributions are horizontally uniform. Under these conditions the well-established theory for flow over flat terrain may be used. Flow over homogeneous flat terrain is discussed in detail in Appendix 2.

There are three major points to keep in mind about flow over homogeneous flat terrain:

- Wind power is maximum over smooth surfaces.

- Average diurnal wind speed near the surface has a daytime maximum, but this may or may not be true at hub height (see Figure 1.5).

- There ma be nocturnal jets and large nocturnal wind shears above the surface.

Figure 5.1 shows wind speeds at 15,65 , and $115 \mathrm{~m}$ as a function of surface roughness; assuming the depth (h) of the PBL is $1000 \mathrm{~m}$, there is neutral stability and the wind speed at $\mathrm{h}$ is $20 \mathrm{~m} / \mathrm{sec}$ (see Appendix 2 for details). The benefits of siting in smooth terrain are obvious. Note also that twothirds of the mean wind shear is below $65 \mathrm{~m}$. At any given instant, however, the fluctuating wind shear due to turbulence could look significantly different.

The wind speed near the surface (e.g., $\leq 10 \mathrm{~m}$ ) wi 11 , on the average, be greater in the daytime than at night. This is because of the dramatic effects that upward or downward heat fluxes can cause. Even a very slight upward heat flux that occurs as a result of solar heating of the surface enhances turbulent exchange of momentum in the vertical; i.e., an upward heat flux causes more of the higher wind speed air from above to be transported near the surface than in neutral or stable conditions. In the opposite way, even a very slight downward heat flux suppresses vertical exchanges of momentum so that the nearsurface layers exchange less low momentum air for high than in unstable conditions. Therefore, the nocturnal winds near the surface are light.

The interpretation of the diurnal wind variation inferred from nearsurface wind speeds may not hold at the level of significance for large wind turbines. This is because the diurnal variation of the PBL height is 


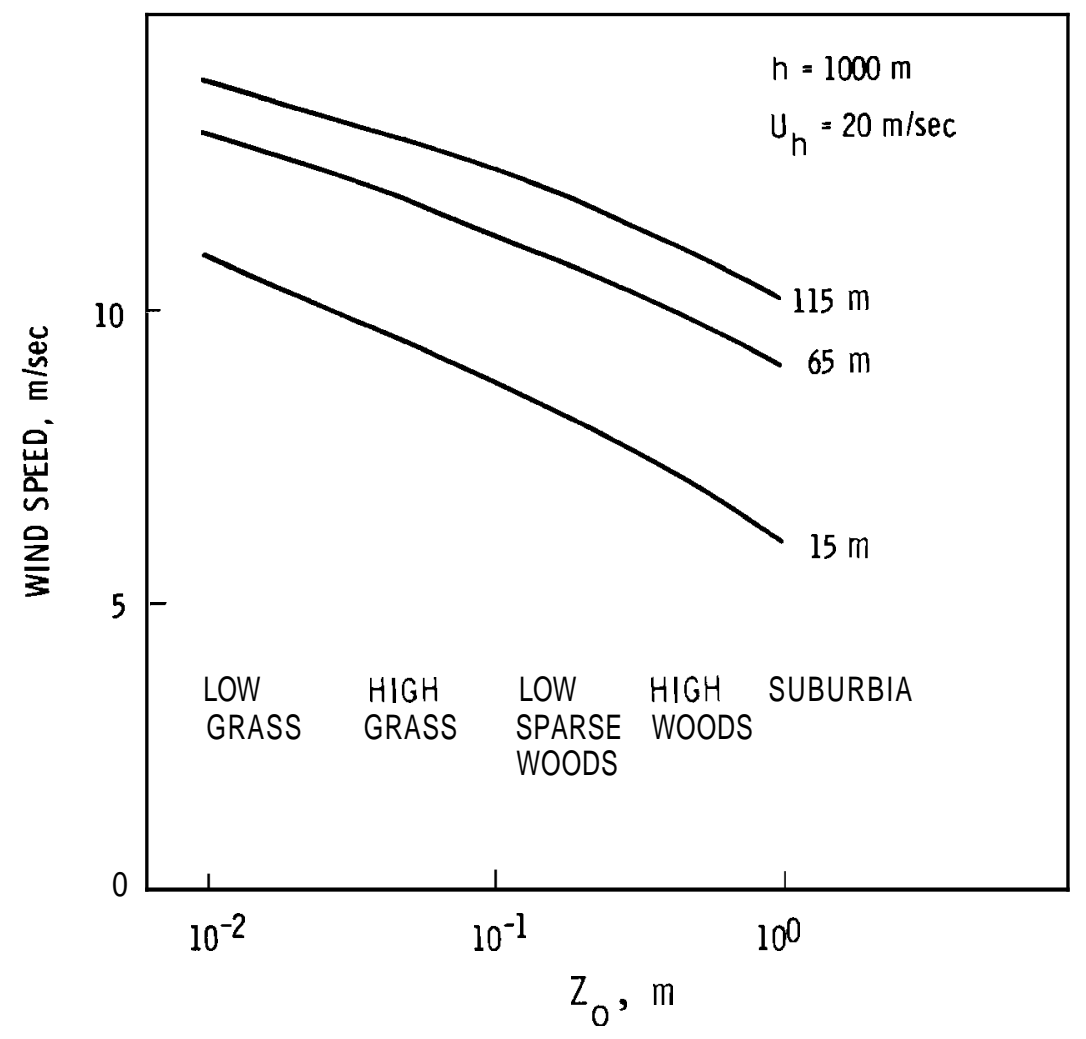

FIGURE 5.1. Wind Speed at Three Levels as a Function of Roughness Length. A wind speed of $20 \mathrm{~m} / \mathrm{sec}$ at the top of a 1000-m-deep neutrally stable PBL has been assumed. $u_{*}$ was computed from Appendix 2, Equation A-2.32.

important. The following is a crude argument (see Appendix 2 for details): Consider the PBL of height $h$ and wind speed at the top of the PBL of $U_{h}$. Let $U_{h}$ be the same both day and night and let $h$ be large (say $2 \mathrm{~km}$ ) during the day, but small (about $500 \mathrm{~m}$ ) at night. The wind speed at $10 \mathrm{~m}$ is higher during the day than at night so the average wind shear between $10 \mathrm{~m}$ and $\mathrm{h}$ is less during the day than at night. The fact that $h$ is low at night further increases the wind shear. The wind speed at hub height of $65 \mathrm{~m}$ is approximately(a)

$$
U_{65}=U_{10}+\frac{U_{h}-U_{10}}{h-10}(65-10)
$$

(a) This assumes that the wind shear is constant above $10 \mathrm{~m}$ for the sake of the argument. In fact the wind shear in unstable conditions decreases with height and in stable conditions it is approximately constant. See Appendix 2, Equations A-2.28 and A-2.29. 
Whether or not the wind speed at hub height is greater or less in the daytime than at night depends, in part, upon the diurnal oscillation of the boundary layer height. Since the boundary layer height is not obtainable by routine inexpensive means, the interpretation of the diurnal wind distribution at hub height from near-surface wind data is impossible. Furthermore, Appendix 2 shows that even ifit is known that the wind profile obeys a given universal law, practical ways of estimating the effects of stability are too crude to adequately predict hub-height winds from near-surface data.

The problem is complicated further by the low-level nocturnal jet (also discussed in Appendix 2) that sometimes forms. When the daytime heating stops, nighttime cooling at the ground establishes a low-level temperature inversion that decouples the remnants of the day's boundary layer from the nocturnal boundary layer that forms in the lowest 50 to $500 \mathrm{~m}$. The winds i $\mathrm{n}$ the daytime PBL are subgeostrophic in speed because of the frictional coupling with the earth's surface. When these winds become decoupled from the surface friction, the wind vector begins an inertial oscillation (Equations A-2.25, A-2.26) around the geostrophic wind vector. Therefore, during the night $U_{h}$ may actually be greater than $U_{h}$ during the day, increasing the chances that the hub-height wind speeds at night will exceed the daytime hub-height wind speeds.

Over large-scale, gently sloping terrain the diurnal heating and cooling of the surface forces a diurnal oscillation of the thermal wind (see Appendix 2 for the thermal wind discussion). The thermal wind vector in this case is the vector difference between the geostrophic wind at the top of the daytime PBL and the surface geostrophic wind. The warmer air is on the right side of the thermal wind vector (in the northern hemisphere) so if the land slopes from high elevation in the west to lower elevation in the east, the thermal wind vector will point south in the daytime and north at night. If the geostrophic wind above the top of the daytime PBL is directed down the slope, in this case to the east, then the difference between the wind vector in the daytime PBL and the nighttime low-level geostrophic wind vector is a maximum and the amplitude of the resulting acceleration and inertial oscitlation of the winds 
just above the top of the nocturnal boundary layer is a maximum (Holton 1967, Bonner and Peagle 1970). This situation is described in Figure 5.2.
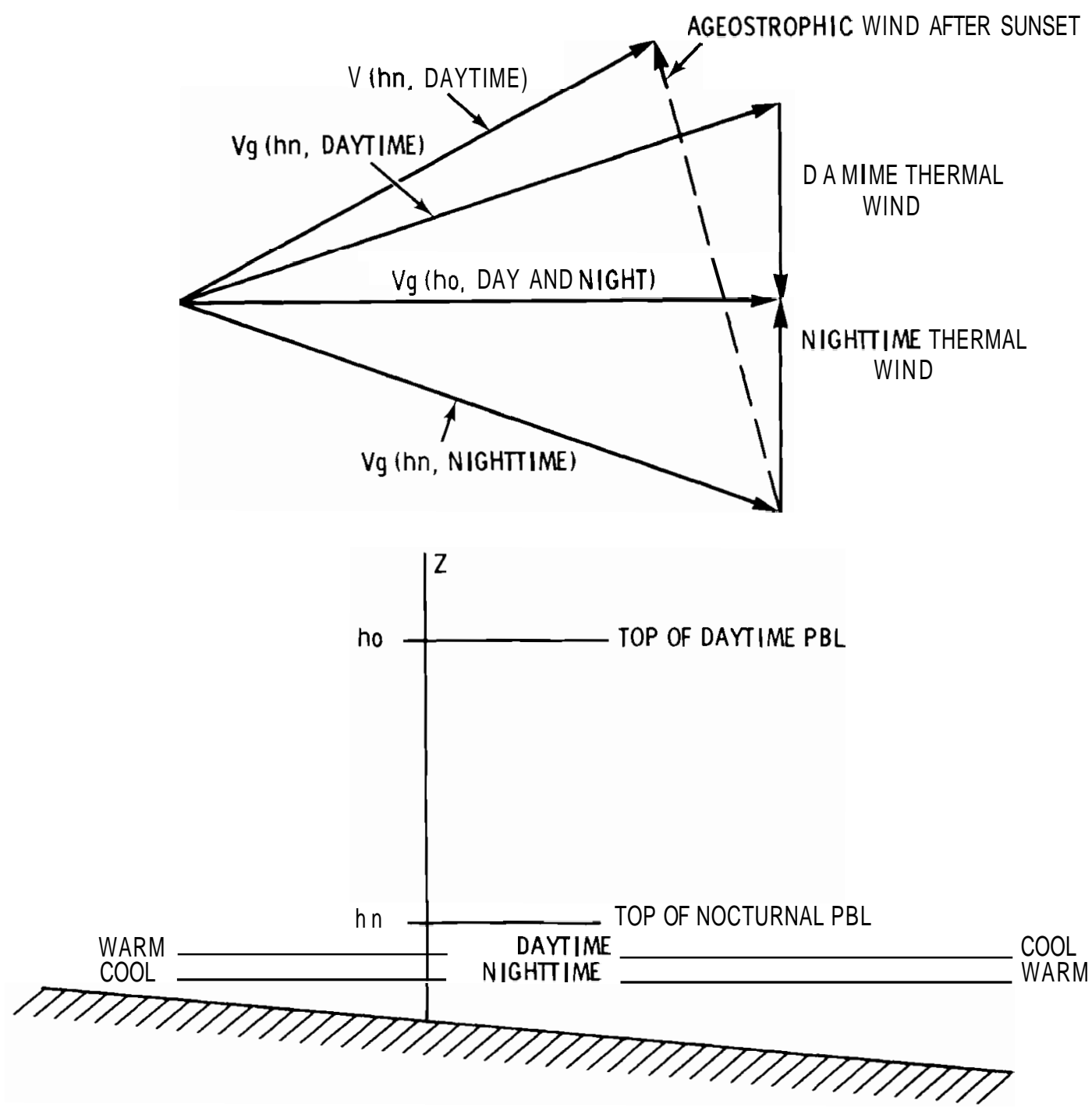

FIGURE 5.2. The Configuration of Geostrophic Wind and Terrain Slope That Results in the Maximum Ageostrophic Wind at the Top of the Nocturnal Boundary Layer Shortly After Sunset When the Newly Formed Nocturnal Boundary Layer Becomes Decoupled From the Remnants of the Daytime Boundary Layer Above. The large ageostrophic wind component results in a large acceleration of the nocturnal winds at the top of the nocturnal boundary layer. 
The enhancement of the nocturnal wind maximum by the effects of terrain slope is one suggestion for the frequent occurrence of the low-level nocturnal jet over much of the Central and Great Plains states. Based on rawinsonde data, which do not have the ability to resolve many near-surface features, Bonner (1968) developed a climatology of the frequency of occurrence and speeds of the low-level jet. Recent work (Sisterson and Hicks 1979) suggests that lowlevel nocturnal wind maxima are much more frequent than the older analyses suggest.

The slope-enhanced nocturnal jet influences the winds a WECS will experience by changing the external parameter that governs flow in the PBL; it changes $U_{h}$. A somewhat steeper gentle slope, which nevertheless satisfies the definition of $f l a t$ with respect to small-scale irregularities, influences the flow within the PBL directly. During the day the warm air rises upslope, or at least has a force component upslope that weakens an externally forced downslope motion. These are called anabatic winds. At night the opposite drainage winds occur, which are called katabatic winds. The exact relationship between diurnally varying slope winds and the PBL structure is not clear, so predictions of the magnitude and depth of the anabatic and katabatic wind components are difficult. However, these slope winds are regular and can often be seen in a climatological record as a diurnal superposition on the large scale flow.

\subsubsection{Homogeneous Flat Terrain With Obstacles}

An obstacle that is significant to a large wind turbine situated on flat land could be manmade, such as a large barn, silo, storage tank, or shelterbelt (a windbreak made of trees), or natural such as an isolated rock outcropping or bluff. Wind turbines as obstacles are considered in Chapter 9.

Most data on the measurement of wakes behind obstables come from wind tunnel studies. Studies based on full-scale obstacles in the boundary layer are extremely scarce so the extent to which wind tunnel modeling may be applicable is uncertain (see Chapter 4). Certainly more work needs to be done on flow around obstacles in a stably stratified PBL.

Classical studies of wakes around obstacles, such as rectangular blocks, in wind tunnels produced a picture of wake behavior that is now called the 
niomentum wake behavior. (Another picture--vortex wake behavior--will be discussed a 1ittle later.) In the picture of the momentum wake, flow impinging on the block accelerates over and around it. The flow separates at the crest of the obstacle and vortices are shed into the airstream causing an increase in the turbulence behind the obstacle. Because there is a pressure minimum behind the obstacle, the separation streamline wil1 bend downward (Plate 1971). The curvature of the streamline and hence the reattachment point downwind of the obstacle depend upon the pressure force and centrifugal force on an element following the streamline. The separation bubble behind the obstacle is a region of momentum deficit (reduced wind speeds compared to the free stream flow) and turbulence excess (compared to the free stream flow). Beyond the reattachment point of the flow, turbulent mixing re-energizes the wake region to free stream values of wind speed and turbulence intensity.

In the case of a porous obstacle such as a shelterbelt, some flow leaks through the obstacle. Porosity is the ratio of the open area on the upwind side of the obstacle to the total cross sectional area on the upwind side. A porous shelterbelt then allows some air to pass through causing less wind speed reduction behind the obstacle. However, the pressure drop behind a porous obstacle is less than for a solid one so the separation streamline does not curve downward as quickly; hence, the protected region is larger.

The picture of a vortex wake is substantially different. A very good description of the properties of vortex wakes has been provided by the wind tunnel studies behind blocks and heniispheres by Hansen and Cermak (1975). When flow encounters an obstacle, sheets of streamwise vorticity are shed at the point of flow separation. At some distance downwind of the obstacle the vorticity rolls up to form a discrete line vortex. A horseshoe vortex forms as vortex lines associated with the vertical wind shear upwind of the obstacle wrap around the obstacle and trail downwind of the obstacle on either side. A roof corner vortex forms as a vortex interacts with a building corner, rolls up, and trails downwind. Figure 5.3 is a conception of these vortices. Note that the sense of rotation of both types of vortices is such that there is downward motion near the center of the wake and upward niotion on the outer 


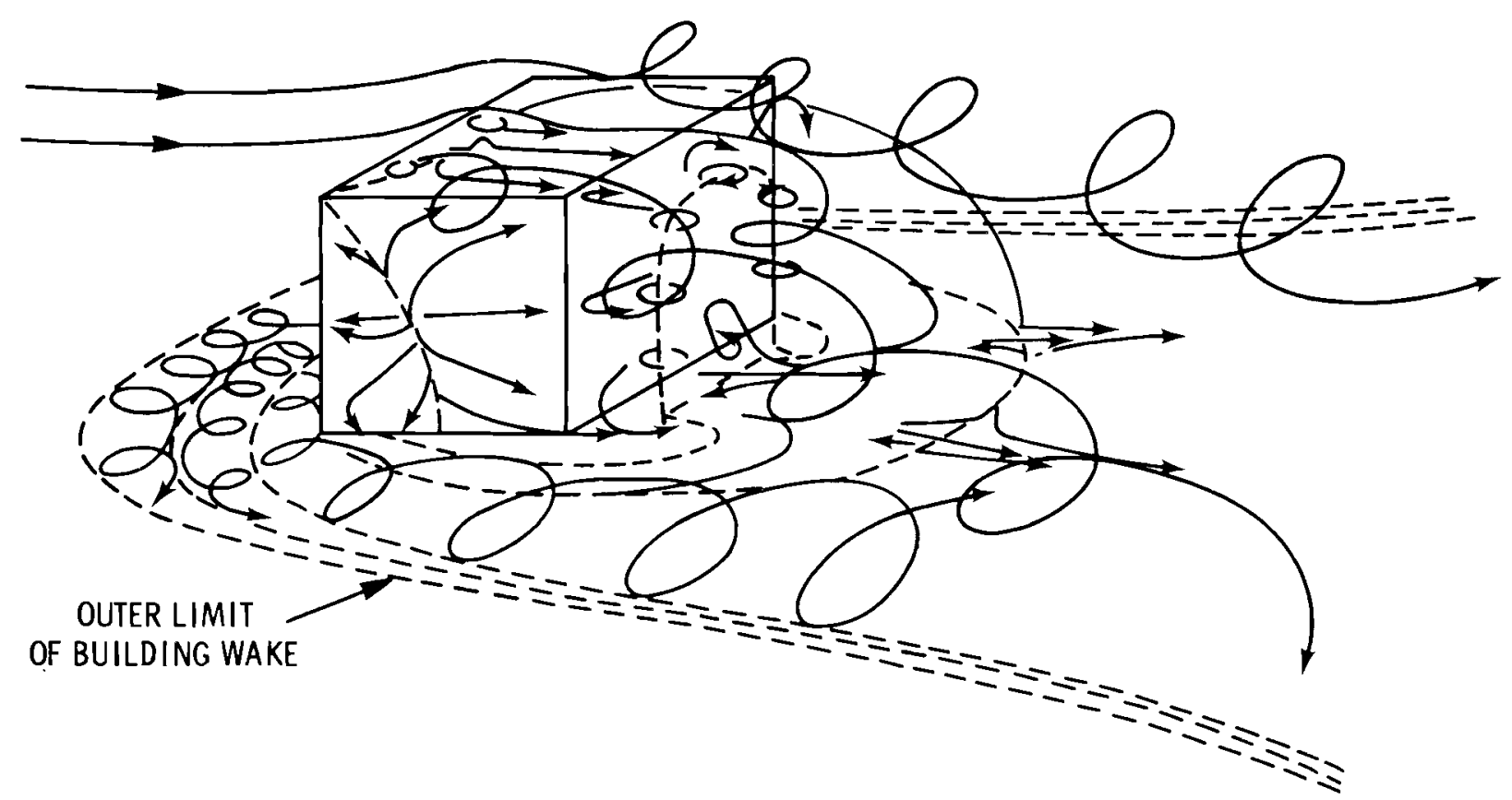

FIGURE 5.3. Flow Over an Obstacle That Produces Horseshoe Vortices and Roof Corner Vortices

portions of the wake. In the presence of wind shear where the wind speed increases with height and the turbulence intensity decreases with height, the vortex motion will advect high momentum and less turbulent air downward from above the wake into the center of the wake. As we shall see this leads to a negative momentum deficit (i.e., higher wind speed in the wake than the free stream wind speed at the same level) and a negative turbulence excess (lower turbulence intensity in the wake than at the same level in the free stream).

For any given obstacle the wake may have properties of both momentum wakes and vortex wakes. Longitudinal trailing vortices are, however, very stable long-lived entities. Consequently the vortex wake characteristics increasingly dominate the wake characteristics at points increasingly far downwind of the obstacle. We shall also see that the orientation of an obstacle to the wind can have dramatic effects on the formation of trailing vortices and therefore wake behavior.

We will look now at the structure of some of the wakes reported by Hansen and Cermak (1975). Figure 5.4a shows lateral profiles of the mean longitudinal 
velocity deficit behind a rectangular block at the level $\mathrm{z} / \mathrm{H}=0.94$ where $\mathrm{H}$ is the height of the block and $z$ is the height above the flat surface. Figure 5.4b shows lateral profiles of turbulence intensity excess. (a) This figure presents a usual picture of a wake that is dominated by momentum wake characteristics. The momentum deficit is almost $50 \%$ just behind the obstacle where the turbulence intensity is also quite high. The two-lobed appearance of the wind speed and turbulence intensity profiles nearest the block reflects the presence of small vortices around the upper corners of the block. This momentum wake was imperceptible at a downstream distance of $20 \mathrm{H}$.

Figure 5.5 shows the same profiles but with the building rotated $47^{\circ}$. This orientation produced stronger roof corner vortices than any other orientation. The structure of the wake is asymmetric because the roof corner vortex trailing from the longer top edge of the block facing upwind produces the strongest vortex. The downward motion of the strong vortex, by advecting higher momentum and less turbulent air downward from above the wake, is responsible for the rapid obliteration of the momentum deficit on the right-hand side of the wake. The left side recovers also very quickly but not as quickly as the right side because the vortex on the left is not as strong. The outstanding feature of Figure 5.5 is the persistence of this wake compared to the wake of Figure 5.4. There is up to a $4 \%$ increase in the wind speed over the free stream value at distances of $80 \mathrm{H}$. There was still evidence of the wake at the end of the wind tunnel at $96 \mathrm{H}$.

The decay of the wakes behind the rectangular block buildings in the two orientations is summarized in Figure 5.6. It is evident that the wake longevity is very sensitive to the production of the trailing roof corner vortices, which depends upon the orientation of the obstacle to the flow.

Vortex wakes due to horseshoe vortices were studied by placing a hemisphere in the wind tunnel, which perhaps we can think of as a small smooth hill in the atmosphere. Profiles of the velocity deficit and turbulence

(a) This is not in fact the turbulence intensity but only the standard deviation of the longitudinal component of the fluctuating winds normalized by the wind speed. 


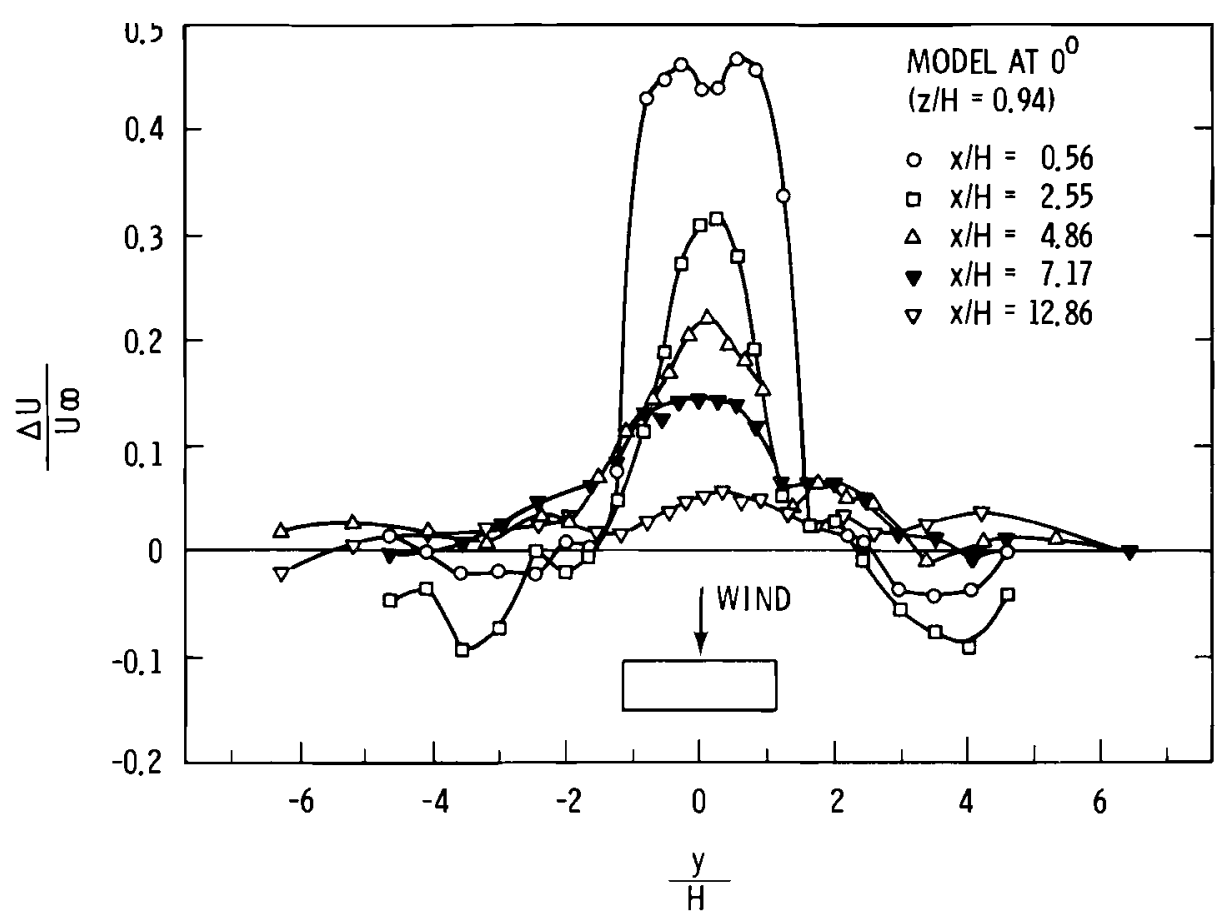

(a)

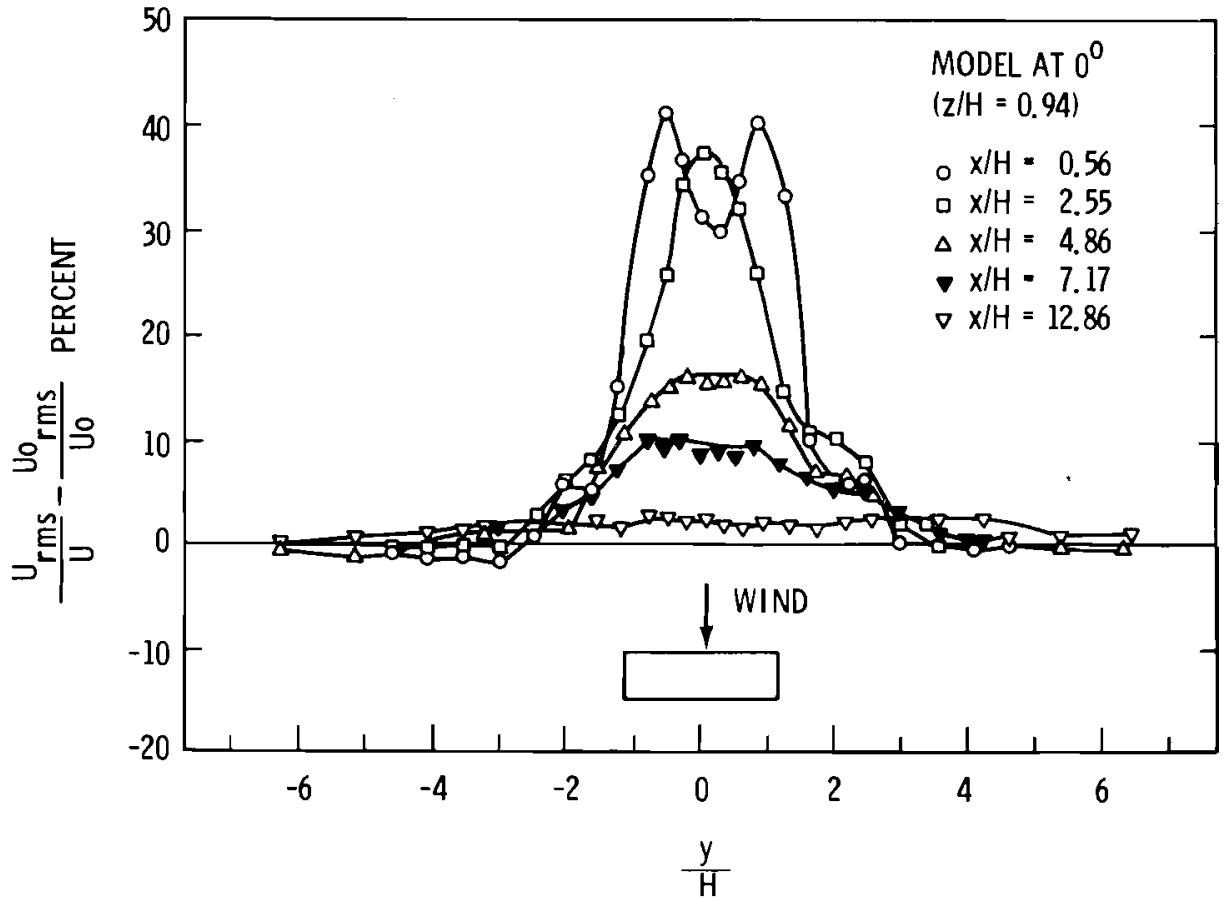

(b)

FIGURE 5.4. Lateral Profiles of Mean Velocity Deficit (a) and Turbulence Excess (b) in the Wake of a Building at $0^{\circ}$. The block is $0.065 \mathrm{~m}$ high by $0.16 \mathrm{~m}$ long by $0.49 \mathrm{~m}$ wide. 


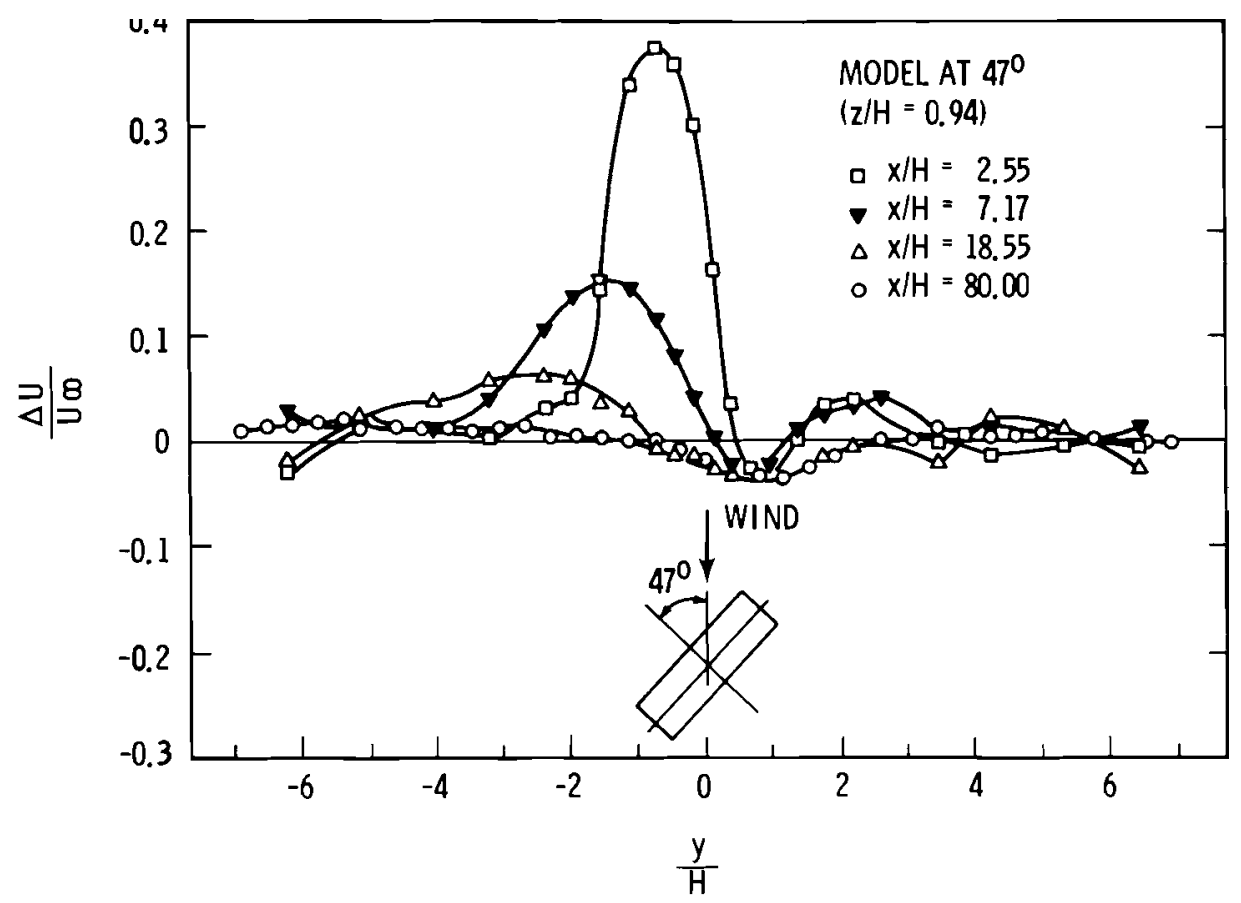

(a)

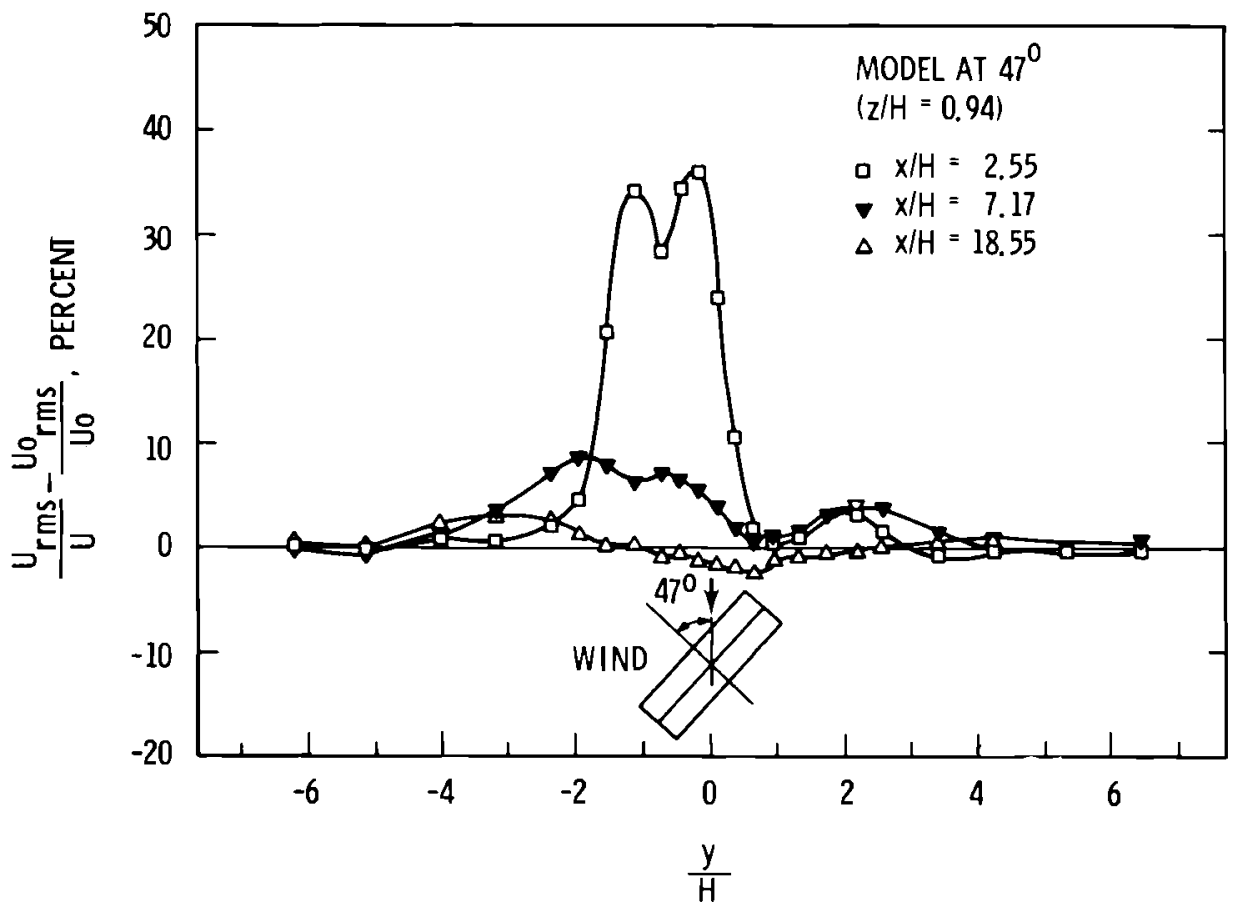

(b)

FIGURE 5.5. Lateral Profiles of Mean Velocity Deficit (a) and Turbulence Intensity Excess (b) in the Wake of a Building at $47^{\circ}$ 


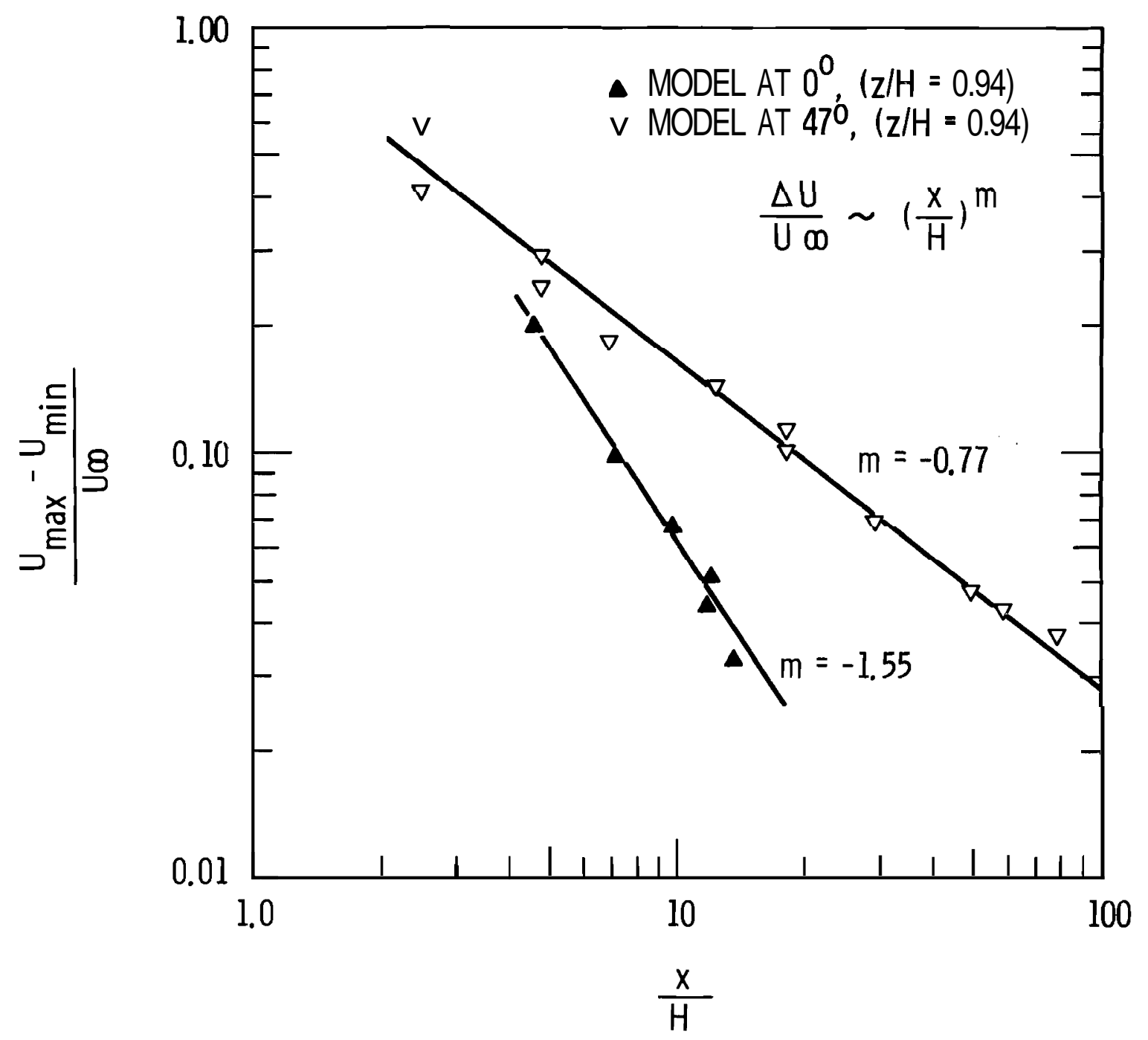

FIGURE 5.6. Decay of the Maximum Mean Velocity Difference (Across a Lateral Profile) Behind the Block Building with Downwind Distance for Two Wind Directions

excess are shown in Figure 5.7. These measurements were made at a level $z=0.364 R$ where $R$ is the radius of the hemisphere. The vortex wake signature is apparent in these profiles as well.

Another view of a wake profile behind a hemisphere is shown in Figure 5.8. The free stream wind speed was $4.9 \mathrm{~m} / \mathrm{sec}$ in this simulation compared to $16.2 \mathrm{~m} / \mathrm{sec}$ for the flow conditions of Figure 5.7. From comparing the velocity 


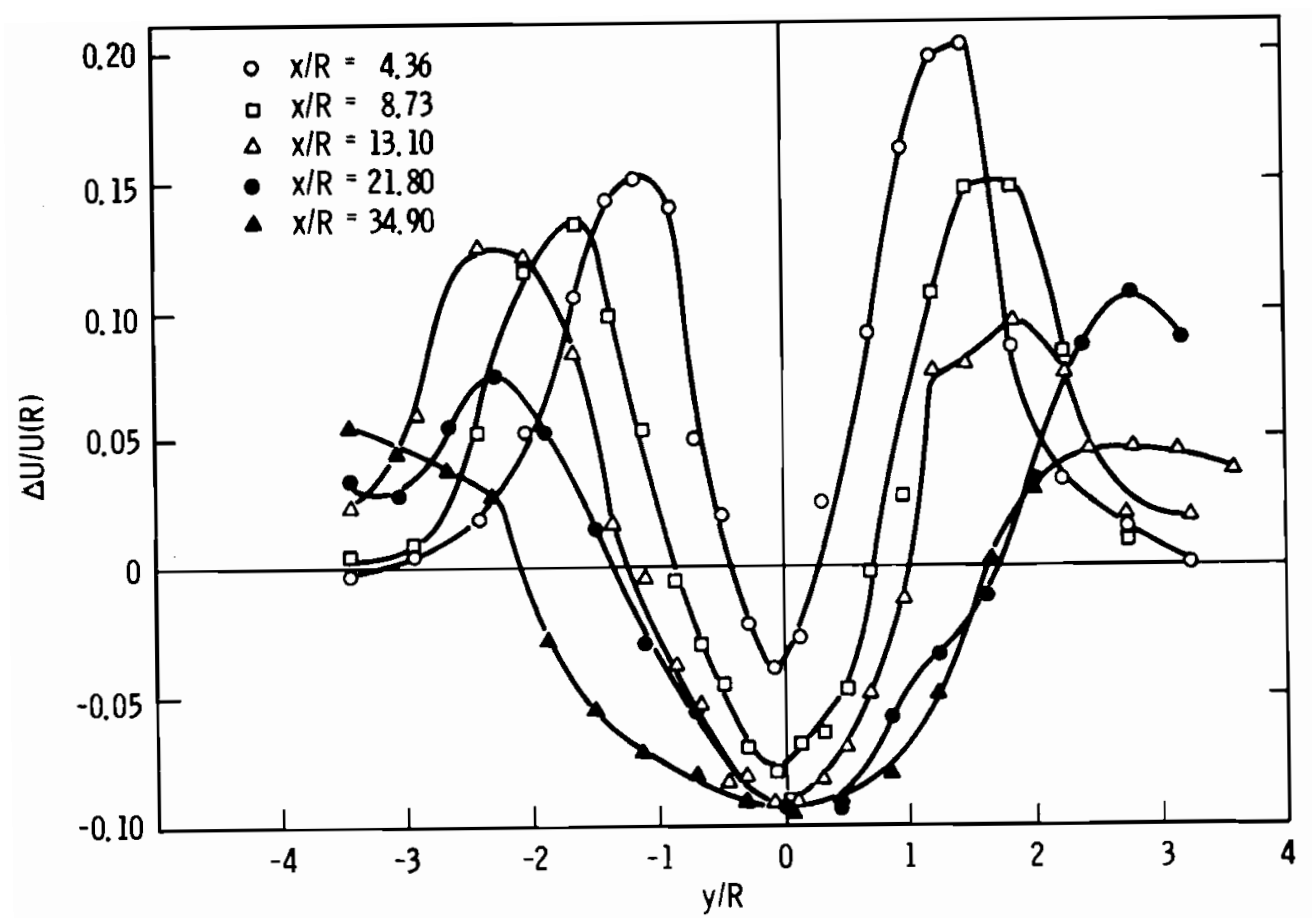

(a)

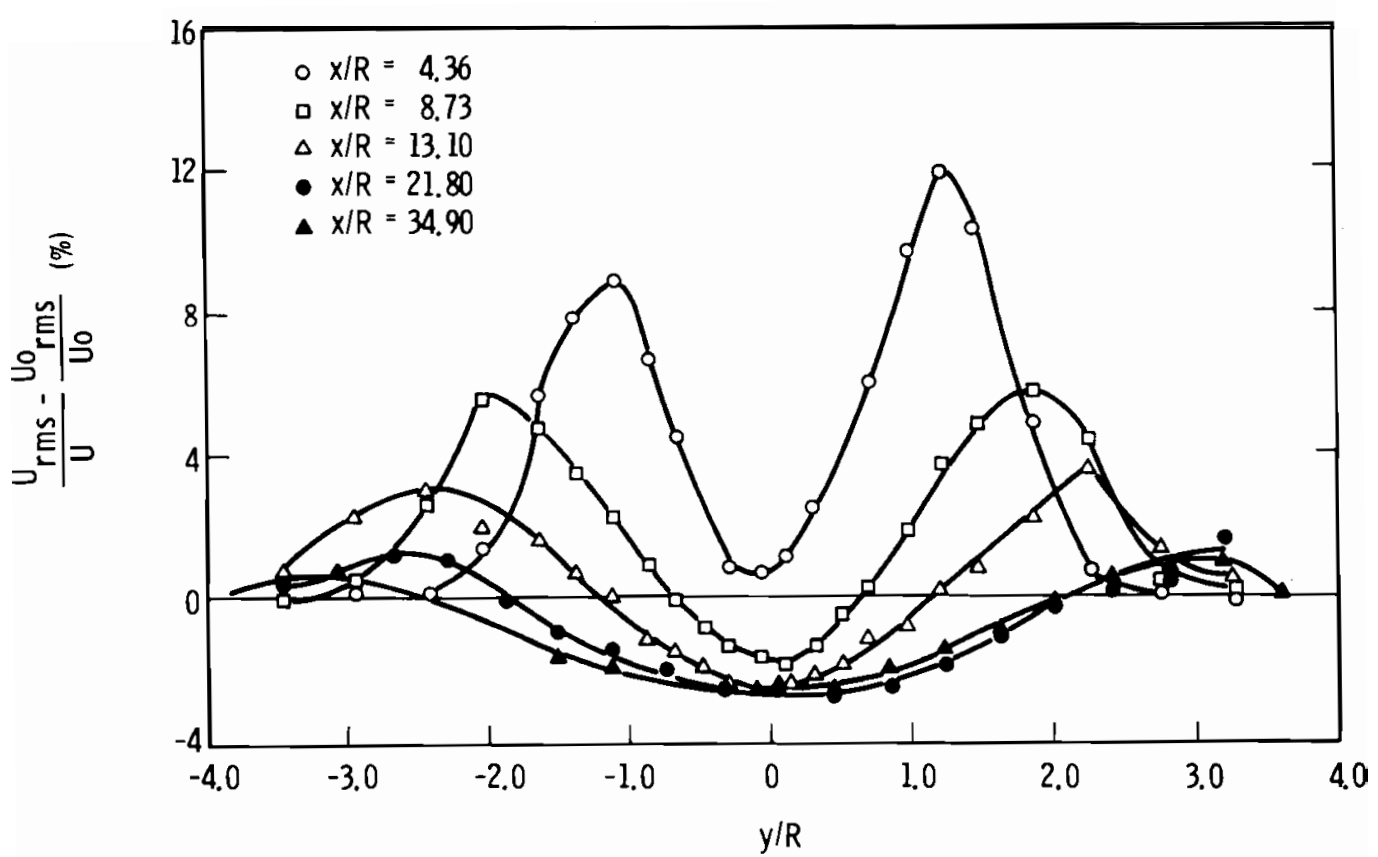

(b)

FIGURE 5.7. Lateral Profiles of Mean Velocity Deficit '(a') and Turbulence Intensity Excess (b) Measured With a Hot-Film Anemometer in the Wake of a Hemisphere at Height $z / R=0.364$. Hemisphere radius is $0.14 \mathrm{~m}$. 


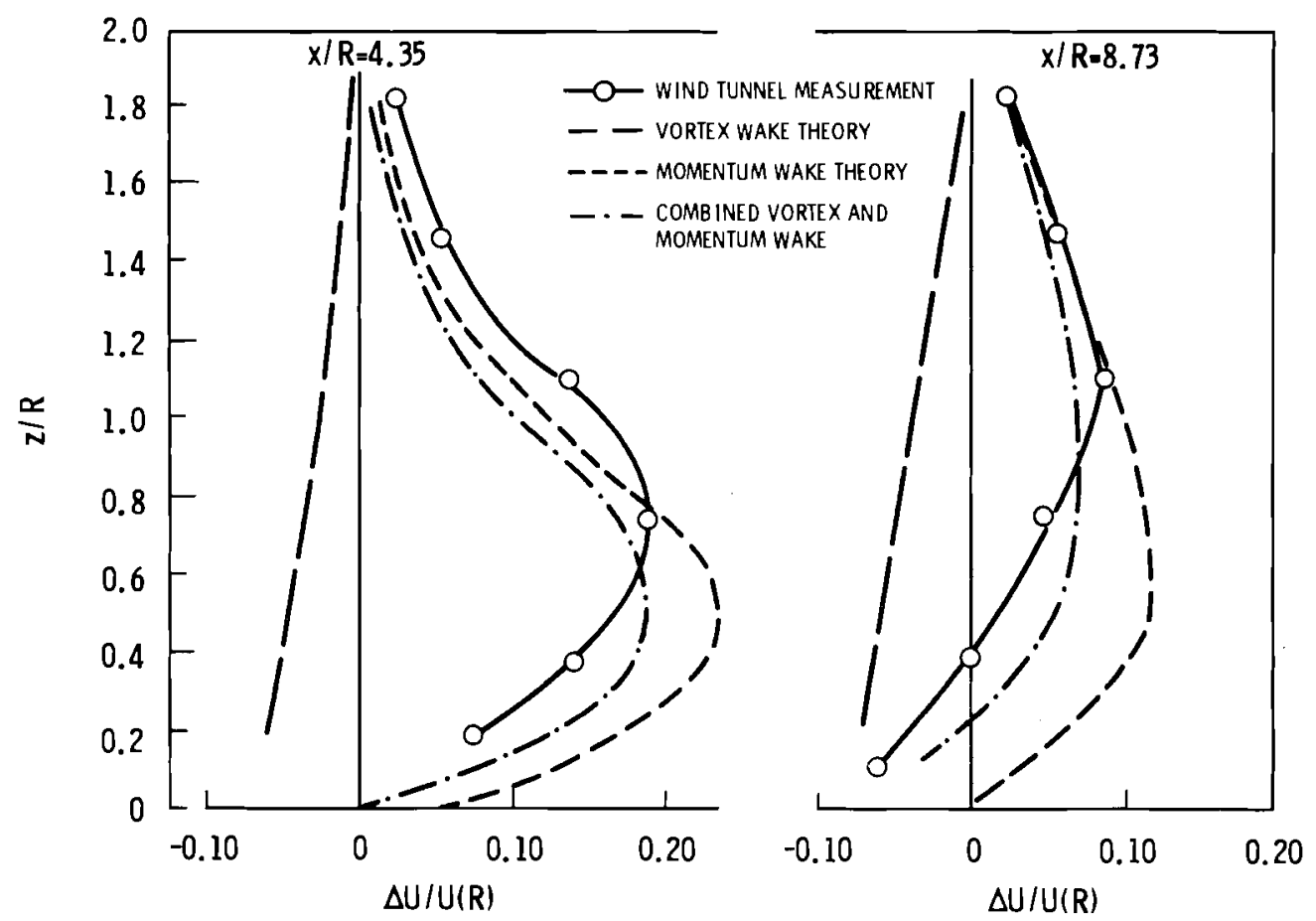

(a)

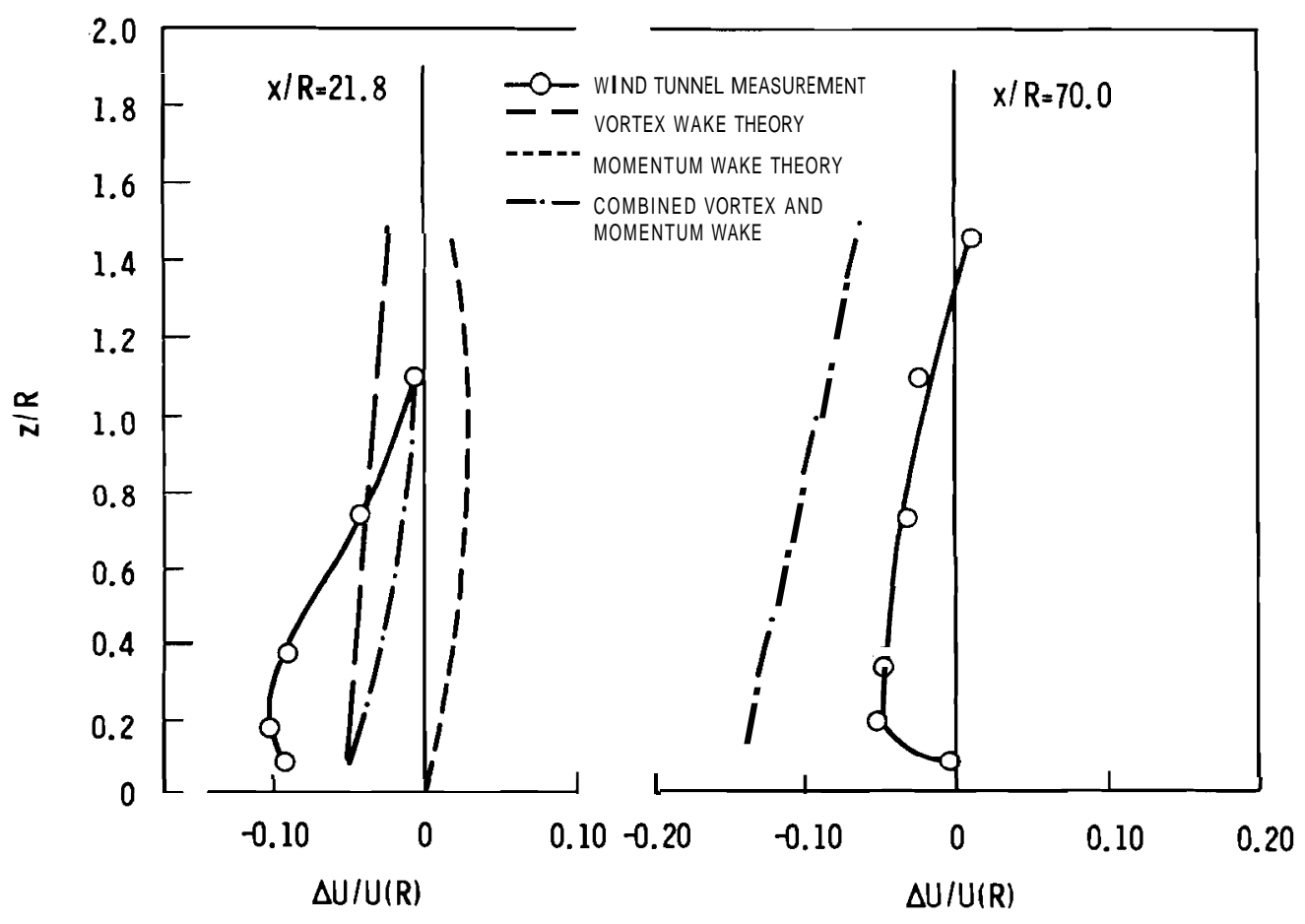

(b)

FIGURE 5.8. Vertical Profiles of Velocity Deficit on the Hemisphere Wake Centerline 
deficit behavior at $z / R=0.364$ in Figure 5.8 to Figure 5.7 we can see that in the wind tunnel there is a Reynolds number dependence for the vortex wake. The higher the Reynolds number the sooner the wake takes on the characteristics of the vortex-dominated wake.

Two explanations for the Reynolds number dependence were suggested by Hansen and Cermak (1975): 1) at high Reynolds numbers the shedding of the horseshoe vortex pairs may occur later than for low Reynolds numbers, the twin vortices would therefore be closer to the wake centerline, and hence the effects of the vortex interaction with mean wind shear would be stronger nearer the hemisphere; or 2) the vortex roll-up process may be dependent on the Reynolds number. In any case one might infer that in the atmosphere where the Reynolds number exceeds that in the wind tunnel by several orders of magnitude, the vortex behavior of wakes may be even more prevalent than these model results show.

The influence of surface roughness on the wake behind the hemisphere is shown in Figure 5.9. The power law coefficient (see Appendix 2) for the rough case was approximately 0.25 and for the smooth cases was approximately 0.12 . The velocity excess along the wake centerline is plotted on the ordinate. The rougher surface has higher turbulence intensity, which dissipates the wake more quickly. In addition, the flow over the rough surface has greater vorticity than the mean flow because the mean wind shear is greater. This means that the trailing vortices are stronger so the wake takes on vortex wake characteristics sooner for the rough case.

The data presented here are time averages. Since the wake meanders due to lateral velocity fluctuations in the ambient flow, these data do not present a true picture of the wake structure at any specific instant. Even though a vortex is growing and weakening at great downwind distances, the fact that the effects of a vortex wake are observable at 60 or more obstacle heights downwind (at least in the wind tunnel) indicates that we must consider the effects of the vortex that may exist at these distances. Since the time-averaged vorticity and the spectrum of the longitudinal velocity were imperceptibly different from the free stream beyond $20 \mathrm{H}$ in Hansen's study, we can conclude that the 


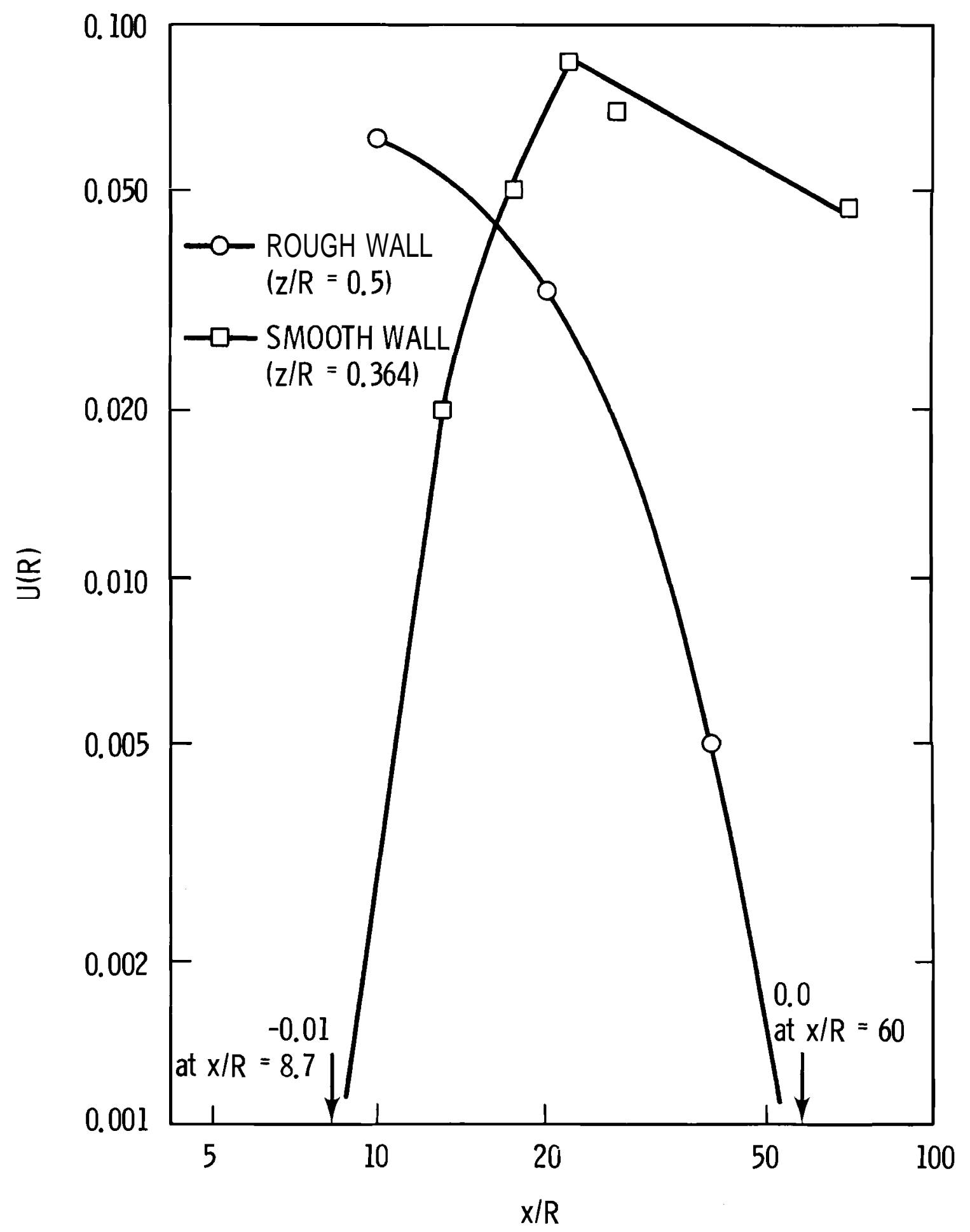

FIGURE 5.9. Comparison of the Decay of the Centerline Velocity Excess in the Wakes of Hemispheres in the Smoothand Rough-Wall Boundary Layers 
vortices at great distances are too weak to be of any significant concern to large wind turbines. However, the lateral velocity variations of the vortex and the meandering of the vortex may create a moderate increase in gustiness and wind direction fluctuations compared to the free stream flow. Such fluctuations may impact the frequency of wind turbine adjustments to changing flow conditions.

The data set of Hansen and Cermak (1975) is limited to only a few cases and so its broad applicability to real atmospheric flows is limited. Other wind tunnel wake studies have primarily looked at features of the momentum wake, where there is a velocity deficit and an increase in turbulence in and shortly downwind of a flow separation zone.

The data on the size of the flow separation zone and the momentum wake as a function of rectangular block geometry reveal more detail on wake structure. Table 5.1 (Meroney 1977) shows wind tunnel data behind rectangular block buildings placed normal to the flow. It is evident that the wider the obstruction to the flow the larger the wake region is. However, when an obstacle is very wide, a larger fraction of the wake flow goes over than around the obstacle The wake does not spread laterally as rapidly for a wide obstacle, as data of Woo et al. (1977) show. The data of Woo also show the vertical extent of wakes behind blocks of differing widths. The wakes are less than $3.5 \mathrm{H}$ high for both wide and not-so-wide blocks, however, the momentum deficit behind the wide block is greater at greater heights for greater distances downstream. Leutheusser and Baines (1967) show that for a given width-to-height ratio, the deeper the block is made in the downstream direction the shorter the wake region becomes.

The effects of atmospheric stability on wakes can only be surmised since measurements to confirm even neutral stability wind tunnel simulations are lacking. In unstable conditions turbulent transfer is enhanced, which causes more rapid dissipation of the wake, but the wake extends through a deeper layer. For stable conditions, flow separation zones are smaller due to the gravitational restoring force. More flow is forced around obstacles and the wake region should not be as high as for neutral or unstable conditions. The turbulence is damped out more quickly but gravity waves may propagate for long distances downstream. 
TABLE 5.1. Wake Behavior of Variously Shaped Buildings and Shelterbelts (Meroney 1977)

Downwind Distances (in terms of buiding heights)

\begin{tabular}{|c|c|c|c|c|c|c|c|c|c|}
\hline \multirow[b]{2}{*}{$\begin{array}{l}\text { Building Shape } \\
\text { (width : height) }\end{array}$} & \multicolumn{3}{|c|}{$5 \mathrm{H}$} & \multicolumn{3}{|c|}{$10 \mathrm{H}$} & \multicolumn{3}{|c|}{$20 \mathrm{H}$} \\
\hline & $\begin{array}{c}\text { Percent } \\
\text { Speed } \\
\text { Decrease } \\
\end{array}$ & $\begin{array}{c}\text { Percent } \\
\text { Power } \\
\text { Decrease } \\
\end{array}$ & $\begin{array}{c}\text { Percent } \\
\text { Turbulence } \\
\text { Increase }\end{array}$ & $\begin{array}{c}\text { Percent } \\
\text { Speed } \\
\text { Decrease } \\
\end{array}$ & $\begin{array}{c}\text { Percent } \\
\text { Power } \\
\text { Decrease } \\
\end{array}$ & $\begin{array}{c}\text { Percent } \\
\text { Turbulence } \\
\text { Increase } \\
\end{array}$ & $\begin{array}{c}\text { Percent } \\
\text { Speed } \\
\text { Decrease } \\
\end{array}$ & $\begin{array}{c}\text { Percent } \\
\text { Power } \\
\text { Decrease } \\
\end{array}$ & $\begin{array}{c}\text { Percent } \\
\text { Turbulence } \\
\text { Increase } \\
\end{array}$ \\
\hline 4 & 36 & 74 & 25 & 14 & 36 & 7 & 5 & 14 & 1 \\
\hline 3 & 24 & 56 & 15 & 11 & 29 & 5 & 4 & 12 & 0.5 \\
\hline 1 & 11 & 29 & 4 & 5 & 14 & 1 & 2 & 6 & -- \\
\hline 0.33 & 2.5 & 7.3 & 2.5 & 1.3 & 4 & 0.75 & -- & -- & -- \\
\hline 0.25 & 2 & 6 & 2.5 & 1 & 3 & 0.50 & -- & -- & -- \\
\hline \multirow[t]{2}{*}{$\begin{array}{l}\text { Height of the wake } \\
\text { flow region (in } \\
\text { building heights) }\end{array}$} & & 1.5 & & & 2.0 & & & $3 . C$ & \\
\hline & \multicolumn{9}{|c|}{ Downwind Distances (in terms of shelterbelt heights) } \\
\hline $\begin{array}{l}\text { Porosity }{ }^{(a)} \text { (open } \\
\text { area: total area) }\end{array}$ & $\begin{array}{c}\text { Percent } \\
\text { Speed } \\
\text { Decrease } \\
\end{array}$ & $\begin{array}{c}\text { Percent } \\
\text { Power } \\
\text { Decrease } \\
\end{array}$ & $\begin{array}{c}\text { Percent } \\
\text { Turbulence } \\
\text { Increase }\end{array}$ & $\begin{array}{c}\text { Percent } \\
\text { Speed } \\
\text { Decrease } \\
\end{array}$ & $\begin{array}{c}\text { Percent } \\
\text { Power } \\
\text { Decrease } \\
\end{array}$ & $\begin{array}{l}\text { Percent } \\
\text { Turbulence } \\
\text { Increase }\end{array}$ & $\begin{array}{c}\text { Percent } \\
\text { Speed } \\
\text { Decrease }\end{array}$ & $\begin{array}{c}\text { Percent } \\
\text { Power } \\
\text { Decrease } \\
\end{array}$ & $\begin{array}{c}\text { Percent } \\
\text { Turbulence } \\
\text { Increase }\end{array}$ \\
\hline
\end{tabular}

$0 \%$ (no space

between trees)

40

78

18

15

39

18

9

15

foliage such as

pine or broadleaf

trees)

80

99

9

40

78

$-$

12

32

$40 \%$ (with dense

foliage such as

Colorado Spruce)

70

97

34

55

90

$--$

20

49

Zone (in terms of

shel terebelt

height)

3.0

3.5

(a) Determine the porosity category of the shelterbelt by estimating the percentage of open area and by associating the foliage with the acceleration rate. 


\subsubsection{Flat Terrain With Roughness Changes}

Consider flow from smooth ocean onto densely forested land. The flow near the surface decelerates as it encounters the increased drag of the forest. Mass continuity requires a vertical velocity; the PBL height $h$ therefore increases. Unless there is a wind speed maximum within the PBL, every level experiences some deceleration near the coast from this dynamic adjustment.

Near the surface the velocity and turbulence structure of the flow adjust to the properties of the near surface. Most research (theoretical, experimental, numerical) has concentrated on this aspect of the wind and turbulence structure for flow normal to an ideal discontinuity in roughness. A region of adjustment forms and propagates upward in the downstream direction (separate from the dynamic adjustment mentioned in the previous paragraph). This region is the internal boundary layer (IBL). Within the IBL is a transition layer where the adjustment to the new surface occurs and an equilibrium layer where the adjustment is complete.

The pioneering work on IBL growth was by Elliott (1958) who found that the IBL thickness (6) grew as $\delta \sim x^{0.8}$ where $x$ is the distance downstream from the discontinuity. Peterson (1972) has shown that in the transition region advection and diffusion of turbulent kinetic energy affect the momentum balance He also found (Peterson 1969) that the height of the equilibrium layer is about $0.18 \delta$. The velocity profile is essentially that of the upstream region for $z / \delta>0.8$ and for $z / \delta<0.1$ it is that of the downstream equilibrium region. For $0.1<z / \delta<0.8$ the velocity profile adjusts to the log profiles at top and bottom, and exhibits maximum (minimum) shear for transitions from smooth (rough) to rough (smooth) at $z / \delta \approx 0.3$. The turbulence characteristics of the upper 75 percent of the IBL most resemble the upstream flow. At the transition from smooth to rough, the turbulence intensity may be twice that of the equilibrium value over the rough surface but drops down to near equilibrium within $x / z_{0} \sim 10^{3}$.

For application to a large WECS that extends to $100 \mathrm{~m}$ above ground, internal boundary layer theory must be taken lightly. An IBL theory may 
predict $\delta \sim 100 \mathrm{~m}$ for $\mathrm{x} \sim 1500 \mathrm{~m}\left(\right.$ or $500 \mathrm{~m}$ ) for downstream roughness $\mathrm{z}_{0} \sim 0.01 \mathrm{~m}$ (or $1 \mathrm{~m}$ ). At these heights and distances the assumptions of the theories break down. In the unstable to near-neutral PBL the large momentum-transporting eddies, which scale as the height of the PBL, dominate the mixing. This effects a more rapid adjustment to the new surface although not enough is known to say just how fast.

In the simplest case of a sharp transition from rough flow to smooth, choose a site as far into the smooth area as possible, say at least $x \sim 1 \mathrm{~km}$, to obtain the maximum power. There may be an area of reduced mean shear nearer the transition but the loss of wind at the upper part of the disk due to moving nearer the transition is not worth the reduction in wind shear.

Flow from smooth to rough is more difficult to describe, but dealing with this case may be necessary in coastal areas. The best location from wind power considerations is as near the smooth region as possible; however, the turbulence intensity may be high near the surface. The flow from over water to over forest may even be decelerated enough at the boundary to cause flow separation. If the site is moved back there may come a point where the bottom of the rotor is in fully adjusted rough flow while the top is still in the smooth flow velocity profile. This point of maximum shear is somewhere between $x \sim 300 \mathrm{~m}$ and $\mathrm{x} \sim 1500 \mathrm{~m}$. An estimate of the worst possible mean wind shear across the disk may be made from curves like Figure 5.1. Moving back further simply leads to decreasing available power. If height of the wind turbine is elective, higher placement is more justifiable in this case.

If the flow strikes the discontinuity obliquely, the flow component parallel to the roughness change will remain approximately the same while the normal component accelerates or decelerates. Hence, directional wind shear results.

If the transition is not an ideal discontinuity but a slow transition or even a multiple roughness element transition, the same principles apply but use of theory to quantify the IBL structure is even less justifiable. Field observations must be made. 


\subsubsection{Flat Terrain With Horizontal Temperature Gradients}

Horizontal temperature gradients over truly flat land form because of different heating and cooling rates of the atmosphere at different locations. The heating and cooling of the earth's surface is controlled primarily by the radiative balance of the surface and the turbulent heat flux from the surface into the atmosphere. A more reflective surface will not heat as quickly as will a dark surface. However, a dark vegetated area will remain cooler than a dry sandy area because the transpiration of plants returns much of the heat to the atmosphere as latent heat. The heat flux is also dependent upon wind speed. The wind speed and heat flux change in complex ways as both are linked through feedback loops involving boundary layer structure.

Only horizontal temperature gradients that have a consistent or climatological impact upon the wind are of concern to the siting of WECS. In this section, two effects of horizontal temperature variations are discussed:

- horizontal variations in thermal wind

- sea breeze circulation.

The diurnal variations in thermal wind over sloping terrain and the influence on the nocturnal jet were discussed in Section 5.2.1.

\section{Horizontal Variations of Thermal Wind}

The horizontal variation on the mesoscale of the thermal wind can cause areas of enhanced or reduced winds. (See Appendix 2 for a discussion of the thermal wind equations.) Consider a well-mixed PBL of cool marine air flowing onshore where the earth is hot. An internal boundary layer of warming air will form at the shoreline. If the shoreline is contoured, as in the bay shown in Figure 5.10, the contours of the thermal wind will roughly follow the coast. If the geostrophic wind above the PBL does not change at the coast, the surface geostrophic wind can be computed with the thermal wind relationships. The difference in surface geostrophic wind between the two sides of the bay can be quite large. The actual surface winds, however, will be much less different due to advection of momentum. However, there is still acceleration of the flow on one side and deceleration on the other side as the streamlines of Figure 5.10 show. Figure 5.10, which is overly simplified, is provided 


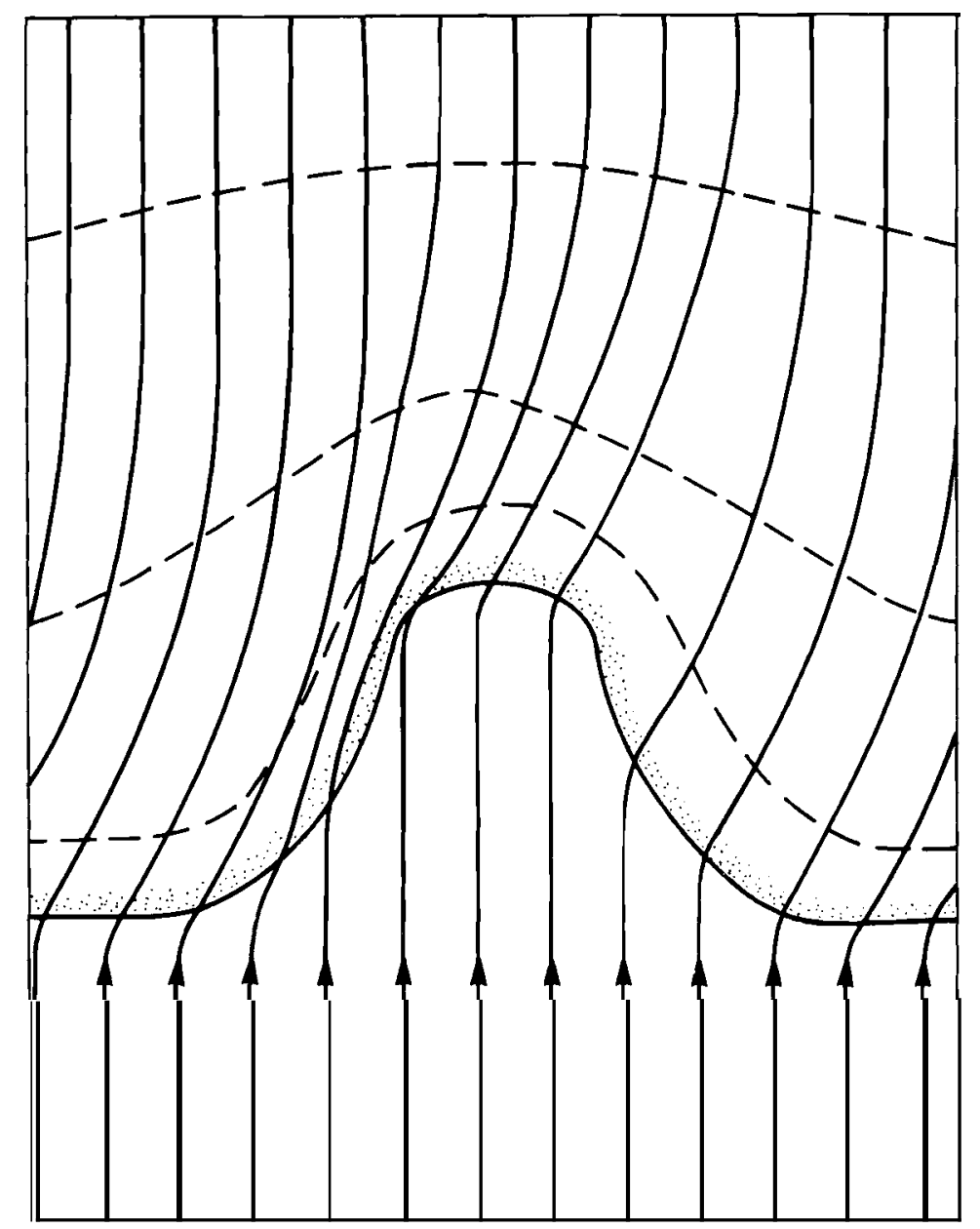

FGURE 5.10. Streamlines of the Thermal Wind (dashed) and of the Wind (solid) for Onshore Flow from Cool Ocean to Hot Land

simply to suggest that low-1evel baroclinic effects be considered. Baroclinic effects on winds are also discussed in Chapters 3 and 4.

$\underline{\text { Sea-Breeze Circulation }}$

Sea and lake breezes are qualitatively well-known examples of the diurnal variation effects of near-surface temperature gradients. During the day, heating of the inland mixed layer results in an increased offshore pressure gradient. Under appropriate large-scale synoptic conditions, marine air will 
move inland behind the sea-breeze front (Figure 5.11). The sea-breeze front is a horizontal convergence zone that moves inland at about 1 to $2 \mathrm{~m} / \mathrm{sec}$. The convergence zone is frequently the location of a line of cumulus clouds. Above the maritime mixed layer a return flow seaward completes the circulation. Wind speeds at $100 \mathrm{~m}$ within a well-developed sea breeze are about 5 to $7 \mathrm{~m} / \mathrm{sec}$. The depth of the sea breeze is about that of the inland mixed layer, since it is the heating of this layer that causes the pressure drop inland. The wind speed maximum is near the surface and may extend from a few $\mathrm{km}$ to $100 \mathrm{~km}$ inland. At night, an analogous land breeze blows back out to sea, but it is much weaker since the temperature gradients are weaker and the nocturnal boundary layer over land is shallow.

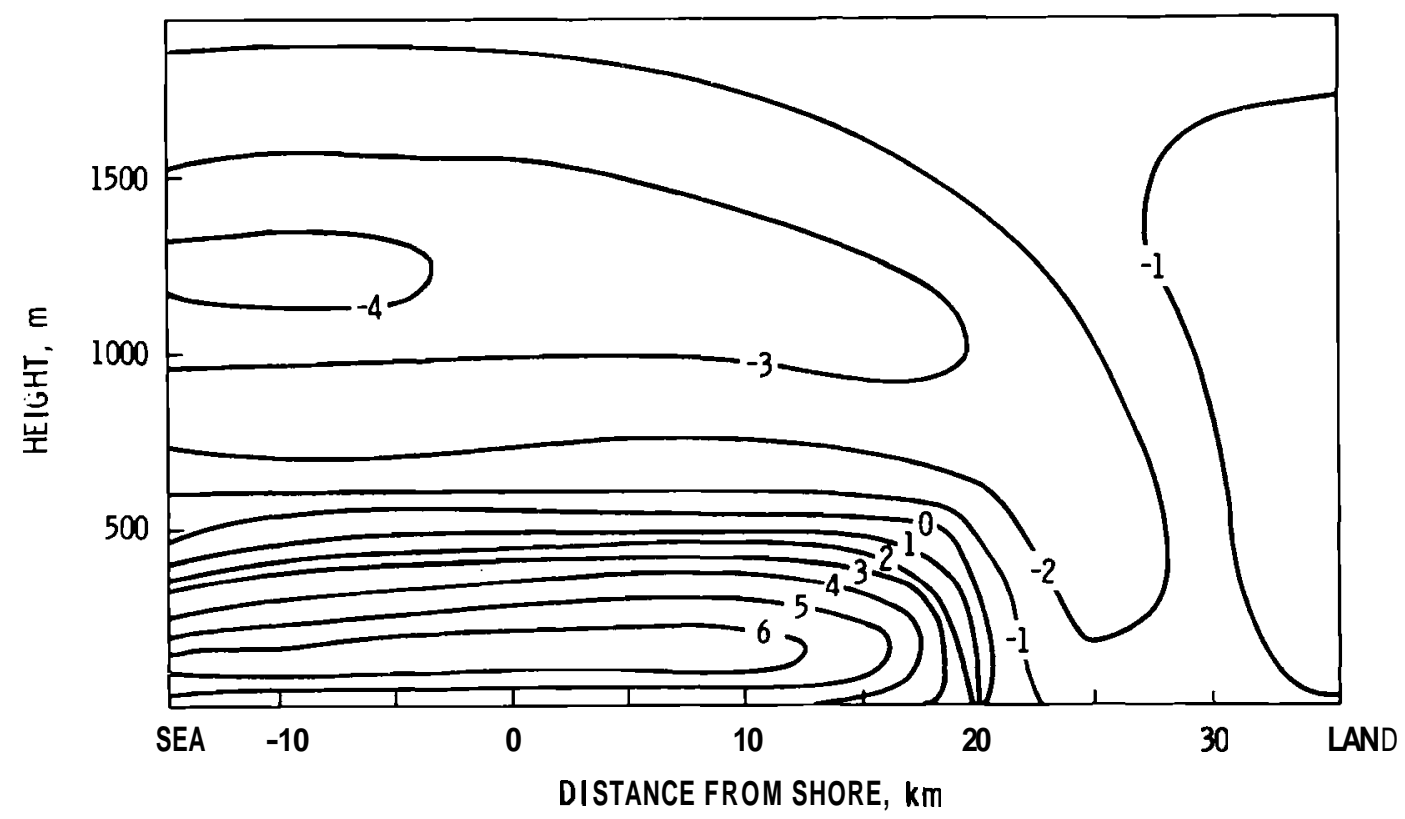

FIGURE 5.11. Cross-Section of a Well Developed Afternoon Sea Breeze, Contours are of the Onshore Component of the Wind in $\mathrm{m} / \mathrm{sec}$

Many complications alter the simplistic sea-breeze picture. Among the complications are: the synoptic scale wind system, the shape of the coastline, coastal topography, the past history of the sea breeze, and the Coriolis effect. 
By far, the synoptic-scale wind system has the largest effect on the sea breeze. A too-strong offshore wind advects wam air over the water, thereby destroying the favorable pressure gradient; a too-strong onshore wind blocks the necessary return circulation aloft. Therefore, the sea breeze usually occurs during high pressure episodes when the synoptic-scale pressure gradient is weak.

An irregular coastline causes the horizontal variations of the thermal winds discussed above. The situation becomes very complex because of the temporal variations that are part of the developing sea breeze. A complex mesoscale numerical model may be needed to-interpret these effects if sufficient wind data do not exist.

Coastal topography influences the sea breeze in two ways. The cool marine air may be channeled along valleys and through passes in coastal mountain ranges. Cool marine air cascading down the inland side of a pass can be a significant source of winds in inland valleys. The heating of the slopes of coastal hills induces upslope flow that can interact with the return flow; sometimes it is weakened and sometimes it is reinforced. If there is significant change of wind direction with height, the air lifted by coastal hills can be transported in many directions, thereby complicating the circulation.

The past history of the sea breeze is important because the air offshore may not be the cool marine air mass that one expects. It may be air pumped out to sea by a strong sea breeze of the preceding day.

The sea breeze takes all day to develop. The time and distance scales of the circulation are large enough that the Coriolis effect can deflect the air mass moving toward the coast. Therefore, a west coast in the northern hemisphere may experience a westerly sea breeze becoming northwesterly by late afternoon.

\subsection{SMALSCALE TERRAN}

In this section we describe the effects that small-scale features have upon the flow in the PBL. A topographical feature is defined as small scale when it is small compared to the depth of the PBL. Small-scale features may 
be elevated (i.e., small cliffs, ridges, or hi11s) or lower than surrounding terrain (i.e., small val leys, gulleys, canyons, or depressions). Study of the flow over small-scale features is simplified for two reasons. First, the flow structure above the top of the PBL need not be considered. Second, turbulent transport of momentum due to the largest eddies occurs on a time scale smaller than the time for flow to cross the feature. Therefore, attempts to understand the effects of these features upon an inviscid flow of assumed upstream character are not wholly unreasonable. Nevertheless, for siting of WeS there is no substitute for measurement.

\subsubsection{E Evated Features}

An elevated feature is any one in which the surface of interest is higher than the surrounding region. This is somewhat dependent on your perspective, since, to one view, a ridge may be a valley wall. However, when considering ridges the primary concern is with flow over and around the ridge or hilltop. Flow over small-scale terrain features resembles flow around obstacles but wind energy research has attempted to look for positive attributes to these natural obstacles.

In this section we discuss flow over:

- small ridges

- small cliffs

- small hills.

W consider a ridge or a cliff as a feature with one dominant axis that extends laterally far enough on both sides of a point of interest so that impinging flow normal to that axis is forced over the crest and there is no avenue for leakage around the side. Small cliffs and ridges may be considered long if the point of interest is more than 10 ridge or cliff heights away from the end (or some other major change) of the feature.

\section{$\underline{\text { Small Ridges }}$}

Because the wind over flat country normally increases with height, wind over small ridges is stronger than over flat country. More important, though, 
is the acceleration of flow over the ridge that depends on its shape. Figure 5.12 illustrates this. Figure 5.12 shows a hypothetical profile for flow normal to a long ridge. The ridge is $30 \mathrm{~m}$ high with average slopes of $1 / 3$. The upstream wind profile is a neutral stability logarithmic wind profile. The upper portion of the ridge-modified profile was inferred from wind tunnel measurements over a model of a similar ridge, and the lower part of the modified profile agrees roughly with Frenkiel's (1962) measurements in the first $40 \mathrm{~m}$ of flow over a ridge. The mass flow across this hypothetical ridge is conserved so it is at least a physically plausible flow.

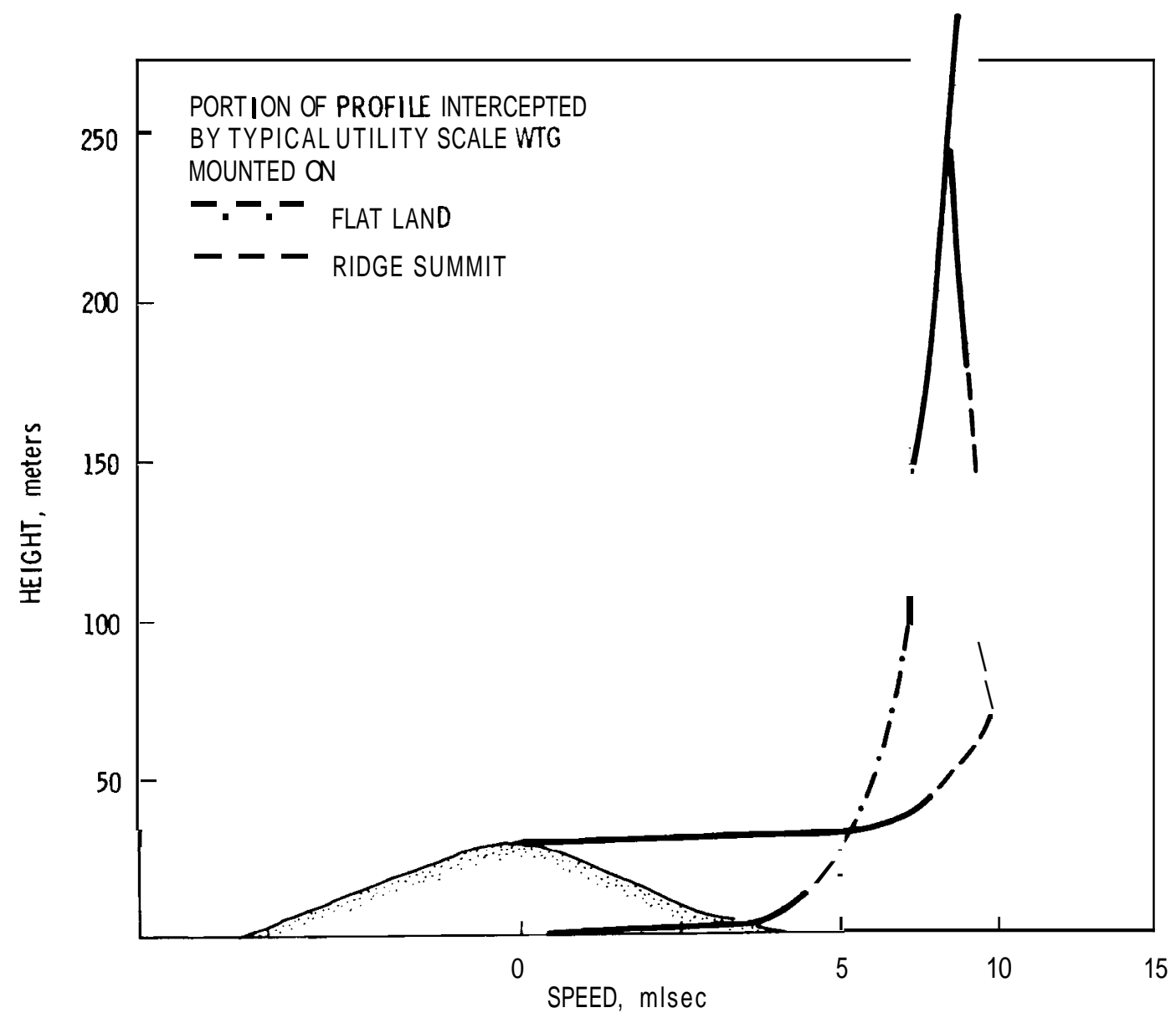

FIGURE 5.12. Hypothetical Wind Speed Profiles Over a Small Two-Dimensional Ridge. The dashed or dash-dot sections of each profile are regions intercepted by a typical large wind turbine. 
The acceleration in the lowest hundred meters over the ridge is apparent. Also shown in Figure 5.12 are the sections of the flat land and ridge profiles that might intersect a large WECS. Note that the mean wind shear over the rotor disk of a WECS is considerably reduced over the hill.

The profiles of the wind speed cubed were integrated over the circular disks of an assumed horizontal axis wind machine to compare the power in the wind (not the power a given machine might extract) over flat land, over flat land with a $30 \mathrm{~m}$ taller tower, and over the ridge. A higher tower produces a $20 \%$ increase in available wind power but siting on the ridge produces a $100 \%$ increase in power in the wind. Therefore, $80 \%$ of the increase can be attributed to the acceleration due to the shape of the ridge.

Frenkiel (1962) studied flow over over a ridge and a hill in Israel. Each feature was about $500 \mathrm{~m}$ tall, which is perhaps not small in the context used here. He did not anticipate the sizes of current large WECS when he formulated his criteria for good WECS sites. His chief criterion was that the best WECS sites would have a minimum wind shear between $40 \mathrm{~m}$ and $10 \mathrm{~m}$. Frenkiel concluded that the flow structure of the lowest $40 \mathrm{~m}$ is very sensitive to the shape of the terrain in the nearest few hundred meters of the point of interest. Specifically he claims that a slope of about $30 \%$ running smoothly to the summit will produce maximum energy and minimum shear. His measurements indicated that if the last hundred meters of slope flattens out, the ratio $\mathrm{U}_{40} / \mathrm{U}_{10}$ increases. This may be interpreted as a deceleration of the very local near-surface winds (if the deceleration is large enough on a flat-topped ridge there may be flow separation), but the flow that is accelerated by the larger scale features of the ridge is still speeding along at levels seen by large WECS.

Frenkiel concluded that there was no perceptible wind speed dependence to his estimation of the wind ratio. He found that the influence of the slopes more than a few hundred meters from the summit was observable but not nearly as important as the slope in the neighborhood of the summit. The wind ratios he measured were a strong function of stability but it is not possible to sort out whether or how the acceleration is affected by stratification from the stratification effects on wind profiles over upstream flat land. 
Bradley (1980) reports a series of measurements in nearly neutral stratification upwind and on the summit of a ridgelike hill near Canberra, Australia. Black Hill rises $170 \mathrm{~m}$ above the surrounding forested plain and has a $1.2 \mathrm{~km}$ cross-section through the base. One purpose of Bradley's experiment was to collect a data set of mean flow and turbulence characteristics over the hill for comparison with numerical and analytical models of flow over hills. Even though Black Hill does not meet the criteria of a small ridge required by many models, reasonably good comparisons were obtained between models and data under appropriately restricted conditions. It is significant to note, however, that even under conditions of nearly neutral stability, (as inferred from a nearly negligible heat flux $)$ the presence of a low-level $(<500 \mathrm{~m}$ above the plain) temperature inversion marking the top of the planetary boundary layer dramatically affected the measurements. Bradley states: "Several occurrences of the distortion of [wind] profiles by the low-level inversion have been observed but were not consistent, sometimes resulting in strongly accelerated flow at the upper levels on the tower, and at other times strong retardation." This indicates that a ridge may at some times behave like a small ridge, as defined in this report, and at other times behave like a large scale feature. Furthermore, the inconsistent behavior indicates that limited short-term field measurements, even under what are believed to be prevailing flow conditions, can yield data from which erroneous conclusions could be drawn.

A number of numerical and analytical models have been developed for studying flow over small ridges. Some of these models were used on specific hill shapes chosen for computational convenience since the main purposes of the models were to illuminate specific flow features. Consequently, not all models are immediately applicable to energy problems, although some general results can be noted. Frost et al. (1975) computed velocity development over a semi-elliptical hill, using momentum equations on a coordinate system conforming to flow stream 1 ines. The conservation of turbulence kinetic energy was used to close ${ }^{(a)}$ the system of equations. For an ellipse similar to

(a) When the momentum equations are averaged to deal with averaged and not instantaneous quantities, the averaging process results in new unknown terms. Further equations and assumptions for these new unknowns must be made in order to provide a closed set of equations. See Appendix 2. 
Black Hill, the solution of Frost et a1. (1975) predicts a velocity maximum at about $0.3 \mathrm{H}$ where $\mathrm{H}$ is the height of the hill, and an increase of wind speed (relative to the same height above the plain) of $76 \%$. Taylor (1977) uses a finite difference model for flow over gentle topography that solves the momentum, continuity, and turbulence energy equations. Jackson and Hunt (1975) provide an analytical solution by separating the flow into two regions with a maximum velocity perturbation at the interface between the two layers. The height of the interface is described by:

$$
1 \ln \left(1 / z_{0}\right)=2 k^{2} L
$$

where 1 = height of perturbation maximum above the ridge crest

$\mathrm{k}=$ von Karman constant (see Appendix 2)

$L=$ length from crest to point where surface elevation is $\mathrm{H} / 2$

$\mathrm{z}_{0}=$ roughness length.

For the Black Hill study, Bradley (1980) used $k=0.4, L=275 \mathrm{~m}$, and $z_{0}=1.14 \mathrm{~m}$ from which $1=28 \mathrm{~m}$. The increase in speed according to the Jackson and Hunt theory may be approximated as $2 \mathrm{H} / \mathrm{L}$.

Bradley (1980) made some comparisons between his data and the theories and models of Frost et a1. (1975), Taylor (1977) and Jackson and Hunt (1975) by scaling the model results to fit Black Hill geometrical and roughness parameters. Some of these comparisons are shown in Figure 5.13. The upstream wind profile and ridge crest wind profile data of Bradley in Figure 5.13 represent the average of three contiguous runs spanning $90 \mathrm{~min}$. The upstream wind profile measurements only extended to a height of $18 \mathrm{~m}$ above the displacement height of $7 \mathrm{~m}^{(\mathrm{a})}$ but Bradley assumed that a logarithmic wind profile could be used up to $90 \mathrm{~m}$. The results show generally good agreement between the data and Frost et al. (1975) and Taylor (1977) model predictions. The detail of the position and structure of the small jet at about $28 \mathrm{~m}$ were not predicted by those models but its position is well predicted by the Jackson and Hunt model, as is the magnitude of the wind speed at the crest.

(a) The logarithmic wind profile extrapolates to 0 wind speed at about $7 \mathrm{~m}$ above ground, within the forest canopy. See Appendix 2 for details of the displacement height concept and wind profile descriptions. 


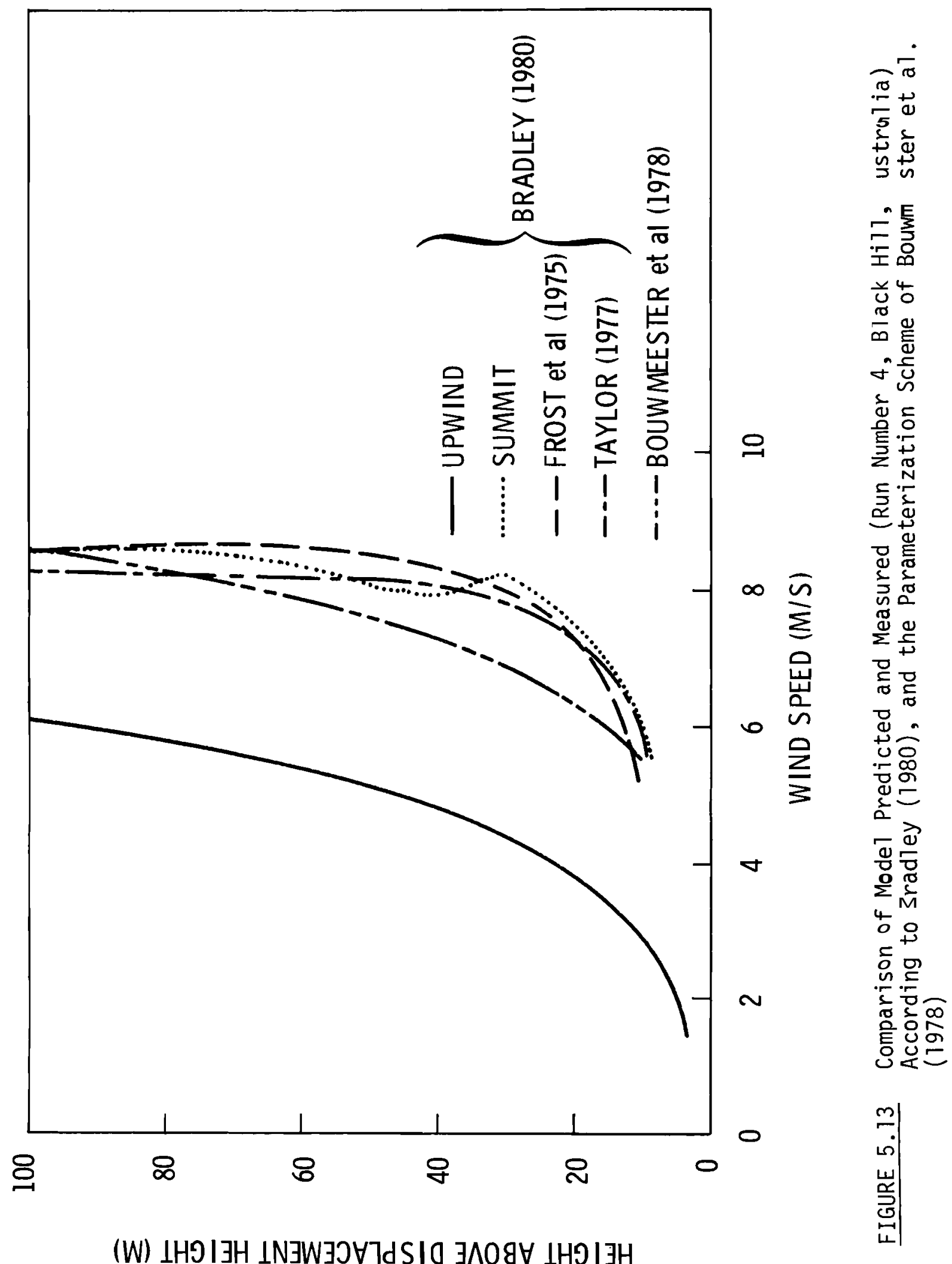


Wind tunnel measurements over generic hill shapes provide another source of data on flow over isolated hills. As described in Chapter 4, complete dynamic similarity is not achievable between wind tunnel studies and fullscale atmospheric flows, especially stratified flows. Information on flow over small hills in neutral flows is usable. The following discussion is based on work on flow over triangular ridges reported by Meroney et a1. (1978).

A simple empirical parameterization scheme for estimating the velocity profile over two-dimensional triangular ridges has been developed (Bouwmeester et al. 1978): define a speed-up factor for flow over the crest of the ridge as

$$
S\left(Z^{\prime}\right)=\frac{U_{c}\left(Z^{\prime}\right)}{U_{0}\left(Z^{\prime}\right)}
$$

where $Z^{\prime}=Z / H$ is the normalized height

$$
\begin{aligned}
& Z=\text { height above terrain } \\
& H=\text { ridge height above plane } \\
& U_{C}=\text { wind speed over ridge crest } \\
& U_{0}=\text { wind speed over flat terrain. }
\end{aligned}
$$

Assume that the wind profile over the ridge and the plane can be described with a power law (see Appendix 2) with $\alpha_{c}$ and $\alpha_{0}$ the power law exponents over the ridge crest and plane respectively. Then

$$
S\left(Z^{\prime}\right)=S(1) Z^{\prime}\left(\alpha_{c}-\alpha_{0}\right)
$$

where $S(1)$ is the speed-up factor at a height of $\mathrm{H}$ above the ridge (or $2 \mathrm{H}$ above the plane). To use Equation (5.2), an expression for $\alpha_{c}-\alpha_{0}$ is needed. Two approaches are possible. The first specifies that at some height, $Z_{T}^{\prime}$, the speed-up factor will be 1 ; i.e., $S\left(Z_{T}^{\prime}\right)=1$. Then Equation (5.2) yields

$$
\alpha_{C}-\alpha_{0}=\frac{-\ln S(1)}{\ln Z_{T}^{1}} \text {. }
$$


Wind tunnel measurements suggest that the speedup over a small ridge extends to $7 \leq Z_{\top}^{\prime} \leq 10$. Another option, which Meroney et al. (1978) derives from field data of Bradley (1980), uses a linear fit to wind tunnel data to arrive at

$$
\alpha_{C}-\alpha_{0}=\frac{1-S(1)}{2.3} \text {. }
$$

$S(1)$ depends upon the geometry of the ridge. Wind tunnel measurements of $S(I)$ are presented in Figure 5.14 for different upwind and downwind slopes of the ridge. $L_{u}$ and $L_{d}$ in the figure are twice the horizontal distance from the ridge crest to the point on the ridge where the elevation above the plane is $1 / 2 \mathrm{H}$; this definition of slope is to be used if the ridge is not perfectly triangular.

The speedup factor plotted in Figure 5.14 depends upon the wind shear of the upwind profile, and specifically the data of Figure 5.14 are for $\alpha_{0}=0.13$. The following relation is suggested as a correction to $S(I)$ as a function of $a_{0}$.

$$
S(1)_{\alpha_{0}}=S(1)_{\alpha_{0}}=0.13\left\{\frac{1.15+\alpha}{1.28}\right\}
$$

This technique is very simple to use and reasonably effective. It is not applicable if $Z^{\prime}<0.05$ or if the hill height is a large fraction of the boundary layer height. The technique may not work too well over very rough terrain as the comparison in Figure 5.13 shows, largely because a large powerlaw coefficient of the upwind profile (a \% 0.32 for the profile in Figure 5.13) strains or exceeds the useful limits of Equation (5.5). The technique does appear to be usable over hills of a variety of shapes; however, hills with extended regions of flatter slopes near the summit show a significant reduction in the speedup near the crest.

The influence of stability on the speedup over a small ridge is uncertain because it is difficult to judge which effects are due to stratification 


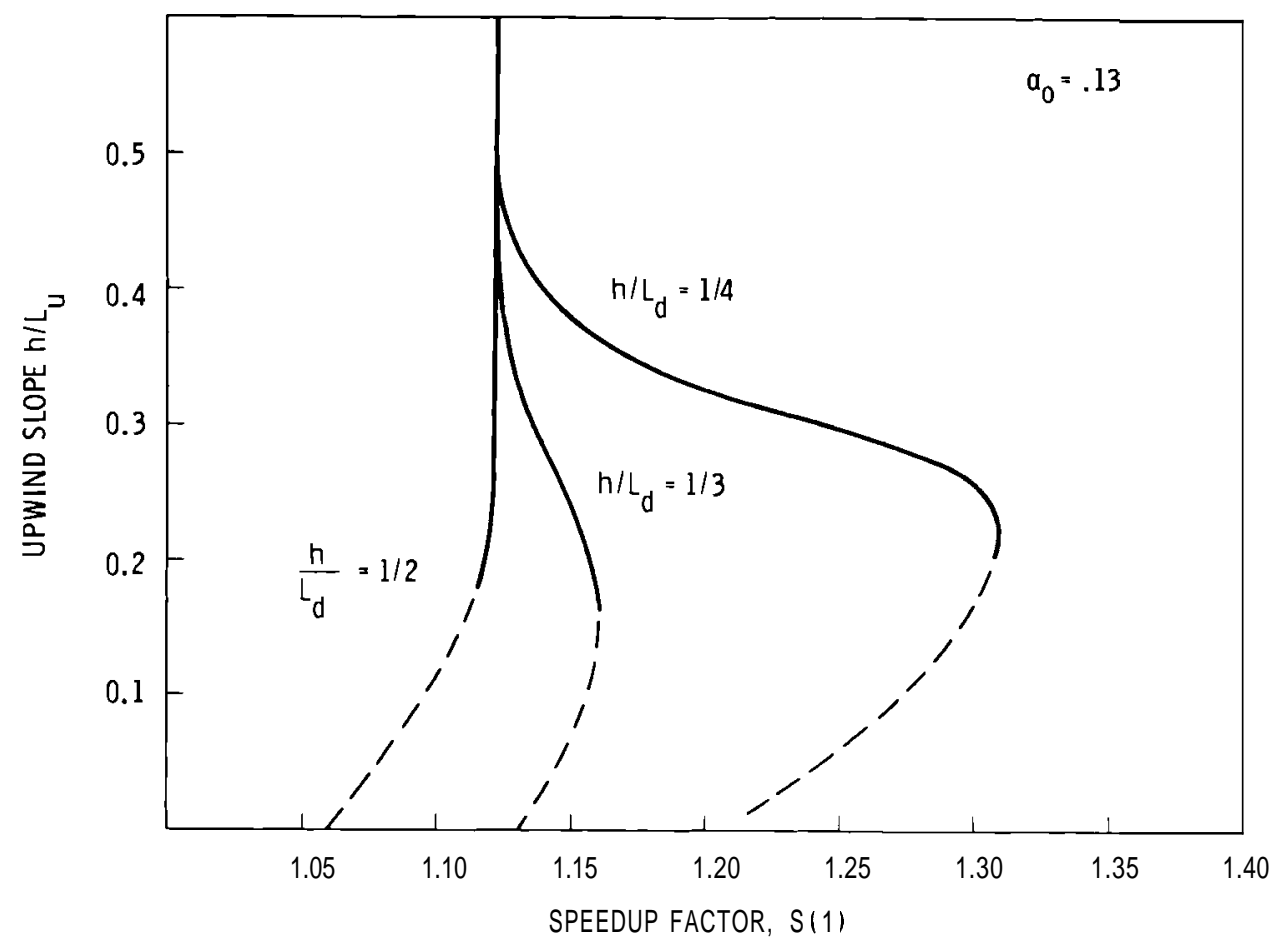

FIGURE 5.14a. The Effect of Upwind Slope on the Speedup Factor for $\alpha_{0}=0.13$

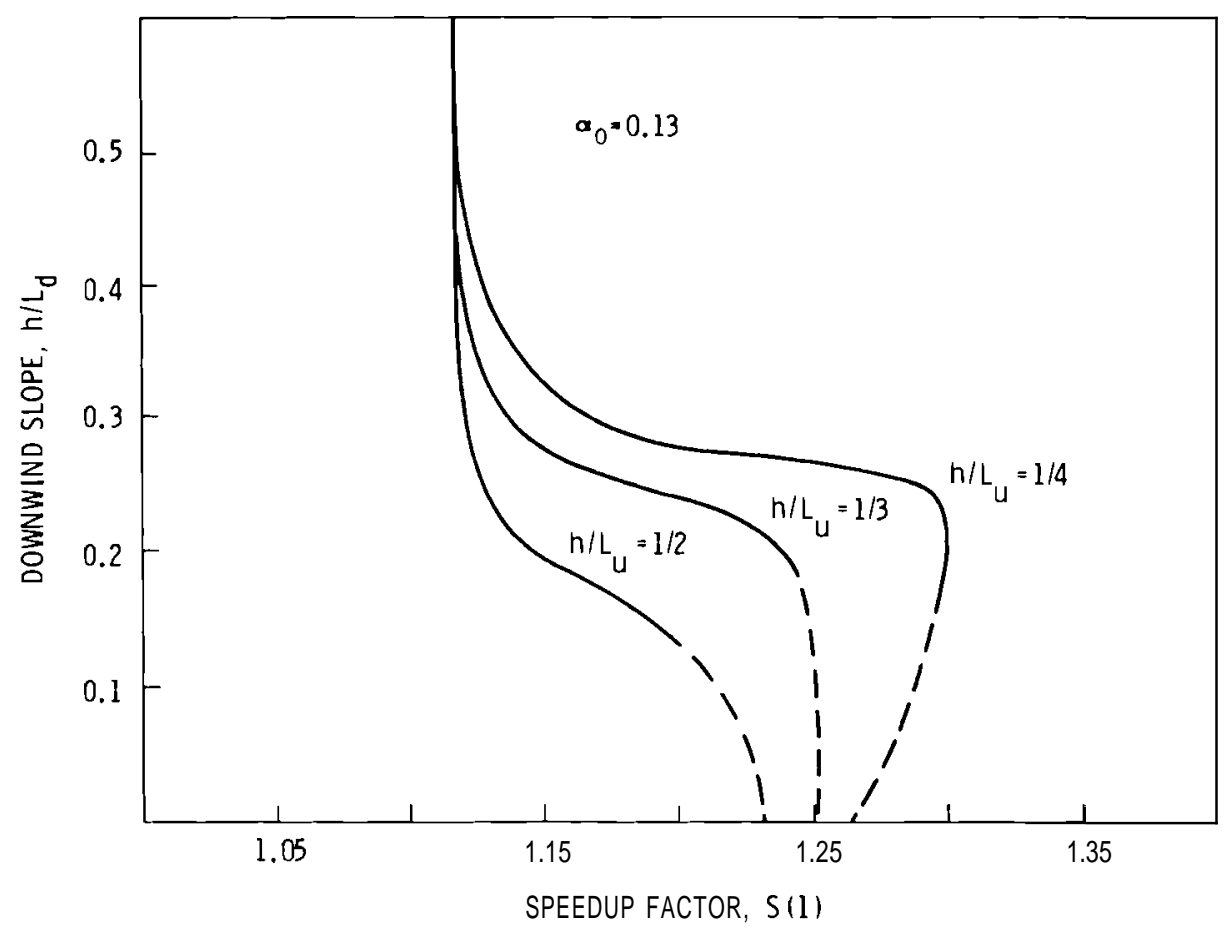

FIGURE 5.14b. The Effect of Downwind Slope of the Speedup Factor for $\alpha_{0}=0.13$ 
alone and which are due to the upwind wind profile. In the wind tunnel, Meroney et al. (1978) find the stability effects are small with a slight increase i $n$ speedup for unstable conditions and a slight decrease for stable conditions. If a strong temperature inversion confines the flow to a shallow layer, the speedup will increase due to a venturi effect. As an approximation based on the depth of the accelerated layer in a neutral-boundary layer this could happen for hill heights of $1 / 10$ to $1 / 7$ the height of the boundary layer. In the field, Bradley (1980) reports inconsistent effects in nearly neutral stratification caused by temperature inversions beneath two hi11 heights above the surrounding plain.

Flow over a long small ridge accelerates to a maximum velocity at or near the ridge crest and decelerates as it moves down the lee side. For weak flows the air can negotiate the passage over the ridge without separating. However, at higher speeds, depending upon the ridge geometry, the flow separates.

The flow can separate on the upwind face of the ridge when the interaction with the hill produces a sufficiently large pressure gradient counter to the mean flow. A circulating eddy forms at the base of the ridge. The separation bubble on the upwind side of the ridge extends up wind to two ridge heights but is constrained by the topography from extending up the ridge face to the crest.

The flow also separates at the crest of the ridge. If the ridge has a sharp crest, the flow will separate right at the crest; if the ridge has a smooth or rounded crest, the flow usually separates at some point down the lee side from the crest due to the deceleration of the flow. The location of the lee side separation is a function of the Reynolds number in the wind tunnel. It can also be expected that it is a function of atmospheric stability; unstable stratification increases the chance of flow separation at the crest or higher up the lee slope, whereas stable stratification inhibits flow separation or causes the point of separation to move farther down the lee side. The downwind separation eddy behaves like the wakes discussed in Section 5.2.2; the wake effects extend up to $20 \mathrm{H}$ downwind, less : instable conditions, more in stable conditions. 
The maximum velocity is attained just before the flow separates. The occurrence of flow separation for triangular ridges depends most critically on the upwind and downwind slopes. Meroney et a1. (1978) therefore suggest

Figure 5.15 as a criterion for geometry leading to flow separation and hence of maximum speedup over the crest.

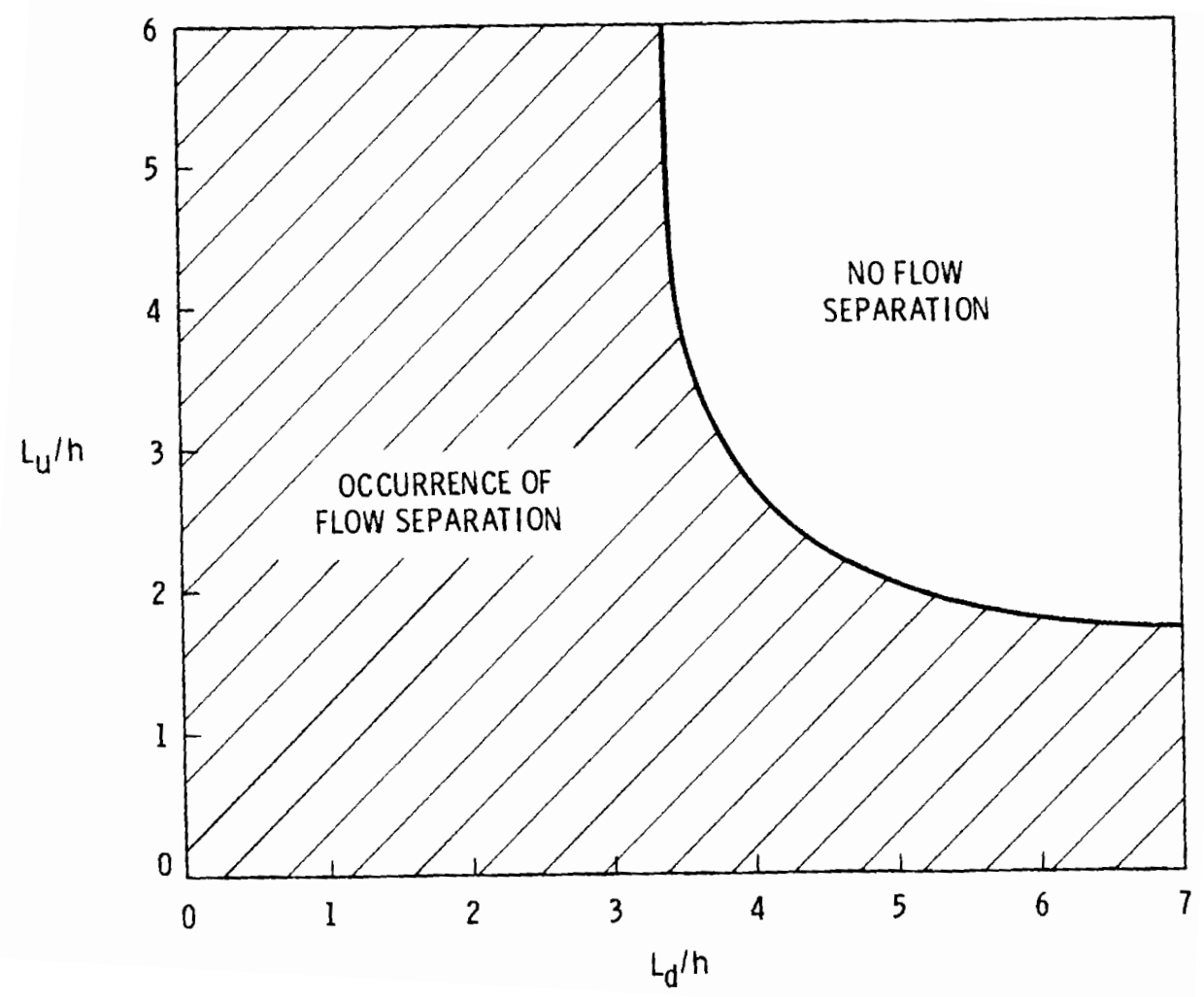

FIGURE 5.15. Criterion for Flow Separation Over Two-Dimensional Hills

The turbuience in a flow over a small hill behaves like turbulence in a strained flow. There is a reduction in the longitudinal component of the turbutence intensity. Downwind of the crest, turbulence production increases and the turbulence intensities exceed upwind values. Figure 5.16 shows wind tunnel measurements of the longitudinal component turbulence intensity for flow over two ridges; one has a slope of $1 / 20$ and the other a slope of $1 / 4$.

Departure from strict two-dimensionality of the ridge may be exploitable. For example, wind turbines sited along the crest of a long small ridge can use 


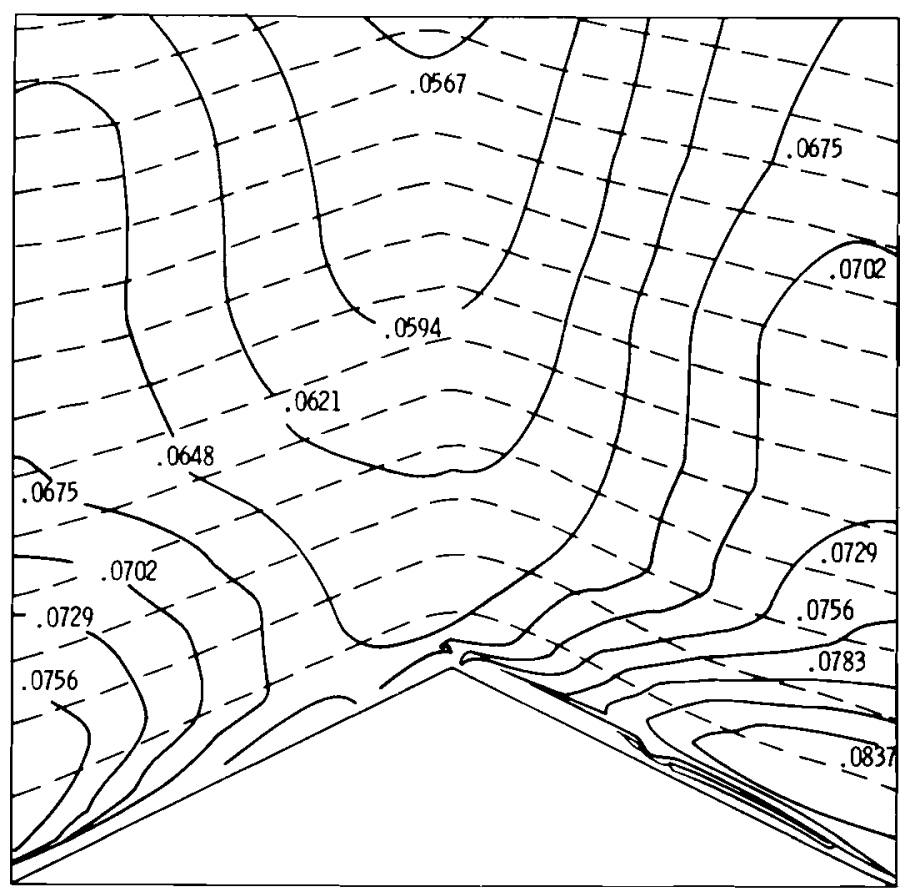

(a)

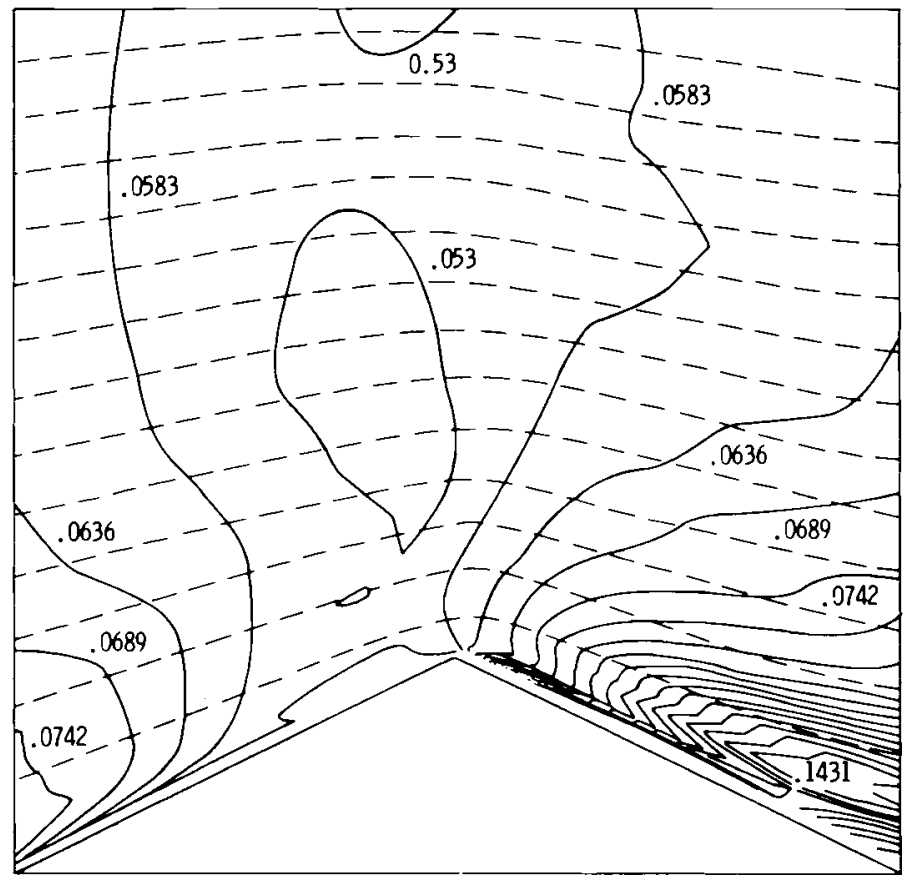

(b)

FIGURE 5.16. Contours of Longitudinal Turbulence Intensity With Superimposed Stream1ines (Broken Lines). The normal ized roughness - length is $\mathrm{z}_{0} / \mathrm{h}=1.5 \times 10^{-4}$. Part (a) is for a symmetrical ridge of slope $1 / 20$; part (b) is for a slope of $1 / 4$. 
small concave sections of the ridge to collect even more wind. Flow will pass around the ends of a long ridge, especially in stably stratified flow, so ridge shoulders may provide good sites for wind turbines. However, if flow comes over and along the ridge toward the shoulder, there could be high turbulence and flow separation. If the wind is blowing along the ridge, up toward the shoulder, the ridge divides the flow much as a hill does and so that case should be considered as a hill.

If the flow is oblique to the ridge, the normal component will still accelerate. Since the speedup effect is a function of height, oblique flow may lead to some directional wind shear. The deviations in wind direction will probably also increase. Finally, it is probable that the small ridge considered is not isolated. The flow over a ridge that is near and upwind of a larger ridge will respond to the larger ridge. The flow may even be decelerated at the small upwind ridge. In a stable PBL, surrounding ridges will excite Kel vi n-Helmholtz gravity waves that can increase turbulence and gustiness at a WECS site. If the ridges are closely spaced and steep, flow separation eddies may fill the intervening valleys. The separation streamlines then form a much more level surface. The flow responds to this modified surface so the acceleration effects at the ridge crests are reduced.

\section{Small Cliffs}

A small cliff is considered here to be a step where the flow must adjust to a new persistent downstream elevation. The principles of flow over small cliffs do not differ much from those for flow over small ridges.

Flow up and over the cliff is the situation of primary interest, because there is no advantage to flow diverted down an escarpment. Under stable atmospheric conditions there may be some acceleration perceptible near the crest of the cliff as dense air cascades down the face of the cliff. In that case there would be little turbulence near the crest of the cliff, but there would be a great deal at the base.

Figures 5.17 through 5.20 illustrate the speedup of the mean flow (see small ridges for discussion of speedup) and turbulence intensities of flow over two-dimensional escarpments of different slopes. These data come from 

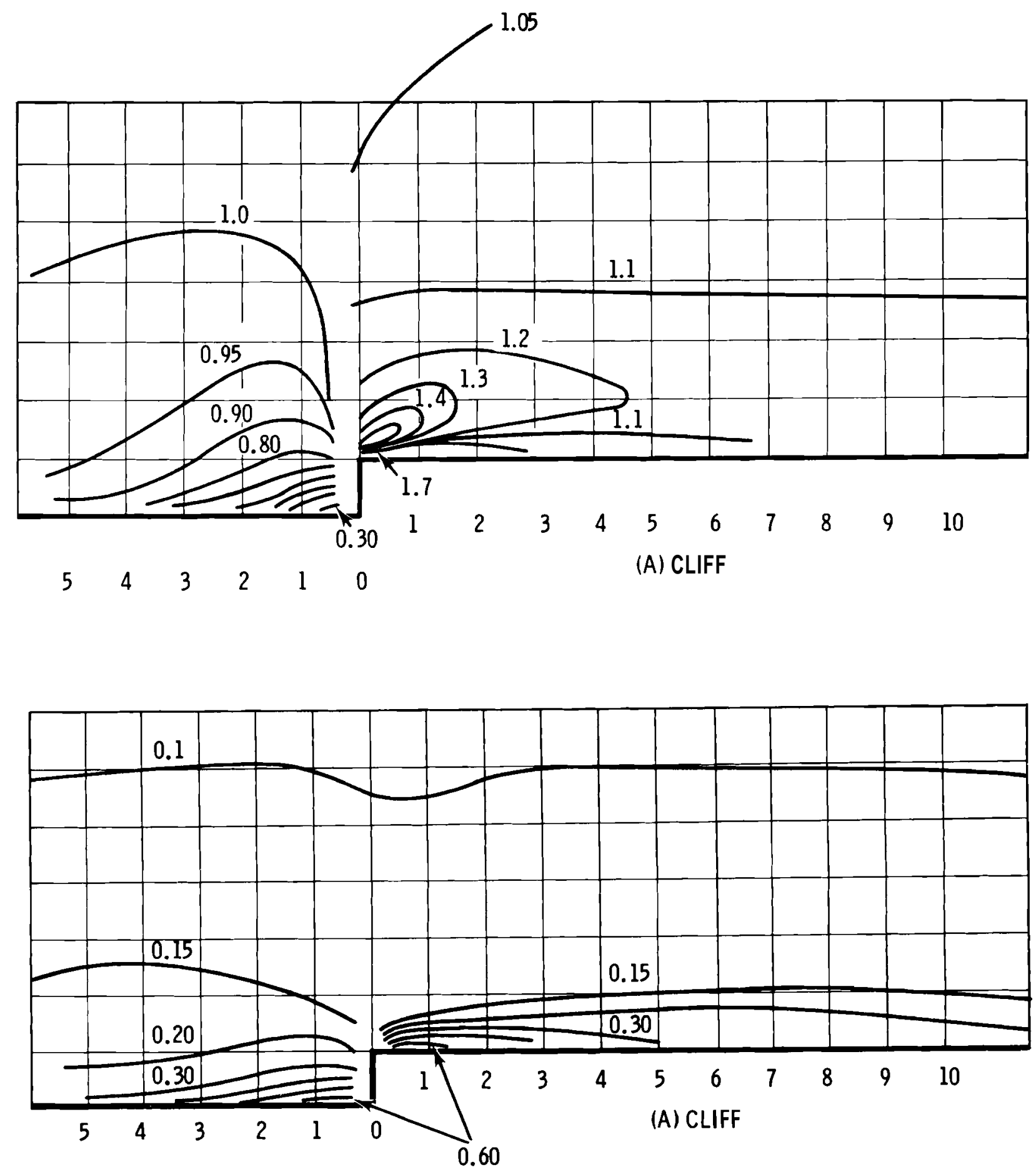

FIGURE 5.17. Contours of the Speedup of Upstream Winds (Upper) and Contours of the Turbulence Intensity (Lower) for Flow Over a Cliff (Bowen and Lindley 1977) 


\subsection{5}
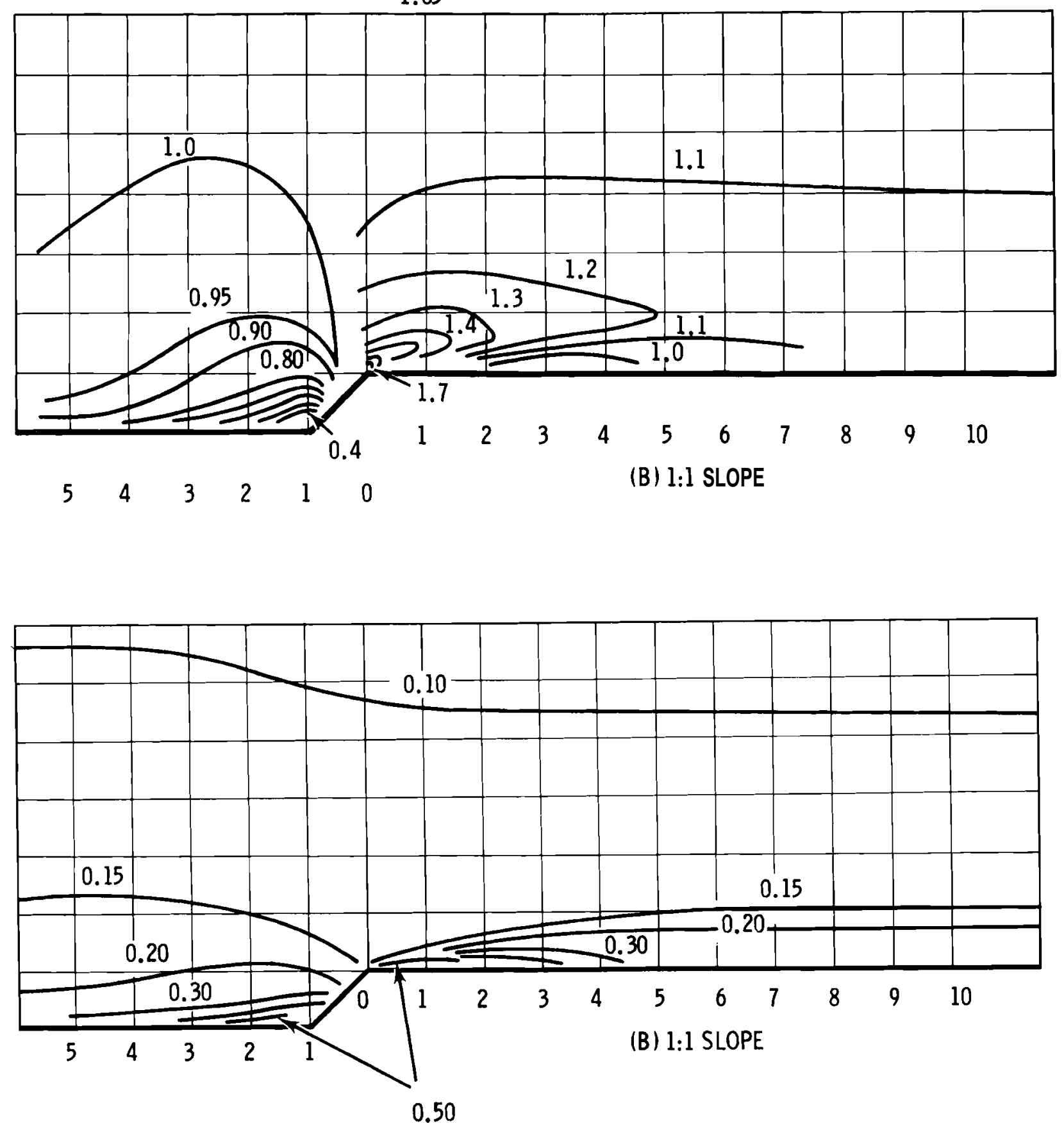

FIGURE 5.18. Contours of the Speedup of Upstream Winds (Upper) and Contours of the Turbulence Intensity (Lower) for Flow Over an Escarpment With 1:1 Slope (Bowen and Lindley 1977) 

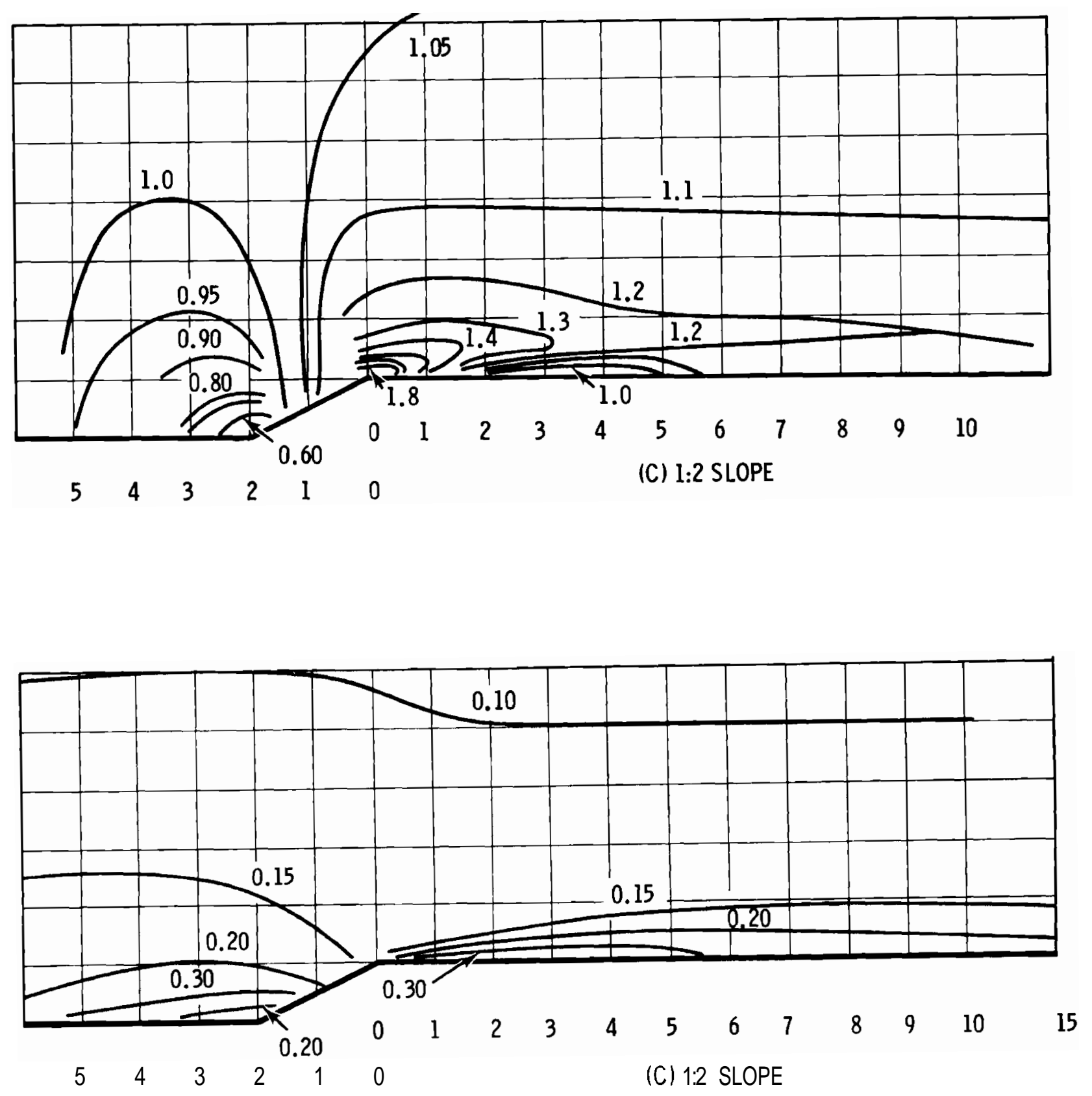

FIGURE 5.19. Contours of the Speedup of Upstream Winds (Upper) and Contours of the Turbulence Intensity (Lower) for an Escarpment With 1:2 Slope (Bowen and Lindley 1977) 

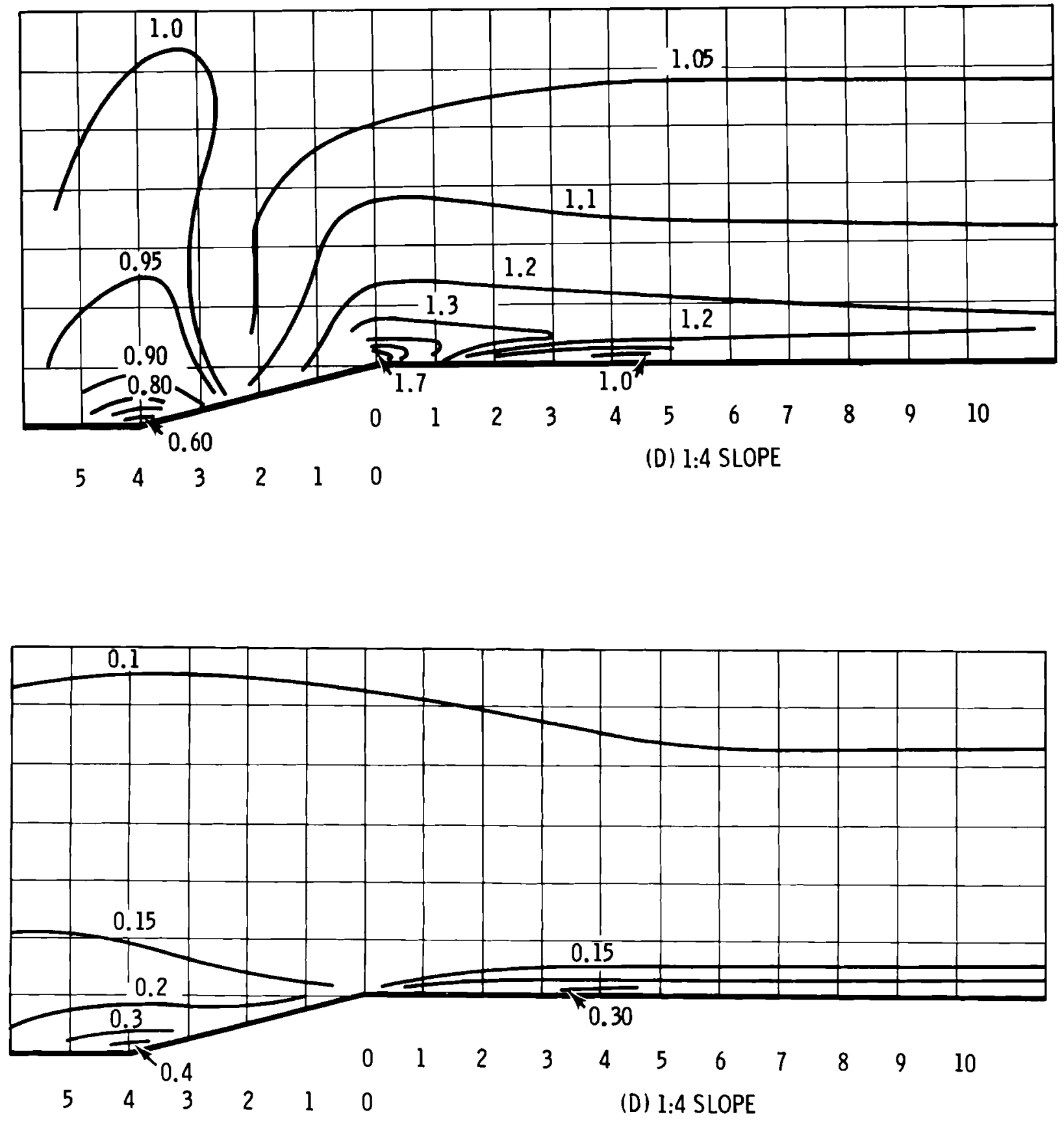

FIGURE 5.20. Contours of the Speedup of Upstream Winds (Upper) and Contours of the Turbulence Intensity (Lower) for an Escarpment With 1:4 Slope (Bowen and Lindley 1977) 
wind tunnel measurements (Bowen and Lindley 1977). The upstream wind profile follows a $1 / 6$ power law with the wind speed at the top of the boundary layer of $20 \mathrm{~m} / \mathrm{sec}$. The upstream turbulence intensity is 0.25 at $0.2 \mathrm{H}$ decreasing to 0.16 at $2 \mathrm{H}$, where $\mathrm{H}$ is the height of the escarpment.

The structure of the flow over each model is similar with major differences occurring only in the wake region near the ground and behind the crest. A blocking effect due to the escarpment is visible to at least $5 \mathrm{H}$ upstream. The maximum speedup factor of about 1.7 was observed for each model above the crest at $\mathrm{z} / \mathrm{H}=0.2$. The similarity of the vertical wind profiles at the crest of each escarpment is attributed to the flow separation upstream of the steeper slopes; the separation streamline that outlines the upstream eddy establishes an effectively smaller slope for the mean flow to respond to. Downstream from the crest in the wake region the effects of the escarpment slope are greater. The turbulent and high wind shear wake grows to a depth of $0.4 \mathrm{H}$ for the $1 / 4$ slope escarpment and grows to $1 H$ for the steeper slopes. Note that above the wake region the turbulence intensity is less than the freestream levels. For the $1 / 4$ slope everywhere downstream of the crest the turbulence intensity levels are smaller than upstream values. In the wake region the turbulence spectrum shifts toward higher frequencies because of the sma 11scale turbulence generated by the small terrain feature. The wake is insignificant at a downstream distance of about $10 \mathrm{H}$. The wind tunnel measurements show the enhancement of the winds of at least $10 \%$ occurs below $3 \mathrm{H}$ and extends downwind beyond $10 \mathrm{H}$; this has also been noted in field measurements by Bowen and Lindley (1974).

Stability and roughness effects on flow over escarpments are similar for cliffs and small ridges. The speedup factor is higher near the crest and near the ground simply because the initial wind shear of the upstream profile is greater over a rough surface. Of course, the total power in the wind is greatest over a smooth surface. However, rough surfaces cause somewhat deeper flow separation eddies and wakes, as does unstable stratification. Stable conditions inhibit flow separation, but the wake will not dissipate as quickly as it would if the stratification were unstable. 
Any concave curvature of a cliff facing upwind may be exploitable as with the small ridge. Winds oblique to the cliff may create directional wind shear and increase wind direction fluctuations. Flow along a cliff will probably produce very gusty wind behavior at the crest of the cliff. Flow down a cliff may be somewhat more variable than flow over flat land due to fluctuations in a separation eddy at the base of the cliff but offers no great hazards and no benefits to WECS.

Small Hills

Small hills are similar to small ridges but flow goes around as well as over them. Therefore, the speedup of the wind is less over a hill than over a ridge of the same slope. However, a hill is more exposed from all directions. If the prevailing winds are not confined narrowly to one direction, a hill will probably produce more power than a ridge of the same height in the same wind regime (Golding 1955).

Table 5.2 lists Frenkiel's criteria (see small ridges section) for hills. Frenkiel concludes, and wind tunnel studies support, that a slope of about $30 \%$ running to a pointed summit is optimum for a WECS placement at the summit.

TABLE 5.2. Frenkiel's WECS Site Suitability Based on Slope of $\mathrm{Hill}$ and Wind Gradient in Lowest 40 Meters

\begin{tabular}{|c|c|c|c|}
\hline & Slope \% & $\begin{array}{l}\text { Power Law } \\
\text { Index a }\end{array}$ & $\begin{array}{c}\text { Wind Speed Ratio } \\
U_{40 / U 10}\end{array}$ \\
\hline Optimum & 30 & $<0.03$ & $<1.05$ \\
\hline Very Good & 20 smooth & $<0.07$ & $1.05-1.10$ \\
\hline Good & $\begin{array}{l}10 \text { smooth } \\
20 \text { rough }\end{array}$ & $<0.10$ & $1.10-1.15$ \\
\hline Fair & $\begin{array}{l}5 \text { smooth } \\
20 \text { very rough } \\
<30 \text { with gradual slope } \\
\text { within } 50 \mathrm{~m} \text { of summit }\end{array}$ & $<0.14$ & $1.15<1.20$ \\
\hline Avoid & $\begin{array}{r}<5 \\
>50\end{array}$ & $<0.14$ & $>1.20$ \\
\hline
\end{tabular}


Since flow goes around a hill as well as over, especially in stable stratification, if there is a prevailing wind direction a WECS could be placed on the sides of the hill tangent to the prevailing wind direction. There is danger here that flow separation and lee wake effects could engulf a WECS if the flow were perpendicular to the prevailing direction. Flow separation may form on a flat-topped hill at the upwind edge of the top, at the summit of a sharp hill, or possibly slightly downwind of the summit of a round-topped hill The separation eddy extends farther downhill if the downwind face is steeper.

Like the small ridges, hills may be strongly affected by neighboring hills, especially in a stable PBL or on a hill close to and upwind of a large hill or ridge.

\subsubsection{Flow Over Depressions}

A depression is one in which the surface of interest is lower than the surrounding region. Channeling of the flow by the surrounding topography is the characteristic terrain-flow interaction. The depth of a small depression is approximately less than or equal to the height of the large WES rotors. Therefore a large WES placed in a small depression would experience the winds through the entire vertical extent of the depression channel and some at the height of the surrounding ridge or plateau. We again distinguish between long features such as small valleys and canyons from short features such as small basins and gaps.

\section{Small Valleys and Canyons}

The airflow over small valleys and canyons depends upon the orientation to the wind, stability, irregularities in width, steepness of the walls, roughness, and slope of the valley floor.

Air that is channeled along and up a small valley or canyon will feel a favorable pressure gradient due to the narrowing of the valley walls and the slope of the valley floor. Whether the flow accelerates or not depends upon the rate of production of turbulence along the valley walls. Turbulence extracts mean flow momentum and tends to balance the effects of the pressure 
gradients. For short narrow valleys, unless the valley walls are very jagged causing large regions of separated flow and wakes, increased turbulence production will not significantly affect the momentum balance. Flow will then accelerate through and (unless the PBL is very stably stratified) over the constriction. Therefore there can be some local enhancement of valley bottom winds. However, in virtually all cases of winds through small valleys, the winds will be greatest along the neighboring ridges or mesas.

Flow at any angle from the axis of the valley will turn slightly along the axis of the valley at the bottom but return to its original course on the downwind side. Depending on the ridge shape, roughness, and stability, the flow may separate. If separation occurs, the valley bottoms will be embroiled in gusty lee wakes and there will be large wind shear at the separation streamline near the ridge or mesa level.

At night cool air slowly drains into and down small valleys. If the winds at night are strong and up-valley, they will overpower the downslope wind. The katabatic forces might still be present near the surface in this case. If so the wind would be directed up-valley with enhanced wind shear. If a valley bottom collects cool stagnating air, the valley bottoms would be very calm while above the rims at ridge level or mesa level the winds may be much stronger. Therefore, large nocturnal wind shears might be expected in shallow valleys.

In summary, there is no reason to site large WES in small valleys or canyons. Wind shear or turbulence over a rotor disk could be great and the total annual wind power in the valley will be less than that over the surrounding ridges or mesas.

$\underline{\text { Small Basins }}$

Basins are low depressions like valleys but with no dominant axis. They are more nearly circular. An air column flowing over a small basin will stretch, causing deceleration; this is just the opposite of the acceleration that occurs over elevated features. Small basins are therefore regions of 
reduced wind resource. At night they can collect a very stagnant pool of cool air. A large WECS placed in a basin will experience considerable wind shear and no flow enhancement, similar to a site in a small valley.

\section{Small Gaps}

A gap in a small ridge will have its base at a level above the foot of the ridge, or a gap may be a narrow separation between two distinct small ridges with its base at the same level as the base of the ridges. Winds can sometimes be quite strong in a gap. Gaps provide a way for flow impinging upon a ridge to get through the barrier without going over it. There are times that gaps can enhance even light winds. For example, in fairly calm conditions and when cool air is pooling behind a ridge, the air will drain out through the gap. Small-scale gaps, however, are narrow. Therefore turbulence and vortex shedding from the nearby gap walls will make small gaps unattractive as large WECS sites unless the wind continually drains directly through the gap. Since we are talking about small-scale features that have no climatological impact on the large-scale flow, this situation probably does not exist.

\subsection{ROW OVR LARGE-SCALE TERRAIN FEATURES}

Here we describe the effects that large-scale terrain features have on a flow. A large scale-feature has vertical dimensions that are a significant fraction of or exceed the depth of the PBL. The elevated large-scale features that primarily block or divert the flow include mountains, ridges, high passes, large escarpments, mesas, and buttes. Large-scale depressions include deep valleys, canyons, gorges (a gorge is a deep cut extending through either a large ridge or an entire range of mountains), and basins. As with the smal1scale features, the flow properties are different depending on whether or not the features have a long axis.

Large-scale features will affect large volumes of air flow. Any enhancement of winds is likely to occur over a large region capable of accomodating numerous large WECS and therefore represents a significant contribution of total energy. The discussions of flow over small-scale features may assist in 
siting one or a few machines in a given WECS cluster site. Large-scale features may be used in selecting sites where large groups of machines may be located. However, when siting individual machines on a large-scale feature, small-scale features that are a part of but dwarfed by the larger feature must also be considered.

In analyzing the flow over a large-scale feature the structure of the flow above the PBL is important. Unlike the case of flow over a small-scale feature, we cannot use the concept of frozen turbulence being advected over the feature. Production of turbulence kinetic energy, its transport, and its dissipation significantly affect the momentum budget within the PBL.

Defining the PBL is a significant problem. For example, consider an offshore PBL: There is a well-mixed layer of nearly neutral stability $1 \mathrm{~km}$ deep. Stratus clouds form under and are confined by the temperature inversion that marks the top of the marine PBL. As onshore flow encounters coastal mountains, the clouds and the mixed-layer air follow the low gorges through the mountains; the mountain summits remain cloud free. Air that was formerly in the stable layer above the offshore PBL interacts with the seaward face of the mountain range and forms a turbulent mountain boundary layer. Since this portion of the mountain boundary layer retains the stable stratification of the offshore PBL, breaking Kelvin-Helmholtz waves and/or larger scale gravity waves aloft are present.

Air moving through different valley systems at different elevations may be of different potential temperatures. A deep trough gravity wave, which probably carries potentially warm air, can forcefully mix up several independent air currents and thereby form statically unstable columns of air. The resulting adjustment, where warm air rises and cool air sinks, causes unsteady and very turbulent winds.

The flow over large-scale features is very complex and extremely nonlinear. Our most sophisticated methods of simulating and interpreting flow in complex terrain are usually inadequate. Therefore the measurement program required to pinpoint and evaluate the wind resource must be of a larger space and time scale than that for small-scale features. 


\subsubsection{Flow Over Large-Scal e El evated Features}

In this section we discuss flow over large ridges and mountains, over large-scale cliffs, and flow through large passes. W consider large passes as elevated features rather than depressions primarily because the pass represents a potential energy barrier for the flow to cross.

\section{Large Mountains and Ridges}

Research and measurements over large-scale long ridges or mountains have not made a great deal of distinction between the two. Though they are considered together here, the more total obstruction of flow by long ridges may have greater effects.

For comparison and reference, recall the major effect of small-scale hills and ridges: Winds at the summits of small hills and ridges are stronger than those over flat land because the summits are higher in the air flow. Winds are enhanced by an acceleration that is dependent upon the surface geometry. The speedup is greater over a ridge of a given slope than over a hill of the same slope. The optimal slope of a smooth hill is about $30 \%$. The wind is also enhanced on the shoulders of ridges and the sides of hills. Leeslope wake regions experience gusty turbulent winds and may be in flow separation zones.

The different effects of large ridges and mountains can be illustrated by the experience of the Smith-Putnam project (Putnam 1948). This team sited a large wind turbine on Grandpa's Knob in Vermont but the site produced less than $30 \%$ of the power they predicted it would. Putnam concludes "We have found no criteria by which to make an economically useful quantitative prediction of the effects of topography upon wind flow." A decade later Golding (1955) concurred with Putnam. Putnam's statement is still basically true today.

The Putnam team (hereafter referred to as Putnam) first assumed that the wind increases with height in mountainous country. (a) Putnam estimated the annual average free-stream winds at the level of Grandpa's Knob, that is

(a) should recall here that the power in the wind depends on density. A WBCS at the $750 \mathrm{mb}$ level will only intercept roughly $75 \%$ of the power as will a WECS in an identical wind speed resource at $1000 \mathrm{mb}$. 
upstream winds undisturbed by the presence of the Knob, to be $24 \mathrm{mph}$. Grandpa's Knob is a $2000-\mathrm{ft}$ summit on the windward side of the roughly $4000-\mathrm{ft}$ Green Mountains. Later analysis estimated that the free-stream winds at the summit level of Grandpa's Knob were actually only $19 \mathrm{mph}$. Putnam concluded that the Green Mountains influence the wind field well to windward of the crest of the range. This influence is manifested in a partial blocking of the flow with subsequent diversion of flow around the north and south ends of the range as well as over the crest.

If we accept that the free-stream winds at the 2000-ft level are retarded by a very large-scale blocking effect of the Green Mountain range, what about the effect of Grandpa's Knob? Do the free-stream winds (here free-stream winds are those unaffected by Grandpa's Knob but not necessarily unaffected by the Green Mountains) accelerate over the summit? They would accelerate over a well-shaped small-scale feature due to compression of streamlines. Putnam found that the hub-height mean wind speed was actually about $17 \mathrm{mph}$. If the $19 \mathrm{mph}$ free-stream wind speed estimate is accepted, we must conclude that Grandpa's Knob on the average actually retarded the free stream flow over the summit by $10 \%$. Putnam expected a speed-up of $20 \%$. The $19 \mathrm{mph}$ estimate could be in error; however, ifit is too low we must conclude that Grandpa's Knob slows the free stream down even more than $10 \%$, and if it is too high the very large-scale blocking effect of the Green Mountains must be correspondingly larger.

If Grandpa's Knob retards the flow over it, the retardation must operate generally on a vertical scale deeper than the height of a large WECS. The slopes near the summit of the knob are about $30 \%$ or nearly ideal by Frenkiel's criteria. The ratio of the winds between $140 \mathrm{ft}$ and $50 \mathrm{ft}$ is 1.08, which gives a power law coefficient of $a=0.07$. This agrees fairly well with Frenkiel's criteria for flow over hills and so is an affirmation of his conclusion that it is the local near-summit topography that determines the shape of the near-surface velocity gradient. Table 5.2 then may be used as an aid in finding a fairly constant with height wind profile. However, speed-up or retardation of free-stream velocity over large-scale mountains or ridges occurs on scales that are large compared to a WECS, difficult to measure, and nearly impossible to predict. 
Wahl (1966) also studied the relation between free-stream winds, mountainsummit winds, and valley-station winds. His studies were carried out in Europe. Wahl correlated, as well as possible, wind speed data (5 minutes) from six mountain summits in Germany with rawinsonde data (2 minutes or 500 vertical meters) for four lower-level stations. The rawinsonde stations were within 20 to $160 \mathrm{~km}$ of the mountains. Wahl found:

1. About $50 \%$ of the variance of summit winds can be explained by the simultaneously observed wind speeds at the same level in the free atmosphere. Only $15 \%$ of the variance can be explained by the simultaneously observed winds observed at lower levels in the valleys.

2. For a given wind speed in the free atmosphere, the average of the corresponding summit winds is highest in winter and lowest in summer, and in between for spring and fall.

3. Summit wind speeds have small diurnal variations, but this may vary somewhat with location of mountain and time of year.

4. Correlation in wind direction between summit and free air flow is specific to a given mountain.

Wahl developed a statistical relationship between the mean winds of a "typical" mountain summit and the free atmosphere flow at the same level as shown in Figure 5.21. His results suggest that the higher summit wind speeds tend to average about half of the free atmosphere speeds. This suggests that the expected narrowing of stream lines over large-scale obstacles and the resulting acceleration are overcompensated by drag effects. However, at low wind speeds a mountain creates its own circulation system since summit winds tend to exceed those of the free air. Wahl also notes that even when the free atmosphere is calm, substantial winds may be observed at a summit.

Putnam's and Wahl's evidence suggests that large-scale ridges and mountains may actually retard the flow over their summits when compared to the free stream flow at the same level. This may not be universally true, but even if it were, it is not a reason to disqualify mountains and ridges as WECS 


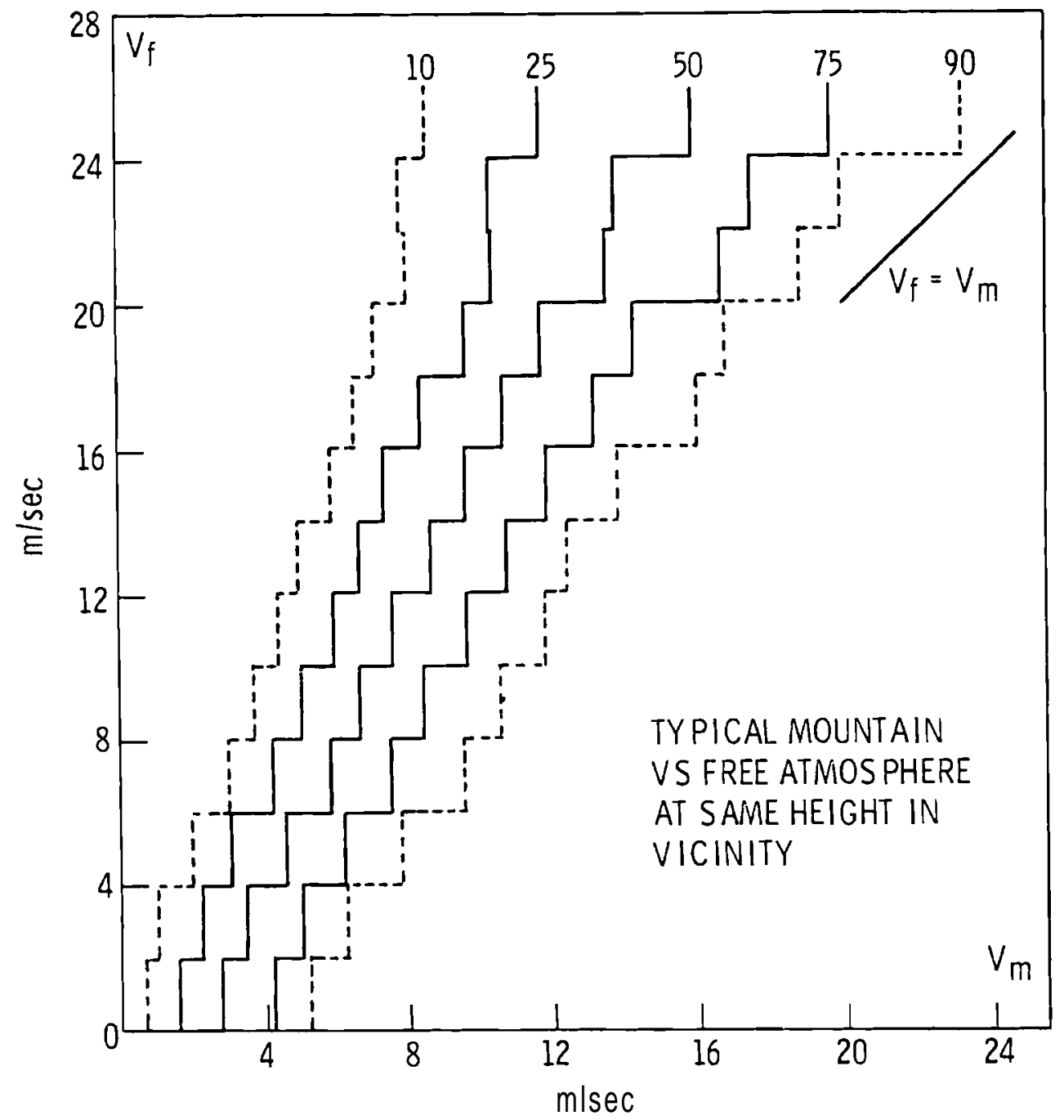

FIGURE 5.21. Windspeed $V_{m}$ (as Measured at a Mountain Observatory) Versus $V_{f}$ (Estimated for the Same Level in the Free Atmosphere, From Rawin Soundings in the Vicinity of the Peak). Climatic mean frequency distributions for $V_{f}$-classes (in $2 \mathrm{~m} / \mathrm{sec}$ steps) are indicated by lines numbered 10 and 90 for the lowest and highest decile, 25 and 75 for lower and higher quartile, and 50 for median value. For example, a speed of $V_{f}=16 \mathrm{mps}$ produces a mountain speed of $V_{m} \leq 10 \mathrm{mps}, 50 \%$ of the time

sites. Mountains and ridges still experience higher winds than lower areas; even with 17-mph average winds Grandpa's Knob is an adequate wind site provided that wind turbines are reasonably well suited to the resource. The SmithPutnam project did not adequately measure the resource before the project was committed to the site. 
A number of complex physical mechanisms are activated when flow interacts with large-scale mountains and ridges, such as blocking and channeling, gravity waves, thermal forcing and turbulence. These mechanisms are discussed below.

- Blocking and Channeling

When flow impinges upon an obstacle, air is diverted around and over it. Some portions of the air stream may not have sufficient energy to pass over the obstacle; as a result, some air may stagnate upstream of the obstacle until it receives a more energetic push. This is blocking. The air that is blocked by a ridge will move along the ridge in response to the pressure gradient parallel to the ridge. This is channeling.

The amounts of air that go around and over the obstacle depend upon the wind and temperature structure of the impinging flow. Consider the following simplistic example of flow over a long ridge normal to the direction of flow: Assume that at some level above the ridge crest, the disturbance of the ridge is not felt and the streamlines at this level are horizontal. In this instance, the height of a column of air crossing the ridge is reduced over the crest, and the conservation of mass requires that the air speedup over the ridge and return to its original speed on the lee side. Since a stably stratified flow resists vertical displacements one could argue that the level of no disturbance, or influence height, is less in the more stably stratified flow (Bavidson 1964). The speedup at the ridge crest would therefore be greater. If, however, the atmosphere is too stable, the kinetic energy of the lower level flow may be insufficient to carry the flow to a higher potential energy state at the ridge crest, and may totally block the lower level. Therefore less air passes between the ridge crest and the influence height and less speedup occurs.

The degree of imbalance between the kinetic energy and the potential energy of the lower layer (e.g., the lower layer could be the mixed layer, or some other layer over which a suitable average may be taken) of a flow is expressed in terms of an internal Froude number (F), one form of which is defined as a ratio of inertia and gravity forces:

$$
F=\frac{U}{\left[\frac{\Delta \theta}{\theta} g h\right]^{1 / 2}}
$$


where

$$
\begin{aligned}
U= & \text { wind speed of lower layer } \\
\mathrm{h}= & \text { depth of lower layer } \\
\mathrm{g}= & \text { acceleration of gravity } \\
\theta= & \text { average potential temperature of lower layer } \\
\Delta \theta= & \text { the difference in average potential temperature of the layer above } \\
& \text { the lower layer but beneath the influence height, and the average } \\
& \text { potential temperature of the lower layer. }
\end{aligned}
$$

If the kinetic energy of the low-level winds upwind of a peak is small (the Froude number is small) a parcel of air may arrive at the summit with much of its kinetic energy exchanged for potential energy. If the low-level kinetic energy is too small, a ridge will totally block passage of low-level air over the summit. Perhaps then air from a level midway up the ridge will pile up against the windward side of the ridge and spill over the backside like water over a dam. The pileup and consequent slowdown of wind speed extends upstream of the peak, but how far is difficult to guess.

The flow normal to the ridge determines whether the flow will cross the ridge or be channeled. If the flow is oblique to the ridge but with insufficient kinetic energy normal to the ridge, the flow will be blocked and will be channeled along parallel to the topography.

- Gravity Waves

Mountains excite gravity waves in the stable flow above the PBL (see Figure 5.22). The gravity waves above the ridge or peak transport and disperse energy of the surface disturbance to high levels. Smith (1978) concludes that the gravity wave mechanism is probably the major source of atmospheric drag over the Blue Ridge of West Virginia.

It is possible that the significant disturbance of the peak may be limited to confluence of air on the downwind side of the peak as air that is forced over the top accelerates downward to combine with streams flowing around the peak; the resul-t is a wind speed maximum and high wind variability on the lee slope of the peak. 


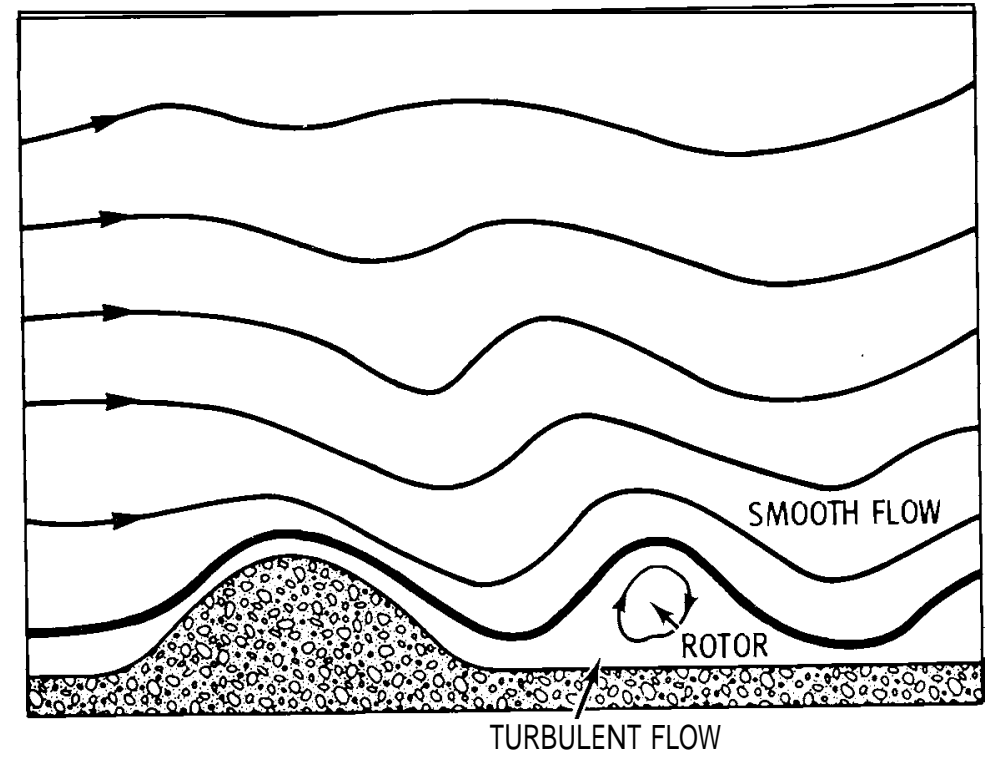

FIGURE 5.22. Gravity Waves Forced by Flow Over a Ridge

Under some conditions gravity wave disturbances will propagate for long distances downwind of the peak as lee waves. For lee wave trains to form downstream of the peak, the air flow must consist of at least two layers with stable flow forming either the lowest layer or sandwiched between two lessstable layers (Scorer 1949). If the stability, the wind shear, or wind direction of the upstream flow is even slightly variable, the wind and turbulence at a point on the lee slope can vary as the wavelengths or amplitudes of the lee waves change.

Gravity waves above and downstream of mountains are often made visible when moist air, rising and cooling, condenses to form clouds. Wave clouds are Ienticular, have a very smooth appearance as opposed to the fluffy look of cumulus clouds or the ragged look of high cirrus clouds, and sunlight shining through the wave cloud often creates a diffuse spectrum of colors. Wave clouds may be seen at the crest of each wave in the wave train. The lee waves may exist in turbulent layers nearer the ground, in which case the cloud will appear more like a low-level cumulus cloud. However, the observer will recognize it as a wave cloud because portions of the cloud will appear to be moving 
very fast, with the mean wind; however, the wave cloud itself retains its position relative to the ground determined by the crest of the wave. Sometimes the wave will be of sufficient amplitude to form a turbulent rotor (see Figure 5.22) that will sit in one place and spin. This may be made visible by a very turbulent cloud.

Topography generates a spectrum of lee waves; the more asymmetrical the mountain or ridge is, the broader the band of wavelengths it excites. Asymmetry also affects the phase of the lee waves; if the lee slope is steepest, the crest of the first lee wave will be nearer the summit than if the windward slope is steepest. In very rugged terrain each peak and ridge generates waves that will combine to form a wave pattern that is fixed with respect to the topography and show little variation in the position of wave crests as flow thermal structure changes; amplitudes, however, may change. If wind direction changes, the wave pattern will change since the streamwise topography is different.

Strong wind events associated with gravity wave mechanisms sometimes occur in the lee of mountain ranges. For example, just east of the Front Range of the Rocky Mountains, near Boulder, Colorado, surface wind events occur that have gusts exceeding $45 \mathrm{~m} / \mathrm{sec}$ (Brinkman 1973, Scorer and Klieforth 1959). These events can happen when a strong wind exists in a stable layer at mountain-top level and when the upper troposphere is less windy and less stable. In this case the flow upwind of the summit is partially blocked and the amplitude of the lee trough (see Figure 5.22) becomes very great with correspondingly strong surface winds at the base of the trough.

Another mechanism that causes strong lee slope wind behavior is analogous to the hydraulic jump of fluid dynamics. In the atmosphere this is frequently referred to as a pressure jump since the passage of the jump causes sharp changes in the pressure. In the atmosphere the hydraulic jump consists of a shallow, fast-moving (shooting flow) layer of dense air upstream of the jump; downstream of the jump the layer abruptly becomes thick and slow-moving (tranquil flow). Ball (1956) applied the theory of hydraulic jumps to observations of katabatic winds in Antarctica and noted quick changes from violence to calm 
as the pressure jump moved upwind in response to changing conditions upwind. He also reported that blowing snow and the roar of the wind just upslope from the jump were observable from the tranquil flow sector.

To apply hydraulic jump theory to the atmosphere also requires assumptions about layered structure. Usually, the PBL beneath the capping temperature inversion is considered to be the dense lower layer. If the flow accelerating down the slope reaches a point where the Froude number, $F$, is greater than one, the advection speed of long gravity waves represented by the numerator of $F$ (Equation 5.6) exceeds the gravity wave propagation speed represented by the denominator of $F$. Where this occurs mass builds up near the jump and kinetic energy of the upstream flow, represented by the numerator of $F$, is transformed into potential energy of the downstream flow shown in the denominator of F. Turbulence kinetic energy is produced at the jump, some of which is radiated away in gravity waves downstream of the jump where $F<1$. The location where the Froude number becomes critical is very sensitive to upstream velocity and temperature structure as well as the topography since slope controls the downward acceleration of dense air. As the upstream velocity and temperature structure changes, the location of the jump changes resulting in I ulls and violent periods at a point.

- Thermal Effects

Thermal effects of large mountains and ridges can modify the flow also. Large mountains and ridges heated by solar radiation, particularly in the warmer months, can become elevated heat sources. Low-lying surface air near the mountain slopes is forced to move upslope (anabatic wind). Thermals and cumulus clouds may form above the summit. This upslope convection favors the development of a large separation eddy to the lee of the mountain.

Mountains can be cold sources as they radiate energy away, at night or from snow and icefields. The downslope katabatic winds that form suppress flow separation. Lee waves, which often accompany katabatic forcing, and down-drafts accompanying heavy rain also suppress flow separation (Scorer 1955). Katabatic winds also can fill the valley bottoms surrounding a peak 
with cold stagnant air. Mountain summits then are a more continuous source of wind power than valleys or bases of mountains that can suffer from stagnation.

Isolated peaks that are not as tall as the daytime thermal boundary layer (mixed layer, see Appendix 2) is deep frequently experience nocturnal wind speed maxima. During the day, the boundary layer is deeper than the peak is tall. If the boundary layer is well-mixed, the mixed layer wind speed will be less than the geostrophic wind speed above the top of the PBL. At night the depth of the thermal boundary layer over the surrounding lower elevation terrain is less than the height of the summit. The summit then experiences the geostrophic winds in a stably stratified flow accelerated (or retarded depending on the specific geometry and flow conditions) over the peak.

- Turbulence

The behavior of turbulence has more impact on the acceleration of flow over a large-scale feature than it does for flow over a small-scale feature. The flow over a large feature is of a sufficiently long time scale that turbulence is not simply advected, without impact, over the terrain. There is time for turbulence to respond to the feature and to interact with the mean flow. An increase of turbulence intensity over the mountain signifies that momentum is being extracted from the mean flow and dissipated in the near-surface shear layers. This is a more important momentum sink if the flow is not blocked and thereby separated from the lower slopes of the ridge or mountain.

Putnam noted reverse flagging of trees (see Chapter 6 for discussion of flagged trees) on the lee sides of some peaks in the Green Mountains. This not only indicates the presence of persistent flow separation eddies but also indicates that those eddies are quite strong.

Large Cliffs

Large-scale cliffs may be found on coastlines or as a face of a mesa or butte. Coastal cliffs and mesas can be considered long two-dimensional features, but a coastal promontory or butte is considered short.

There are few appropriate measurements over these kinds of features. For flow normal to long cliffs there must be acceleration over the crest, but the 
details of the shape or extent of the speedup are not now known. It is quite likely that a large separation eddy will form at the bottom of the cliff. If the winds are gusty or if the cliff is being heated by the sun, this eddy may sometimes shed vorticity into the flow to be carried up and over the cliff. One should also expect, depending on the cliff profile, flow separation somewhere along the top edge of the cliff. How deep this eddy is will depend in part upon the stability and the nature of the flow separation eddy at the base of the cliff. Until a wider body of knowledge is available, it is assumed that there is a good chance of strong turbulence at the levels seen by large WECS within $1 \mathrm{~km}$ of the edge of a large cliff. Beyond this distance, the advantage of the cliff effect may be lost because the entire boundary layer will adjust to the presence of the cliff. Measurements should be used to evaluate the quality of the resource.

The same comments apply to promontories and buttes. However, the flow separation eddies at the bases of these features may shed around them and not over. The wakes of buttes will be especially turbulent and must be avoided. Since some flow is diverted around as well as over, the flow separation eddy at the upwind edge of the cliff crest may not be as severe as for a long cliff. In essence then, these features' greatest advantage is that they serve as tall towers for a large WECS, but they do not provide much dynamic enhancement of wind speed. A butte may be especially helpful if located in an area where winds are already enhanced by valley funneling or some other large-scale enhancement.

\section{$\underline{\text { Passes }}$}

Passes are notches across a mountain barrier or between mountains. A pass can be considered as an elevated feature since the base of a pass is substantially elevated above the approaching valleys and $i t$ represents a potential energy barrier. The crest of a pass may be as narrow as several hundred meters in rugged terrain (if smaller it should be considered a small-scale gap) up to some tens of kilometers wide. The length scales through the pass have similar ranges. 
Passes are potentially good wind energy sites. If a pass is open to the wind, air will be forced over the crest, around the adjacent peaks, and through the pass. The confluence can result in an acceleration through the pass. When cold air is blocked behind ridges it will rush through the pass like water through a hole in a dam. The particular characteristics of a particular pass and the free stream flow will combine to produce its own unique wind system.

A narrow pass, for example that of Figure 5.23, will produce a core of enhanced winds. The drag of the floor and nearby walls of the pass (especially so in a V-shaped pass, less so in a U-shaped pass) may cause the jet to be located above or near the top of the rotor of a large WECS. The WECS might then be in a very turbulent and sheared environment. However, if the approach to the crest of the pass is smooth and of the right slope, local low-level acceleration may reduce the wind shear somewhat. Therefore, Frenkiel's criteria are probably applicable to passes.

An actual core of high winds that does not fill the entire pass might be found in a medium-sized pass; see Figure 5.23. This might occur, for example, if the approaching valley turns sharply near the pass. Inertia of the flow prevents the winds from following the exact center of the channel. If the jet piles into the walls of the pass a significant amount of turbulence could be produced.

In very large passes, the wind will generally be enhanced, provided of course that the pass opens to the prevailing wind. Beyond this the pass might behave much like nearly flat terrain. An analysis of the small-scale features within the pass will be necessary to locate the wind maxima, turbulence characteristics, and any diurnal effects.

\subsubsection{Large-Scale Depressions}

Valleys and Canyons

Valleys and canyons are generally of two types: V-shaped because of stream erosion or U-shaped from glacial erosion. Combinations of the two also exist. U-shaped valleys will accomodate more large wind machines per area than a V-shaped valley since more machines can be placed at safe distances from potentially very turbulent flow near valley walls. 


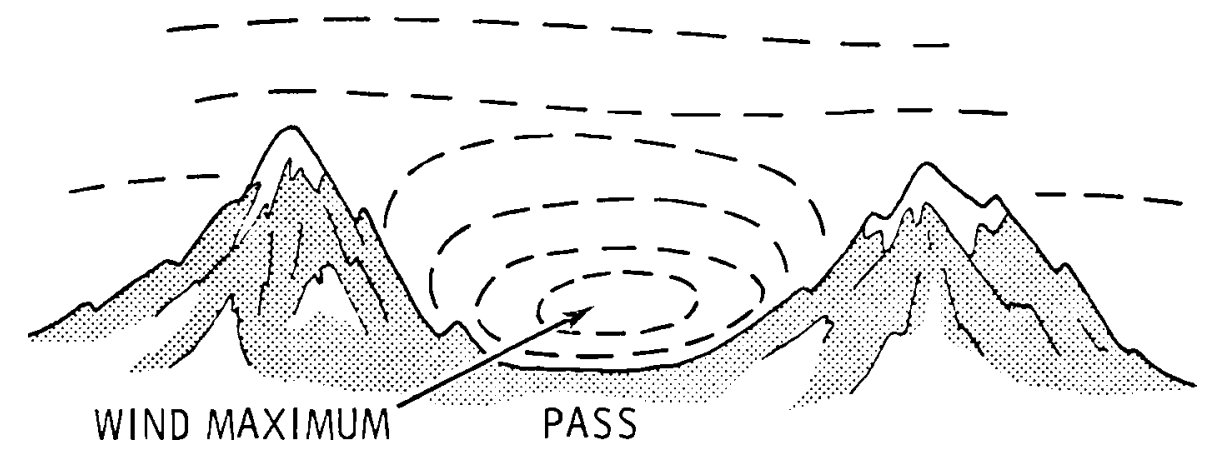

(a)

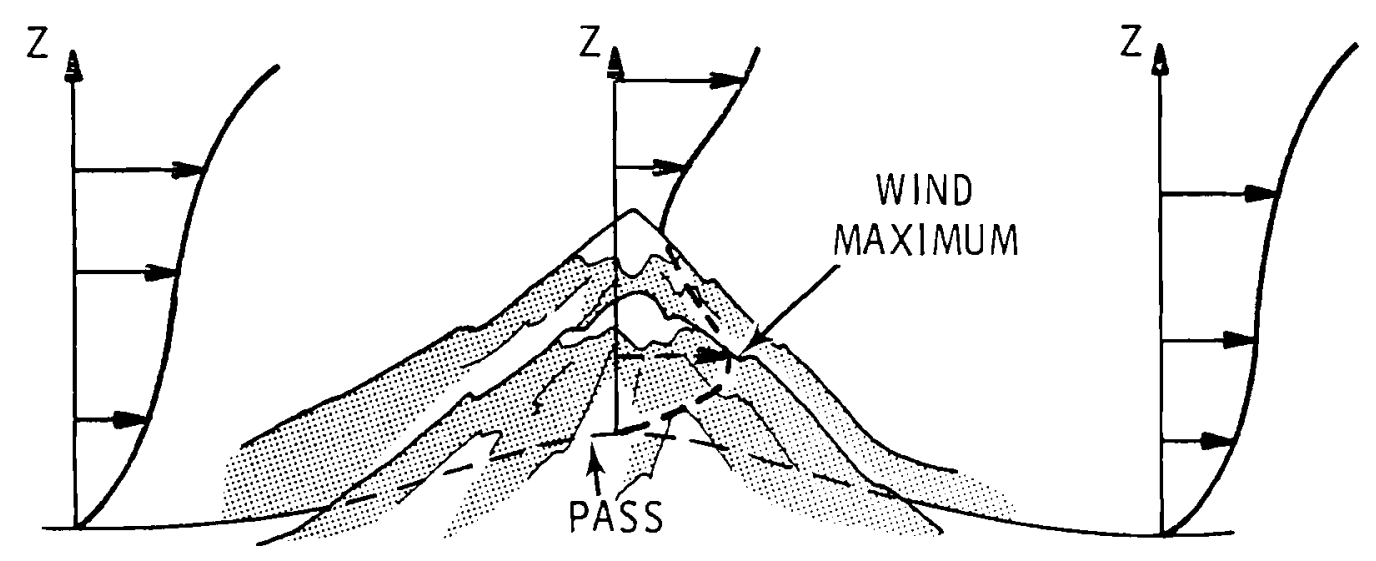

(b)

\section{FIGURE 5.23. A Schematic of the Wind Pattern and Velocity} Profile Through a Mountain Pass

Four basic types of flow patterns occur in valley systems. The first is known as a mountain-valley wind and has been studied extensively. This is an anabatic wind where the winds are channeled by the valley. In broad valleys distant from mountainous terrain these winds may be seen in climatological records; however, larger circulations only occur where there is substantial vertical relief. The mountain-valley winds occur when the large-scale winds over the area are weak and the daily heating/cooling cycle dominates. This would happen most in warmer mont'hs, May to September, and when a high-pressure system with clear skies is present. 
The daily sequence of mountain-valley winds is shown in Figure 5.24. By sunrise cold air has filled a portion of the valley and a strong temperature inversion exists. At the surface there is a wind from the mountain. Shortly after sunrise the valley slopes warm. Upslope winds (white arrows) and the continuation of the mountain winds (black arrows) combine (Part A). By forenoon (Part B) the nocturnal temperature inversion has been obliterated and the upslope winds on the valley walls are strong. The up-valley winds begin. By early afternoon (Part C) the valley wind is fully developed. By late afternoon (Part D) the slope winds have ceased and the winds are strictly up-valley. The valley is still warm. Shortly after sunset the ridges are cooling (Part E). Downslope winds begin but the winds at the bottom of the valley are still upslope. By early night (Part F) the downslope winds are well developed and the valley wind is shut off. In the middle of the night the valley bottom is cold (Part $G$ ) and the mountain winds begin. During the late night (Part H) the mountain winds fill the valley with cold air once again.

The mountain wind is part of a stable drainage flow, but the shape of the wind profile is difficult to predict. There is a trade-off between maximum acceleration of the coldest, densest air flowing nearest the surface and the effects of surface friction. A velocity maximum at some level above the surface could therefore be expected, depending on the depth of the drainage flow. The depth of the mountain wind will depend upon the shape of the valley system and the surrounding mountains (since the question is basically how much air can be cooled and how big a container the valley is). Similarly, different tributaries of cold mountain air may arrive at a given location at different times. Mountain winds thus may be intermittent. The mountain wind accelerates down the valley. If in the downstream direction the valley slope lessens or if the source of cold air from the valley walls diminishes (as it would if the ridges diminish in elevation downstream) friction will reduce the speeds of the winds. Hence a velocity maximum part way down the valley is expected. Behind a constriction in the valley, cool air will pile up and slow down but the downwind side of the constriction may see very accelerated flow from the pressure head analogous to that at a hydroelectric facility. A mountain wind current will not necessarily be maximum at the bottom of the valley. It may be near a side if the current is negotiating a sharp turn in the valley. 


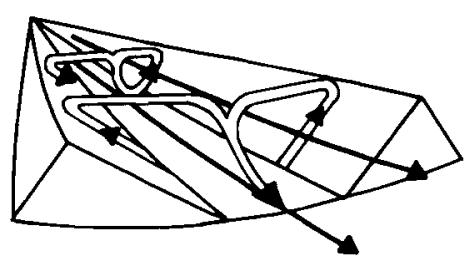

(A) SUNRISE (EARLY MORNING)

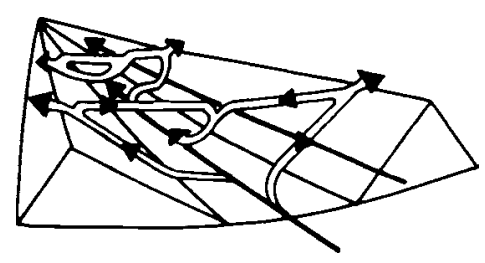

(C) EARLY AFTERNOON

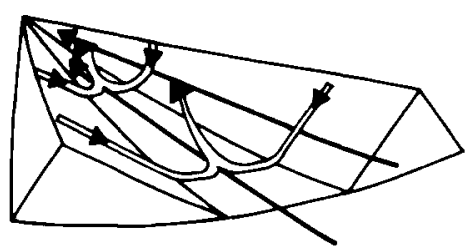

(E) EVENING (AFTER SUNSET)

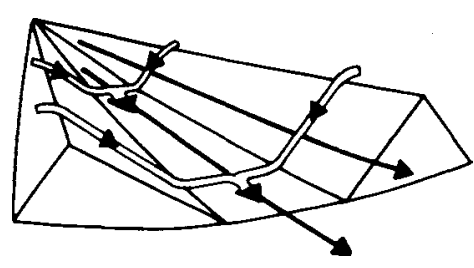

(G) MIDDLE OF NIGHT

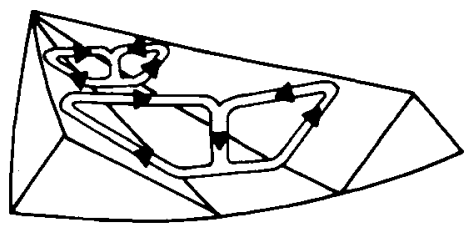

(B) FORENOON

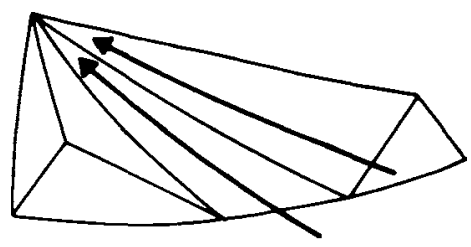

(D) LATE AFTERNOON

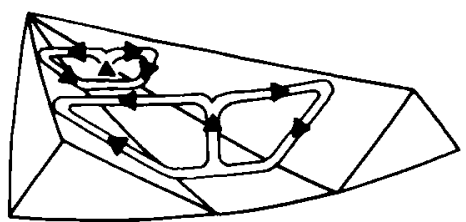

(F) EARLY NIGHT

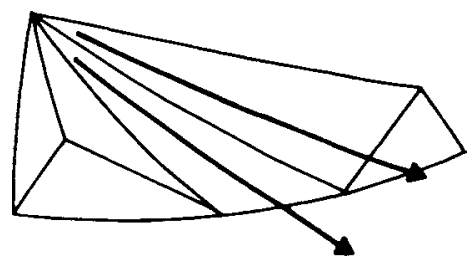

(H) LATE NI GHT

FIGURE 5.24. The Daily Sequence of Mountain and Valley Winds (Reprinted by Permission of the American Meteorological Society)

The daytime wind blowing up the valley tends to be more sensitive to factors such as heating by the sun (the driving force for this wind) and the winds blowing above the valley rim. As a result, the valley winds are more variable, and often weaker, than mountain winds. Valley winds are normally greatest along the side slope most directly facing the sun. Figure 5.25 shows potential WECS locations that take advantage of mountain and valley winds.

A second type of valley wind behaves very much like the mountain wind current but the initiating mechanism is different. This occurs if a large 


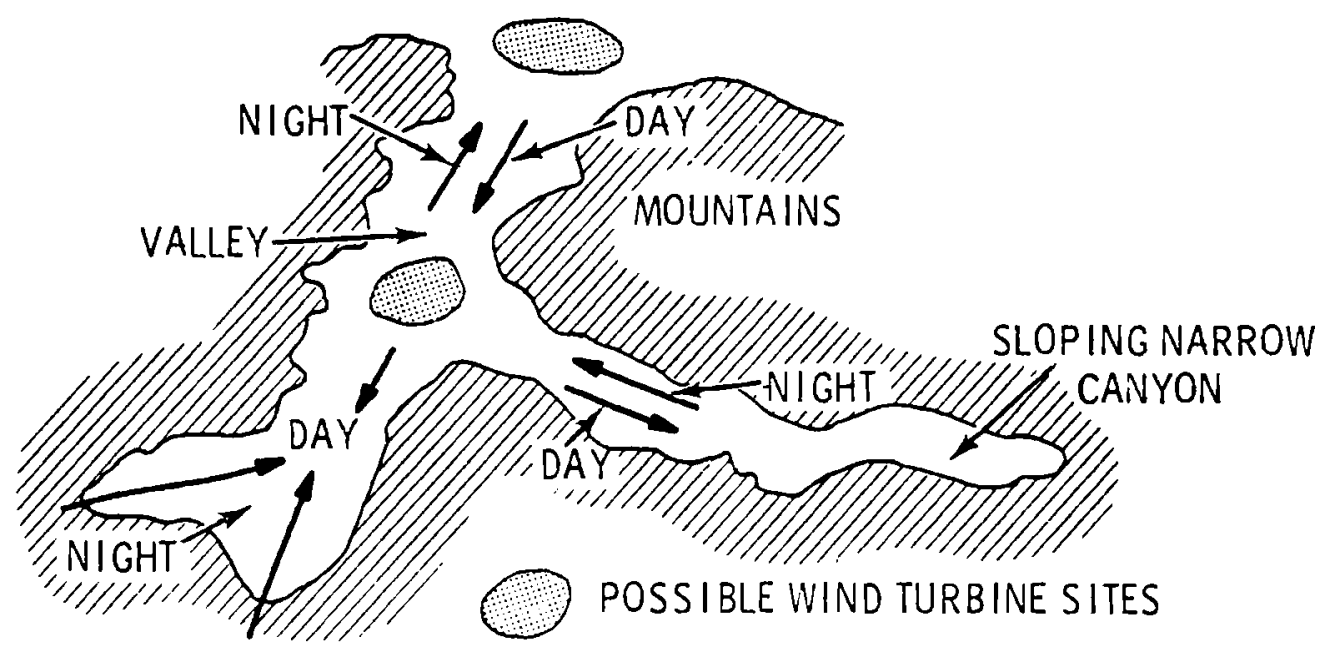

FIGURE 5.25. Possible WECS Sites in Sloping Valleys and Canyons Where Mountain/Valley Winds Develop

mass of cold air enters the valley, not by radiational cooling of the valley walls but from behind a pass near the head of the valley. This can occur along the coastal areas where the sea breeze pushes a cold air mass inland through passes in coastal mountains. It can also occur quite dramatically along the southeast Alaskan coast in winter and spring where cold continental air is separated from the warmer air of the Pacific by the coastal mountains, which are almost $6000 \mathrm{~m}$ high. This air is occasionally pushed over the high passes and cascades seaward maintaining its cold temperatures during its passage over the glacier fields. The winds at the coastline have exceeded $50 \mathrm{~m} / \mathrm{sec}$. Katabatic winds such as these may pass through a hydraulic pressure jump. The winds at such a point can go suddenly from mild to violent to mild again.

The third type of flow pattern, which is probably the most important, occurs when moderate-to-strong winds are parallel to (or within about $35^{\circ}$ of) the valley axis. In this case, broad valleys surrounded by mountains can effectively channel and accelerate the large-scale wind.

Figure 5.26 shows possible wind sites where valley channeling enhances the wind flow. Part (a) presents a funnel-shaped valley on the windward side of a mountain range. The constriction (or narrowing) near the mouth produces 


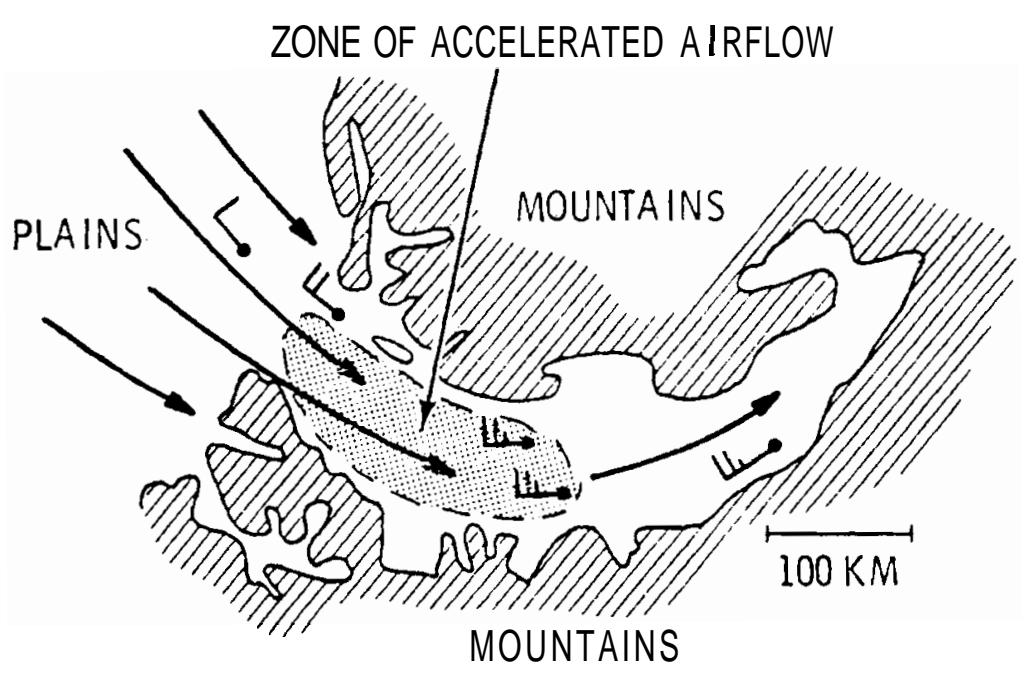

(a)

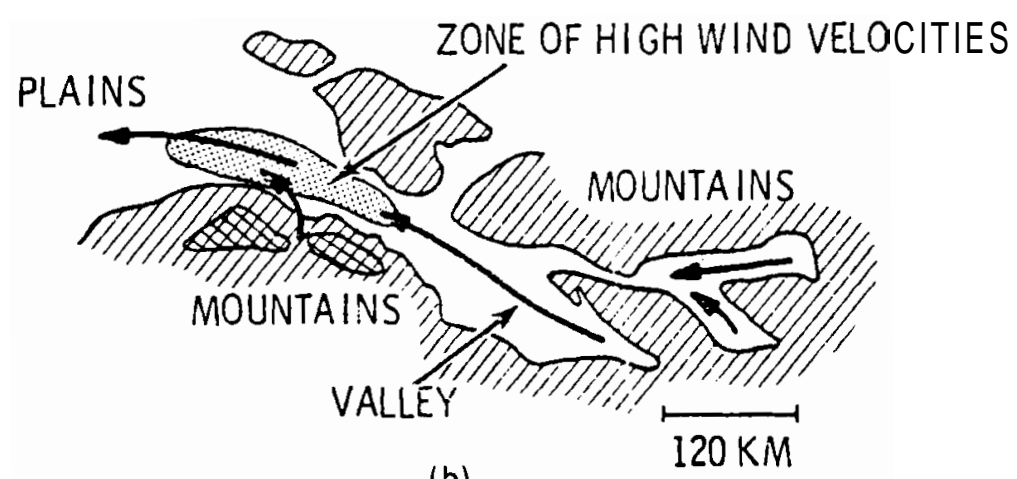

(b)

FIGURE 5.26. Possible WECS Sites Where Prevailing Winds Are Channeled by Valleys

a zone of accelerated flow. In this example the valley is large and open to the wind. Part (b) shows a narrow valley in the lee of a mountain range. It is parallel to the wind and constricted slightly near its mouth.

A valley that is both parallel to the prevailing wind and experiences mountain/valley winds will provide sites that are dependable sources of power. Moderate-to-strong prevailing winds in winter and spring will drive the WECS. During the warmer months, mountain/valley winds, though they are weak, may be strong enough to be utilized. 
The fourth type of valley flow occurs when the wind is perpendicular to the valley (or crosses it at an angle greater than $35^{\circ}$ ). A turbulent valley eddy may be set up by a combination of solar heating and cross-valley winds. If the valley is narrow, the flow in the valley will be weak except for occasional gusts associated with lee effects of the ridge and downward momentum transport by the thermally driven valley eddy. If the valley is wide, the flow should be analyzed from the viewpoint of a large-scale ridge.

\section{Gorge}

A gorge, as considered here, is a very deep passage through large mountains or even an entire mountain range. It is very much like a pass except the pass has a crest, which is a potential energy barrier. It is very much like a valley except $i t$ is flat enough that slopewind forces are not as significant.

Because gorges are so deep, any pressure gradient across the mountain range can cause a great deal of flow enhancement through the gorge. Such pressure gradients can arise from winds being blocked by the ridges around the gorge. They may also come from temperature differences across the mountain range. This may be especially prevalent near coastal mountain ranges. For example, the Columbia River gorge east of Portland, Oregon gets strong winds from the onshore storm winds, the summer sea-breeze (cool air offshore), or winter high-pressure periods (cool air east of the gorge).

A favorable pressure gradient drives air into the mouth of a gorge. The air accelerates and turbulence production increases until finally equilibrium is nearly reached. Near the exit of a gorge the pressure gradient decreases, the flow spreads out and decelerates. Compared to those in a pass or a wide valley the levels of turbulence may be higher and more ubiquitous in a gorge. Otherwise siting considerations for a gorge are much like those for a pass or valley.

\section{Large Basins}

Large basins are depressions surrounded by higher terrain. They have no particular long axis, so unlike valleys, downslope wind is not channeled except by small-scale features. Like valleys, basins may have well-developed 
diurnal wind cycles during warmer months. Nighttime flow into a large basin (such as the Columbia Basin in southeastern Washington) may average 5 to $10 \mathrm{~m} / \mathrm{sec}$ for several hours during the night. Afternoon flow out of a basin is generally lighter and more variable, averaging 2 to $7 \mathrm{~m} / \mathrm{sec}$. This is because during the daytime upslope conditions the mixed layer is deep and coupled strongly to the large-scale winds over the basin.

Basins can, especially in winter, fill with cold air that stagnates. It then requires a vigorous storm to move the cold stagnant air out of the basin, or at least stir up the air in the basin enough that after the storm passes the winds blow until the cold air settles back to stagnation. Clearly this is not ideal for large WECS.

It is then only the very large shallow basins that are along principal storm tracks that could be of any use to wind power. The potential of such basins will show up clearly in a regional wind energy resource assessment (see Appendix 1). In that case the siting problem reduces to analysis of sma11scale features within the basin. If the basin is too small to show up on a regional resource analysis, it is probably too small and too subject to periods of stagnation to be of value for a large WECS cluster site.

\subsection{SUMMARY AND RECOMMENDATIONS}

There is very little quantitative understanding of the behavior of wind over terrain. Measurements are generally lacking and the scant theory avai 1able is therefore difficult to verify. Wave been able to make some general qualitative statements about flow over terrain. Such statements serve only as a guideline to assist in the placement of measurement platforms and in the interpretation of measurements and model results. The points noted below reiterate the highlights of topographical indicators.

Homogeneous Flat or Gently Sloping Terrain

$\mathrm{N}$ real terrain (except water surfaces) is flat at all scales of motion. Large-scale gentle slopes influence the entire PBL structure in a manner similar to that of an external parameter. Within the PBL the terrain may be considered 
flat if it is free enough of surface irregularities to establish the logarithmic wind profile. The criteria suggested by Frost (1979) are generally adequate. However, in stably stratified flow the effects of terrain irregularities may persist for long distances downstream and flow at a point may never achieve true steady state.

Given that the terrain may be considered flat, the most relevant siting consideration is that wind power is greater over smooth terrain than rough. Diurnal variations in stability cause $10-\mathrm{m}$ winds to be maximum in the daytime. Diurnal variations in stability and boundary layer depth make it difficult to predict the phase and amplitude of the diurnal modulation of wind speed at hub height. The nocturnal jet which has a theoretical basis over truly flat terrain can be enhanced over gently sloping terrain. The nocturnal jet is both a resource underestimated by surface wind speeds and a source of significant wind shear. Daytime upslope and nighttime downslope wind circulations are superimposed on the large-scale flows and are evident in climatology. The strength and depth of these circulations are difficult to predict.

\section{Flat Terrain With Obstacles}

The structure of wakes behind obstacles will depend upon obstacle geometry, roughness, stability and the interaction with other nearby wakes. Depending on the orientation of an obstacle to the flow, trailing longitudinal vortices are formed that impart unique characteristics to the wake. However, wake structure details need to be verified in the real atmosphere. Until more experience is obtained, we submit that a large wind turbine should avoid those regions defined as the classical momentum wake. As a rough guideline, momentum wakes extend roughly $20 \mathrm{H}$ downwind and grow to $3 \mathrm{H}$ in the vertical before dissipating. The meandering vortex wake, which extends further downstream than the momentum wake, could result in increased gustiness and wind direction fluctuations that may affect operations of WECS but not structural integrity.

Flat Terrain With Roughness Changes

The principles of the flow modifications from rough to smooth surfaces and vice-versa predict an internal boundary layer that grows in thickness as 
$\delta \sim x^{0.8}$. However, these theories were never intended to apply through layers as deep as the layers occupied by large wind turbines. It is more appropriate to think in terms of adjustment caused through the turnover of the largest eddies in the PBL. The magnitudes of mean wind shear across the rotor disk that could be encountered can be determined using Figure 5.1. In addition, the following points should be considered:

- For transitions from rough to smooth, the wind power is maximum in the fully adjusted smooth flow. Therefore, the WECS site should be at least $1 \mathrm{~km}$ into the smooth region.

- For transitions from smooth to rough, the wind power is maximum nearest the smooth flow. There may be enhanced turbulence or wind shear within $1 \mathrm{~km}$ of the transition.

- Roughness changes oriented obliquely to the wind can be a source of vertical shear of wind direction.

\section{Flat Terrain With Horizontal Temperature Gradients}

Baroclinic enhancement of winds due to horizontal temperature gradients and the more complicated sea-breeze circulation were discussed. The effects of thermal wind variations should be considered, although it is unlikely that the true location of wind enhancements can be anticipated without assistance from numerical models or historical data, because the thermal wind will vary temporally as well as spatially.

If the seasonal and diurnal characteristics of the sea breeze match the anticipated electrical load, the sea breeze may be useful as a wind resource. It is generally weak, but if the forcing is from a long coastal area, topographical channeling of the flow may amplify the resource locally. The sea breeze can be clearly seen in climatological records of stations, but one should not become complacent when screening a region. The sea breeze, though well understood in principle, is sensitive to many parameters including synoptic flow conditions, coastal shape and topography, and the previous day's sea breeze. Therefore, a brief measurement survey of a remote site may show very good correlation with a long-term station that does not prove true in a longer measurement program. 
Small Ridges

A small ridge is one whose height is small compared to the depth of the PBL $(H / h \bumpeq 0.1)$. Ridges provide two benefits: an effective increase in hub height relative to surrounding terrain and a speedup of the flow over the ridge crest. The ideal flow is normal to a ridge with slopes of about $25 \%$ extending right up to the crest. Gentler slopes than those of about $25 \%$ provide less speedup, and steeper slopes provide less speedup also because the flow separates. A parameterization scheme (Bouwmeester et al. 1978) is available to estimate wind profiles over small ridges and many nunierical models could be applied to simple cases. Turbulence intensities at the crest are decreased from the free stream values but on the lee side the intensities exceed the free stream values. Any concave curvature toward the upwind direction might provide additional funneling of wind over the ridge. If the flow is along the ridge, the downwind shoulder may experience the high turbulence intensities of the lee separation eddies; the upwind shoulder should be regarded as a hill. Small ridges upstream of larger ridges should be avoided since the flow will primarily respond to the larger ridge. Extensive separated eddies will exist between closely spaced steep small ridges.

Small Cliffs

A small cliff facing upwind can cause a speedup of the upstream winds of at least $10 \%$ below a height of $3 \mathrm{H}$ and up to $70 \%$ at about $2 \mathrm{H}$. Some speedup of the wind persists to more than $10 \mathrm{H}$ downwind where the wake is mostly dissipated. Although the speedup at the crest of an escarpment is only weakly dependent on slope of the escarpment, the occurrence of higher turbulence and high wind shear in the wake region is associated with steeper slopes. Based on the wind tunnel data of Figure 5.20, an escarpment with a slope of $25 \%$ and up to $20 \mathrm{~m}$ high would provide exploitable enhancement of winds for a wind turbine with a rotor that does not come nearer to the surface than $10 \mathrm{~m}$. Steeper slopes may still provide some enhancement with minimum hazard if the turbine is sited between $6 \mathrm{H}$ and $10 \mathrm{H}$ downstream of the crest. Measurements are advised to determine the extent of the turbulent wake region. 
Small Hills

The speedup over hill is less than over a ridge of the same slope because the steeper slope diverts more flow around the hillsides. If there is no dominant wind direction, the annual wind power over a hill of a given height may exceed the annual wind power over a ridge of the same height. The optimum hill slope is about $30 \%$. The slope should lead smoothly and continuously to a small, not too flat, summit. Rougher surface causes less speedup. The sides of a hill are possible secondary sites, but lee wake and flow separation effects are more severe than on ridge shoulders.

Small Valleys and Canyons

Large WECS should not be sited in a small valley or canyon. The valley top wind resource is generally reduced in the valley. Light and variable winds are expected in a small valley in the daytime. Large WECS will experience large wind shear between the valley bottom and ridge or plateau level.

Small Basins

Large WECS should not be sited in small basins because the wind resource is reduced and wind shear seen by large WECS may be increased.

Small Gaps

Gaps can enhance the winds if the flow is nearly normal to ridge or if cool air pools behind the ridge. These conditions are not expected to occur often enough to be of any advantage. Therefore, small-scale gaps are probably too turbulent for large WECS.

Large-Scale Mountains and Ridges

Anywhere in mountainous country the winds can change rapidly and sometimes violently with just small changes in the free stream flow conditions. As wind directions shift slightly lee waves will change and combine in new and possibly very different superpositions. As thermal stratification changes so will the response of the atmosphere to large-scale terrain features. Likewise slight changes in the large-scale flow pattern can greatly influence the anabatic and 
katabatic winds affecting a given summit. Measurements at a given mountain are required in order to obtain a true understanding of the local climatology. The situation is well summed up by Wahl (1966):

It is very difficult to relate wind speed on a mountain in a general way, applicable to any 'typical mountain, ' to any one distinct topographical feature. While the small-scale variations, for example, gustiness, directional stability, etc., are certainly most dependent on the immediate terrain surrounding the specific peak, most effects on the larger-scale flow character, e.g., relation between mean speeds and the free flow in the vicinity, will depend on medium and largescale features of the terrain, such as its relation to neighboring mountain chains, overall geographic locations with repect to the gross flow patterns in the atmosphere, and so on. All these effects will combine, for a particular summit, in some specific way which makes up that very specific wind pattern observed on top of that very special mountain summit.

In particular, one should consider the following items:

- The characteristics of the wind resource must be obtained by measurement.

- The ideal ridge is normal to the prevailing wind.

- Ridge and mountain summits and upper slopes have stronger winds and less diurnal wind variation than a valley.

- Shoulders of peaks and ridges may be good sites.

- Within 10 to $30 \mathrm{~km}$ downwind of a summit there may be very strong and very turbulent winds if large upwind ridges force large amplitude gravity waves.

- Flat-topped ridges or mountains may produce wind shear and flow separation problems for large WECS.

- Near summits of mountains and ridges, Frenkiel's results can be used to estimate the vertical wind speed gradient.

- High shoulders downwind of summits on long ridges are worth investigating although the wind may turn out to be too gusty and variable.

- Flow near small mountains or ridges upwind of larger ridges or mountains may be influenced by blocking of the larger features. 
- Thermal forcing, anabatic and katabatic winds influence flow over and downwind of the crest.

On 7arge-scale features, smaller scale features (rock outcroppings, small gaps, small ravines, small hills) that influence winds locally should be considered.

\section{Large-Scale Cliffs}

The higher elevation in wind flow and the possible speedup at WECS level are advantages of locating WECS on large-scale cliffs. Lee sides of buttes should be avoided. Turbulence on top of large cliffs may be severe; this phenomenon is not we11 understood. Resource quality should be evaluated with measurements. Winds nearly parallel to cliff may be very gusty. The concave curvature of the upwind face of cliff should be exploited.

\section{$\underline{\text { Passes }}$}

The pass should be open to the prevailing wind to capitalize on valley and ridge channeled flow or ridge blocking of flow. Adjacent ridges should be high, the higher the better. A very narrow pass may be very turbulent. A U-shaped pass is preferable to a V-shaped pass. Apply Frenkiel's criteria (Table 5.2) to the slope up to the crest of pass to help minimize mean wind shear. The wind maximum may not be in the center of the pass but there may be significant turbulence near the walls.

Valleys and Canyons

Valleys channel flow (especially stably stratified flow). Select wide valleys parallel to the prevailing wind or long valleys extending down from mountain ranges. Choose sites in constrictions in the valley or canyon where the wind flow might be enhanced, but not in constrictions narrow enough to cause turbulence hazards. Avoid extremely short and/or narrow valleys and canyons, as well as those perpendicular to the prevailing winds. Choose sites near mouths of valleys to capitalize on prevailing flow into valleys. Consider the thermal structure and inertia of the flow and how it will interact with the valley slope and turns of the valley. 


\section{Gorges}

Gorges develop strong winds when a pressure gradient across a mountain barrier exists. The pressure gradient through a gorge arises from the blocking effect of mountains or temperature difference across mountains. Gorges should be aligned with prevailing winds. Turbulence in a gorge may be high. Constriction of the gorge may enhance flow further. Consider how inertia of flow will affect flow interaction with turns of the gorge.

\section{$\underline{\text { Large Basins }}$}

Only very large, shallow inland basins that clearly demonstrate wind energy potential visible in a regional resource assessment should be considered. Smaller basins are subject to light winds and stagnation. For large basins, the siting problem reduces to analysis of smaller scale features within the basins. 


\section{REFERENCES}

Ball, F. K. 1956. "The Theory of Strong Katabatic Winds." Australian Jour. of Physics 9(3):373-396.

Bonner, W. D. 1968. "Climatology of the Low-Level Jet." Mon. Wea. Rev. $96: 833-850$.

Bonner, W. D. and J. Peagle. 1970. "Diurnal Variations in Boundary Layer Winds Over the South-Central United States in Summer." Mon. Wea. Rev. $98: 735-744$.

Bouwmeester, R. V. B., R. N. Meroney and V. A. Sandborn. 1978. Part 1 of Sites for Wind Power Installations: Wind Characteristics Over Ridges. RL0/2438-78/1. Available from National Technical Information Service. Springfield, Virginia.

Bowen, A. J. and D. Lindley. 1974. "Measurements of the Mean Wind Flow Over Various Escarpment Shapes." In Proceedings of Fifth Australian Conference on Hydraulics and Fluid Mechanics, pp. 211-219, University of Canterbury, New Zealand.

Bowen, A. J. and D. Lindley. 1977. "A Wind Tunnel Investigation of the Wind Speed and Turbulence Characteristics Close to the Ground Over Various Escarpment Shapes." Boundary Layer Meteorology 12:259-271.

Bradiey, E. F. 1980. "An Experimental Study of the Profiles of Wind Speed, Shearing Stress and Turbulence at the Crest of a Large Hill." Quart. J. Roy. Met. Soc. 106:101-123.

Brinkmann, W. A. R. 1973. A Climatological Study of Strong Downslope Winds in the Boulder Area. NCAR Cooperative Thesis No. 27, University of Colorado and the Laboratory of Atmospheric Science and GARP Task Group, NCAR, Boulder, Colorado.

Davidson, B. et al. 1964. "Sites for Wind Power Installations." Tech. Note No. 63, World Meteorological Organization, Geneva, Switzerland.

Elliott, W. P. 1958. "The Growth of the Atmospheric Internal Boundary Layer." Trans. Amer. Geophys. Union 39:1048-1054.

Frenkiel, J. 1962. "Wind Profiles Over Hills (In Relation to Wind Power Uti1ization)." Quart. J. Roy. Met. Soc. 88:156-169.

Frost, W. 1979. Handbook of Wind Turbine Generator Siting Techniques Relative to Two-Dimensional Terrain Features. RL0/2443-77/l. Available from National Technical Information Service, Springfield, Virginia. 
Frost, W., W. L. Harper and G. H. Fichtl. 1975. "Analysis of Atmospheric Flow Over a Surface Protrusion Using the Turbulence Kinetic Energy Equation." Boundary-Layer Met. $8: 401-407$.

Golding, E. W. 1955. The Generation of Electricity by Wind Power, E. and F. N. Spon. Ltd., London. (Reprinted with additional material from R. I - Harris, 1976.)

Hansen, A. C. and J. E. Cermak. 1975. Vortex-Containing Wakes of Surface Obstacles. Colorado State University Research Report No. CER75-76ACH-JEC16, Fort ColTins, Colorado.

Holton, J. R. 1967. "The Diurnal Boundary Layer Wind Oscillation Above Sloping Terrain." Tellus 19:199-205.

Jackson, P. S. and V. C. R. Hunt. 1975. "Turbulent Wind Flow Over a Low Hill." Quart. J. Roy. Met. Soc. 101:929-955.

Leutheusser, H. J. and W. D. Baines. 1967. "Similitude Problems in Building Aerodynamics." In Proceedings ASCE, pp. 35-49, Jour. of Hydr. Div.

Meroney, R. N. 1977. "Wind in the Perturbed Environment: It s Influence on WECS." Paper presented at American Wind Energy Association Conference, Boulder, Colorado, May 11-14, 1977, Colorado State University, Fort Col 1ins, Colorado.

Meroney, R. N., V. A. Sandborn, R. J. B. Bouwmeester, H. C. Chien and M. Rider. 1978. Sites for Wind Power Installations: Physical Modeling of the Influence of Hills., Ridazes and Complex Terrain on Wind Speed and Turbulence. RL0/2438-77/3. Available from National Technical Information Service, Springfield, Virginia.

Peterson, E. W. 1969. "Modification of Mean Flow and Turbulence Energy of a Change in Surface Roughness Under Conditions of Neutral Stability." Quart. J. Roy. Met. Soc. 96:561-575.

Peterson, E. W. 1972. "Relative Importance of Terms in the Turbulent-Energy and Momentum Equations as Applied to the Problem of a Surface Roughness Change." J. Atmos. Sci. 29:1470-1476.

Plate, E. J. 1971. Aerodynamics Characteristics of Atmospheric Boundary Layers. TID-25465, U.S. Atomic Energy Commission, Washington, DC. p. 190.

Putnam, P. C. 1948. Power from the Wind. Van Nostrand Reinhold Co., New York.

Scorer, R. S. 1949. "Theory of Waves in the Lee of Mountains." Quart. J. Roy. Met. Soc. 74:41-56. 
Scorer, R. S. and H. Klieforth. 1959. "Theory of Mountain Waves of Large Amplitude." Quart. J. Roy. Met. Soc. 85:131-143.

Sisterson, D. L. and B. B. Hicks. 1979. "Application of Power Laws for Wind Energy Assessment." In Proceedings of the Conference and Workshop on Wind Energy Characteristics and Wind Energy Siting 1979. American Meteorological Society, Boston, Massachusetts,

Smith, R. B. 1978. "A Measurement of Mountain Drag." J. Atmos. Sci. $35: 1644-1654$.

Taylor, P. A. 1977. "Some Numerical Studies of Surface Boundary-Layer Flow Above Gentle Topography." Boundary-Layer Met. 11:439-465.

Wahl , E. W. 1966. Wind Speed on Mountains. AFCRL-66-280, Final Report Project No. 8624. University of Wisconsin, Madison, Wisconsin.

Woo, H. G. C., J. A. Paterka and J. E. Cermak. 1977. Wind-Tunnel Measurements in the Wakes of Structures. NASA CR-2806, National Aeronautics and Space Administration, Washington, DC. 


\subsection{BIOLOGICAL INDICATORS OF WIND POWER POTENTIAL}

In areas of persistent winds, physiological and biochemical processes can cause permanent or developmental changes in plants. The changes can be morphological, i.e., changes in the shape of the plant, or anatomical, i.e., changes in plant cell structure. Among the many effects of wind on plant growth, the effects of wind on the morphology and anatomy of trees are the most useful for wind power prospecting (Hewson, Wade and Baker 1979). Based on these effects, information on the wind can be found on varying space scales:

- small, where the wind has permanently deformed a single tree or shrub,

- regional, where the wind has deformed a number of trees that may provide a mean streamline map for the flow,

- large, where appropriate use of remote sensing techniques may show observable effects of wind on entire plant communities (Rosenfeld and Maule 1979).

Astute observations of the ecological community of a site can provide useful qualitative information about the winds in and around a site, but such interpretations must be made with caution. Wade and Hewson (1980) have established, on a quantitative basis, the effects of wind on certain genera of trees. The remainder of this chapter describes the interpretation of wind effects on trees as a wind prospecting technique. Much of what follows is taken from Wade and Hewson (1980). The reader should refer to the original source for complete details.

\subsection{TREES AS INDICATORS OF MEAN WIND SPEED}

Putnam (1948) observed that tree deformation appeared to be a function of the mean annual wind speed. Hewson et al. (1977) confirmed this and calibrated (1979) three indices of tree deformation against a number of wind characteristics, including mean annual wind speed, mean growing season wind speed, mean dormant season wind speed, and percentage of winds from the prevailing direction. 
The mean annual wind speed was the wind characteristic best correlated with each index of tree deformation. These calibrations were made for two species of trees: the Douglas fir (Pseudotsuga menziesii), which is not a true fir, and Ponderosa pine (Pinus ponderosa).

Similar techniques were applied by Wade and Hewson (1980) to several genera of trees. These include Pinus (pines), Pseudotsuga (Douglas fir),

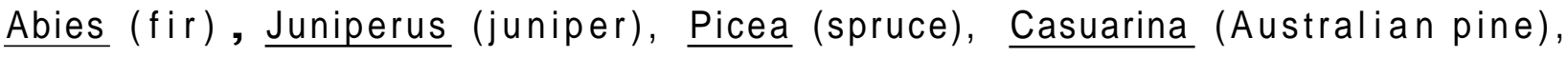
Acer (maple), Legumes (mesquite-locust), Quercus (Oak), and Ulmus (elm).

Figure 6.1 illustrates the distribution of five genera of trees over the United States and indicates a broad potential for the use of biological indicators of wind. Wade and Hewson (1980) also discuss, on a less quantitative basis, wind effects on cedar, cypress, hemlock, larch, hickory-pecan, hackberry, cherry-plum, poplar, ash, and birch, trees, and on shrubs, grasses, insects, animals, and man. Only the calibrated genera are discussed below.

\subsubsection{Description of the Indices of Wind Deformation}

The Griggs-Putnam Index is historically the first index of wind effects on coniferous trees and is based on the pioneering work of R. F. Griggs and P. C. Putnam (Putnam 1948). Griggs was a botanist who collaborated with Putnam in the survey of wind power potential in New England before the instalIation of the Smith-Putnam wind turbine on Grandpa's Knob in Vermont. This index (shown in Figure 6.2) is a subjective scale based on the degree of response by the tree to the wind. The index is broken into eight classes:

Class 0 , No Effect. Careful examination of needles, twigs, and branches indicates that the wind has had no noticeable influence on the tree.

Class I, Brushing. The small branches and needles appear bent away from the prevailing wind direction. The tree crown may appear slightly asymmetrical if carefully examined.

Class II, Light Flagging. The small branches and the ends of the larger branches are bent by the wind, giving the tree a noticeably asymmetric crown. 


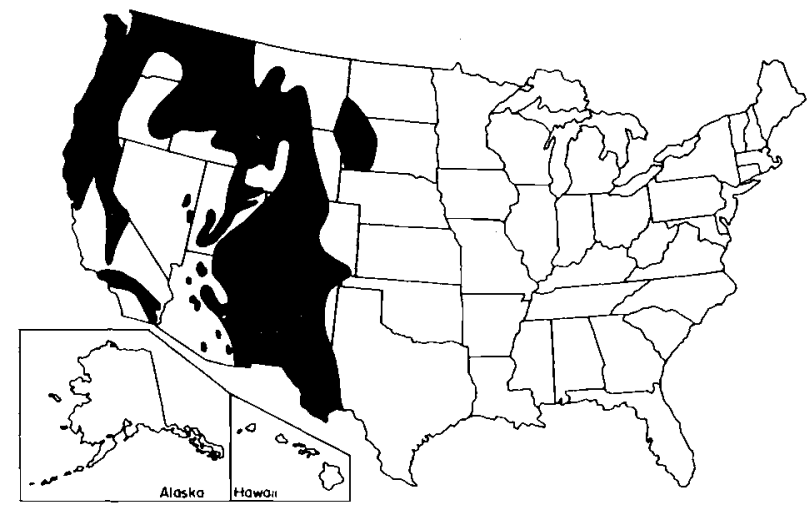

THE DISTRIBUTIONOF DOUGLAS-FIR AND PONDEROSA PINE IN THE UNITED STATES.

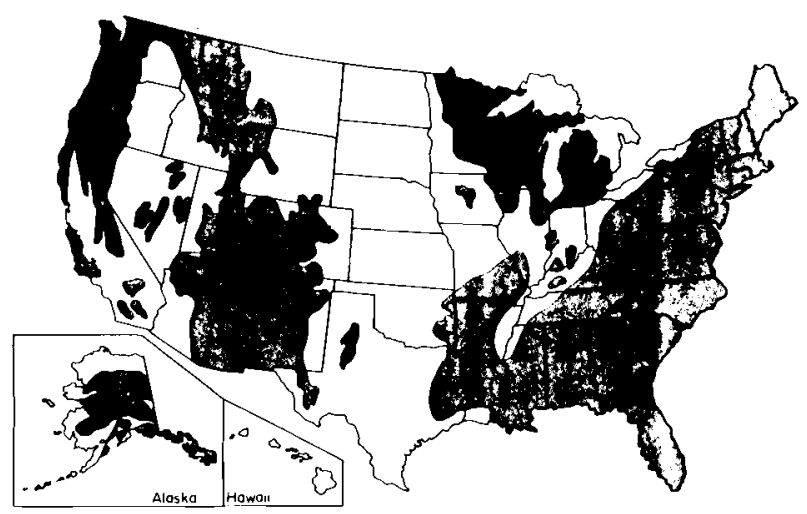

THE DISTRIBUTION OF PINES. HEMLOCK AND SPRUCE IN THE UNITED STATES

A

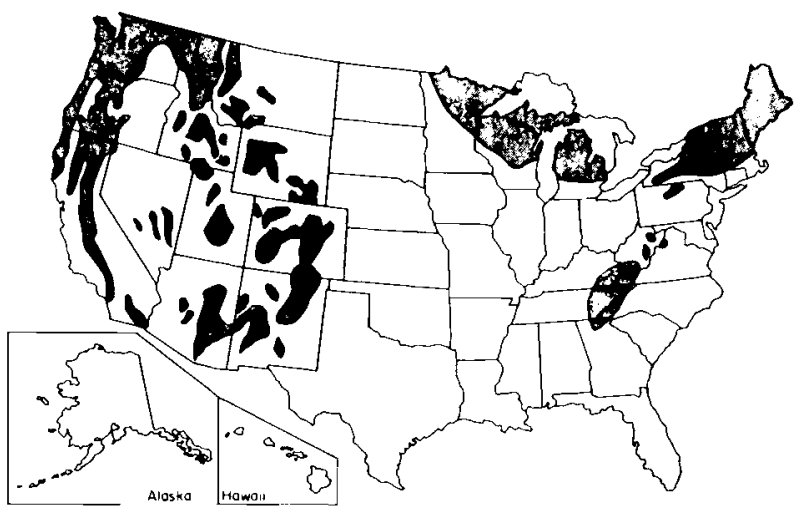

THE DISTRIBUTION OF FIR IN THE UNITED STATES.

C

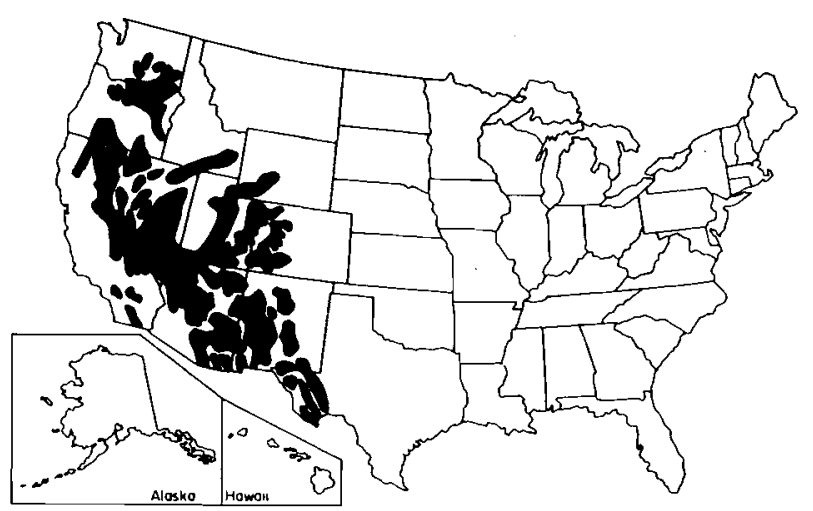

THE DISTRIBUTION OF SEVERAL SPECIES OF JUNIPER IN THE WESTERN UNITED STATES.

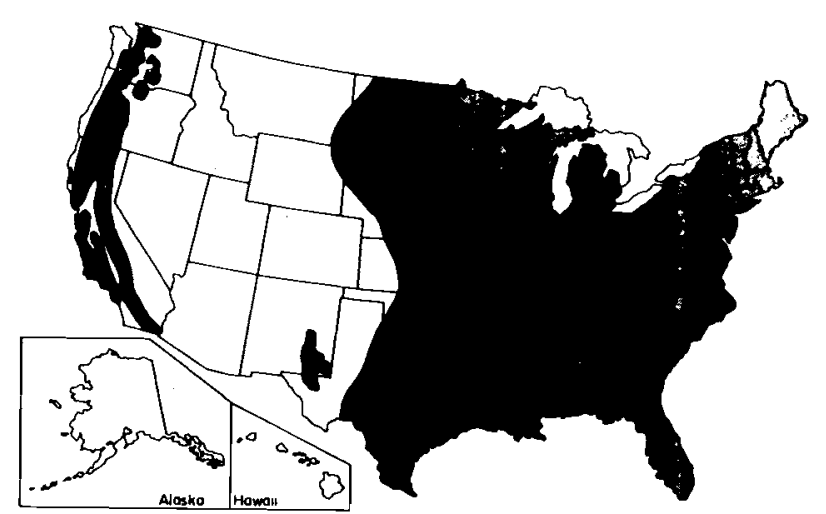

THE DISTRIBUTION OF SELECTED SPECIES OF OAKS IN THE UNITED STATES.

E

FIGURE 6.1. Distribution of Trees in the United States Being Calibrated for Use as Indicators of Wind Power Potential 


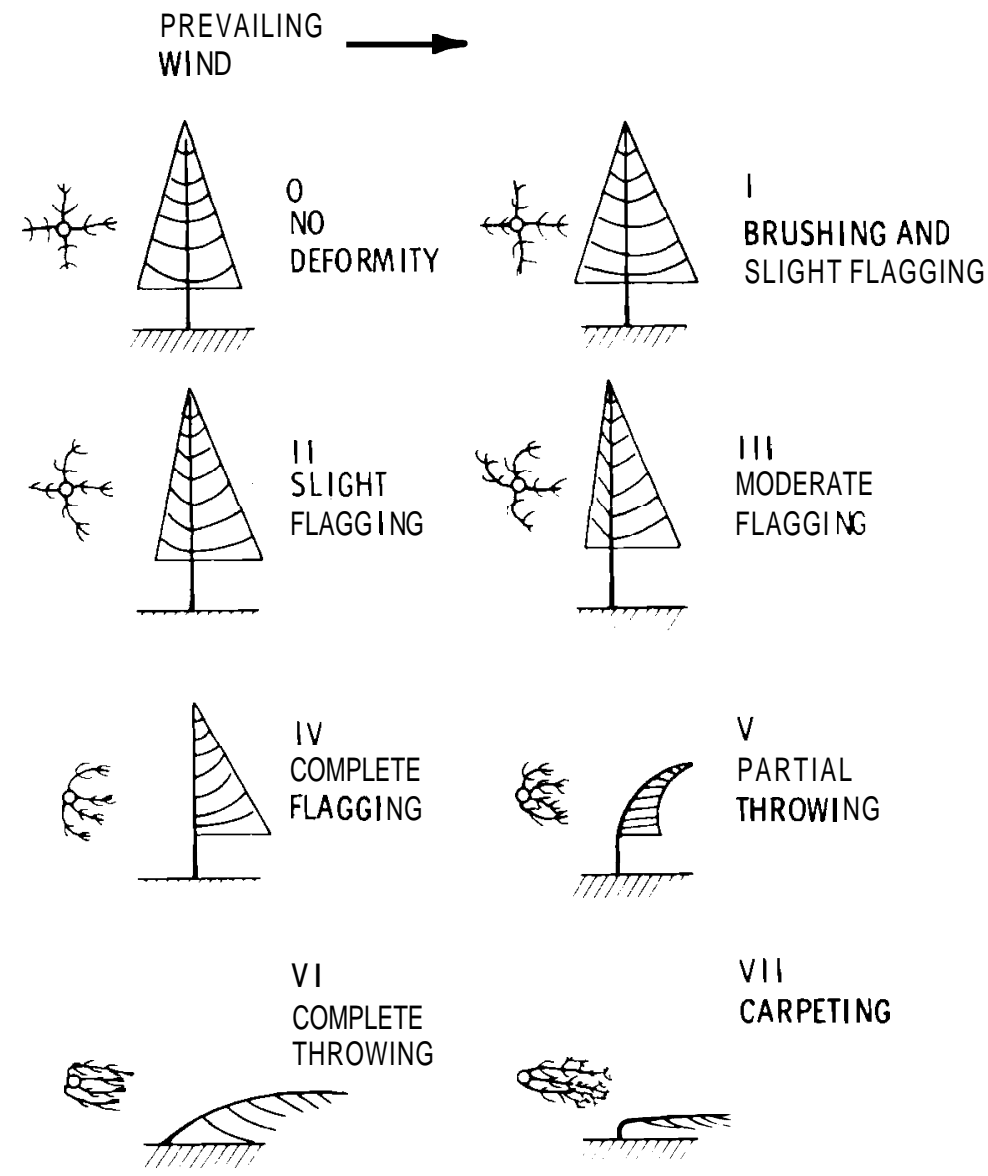

FIGURE 6.2. The Griggs-Putnam Index of Wind Deformation Based on the Degree of Permanent Bending of Needles, Twigs, Branches and Trunks of Coniferous-Type Trees

Class III, Moderate Flagging. The large branches are bent toward the leeward side of the tree, giving the tree a nearly one-sided crown.

Class IV, Strong Flagging. All the branches are swept to the leeward and the trunk is bare on the windward side. The tree resembles a banner.

Class V, Partial Throwing. (a) A partially thrown tree is one in which the trunk, as well as the branches, are bent to the lee. The trunk may

(a) Throwing should not be confused with the type of throwing caused by root break or soil failure. Throwing, as defined here, can be differentiated by examining the angle of the trunk with ground surface. Root break or soil failure causes trees to tilt, and thus, the trunk projects from the ground at an angle. Throwing, caused by persistent winds, results in the trunk rising perpendicular from the ground and bending above the ground. 
be bent in a concave or convex fashion, but rises vertically near the ground and the degree of bending increases near the top of the trunk.

Class VI, Complete Throwing. The tree grows nearly parallel to the ground and along the path of the prevailing wind. The larger branches on the leeward side may extend beyond the tip of the trunk.

Class VII, Carpeting. The wind is so strong or accompanying conditions so severe (e.g., ice is present) that the tree takes the form of a shrub. Upright leaders are killed and lateral growth predominates. The crown grows across the ground like a prostrate shrub.

The Barsch Index (Barsch 1963) is similar to the Griggs-Putnam Index, but is used with broadleaf trees (see Figure 6.3).

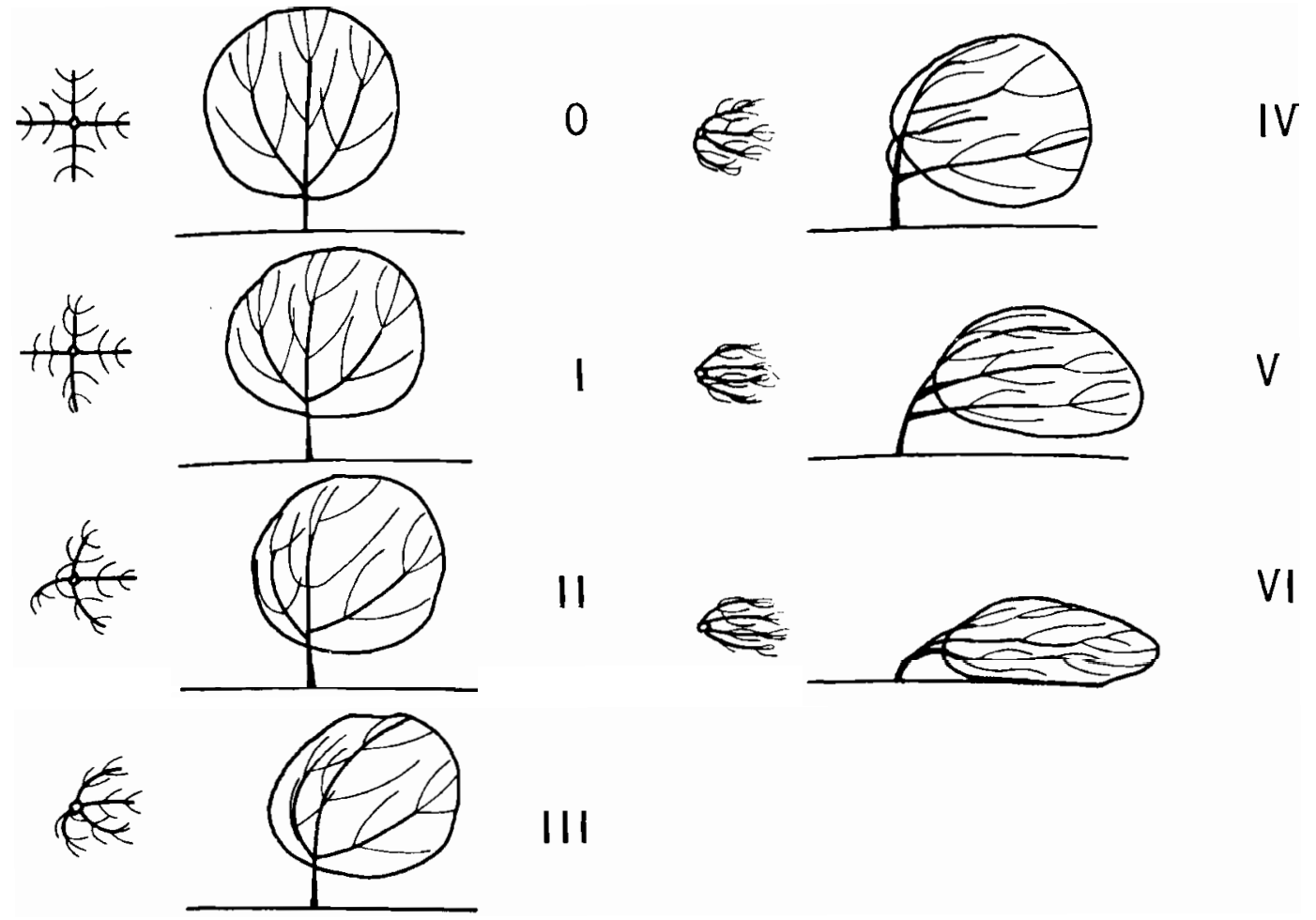

FGURE 6.3. The Barsch Rating of Wind Deformation 
The deformation ratio, an index resembling the Griggs-Putnam Index, represents the amount of crown asymmetry and trunk deflection of trees caused by the wind (see Figure 6.4) The equation used to compute the deformation ratio (D) is:

$$
D=\frac{A}{B}+\frac{C}{45^{\circ}}
$$

where

for conifers, $A$ is the angle formed by the crown edge and the trunk on the leeward side, $B$ is the angle formed by the crown edge and the trunk on the windward side, and $C$ is the average angle of trunk deflection, and where

for hemispherically crowned trees, $A$ is the distance between the crown perimeter on the leeward side, $B$ is the distance between crown and perimeter on the windward side, and $C$ is the angle between the leeward crown perimeter and the trunk.

tiowever, $A / B$ is defined to lie within the range $1 \leq \frac{A}{B} \leq 5$. As a result the minimum value of $D$ is 1 , which corresponds to no crown asymmetry or stem deflection. Since the maximum possible value of $\mathrm{C}$ is $90^{\circ}$ for a tree growing along the ground, the maximum value of the deformation ratio is 7 . The deformation ratio can conveniently be determined from photographs taken of a tree from positions at right angles to the direction of flagging.

The compression ratio, a third index of tree deformation, measures the influence of wind on the formation of reaction wood. Reaction wood is "abnormal" wood laid down on a particular side of the tree when the tree is displaced from the vertical by a force such as the wind. Conifers exposed to the prevailing winds form reaction wood on the leeward side; deciduous trees form reaction wood on the windward side. In conifers, reaction wood is called compression wood. In deciduous trees, it is called tension wood.

The compression ratio may be determined either by looking at a radial cross section of the tree or by taking an increment core from the windward and leeward sides of the tree. Tree cores and radial cross sections are first 

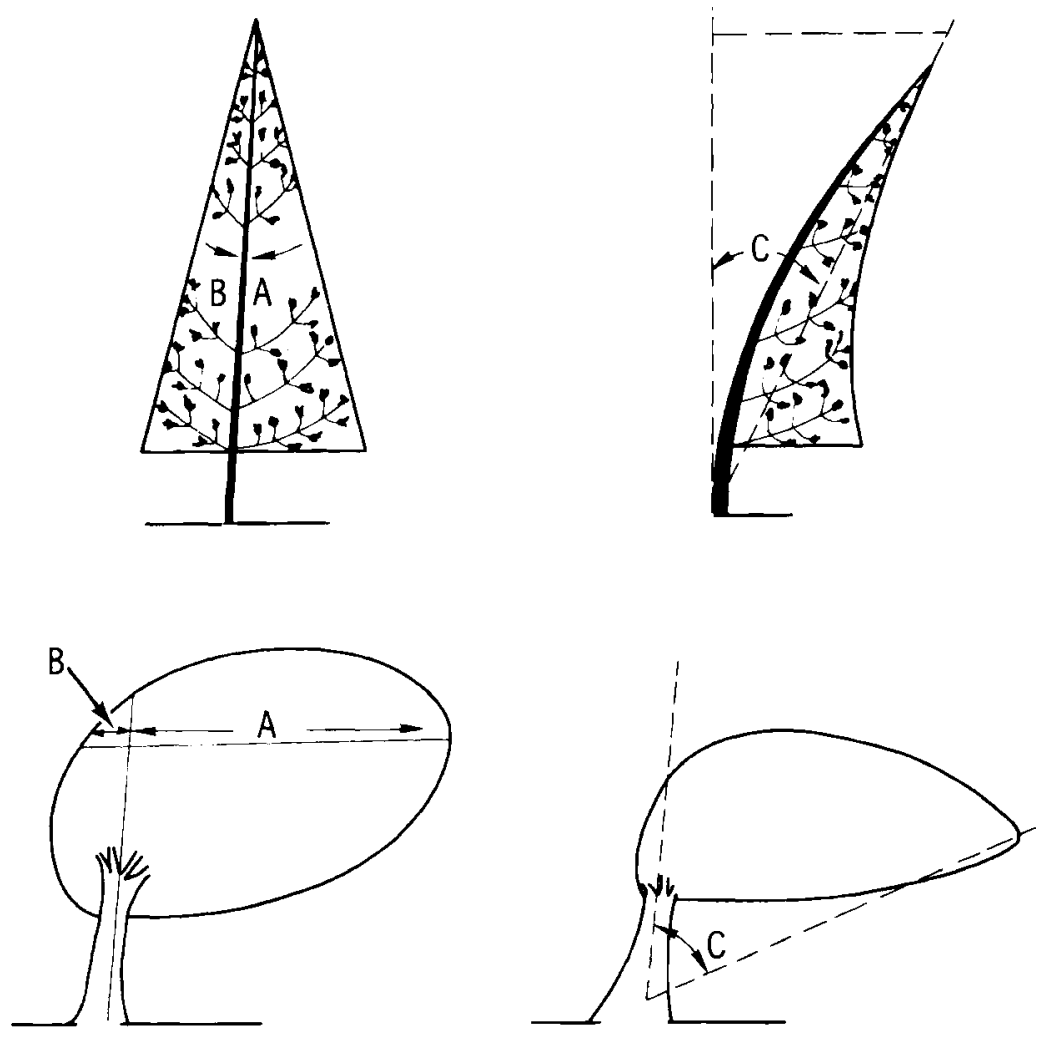

FIGURE 6.4. The Technique for Computing the Deformation Ratio Index for Conifers and Hemispherically Crowned

Trees. The prevailing winds blow from left to right.

cross-dated as shown in Figure 6.5. The growth increments for each year are then measured in the leeward and windward directions. The compression ratio for conifers is the ratio of leeward growth to windward growth. (Calibration of compression ratio for deciduous trees has not yet been attempted.) The compression ratio should be calculated over the last 5 years, since that period would be the most representative of the tree's most recent exposure to the wind.

\subsubsection{Mean Wind Speed and Tree Deformation}

Field measurements were used to calibrate and validate the wind deformation indices. Locations along the coast were not used because salt spray might enhance the wind deformation. In locations with strongly flagged trees and limited existing wind data, anemometers were installed in open areas near 


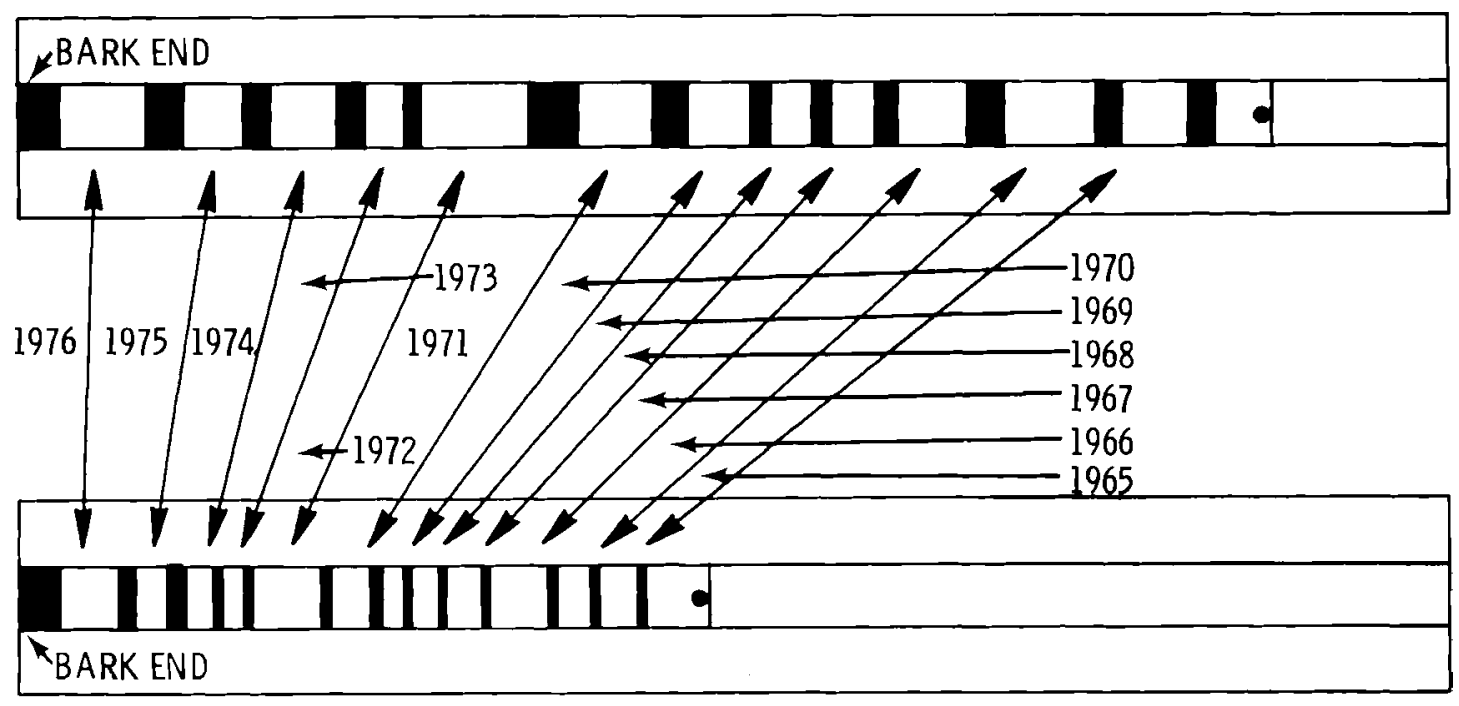

CORE FROM WINDWARD SIDE

OF CONIFER

FIGURE 6.5. Core Samples From the Windward and Leeward

Side of the Tree, Mounted and Cross-Dated

trees. Anemometers were usually placed at a standard height of $10 \mathrm{~m}$ At some of the locations used in the initial calibration, wind data were taken from anemometers recording wind speed and direction continuously on strip chart recorders. At other locations, contact anemometers recording the miles of wind passage were used to measure monthly wind speed. At still other locations with slight or no wind flagging, National Weather Service or airport wind data were used.

Values of the Griggs-Putnam Index $(G)$, the deformation ratio (D), and the compression ratio ( $\mathrm{C}$--for Douglas fir and Ponderosa pine only) were calculated for each field location. Linear regressions of the annual mean wind speed were computed for each index. To validate the indices the mean annual wind speed was predicted for a datum not included in the calibration of the indices. A cross-validation procedure closely related to "jack-knife" 
statistical techniques was used (Quenouille 1956, Gray and Schucany 1972). This technique divides the sample into as many subsets as there are data points. Then, linear regressions are performed for each subset, alternately leaving out one of the points. In this way, an error estimate for each regression relation is obtained for the point not included.

Several measures of the error are possible. The root-mean-square (RMS) error measures the dispersion of the data about the fitted regression line. The square of the correlation coefficient $\left(r^{2}\right)$ measures the fraction of the variance of the measured annual mean wind speeds that is explained by the regression relation. Confidence intervals are another measure. If one makes a prediction using a given index and regression equation, one can be $95 \%$ confident (i.e., run a $5 \%$ chance of being wrong) that the predicted annual mean wind speed differs from the true annual mean wind speed by less than the amount specified by the 95\% confidence interval (see Figure 6.6). Yet another measure is the percentage of observations that differ from the predicted value by more than a specified interval, e.g., $1 \mathrm{~m} / \mathrm{sec}$. The regression coefficients and these measures of error are shown for ten genera of trees in Table 6.1.

The Griggs-Putnam Index or the Barsch Index, in general, provides the best estimate of mean annual wind speed. The deformation ratio provides a second check or can be used when only photographs are available (as from a helicopter survey). The compression ratio provides a third estimate, which could be the only estimate if only a tree stump is available.

To assist in identifying the various genera of trees and to visualize the deformation associated with each value of the Griggs-Putnam Index or the Barsch Index, Wade and Hewson (1980) provide illustrations and brief narratives in handbook form. Figure 6.7 is an example illustration for the genus Pinus, and Figure 6.8 is an example illustration for genus Quercus.

\subsection{NDICATORS OF PREVAILNG WND DIRECTION}

In mountainous areas, winds are complex and the sparse wind data available provide little information on wind direction. One technique for determining the mean wind direction is by observing flagged trees, the branches of which 


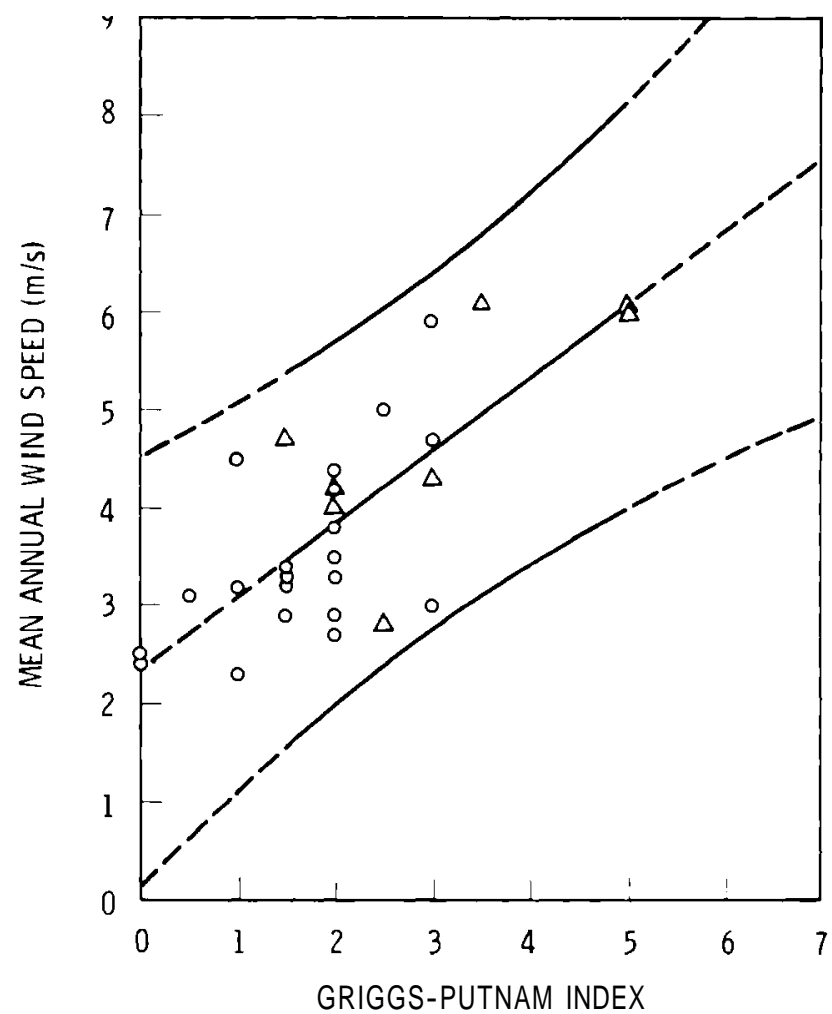

FIGURE 6.6. The Relationship Between the Griggs-Putnam Index for Ponderosa Pine and Mean Annual Wind Speed. The solid lines are the regression relationship and $95 \%$ confidence limits for ponderosa pine data indicated by triangles. The open circles represent data for other species of pines found throughout the United States including pinion pine $(P I)$, shortleaf pine (PE), eastern white pine (PS), pond pine (PO), loblolly pine (PT), lodgepole pine (PL), pitch pine (PR), and shore pine (PC).

grow away from the prevailing wind direction. Holroyd (1970) mentions that phototropism (directed growth as a result of differential illumination) may cause bending of branches but the direction of bending is usually random. With wind-flagged trees, all branches in the same neighborhood point in a uniform direction. As discussed above, conifers exposed to strong winds may form compression wood on the downwind side of the tree trunk, whereas deciduous trees may form tension wood on the upwind side.

The direction of flagging can be marked on a topographic map after the direction has been determined. In this way, flow patterns of the area can be 
TABLE 6.1. Statistical Results and Error Analvsis for the Calibration of the Indices

G (Griggs-Putnam Index), B (Barsch Index), D (Deformation Ratio), and

C (Compression Ratio) With Mean Annual Wind Speed (Wade and Hewson 1980)

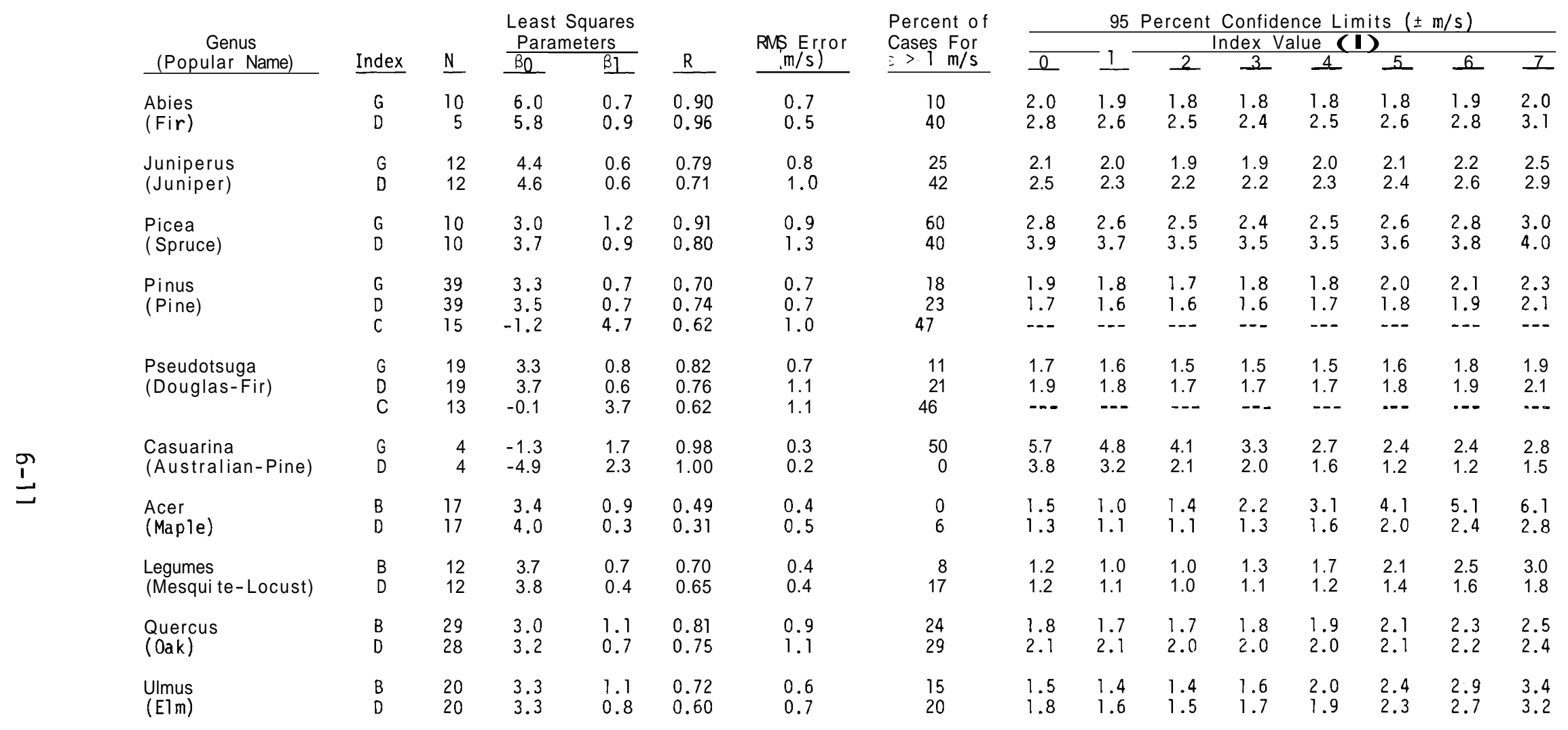

\footnotetext{
$\mathrm{N}=$ Number of data points

$V_{P}=$ Predicted annual mean wind speed

$V_{0}^{P}=$ Observed annual mean wind speed

${ }^{3} 0$ and $B_{1}=$ Regression coefficients

$v_{p}=\beta_{1} \mathbf{I}+\beta_{0}$ where $\mathbf{I}=$ index value

$\varepsilon=V_{p}-I_{0}$

$\mathrm{R}=$ Correlation coefficient

RMS $\left.=\frac{-\frac{1}{N}}{-N} \varepsilon^{2}\right]^{\frac{1}{2}}$
} 

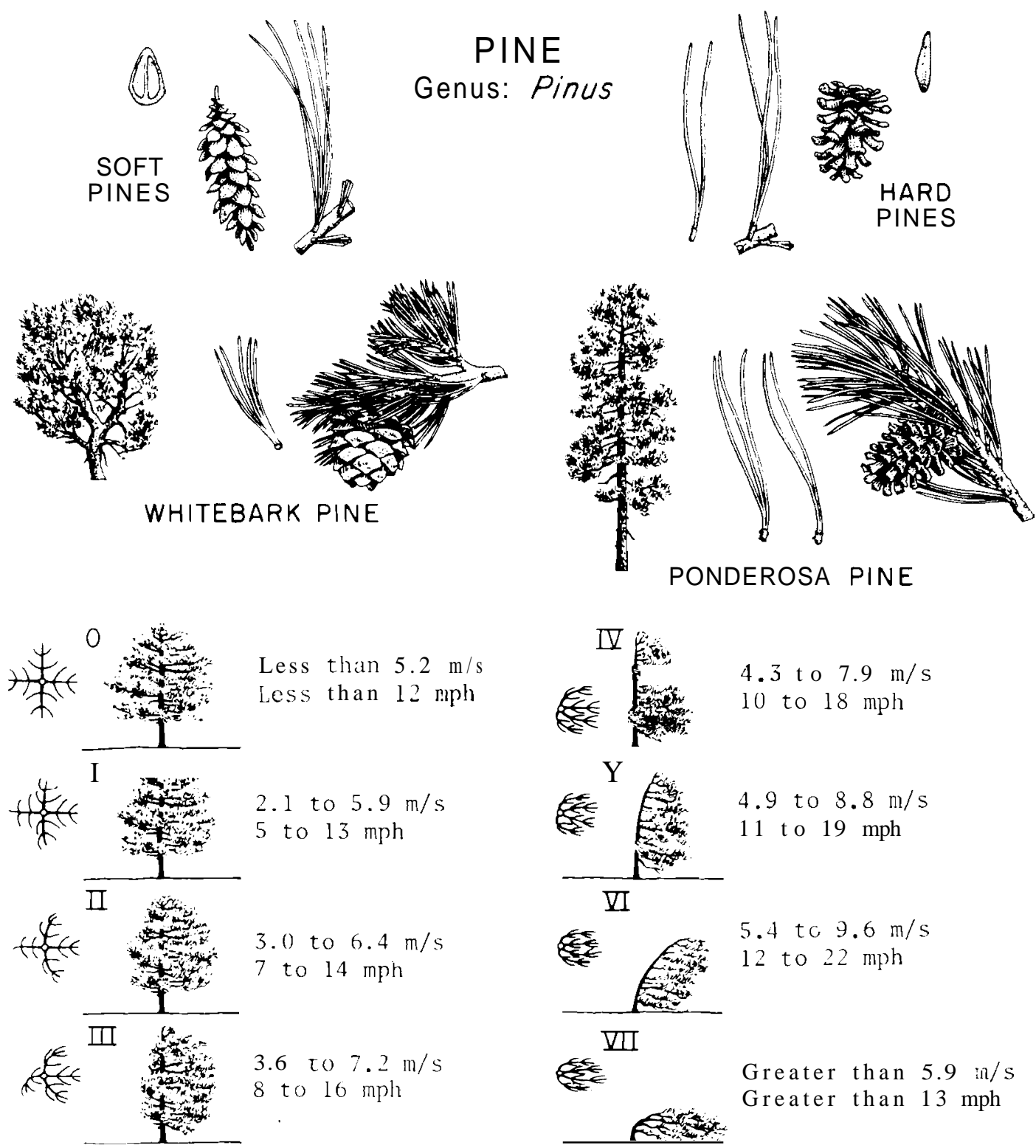

NOTE: Soft pine needles are usually in bundles of 5 and occasionally 1 to 4. Their cones have a stalk. !lard pine needles usually arc in bundles of 2 to 3 , occasionally 5 to 8 . Their cones arc often armed with prickles. Pines growing at high elevations or along the coast are likely to be also affected by wind-blown sand, salt, ice, and snow which would add $t o$ the wind deformation.

The relationships between mean annual wind speed $(\bar{V})$ and the indices of wind deformation are:

$$
\begin{array}{cccc}
\overline{\mathrm{V}}=.7 \mathrm{G}+3.3 & \text { or } & \overline{\mathrm{V}}=.7 \mathrm{D}+3.5 \\
(\mathrm{rms} \text { error }= \pm 0.7 \mathrm{~m} / \mathrm{s}) & & (\text { rms error }= \pm 0.7 \mathrm{~m} / \mathrm{s})
\end{array}
$$

FIGURE 6.7. Guide to Wind Deformation Indices for Pinus (Wade and Hewson 1980) 


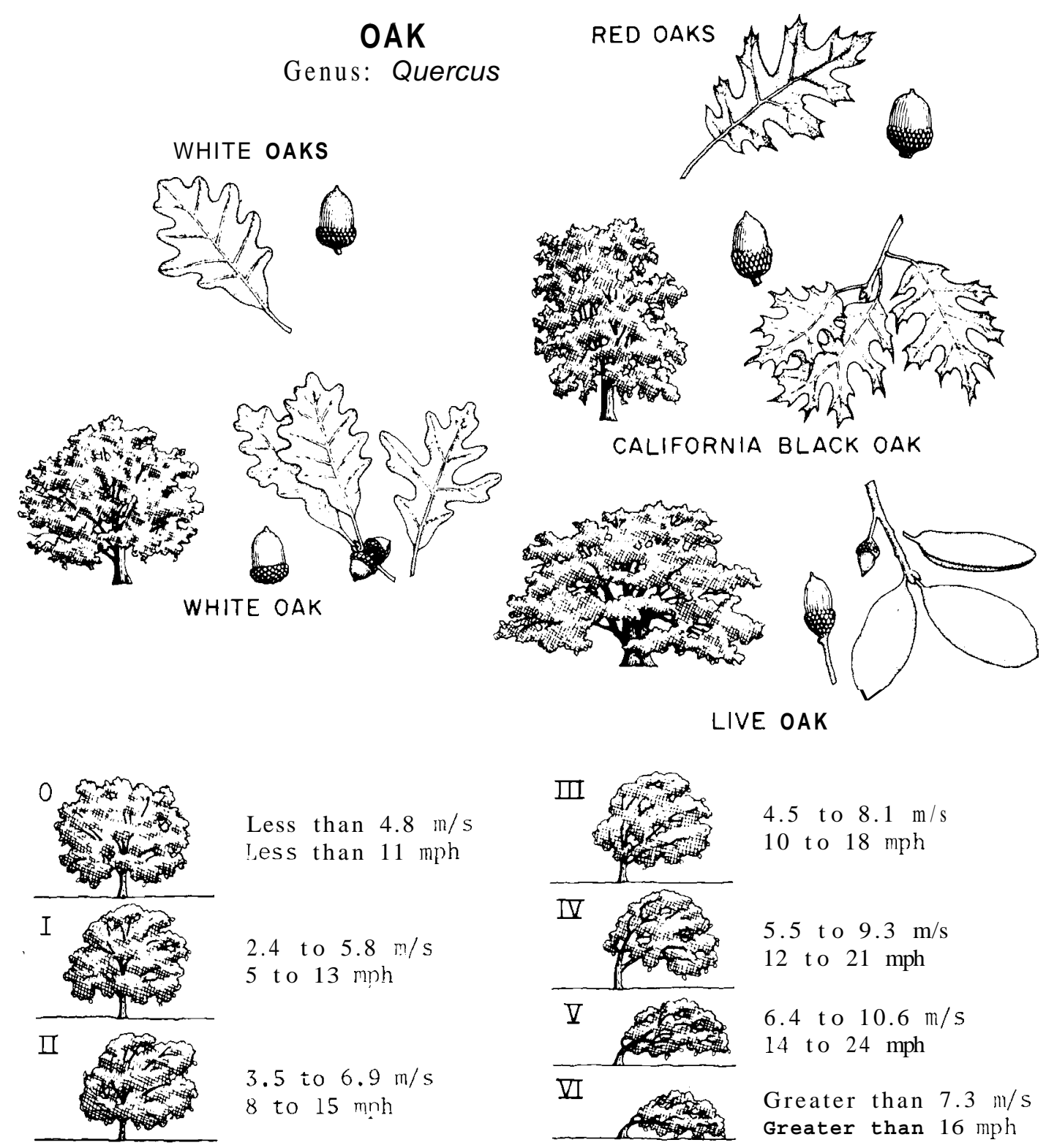

NOTE: Live oaks are evergreen and, thus, should provide better evidence of winter wind than other oaks. Most white oaks have rounded lobes and sweet acorns. Most red oaks have spiny lobes and bitter acorns. Certain species of oak which prefer moist bottomland soil are unlikely to be found in windy locations. These include Bur Oak, Swamp Oak, Shumard Oak, and Laurel Oak.

The relationships between mean annual wind speed $(\bar{V})$ and the indices of wind deformation are:

$$
\begin{array}{ccc}
\overline{\mathrm{V}}=1.1 \mathrm{~B}+3.0 \quad \text { or } & \overline{\mathrm{V}}=.7 \mathrm{D}+3.2 \\
(\text { rms error }= \pm 0.9 \mathrm{~m} / \mathrm{s}) & (\mathrm{rms} \text { error }= \pm 1.1 \mathrm{~m} / \mathrm{s})
\end{array}
$$

FIGURE 6.8. Guide to Wind Deformation Indices for Quercus (Wade and Hewson 1980) 
noted. However, flagged trees reflect the prevailing direction of the strongest persistent winds, which may occur at one location during part of the year only. The wind system that produces flagging at one location may not produce flagging at another location. For example, in the Columbia River gorge, strong easterly winter winds cause crowns to project to the west at the west end of the gorge, but strong westerly spring and summer winds cause crowns to project east at the east end of the gorge (Lawrence 1939). If the prevailing winds were assumed to occur at the same time, then the winds in the gorge would be blowing from the center out in both directions. Since this situation is not probable, the season in which flagging occurs must be determined.

\subsubsection{Winter Wind Flagging}

Lawrence (1939) provided some guidance on a method for determining the season during which wind flagging occurs. Winter flagging is characterized by a tattered appearance, with the longest branches projecting downwind. The windward side may have no branches or may have only small recently formed twigs and branches that are irregularly distributed about the stem. The branch ends on the windward side appear as if they have been clipped by a gardener's shears. Winter flagging may be caused by ice and breakage from severe winds, dessication caused by strong, dry winter winds and wind sway that disrupts roots on the windward side, resulting in greater growth the following spring on the leeward side of the tree.

\subsubsection{Growing Season Wind Flagging}

Trees exposed to strong winds during the growing season have branches that appear to have been entrained in the prevailing winds. Trunks are often bowed slightly leeward. Well-developed branches rise on all sides of the tree, but those emerging from the side facing the prevailing winds have been swept around toward the leeward side.

\subsubsection{Flagging By Winds During Both Growing and Winter Season}

Trees subjected to strong winds during the winter and the growing season will exhibit the flagging effects of both seasons. If the prevailing wind 
directions of each season are opposite (i.e., east in winter and west in summer, as in parts of the Columbia River gorge) long branches are found only in the directions perpendicular to the prevailing wind directions. If both winter and growing-season winds come from the same direction, the trees commonly exhibit only the growing-season form of flagging.

\subsection{INDICATORS OF SEVERE WIND OR ICE DAMAGE}

Trees record evidence of severe wind and ice, both in their outward appearance and in the wood tissue of the trunk. Trees can, therefore, be used as indicators of these destructive forces, which may present severe problems for wind turbines, their support structures, and the power transmission lines from the turbines.

Broken branches, wind-throw (leaning trees) and blow-down are all external evidence of severe winds, Other external evidence may include:

- fallen trees

- leaning trees

- trees with broken branches or tops

- dead branches on the ground

- trunks with gnarled knots where branch stubs have healed imperfectly.

An internal indicator of tree stress is the configuration of the tree's rings. Pillow (1931) found a change in the tree ring structure of pine trees in Florida exposed to a hurricane that occurred in 1926. The most recent ring on the windward side was much narrower in each tree the following spring. Rings on the leeside were much wider. By 1928, this asymmetric growth had disappeared. This narrow streak of abnormal ring structure was suggested to be due to a temporary bending of the tree by the severe wind.

Wald (1934) used trees and other plants to map locations of possible heavy ice loads. The patterns of suppressed tree rings (narrow rings) followed by released tree rings (wide rings) were used to interpret which trees had been subjected to defoliation by severe wind or ice loading. These indicators do not exclusively result from severe weather, however. Patterns of narrow 
and wide rings may be the result of the tree having reduced growth, crowding or shading by nearby trees and their subsequent release, or removal of competitors. Insect or fire damage could also produce an alternating pattern of narrow and wide rings.

Thus, both external and internal evidence should be examined if trees are used as indicators of severe wind and ice damage.

\subsection{SUMMARY AND CONCLUSIONS}

Observation of wind-deformed vegetation can provide useful, and indeed critical, information in a WECS siting effort. In the candidate resource area evaluation stage (see Chapter 2), observation of the directions of flagging lends support to other methods of analysis of flow through the region.

Sampling of individual trees provides some "ballpark" guidelines on the amount of wind resource available at a number of different locations. As a tool used in the potential candidate site screening stage, observation of trees may be useful in pointing out drawbacks of a potential candidate site. For example, flow separation in the lee of hills is sometimes noted by a reversal in the direction of wind flagging on the downwind side of a hill. Ice or severe wind damage within a tree's lifetime may suggest dropping a site from consideration for alternative sites with a more benign climate. This very site-specific information may not be obtainable in any other way. Finally, in the sitedevelopment stage, use of vegetation may be coordinated with other techniques to pinpoint areas of significant wind resource within the boundaries of the cluster site. There are, of course, limitations and cautions in the use of this technique.

The use of trees as indicators of wind velocity is subject to a number of practical limitations that have a bearing on the method of selecting indicator trees. Of greatest concern is the tree's exposure to the wind. Trees selected as indicators must be well exposed to the prevailing winds. Seldom do trees in a forest extend far enough above the canopy to be in an air stream undisturbed by the other trees. High winds will suppress the growth of new shoots 
above the canopy so that smooth, evenly topped canopies are, at least, a qualitative indicator of windy areas. However, isolated trees or those in small, widely spaced groups should be favored as wind velocity indicators.

All trees should be of nearly the same height if they are going to be used to compare several locations. Wind speed increases dramatically with height up to $10 \mathrm{~m}$. Above $10 \mathrm{~m}$, this change in wind speed, although significant, is less pronounced so trees selected should be $10 \mathrm{~m}$ or taller.

All trees should be of the same species if they are being used to rate potential sites. Douglas fir and Ponderosa pine react very similarly to the mean annual winds above $5 \mathrm{~m} / \mathrm{sec}$. Below that speed, Douglas $\mathrm{fir}$ are somewhat more sensitive to wind than the Ponderosa pine.

Salt damage in coastal areas ma enhance the degree of deformation. Coastal trees deformed by wind may be compared to one another, but should not be compared to wind-deformed trees in noncoastal locations.

Factors besides wind may cause a characteristic one-sided crown. The growth of two trees side by side, for example, will cause both trees to have an asymmetric crown. If one tree is removed, the other will look wind-flagged. Always look for evidence of past competition. Soil or snow creep, an occasional severe ice storm, fires, insect damage, pruning, or logging damage may all result in asymmetric crown forms that are not associated with wind.

Many factors can affect the amount of compression wood besides wind. Younger trees or fast-growing trees will put on more compression wood than older or slower-growing trees. Coniferous trees growing on slopes put extra wood on the uphill side of the stem or on the downhill side when soil or snow creep is occurring. Trees that have been tilted also put on compression wood in response to displacement from the vertical.

The character of the crown strongly affects the amount of compression wood. Trees with deep crowns are less affected than those with crowns only in the upper portion of the tree. The number and weight of branches on each side of the tree will also affect the amount of compression wood that is formed. 
It should be emphasized that while wind-flagged trees indicate strong winds, a lack of flagging does not necessarily indicate that the wind is not strong. There may be situations where strong winds come from three or more directions, but their persistence is insufficient from any of the directions to cause noticeable deformation. Also, at locations where afternoon winds may be strong but decrease considerably at night, trees are unlikely to be winddeformed.

There is error in the process of estimating the Griggs-Putnam Index value, which may result in $20 \%$ to $40 \%$ error in the estimate of the annual mean wind speed. This error can be reduced by having several people estimate the index value, using more than one tree at a site and using all three indices.

The relationships presented here between mean annual wind speed and the indices of wind deformation should be used only as rough estimates. Trees selected for calibration span much of the country, but calibrations can vary locally (Zambrano 1980). Some calibrations are based on just one year's data. The error in determining mean annual wind speed with only one year's data may be $\pm 10 \%$ (see Chapter 2 and Appendix 3 ). Additional uncertainty in the calibrations may result from the error introduced by the use of contact anemometers, an error which may approach $\pm 11 \%$ of the annual mean wind speed at some of the locations. It is best to interpret the tree deformation in a local region as a relative indicator of wind speed.

The study of tree deformation indicates that trees are a useful tool for determining prevailing wind direction, identifying areas where severe wind and/or ice loads may occur, and for estimating mean annual wind speed. Estimates of mean annual wind speed based on wind-deformed trees, although subject to some uncertainty, are simple, quick, and inexpensive criteria for identifying locations where more detailed wind measurements are justified, and as a guide for preliminary ranking of sites in terms of wind power potential. 


\section{REFERENCES}

Barsch, D. 1963. "Baumform und Landschaft: Eine Untersuchung des windeinflusses auf Baumform und Kulturland schaft am Beispiel des Mistralgebietes im franzosischen Rhonetal." In Freiburger Geographische Hefte, Heft 1, edited by F. Bartz and W. Weischet. Freiburg, Federal Republic of Germany.

Curtis, J. 1941. "Some Observations of Wind Damage." J. For. 41:877.

Gray, H. L. and W. R. Schucany. 1972. The Generalized Jack-Knife Statistic. Marcel Decker Inc., pp. 308.

Hewson, E. W. and J. E. Wade. 1979. A Handbook on the Use of Trees as Indicators of Wind Power Potential. RL0/2227-79-3. Available from National Technical Information Service, Springfield, Virginia.

Hewson, E. W., J. E. Wade and R. W. Baker. 1977. Vegetation as an Indicator of High Wind Velocity. RL0/2227-T-24-772. Avai Table from National Technical Inforniation Service, Springfield, Virginia.

Hewson, E. W., J. E. Wade and R. W. Baker. 1979. Vegetation as an Indicator of High Wind Velocity. RL0/2227-T-24-79-1. Available from National Technical Information Service, Springfield, Virginia.

Holroyd, E. W. III. 1970. "Prevailing Winds on Whiteface Mountain as Indicated by Flag Trees." Forest Science 16:222-229.

Lawrence, D. B. 1939. "Some Features of the Vegetation of the Columbia River Gorge with Special Reference to Asymmetry of Forest Trees." Ecol. Monogr. 9(2):217-157.

Pillow, M. 1931. "Compression Wood Record Hurricane." J. For. 29:575.

Putnam, P.C. 1948. Power from the Wind. Van Nostrand Co. Inc., New York, p. 224.

Quenouille, M. 1956. "Notes on Bias Estimation." Biometrica 43:353.

Wade, J. E. and E. W. Hewson. 1980. A Guide to Biological Wind Prospecting. DOE/ET/20316-80-2. Available from National Technical Information Service. Springfield, Virginia.

Wald, W. 1934. "Prediction of Hoar Frost Areas on the Basis of a Geobotanical Map." J. of the Soc. for Electrotechnology 52:17.

Rosenfeld, C. L. and P. A. Maule. 1979. "Remote Sensing Applications to Wind Power Facility Siting." In Proceedings of the Conference and Workshop on Wind Energy Characteristics and Siting, 1979. American Meteorological Society, Boston, Massachusetts. 
Zambrano, T. G. 1980. Assessing the Local Windfield With Instrumentation. PNL-3622, Pacific Northwest Laboratory, Richland, Washington. 


\subsection{GBOMORPHOLOGCAL NDICATORS OF WND POWER POIENIIAL}

When winds interact with and alter the earth's surface, the geomorphological features that result are called eol ian landforms. Especially in arid regions where vegetation is sparse, winds can erode the surface, transport sand and dust, and deposit sediment. The erosional and depositional eolian landforms and the characteristics of the transported sediments are indicators of the history of the winds that caused these landforms. Since eolian landforms usually exist in areas where people do not live and where wind data are not likely to have been gathered, a correct interpretation of the history ma provide useful auxiliary wind data to assist in wind prospecting.

Geomorphological interpretative techniques can provide information quickly, with minimal effort. For example, significant information can be obtained from satellite data or from high altitude stereoscopic photographs. Although these data are easily accessed, interpretation of these pictures requires an expert in remote sensing imagery. However, sand samples from eolian landforms can be collected on one or more site visits and quickly analyzed.

The information gained using these techniques is at best somewhere between quantitative and qualitative. Erroneous qualitative conclusions can even be drawn if careful attention is not paid to the restrictions of the techniques. For example, proper interpretation of eolian landforms requires substantial a priori knowledge of the regional climatology. Still, these techniques represent easily obtainable input for cross checking one's concepts of regional flow features and may stimulate new ideas about the flow over terrain.

\subsection{BOUAN LANDFORMS}

Eolian landforms are generally either sand dunes or deflation areas. When blowing sand enters an area where either the nature or configuration of the ground or vegetation interferes with the wind, it accumulates into dunes. of the various types of dunes, three are related to topography: 
- climbing dunes, where the wind rises over a sharp topographic break

- falling dunes, where sand is swept over a cliff and falls into a sheltered hollow

- sand sheets or dunes, where the wind, after sweeping sand through a topographic gap, diverges across a wide plain with a consequent slacking of speed. Sand can also accumulate on wide, flat plains to form persistent dunes.

Sand dunes in deserts take different forms (see Figure 7.1 ), and the mechanisms for generating them are complex and not completely understood. The type and quantity of vegetation significantly influence the development of dunes; however, active dunes that experience frequent strong winds do not allow the vegetation to persist and stabilize the dune. In these instances, heat, moisture, sand supply and sand characteristics also influence the development of dunes. Specific dune characteristics only partly reflect wind influences, such as temporal changes in regional wind patterns, differences in dune height and orientation to the wind, differences in position and orientation to the surrounding topography, and wind interaction with different sizes, shapes, and densities of sand grains.

Geflation areas are erosional features, such as blowouts, scour streaks and playas. Blowouts are saucer-shaped hollows formed by wind erosion. Scour streaks are similar to blowouts, but are elongated along the direction of strongest winds. Scour streaks are usually found in dry areas of easily erodible soil; however, their occasional occurrence in nearly rock-hard surfaces is a sure indication of very high wind speeds. Playas are depressions that collect water, as well as the silt and salts transported by water, during the wet season. In the dry season, playas are dry and subject to wind erosion. The alkaline sediments blown out of the playa settle out downwind of the playa.

Weathering processes on the earth's surface act to reduce coarse-grained sediments to fine-grained sediments. The wind transports sediments through three general processes. Fine-grained sediments that do not settle out quickly travel in suspension. Heavy-grained sediments tumble along the surface. Intermediate-sized grains saltate, that is, airborne grains collide with the 
A. BARCHAN
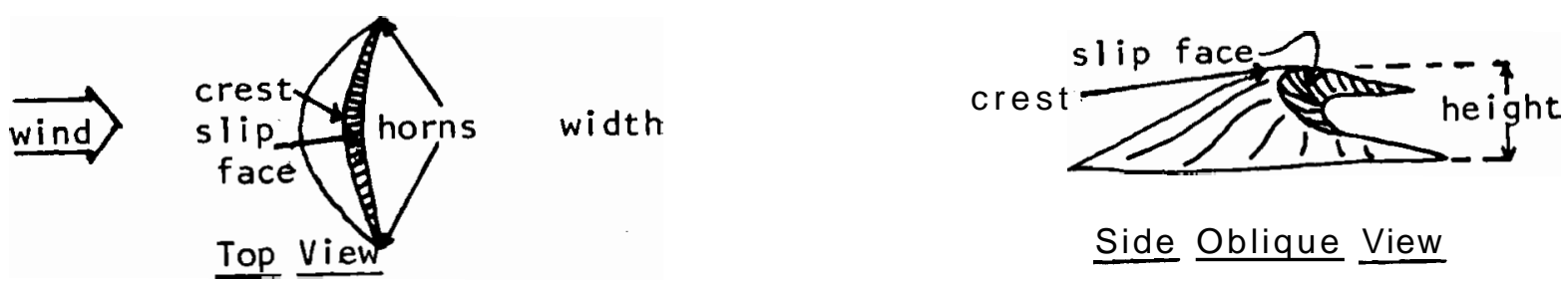

Side Oblique View

B. TRANSVERSE

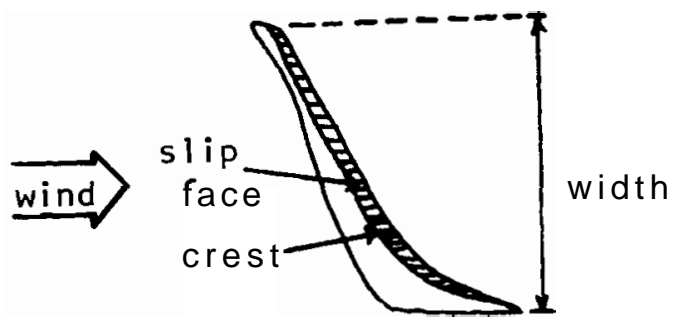

Iop View

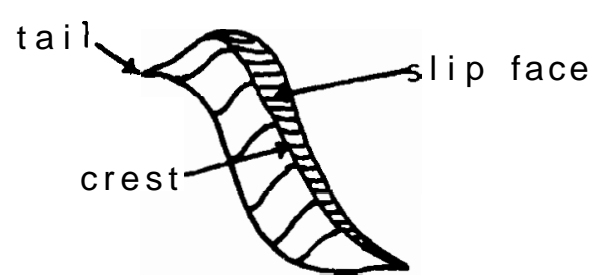

Side Oblique View

c. LONGITUDINAL

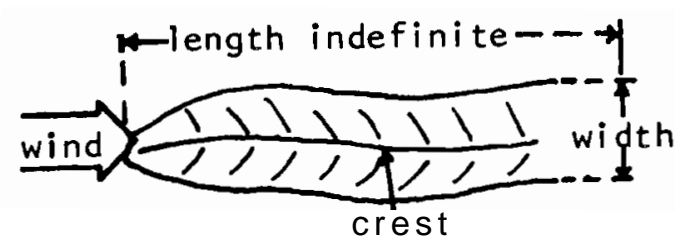

Iop View

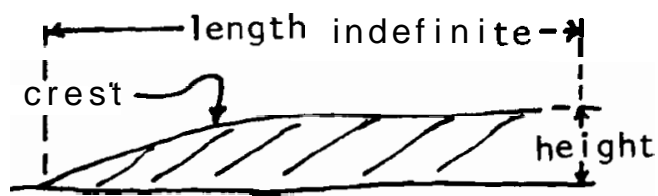

Side Oblique View

\section{PARABOLIC}

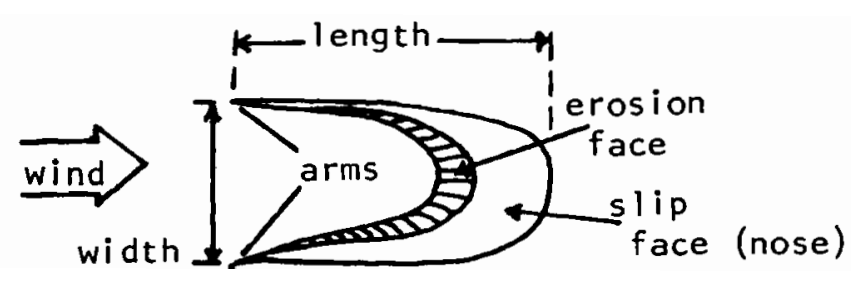

Top View

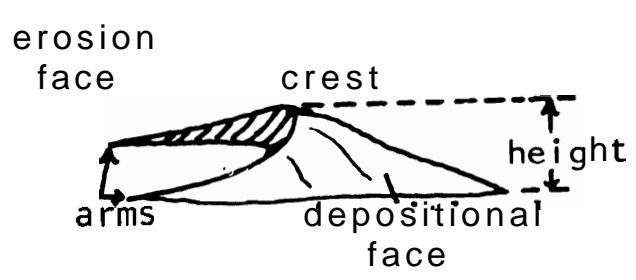

Side Oblique View

FIGURE 7.1. Diagram Showing Plan and Profile Views of Simple Dunes (Marrs and Gaylord 1979) 
surface, which causes the same or new grains to become airborne. The wind accelerates the grains before the next collision with the surface. Since more wind energy is required to transport larger grains than smaller grains, the rate of transport and the characteristics of the transported material should be related to the transporting wind regime.

Usually eolian landforms and transport have been studied as a function of the wind regime (Bagnold 1941, Lettau et al. 1978). However, in this section, the inverse problem is studied: eolian indicators are used to help determine the wind regime.

\subsection{ESTIMATING WIND SPEEDS}

Wind speeds can be estimated from the size distribution of sand grains or from the migration of dunes. However, both approaches require additional information on the area's climatology to ensure the accuracy of the estimates.

\subsubsection{Sand Size Distribution and Wind Speed}

As the wind speed over a sandy surface increases, the surface stress also increases until it is sufficient to begin moving sand. The wind speed at which this occurs is the fluid threshold velocity (Bagnold 1941). However, once grains are saltating, less energy is required from the wind to maintain sand transport because the energy contained in the transported grains that collide with the surface helps perpetuate saltation. The speed necessary to sustain sand transport, once the transport has been initiated, is called the impact threshold velocity (Bagnold 1941).

When the wind speed equals the impact threshold velocity, the following equation is a valid description of the wind profile (see Appendix 2):

$$
U(z)=\frac{u_{\star}}{k} \ln \frac{z}{z_{0}}
$$

where

$$
\begin{aligned}
& U=\text { the wind speed at height } z \\
& u_{*}=\text { friction velocity } \\
& k=0.35, \text { von Karman constant } \\
& z=\text { height above ground } \\
& z_{0}=\text { surface roughness. }
\end{aligned}
$$


The friction velocity $\left(u_{\star}\right)$ represents a velocity scale of turbulence, related to the turbulent momentum flux or stress $(\tau)$ as:

$$
u_{*}=\sqrt{\tau / \rho}
$$

where

$$
p=\text { density of air. }
$$

Given the above, a threshold friction velocity would be $u_{*}$.

If the results of Nikuradse (1933) are used to estimate the roughness length $\left(z_{0}\right)$, the following equation may be used:

$$
z_{0}=d / 30
$$

where

$d$ is the diameter of the sand grain

$1 / 30$ :s a proportionality constant.

If grain sizes vary, the diameter of the coarsest grains is used; i.e., the 95 th percentile of the sand size distribution.

In Bagnold's theory, the threshold friction velocity $\left(u_{* \top}\right)$ depends on the density and diameter of the sand grains; that is,

$$
u_{\star T}=A \sqrt{\frac{\sigma-\rho}{\rho} g d}
$$

where

A is a proportionality constant that equals 0.08 for the impact threshold velocity and 0.1 for the fluid threshold velocity

$\sigma$ is the density of the sand

$g$ is the acceleration of gravity.

A family of curves derived using Equation (7.4) is shown in Figure 7.2. To extrapolate the threshold friction velocity to any height near the surface, use Equation (7.1). 


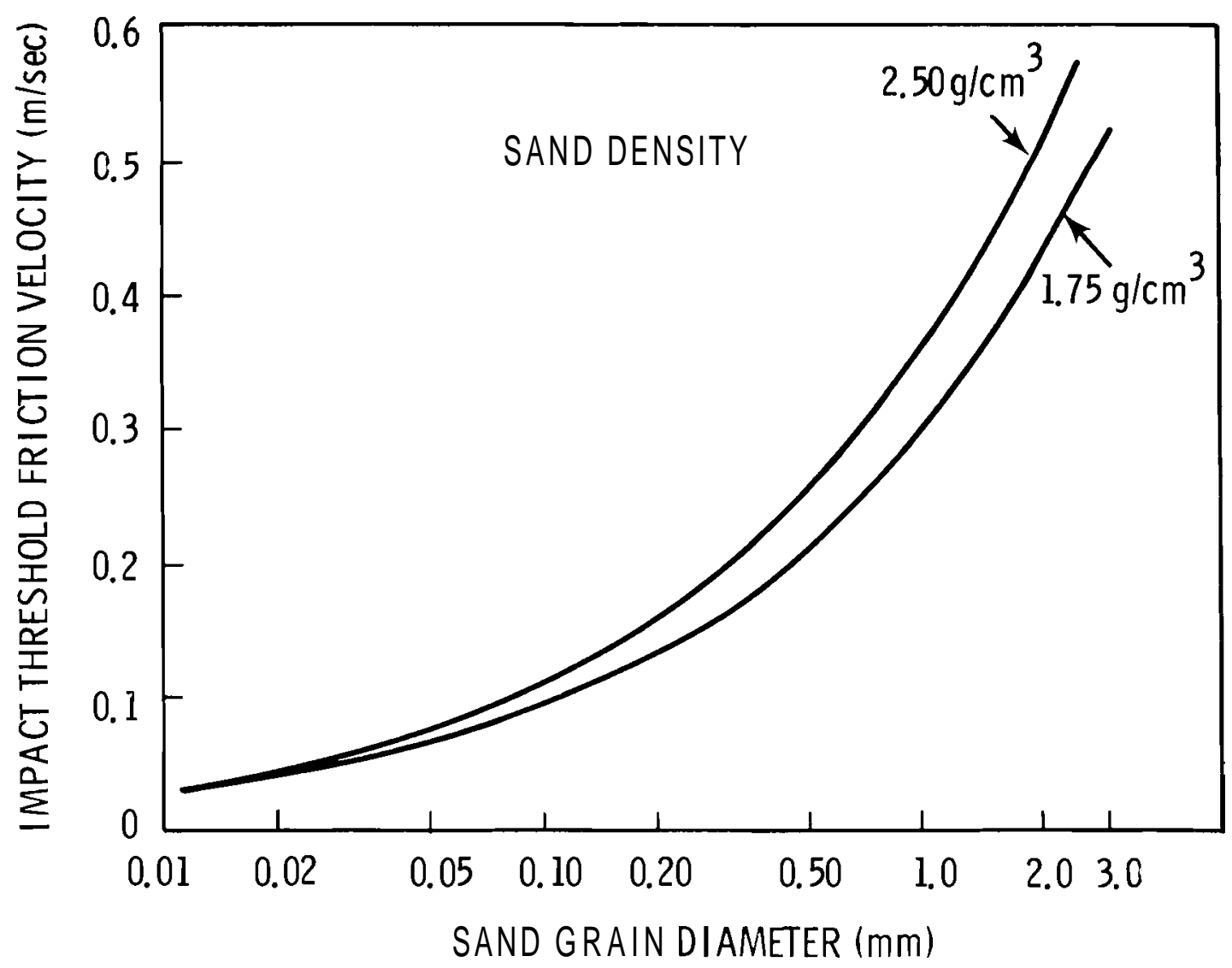

FIGURE 7.2. Threshold Velocity as a Function of Sand Grain Diameter and Sand Density (in $\mathrm{g} / \mathrm{cm}^{3}$ ).

As wind speed increases over a dune surface, the smallest grains begin to saltate, and the surface sand distribution becomes correspondingly coarser as the smaller grains are transported by the wind. When the winds become strong enough, the impact threshold velocity of the coarse grains is reached and the coarse grains begin to saltate. Figure 7.3 shows the behavior of the wind profile over a dune surface for several different wind speeds. Curve $A$ of Figure 7.3 shows the wind profile at the impact threshold velocity of the coarse grains. The velocity profile projects $0 \mathrm{~m} / \mathrm{sec}$ at a roughness height dictated by the size of coarse surface grains. (Note: a straight line in Figure 7.3 indicates a logarithniic wind profile.)

As the wind speed increases more, the intensity of saltation increases as does the drag on the airstream. Curves $B$ and $C$ of Figure 7.3 show that this increase occurs at a rate that limits the wind profile to that of the impact 


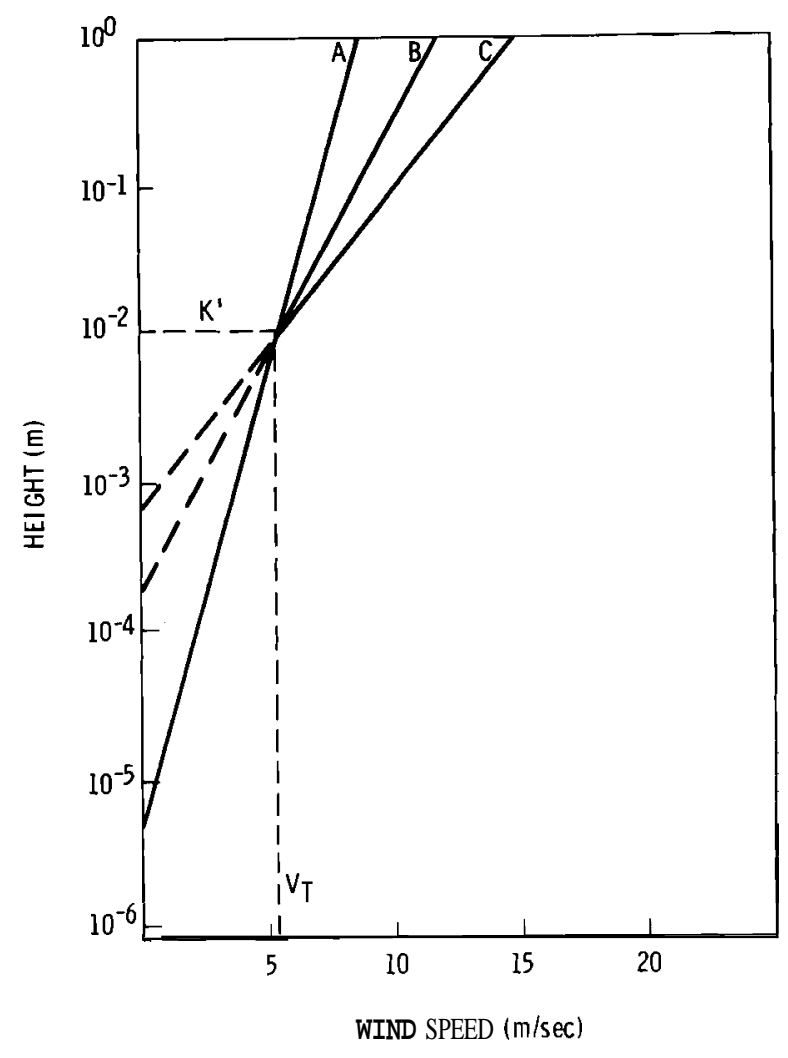

FIGURE 7.3. Structure of Three Wind Profiles Over a Dune. Dashed lines are extrapolations of upper portions of wind profiles in which the wind speeds exceed the impact threshold velocity. Upper profiles meet at a height of $0.01 \mathrm{~m}$ at the threshold velocity $\mathrm{V}_{T}$, and follow impact threshold velocity profile beneath $0.01 \mathrm{~m}$.

threshold velocity below a height that Bagnold calls $k^{\prime}$. This height is on the order of $0.3 \mathrm{~cm}$ for very well-sorted sands and $1 \mathrm{~cm}$ over typical dune sands. A 11 wind profiles focus at the point $\left(v_{T}, k^{\prime}\right)$, which Bagnold calls simply the threshold velocity, $V_{T}$ (as opposed to impact threshold or fluid threshold). Equations (7.1), (7.3), and (7.4) and an estimate of $\mathbf{k}^{\prime}$ can be used to estimate $v_{T}$ as

$$
V_{T}=\frac{A}{k} \sqrt{\frac{\sigma-\rho}{\rho} g d} \ln \frac{30 k^{\prime}}{d}
$$

However if the wind profiles are extended logarithmically to 0 wind speed, the increased intensity of saltation forces the wind profile above $k^{\prime}$ to behave as if the surface were rougher than would be determined from the grain sizes. In 
fact, Bagnold found these effective roughness lengths to be related to the development of sand ripples (see Section 7.2.3).

The smallest sand grains present in an eolian sample are those that are just slightly too large to be picked up and transported away from the dune area in suspension. Bagnold estimates that a horizontal wind velocity that is five times the settling velocity in air for a given grain size will sustain suspension transport. Essentially all mass transport of dune sand occurs below $z=0.1 \mathrm{~m}$. Any sand that is removed by suspension transport must be entrained by the winds near the $0.1-\mathrm{m}$ level. One can therefore look up the settling velocity in air for the minimum grain size and multiply this velocity by five to get an estimate of the horizontal wind velocity at roughly the 0.1-m level. If the $z_{0}$ based on the coarse grain sizes and Equation (7.1) are used, the wind speed can be adjusted to other levels.

How the effects of surface moisture, cohesion, and vegetation change the simple descriptions of sand motion is not clear. Therefore, in the following discussion, the samples are assumed to come from active dunes (i.e., not stabilized by vegetation) in an arid region. If the dunes are to remain active, the sand must move often enough, even though perhaps only at the right time of year, to prevent stabilization of the dune by encroaching vegetation. The time scale of stabilization by vegetation will partially determine how frequently the wind must blow.

The distribution of sand grain sizes shown in Figure 7.4 is typical of the Ferris Dune Field in Wyoming. The distribution reflects the narrow range of wind speeds that preserves the distribution. If the mean winds were to increase in strength, the smaller particles would be carried away and the size distribution would shift toward the coarser fraction. If the mean wind speed were to decrease, the coarser sands could not be moved, finer grained sands formed by weathering wo 't accumulars and the size distribution would shift toward the finer grained fraction (Marrs and Gaylord 1979).

To obtain grain size distributions, sieve techniques can be used, but a preferred technique is to measure the settling velocities in water. However, if sieve techniques are used, corrections must be made to account for grain 


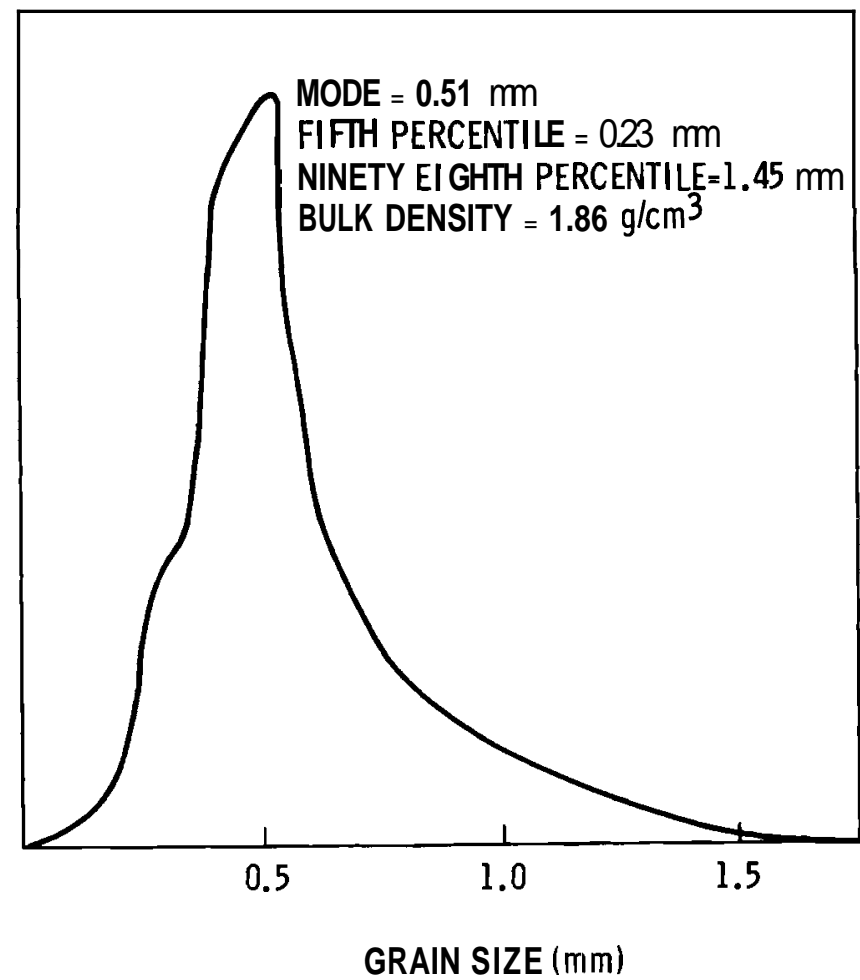

FIGURE 7.4. Grain Size Distribution of a Typical Sample of the Wyoming Ferris Dune Field (Marrs and Gaylord 1979)

shape and specific gravity (Bagnold 1941). Figure 7.5 compares the settling velocities of quartz spheres in water and in air.

Wind speed estimates related to the saltation of sand, or to the suspension transport of sand, for different grain sizes, can provide estimates of the wind speeds that can form and maintain sand size distribution (Marrs and Gaylord 1979). The threshold wind speeds necessary to initiate saltation of a certain-sized grain of sand are calculated using Equations (7.1) and (7.4).

The wind speed required to maintain suspension transport of a given grain size is five times the settling velocity, which is derived from Figure 7.5. If assumptions are made about the relation between the sand transport process and the features of the sand size distribution, such as size of the coarsest, finest, or modal grains, then a qualitative estimate of the frequency of occurrence of a given wind speed can be made. The following paragraphs discuss estimates of wind speeds based on the sand size distribution in Figure 7.4. The estimates are summarized in Table 7.1. 


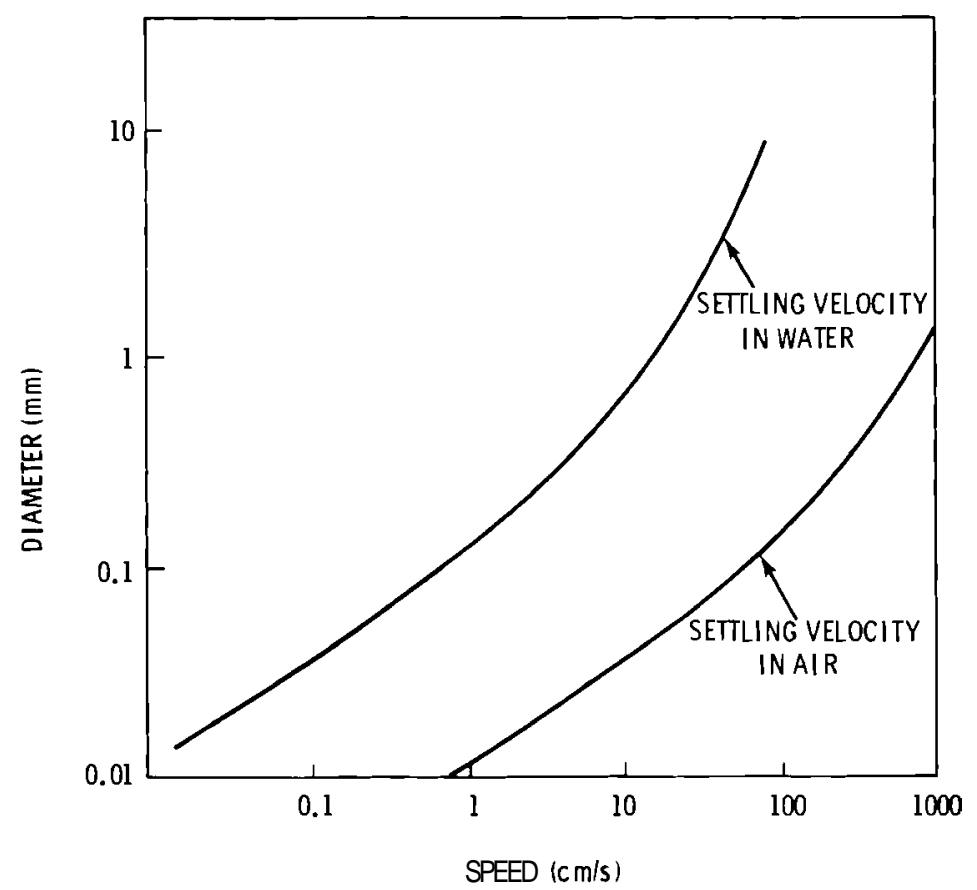

FIGURE 7.5. Settling Velocities in $\mathrm{cm} / \mathrm{sec}$ of Quartz Spheres in Water and Air (From Rouse) 1937)

\section{TABLE 7.1. Wind Speeds Inferred From Eolian Sand Size Distribution Shown in Figure 7.4. All units are SI.}

\begin{tabular}{|c|c|c|c|c|}
\hline $\begin{array}{l}\text { Physical Link } \\
\text { To Eolian } \\
\text { Processes } \\
\end{array}$ & $\begin{array}{l}\text { Approximate Mode Of } \\
\text { Sand Moving Wind } \\
\text { Energy Distribution }\end{array}$ & $\begin{array}{r}\text { Frequ } \\
\text { To } \\
\text { Acti }\end{array}$ & $\begin{array}{l}\text { Enough } \\
\text { ain } \\
\text { unes }\end{array}$ & $\begin{array}{c}\text { Occasional } \\
\text { Extreme Winds }\end{array}$ \\
\hline $\begin{array}{c}\text { Method of } \\
\text { Calculation }\end{array}$ & $\begin{array}{c}\text { Threshold of } \\
\text { Modal Size Grains } \\
\end{array}$ & $\begin{array}{l}\text { Threshold of } \\
\text { Largest Grains }\end{array}$ & $\begin{array}{c}\text { Suspension of } \\
\text { Smallest Grains } \\
\end{array}$ & $\begin{array}{c}\text { Suspension of } \\
\text { Modal Size Grains }\end{array}$ \\
\hline$u_{\star T}$ & 0.22 & 0.38 & & 0.38 \\
\hline$z_{0}$ & $4.8 \times 10^{-5}$ & $4.8 \times 10^{-5}$ & $4.8 \times 10^{-5}$ & \\
\hline $\begin{array}{l}\text { Settling } \\
\text { Velocity }\end{array}$ & & & 1.6 & 3.8 \\
\hline $\mathrm{U}_{0.1}$ & & & 8.0 & 19.0 \\
\hline $\begin{array}{l}V_{T}=V_{T}(z=0.01, \\
\left.u_{\star T}, z_{0}\right)\end{array}$ & & & & 5.8 \\
\hline Effective $u_{\star}$ & & & & 2.0 \\
\hline Effective $z_{0}$ & & & & $3.6 \times 10^{-3}$ \\
\hline$U_{10}$ & 7.7 & 13.3 & 12.8 & 45.0 \\
\hline Uncertainty & $?$ & $?$ & $?$ & $?$ \\
\hline $\begin{array}{c}\text { Frequency of } \\
\text { Occurrence }\end{array}$ & & $?$ & & $?$ \\
\hline
\end{tabular}


The mode of the sand distribution must be maintained by, and reflect something like, the mode of the "sand-moving" wind energy distribution. (The mode of the sand-moving wind energy distribution is not necessarily the same as the mode of the wind energy distribution.) Therefore, a calculated threshold velocity based on the mode of the sand size distribution is an indicator of the most frequent sand-transporting winds. (This estimate for the Ferris Dune Field is shown in Table 7.1). However, this information might mean that the sand-moving winds are very vigorous but only occur in 1 or 2 months of the year. Or, it may mean that high winds occur more frequently when the ground is wet and/or frozen.

Sand that is taken up in suspension transport is removed quickly. The complete removal of the very small grains formed by weathering is a necessary condition for maintenance of active dunes. Frequent motion of the coarse grains is also necessary to maintain active dunes. Therefore, the suspension and removal of small grains is probably due to wind speeds near those that activate saltation of the coarse grains, and the wind speed estimates based on the threshold velocity of the coarse grains and the suspension of the small grains should be fairly close to each other (see Table 7.1).

When occasional extreme winds occur, larger grains of sand can be suspended and removed. Abnormally high winds cannot occur very often without removing small-to-medium sized grains, thereby pushing the mode of the size distribution toward the coarser fraction. Therefore, an estimate of the wind speeds necessary to suspend sand grains just smaller than the modal size will give an indication of the extreme wind speeds in an area. However, in this situation the surface roughness is increased and the wind profile behaves like curve $\mathrm{C}$ of Figure 7.3. I I this case, to adjust the $0.1-\mathrm{m}$ velocity estimate to some other height one should estimate $V_{\mathrm{T}}$ from Equation (7.5) and assume a $k^{\prime}$ to determine a $z_{0}$ to use in Equation (7.1). This makes the estimate of the extreme winds higher than if the roughness were due to coarse grains. This estimate is also listed in Table 7.1.

The numbers shown in Table 7.1 seem reasonable for sand-moving winds in a Wyoming dune field. However, no good way exists to estimate the frequency of 
occurrence of winds in these speed ranges. Although winds of these speeds probably do occur, some external indication of the regional climatology needs to be used, along with an understanding of the local ecology, to judge whether these wind speeds are in any way indicative of year-round wind energy potential.

\subsubsection{Dune Migration and Wind Speeds}

Following Bagnold's theory, the mass transport rate of wind-blown sand (q) is :

$$
\begin{aligned}
q & =a u_{*}^{3} \text { for } u^{u_{1}} \boldsymbol{J}_{T} \\
& =0 \quad u^{*}<U_{T}^{*}
\end{aligned}
$$

where

$$
\alpha=c \frac{\rho}{g} \sqrt{\frac{d}{D}}
$$

and

$$
\begin{aligned}
u_{T}= & \text { impact threshold friction velocity } \\
u_{\star}= & \text { friction velocity } \\
C= & 1.5 \text { for nearly uniform sand, } 1.8 \text { for typical dune sand and } 2.8 \\
& \text { for sand with a wide range of grain sizes } \\
d= & \text { diameter of modal-sized grains } \\
D= & 2.5 \times 10^{-4} \mathrm{~m} \\
\rho= & \text { air density } \\
g= & \text { gravitational acceleration. }
\end{aligned}
$$

There are alternative theories or descriptions as well (Lettau et al. 1978). Kawamura (1951) suggests:

$$
q=c_{1} \frac{\rho}{g}\left(u_{\star}-u_{\star}\right)\left(u_{\star}+u_{\star}\right)^{2} \text {. }
$$

Based on wind tunnel experiments with relatively uniform sand of $4.4 \times 10^{-4} \mathrm{~m}$ grains and where $u$ was experimentally found to be about $0.30 \mathrm{~m} / \mathrm{sec}$, Belly (1962) estimates $C_{1}^{\top}=3.1$. 
Lettau (1978) suggests another formula:

$$
q=c_{2} \frac{\rho}{g} \frac{d}{D}{ }^{n} u_{*}^{2}\left(u_{\star}-u_{\star T}\right)
$$

Lettau showed this equation fit the data of Belly (1962) well if $C_{2}(d / D)^{n} \rho / g=6.5$, but provided no means to generally determine $n$ or $C_{2}$. For the remainder of this discussion Bagnold's description (Equation 7.6) will be used. However, it is worthwhile noting that for wind speeds near the threshold velocity Equation (7.6) produces the highest estimate of the sand transport rate, or conversely, for a given sand transport rate Equation (7.6) implies the lowest value of $u_{*}$.

The magnitude of the average sand transport rate vector $\langle\vec{q}\rangle$ can be approximated by observing net dune migrations over a period of years, using the following equation

$$
\langle\vec{q}\rangle=\frac{x \sigma H}{t}
$$

where

$\mathrm{x}=$ distance of dune migration

$\mathrm{a}=$ bulk sand density

$\mathrm{H}=$ height of dune

$\mathbf{t}=$ time interval between observations.

If the wind always blows in the same direction at the same speed and the dune never becomes stabilized, Equation (7.6) could be used to determine the wind speed from the observed transport of sand. However, Equation (7.6) shows that a few episodes of high winds can move a great deal of sand. In addition, winds that blow back and forth move sand through a larger distance than the net migration suggests. Therefore, Equation (7.6) is not appropriate for estimating wind speed as a function of sand transport since the conditions required for it to be accurate rarely exist in nature.

Equation (7.6) can be used with a wind distribution to derive a relation between $\langle\vec{q}\rangle$ and the long-term mean wind speed $(\bar{V})$. This is done by evaluating the integral 


$$
\langle\vec{q}\rangle=\int_{u_{\star}}^{\infty} \int_{0}^{2 \pi} \int_{0}^{1} \vec{q}\left(u_{\star}, \theta, A\right) p\left(u_{\star}, \theta, A\right) d A d \theta d u_{*}
$$

where

$\vec{q}\left(u_{\star}, \theta, A\right)$ is the vector rate of sand transport as a function of wind speed $\left(u_{\star}\right)$, wind direction $(\theta)$, and dune activity $(A)$;

A is a function ranging from 0 to 1 indicating the degree of the dune's activity as influenced by vegetation and moisture;

$p\left(u_{\star}, \theta, A\right)$ is the normalized joint probability density function.

The integration over wind speed starts at $u_{T}$ because for wind speeds smaller than $U_{*_{T}}$ no sand is moved. For a given wind speed distribution, the effects of wind ${ }^{\top}$ direction and periods of diminished dune activity only reduce the value of the integral in Equation 7.9. That is, if we estimate $\langle\vec{q}\rangle$ as

$$
\langle\vec{q}\rangle=\int_{u_{\star}}^{\infty} q\left(u_{*}\right) p\left(u_{*}\right) d u_{\star}
$$

where the vector sand transport rate is replaced by the scalar sand transport rate (e.g., using Equation 7.6), then the value of $\langle\vec{q}\rangle$ obtained in Equation (7.10) will be greater than or equal to that obtained using Equation (7.9). The converse is more important. If one knows the shape of the wind speed distribution (i.e., Rayleigh, Weibull, etc.), then a measured value of $\langle\vec{q}\rangle$ must indicate the minimum mean wind speed in a dune area.

If the distribution of friction velocities is a Rayleigh distribution, (a) then the integral in Equation (7.10) can be solved analytically (see Appendix 4). The results are easiest to display graphically using the following equation:

$$
\langle\vec{q}>=a u, f(\beta)
$$

(a) The quality of this assumption is highly suspect. The general skewed shape of the Rayleigh distribution is no doubt appropriate; however, the results will be sensitive to the shape of the tail of the distribution of which little is known. Nevertheless, the Rayleigh distribution is a convenient one-parameter distribution. 
where $\beta=\bar{u}_{\star} / u_{*}, \bar{\varphi}$ is the long-term average friction velocity and $f(B)$ is shown in Figure 7.6. To obtain an estimate of the minimum mean wind speed, take the estimate of $\langle\vec{q}\rangle$ obtained from dune migration rates, analyze the sand so that a and $u_{*_{T}}$ can be estimated, solve Equation (7.11) for $f(\beta)$, look up $B$ in Figure 7.6, and multiply $\beta$ by $u_{\star_{T}}$ to get $\bar{u}_{*}$. Given an estimate of $\bar{u}_{\star}$ and $k^{\prime}$ (see Figure 7.3), an estimate of the mean wind speed at some height near the surface can be made using

$$
\bar{V}(z)=v_{T}+\frac{\bar{u}_{\star}}{k} \ln \frac{z}{k^{\top}}
$$

For small $\beta$, errors in the estimate of $\langle\vec{q}\rangle$ become large errors in the estimate of $\bar{V}$.

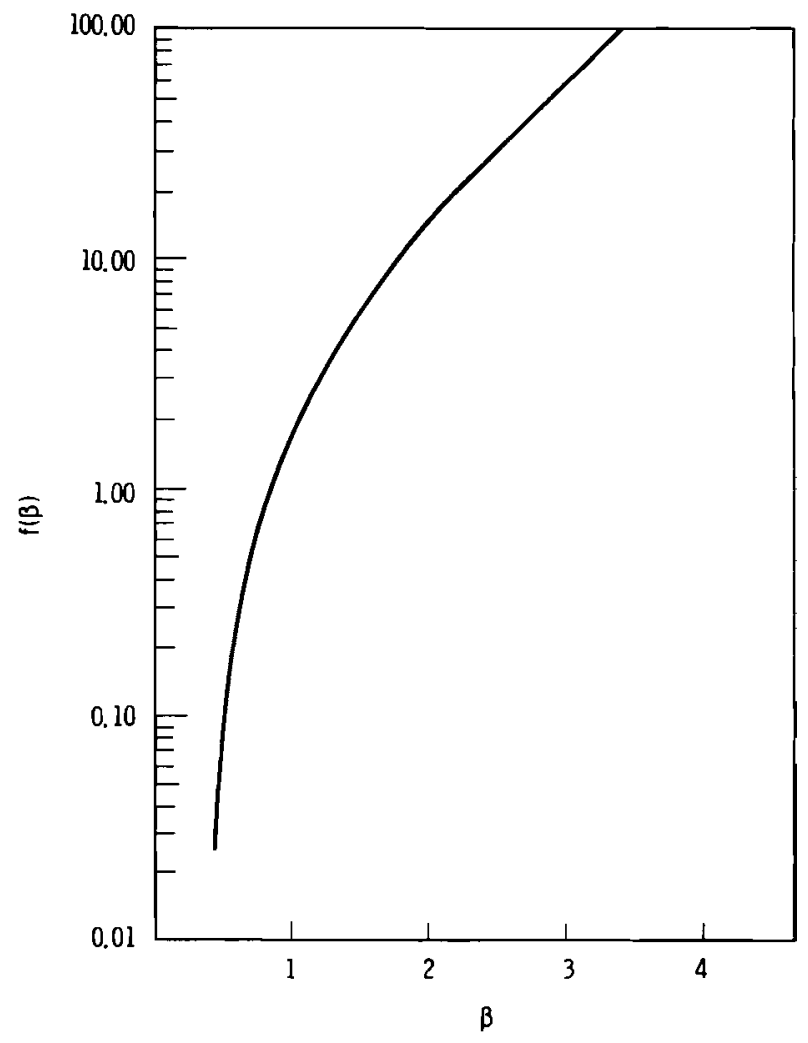

FIGURE 7.6. Assuming a Rayleigh Distribution of Wind Speeds, Equation 7.11 Shows That the Average Sand Transport Rate Depends on $f(B)$, Which is Plotted Here and Described Analytically in Appendix 4. 
The relation between an assumed Rayleigh distribution mean wind speed and the observed net rate of transport of sand have been computed for the Ferris Dune Field. Marrs and Gaylord (1979) observed $\langle\vec{q}\rangle=8.9 \times 10^{-3} \mathrm{~kg} \mathrm{~m}^{-1} \mathrm{sec}^{-1}$ for these dunes. This implies a minimum annual mean friction velocity of $0.25 \mathrm{~m} / \mathrm{sec}$ and a minimum annual wind speed at $10 \mathrm{~m}$ near $11 \mathrm{~m} / \mathrm{sec}$. Marrs and Gaylord had additional data that indicate that roughly $75 \%$ of the winds above the threshold velocity are westerly and the remaining $25 \%$ are easterly. Thus, the net sand movement is about one half of the total sand movement. Given this supplementary data, $\langle\vec{q}\rangle=1.8 \times 10^{-2} \mathrm{~kg} \mathrm{~m}^{-1} \mathrm{sec}^{-1}$ and the estimates of the minimum mean wind speed would be raised to about $12 \mathrm{~m} / \mathrm{sec}$. This estimate could be raised more if the wind is strong but the sands do not move because of moisture or frozen surfaces. The accuracy of Bagnold's theory and the assumption of the Rayleigh distribution determine the accuracy of the estimate.

\subsubsection{Wind Speed and Other Eolian Activities}

Wind blowing over sand organizes the sand into ripples, transverse to the wind direction. Bagnold (1941) related this process to the mean trajectory of saltating sand grains. That is, when a surface grain becomes airborne as a result of a collision with other saltating sand grains, it rises nearly vertically from the surface, picks up forward momentum from the wind (thereby causing drag on the windstream), and collides with the surface again one ripple wavelength downstream. The wavelength of the ripples then depends upon the momentum transfer from the air to the saltating sand. The momentum transfer in turn depends upon the friction velocity $\left(u_{*}\right)$. As the wind speed increases, the wavelength increases. Bagnold (1941) found, however, that when the wind speed reaches about three times the threshold velocity $\left(V_{T}\right)$, the ripple flattens out and disappears. Sharp (1963) found that both ripple height and spacing are influenced by grain size. Coarse or poorly sorted sands form higher ripples than we11-sorted, fine-grained sands.

Ripple observations as a wind energy siting technique are very limited, although ripples can provide clues for interpreting local topographic enhancements. For example, after a strong wind event from the major power-producing wind direction in areas of similar sands, areas where ripple wavelengths are 
1arger would have topographically enhanced wind speeds. This technique only works after a frontal passage where winds die down quickly or where precipitation deactivates the sand quickly, so the record of the peak winds is preserved. If the wind dies off slowly, the ripples at all locations will indicate the dying winds that were just strong enough to maintain sand motion, and all areas will show the same final wavelength, assuming the sand type was the same in all locations. Alternatively, the spatial variations in ripple wavelengths at the height of a wind event could be observed to estimate wind speeds. The only advantage this method could have over making short-term (say 10 minute) handheld anemometer measurements at several locations is that ripples may represent a wind speed average of an hour. However, this has not been established yet.

Since sand ripples result from the most recent sand-moving winds, they are not useful for making estimates of the long-term wind speeds. Furthermore, they are subject to the severe constraints as discussed above, when used as indicators of short-term spatial variations in wind speed. In most instances, a wind prospector should simply be cognizant of the physics of sand ripples and incorporate sand ripple indicators only if other indicators or measurements are lacking.

Deflation features, such as playas, sometimes provide qualitative indicators of wind speed. Carlisle and Marrs (1978) noted that in regions where playas are forming, the playas are more numerous and more closely spaced in regions of higher wind speeds. The length of the sediment plume downwind of a playa is also longer in areas of higher wind speeds. However, both of these indicators are sensitive to vegetation, moisture, and soil type, so they must be used cautiously (Marrs and Gaylord 1979).

\subsection{BOUAN NDICATORS OF WND DIRECTION}

Eolian landforms that form in regions of persistent winds are indicators of wind direction. Each type of sand dune shown in Figure 7.1 is related to the wind direction in a specific way. Barchan, transverse, and parabolic 
dunes are asymmetric, form perpendicular to the dominant wind direction, and indicate a primary direction of flow. Barchan dunes are distinguishable from parabolic dunes because parabolic dunes are in transition between active and stabilized states, so the arms are stabilized with vegetation. The horns on a barchan dune are fully active. The transverse dune has a slip or avalanche face on the downwind side. Longitudinal dunes, which lack the slip face of the transverse dune, are symmetrical. This symmetry may be caused by the wind blowing from two directions (Marrs and Gaylord 1979).

Simple dunes (see Figure 7.1) form only when the sand-moving winds are nearly unidirectional. When two or more wind directions are prominent, the dunes will take on complex forms. Even so, dunes respond in a predictable fashion (Marrs and Gaylord 1979). If the wind direction varies seasonally, dunes in the most active portion of the dune field will begin to change their orientations as the winds shift from season to season.

Deflation areas can also be indicators of wind direction. The downwind sediment plumes of playas are obvious indicators. In addition to these plumes, playas tend to develop (over time) a long axis normal to the responsible wind direction. The long axis is a useful indicator when the sediment plume has been rendered unobservable by cultivation or other cultural activities (Marrs and Gaylord 1979). Progressive wind erosion, such as migration of blowouts, forms scour streaks. The long direction of these features lies along the dominant wind direction.

Patterns of snow accumulations can indicate prevailing wind directions. Cornices, or buildups of snow that sometimes become overhangs, form on the lee sides of ridges. The windward side of the ridge may be blown clear of snow, although one must be careful not to confuse these effects with the processes of sublimation and runoff.

Wind direction information obtained from eolian landform indicators can be plotted on topographic maps to help determine regional wind patterns. In regions where the topography is gently sloping but the features have horizontal length scales larger than $10 \mathrm{~km}$, such as might be found in the high plains 
states, it is not easy to estimate the effects of topographical control on the flow. Eolian direction indicators can supply significant information for this.

\subsection{CONCLUSIONS AND RECOMMENDATIONS}

Wind, as a geomorphological agent, can create eolian landforms from which the wind history over a wide range of time scales can be interpreted. Eolian landforms and their changes in time, such as dune migration, can be examined, using satellite remote-sensing and aerial photography. Both of these data have been collected and archived for most of the United States (see Chapter 10). Quick access to the data and the rapid interpretation of eolian landforms by a trained expert are the advantages that justify spending time investigating eolian landforms as indicators of wind power potential.

Eolian features, whether they are depositional such as sand dunes, or deflation features such as blowouts or playas, indicate substantial winds. However, these features can be created by a few wind events. For this reason, identified eolian features should be confirmed as currently active features by site visits. Sand grain size distributions may be analyzed for indications of wind speeds that occur with a significant, although indeterminate, frequency. Dune migration rates, observable through differences in past and current aerial photographs, provide an indicator of mean wind speed. Regular observations of dune position provide information on the temporal variability of the wind resource that may be of value in interpreting the climatological representativeness of a given year of data. Under some very restrictive conditions, sand ripple wavelengths, which are longer at high wind speeds than at lower wind speeds, can be used as a short-term indicator of local topographic enhancement of winds. Due to the great uncertainties in the use of eolian features for estimating wind speeds, the estimates should be regarded as relative indicators rather than indicators of absolute wind speeds.

Mean wind directions and mean wind patterns in a region can be mapped on topographic maps using eolian landforms as wind direction indicators. However, 
the temporal nature of the geomorphological processes should be reviewed before a wind pattern is described. Consider this example: two broad valleys merge. One valley runs down from the northwest to the southeast and the other runs down from the southwest to the northeast. The sand dunes in the northern valley are active during the fall when northwesterly winds blow down the valley. In the southern valley, the northwesterlies blow across the valley in the fall. As a result the winds are light down in the valley and the sand dunes are not being moved by the wind. In the spring, the situation is reversed with the southern valley dunes being moved by the southwesterly spring winds along the valley while the northern valley is sheltered. To conclude that a large confluence and possibly an acceleration of airstreams occur at the junction of the valleys would be erroneous. Therefore, one must be careful to determine that eolian landforms being used to describe a wind pattern were formed at the same time and do not represent components of several wind patterns.

Information gained from the use of geomorphological indicators is sometimes helpful in forming an integrated picture of the wind regime in a datasparse area. However, the great uncertainty of the technique should be compared with the effort expended. Aerial photographs can be obtained (see Chapter 10) during the region of interest analysis and candidate resource area evaluation stages of the wind prospecting strategy (see Chapter 2) for purposes other than for examining eolian landforms. If eolian indicators are found in the photographs, interpret the photographs as quickly as possible and use techniques described in this chapter only as part of site visits that include other activites, e.g., setting out anemometers or inspecting terrain feasibility. In this way, information can be added to the data base of the site without undue delay and expense. 


\section{REFERENCES}

Bagnold, R. A. 1941. The Physics of Blown Sand and Desert Dunes. William Morrow and Co., New York. 1973 ed., John Wiley and Sons, New York.

Belly, P. Y. 1962. Sand Movement by Wind. Technical Report, Hydraulic Engineering Laboratory, Wave Research Projects, University of California, Berkeley, California.

Carlisle, W. J. and R. W. Marrs. 1978. Airflow Over Part of the Southern High Plains Interpreted From Landsat. RL0/2343-78/4. Available from National Technical Information Service, Springfield, Virginia.

Kawamura, R. 1951. Study on Sand Movement by Wind. Volume 5 . Institute of Science and Technology, University of Tokyo, Japan.

Lettau, H. H. and K. Lettau (eds,). 1978. Exploring the World's Driest Climate. IES-101, Center for Climatic Research, Institute for Environmental Studies, University of Wisconsin, Madison, Wisconsin.

Marrs, R. W. and D. R. Gaylord. 1979. A Guide to the Interpretation of Windflow Characteristics From Eolian Landforms. RLO/2343-79/2. Avai 1able from National Technical Information Service, Springfield, Virginia.

Nikuradse, J. 1933. "Stromungs gesetze in rauhen Rohren." VDI-Forschungsheft (361).

Rouse, H. 1937. Nomogram for the Settling Velocity of Spheres, Exhibit D. Prepared for the Committee on Sedimentation by the National Research Council, Division of Geology, Washington, DC.

Sharp, R. P. 1963. "Wind Ripples." J. Geol. 71:617-636. 


\subsection{SOCIAL AND CULTURAL INDICATORS OF WND POWER POIENIIAL}

Valuable supplementary wind input can be inferred from human interaction with, and behavior changes because of, the wind. Techniques using social and cultural indicators to infer information about wind are highly subjective and difficult to quantify. The information derived from these techniques is not absolute and should not be a replacement for wind data. Instead, the information should be integrated into the wind prospector's qualitative feel for a given region.

These techniques do not and should not require a great commitment of time and effort. Instead, these techniques require an attitude of continual observance for clues of wind effects. The indicators discussed below give a glimpse of the wide range of clues that the astute observer might be able to recognize.

First, people do not like to live in high wind areas. If a town or home is sited in an obviously sheltered location and other conveniences such as water access or road service are less available than if the town or house were located in a less sheltered area, the area surrounding the sheltered location may be windy. Often, people grow shelterbelts on the upwind side of an area they wish to protect, if they cannot avoid windy areas.

Land use can also indicate wind characteristics. Land cannot be cultivated for long in a windy area before the land will no longer sustain crops. Therefore, in regions of mixed open rangeland and cultivated fields, the range areas are more likely to be windy. The border between crop and rangeland can also be noted on small space scales, as when cultivation exists in small protected areas or on the lee sides of small ridges but not on the windy crests of ridges.

Past use of wind power for local water pumping or small-scale electric generation may indicate a wind resource. Water pumpers were often sited down in small valleys and ravines, because those sites were nearer the water table. If there was enough wind for water pumping in these poorer sites, then the surrounding high ground is probably a viable resource area. Snow fences may also indicate windy areas. In semi-arid regions snow fences are used to control drifting snow. 
Signs along roadways often reflect wind potential. "Blowing sand" or "blowing snow" warning signs probably indicate local topographic enhancement of winds. "High winds likely" or "gusty winds likely" indicate strong turbulent winds that are probably more a hazard to avoid than a boon. State highway patrols can be a source of information on wind because of their continual concern with road conditions. Accidents where trucks carrying light bulky loads (such as mobile homes) have had their loads blown off may be considered along with the concurrent synoptic conditions. The extrapolation of the information contained in these roadside indicators to the surrounding off-road areas requires an experienced meteorologist.

Wind damage to buildings, billboards, power lines, etc, can also be considered. Damage to some structures may indicate extreme wind speeds or extreme turbulence to be avoided. On the other hand, some structures may be poorly situated in locations where turbulence is particularly enhanced. In the latter case, surrounding areas could have strong but more benign wind.

Long-time residents of an area, especially those that work outdoors and cover a Targe territory, can sometimes provide useful information. However, people tend to remember specific discrete wind events rather than average conditions. Even so, a resident may be able to say with considerable confidence that region $\mathrm{x}$ has more wind than region $\mathrm{y}$. If region $\mathrm{y}$ is near an existing anemometer, then some useful information may be available to incorporate into the candidate resource area evaluation (see Chapter 2).

Several social and cultural indicators of wind power potential have been discussed, but the list could be expanded. The sharp observer will look for, recognize, and evaluate the information provided by these indicators. For example, consider two siting engineers traveling to a proposed site. One is an alert observer and notices many social and cultural indicators along the way. The second thinks only about getting a look at "the site." The first engineer is in a better position than the second to integrate information obtained from the region into a broader perspective of the nature of the site's wind resource. Although these indicators are difficult to quantify, they do contribute to the understanding of the wind flow in a region and near a potential WES site. 


\subsection{WIND TURBINE WAKES AND CLUSTER DESIGN}

To exploit significant quantities of wind energy effectively, utilities will need to site large numbers of wind turbine generators. These machines will be arranged in clusters, since land availability, the size of wind resource areas, the costs of installation and maintenance will favor siting in compact groups. Siting several machines in a limited area requires knowledge of how closely wind machines can be placed and of how the machines' geometric configuration affects overall cluster performance. If a cluster is well-designed, problems detrimental to performance can be avoided.

Wakes, which are created when an object is placed in a flow field, are characterized by a lower mean wind speed, a modification to the wind profile and a change in the turbulence structure. (a) Wakes affect wind turbine performance by reducing:

- energy production because the available kinetic energy in the wake is less than in an undisturbed flow

- energy production because wake turbulence may adversely affect aerodynamic performance

- operational life because wake turbulence may affect structural integrity.

The turbulence in the wake and in the ambient flow eventually eliminates the disturbance and the flow returns to the characteristics of the free stream. How quickly and how completely this restoration to free stream conditions occurs is the major determinant of cluster design.

When air flows through a wind turbine, two things happen: a fraction of the available kinetic energy in the air stream is removed by the turbine and converted to shaft work, but a portion of the ordered kinetic energy of the mean flow is converted into the more random motions of turbulence. Both of these processes reduce the available kinetic energy in the wake flow.

(a) For a thorough review of wind turbine wake problems and an extensive bibliography, see Riley et al. 1980.

(b) Technically, the existence of turbulence in a flow reduces thermodynamic availability by only a small amount. This reduction in availability is due to the conversion of kinetic energy to heat by the fluid's viscosity. If the wind turbine were small enough and responsive enough, the kinetic energy of turbulence could be converted to shaft work. 
Turbulence in the wake has many sources. Some is produced in the boundary layers that form on turbine blades. Some turbulence results from the vortices shed by the blades, and some is generated by air flow separating from the turbine nacelle and support structure. Finally, some turbulence is created by the mean velocity gradient of the wake itself.

The wake of a wind turbine can be divided into three regions. Near the turbine, the wake is very nanhomogeneous; most of the air passes undisturbed through the plane of the wind turbine rotor and flow perturbations are concentrated in the wakes of the rotor blades and the turbine support structure. The evolution of the near wake is governed by the turbulence characteristics of the wake itself. As flow proceeds downstream of the wind turbine, the individual wakes merge, marking the beginning of the mid-wake region. In the mid-wake region, wind speed and turbulence profiles are smoother, but the shapes of these profiles change. Here, the wake is changing in response to wake turbulence and the turbulence characteristics of the undisturbed flow. The structure of the wake continues to evolve as wake turbulence levels come into equilibrium with turbulence production rates associated with the local mean velocity gradient. Once this equilibrium is achieved, the far-wake or region of self-similarity is reached. In the self-similar region, the shape of the mean velocity or turbulence intensity profiles remains constant with increasing downstream distance; only the magnitude of the wake disturbance changes. In the region of self-similarity, the wake has only a weak memory of how it was created and its decay is largely governed by the ambient turbulence. (a)

\subsection{MODELING SINGLE TURBINE WAKES}

Modeling the behavior of the near and mid-wake is very difficult since the structure and evolution of each of the individual wakes making up the flow must be accurately described. In these regions, wake behavior will be very dependent on the characteristics of the wind machine that formed it. Modeling the far wake is much easier, since the details of the wake structure in this

(a) See Section 9.2.2 for more discussion of this point. 
region are nearly independent of how the wake was formed. In the self-similar region, the wake of a wind turbine decays in the same manner as the wake of a bluff body or as a nonbuoyant jet. Thus, the results of experiments on flows that bear little resemblance to the flow through a wind turbine (but produce similarly-looking wakes) can be applied to the problem of wind turbine wakes.

\subsubsection{Numerical Modeling of Wind Turbine Wakes}

Recently, several approaches have been used in modeling wakes of a wind turbine (Lissaman 1977, Walker and Lissaman 1978, Sforza et al. 1979, Crafoord 1979, and Taylor 1980). Of these approaches, Lissaman's model is the most developed, and it will be discussed in some detail. Lissaman's model is based on the presumed analogy between a wind turbine wake and a jet-like flow (see Figure 9.1 for a schematic of the physical assumptions inherent in the model).

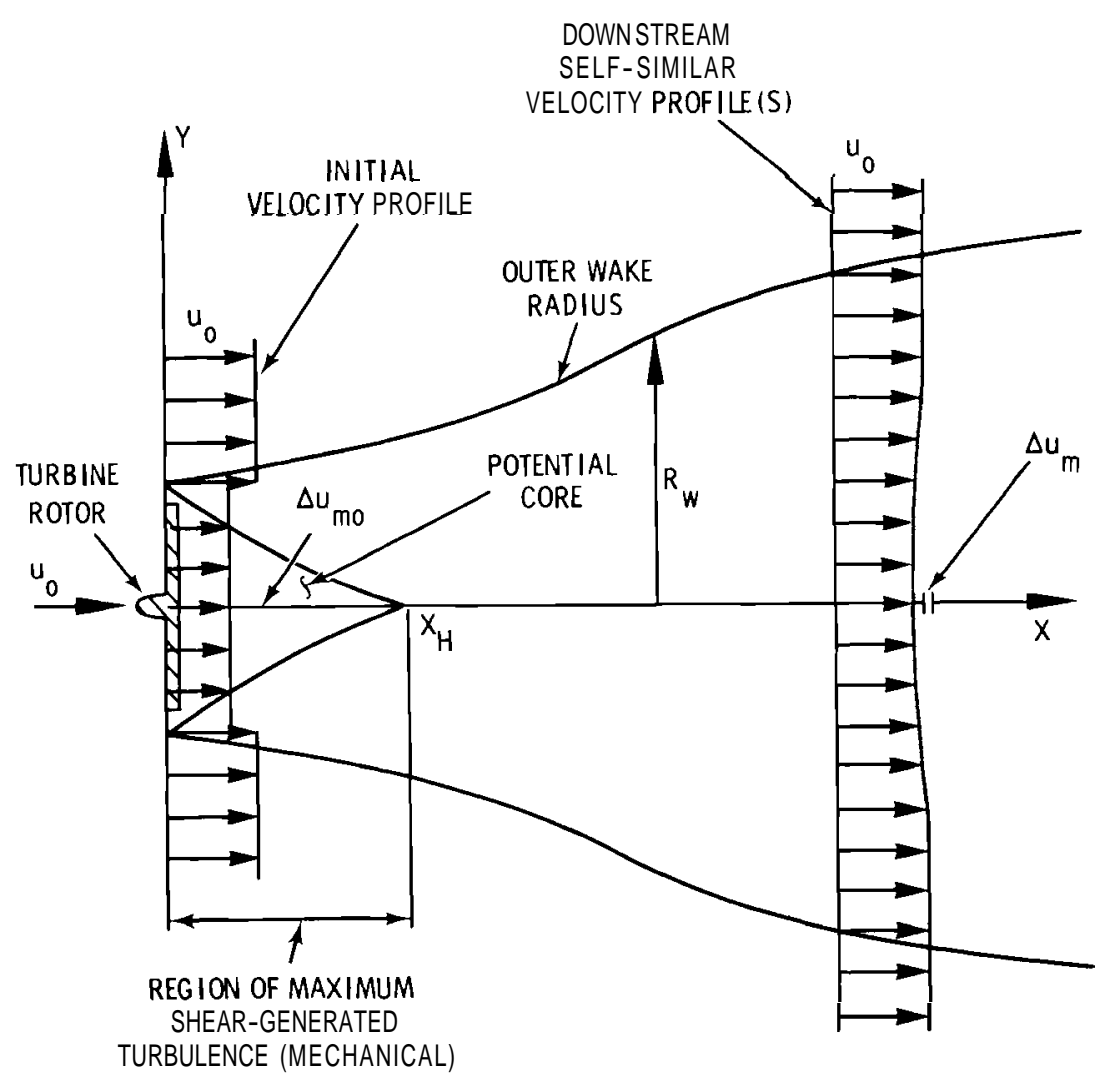

FIGURE 9 _ - The Structure of the Wake Assumed i n Lissaman's (1977) Mode1 
In the model, the wind turbine is represented by a jet of fluid entering an ambient flow. The jet has a uniform velocity profile, and the velocity at every point in the jet is less than the undisturbed flow. The model predicts the evolution of the initial "top hat" velocity profile of the jet (see Figure 9.1). This evolution is assumed to occur in four stages. First, the core region, marked by a uniform velocity deficit, is erased by the turbulence generated in the shear layer that separates the core from the undisturbed flow. Second, the shape of the velocity profile evolves into a self-similar one. In stages three and four, the velocity profiles are self-similar. However, decay of the wake in stage three is governed by both the wake and the ambient turbulence levels, whereas wake decay is assumed to be governed only by ambient turbulence in stage four.

The shape of the velocity deficit in all four regions is assumed to be the same as velocity deficits in the top hat jet problem as solved by Abramovitch (1963). These profiles are defined by the maximum velocity deficit in the wake, $\Delta u_{m}$, and by the wake radius, $R$. At distances sufficiently far from the wind turbine, the momentum thickness, $\theta_{\mathrm{m}}$ represented by the integral

$$
\theta_{m}=2 \pi \int_{0}^{\infty} \frac{u}{u_{0}}\left(1-\frac{u}{u_{0}}\right) r d r
$$

will remain constant, where $u$ is the wind speed at distance $r$ from the center of the jet and $u_{0}$ is the unperturbed speed. In Lissaman's model, (9.1) is assumed to hold at every stage of wake evolution and thus provides a relationship between the wake velocity profile and the wake radius. The equations defining how the wake size changes with downstream position, wake turbulence and ambient turbulence are empirical; they are assumed to be the same as for the top-hat jet. The intial value for $\Delta u_{m}$ at the plane of the wind turbine rotor is determined from the drag coefficient of the rotor. Ground effects are simulated by classical reflection techniques. (a)

(a) A mirror image of the wind turbine wake is assumed to grow from a point the same distance below ground level as the real turbine is above ground. As the two wakes intersect, the wind speed deficits $\left(u-u_{0}\right)$ are added to give the actual deficit. This is a common approach in potential flow theory. 
Wind turbines require a supporting tower, and the tower wake can affect downstream machines. In Lissaman's model, tower wakes are simulated using empirical decay relationships for two-dimensional circular cylinders. The effects of wind shear in the atmospheric boundary layer are accounted for by assuming the boundary layer wind profile obeys a power lav relationship.

Lissaman's model has many attributes. It is simple, flexible, and backed by plausible physical assumptions. It is easily extended to simulate cluster performance if the wind speed deficits in intersecting wakes are assumed to be additive. The main questions about the model center on the realism of the jet analogy and on the additivity of wind speed deficits. The jet analogy is most questionable in the near wake region. In this region, the wake structure of a real turbine is very different from the assumption of a uniform velocity coreflow surrounded by a shear layer. The importance of this lack of realism depends on how quickly the transition from the inner wake to the outer wake occurs, since the speed of this transition determines how close to a machine the Lissaman model can be applied. The accuracy of the model in both the near and far wake regions can only be determined through comparisons with experiments.

\subsubsection{Physical Modeling of Wind Turbine Wakes}

Physical modeling of wind turbine wakes may be accomplished by using a scaled version of the actual wind turbine or by using a physical analog to represent the turbine. A physical analog may not look like a real turbine, but it must produce a similar wake. Several studies have been conducted in Europe in which the wakes of scale-model turbines have been examined. These studies have included both vertical (Builtjes 1979) and horizontal-axis turbines (Vermeulen 1978; Alfredsson and Dahlberg 1979). Experiments on the characteristics of nonrotating physical analogs have been performed by several investigators. Sforza et al. (1979) used a perforated disk and compared wake decay measurements with their numerical model of wake dynamics. Milborrow (1980) modeled a wind turbine by attaching a wire gauze disk to the downstream portion of a shrouded propeller anemometer. Builtjes (1979) modeled the wake characteristics of vertical- and horizontal-axis machines using, essentially, a shrouded tea strainer. 
When using scale models to study wake behavior, the principal objective is to maintain kinematic and dynamic similarity (see Chapter 4). Kinematic similarity is maintained by matching the tip-speed ratio of the model and the prototype. Dynamic siniilarity is achieved by matching Reynolds numbers.

Kinematic similarity, which is very important for proper wake simulation, is easily achieved. The distinguishing feature of the near-wake region of a wind turbine is the wake shed from the rotor blades. Similarity requires that this wake have the right pitch, i.e., the distance between successive tip vortices should bear the same relationship to the rotor diameter in the model as in the prototype. This pitch ratio is $2 \pi / n \lambda$ where $X$ is the tip-speed ratio ( $\lambda$ is the ratio of tip speed to the approaching wind speed) and $n$ is the number of blades. Kinematic similarity will be met if the tip-speed ratio is the same for both the model and the prototype.

Achieving dynamic similarity is more difficult. Previous modeling experiments have been conducted with models 25 to 100 times smaller than the prototypes. Since the models are run at wind speeds comparable to those found in nature, the Reynolds number discrepancies are considerable. When operated at low Reynolds numbers, most air foils will stall at fairly low angles of attack. Builtjes, Vermeulen, as well as, Alfredsson and Dahlberg used air foil profiles with good low Reynolds number performance in building their models. Even with these precautions, Builtjes and Vermeulen observed that a large fraction of their blade span was in a stall condition, as evidenced by the low coefficient of performance and high drag characteristics of their models. Alfredsson and Dahlberg seemed to get better performance with their blade design. The coefficient of performance of their model was closer to that of a full-size machine. (a)

A7though modeling with scaled-down versions of actual machines is preferred to other approaches, it is not feasible to use rotating models in simulating the performance of a large cluster. Rotating models cannot be made very small and still perform like full-size machines. Thus, simulating the behavior of

(a) Having a good portion of the blade in a stall condition is undesirable because it means that the characteristics of the wake could be sensitive to Reynolds number changes. Reynolds number sensitivity means that extrapolating model results to the prototype is more risky. 
a large cluster with rotating models requires a very large flow facility. The desire to physically model cluster performance has motivated the search for a nonrotating physical analog to a wind turbine that can be made small enough to enable the simulation of cluster performance.

Objects such as tea strainers, perforated disks, or propeller anemometers are not necessarily realistic analogs of a rotating wind turbine. In the near-wake region, this is certainly the case. The characteristics of the wake near a wind turbine bear no resemblance to the wake behind a wire gauze, for example. However, in the region of wake self-similarity the evolution of the mean velocity profile is presumably governed by the shape of the profile and the ambient turbulence. In this region the wake has only a weak memory of how it was generated, and wake behavior can be modeled by any device that produces an identical velocity profile.

An excellent example of the use of nonrotating models is the work of Builtjes (1979). Buil tjes modified his nonrotating model until he achieved a satisfactory representation of the mean velocity profile behind a rotating model of the type of wind turbine he was trying to represent. Figure 9.2 compares measurements of the decay of the wake centerline velocity deficit behind a $0.2 \mathrm{~m}$ rotating model of a Darrieus rotor and two tea-strainer models of different diameter. Figure 9.3 compares measurements of the available power ratio

$$
\frac{P}{P_{0}}=\frac{2}{R_{t}^{2}} \int_{0}^{R_{t}}\left(\frac{u}{u_{0}}\right)^{3} r d r
$$

for the rotating model and the tea strainers. $P / P_{0}$ is a measure of the reduction in available wind power averaged over the rotor disk since $R_{t}$ is the radius of the model turbine or the nonrotating analog. The two figures show reasonably good agreement between the rotating and nonrotating models for this particular quantity. 


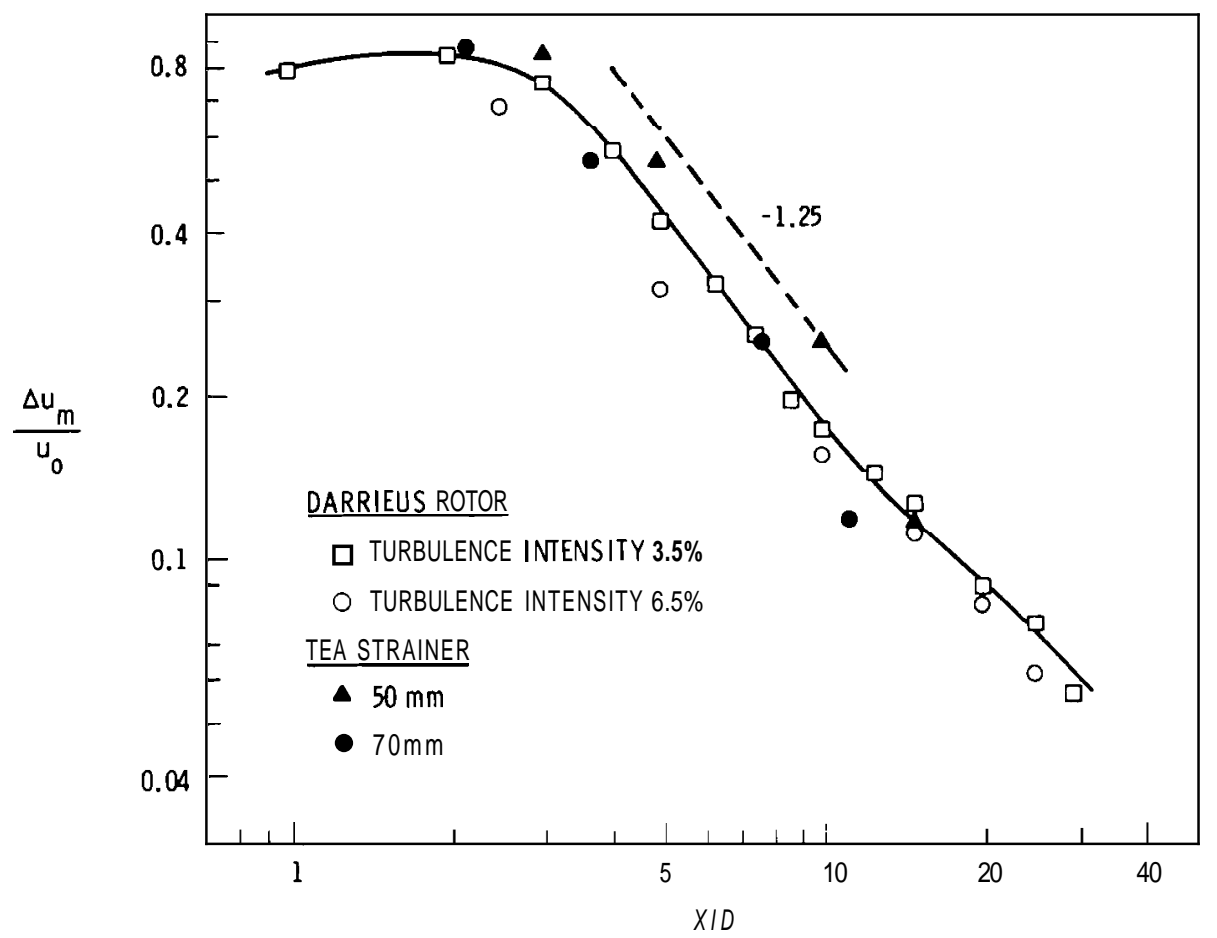

FIGURE 9.2. Wake Centerline Velocity Deficits Behind a 0.2 m Rotating Model of a Darrieus Rotor and Two Tea-Strainer Models of Different Diameters. Downstream distance is expressed in terms of model diameter. The dashed line has a slope of -1.25 (Builtjes 1979).

Although nonrotating analogs appear capable of mimicing some of the wake characteristics of a rotating model, there are still some uncertainties about their ability to represent the wake characteristics of a full-size machine operating in the atmospheric boundary layer. The rotating models used by Builtjes had higher drag coefficients than a full-size machine, and large portion of the blade span of the rotating models was in a stall condition. Thus, the wake behind the model was largely caused by flow separating from the blades and the support structure. Because of this, the mechanics of wake generation for the rotating model are not much different from those of a wire gauze. In the case of a full-size machine there should be little, if any, flow separation from rotor blades.

There are other uncertainties. The measurements reported by Builtjes were performed in a low turbulence wind tunnel having a very uniform velocity 


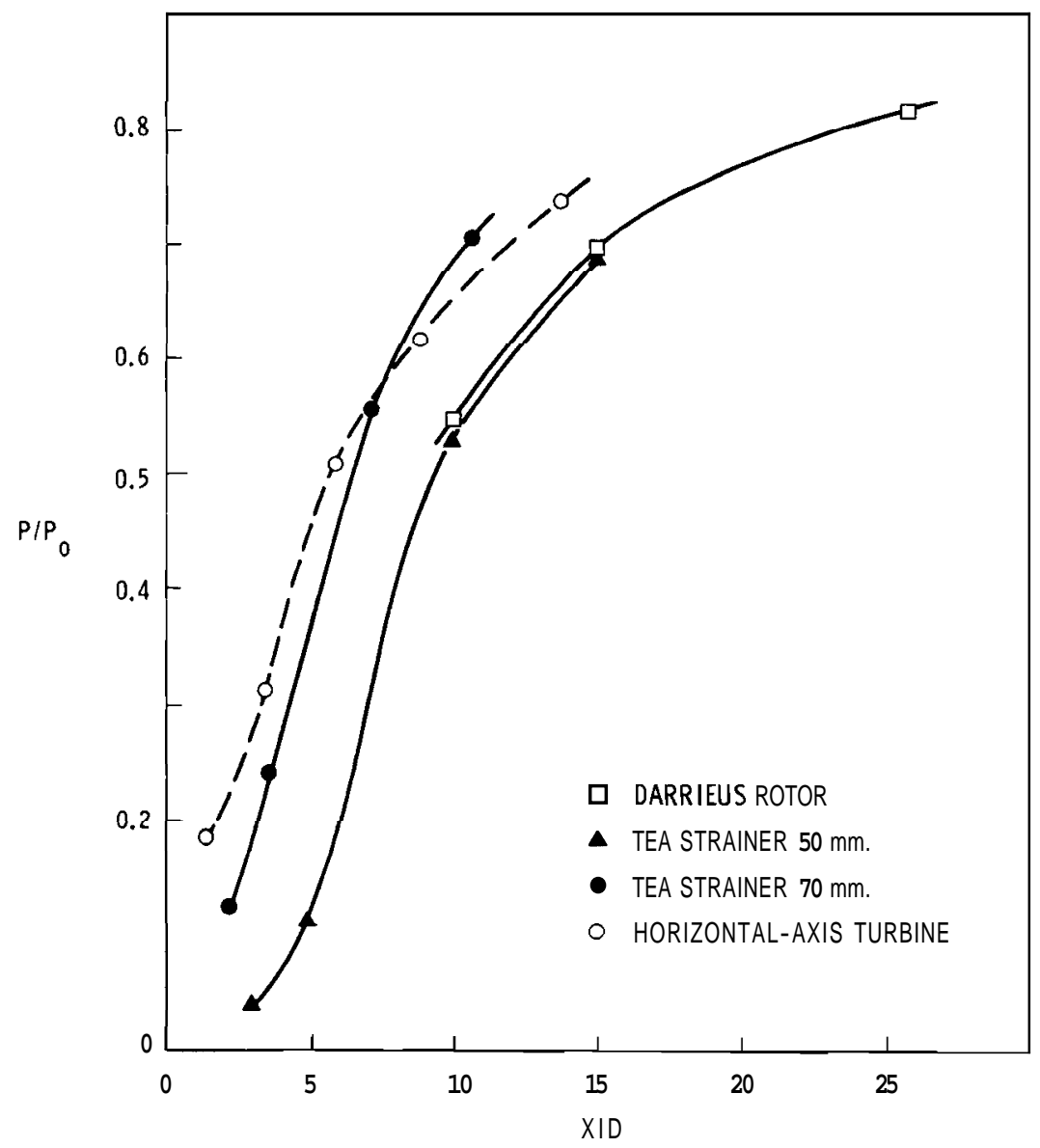

FIGURE 9.3. Available Power Ratios Behind a $0.2 \mathrm{~m}$ Rotating Model of a Darrieus Rotor and Two Tea-Strainer Models of Different Diameters (Builtjes 1979)

profile. It is possible that in a turbulent flow or in a turbulent boundary layer flow, wake decay behind the rotating model could differ from the tea strainer. Finally, the drag coefficient of a porous disk or of a tea strainer will not vary a great deal with wind speed. This is not the case for a variable pitch wind turbine. The output of such a turbine is designed to remain constant above a certain wind speed; therefore, it will spill more and more power as wind speed increases. Since the available power ratio (see Equation 9.2) at a given distance downstream will increase with speed, the effective drag coefficient will decrease. Modeling wake decay over the operating range of a variable pitch turbine could require the use of several nonrotating models. 


\subsubsection{General Behavior of Wind Turbine Wakes}

Since measurements behind full-size turbines under operational conditions are very difficult to make, few measurements have been reported. Lissaman (1979) has reported measurements that were made in Sweden of mean velocity profiles in the wake of a full-size horizontal-axis wind turbine. The measurements show incredibly good agreement with Lissaman's model, but how the measurements were made or whether the numerical model was tuned to improve agreement was not stated. (a) Most of our knowledge of wind turbine wake decay has resul ted from physical model ing studies.

Figure 9.4 compares measurements of centerline velocity deficit as a function of downstream distance for rotating models of vertical- and horizontalaxis wind turbines. These measurements are compared with field measurements of the velocity deficit behind a 5.3-m Darrieus turbine. The physical models definitely indicate the existence of a near-wake region where $\Delta u_{m}$ remains constant. In the region of the wake where the centerline velocity deficit is decaying, both the model and full-scale results show the velocity deficit to decrease approximately as $(x / 0)^{-1.25}$. Model results show the length of the constant $\Delta u_{m}$ region to be a fairly strong function of the ambient turbulence level. For the full-scale measurements, turbulence intensities were estimated to be between 20\% and 30\% (Vermeulen et al. 1979).

It is important to note in Figure 9.4 the difference between the initial velocity deficit for the model turbines as compared to the full-scale Darrieus. For the model turbines, $\Delta u_{m} / u_{0}$ is about 0.8 to 0.9 immediately behind the turbine. For the full-size turbine, the initial deficit is about 50\%. The differences between the initial velocity deficits result from the higher drag characteristics of the model turbines. This higher drag, of course, affects wind speed recovery behind the turbines. For the model turbines, centerline

(a) Since simple models, such as Lissaman's wake model, have a certain number of adjustable parameters, it is common procedure to tune the parameters using results from base-line experiments. 


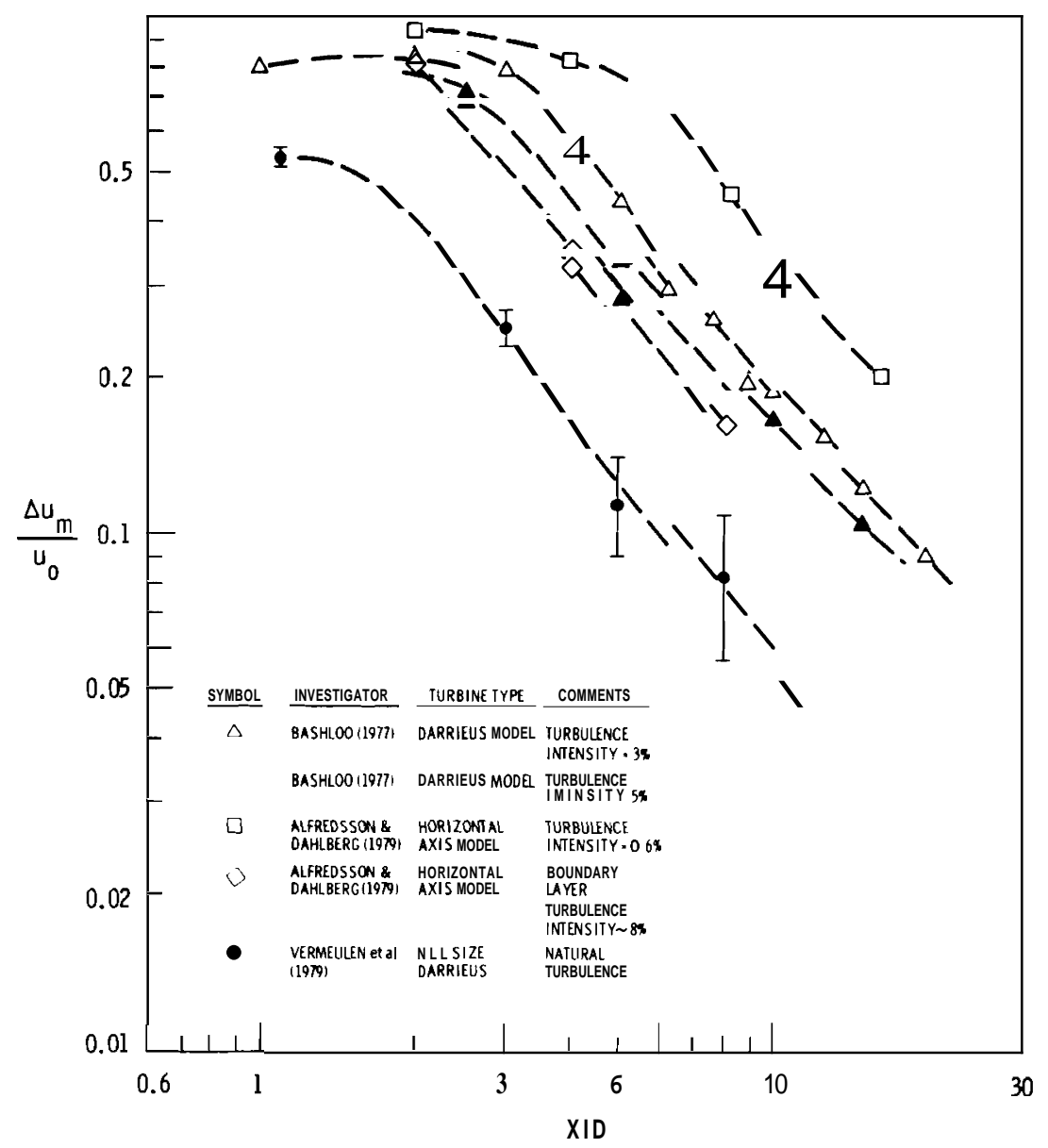

FIGURE 9.4. Wake Centerline Deficits Behind a Model Wind Turbine and a Full-Scale 5.3-m Darrieus Wind Turbine as a Function of Downstream Distance Measured in Terms of the Rotor Diameter. Error bars indicate the amount of scatter in the measurements.

velocities range from $20 \%$ to $70 \%$ of undisturbed values at a distance five diameters downstream of the turbine. For the full-size turbine, the centerline wind speed has recovered to within $90 \%$ of its upstream value at the same distance.

Figure 9.5 compares predictions of $\Delta u_{m} / u_{0}$ using Lissaman's model with the full-scale Darrieus measurements of Vermeulen et a1. (1979). Model results are shown for various values of the turbulence parameter, a. In Lissaman's - model, a is assumed to be identical to the turbulence intensity of the undisturbed flow. Good agreement is achieved for a $=0.20$. This value is about the same as the reported turbulence intensities. 


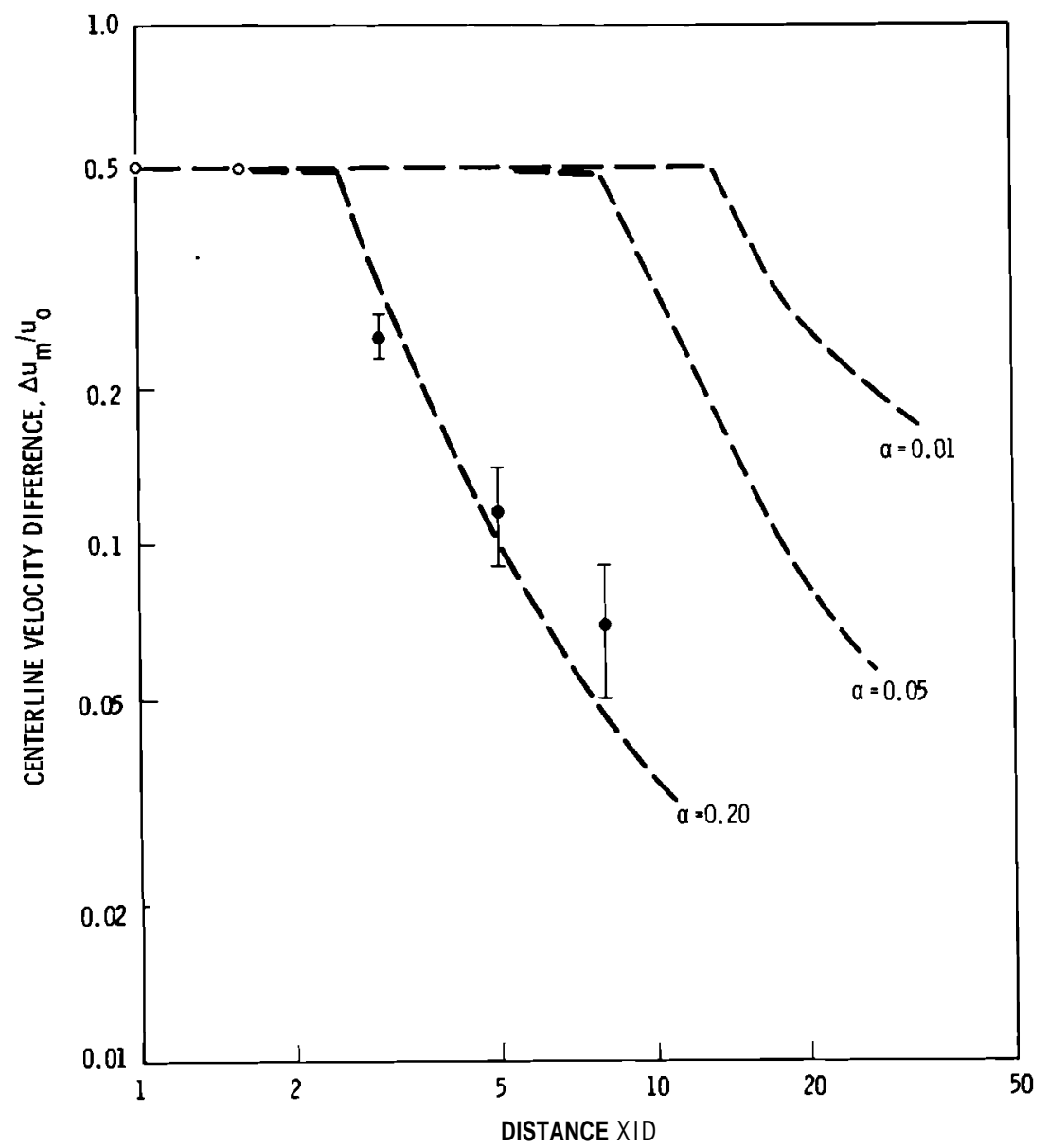

FIGURE 9.5. Predicted Centerline Velocity Deficits Using Lissaman's Model (Dotted Lines) for Various Values of the Turbulence Intensity, a, Compared With Full-Scale Darrieus Measurements (Vermeulen et a1. 1979). The error bars indicate the amount of scatter in the measurements.

Most experiments on wake behavior report measurements of the wake centerline velocity deficit. However, a real wind turbine is affected by the wind flowing through the entire rotor disk. Lift on a blade surface is proportional to the square of the relative wind speed between the blade and the air; therefore, lift increases dramatically as distance from the hub increases. Concentration on the decay of the centerline velocity deficit overstates the effect of wind turbine wakes on downstream machines. The wind behavior over the outboard half of the rotor disk is what is really important to machine performance. 
Figures 9.6, 9.7 and 9.8 are plots of $\Delta u / u_{0}$ as a function of downstream distance for three radial positions: $\quad r / R_{t}=0.0, \quad r / R_{t}=0.5$ and $r / R_{t}=1.0$ where $R_{t}$ is the radius of the rotor disk. The data are taken from Alfredsson and Dahlberg (1979) and are based on their tests of a 0.25-m horizontal axis turbine. Figure 9.6 shows wake decay for a uniform approach wind speed and a low turbulence level. The behavior of a wind turbine wake at low levels of ambient turbulence is relevant to an actual installation, because a significant portion of the rotor disk of large turbines could be above the top of the atmospheric boundary layer at night. Figure 9.7 shows wake decay for a uniform approach profile but with grid-generated turbulence. Grid-generated turbulence decays as the distance from the grid increases; therefore, ambient turbulence intensities were approximately $5 \%$ at $X / D=4$ and $3 \%$ at $X D=12$. Figure 9.8 shows wake decay for more natural turbulence. For this test, the model was run in a boundary layer having a depth of $0.6 \mathrm{~m}$. Hub height was $0.18 \mathrm{~m}$ ( $30 \%$ of the boundary layer depth). (a) The outboard sections of the model turbine blades are probably not stalled; thus behavior of the outer portion of the model turbine wake should be more similar to a full-size machine than the behavior of the wake core.

In Figures 9.9 and 9.10, velocity profiles measured by Alfredsson and Dahlberg (1979) are compared to profiles predicted by Lissaman's model (Bark 1979). Comparisons are made for conditions of low ambient turbulence and for grid-generated turbulence. With low ambient turbulence levels, agreement is fairly poor for downstream distances greater than eight diameters. (b) This lack of agreement suggests that parameterization of the effects of wake turbulence on wake decay, which is the dominant mechanism at very low ambient turbulence levels, needs improvement. The model does a much better job when ambient turbulence levels are higher, as Figure 9.10 shows.

(a) Although turbulence levels varied across the rotor disk for the boundary 1ayer runs, there was little difference between decay rates at the top of the rotor disk compared to the bottom.

(b) Failure of the model to predict wake decay near the centerline of the near-wake region is discounted because of the probable differences in wake structure between the model and a full-size machine. 


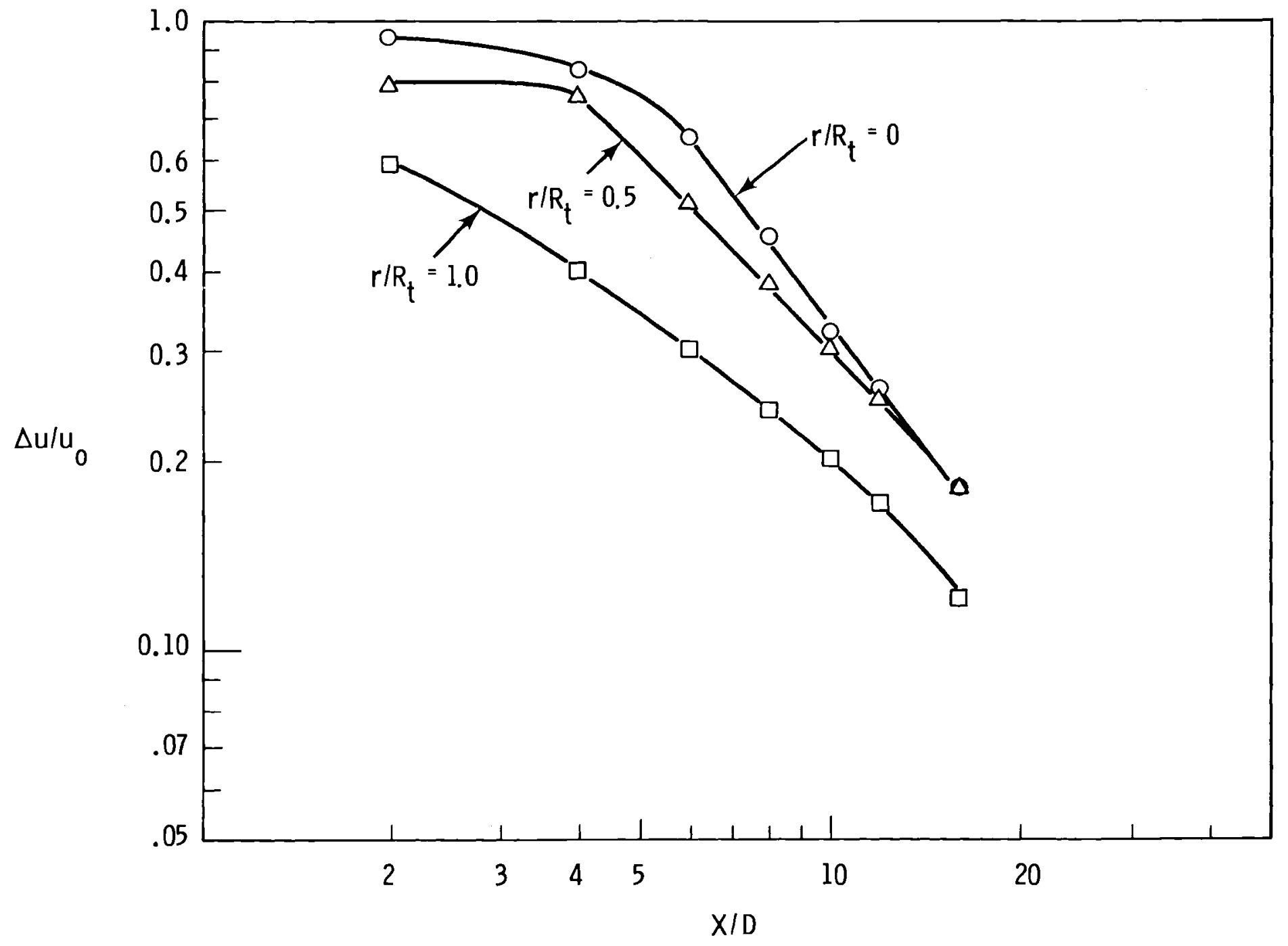

FIGURE 9.6. For Low Levels of Ambient Turbulence ( 0.6\%): Wake Velocity Deficits Behind a $0.25-\mathrm{m}$ Horizontal-Axis Model Wind Turbine at Various Downstream Distances, Measured in Terms of the Rotor Diameter, and at Three Positions Within the Wake, Measured in Terms of Rotor Radii From the Wake Centerline (Alfredsson and Dahlberg 1979) 


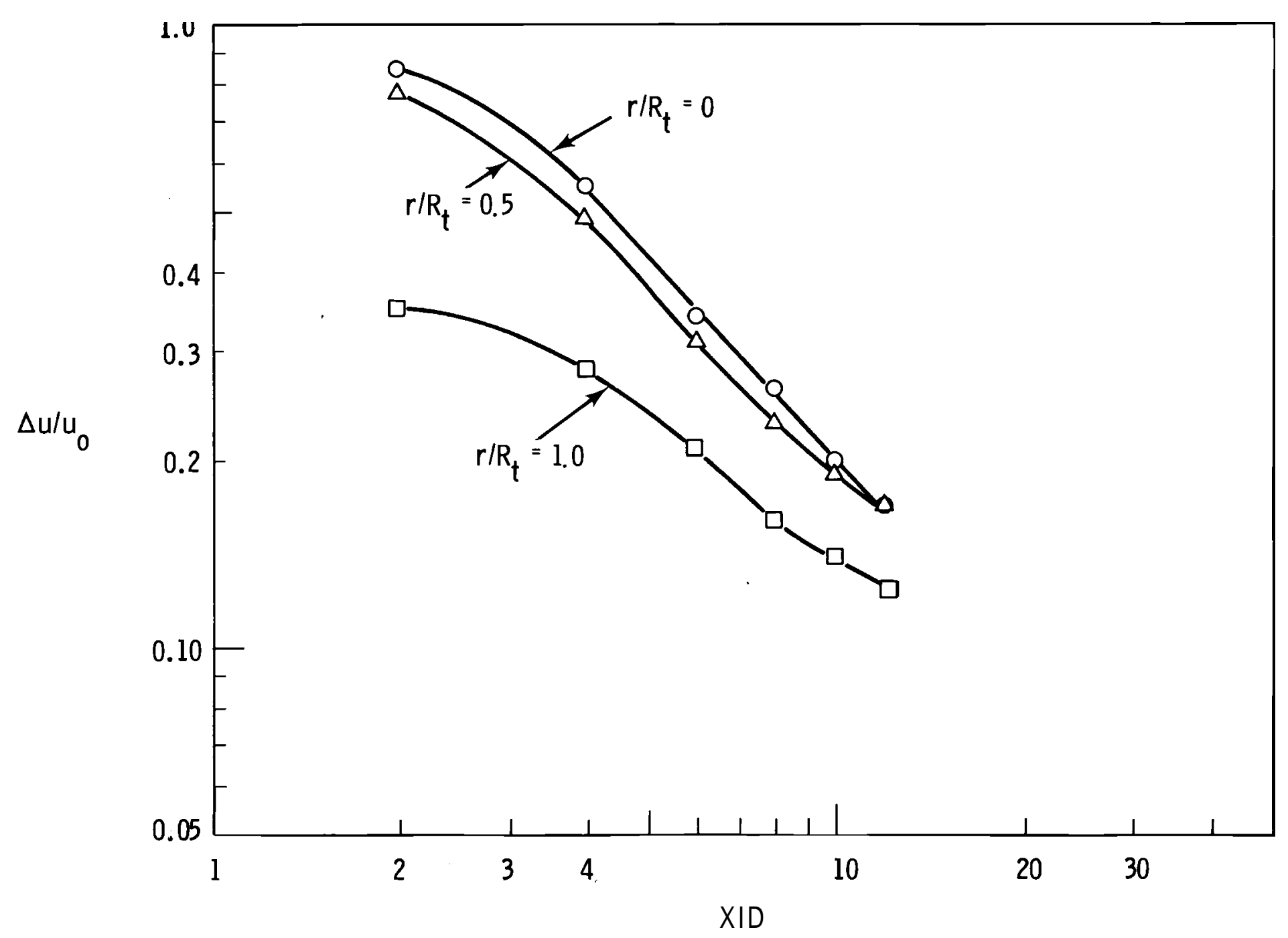

FIGURE 9.7. For Decaying Grid-Generated Turbulence $(5 \% \propto X / D=4,3 \% \odot X / D=12)$ : Wake Velocit y Deficits Behind a $0.25-m$ Horizontal-Axis Model Wind Turbine at Various Downstream Distances, Measured in Terms of the Rotor Diameter, and at Three Positions Within the Wake, Measured in Terms of Rotor Radii From the Wake Center1ine (Alfredsson and Dahlberg 1979) 


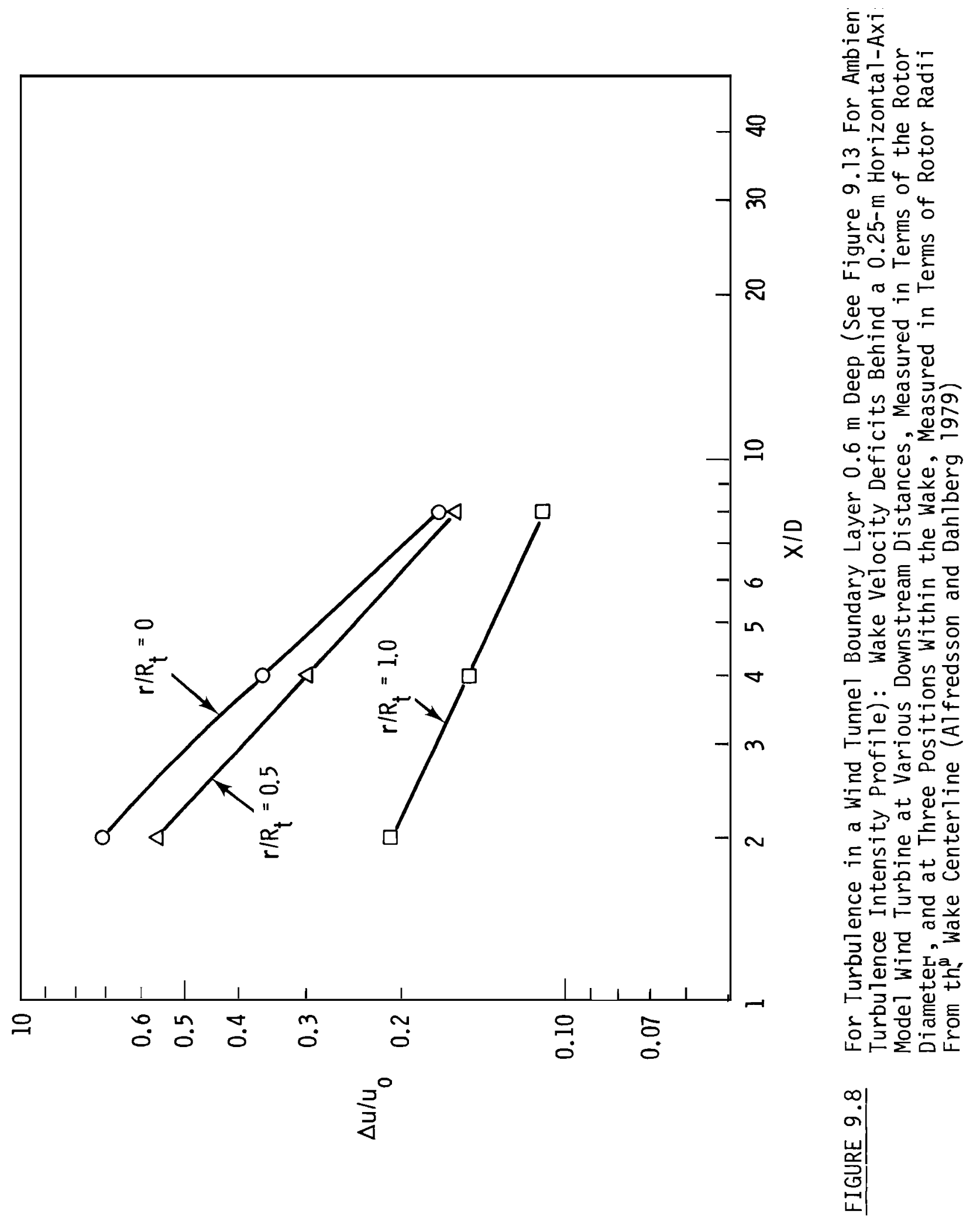




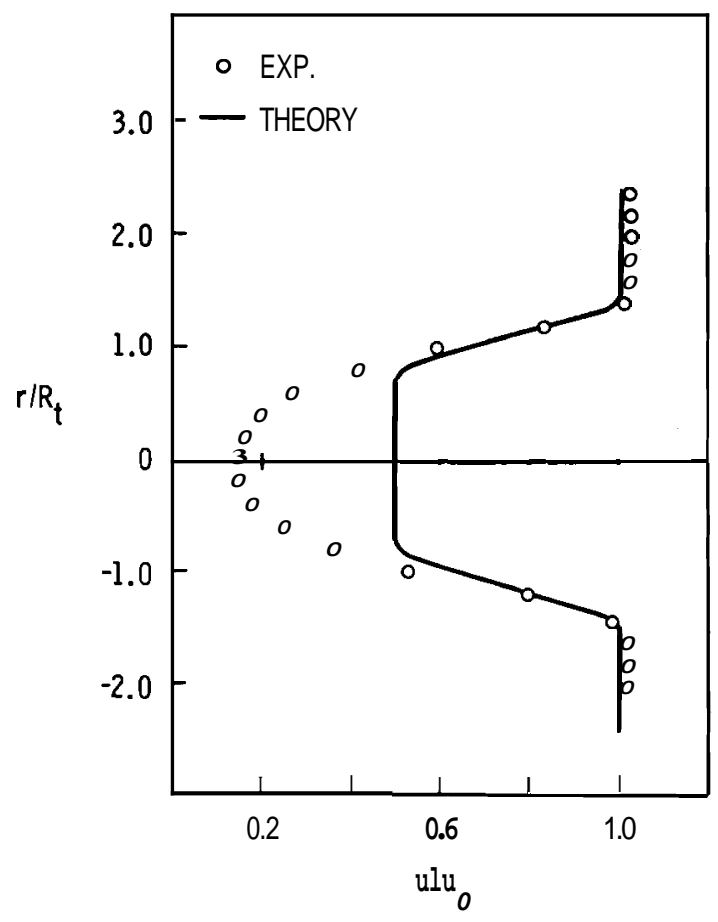

A

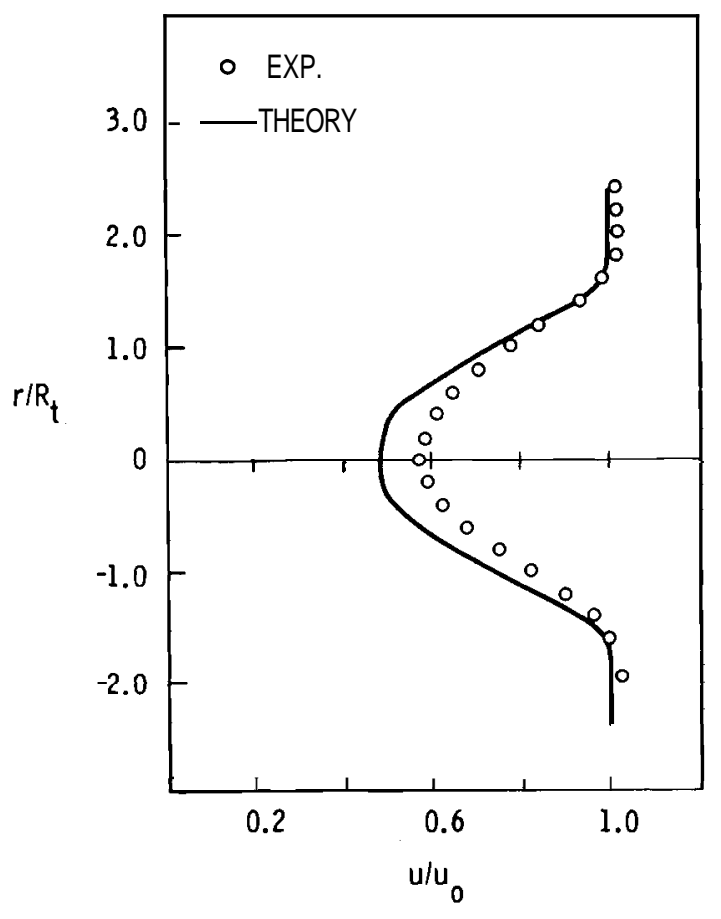

B

FIGURE 9.9. Lateral Velocity Profiles in Low Ambient Turbulence Conditions: Comparison Between Predictions of the Lissaman Model and Measurements Behind a 0.25-m Horizontal-Axis Model Wind Turbine. Downstream positions in terms of rotor diameter are: a) $x=4 D$, b) $x=8 D, c) x=12 D, d) x=16 D$ (Bark 1979). 

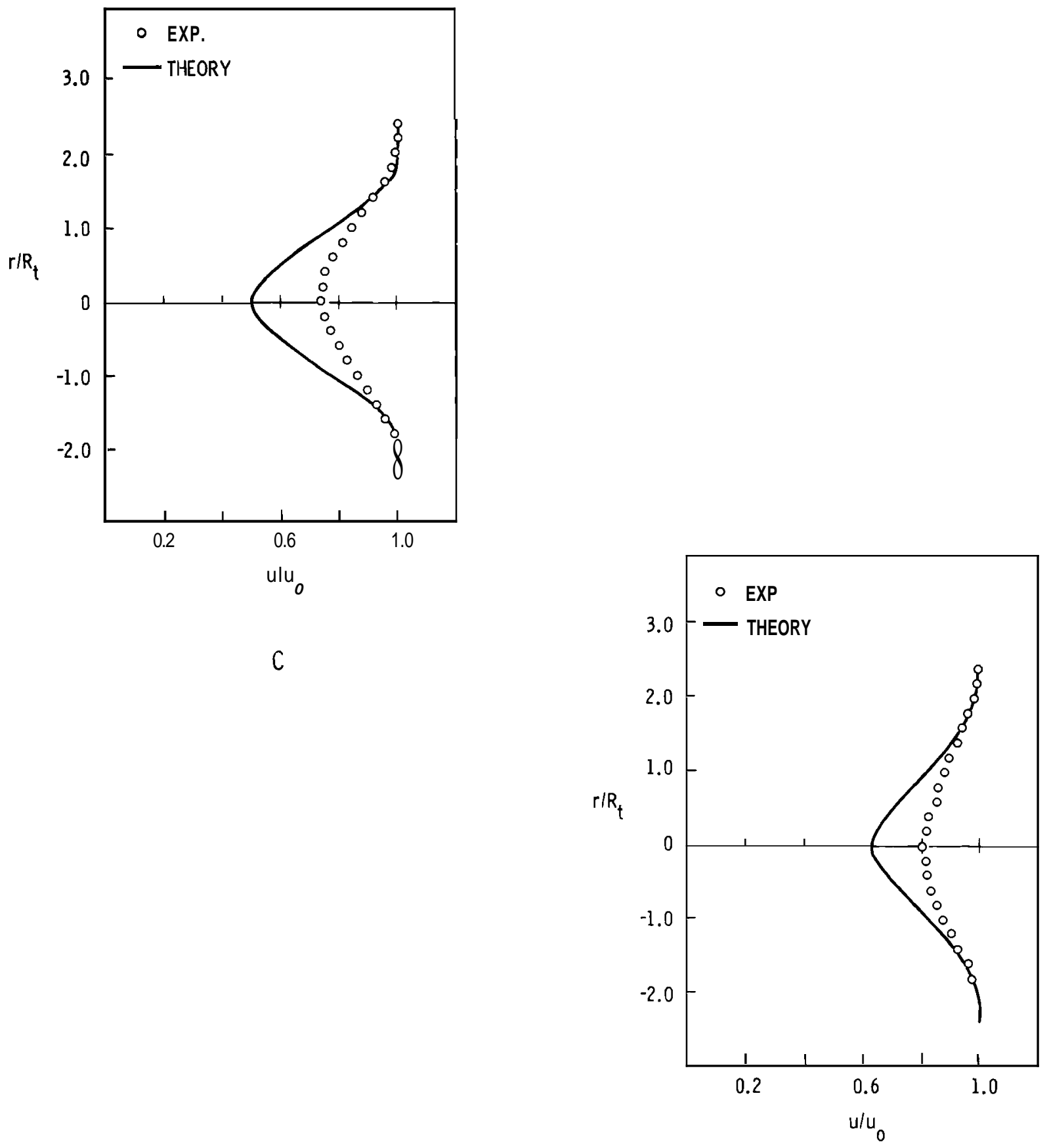

D

FIGURE 9.9. (cont.) 


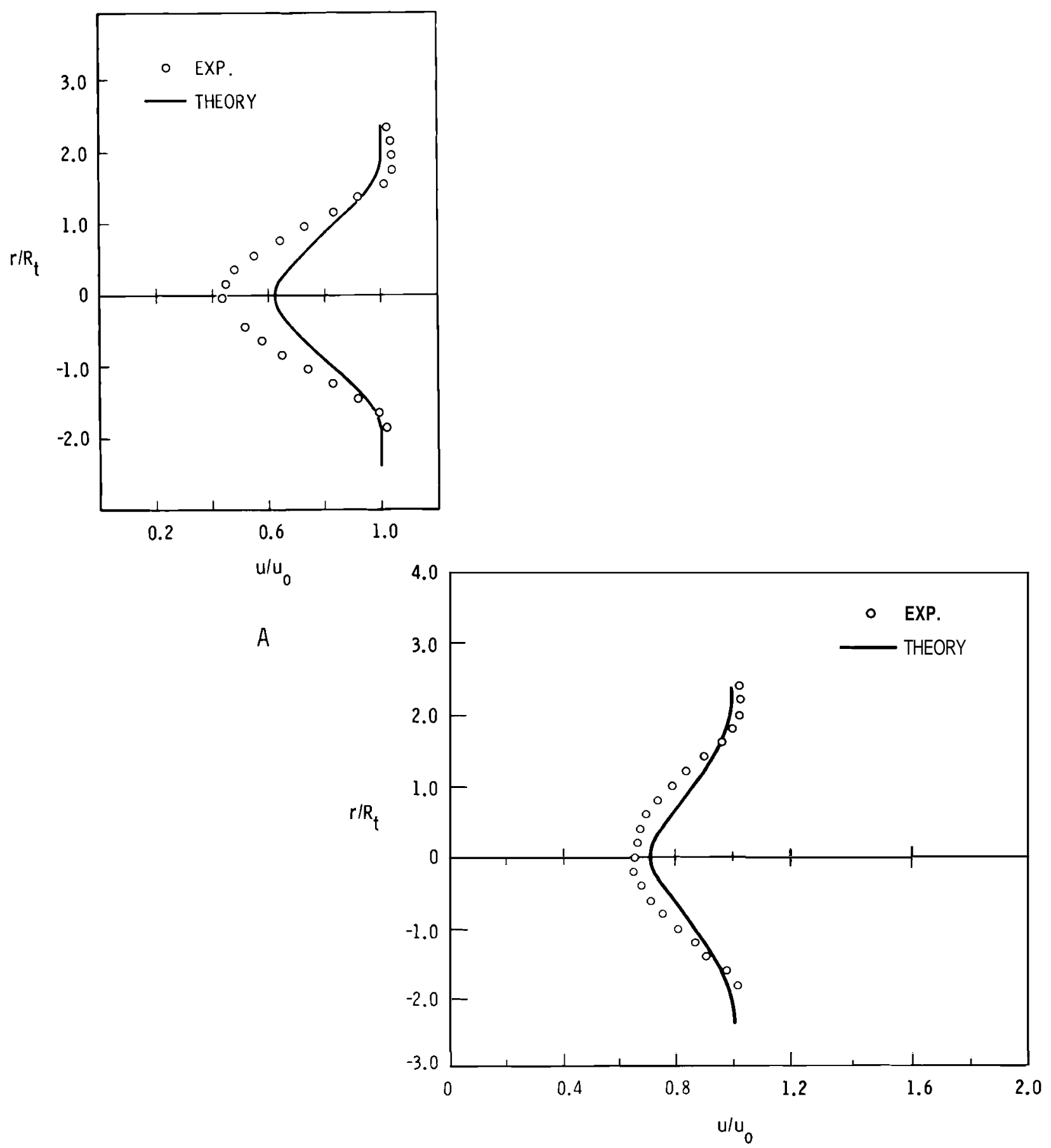

B

FIGURE 9.10. Lateral Velocity Profiles in Decaying Grid-Generated Turbulence: Comparison Between Predictions of the Lissaman Model and Measurements Behind a $0.25-\mathrm{m}$ Horizontal-Axis Model Wind Turbine. Downstream positions in terms of rotor diameter are: a) $x=4 D$, b) $x=6 D, c)=8 D, d) x=10 D($ Bark 1979). 

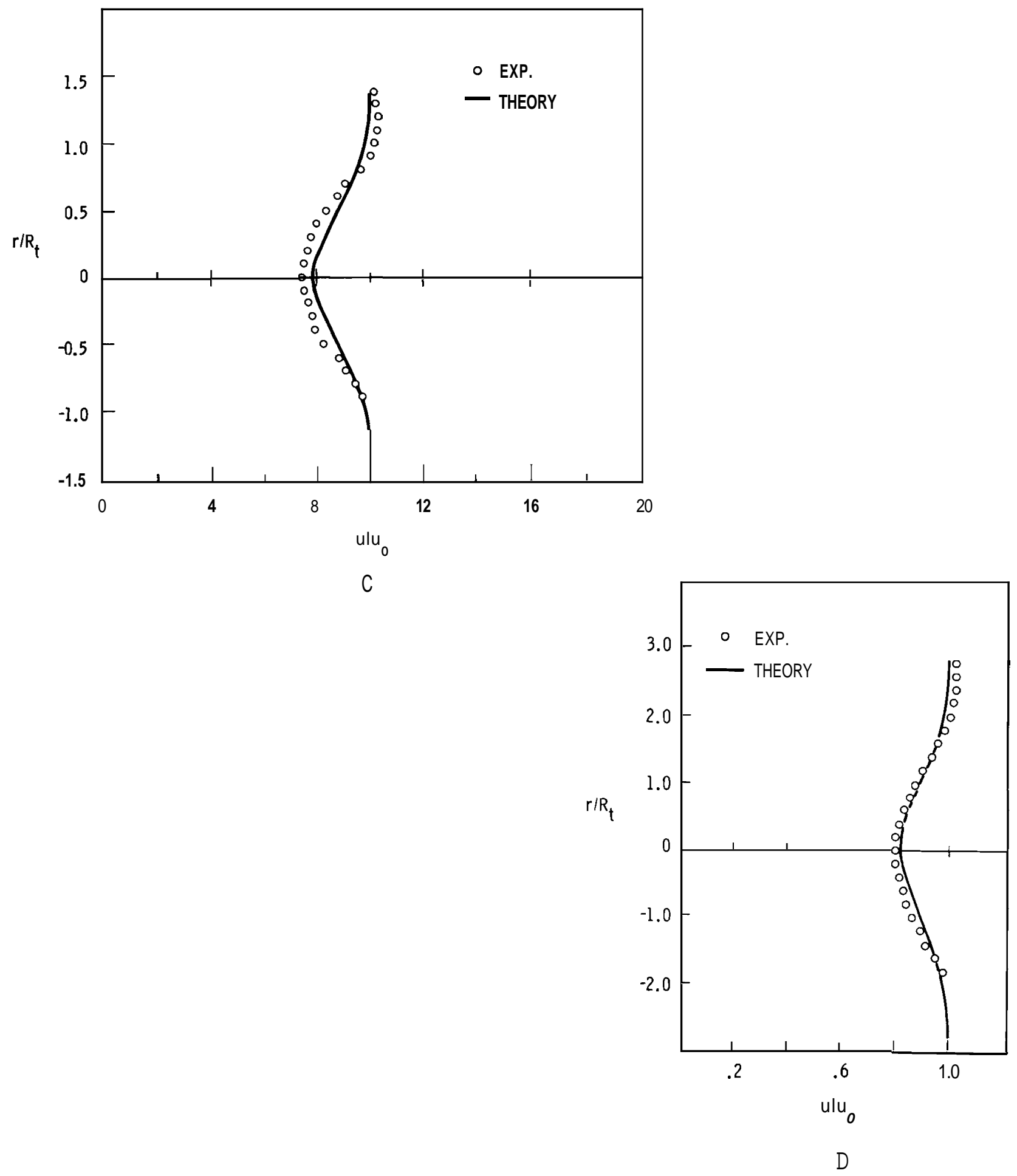

FIGURE 9.10 . (cont.) 
Figures $9.11,9.12$ and 9.13 depict profiles of the stream-wise component of turbulence intensity across the wake of a model turbine (Alfredsson and Dahlberg, 1979). Three ambient conditions are depicted: low turbulence, grid-generated turbulence, and boundary-layer turbulence. Figure 9.11 shows that with low levels of ambient turbulence, high turbulence levels in the wake persist for at least 8 to 10 diameters. At eight diameters, the tip vortices still persist (note peaks at $r / R_{t}=1.0$ ). With higher ambient turbulence levels, evidence shows that the tip vortices decay more rapidly, but turbulence levels seem to remain significantly higher than ambient levels for downstream distances less than 10 diameters.

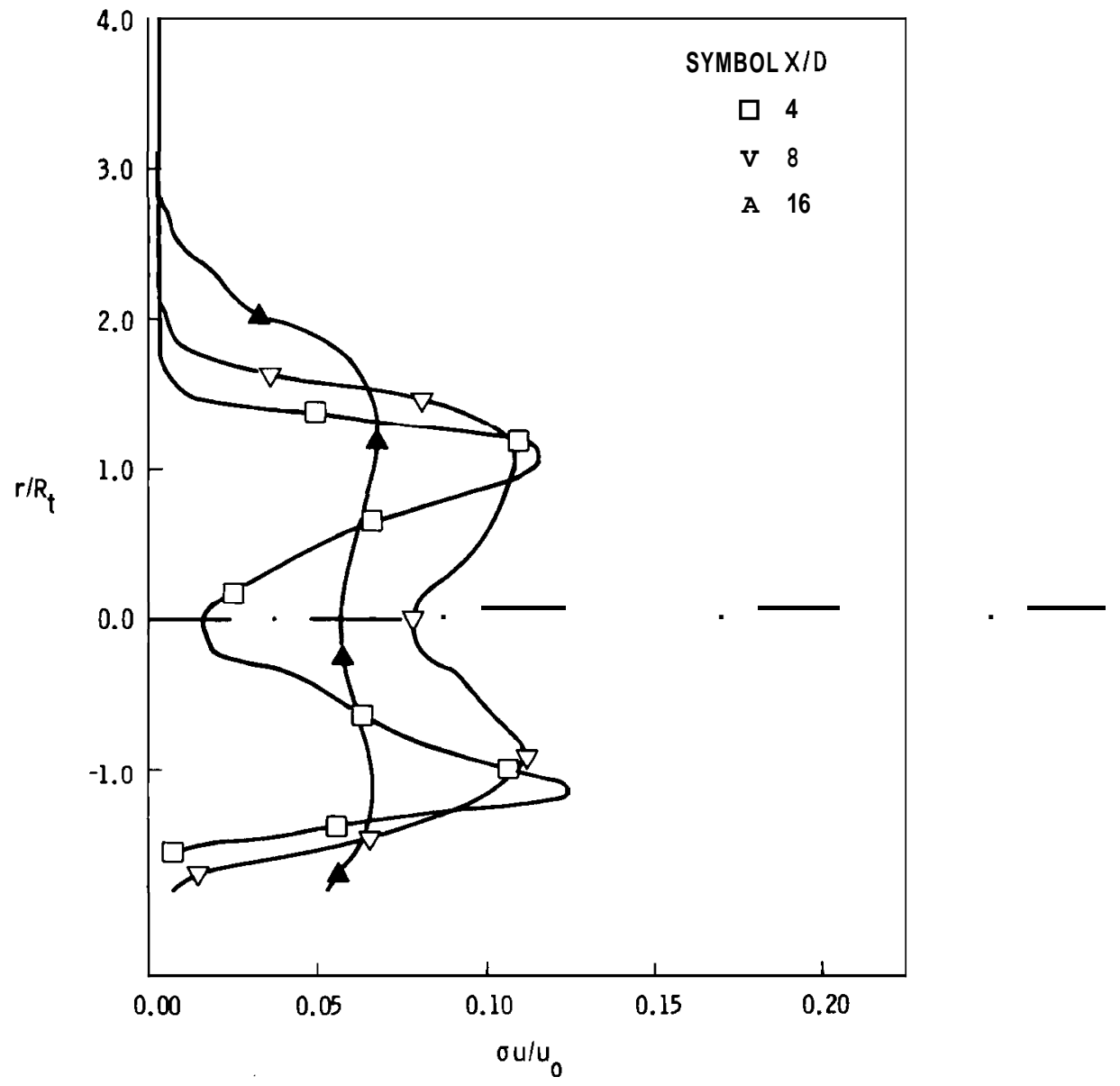

FIGURE 9.11. For Low Ambient Turbulence Intensity ( $20.6 \%$ ): Profiles of Turbulence Intensity Measured at Lateral Positions Measured in Terms of Rotor Radii and Downstream Distances Measured in Terms of Rotor Diameters. The symbols are a small sample of the 60 measurements made across the wake at each downstream location. The model is a $0.25-\mathrm{m}$ horizontal-axis wind turbine (Alfredsson and Dahlberg 1979). 


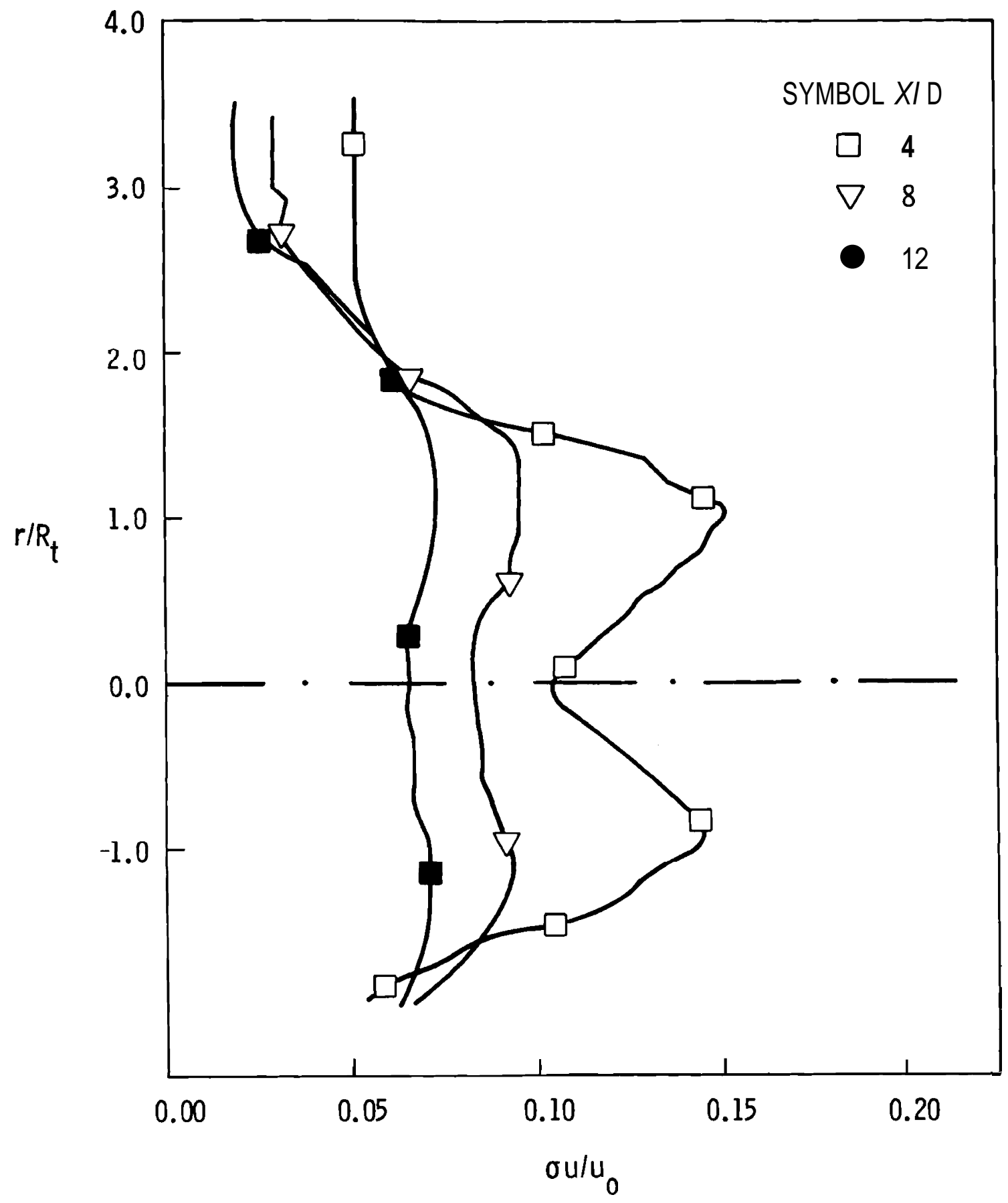

FIGURE 9.12. For Decaying Grid-Generated Turbulence (ou $/ U=5 \% @ X / D=4$, $3 \%$ Q X/D = 12): Profiles of Turbulence Intensity Measured a $t$ Lateral Positions Measured in Terms of Rotor Radii and Downstream Distances Measured in Terms of Rotor Diameters. The syrnbols are a sma 11 sample of the 60 measurements made across the wake at each downstream location. The model is a $0.25-\mathrm{m}$ horizontal-axis wind turbine (Alfredsson and Dahlberg 1979). 


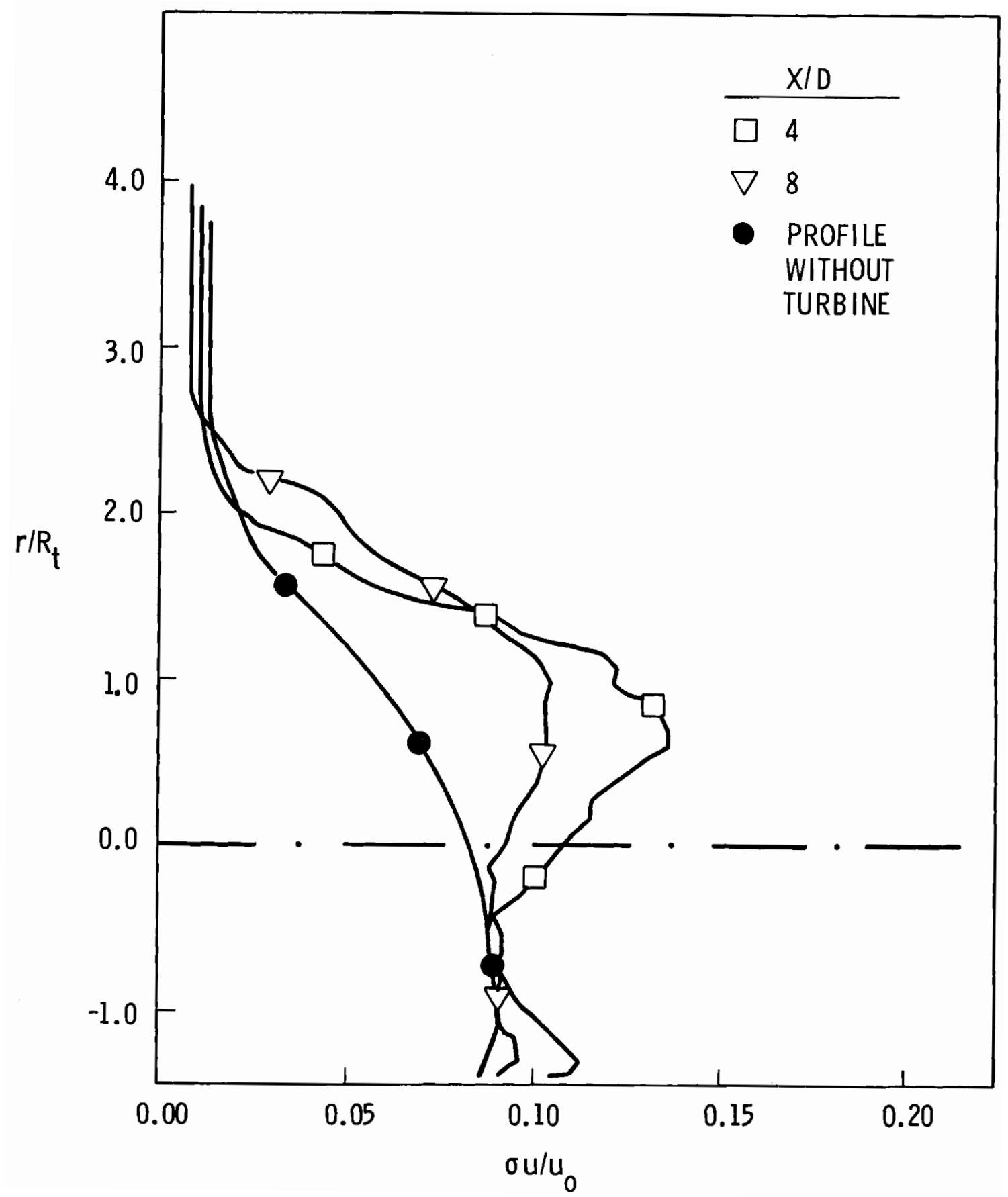

FGURE 9.13. For Turbulence in a Wind Tunnel Boundary Layer $0.6 \mathrm{~m}$ Deep: Vertical Profiles of Turbulence Intensity Measured at Separations From Hub Height Measured in Terms of Rotor Radii and Downstream Distances Measured in Terms of Rotor Diameters. The symbols are a small sample of the 60 measurements made across the wake at each downstream location. The model is a 0.25-m horizontal-axis wind turbine (Alfredsson and Dahl berg 1979). 


\subsection{WAKE INTERACTIONS AND CLUSTER PERFORMANCE}

\subsubsection{Approximate Numerical Models}

The first performance estimates for wind turbine clusters were those of Templin (1974). Templin's analysis was for an infinite array on flat terrain. The wind turbines were treated as additional surface roughness. For a sufficiently large array of regularly spaced machines, the atmospheric boundary layer will come into equilibrium with the increased surface drag due to the machines. Once equilibrium is reached, the increase in energy flux from the large-scale flow to the surface wi11 be proportional to the energy removal by the wind turbines. Figure 9.14 summarizes Templin's results. The figure shows cluster efficiency, $n$, as a function of machine spacing. Cluster efficiency is the ratio of the average power output from an individual machine in the cluster to the power output of an isolated unit. Figure 9.14 suggests that a spacing of 10 diameters would result in a cluster efficiency of less than $50 \%$.

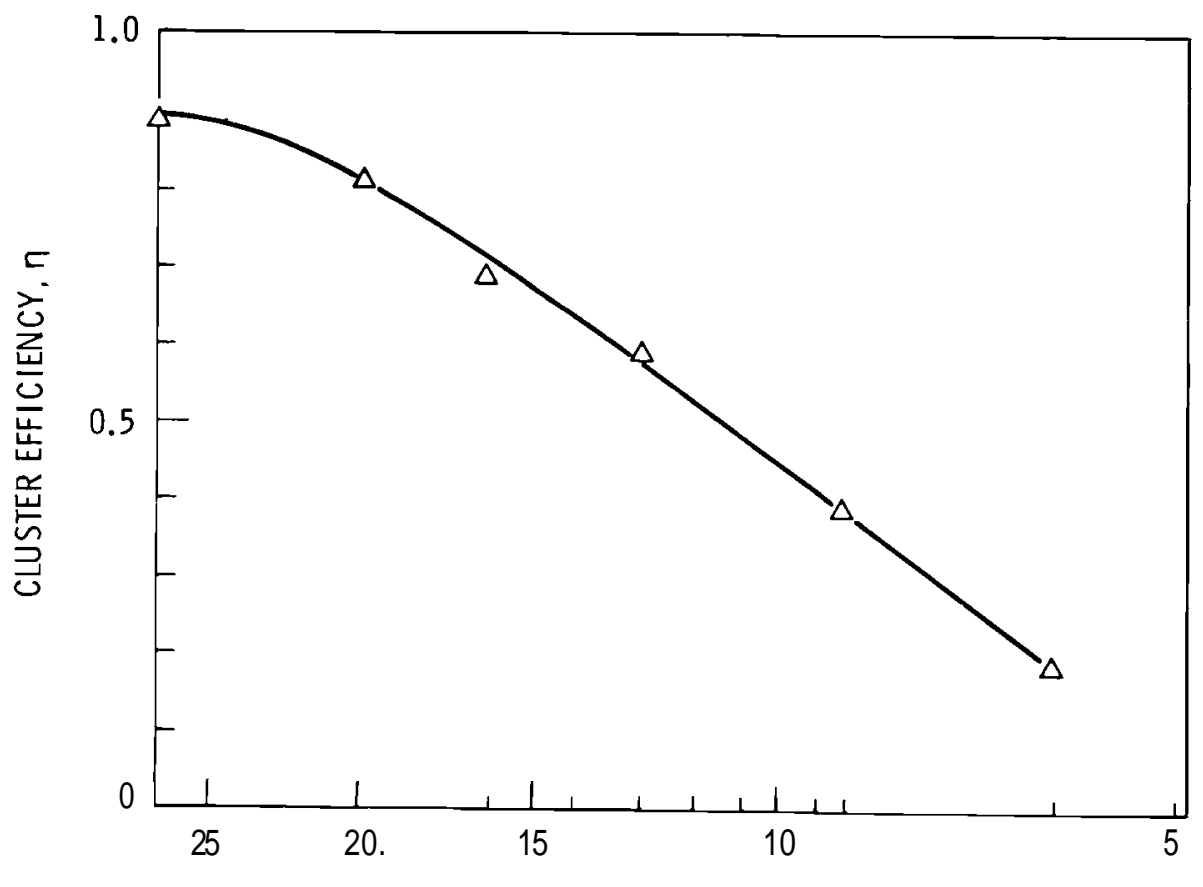

TURBINE SPACING IN ROTOR DIAMETERS

FIGURE 9.14. Cluster Efficiency as a Function of Wind Turbine Spacing Based on Hypothesis of Templin (1974) (After Builtjes 1979) 
A real cluster, of course, is not infinite, and there have been attempts to modify Templin's approach accordingly (see Riley et al. 1980). However, all of these approaches are primarily of academic interest. They certainly cannot be used in designing cluster layout, and it is even questionable whether they can be used for providing rough estimates of cluster performance. The basic assumption of these models is that the relationship between turbine drag and power output is constant. It is also assumed that the drag coefficient of an individual turbine is independent of wind speed. Neither of these assumptions is true. For example, the power output of a variable pitch machine is constant for wind speeds above rated. With sufficiently high wind speeds, there could be no reduction in the output of a cluster of these machines.

\subsubsection{Cluster Design Using Lissaman's Model}

At present, Lissaman's jet-analogy model (see Section 9.1.1) is the most practical for analyzing the performance of wind turbine clusters. (a) Its application is very straightforward (Lissaman 1979); the velocity deficits of intersecting wakes are added to produce the three-dimensional wind field at any point in the cluster. Power output is assumed to be related to the average wind speed through the rotor disk. (b) In previous applications, turbine power output has been assumed to be a constant fraction of the cube of the disk-average wind speed. This may not be a good assumption for many turbine designs, and the relationship between turbine output and the cube of the disk-averaged wind speed may have to be a function of wind speed. Making such a change to the model, however, would not be difficult.

Once the cluster has been layed out, cluster efficiency can be computed by applying Lissaman's model. I $n$ this application, cluster efficiency $n_{r}$ is defined as the total energy production of the cluster divided by the cluster's total rated energy production. Computed values of $n_{r} w i l l$ be a function of wind speed, wind direction, wind shear in the atmospheric boundary layer and boundary layer turbulence. In Lissaman's model, wind direction is assumed to be constant with height. The variation of wind speed with height is represented by

(a) New modeling approaches are being developed and this situation could change (e.g., see Crafoord 1979 or Taylor 1980)

(b) Actually, a weighted average should be used since most of the blade lift is generated along the outer third of the blade length. 
the power-law approximation of the boundary-layer wind profi le $\left(u=u_{r}\left(z / z_{r}\right)^{n}\right)$, and the ambient turbulence is represented by its intensity (a). Both $n$ and a are a function of stability. Given the approximations inherent in Lissaman's model, consideration of three broad stability regimes should be sufficient to specify the power law coefficient or the turbulence intensity. Recommended values of $\mathrm{n}$ and a for these regimes are given in Table 9.1.

TABLE 9.1. Recommended Values of Power Law Coefficient and Turbulence Intensity for the Lissaman Wind Turbine Wake Model

\begin{tabular}{|c|c|c|}
\hline $\begin{array}{l}\text { Stabi 1i ty } \\
\text { Type }\end{array}$ & $\begin{array}{c}\text { Power Law }(a) \\
\text { Coefficient } n \\
\end{array}$ & $\begin{array}{l}\text { Turbul ence } \\
\text { Intensity a }\end{array}$ \\
\hline $\begin{array}{l}\text { "Stable" } \\
\text { (nighttime) }\end{array}$ & 0.28 & 0.01 \\
\hline $\begin{array}{l}\text { "Neutral" } \\
\text { (early morning } \\
\text { early evening, } \\
\text { or winter day) }\end{array}$ & 0.18 & 0.10 \\
\hline $\begin{array}{l}\text { "Unstable" } \\
\text { ( spring, summer } \\
\text { or autumn day) }\end{array}$ & 0.13 & 0.25 \\
\hline
\end{tabular}

(a) See Figure A-2.9.

Table 9.2 outlines a procedure for determining cluster design and predicting cluster performance based on Lissaman's model. The procedure assumes that time-series data on hub height winds are available and that time-series estimates of cluster energy production are desired. Time series of estimated energy production from any machine in the cluster can also be obtained if this is desired.

The first step in the procedure is to determine the prevailing wind direction or directions. The layout for the cluster (step 2) is determined by maximizing $n_{r}$ for the prevailing direction. Usually, machine locations will 
be 1 imited by topography and engineering constraints. Step 2 suggests maximizing $n_{r}$ with the power law coefficient and turbulence intensity appropriate to stable conditions. The justification for this suggestion is that wake interaction effects will be largest for this condition. However, the cluster could be optimized for daytime conditions if degradation of nighttime performance is of no concern. (a) Optimization of the cluster for neutral or unstable conditions will result in the closer spacing of machines.

\section{TABLE 9.2. A Procedure for Cluster Design and Performance Evaluation Based on the Lissaman Wake Model}

\begin{tabular}{|c|c|}
\hline Step & Activity \\
\hline 1 & $\begin{array}{l}\text { Determine prevailing wind } \\
\text { direction }(s) \text {. }\end{array}$ \\
\hline 2 & $\begin{array}{l}\text { Optimize layout for prevailing } \\
\text { direction and stable stratifica- } \\
\text { tion (see Table 9.1). }\end{array}$ \\
\hline 3 & $\begin{array}{l}\text { Create look-up table for cluster } \\
\left.\text { efficiency ( } \eta_{r}\right) \text { as a function of } \\
\text { wind speed, wind direction and } \\
\text { stability (see Table 9.1) using } \\
\text { Li ssaman model. }\end{array}$ \\
\hline 4 & $\begin{array}{l}\text { Create time series of cluster } \\
\text { energy production using time } \\
\text { series of wind speed, direction, } \\
\text { and by multiplying the cluster's } \\
\text { rated output by the appropriate } \\
\text { values of } n_{r} \text {. }\end{array}$ \\
\hline
\end{tabular}

In step 3 a look-up table is created for $n_{r}$. This is accomplished by running the model at all three stabilities for a number of wind directions and wind speeds sufficient to define $n_{r}$ as a function of speed, direction and stability. Once this has been done it is simple to create time series of cluster output using time series information on wind speed and direction--if

(a) Some daytime conditions in winter may also be stable. 
the stability is known. Table 9.1 provides a suggestion of how stability might vary with time of day and season for all but the more southern portions of the U.S.

Potential users should bear in mind the limitations of the Lissaman model. The model is not thoroughly verified. The accuracy of the jet-analogy for turbine wakes is still unknown, although agreement between model predictions of the decay of the centerline velocity deficit and full-scale measurements look encouraging. Nevertheless, the propriety of adding velocity deficits to determine wind profiles deep in wind turbine clusters has not been verified (however, see Section 9.2.3). There is also evidence that the model may improperly handle wake decay when decay is controlled by wake-generated turbulence. Wake turbulence may be the dominant mixing mechanism in large clusters (Milborrow 1980). Finally, the model neglects possible topographic influences. The accuracy of Lissaman's model in situations where local terrain relief is comparable to hub height is completely unknown.

\subsubsection{Cluster Design Using Physical Modeling}

Another option for evaluating the performance of cluster designs is physical modeling. This approach is particularly popular in Europe (Builtjes 1979) and in the United Kingdom (Mi Jborrow 1980). The physical modeling approach would seem to be especially attractive when there is significant terrain relief within the wind turbine cluster. The applicability of physical modeling to cluster design hinges on the existence of an adequate nonrotating physical analog of a full-size wind turbine.

In Sections 9.1.2 and 9.1.3, comparisons of a nonrotating analog were made with a rotating model turbine in a shear-free flow. Comparisons of mean profile behavior were good, but there were several questions about a nonrotating analog's similarities to a full-size machine. One of the most serious of these questions is in the drag characteristics. The drag coefficient of a tea-strainer or any similar object is not a strong function of wind speed. The drag coefficient of a wind turbine, on the other hand, does change as wind speed changes. No single nonrotating physical model is capable of simulating 
the drag characteristics of a wind turbine over its full range of operating conditions; several models would have to be used to accomplish a thorough study of cluster behavior.

An additional complication in the application of physical modeling to the evaluation of cluster performance is in properly interpreting the results. In previous physical modeling studies of cluster behavior (Builtjes 1979 or Milborrow 1980), power output has been deduced either by assuming it to be proportional to the $3 / 2$ power of the drag force on the turbine simulator (Builtjes) or by assuming it to be proportional to the cube of the wind speed through the simulator (Milborrow). In reality, power output is a variable function of flow through the rotor disk. It is also not related to the drag force on the turbine in a simple way. The output of each machine in the modeled array can only be determined through knowledge of the wind conditions at each simulator. If, for example, the mean wind speed through the simulated rotor disk were known, power output could be determined from the operating characteristics of the machine. For Milborrow's shrouded anemometers, knowledge of the mean flow through the rotor disk is easy to obtain since that is the quantity measured. Determining mean wind speeds through Builtjes' tea-strainers is possible if the force on the tea-strainers can be measured with sufficient accuracy. If such measurements are feasible, the average wind speed can be determined by applying the relationship between drag force and the square of the mean wind speed.

In summary, physical modeling of cluster performance is feasible if the following conditions are met with sufficient accuracy: (a)

- The device used to simulate wind turbine wakes must produce a mean velocity defect that closely matches the behavior of a real wind turbine.

- Turbulence characteristics of the simulated wake must be sufficiently close to the real wake that the simulated wake responds to changes in ambient turbulence and wind shear in a realistic manner.

- The wake of the simulator should match the wake of a real turbine over a realistically useful range of downstream distances. This range must be known.

(a) An analogous set of conditions should be met by a numerical model of cluster performance as well. 
- The behavior of the simulated wake as a function of approach wind conditions (speed, shear, turbulence) must match that of the full-size turbine. This requirement could dictate the use of different simulators for different wind speed ranges.

- Proper turbine performance characteristics must be used in computing the power output of each machine in the cluster. This requires knowledge of wind conditions at each simulator.

- The flow facility used in modeling cluster performance must produce conditions typical of the atmosphere. Conclusions must be limited to those atmospheric situations that can be adequately modeled (see Chapter 4).

Another way that physical modeling can be used in attacking the cluster problem is in the testing and the tuning of simple numerical models. Figure 9.15 compares results of a physical modeling study of cluster performance with predictions of the Lissaman model (Builtjes 1979). The physical modeling simulation was of an array of 49 tea-strainer simulators and was intended to simulate cluster output as a function of wind direction. The simulators were arrayed in a staggered grid with 4 diameters between each row. Thus for a wind direction of $0^{\circ}$ or $90^{\circ}$ the spacing between machines was 8 diameters; the minimum spacing between machines was 5.7 diameters for a wind direction of $45^{\circ}$. Figure 9.15 shows the ratio of average power output from a machine in the cluster to the power output of an isolated turbine. A constant relationship was assumed between drag and power output.

In applying Lissaman's model, Builtjes (1979) tuned the free parameters in the model to get the best match between wake characteristics measured behind an isolated tea-strainer in the wind tunnel boundary layer and the evolution of these characteristics as predicted by the model. When the numerical model was applied to the tea-strainer array, the computed power ratio was somewhat larger than the measured ratio. However, the model seems to simulate wind direction sensitivity fairly well. The ability to simulate directional similarity appears to verify the way in which intersecting wakes are handled in the model--at least for tea-strainers. 


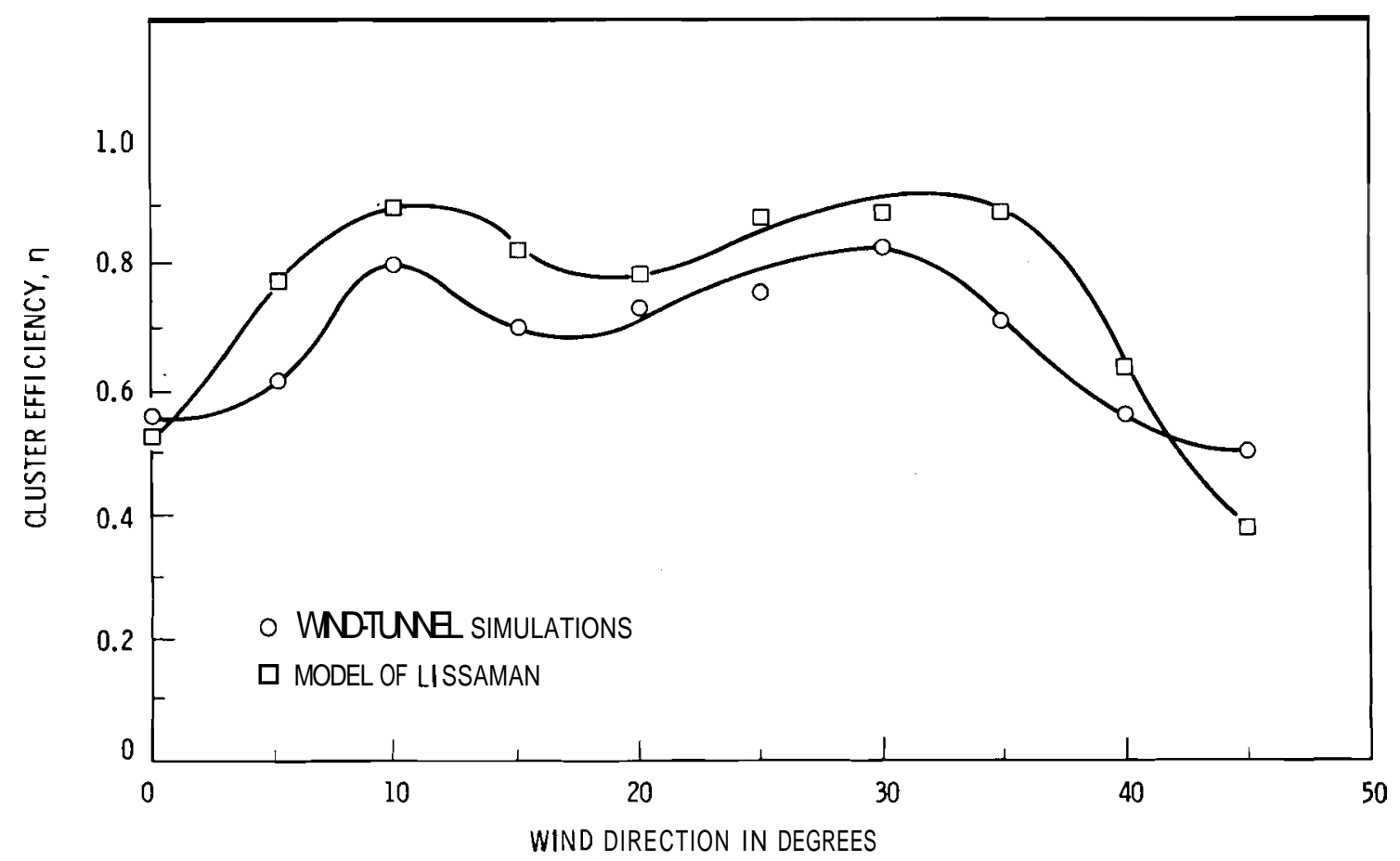

FIGURE-9.15. Cluster Efficiency as a Function of Wind Direction. The model array consists of staggered rows of wind turbine with rows being 4 diameters apart. Therefore machine spacing for $0 "$ and $90^{\circ}$ wind directions is 8 diameters and is 5.7 diameters for a wind direction of $45^{\circ}$. Forty-nine tea-strainers were used in the physical model (Builtjes 1979).

\subsection{CONCLUSIONS AND RECOMMENDATIONS}

\subsubsection{Numerical and Physical Modeling Approaches to Cluster Evaluation}

Cluster performance can be simulated by existing numerical models or by physical modeling. At the present time, the most developed numerical model for simulating cluster performance is the model of Lissaman (1977). The model is discussed briefly in Section 9.1.1 and is compared to a sample of wake measurements in Section 9.1.3. The use of the model in evaluating cluster performance is described in Section 9.2.2. As indicated in Section 9.2.2, some changes in the way wind turbine power output is computed are needed in order to simulate the performance of actual machines. However, the changes required are minor. 
Lissaman's model is not thoroughly verified. It does, however, appear to treat wake decay over flat ground in a realistic manner--there is reasonable agreement with a limited number of laboratory and field measurements. The primary failing seems to be in the prediction of wake decay under conditions when ambient turbulence levels are low. How realistically the model handles wake interaction and predicts cluster performance when local terrain relief is not small compared to hub-height distances is not known.

Physical modeling can also be used to simulate cluster performance. However, there are problems in finding a realistic nonrotating analog of a full-scale wind turbine. Different types of models may be required to simulate wind turbine wake characteristics over different wind speed ranges. Because the relationship between wind speed (or wind speed cubed) and power output is not a simple one, average wind conditions across the rotor disk of each machine in the cluster must be known in order to compute power output for the cluster. (a) Physical modeling of cluster behavior in nonflat terrain should be superior to existing numerical models (assuming the wind turbine simulator can be made small enough to have the proper scale relationship to the terrain). However, physical modeling studies are more expensive than numerical simulations. In addition, no one has yet attempted to simulate a wind turbine cluster where the drag and power coefficients of the turbines has been a strong function of wind speed. Finally, one of the most useful roles for physical modeling is in the testing and tuning of simple numerical models. These points are discussed in greater detail in Section 9.2.3.

\subsubsection{General Spacing Guidelines for Large Wind Turbines}

Based on results of numerical and physical modeling studies of wind turbine wake decay, several general guidelines on turbine spacing can be given. The reader should be aware that these recommendations are based primarily on wind tunnel simulations of rotating models. Very few of these observations have been confirmed through field measurements on full-size machines. Conclusions on cluster performance are not based on experience since no large clusters have been built.

(a) Remember, at sufficiently high wind speeds there could be no degradation of turbine performance within a cluster of variable pitch machines. 
Spacing machines at distances of 10 to 15 diameters should result in no appreciable degradation of wind turbine performance. Wind tunnel simulations have shown mean wind speed recoveries of 80 to 90 percent in the wakes of isolated machines even at very low turbulence levels. (a) Since the performance curves of most large wind turbines show a nearly linear relationship between disk-average wind speed and power output (see Figure 1.11), a 10 to 20 percent reduction in wind speed will cause only a small reduction in turbine output. Wake turbulence should also be no problem. Turbulence intensities should be comparable to boundary-layer turbulence levels and rotor tip vortices should be unimportant.

A conservative approach is to avoid spacing machines closer than about 5 diameters in the prevailing wind direction. At distances closer than this, the performance penalty starts to become large. Turbulence from upwind machines could also be a problem at close spacing. However, like any other general guideline there are situations where it could be profitable to violate the "rules". If the wind resource is large and the area of available land is small, spacing machines at distances closer than 5 diameters might be economically justified.

Spacing machines at a distance of 7 to 8 diameters in the prevailing wind directions is probably acceptable; however, modeling studies indicate that a performance penalty will be paid under some atmospheric conditions. When atmospheric turbulence levels are reasonably high, however, the velocity deficit across the rotor disk will be negligible (see Section 9.1.3). Wake turbulence may not be negligible at a 7 to 8 diameter spacing, particularly at night when ambient turbulence levels are low. Wind tunnel models have shown evidence of wake vortices 8 to 10 diameters downstream of a scale model turbine. The turbulence intensity levels in the wakes of isolated turbines are not particularly high. Turbulence intensities should rarely exceed values typical of natural boundary-layer turbulence--20 to 30 percent. However, the spectral characteristics of wake turbulence are unique (due to tip vortex shedding) and could be troublesome. We have no firm information on the

(a) In interpreting the results of wind tunnel tests of model turbines, their high drag characteristics should be considered. At distances less than 10 diameters, model turbines will show higher velocity deficits than full-size machines. 
turbulence characteristics that could be expected within large clusters; but Builtjes (as quoted in Riley et al. 1980) has reported turbulence intensity levels as high as 50 percent within large arrays of simulated wind turbines. Whether wake turbulence is a problem in wind turbine siting is unknown a the present time. The answer must await further analysis of the effects of turbulence on aerodynamic performance, noise generation, and structural integrity as well as experience in the operation of clusters. 


\section{REFERENCES}

Abramovitch, G. N. 1963. The Theory of Turbulent Jets. Massachusetts Institute of Technology Press, Cambridge, Massachusetts.

Alfredsson, P. H. and J. O. Dahlberg. 1979. A Preliminary Wind Tunnel Study of Windmill Wake Dispersion in Various Flow Conditions. Technical Note FFA-AV-1499, Part 7. The Aeronautical Research Institute of Sweden, Stockholm, Sweden.

Bark, F. H. 1979. A Preliminary Evaluation of a Model Proposed by P. Lissaman for the Wake Behind a Rotor in a Turbulent Flow. Technical Note FFA AU-1499. Part 2. The Aeronautical Research Institute of Sweden, Stockholm, Sweden.

Boschloo, G. 1977. Wake Structure of a Darrieus Rotor. TNO Report 77-07244 (Dutch), Organization for Industrial Research (TNO), Apeldoorn, The Netherlands (referenced in Vermeulen et a1. 1979).

Builtjes, P. J. H. 1979. Wind Turbine Wake Effects. TNO Report 79-08375, Organization for Industrial Research (TNO), Apeldoorn, The Netherlands.

Crafoord, C. 1979. Interaction in Limited Arrays of Windmills. DM-26, Department of Meteorology, University of Stockholm, Stockholm, Sweden.

Lissaman, P. B. S. 1979. "Energy Effectiveness of Arbitrary Arrays of Wind Turbines." J. of Energy 3:323-328.

Milborrow, D. J. 1980. "The Performance of Arrays of Wind Turbines." J. Industrial Aero. 5:403-430.

Riley, J. J., E. W. Giller, M. D. Coon and J. C. Schedvin. 1980. A Review of Wind Turbine Wake Effects. Flow Research Co., Kent, Washington.

Sforza, P. M., M. Smorto and P. Sheerin. 1979. "Wind Turbine Generator Wakes." AIAA Paper No. 79-0113, California Institute of Technology, Pasadena, California.

Taylor, P. A. 1980. On Wake Decay and Row Spacing for WECS Farms. AQRB-80-001-L, Air Quality and Inter-Environmental Research Branch, Atmospheric Environment Service, Downsview, Ontario, Canada.

Templin, R. J. 1974. An Estimate of the Interaction of Windmills in Widespread Arrays. LTR-LA-171, National Research Council of Canada, Ottawa, Canada.

Vermeulen, P. 1978. A Wind Tunnel Study of the Wake of a Horizontal Axis Wind Turbine. TNO report 78-09674, Organization for Industrial Research (TNO), Apeldoorn, The Netherlands. 
Vermeulen, P., P. Builtjes, J. Dekker and G. L. V. Bueren. 1979. An Experimental Study of the Wake Behind a Full Scale Vertical Axis Wind Turbine. TNO Report 79-06118, Organization for Industrial Research (TNO), Apeldoorn, The Netherlands.

Walker, S. N. and P. B. S. Lissaman. 1978. Wind Flow Characteristics in the Wake of Large Wind Turbines. AV-FR-8147, AeroVironment, Inc., Pasadena, California. 


\subsection{MEASUREMENTS AND INSTRUMENTATION}

Data are required at every stage of a site selection program, but deciding how to collect the data in the most cost-effective way is a problem. The intended use of the data determines the manner in which data are gathered and processed and the instrumentation used. Chapter 2 outlined a variety of data-related problems to be encountered at different stages of the site selection process. In this chapter measurements and measurement systems necessary to collect the data required for a specific problem are described. Although wind measurements are the primary focus of the chapter, the use of icing measurements and the use of satellite and aircraft imagery of the earth's surface with the biological, geomorphological, and social and cultural indicator techniques is also presented.

\subsection{CONCEPTS OF A MEASUREMENT SYSTEM}

A measurement system consists of a sensor that produces a signal related in some way to changes in the physical variable of interest and a means of translating the signal into an interpretable record. What comes out at the end depends upon the properties of the system and its deployment in the field. Consider a constant-temperature hot-wire anemometer for measuring wind speed. (a)

The hot-wire anemometer works on the physical principle that wind blowing over a heated wire cools the wire in a way that depends upon the wind speed. The wire is heated by electrical resistance and the resistance, which is a function of the wire temperature, is monitored. Clearly the sensor is telling something about the flow passing it; but exactly what is it saying? The wire provides flow information averaged over the length of the wire since fluctuations smaller than the length of the wire cannot be resolved. The heat transfer is dependent on the temperature fluctuations in the flow as well as velocity fluctuations, so those effects should be measured also (with a second hot wire operated at a different resistance). It takes a finite time for heat to be

(a) The hot-wire anemometer is not an instrument suited for wind energy siting measurements, but it clearly illustrates many problems common to the design of sensing devices; these problems should be considered whatever sensor is being used. 
transferred from the wire to the flow so that there is a high frequency limit to the flow fluctuations that the hot wire can respond to. The structure that supports the wire also transfers heat. This influences the heat tranfer from the wire. Since the supports are larger than the fine wire, the frequency response effects due to the supports appear at lower frequencies than the high frequency limits of the wire alone. The relation between the sensor signal and the flow parameters is incalculable so that calibrations are the only way to quantify the relation. The calibrations differ from sensor to sensor and may change with time so that recalibration is necessary. The signal produced by a sensor is therefore a filtered representation of the time behavior of the variable being studied.

A sensor produces some form of signal, which is then intentionally or unintentionally conditioned as it is transmitted to a recording device. The signal might be a voltage, a frequency, a series of electrical pulses, or mechanical work that turns gears or pulls springs. An analog signal might be digitized and multiplexed for transmission on a frequency modulated (FM) carrier wave. If an analog signal is sent along a cable there will be some signal loss within the cable. Amplifiers and filters may be used along the way. A the end of the transmission process the signal is converted to a form compatible with the recording device if it is not already in a suitable format. Just as the sensor produces a signal that is a modified representation of the measured parameter, the transmission process further modifies the signal.

Finally the signal is recorded. The recording device could be a person noting readings from a meter. It could be either a mechanical or electrical accumulator that keeps a summation of the mechanical or electrical work done between readings by a person. This summation can be recorded with a pen on a moving strip chart so that the time information is always there whether an observer is or not. Either analog or digital signals could be recorded on other more sophisticated magnetic devices or in solid state memory. Each recording option will have its onn peculiar prob?ems. For example, it is well known that when a human reads a meter that is fluctuating at a rate that is about as fast as the eye can track, that observer preferentially chooses convenient numbers depending on the scale; e.g., even numbers, or multiples of 5, 
or integral numbers. Clearly the motion of a pen on strip charts must have a high frequency limit, and strip charts frequently keep time incorrectly.

Four criteria used to judge measurement systems and their components are:

- responsiveness

- accuracy

- precision

- reliability.

Responsiveness is the ability of a sensor or system to follow high frequency changes in the measured variable; in other words, its ability to measure fluctuations. Wind sensor responsiveness may be characterized by:

- a distance constant, which is the amount of air passage required to make a $63 \%(1-1 / e)$ response to a change in the wind (speed or direction)

- a threshold speed, which is the lowest wind speed at which the sensor responds.

Accuracy and precision of measurements are two separate measures of system performance that are often treated ambiguously. Accuracy refers to the mean difference between the output of a sensor and the true value of the measured variable. Precision refers to the dispersion about that mean. For example, a system performing an ensemble of measurements on a fixed variable may produce the same measured value every time but produce a value that is $50 \%$ off. That system has a high precision but low accuracy. Another system making an ensemble of measurements may produce measurements with no mean error but the dispersion of individual measurements may be $50 \%$ of the mean. In this case the system demonstrates high accuracy but low precision. For the conventional wind measuring systems to be discussed below, the precision is usually high so that accuracy is the principal concern. One should be particularly aware that system accuracy can be a function of wear or, equivalently, time. Periodic cal ibrations are therefore required.

Instrument system reliability is more difficult to quantify than either response or accuracy. Basically, reliability is a measure of an instrument 
system's ability to produce useful data and the period of time over which the system will operate properly. Reliability may be built into a system by making it simple and rugged, or it may be obtained from a lightweight, complex system by careful maintenance and frequent checks of system operation.

The best indicator of a system's reliability is its performance record. Systems that have been used for years in most applications are likely to be reliable, although there may be exceptions. Newly developed systems may be reliable, but some caution is necessary because their past performance records will be limited. Simplicity is another possible indicator of system reliability. Systems with a few simple parts often tend to be more reliable than a system with a large number of complex parts. However, there are also many exceptions.

In assessing the reliability of a system, particular attention should be given to the recording device and to possible problems related to its failure. Recording device failure during a data collection period may result in the loss of all data for the period, or it may result in the loss of data for the portion of the period following the failure.

A measurement produces a series of data that is processed and modified through an entire system consisting of sensors, transmitters, and recorders. The choice of a measurement system will always begin with the accuracy and reliability requirements of the final data product. Knowing that, the system can be chosen to do an adequate job for the least cost. Because a system consists of a series of components, calibrations of the entire system as well as individual components must be considered.

\subsection{WIND SENSING SYSTEMS}

In this subsection, the wind sensors used in meteorology are presented. The type of wind sensor one should select depends upon the problem. The wind data needed for siting studies (see Chapter 2) range from long-term data to some turbulence data. The scales of turbulence that affect the site selection problem (see Appendix 2) are large enough that the very sophisticated turbulence measuring equipment is not necessary. Some of the wind sensing systems 
that are not useful for siting will be briefly mentioned at the end of this section because some individuals that have heard of these systems may not know their proper role in atmospheric measurements. Primarily the emphasis will be on the systems that have application for siting studies, and they are:

- cup anemometers

- wind vanes

- propeller anemometers

- tethered balloon instrument systems

- kites and kite anemometers.

In addition, remote sensing of the winds using radar, lasers, acoustic radars, and satel1ites wil1 be discussed.

\subsubsection{Cup Anemometers}

Cup anemometers use their rotation, which is proportional to wind speed, to generate a signal. Miniature ac or dc generators produce a continuous signal that is directly proportional to wind speed and can be transferred to a recorder with minimal electronic processing. Some signal generation devices produce a train of pulses. In the "contact anemometer", revolution of the anemometer shaft is geared down so that contacts of a switch (or mechanical movements of an odometer) are closed after a certain distance of wind stream has passed through the anemometer, e.g., 1/60 mile. Therefore, the pulse train is slow, and the train of pulses can be used only to produce average wind speeds. The number of pulses in a given time increment is recorded. In this way a time series of averages can be produced, whether the time increment is an hour, a day, or months. Shorter averaging intervals may be used, but ultimately the minimum time interval must be determined by the desired speed resolution, which is then related to the speed change required to produce one extra pulse per time increment. One pulse per revolution of the cup is sufficient for use with strip chart recorders (see Section 10.3.4). Some anemometers produce up to 100 pulses per revolution, thus reducing the minimum allowable averaging increment a hundredfold. This is usually done with a "light chopper." The light from a light source is periodically interrupted between the source 
and a photocell, thereby producing a rapid train of electrical pulses. This train of pulses is fast enough to process electronically and produce a continuous signal. The signal may be interpreted as providing quasi-instantaneous wind speeds or a low-pass filtered signal.

In general, a cup anemometer's responsiveness is determined by it s weight, physical dimensions, and internal friction. If any of these factors is increased, the responsiveness of the sensor will decrease. For example, if turbulence measurements are desired, small, lightweight, low friction sensors should be used. Typically, the most responsive cups have a distance constant of about $1 \mathrm{~m}$. If turbulence spectra are being measured (see Appendix 2), there wi11 be a decrease of spectral energy at higher and higher frequencies due to the filtering of the anemometer in addition to the ambient decrease in spectral energy with increasing frequency. Where turbulence data are not required, the cup anemometer can be larger and heavier; distance constants for these are on the order of 3 to $5 \mathrm{~m}$. This limits the maximum usable data sampling rate for heavy anemometers to no greater than once every few seconds.

The cup anemometer's accuracy, like its responsiveness, is affected by the instrument's weight, dimensions, and friction: the more responsive the sensor the more accurate it can be. This is true for new or newly refurbished sensors, but the accuracy of the lightweight sensors is obtained by sacrificing ruggedness. Therefore, close attention must be given to the wear and tear and consequent changes in the calibrations of the less rugged sensors. For large WECS siting studies, even the most rugged anemometers should be checked and/or serviced every 3 to 6 months.

Anemometer accuracy is variable in a turbulent environment. A typical value reported as the accuracy of a cup anemometer is $\pm 2 \%$ - an estimate obtained in a wind tunnel. In the real atmosphere where the flow is turbulent, the cup anemometer will overestimate an average wind speed because a cup will respond to an increase in wind speed faster than it will for a decrease in wind speed. This error (called overspeed) is worst for the least responsive cup anemometers but decreases with increasing wind speed. Since turbulence 
intensity is not a function of mean wind speed only (see Appendix 2), this error in the real atmosphere is a function of local conditions. It is not yet clear just how severe this error can be for the typical cups that are in use, but an average error of several percent is likely.

Numerous environmental problems plague anemometers and reduce their reliability. The reliability of specific models of anemometers has not been adequately tested in an objective comparison field experiment. Generally, the heavier, slower responding sensors are more durable, but icing or blowing dust can cause sensor failure. If an anemometer ices up, the rotation will slow or cease altogether, causing erroneous wind speed estimates until the sensor thaws completely. Strong winds during heavy ice conditions can break cups or the anemometer shaft. Blowing dust can lodge in the bearings, cause an increase in friction and wear, and thereby reduce anemometer estimates of the wind speed. Dust can prevent the closing of the switch in some contact anemometers so that even if the cups are rotating as they should, too few pulses are registered in a given increment of time. Lightweight cups sometimes blow off during high wind events.

In light of these problems, the assurance of reliability in cup anemometers depends upon calibration and service visits. The frequency of these visits will depend upon the value of the data, i.e., the programniatic cost of a loss of a certain interval of data.

\subsubsection{Wind Vanes}

Wind direction is most commonly obtained from a wind vane. The conventional wind vane consists of a broad tail that the wind keeps on the downwind side of the rotating vertical shaft, and a counterweight at the upwind end to provide perfect balance at the junction of the vane and shaft. Friction at the shaft is reduced with bearings.

Wind vanes generally use one of the following mechanisms for producing a signal: contact closures, potentiometers, or servo-mechanisms. The accuracy required of wind direction data will dictate the choice. Vanes using contact closure are generally low priced. These instruments may have four or eight 
switches, each corresponding to a primary direction sector. The vane's position physically determines which switches are closed. If the vane's construction permits simultaneous closure of two switches, the direction resolution is twice the number of switches. For example, a vane with four switches that can only be closed individually can resolve the wind into one of four 90" sectors centered on $N, E, S$ and W. If two adjacent switches can be closed at the same time, the wind direction can be resolved into one of eight 45" sectors: $N, N E, E, S E, S, S W, W, N W$.

A continuous signal that permits wind direction resolution to within a few degrees is produced by wind vanes using potentiometers to generate signals. However, in these vanes, a small sector may exist in which no signal is produced because of the physical construction of the potentiometer. This gap tends to be wide in 1ess-expensive instruments. The discontinuous nature of the signal, generated when the wind causes the vane to fluctuate back and forth across the gap, can cause serious problems in data recording and analysis. For example, when two wind directions near north, $355^{\circ}$ and $005^{\circ}$, are averaged the result is $180^{\circ}$, which is south rather than north. To avoid such problems, some wind vanes have two potentiometers with gaps located $180^{\circ}$ apart and electronic circuitry to switch from one potentiometer to the other when the gap in the first is approached. A wind vane with this feature is said to have $540^{\circ}$ logic.

Other wind vanes have a servo-mechanism for signal generation. However, extensive electronic signal conditioning is required to produce an unambiguous signal for recording when servo-mechanisms are used.

The responsiveness of wind vanes is also associated with a damping or distance constant. If turbulence is to be measured, the distance constant of the vane should be matched with the distance constant of the anemometer. Some recording options (see Section 10.3) compute an average wind speed over the sampling interval but record only an instantaneous sample of the wind direction, usually taken at the end of the interval. If such a data logger is used, a vane with a slow response should be used to mechanically filter the meaningless small-scale fluctuations. 
Environmental problems - ice, blowing dust, salt - similar to those that affect cup anemometers also affect the reliability of wind vanes. High winds are less of a problem for wind vanes than for cups, unless ice is present, because the vane moves to minimize wind stress. The direction calibrations should not change with wear for a wind vane although responsiveness might. During dusty conditions, wind direction sensors that use contacts to indicate the wind direction sector may fail if dirt particles prevent the contact from closing. Recalibration is therefore less of a problem for wind vanes than for cup anemometers, but periodic inspection and routine service is still necessary to maintain optimal performance.

\subsubsection{Prope17er Anemometers}

Propeller anemometers use the wind blowing into a propeller to turn a shaft that drives a dc or ac generator, or a light chopper to produce a pulse train. The response of propeller anemometers covers a range that is similar to cup anemometers; however, the tendency for overspeeding in gusty wind is less for propeller anemometers.

The familiar aerovane, which is a propeller mounted on the nose of a wind vane that is shaped like an airplane fuselage plus tail, is a durable instrument with a distance constant of a few meters. These anemometers may read low in winds with rapidly changing directions if the response of the vane, which directs the propeller into the wind, is slow.

The " Gill" propeller anemometer is driven by a lightweight styrofoam propelier. (a) One propeller may be mounted at the nose of a vane, or two or three may be fixed along different orientations to resolve wind components. The responsiveness and accuracy of these are more than adequate for the turbulence measurements that might be needed for a large WECS siting program. These propellers are too fragile to be left unattended in remote areas.

The problems of reliability for propeller anemometers are similar to those for cup and vane anemometers.

(a) The Gill Anemometer is manufactured by the R. M. Young Company, 2801 Aeropark Drive, Traverse City, MI 49684. 


\subsubsection{Tethered Balloon Systems}

Instruments may be attached to balloons and allowed to rise to levels not conveniently reachable by other means. The earliest radiosondes telemetered temperature data from the lower atmosphere back to the ground. When largescale use of radiosondes began in the 1930s the instrumentation developed to the point that disposable packages were affordable. When wind data became available through radar tracking of the balloon, those systems became known as rawinsondes. Finally, coming around a full circle, instrument packages exist for use with balloons that are tethered to the surface.

Tethering the balloon serves more of a function than keeping an expensive instrument package from escaping. One of the great advantages of using a balloon system instead of a tower to investigate the boundary layer is the mobility of the system. By the use of a tether the instrument package can be "hung" at any desired location at any level for just about as long or short a time as you like. A tower is not mobile enough and a free-floating balloon is too mobile and uncontrollable. One potential problem with tethered balloon systems, especially those that may have been designed for air quality monitoring work, is the lack of durability of the balloon in high winds.

The tether also allows the more conventional, simple, wind speed and direction sensors to function on the airborne platform. If the sensor is not drifting with the wind, a cup or propeller anemometer will provide a usable Eulerian (fixed-coordinate) wind speed. The balloon is shaped so that it will act like a wind vane. If the instrument package is suspended in a fixed orientation to the balloon, then wind direction information can be obtained from the orientation of the package (sensed by a magnetic compass). Alternatively, a package may have its own wind vane and be suspended so that it is free to pivot beneath the balloon or anywhere along the tether line. The orientation of the package vane then provides the direction information. The former approach provides a slower response to wind direction changes, and that is probably desirable for siting work. The disadvantage is that multiple sensors cannot be used on a single line, as in the latter approach.

Although the tethered balloon system may use cup or propeller anemometers to measure wind speed, the accuracy and response of the entire system are not 
identical to the accuracy and response of a system with the same sensors mounted on a tower. The balloon is unfortunately not rigidly fixed in place. The vertical lift on the balloon, dynamic plus buoyant, is balanced by the weight of the instruments and the vertical component of tension in the tether. The tether follows the shape of a catenary curve. The horizontal force balance on the balloon is between the drag force of the wind and the horizontal component of the tension. In gusty winds these force balances change, causing the balloon to climb and sink and move upstream and downstream. The upstream and downstream motions contaminate the wind speed signals coming from the balloon. The properties of this contamination are a function of the characteristics of the balloon system's response to fluctuations of different frequencies, and the spectrum of the ambient turbulence (see Appendix 2). One could expect the accuracy and precision of mean wind speed measurements from a tethered balloon system to be less than from similar sensors mounted on a tower. Since the balloon motions introduce variance to the wind speed signal, interpretations of turbulence intensity should be made with caution unless one is familiar with both the spectral distribution of variance in the boundary layer (Appendix 2) and with the characteristics of the balloon system.

Signals from tethered balloon systems are typically digitized and multiplexed within the instrument package and telemetered on an $\mathrm{HM}$ carrier wave. Usually, pressure, temperature, wind speed and direction, and perhaps relative humidity are measured. The user should find out for a particular system how often each of these variables is sampled and if the sample represents averages or instantaneous readings.

\subsubsection{Kites and Kite Anemometers}

Kites can serve as wind indicators in two ways. First, they can provide a visual indication of the wind direction and of the steadiness of wind at their level of flight. Anyone who has struggled with adjusting the tail and bridle of old-fashioned crossed-bow paper kites can well imagine that kite aerodynamics have as much to do with unsteady kite flight as do turbulent winds. There are, however, some remarkably stable flying kites on the market. 
Kites can be used to indicate regions of highly turbulent separated flow (see Chapter 5). However, it must be remembered that the kite flies and interacts with turbulence and is not just a passive rider of turbulent gusts.

The second application of kites uses the tension on the line as an indicator of wind speed (Woodhouse 1980). Two transducers are available for turning the line tension into a usable signal - a strain gage that produces a voltage output or a spring-scale translating stretch into wind speed. Both system calibrations are easily checked in the field although both calibrations may change with system wear.

In wind tunnel tests, the tension on the line can be calibrated to within $\pm 2 \%$. It is expected that a kite calibration determined in the wind tunnel will be less accurate in the real atmosphere, just as the cup anemometer behaves differently in the turbulent atmosphere than in the wind tunnel. The kite anemometer concept was tested as part of an international comparison experiment for low-level meteorological instrumentation at the 300-m tower of the Boulder Atmospheric Observatory (BAO) (Kaimal et a1. 1980). In that experiment, analog voltage signals for line tension, line azimuth, and line inclination were sent directly to the BAO computer where they were digitized at $10 \mathrm{~Hz}$. Similar data from Gill and sonic anemometers mounted on the BAO tower were used for comparison. Numerous 20-minute runs were made over a several day period for comparing the average westerly (U) component and the average southerly (V) component of the wind. The results presented by Kaimal et a1. (1980) are reproduced in Table 10.1.

TABLE 10.1. Statistical Summary of Comparison Between Kite Anemometer and BAO Tower. Speeds in $\mathrm{m} / \mathrm{sec}^{-1}$ (Kaimal et al. 1980).

\begin{tabular}{|c|c|c|c|c|c|c|c|}
\hline Variable & Sensor & $\begin{array}{c}\text { No. of } \\
\text { 20-Min } \\
\text { Averages }\end{array}$ & $\begin{array}{l}\text { BAO } \\
\text { Mean }\end{array}$ & $\begin{array}{l}\text { Sensor } \\
\text { Mean }\end{array}$ & $\begin{array}{c}\text { RMS } \\
\text { Difference }\end{array}$ & Correlation & $\begin{array}{l}\text { BAO Mean } \\
\text { Speed }\end{array}$ \\
\hline $\begin{array}{c}U \\
\text { (Westerly) }\end{array}$ & $\begin{array}{l}\text { TALA } 1(\mathrm{a}) \\
\text { TALA } 2\end{array}$ & $\begin{array}{l}25 \\
12\end{array}$ & $\begin{array}{l}0.94 \\
3.89\end{array}$ & $\begin{array}{l}0.97 \\
2.94\end{array}$ & $\begin{array}{l}0.96 \\
1.72\end{array}$ & $\begin{array}{l}0.94 \\
0.92\end{array}$ & $\begin{array}{l}4.31 \\
8.64\end{array}$ \\
\hline $\begin{array}{c}\text { V } \\
\text { Southerly) }\end{array}$ & $\begin{array}{l}\text { TALA } 1 \\
\text { TALA } 2\end{array}$ & $\begin{array}{l}25 \\
12\end{array}$ & $\begin{array}{l}1.12 \\
6.51\end{array}$ & $\begin{array}{l}1.24 \\
5.99\end{array}$ & $\begin{array}{l}0.82 \\
1.15\end{array}$ & $\begin{array}{l}0.98 \\
0.97\end{array}$ & $\begin{array}{l}4.31 \\
8.64\end{array}$ \\
\hline
\end{tabular}

(a) TALA 1 is a small kite used in light winds and TALA 2 is a larger kite used during strong winds. 
The data of Table 10.1 are appropriate for assessing instrument performance where both wind direction and speed are important. For many potential uses of kite anemometers in wind energy applications substantial wind direction error could be tolerated and only the wind speed signal would be of interest. Since some of the rms difference reported in Table 10.1 is due to error in the wind direction, it is not possible to estimate wind speed error from those data.

Comparisons of wind speeds measured by the kite anemometer and the BAO tower were made ${ }^{(a)}$ using preliminary but unvalidated data generated by the BAO computer at the end of each day's measurements. These results are encouraging. Nineteen comparisons of wind speed were made for winds between 5 and $12 \mathrm{~m} / \mathrm{sec}$. Linear regression between tower and kite measurements yield $r^{2}=0.92$, where $r$ is the correlation coefficient, and a standard error of the estimate of $\pm 0.4 \mathrm{~m} / \mathrm{sec}$. It appears that a kite anemometer system can deliver a signal useful for determining the mean wind speed.

Since the response characteristics of the system are not well known, it is premature to attempt to infer any quantitative interpretation of turbulence from the kite signals. This includes using the standard deviation of wind speed measured by the kite to produce a turbulence intensity. There is no doubt that there is some relation there, but the details remain unclear.

\subsubsection{Remote Sensing Systems}

A remote sensing system makes measurements without placing an active sensor at the point of measurement. At the present time, no remote sensing system is free from shortcomings, which limits the potential of these systems for wind energy siting measurements. In some cases these deficiencies arise from the inability of the measurement system to make measurements required; in others, because the systems are too complex, too expensive, too labor-intensive, etc. However, development is continuing on several systems and they may become more useful and accessible in the future. Hooke (1980) provides a

(a) From an unpublished communication from FKG and Associates, Tullahoma, Tennessee. Validated data are those that have been screened and reviewed for quality by the BAO staff. 
thorough review of the applicability of remote sensing instrumentation and techniques for wind energy problems. Further descriptions may be found in Derr (1972). This section will therefore only present the basic concepts.

All techniques for remote sensing of wind speeds work on the same fundamental principle. Some form of propagating wave interacts with some entity at the point of measurement and is monitored at the remote receiving station. The simplest propagating wave and receiver system is visible light and the eye. Watching the evolution of a dust or smoke plume is a form of remote sensing. That principle, developed to its fullest potential, uses two theodolites to triangulate on the position of a pilot balloon, or pibal, as it is moved by the winds. Modern equipment can provide very good digital data on the motion of the balloon as it rises through the lower atmosphere. A meaningful average wind profile can only be obtained from an ensemble of many balloon ascents, since each ascent produces just a string of quasi-instantaneous wind measurements from the turbulent boundary layer. In complex terrain, repeated pibal releases from the same point may result in substantially different trajectories, due to the behavior of the large-scale turbulent eddies and secondary circulations. Therefore, an ensemble average wind speed referenced to a given terrain feature may not be achievable.

Echoes of beams scattered from atmospheric structures or constituents are the basis of most remote sensing techniques. To begin, some kind of beam is emitted. Microwaves (radar), laser light, or acoustical waves are the usual beam sources. The beam is scattered, dispersed, and attenuated as it passes through the atmosphere. A receiver looks at a sample volume of the atmosphere to capture the portions of the emitted beam that were scattered within that volume.

Radar uses $1-\mathrm{cm}$ to $10-\mathrm{cm}$ wavelength radiation. The scattering of this radiation is strong from hydrometeors (cloud droplets, rain drops, ice crystals) within a certain size range, which depends on the wavelength of the radiation. Small foil strips or chaff can be dumped into a dry atmosphere to provide strong scatterers. In a nonsaturated but moist atmosphere, there is a weak 
scattering of microwaves, primarily from humidity variations. Lidar senses laser light scattered primarily from aerosols and again, the optimal particle size depends upon the wavelength used. However, the coherence of the laser beam is quickly degraded in a turbulent atmosphere by index of refraction variations caused by temperature and humidity variations. To mitigate this undesired effect, long wavelength lasers such as a $10.6 \mu \mathrm{m} \mathrm{CO} \mathrm{C}_{2}$ laser are used. Acoustic waves are scattered from temperature variations and velocity turbulence.

Wind speed may be inferred from the return signals in two ways. Since the scatterers are carried along with the wind and since the concentrations or structure of the scattering regions do not change quickly, compared to the time between samples collected by the receiver, the drift of the scatterers from one sample to the next can be used to infer wind vectors. Alternatively, as the beam is scattered, the frequency of the radiation will be Doppler shifted, due to the velocity of the scatterer. Measuring the Doppler shift yields the component of wind velocity between the receiver and the scatterer. Measuring all three components of the wind speed requires that three noncolinear receivers be focused on the sample volume.

The techniques do not work if scatterers are not present, if the air is too free of aerosols for laser techniques, if there are no temperature fluctuations (and there are none in a neutral stability layer--see Appendix 2) for monostatic acoustic sounder techniques, ${ }^{(a)}$ or if the air is so dry that there are no humidity fluctuations or hydrometeors for radar techniques.

The range of all echo-sounding techniques is limited by the attenuation of the emitted and scattered beams, and the weak signals produced by interaction of the beam and the scatterer. Efforts to increase the range usually attempt to increase the power of the beam. The spatial and temporal resolution of echo techniques depends upon the intersection of the geometrical and temporal characteristics of the emitted beam and the geometrical and temporal characteristics of the region sampled by the receiver.

(a) Monostatic acoustic sounder systems (colocated transmitter and receiver) sense only waves backscattered from temperature fluctuations. Bistatic systems (separated transmitter and receiver) sense waves scattered from fluctuations of temperature and velocity. 
For example, one of several possible configurations of acoustic Doppler systems emits a pulse of sound vertically. The pulse spreads out to fill a solid angle determined by the construction of the transmitter. The radial length of the pulse is just the speed of sound multiplied by the pulse duration. Pulse travel time is, in the same way, proportional to height above ground. Portions of the pulse are scattered in the direction of a receiving antenna. If the scattering occurred within the solid angle scanned by the antenna, the receiver will pick up the signal, determine the Doppler shift, and hence, the component of velocity along the trajectory of the received signal.

A vertically oriented receiver antenna (usually the transmitter antenna also) can sense vertical velocities. Antennas oriented at an angle can be used to sense horizontal wind components when the vertical velocity is known. The angle should be far enough from vertical to contain a resolvable component of horizontal wind speed, but not so far from vertical that the signal becomes too attenuated.

In this system, we see that the pulse width and the width of the region scanned by the antenna determine the horizontal dimensions of the sample volume, and the speed of sound and the pulse duration determine the vertical dimension of the sample volume. Pulses are repeated after all signals from the highest attainable levels above ground have been received. Similar principles are involved in the other echo-sounding techniques.

A technique using laser light that is not an echo sounding technique measures the average component of the wind normal to a beam path through the atmosphere. The index of refraction variations that are to be avoided in laser-Doppler wind sensing techniques are the cornerstone of transverse windsensing techniques using lasers. These variations, due to temperature and humidity structures of various sizes along the beam path, cause the image of the laser to scintillate or twinkle. The turbulent eddies that cause the scintillations do not change appreciably over the time required for the wind to advect those eddies a short distance. Therefore, two receivers at the end of two closely spaced parallel beam paths will see the time series of irradiance 
at the upwind receiver repeated a very short time later in the downwind receiver. A signal correlation computer can produce a real-time readout of the transverse wind component. Lawrence et a1. (1972) used this technique to measure the average wind normal to a $15-\mathrm{km}$ path. Measurements along a $1-\mathrm{km}$ path have shown good agreement between spaced, averaged propeller anemometer readings and the laser derived wind (Beran and Hal 1 1974).

Hooke (1980) concludes that the high costs and demanding operational requirements of remote sensor devices preclude their use in the early stages of the siting process, but that remote sensing devices could be used effectively in the site development phase (see Chapter 2) at the site of a large cluster of WECS. Though these devices may produce sufficient data, they are not necessarily as simple to operate, as reliable, or as economical as the conventional instrumented tower approach. Remote sensing systems in increasing order of expense and operating demands are:

- optical transverse wind sensors

- acoustic Doppler sounders

- Tidar

- Doppler radar.

Only the first two of these are currently commercially available. Finally, Hooke concludes that if user requirements for siting data become more stringent, as inflation increases the cost of conventional siting methods, and as improved technology reduces the cost of remote-sensing alternatives, those alternatives wi 11 become increasingly attractive.

\subsubsection{Specialty Sensors}

Other sophisticated sensors exist for making wind measurements. Among these are: hot-wire and hot-film anemometers, sonic anemometer-thermometers, and vortex anemometers. These instruments are commonly used in research but are not appropriate for use in WECS siting programs. The principles and applications of these specialty sensors are briefly described in this section.

The hot-wire anemometer measures the electrical energy necessary to maintain a constant temperature or current in a resistively heated wire that is cooled by the wind. The hot-film anemometer is a more rugged version of the 
hot-wire that uses a quartz-coated wire. These sensors are useful for providing very accurate data on the very fine scales of turbulence, much finer than are important to wind turbines (see Appendix 2, Section A.2.2.3 for a discussion of the scales of atmospheric turbulence). Hot-wires are very delicate and sophisticated systems that require a great deal of attention. They measure high frequency fluctuations very well but do not measure mean winds well because the signal can drift a significant amount. Hot-films are used only in occasional short-term micrometeorological research experiments where fine structure must be measured.

Sonic anemometer-thermometers are also used in micrometeorology for very fine structure measurements, but are generally not suitable for measuring mean wind speeds. Sound waves propagate relative to the air they move in. The sonic anemometer-thermometer measures the speed of sound in opposite directions across a short gap of several centimeters. The difference in the two speed measurements is twice the component of wind speed in the direction of the measurement. The sum of the two speed measurements is equal to twice the speed of sound and, therefore, proportional to the square root of the temperature.

The vortex anemometer measures the wind speed by measuring the frequency at which vortices are shed from a cylindrical obstruction to the flow. When a small, circular cylinder is placed in a flow, vortex streets form downwind of the cylinder. A vortex street consists of very regular patches of vorticity, shed first from one side of the cylinder, then from the other. The rate at which these vortices are shed depends upon the Reynolds number, Re $=\mathrm{UL} / \mathrm{\nu}$, where $U$ is the wind speed, $L$ is the diameter of the cylinder, and $v$ is the kinematic viscosity of air. A hot-film placed downstream of the cylinder is used to detect the frequency of vortex shedding. Changing calibrations and drift of the hot-film system are not significant problems here, since only a frequency is being detected. The vortex anemometer provided wind measurements for the Viking spacecraft that landed on Mars. However, it is more sophisticated than is necessary for siting measurements. 


\subsubsection{Conclusion}

In this section, we have discussed basic features of several types of wind sensors. The conventional wind speed and direction sensors, i.e., the cup or propeller anemometers and the wind vane, are most appropriate for wind energy siting measurements. This is because of the proven reliability and base of experience with conventional systems, and because the accuracies and responsiveness are appropriately matched to the problem. It is currently felt that conventional anemometry is more practical (both long-term and short-term) for siting measurements than is anemometry using remote sensors. In the future this may no longer be so. Tethered balloon and kite measurements have potential for making short-term wind speed measurements.

\subsection{RECORDING SYSTEMS}

This section discusses methods of handling and recording on-site data produced by wind sensors and their associated transducers. Each method has advantages and drawbacks. The particular problem will define the data requirements, which in turn dictate the choice of recording methods. Based on data storage capabilities, wind data collection systems can be grouped into four general classes (Ramsdell et al. 1980), which are labeled as I, II, III, and IV:

\begin{tabular}{|c|c|}
\hline Class & Data Storage Capability \\
\hline $\mathbf{I}$ & $\begin{array}{l}\text { None; display on a meter; observer } \\
\text { records readings }\end{array}$ \\
\hline I I & Limited to a single storage register \\
\hline II I & $\begin{array}{l}\text { Processed information stored in data } \\
\text { logger with more than one storage } \\
\text { register, but sequence of data is lost }\end{array}$ \\
\hline IV & $\begin{array}{l}\text { Processed or raw information with } \\
\text { sequence retained. }\end{array}$ \\
\hline
\end{tabular}

The characteristics of these data systems are discussed below and are summarized in Table 10.2. The option of relaying data from the site via a telemetry link is also discussed. 


\section{TABLE 10.2. Instrument Systems}

\begin{tabular}{|c|c|c|c|c|}
\hline Class & Recording Device & Data Product & Advantages & Disadvantages \\
\hline I & None & $\begin{array}{l}\text { Display of current } \\
\text { conditions }\end{array}$ & $\begin{array}{l}\text { Low cost, portable, } \\
\text { simple to use. }\end{array}$ & $\begin{array}{l}\text { Humans must record data; this } \\
\text { introduces observer biases. }\end{array}$ \\
\hline II & $\begin{array}{l}\text { Single storage register; } \\
\text { mechanical or electronic. }\end{array}$ & $\begin{array}{l}\text { Integrated statistic } \\
\text { such as monthly mean } \\
\text { wind speed. }\end{array}$ & $\begin{array}{l}\text { Low cost, simple to } \\
\text { use, can be I eft } \\
\text { unattended in remote } \\
\text { locations. }\end{array}$ & Limited utility of data. \\
\hline $\operatorname{III}(\mathrm{a})$ & $\begin{array}{l}\text { Multiple register/ } \\
\text { processing data logger; } \\
\text { solid state memory. }\end{array}$ & Statistics, histograms. & $\begin{array}{l}\text { Preprocessing of data } \\
\text { reduces data analysis } \\
\text { costs. }\end{array}$ & $\begin{array}{l}\text { Choice of outputs must be } \\
\text { carefully made since } \\
\text { preprocessing of data limits } \\
\text { usefulness of data. Higher } \\
\text { cost. }\end{array}$ \\
\hline$I V^{(a)}$ & $\begin{array}{l}\text { Raw data storage or } \\
\text { preprocessed data. } \\
\text { Strip chart, magnetic } \\
\text { tape, magnetic disk, } \\
\text { soli.d state. }\end{array}$ & $\begin{array}{l}\text { Time series of raw data } \\
\text { and/or time series of } \\
\text { sub-sampling interval } \\
\text { statistics. }\end{array}$ & $\begin{array}{l}\text { Maximum flexibility in } \\
\text { use of data. Usable } \\
\text { partial record may } \\
\text { exist if remote system } \\
\text { fails. Easier to } \\
\text { assess quality of data } \\
\text { collected. }\end{array}$ & $\begin{array}{l}\text { More intensive data reduction } \\
\text { effort. Higher cost. }\end{array}$ \\
\hline
\end{tabular}

(a) Consider options of on-site recording or telemetry data link. 


\subsubsection{Class I Systems}

Class I instrument systems do not have any data storage capability. If data are to be collected, a human observer must monitor the system and record the data. Since data collection requires substantial human involvement the cost of data collection per sample is likely to be high. This restricts Class I systems to two kinds of uses:

- short-term intensive measurement programs long-term programs that require only a small portion of the observers regular duties be devoted to reading the instruments.

An example of the first kind is the use of handheld anemometers or kite anemometers with spring scales as part of a series of spot measurements at selected points of interest. There are unique errors associated with this use of a Class I system, regardless of the accuracy of the sensor. These errors are due to the observers attempt to read scales that are fluctuating faster than the eye can track.

Consider the spring-scale kite anemometer. The wind speed is measured by the deflection of a spring, attached to the kite line, with scales marked of $f$ in integral values of $\mathrm{m} / \mathrm{sec}$ or mph. The marks are packed closer together at higher wind speeds because of the nonlinear relationship between wind speed and spring scale extension.

An observer reading the fluctuating spring scale cannot make a single reading to any better than \pm 1 scale division, probably worse at the high wind speed end of the scale. Even if there were no need to take repeated measurements because of the sampling error caused by sampling too few of the largest turbulence eddies (Appendix 2, A.2.2.3), the observer would need to make repeated readings in an effort to statistically minimize what are hoped to be random errors in reading the scale. Observations of this type are well known to err systematically and not strictly randomly. Observers preferentially pick significant scale intervals many times during an ensemble of measurements. In one experiment with the spring-scale kite, 60 samples, one every 5 seconds for 5 minutes, were read from the scale to the nearest $1 \mathrm{mph}$ in about $28 \mathrm{mph}$ winds. Analysis of the series of numbers selected showed that the observer reported an unexpectedly high number of 25 and $30 \mathrm{mph}$ readings. Furthermore, 
the analysis showed that the observer preferred to round downward to those values and not up. In that measurement the observer bias alone contributed about $2 \%$ error in the uncertainty of the mean. From the original 60 samples was derived an artificial synthesis of two separate wind speed measurements using 30 samples, one every 10 seconds for 5 minutes. Therefore, one group consisted of the odd sample and the other group the even sample of the original 60 samples. The means of these two groups differed by $0.6 \mathrm{mph}$. That is, there was an unavoidable error of $2 \%$ just from the choice of samples. It is therefore recommended that, if short-term intensive measurements are to be made with Class I data systems, the users consider and weigh the potential contribution of observer bias and error.

Examples of the second kind of use of Class I data systems include routine brief observations made hourly at National Weather Service offices, airports, military bases, ships, and so on. In programs of this type it is best to adhere to a strict routine schedule of operations and not let observers choose their onn observing times. This is because people tend to selectively notice and record higher wind speed events if they are not regimented by external constraints, such as a schedule.

\subsubsection{Class II Systems}

Class II instrument systems characterize the wind with a single number. The wind-run anemometer is a classic example of these systems. The wind-run anemometer is such a simple system that the storage mechanism and the sensor are frequently built as one unit. The storage unit keeps a running count of the miles of wind that pass through the anemometer. There is no reason why a more complicated statistic could not be accumulated by a Class II system, such as wind speed cubed or the product of wind speed cubed and temperature. However, since the accumulator sacrifices a great deal of temporal information, there is no compelling reason to record anything more than miles of wind. Use of these instruments requires that the storage register or accumulator be read twice and that the time between readings be known. If a wind-run anemometer is used, the average speed for the observation period is found by dividing the wind-run (difference in readings) by the elapsed time between readings.

The average wind speed may be used to approximate the wind speed probability density function (PDF). The Rayleigh distribution is an analytical 
expression that closely resembles many measured wind speed distributions (see Figure 10.1). The mean wind speed is the only parameter required to specify the Rayleigh distribution, which is:

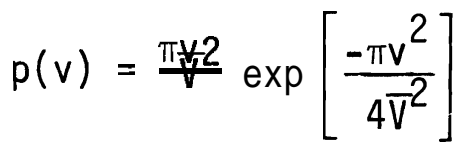

where

$$
\begin{aligned}
p(v) & =\text { probability density of wind speed (fraction per } \mathrm{m} / \mathrm{sec} \text { ) } \\
\mathrm{v} & =\text { wind speed } \\
\nabla & =\text { long term average wind speed. }
\end{aligned}
$$

Class II systems are particularly useful in remote locations where access and power for system operation are limited, and where site visits are infrequent. If the interval between visits is long, there is a greater risk of a costly loss of data due to system or sensor failure. If the instruments are read on a regular basis (weekly or monthly), both the total wind resource and its seasonal variation can be estimated with data from these systems. These instruments could be read as frequently as once an hour to provide diurnal wind information, but such an observation program resembles a Class I use of the system rather than taking advantage of the integrating ability of the Class II system. The user can treat the loss of temporal resolution in Class II systems for wind speed in one of two ways. Assumptions about the diurnal variations in mean wind speed and the distribution of wind speeds about the mean (e.g., Rayleigh distribution) can be made. Or more approximately, the user can simply acknowledge the data as they are: that on the average one site has more wind at the level of the anemometer than another site, and that further assumptions about the structure of the wind resource are not substantiated by the data provided with a Class II system.

There is no reason why a Class II system for wind direction could not be developed; however, there is no good reason to develop one. Wind directions averaged over the time intervals typical of Class I I systems are meaningless for wind energy siting. Wind direction information is valuable if coupled with the simultaneous wind speed information. This requires either a Class III or Class IV system. 


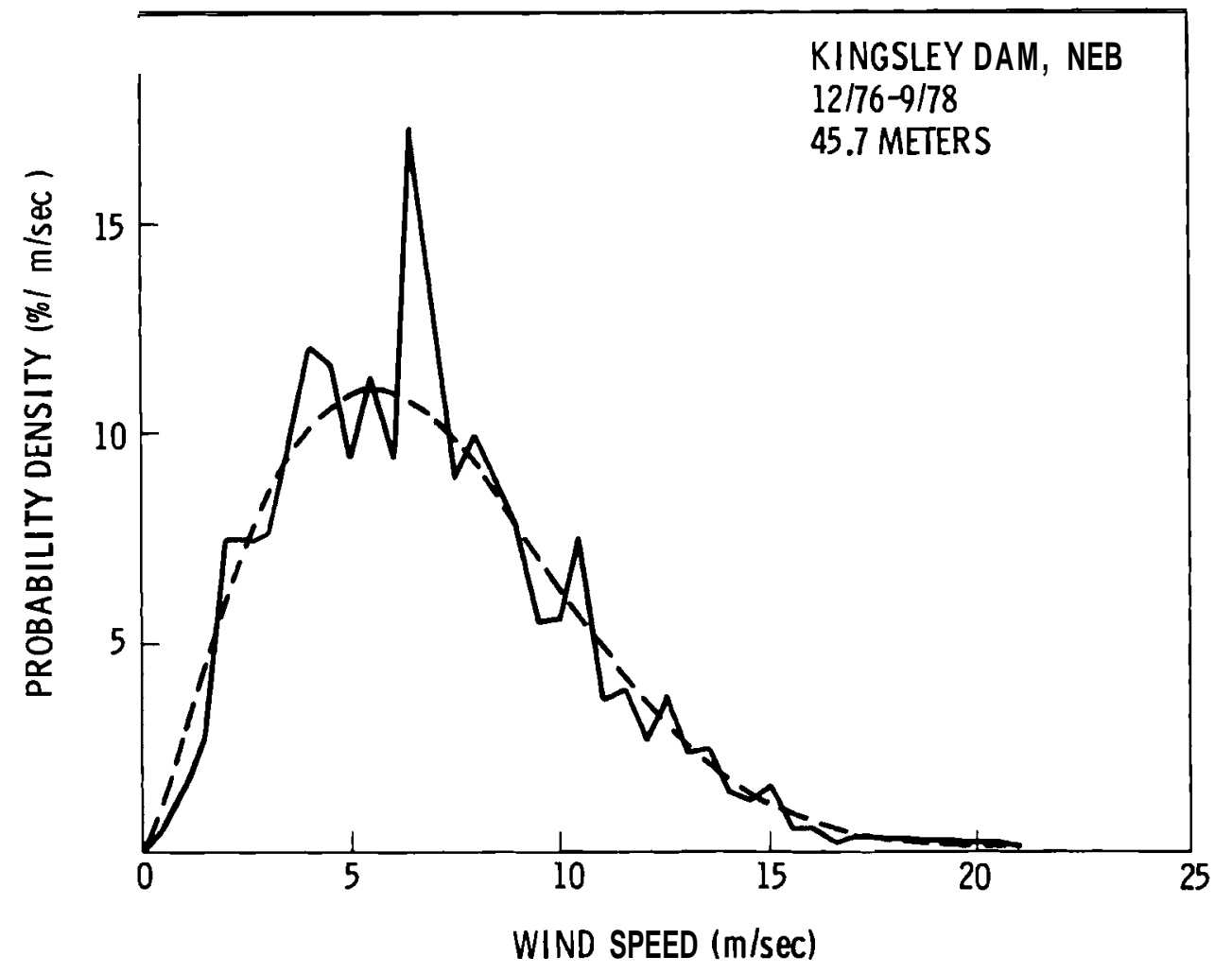

FIGURE 10.1. Wind Speed Frequency Distribution from Kingsley, Nebraska (Solid) and the Rayleigh Distribution (Dashed). A Rayleigh distribution is obtainable with a Class II instrument system.

\subsubsection{Class III System}

Class III systems process data and record it in summaries or histograms. A microprocessor is used to compute the statistics of interest and store the appropriate information in one of many storage registers. By carefully selecting or designing the data logger of Class III systems, it is possible to collect and store very powerful data in a form ready for final analysis and presentation. However, care must be taken to ensure that all needed summaries are formed and stored in the data logger. Because information on individual observations is lost in the summaries, it is not possible to significantly change the types of summaries after data collection. The potential exists, of course, to 
produce data loggers that are user-programmable so that the user can change the data processing routines, if necessary, after 1 month's data have been analyzed. Data from Class III systems typically are retrieved from the memory by some form of "milker" that displays, prints, or transfers the data onto a portable medium such as cassette tapes. The storage registers are then reset.

Direct accumulation of the wind speed frequency distribution is the most fundamental measurement need capable of being fulfilled by Class III systems. The Rayleigh distribution does not always match the shape of the PDF as Figure 10.2 clearly shows. More complicated wind distributions exist. For example, the Weibull distribution (Justus et al. 1976) is a unimodal distribution described by:

$$
p(v)=\left(\frac{k}{c}\right)\left(\frac{v}{c}\right)^{k-1} \exp \left\{-(v / c)^{k}\right\}
$$

where

$$
\begin{aligned}
\mathrm{p}(\mathrm{v}) & =\text { probability density of wind speed (fraction per } \mathrm{m} / \mathrm{sec}) \\
\mathrm{c} & =\text { scale factor related to the mean wind speed }(\overline{\mathrm{V}}) \text { by } \\
\nabla & =c \Gamma(1+1 / \mathrm{k}) \\
\Gamma & =\text { the gamma function } \\
\mathrm{k} & =\text { shape factor related also to the variance of wind speed }\left(\sigma_{\mathrm{v}}{ }^{2}\right) \text { by } \\
\sigma_{\mathrm{v}}{ }^{2} & =c^{2}\left\{\Gamma(1+2 / \mathrm{k})-[\Gamma(1+1 / \mathrm{k})]^{2}\right\} .
\end{aligned}
$$

Note that the Rayleigh distribution is a special case of the Weibull where $k=2$. A Class III system could compute $\bar{V}$ and $\sigma_{v}$, which could then be used to fit a Weibull distribution. From Figure 10.2 it is evident that in some cases the Weibull distribution would not be a good fit.

Direct measurement of the wind speed distribution is the preferred approach. Once the distribution has been measured, one could perform a least-squares fit to a function such as the Weibull distribution function. If the fitted function adequately models the measured distribution, the analytical function provides a concise expression for representing the measured data. (It is interesting to note that the bimodal distribution of Figure 10.2 can be well 


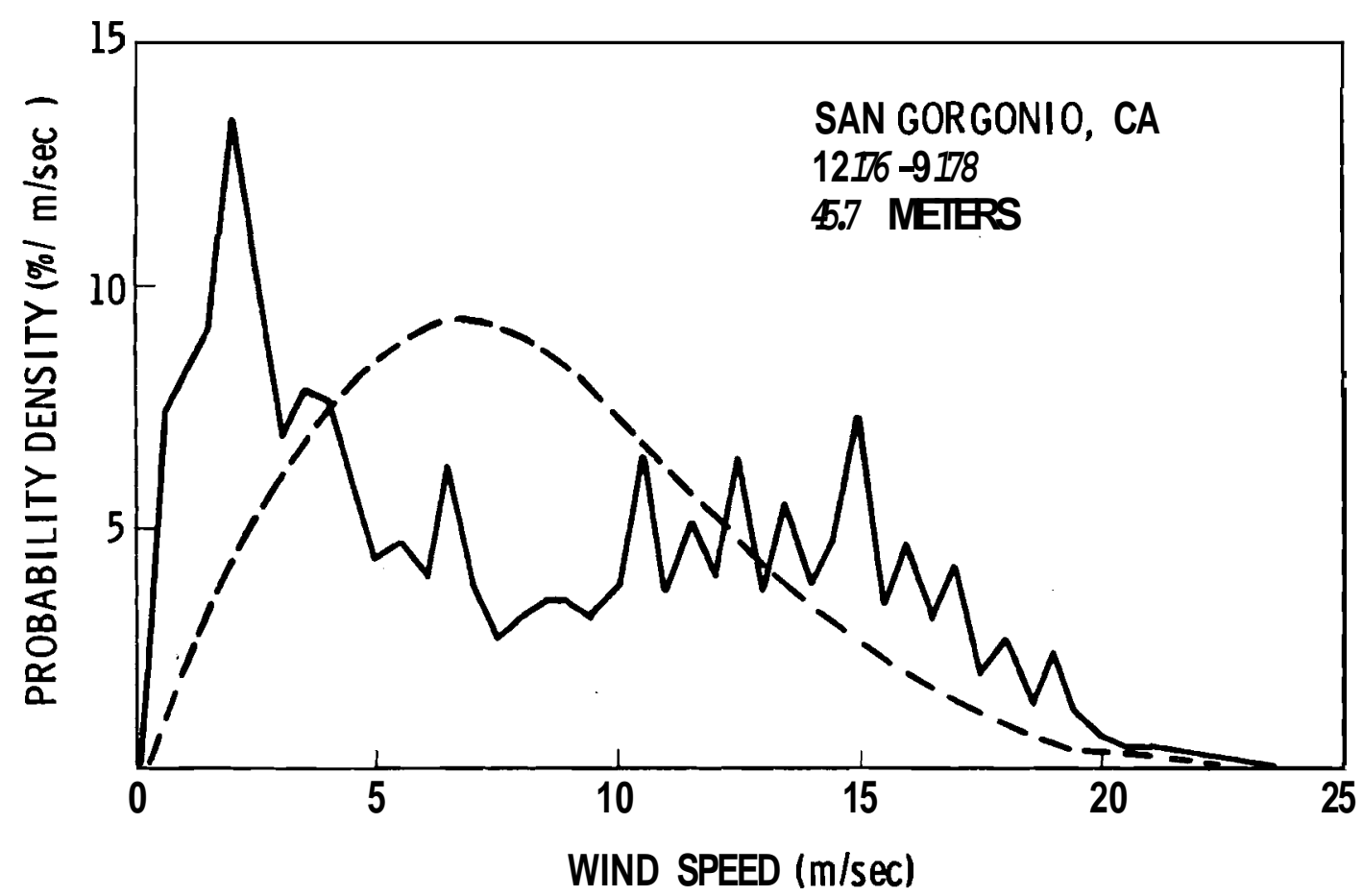

FIGURE 10.2. Wind Speed Frequency Distribution from San Gorgonio, California (Dashed Curve is the Rayleigh Distribution). The actual distribution is obtainable with Class III of IV instrument systems.

represented by the sum of two separate Weibull distributions ${ }^{(a)}$ which suggests two independent wind mechanisms are responsible.) Several commercially avai7able Class III systems are capable of accumulating the wind speed frequency distribution.

The potential for further development of this type of storage system is tremendous and as yet largely undeveloped. It is anticipated that more of these advanced data loggers will be commercially available in the near future. The following are just some examples of the kinds of statistics that could be produced by Class III systems:

(a) Personal communication from N. J. Cherry, Lincoln College, New Zealand. 
produce data loggers that are user-programmable so that the user can change the data processing routines, if necessary, after 1 month's data have been analyzed. Data from Class III systems typically are retrieved from the memory by some form of "milker" that displays, prints, or transfers the data onto a portable medium such as cassette tapes. The storage registers are then reset.

Direct accumulation of the wind speed frequency distribution is the most fundamental measurement need capable of being fulfilled by Class III systems. The Rayleigh distribution does not always match the shape of the PDF as Figure 10.2 clearly shows. More complicated wind distributions exist. For example, the Weibull distribution (Justus et al. 1976) is a unimodal distribution described by:

$$
p(v)=\left(\frac{k}{c}\right)\left(\frac{v}{c}\right)^{k-1} \exp \left\{-(v / c)^{k}\right\}
$$

where

$$
\begin{aligned}
\mathrm{p}(\mathrm{v}) & =\text { probability density of wind speed (fraction per } \mathrm{m} / \mathrm{sec} \text { ) } \\
\mathrm{c} & =\text { scale factor related to the mean wind speed }(\overline{\mathrm{V}}) \text { by } \\
\overline{\mathrm{V}} & =c \Gamma(1+1 / \mathrm{k}) \\
\Gamma & =\text { the gamma function } \\
\mathrm{k} & =\text { shape factor related also to the variance of wind speed }\left(\sigma_{\mathrm{v}}{ }^{2}\right) \text { by } \\
\sigma_{v}{ }^{2} & =c^{2}\left\{\Gamma(1+2 / k)-[\Gamma(1+1 / k)]^{2}\right\} .
\end{aligned}
$$

Note that the Rayleigh distribution is a special case of the Weibull where $k=2$. A Class III system could compute $\bar{V}$ and $\sigma_{v}$, which could then be used to fit a Weibull distribution. From Figure 10.2 it is evident that in some cases the Weibull distribution would not be a good fit.

Direct measurement of the wind speed distribution is the preferred approach. Once the distribution has been measured, one could perform a least-squares fit to a function such as the Weibull distribution function. If the fitted function adequately models the measured distribution, the analytical function provides a concise expression for representing the measured data. (It is interesting to note that the bimodal distribution of Figure 10.2 can be well 


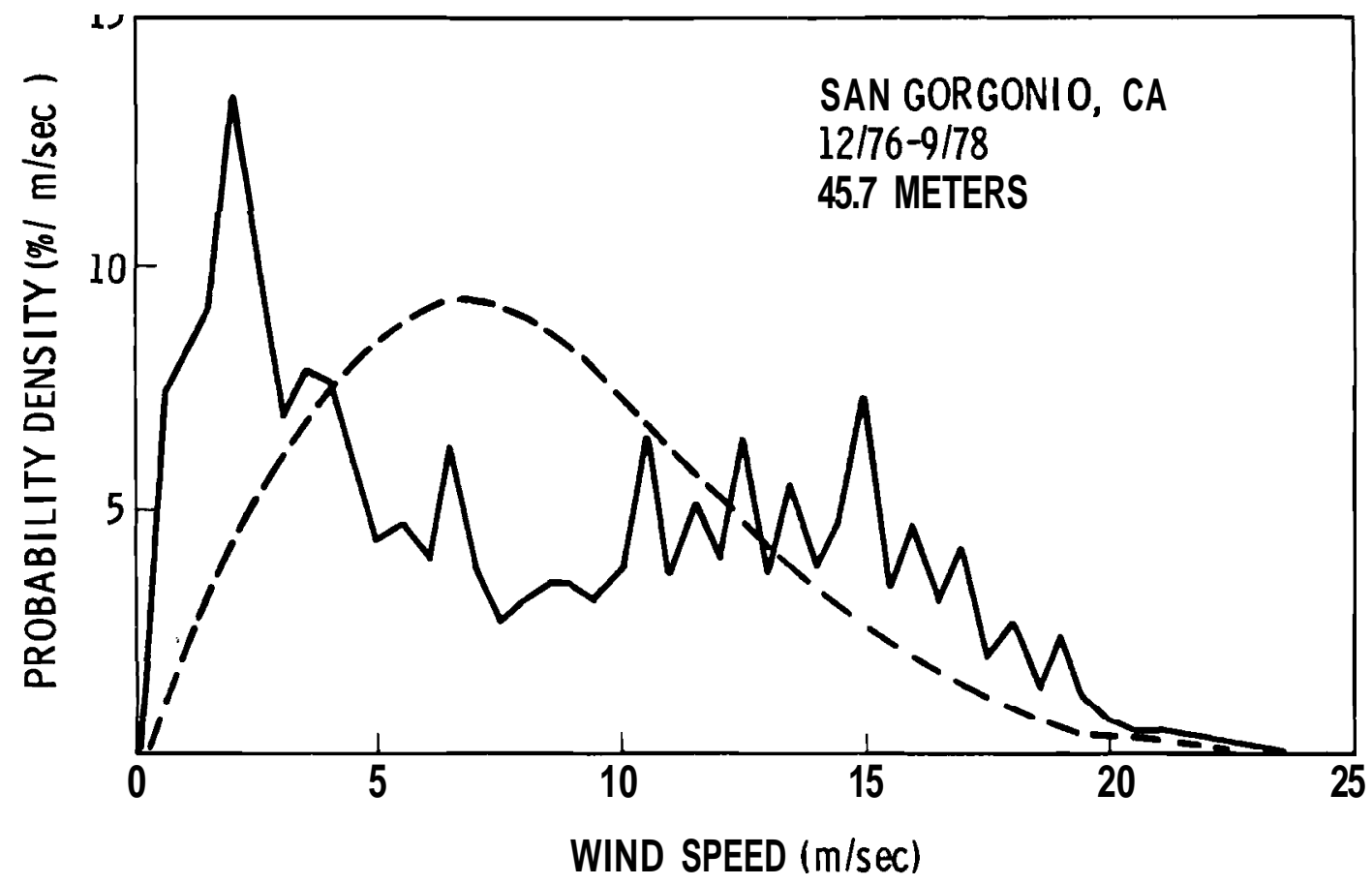

FGURE 10.2. Wind Speed Frequency Distribution from San Gorgonio, California (Dashed Curve is the Rayleigh Distribution). The actual distribution is obtainable with Class III of $\mathrm{N}$ instrument systems.

represented by the sum of two separate Weibull distributions(a) which suggests two independent wind mechanisms are responsible.) Several commercially avai 1able Class III systems are capable of accumulating the wind speed frequency distribution.

The potential for further development of this type of storage system is tremendous and as yet largely undeveloped. It is anticipated that more of these advanced data loggers wi 11 be commercially available in the near future. The following are just some examples of the kinds of statistics that could be produced by Class III systems:

(a) Personal communication from N. J. Cherry, Lincoln College, Nw Zealand. 
- frequency distribution of wind speed (see Figures 10.1 and 10.2) by time of day

- frequency distribution of wind speed-wind direction by time of day

- average diurnal variation of wind speed (see Figure 10.3) or wind direction

- maximum wind speed

- frequency distribution of duration of wind speed episodes within given speed classes (see Figure 10.4)

- frequency distribution of the magnitudes of changes of wind speed or direction within a given time interval (see Figure 10.5)

- frequency distribution of wind shear between two anemometers (see Figure 10.6)

- distribution of subhourly variances of wind speed or wind direction.

It is anticipated that more of these advanced data loggers will be commercially available in the near future. The only limitation on the type and amount of processing that a Class III system is capable of is the amount of time necessary to make the computations that must be completed before the next sample is taken.

One does not wish to produce histograms that are broken down into finer resolution than is necessary but if there is any doubt, a fine resolution should be selected. For example, if time of day information recorded in histograms with time groups 3 hours wide is adequate, there is no need to create statistics for each hour of the day. Hourly histograms may be summed to provide three-hourly histograms but the converse is impossible.

Class III systems share some of the advantages of Class II systems, although they are more expensive. They may be operated in remote regions and visited infrequently since they also can be operated with their own battery power sources. They share the risk of total data loss in any given interval between site visits if the system fails, or partial data loss if the solidstate memory saturates. 


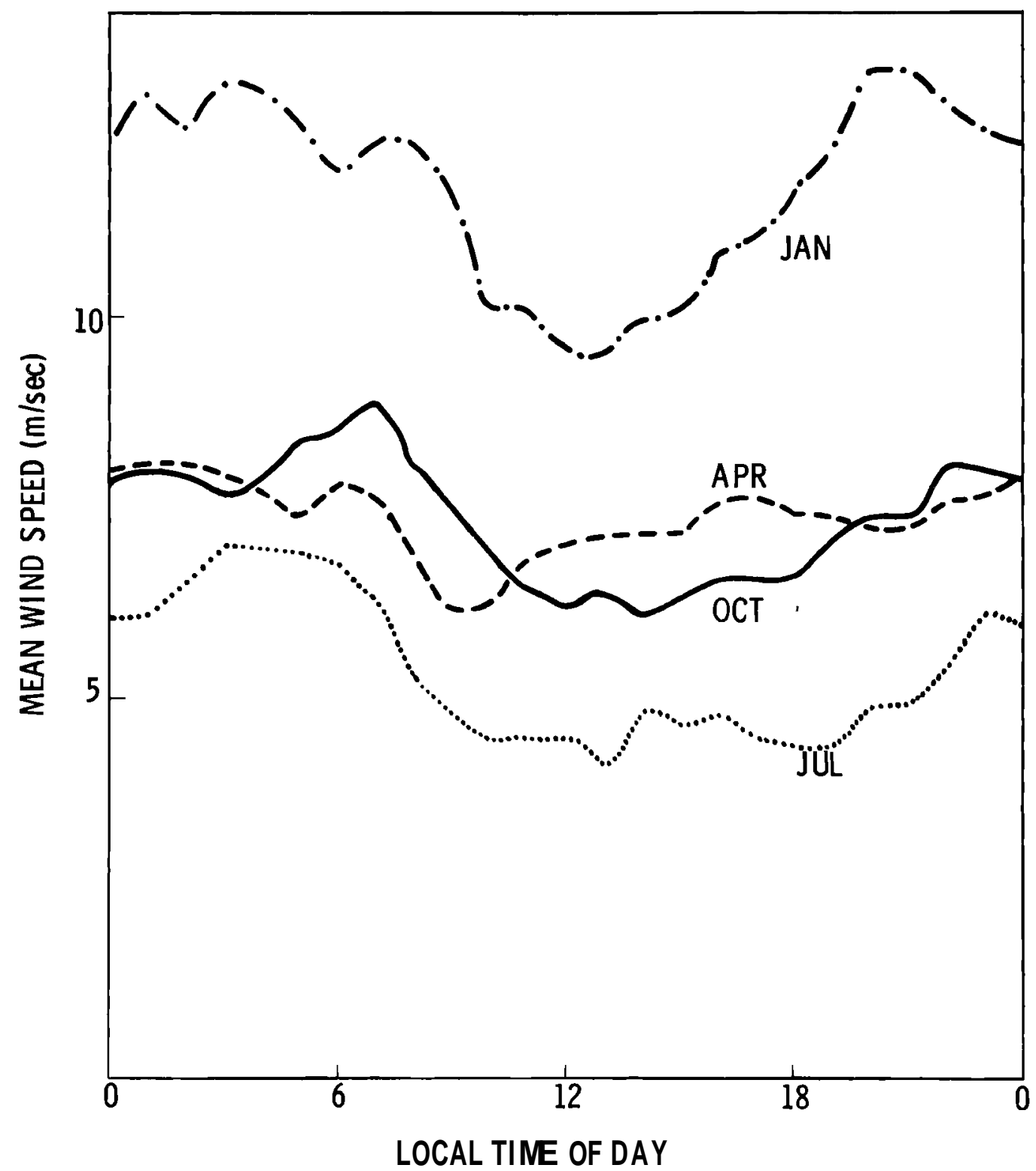

FIGURE 10.3. Diurnal Variations of Wind Speed by Season for Boone, North Carolina. These data are obtainable with either Class III or IV instrument systems.

\subsubsection{Class IV Systems}

Class IV data systems record time-series data of either raw or processed information. Processed information could simply be the average wind speed and direction for every hour or perhaps the mean, variance, skewness, maximum wind 


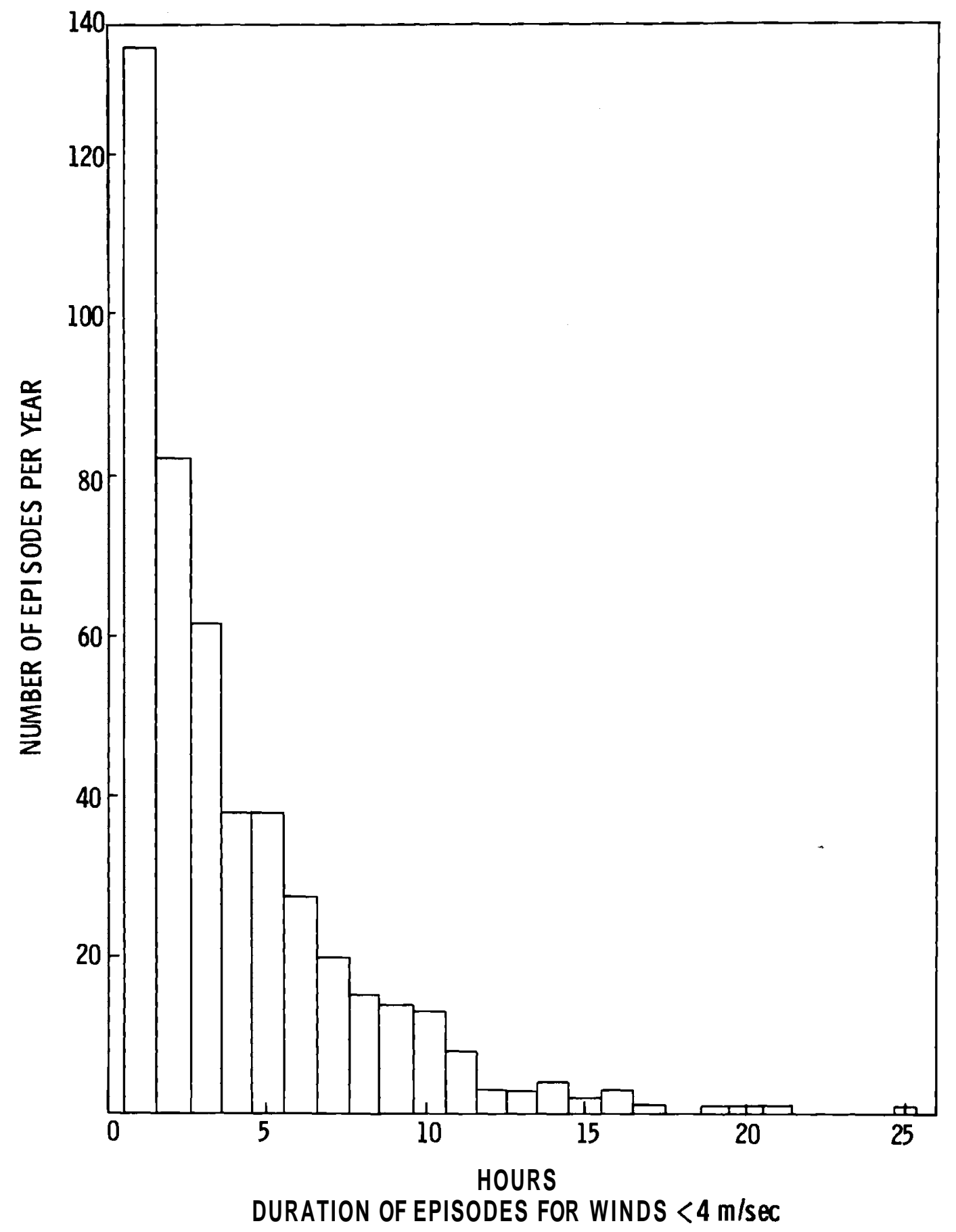

FIGURE 10.4. Wind Speed Persistence in Speed Class. These data are obtainable with either Class III or IV instrument systems. 


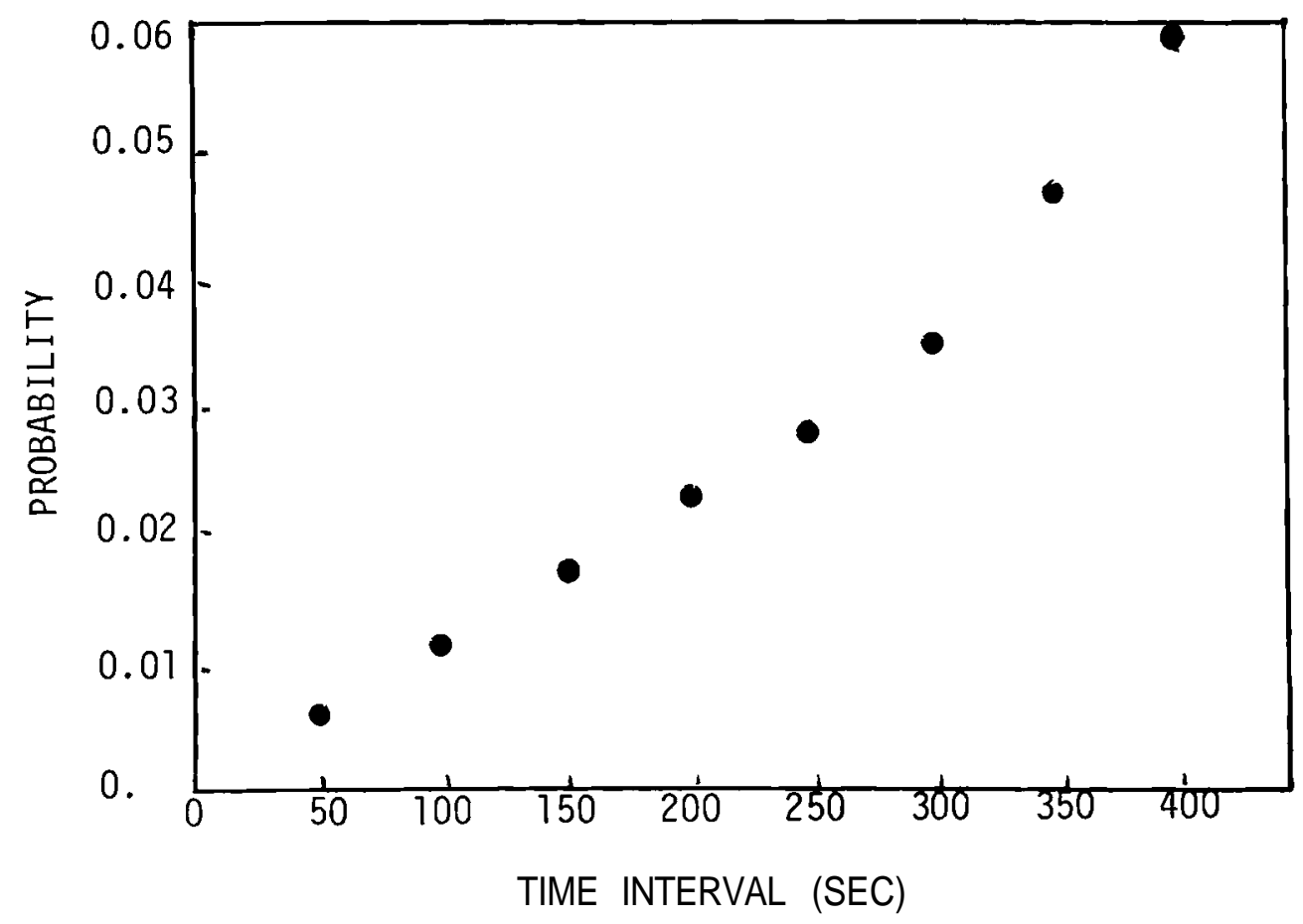

FIGURE 10.5. Probability of Difference Between 50 second Averages of Wind Direction Exceeding $10^{\circ}$ as a Function of comparison Interval (Doran 1979). These data are obtainable with either Class II or IV instrument systems.

speed, maximum gust amplitude, etc., for each hour beginning with 1-sec samples, or maybe 1-min samples. Raw information would simply be instantaneous values of wind speed and direction sampled at discrete intervals or perhaps the signals are recorded continuously. Retaining the sequential information results in more data from Class IV systems than from Class II or III systems.

Class IV systems provide the greatest flexibility for data analysis of all approaches. Any summary that can be programmed into a Class III system may be produced with Class IV system data. If the user anticipates viewing the data in such a multiplicity of ways that a Class III system could not effectively be used to carry out the processing, then Class IV systems provide the more eff cientapproach. In addition, the time series obtained in Class IV 


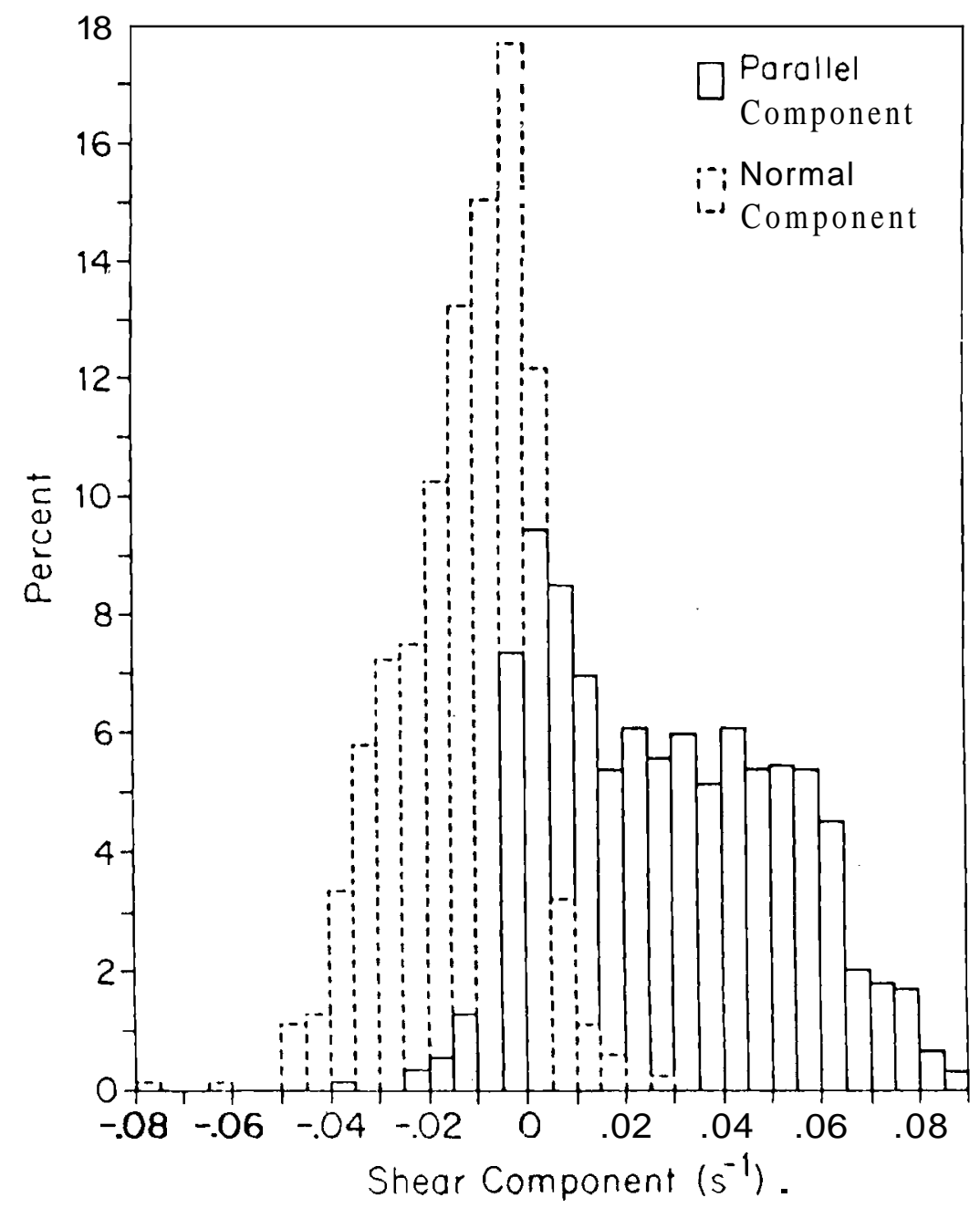

FIGURE 10.6. Frequency Distribution of Parallel and Normal Shear Components (Heald 1979). Positive normal shear is defined as clockwise rotating wind direction with height. These data are obtainable with two anemometers and Class III or IV instrument systems.

systems allows simulations of wind turbine performance with actual data, whereas statistical models would have to be developed to do the same thing with parameters obtained from Class III system data.

Data of a Class IV system may be recorded in a variety of media. Strip charts have always been a widely used method. The primary advantage to recording on strip charts is low cost. Where a high data recovery rate is 
required (e.g., the Nuclear Regulatory Commission Guide 1.23 specifies a $90 \%$ data recovery rate) the strip chart could serve as a low-cost auxiliary to a digital data logger. A secondary but important advantage is that an investigator can quickly look at a long time series and spot potential problems with the data, such as intermittent instrument failure. Intermittent failures are frequently impossible to detect from statistical processing of data stored on magnetic tapes if the investigator does not produce and scrutinize a graph of the time series from the data. (a) In the event of a total system failure, a strip chart retains all data up to the time of failure. This may or may not be true of other recording options. There are also problems with strip charts. Since time is kept by the rate at which the chart is driven, variance or bias in this rate can lead to significant time errors at the end of a week's or month's data collection. A bias can develop over time so that a strip chart that reads $0 \mathrm{~m} / \mathrm{sec}$ when the anemometer is held stationary when the system is set up may read something different when the same procedure is applied 1 month later. In one documented siting exercise (Zambrano 1980), strip chart failure was the major cause of data loss.

If time series are to be processed from strip charts, the strip charts must be digitized i $n$ some way. This is a time-consuming, costly procedure. If a technician digitizes the charts by hand, the observer errors discussed in the previous section are again a problem. For example, much of the spiked appearance to the wind speed frequency distributions shown in Figures 10.1 and 10.2 is attributable to observer bias in digitizing strip charts. Digitizing may also be performed with a digitizer. No matter which method is used, quality control checks on the digitized data should be made.

(a) It is a fact that, with the increase in computing power available to more people, there is a tendency for principal investigators (PI) to assign technicians the task of processing raw data into formats that the investigator can digest. This can and does result in analysis and conclusions based on erroneous data because what would have been an easily recognized problem for the PI was not noticed or even looked at by the less trained technician. The bad data is then thoroughly disguised by the processing. We cannot overemphasize what should be obvious: a trained specialist must look at raw data and, in addition, provide continual assurance that processing routines function as they are supposed to. 
There are a variety of magnetic recording devices, of which the cassette tape is the most common. The primary advantage of a cassette system is its convenience. However, depending on how the data are written onto the tape, cassettes can be somewhat time consuming when reading the data from the cassette onto a computer compatible 7 or 9 -track tape. In the worst case of some telemetered systems (e.g., from a tethered balloon system), the cassette records a real-time signal that is not compressed, so playback of data is also in real time. Other options that are much more sophisticated but circumvent these problems may store the output of an entire array of sensors directly on a 7 or 9 -track computer-compatible tape, or onto a "floppy" magnetic disk. These systems are capable of storing great amounts of data and are in a form to rapidly transfer the data to a computer system.

When functioning properly the magnetic tape or disk storage systems should provide more convenient and reliable service than will strip charts. The initial costs are of course higher but labor costs of data processing are jikely to be less. Many new systems that appear on the market prove to have some problems to work out. The user should attempt to determine if relatively new systems have a proven record of reliability. Some of these data loggers may be more sensitive to harsh or rapidly changing environmental conditions. Therefore, calibration checks through the entire system, onto the tape and not just to a display, should be made. Although the position in the record of data stored serially on a tape should be tantamount to the sample time, recording the time in each record is a further check on the reliability and continuity of system performance.

Solid state memory may also be used to store time-series data. This form of memory may be more reliable than tape data storage, although the cost per bit of storage is higher; therefore, long records can only be obtained by using a long interval between samples. Transferring the data from a field unit to the laboratory for processing is usually done via a cassette tape. Once again, it is wise to determine if a newly available system has a proven record of reliability.

The four classes of instrument systems were summarized in Table 10.2. 


\subsubsection{Remote Access or Telemetry Data Link}

Telemetry of data is an option to onsite recording that deserves serious consideration in a large wind turbine siting program. Two basic approaches are possible:

- ground-to-ground telemetry or direct hookup to a relay station connected with a telephone line

- ground-to-satellite-to-ground telemetry to a buffer data collection center accessible by telephone line.

In these systems an onsite data logger could accumulate raw or processed data. The user may dial up the data relay station to obtain all of the accumulated data and transfer those data directly into a computer file. In fact, the user's computer could be programmed to routinely dial up and obtain the data with no effort required of the user.

The initial equipment costs are higher but the advantages must be weighed against this. Site visits for data collection are unnecessary. If the data are called up every few days system failures may be spotted very soon after they occur instead of not being discovered until the routine site visit is made. Data transfer directly into the user's computer is a distinct advantage that eliminates a major bottleneck of data reduction. As significant wind events occur, such as a major storm, the progress can be monitored nearly in real time. The data may be routinely considered along with the concurrent synoptic conditions; even if this is done casually, it can build up considerable subjective experience with the site. Such experience is useful for the siting program as it progresses and for obtaining data on the ability to forecast winds at the site.

\subsection{ICE DEIECTORS}

Ice formation presents a hazard to nearby personnel from ice thrown of $f$ rotating wind turbine blades and a structural hazard to wind turbines. Water that is in a supercooled liquid state, i.e., the water temperature is $<0^{\circ} \mathrm{C}$, 
will not freeze until it encounters an object that can initiate crystallization. When supercooled cloud or raindrops encounter a solid object they freeze immediately. In this way, large volumes of ice can build up very quickly. If a potential wind site is in an area prone to icing it may be of interest to know the frequency of occurrence of these conditions since a wind turbine may have to be shut down because of icing (Linscott 1978).

The existence of icing conditions is easily detected, but icing rates are not. A common instrument used to detect the presence of ice is a quartz crystal oscillator. The natural frequency of the oscillator changes due to pressure of ice on the crystal and the piezoelectric effect. If the frequency changes a specified amount a binary signal can indicate that ice is present. The number of occurrences of ice could easily be accumulated in a dedicated Class II accumulator (Section 10.3.2) or as part of a Class III system making wind measurements.

\subsection{SATELUTE AND ARRCRAFT MAGRY OF THE EARTH}

Interpretation of features of the earth's surface is frequently made simpler from an aerial perspective and with sophisticated imaging equipment (Rosenfeld and Maule 1979). High altitude imagery is useful for outlining boundaries of vegetation communities, boundaries that may reflect the influence of different wind regimes (Chapter 6). The imagery is useful for locating eolian landforms (Chapter 7). And finally, the high altitude perspective i11uminates some cultural patterns that are not clearly interpretable from the ground-based perspective (Chapter 8 ). Techniques using indicators observed with high altitude imagery are not particularly accurate, reliable, or universally applicable as other techniques but the materials are easily accessed. The investigator can readily obtain the images, quickly analyze them for the limited information they contain, and move on. Note however that interpreting some of the imagery, especially satellite imagery of eolian landforms, requires expertise.

The most readily obtained data are from the:

- Landsat series of satellites

- SKYLAB

- NASA high altitude U2 photography

- U.S. Geological Survey aerial mapping photography. 
Information about and orders for items from the existing data base are handled through two centers:

User Services

Earth Resources Observation Systems (EROS) Data Center

Sioux Falls, SD 57198

(609)594-6511 ext. 151

and

\section{National Cimatic Center \\ Federal Bu ildi ng \\ Asheville, NC 28801 \\ (704)254-0961 ext. 620 .}

The user of these data specifies his region of interest, the time frame, the type of information and products he wants, and any constraints he may have on the quality of the image or cloud cover. A computer search provides a 1isting of the products that meet those criteria, from which the user orders specific products.

Landsat. Satellite coverage from the Landsat series of satellites began in July of 1972 when Landsat-1 (formerly Earth Resources Technology Satellite or ERTS-1) was launched. Landsat-1 served until January of 1978. Since then an identical satellite, Landsat-2, and an improved satellite, Landsat-3, have been launched. A Landsat-4 is scheduled for launch in 1981.

The polar orbit of Landsat satellites at an altitude of $920 \mathrm{~km}$ allows each vehicle to repeat its coverage of a given scene every 18 days. When two satellites are up simultaneously the coverage of a scene is repeated every nine days. The orbits are designed to repeat each scene at the same local time. At the equator on the southward path the local time is about 9:30 a.m.

Each Landsat carries three data acquisition systems:

- multispectral scanner or MSS

- return beam vidicon or RBV (a television system)

- data collection system for relaying ground base environmental data (see Section 10.3.5). 
The MSS is the primary system; it captures images of $185 \mathrm{~km}$ per side (parallelogram) in each spectral band. The spectral bands are:

- Band 4, the green band, 0.5 to 0.6 micrometers wavelength, emphasizes movement of sediment-laden water and delineates areas of shallow water.

- Band 5, the red band, 0.6 to 0.7 micrometers, emphasizes cultural features such as metropolitan areas.

- Band 6, the near-infrared band, 0.7 to 0.8 micrometers, emphasizes vegetation, the boundary between land and water, and landforms.

- Band 7, the second near-infrared band, 0.8 to 1.1 micrometers, provides the best penetration of atmospheric haze and also emphasizes vegetation, the boundary between land and water, and landforms.

- Band 8, emitted (not reflected) infrared band, 10.4 to 12.6 micrometers, measures temperatures between $-13^{\circ} \mathrm{C}$ and $67^{\circ} \mathrm{C}$ and discriminates temperature differences as small as $1.5^{\circ} \mathrm{C}$.

Landsats 1 and 2 used only bands 4 through 7 while Landsats 3 and 4 use band 8 also. The resolution obtainable with Landsats $1-3$ is about $100 \mathrm{~m}$ whereas Landsat-4 will improve upon this figure.

The RBV system on Landsats 1 and 2 consisted of three cameras simultaneously sensing different wavelengths of reflected light. Each scene was $185 \mathrm{~km}$ on a side and the resolution was about $100 \mathrm{~m}$. Beginning with Landsat-3, the RBV system consists of two broadband panchromatic cameras that produce adjacent images. Each camera resonds to reflected light of wavelengths of 0.5 to 0.75 micrometers and covers a $99 \mathrm{~km}$ by $99 \mathrm{~km}$ area, with a total swath width of $185 \mathrm{~km}$. Four RBV images, or subscenes, coincide approximately with one MSS scene. The resolution of the improved RBV system is about $50 \mathrm{~m}$

The user of Landsat data products can choose from a number of options. The digital signals from the satellites undergo some transformations for radiometric and geometric corrections and are then stored on coniputer tape or converted to photographic products. For applications in wind energy prospecting, 
processing of digital data is not likely to be cost effective, but the advantage to processing digital data is that one could employ digital image enhancement techniques. Analysis of the photographic data may be performed from black and white prints from each band or from false-color composites. False-color composites are formed by exposing black and white negatives from several bands through different color filters onto color film.

One use of these data is in the geomorphological indicator techniques. From the photographs, locations of active eolian processes may be noted, e.g., dune fields, playa fields. With the higher resolution RBV data, very large individual dunes may be recognizable. Climate wind directions and wind patterns are observable with these data, subject to the caveats discussed in Chapter 7.

Aerial Photography. Aerial photographs are available for selected areas of the U.S. Much of the country is covered by low altitude photography. High altitude (U-2 research and reconnaissance) photography is available for many locations as well, some of which is made through spectral bands. Local government agencies are a valuable source for locating the small-scale local photo coverage. Local historical photographs may show wind indicators that have since been erased by land use changes. The U.S. Department of Agriculture Aerial Photography Field office maintain files of accessible data and the EROS data center can provide aerial photographs used for mapping.

The use and information content of aerial photography depends upon the scale and quality of photo coverage. High altitude reconnaissance photography provides adequate detail for identification and mapping of small $(10 \mathrm{~m})$ eolian features. When the photographic coverage is in stereo pairs, the ability to interpret the form and migration of eolian features is significantly enhanced. Low-level oblique view and stereoscopic photography can be used to identify wind-deformed vegetation (Rosenfeld and Maule 1979). Topographic features, oftentimes inadequately represented by topographic maps, are easily and quickly screened through low-level reconnaissance. Cultural indicators of wind potential are easily identified and land access problems may be most easily identified from low-level imagery. 
Table 10.3 is a summary of the remote-sensing imagery that is useful to the wind energy prospector.

\subsection{INSTRUMENT DEPLOYMENT AND SITING STAGES}

The measurements required at each stage of the site selection problem, as outlined in Chapter 2, differ. The previous two subsections discussed the available wind sensors and the variety of recording systems that can be used. This section now looks at which systems should be used to fulfill the data collection requirements at each of the five stages of the wind prospecting approach to siting (Chapter 2) and considers some of the problems of deploying those systems. All measurements are assumed to be recorded on-site, but the use of telemetry for data transfer requires no major changes in the arguments. Even if a utility does not follow identically the strategies outlined in Chapter 2 it is very likely that stages resembling those of the wind prospecting strategy will be encountered.

\subsubsection{Analysis of Region of Interest}

By definition, the region of interest (ROI) analysis does not require additional wind data, since the regional wind energy resource assessments and existing wind data are the foundation of this analysis. Satellite and aircraft imagery may be used in the ROI analysis.

10.6.2 Candidate Resource Area Evaluation

The Candidate Resource Area (CRA) evaluation stage seeks to identify the locations of specific, attractive, potential wind turbine cluster sites within an area defined by the ROI analysis. When those sites have been tentatively identified some crude estimate of the potential resource is made. A crude estimate is made because at this stage the expense of towers over 10 to $15 \mathrm{~m}$ high is not justified, and as Appendix 2 clearly shows, vertical extrapolations at the most ideal sites are too uncertain for economic evaluation of wind turbine output.

Chapter 2 outlined two approaches that may be used for the CRA evaluation. The first approach, CRA analysis, requires wind data from several locations to 
TABLE 10.3. Summary of Remote Sensing Products Useful for Wind Energy Site Selection

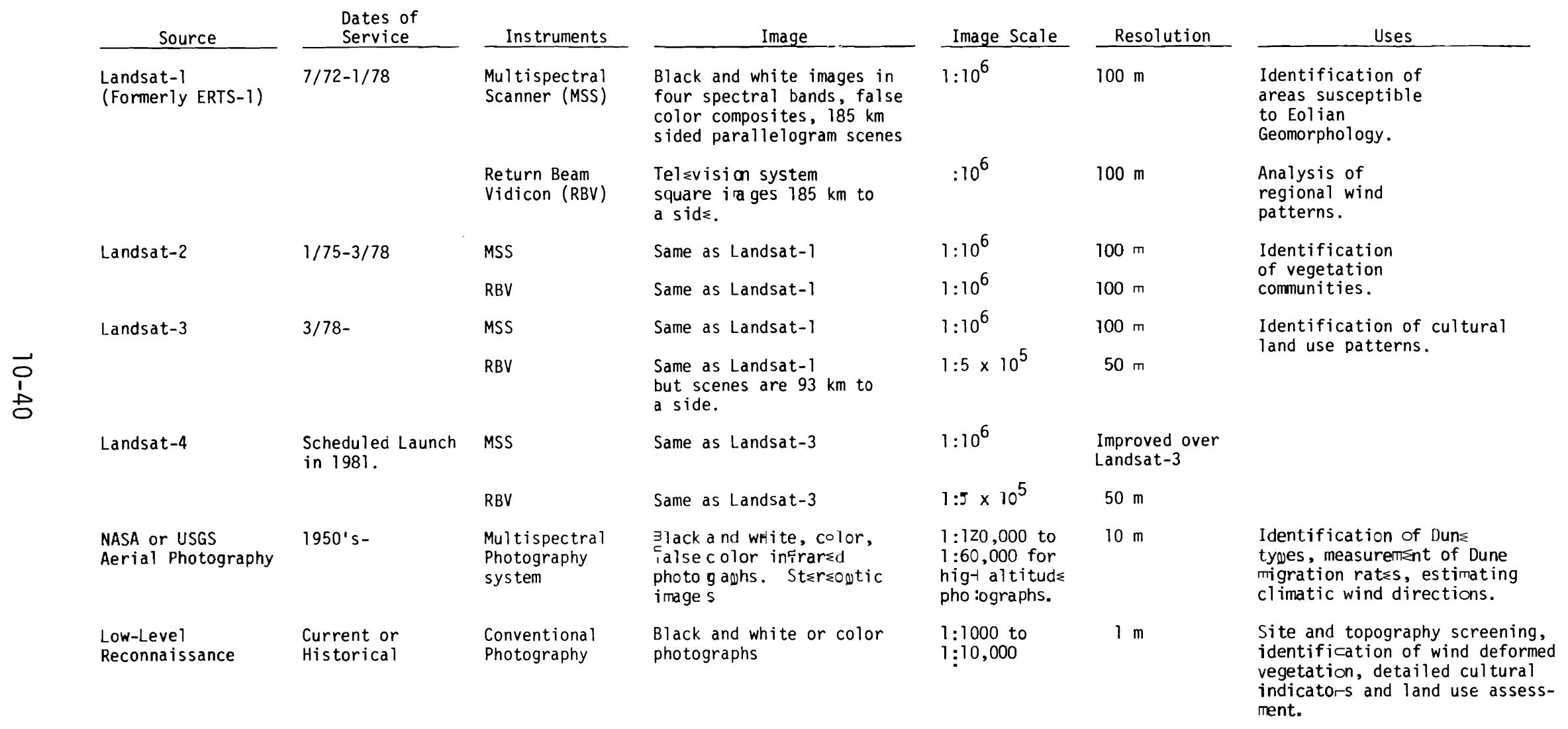


drive a numerical model or use in conjunction with other analysis techniques to determine the flow characteristic throughout an entire area. The second approach, CRA documentation, simply chooses to estimate what sites within a CRA are high wind areas and verify those estimates with wind data. The data requirements are less stringent in the latter case; however, the utility of the data for assessing regional wind patterns from just a few sensors is I imited.

Numerical models only provide solutions for the mean flow, not the turbulent flow (see Chapter 3). Therefore, data collected to drive or compare with a model (or any analysis technique) should be representative of the mean flow and not the turbulent flow. The required measurement is therefore an average that includes a significant sample of the longer period turbulent motions yet is not so long as to be influenced by changing external conditions. The error associated with sampling an insufficient number of the largest turbulent eddies is one form of sampling error. (a) For a given averaging time, sampling error increases with height of measurement above ground and varies with atmospheric stability because the fraction of the variance of velocity due to large-scale eddies increases with height and decreasing stability (Appendix 2.2.3). For measurements in the CRA evaluation stage, averages of 15 minutes to 1 hour are adequate to obtain estimates of the mean wind speed for analysis.

Analysis requires data from several locations recorded synchronously. Therefore, Class IV systems are required to provide time series of averaged wind data. The cheapest system to use would be the strip chart recorder if the user intended to shortcut the analysis of the climatological characteristics of the area. For example, if an investigator feels that he can select climatologically representative cases from the measurement program, he would need only to digitize the strip chart data for those specific hours. However if a more rigorous study of the resource area wind climatology is to be performed, the labor involved in completely digitizing a series of strip charts may be prohibitive. Then the choice of some form of digital data logger is appropriate.

(a) See Wyngaard (1973), or Lumley and Panofsky (1964), for excellent discussions on the sampling error and averaging time requirements in the turbulent boundary layer. 
There are four ways that wind data could be logged or processed to represent hourly averaged data. These are:

- vector average wind speed and direction

- scalar average wind speed and direction

- scalar average wind speed and vector averaged direction

- scalar average speed and instantaneous wind direction.

The differences among the first three are small except in rapidly changing conditions. When wind speed and direction are changing quickly, as when a front passes, the scalar average speed can exceed the vector average speed significantly and the scalar average direction will be closer to the wind direction associated with the lower wind speeds than will the vector average direction. The only significance of this is that models keep account of mass flow through the area, which the vector average process measures, whereas the scalar average process will overestimate this. Since models usually are not run in such nonstationary conditions, the difference can be ignored.

The fourth way listed above, which many data loggers use for convenience, causes more concern. Because of turbulence, any given instantaneous sample of wind direction can be substantially different than any other form of estimating an average direction. While it can be expected that these errors are Gaussian and average out for long-term statistics, the use of that kind of data for a model simulation is wholly unappropriate. If that kind of instrument system must be used, it should at least be equipped with a wind vane with a long distance constant to help filter out as much of the high frequency oscillation as possible.

If the documentation approach is elected, a rough estimate of the magnitude of the resource at specific sites is obtained with instrumentation. Although several sites may be instrumented, the intent is not to interpolate the flow between anemometers. Therefore, synchronous measurements are not required. This implies that either Class II or Class III instrument systems are appropriate.

The applicability of these data must be kept in perspective. If the resource is significantly greater at one site compared to another at $10 \mathrm{~m}$ 
then it is probably true at hub height as well; however, this is not certain. The use of diurnal wind information obtained at $10 \mathrm{~m}$ is of some help in analyzing the characteristics of the site, especially if the interpretation is coupled with an understanding of the effects of topography on the diurnally oscillating boundary layer. However, i $\mathrm{t}$ is premature to use 10-m data from one site in an area to make predictions with much certainty on the phase and amplitude of the diurnal modulation of power output. Finally, the effects of the small-scale site topography on the wind speed at the anemometer site must be understood to properly interpret both Class II and Class II system data. If, for example, there is small-scale topographic enhancement of the wind that influences only a shallow layer near the ground and would not contribute significantly to the output of a large wind turbine, a wind-run anemometer will overestimate the resource. A Class III wind system might show the enhanced winds from a particular direction. The investigator must judge whether or not the data represent mi nor local flow enhancement or a genuine large-scale enhancement.

Anemometers for the CRA evaluation stage are commonly placed at the $5 \mathrm{~mm}$ to 15-m level. The most inexpensive apparatus to support the anemometer (existing towers excepted) are tubular aluminum or steel poles supported by guy wires. These are very portable and easy to erect. The base can be supported with a pile of rocks or be slightly buried if necessary. The guy wires are easily supported with metal fence posts driven into the ground normal to the wire. One of the guy wires should be easily detached so that the entire mast can be lowered to the ground to reach the sensors. Alternatives to pole masts are triangular truss towers, either crankup or in sections, and telephone poles. For $10-\mathrm{m}$ sensors the truss towers are probably not worth their cost. Telephone poles have an advantage over tubular poles in that they are very sturdy and undamageable by heavy icing or wind. Telephone poles can be inexpen sive to install but an auger track is required so there are limitations on where they can be placed (Barnett and Reynolds 1980). Transmission line towers might also be used to support instruments, but there are problems with tower interference with the windflow, and there can be electric field interference with the instrument electronic $\sim$. With any small mast or tower, a spar that protrudes from the mast just a few feet on either side provides a mounting platform for the sensors. 
The choice of the specific location for anemometer masts is dictated by the anticipated flow conditions and local topography. The site should be free of the influence of obstacles (Chapter 5) as much as possible. Small-scale topographic enhancement of winds at $10 \mathrm{~m}$ that is not representative of the winds a wind turbine would experience or of the surrounding region should be avoided, especially if numerical models are to be used.

If estimating the annual mean wind speed is the only intent, as in the simplest form of the CRA documentation program, portable towers could be moved from one location to another. Ramsde11 (1980) shows that rotating one anemometer through four separate locations during a 2-year measurement program provides the same confidence in the estimate of the annual mean wind speed at each of those four sites as if four anemometers were used continuously, one at each site, for a period of 1 year. It is unlikely, however, that this cost saving will be significant for a utility that is siting a large cluster, but small utilities or agencies siting one turbine in a fairly remote location could consider this strategy.

There are a number of common problems that come up when deploying these small instrument systems. Lightweight tubular masts can be:

- blown down by ice and wind loading

- knocked down by cattle

- vandalized.

Very little can be done about the first item except to keep the base and guy wires in adjustment. Ice and wind damage may be an indicator of detrimental environmental conditions if it occurs frequently enough. Barbed wire fence provides protection from cattle. A determined vandal, however, is hard to beat. The first line of defense against vandals is to site the anemometer in a location that is not easily noticed from nearby trails or roads or site it on secure private property. If a tubular pole is used for the mast and is rigged for one guy line to separate in order to lower the tower, a padlock should be used at the separation point. Data loggers are also subject to vandalism. Data loggers my be placed in strong boxes but the strong boxes 
need to be bullet proof. Some investigators have had to resort to burying the data logger. Disguising the data logger as an electrical transformer has also been suggested since transformers do not seem to be frequent targets.

Other failures of the data loggers result from battery failure, extreme temperatures, lightning, electric field interference, and strip chart failure. Battery life is reduced by cold temperature and, for contact anemometers, by high wind speed. Batteries rated at $\mathbf{3}$ months life should be replaced every month to provide an adequate safety margin. Only data loggers specified to withstand the expected temperature ranges should be used, and even then the data loggers should be checked out by reputed reliability or actual tests. Insulated boxes seem to provide little protection without a heat source, which requires too much power for remote locations (Barnett and Reynolds 1980). Direct lightning strikes can ruin the entire system so the only defense is to try and avoid the lightning prone areas; unfortunately those areas are potentially good wind energy sites as well. Strong electric potential fields due to an imminent lightning strike or high voltage power lines can cause interference within the data logger. Careful grounding and shielding alleviates this problem. Strip charts fail when pens clog or dry; thermofax recording solves that. Changes in chart drive speed occur quite often. This is detected by noting whether actual time elapsed between site visits corresponds to the length of chart use. If not, look for evidence to suggest when the time base changed, e.g., ink blobs and crinkled paper may indicate a paper jam. Linear time corrections could be applied. The quality of the correction may be generally checked by studying the diurnal wind signals, but even if the correction is close the data should not be used to drive models.

Icing of the sensors produces either reduced wind speed estimates or, if heavy icing is accompanied by strong winds, the icing can stop the rotation of the sensors which then break under the wind stress (Barnett and Reynolds 1980). Spraying vegetable (non-stick) cooking oil or silicone lubricants on the sensors may help alleviate the icing problems. Intermittent freeze up of the cups is undetectable with Class II data systems; this shows up as an 
anomalous frequency of calm spells in a Class III system record. With a Class IV system genuine calm can probably be separated from icing.

\subsubsection{Potential Candidate Site Screening}

Numerous visits to the site are made in the potential candidate site (PCS) screening stage, during which wind measurements between 10 and $150 \mathrm{~m}$ are made. Kites, kite anemometers, or tethered balloon systems are the usable systems until cost-effective remote sensing systems are proven. The intent of wind measurements in the PCS screening stage is twofold:

- to seek wind profile characteristics that disqualify a site

- to determine whether or not the wind field at the site has a great deal of structure.

The utility of the wind measurements made in this stage must be kept in perspective. Recall that the details of the flow structure over a site will depend on stability in the boundary layer, ambient levels of turbulence, stability of the flow above the boundary layer, wind direction, wind speed, baroclinicity, and so forth. Site visits only sample a meager fraction of the climatology. The assumption that a given day represents prevailing flow conditions is risky since the important knowledge of the flow conditions in the upper-boundary layer and above are uncertain. Therefore, conclusions on the magnitude and subtle spatial variations of the wind resource should not be made on the basis of PCS screening measurements.

The existence of zones of flow separation can easily be determined with kites. Extensive regions of flow separation is a reason to disqualify a site. Kites will fly very erratically in the turbulence within the flow separation region but fly relatively more smoothly in the quiescent stronger winds outside of the zone. The occurrence and extent of flow separation are functions of stability and ambient revels of turbulence, so repeated probing of an area on many visits in different conditions is necessary to adequately determine the extent of a potential hazard. 
Kite anemometers and tethered balloon systems are also useful for determining how much horizontal wind structure exists at hub height over a potential cluster site. A great deal of horizontal structure at the site is not a good indicator since verifying the details of the horizontal structure requires substantially more instrumentation and analysis than in a more benign site and since horizontal structure ma be associated with high levels of turbulence. The presence of a high degree of horizontal wind structure can be determined by flying kite anemometers simultaneously at key points around the site, at hub height. Measurements should be about 30 minute to 1 hour in duration compared to shorter measurements of 15 to 30 minute for near-surface sampling, since sampling error increases with height. Simultaneous measurements are required rather than serial measurements at different locations because to cover several locations serially entails error caused by one, or both, of two sources: sampling error caused by too short of a measurement at any one location or nonstationarity of the mean flow if too long a time is spent covering all locations.

The use of tethered balloons and kites in the candidate site screening stage requires only a few considerations. If a tethered balloon system is to be flown there may be local Federal Aviation Administration regulations to follow. One in particular is that the balloons must be equipped with a pressure altitude sensor to puncture the balloon in the event that the tether line breaks. Some tethered balloons are too fragile to fly in winds stronger than $10 \mathrm{~m} / \mathrm{sec}$. Since one of the chief uses of a kite is to indicate the presence of turbulent flow separation zones, frequent checks should be made of the kites' rigging to ensure that they are in proper aerodynamic trim. If they are not flying properly there may be confusion between high turbulence and moderate turbulence with poor kite performance.

\subsubsection{Candidate Site Evaluation and Site Development}

Candidate site evaluation and site development data requirements are similar, differing only in the number of measurements made at a potential cluster site and perhaps in the sampling frequency. The recommendations and 
rationale for desirable measurement strategies are discussed in Chapter 2. The candidate site evaluation measurements are primarily used to estimate cluster energy production characteristics for economic evaluation. Chapter 2 recommends 1 -min averages of wind speed and direction at a level near hub height as desirable and sufficient, based on current knowledge of wind turbine behavior. The site development stage may require an expansion of the measurement program and some measurements of $5-$ to $10-\mathrm{sec}$ averages at various levels in order to make final decisions on site development and to address certain questions of wind turbine operations. In both stages, icing measurements may be desirable in regions prone to icing.

It is, of course, possible for utilities to depart from the specific measurement recommendations presented in Chapter 2. Recording data from every channel at a candidate site (which might consist of several towers instrumented at more than one level) at a rate of once per minute provides the greatest flexibility in future data analysis, but also produces a lot of data. The data are easily managed if the system records directly onto a computer tape. If cassette tapes are used, more frequent site visits are required, which can be good for ensuring data continuity. Nevertheless, other options exist at a tradeoff in analysis capability. It is conceivable that models could be developed that would be capable of simulating realistic time series and spatial variation of site winds and be driven by parameters computed and stored at a site using Class III data loggers. Until these models and instruments are developed, it is clear that Class IV systems will be required. A Class IV option to the recommendations of Chapter 2 is to sample the signals every 1 to 30 seconds, compute means, variances or other statistics that may be deemed appropriate, and record only the statistics once each hour. After more experience is obtained with various models for predicting wind turbine energy production characteristics at a site (see Section 1.3.2), an option such as this may prove to be of adequate accuracy and simplicity.

Assuming that the measurement recommendations of Chapter 2 are followed, the choice of which type of Class IV system is still a fundamental decision 
requiring careful consideration of the needs and constraints of the siting program. Recording directly onto computer tape simplifies and expedites data reduction and quality control, but the requirements for instrument sheltering are more severe than for other options. If ac power is available at a site there is no difficulty in providing heat to a small instrument shelter. The sheltering problems are more severe when cold conditions are expected and there is no power at a site. If numerous towers are to 'be erected in an area it may be justifiable to establish one central data acquisition shed and telemeter, or transmit via fiber-optic cables, signals from all towers to the shed. The climate of the shed could be controlled from battery banks intermittently charged by a standby generator or a small WECS. Another option is to record on cassette data loggers at each tower and be willing to accept some loss of data during cold weather. In this case, it is best if the data logger keeps a clock running throughout the cold episode and be designed so that when it warms normal function can return automatically without needing to be reset by a technician during a routine tape-changing visit. Independent data loggers have some advantage in redundancy of data collection, however, independent data loggers with independent clocks sometimes lose their time synchronization (Barnett and Reynolds 1980). If time synchronization is important, such as for numerically modeling flow over a site, a centralized clock is required. Environmental considerations, such as these, must be carefully evaluated when selecting a data acquisition system for deployment in the candidate site evaluation and site development stages.

A primary deployment problem in these stages is the placement of sensors on the towers. Tower interference with the wind is a problem for the triangular truss towers used in these stages. The wind flows through and around the tower. The wind accelerates around the sides of the tower causing overestimates of the free steam wind on downwind flanks of the tower. Directly downwind of the tower the winds reaching the anemometer have come through the tower where mean flow kinetic energy has been converted to turbulence causing an underestimate of the free stream wind speed. Gill et al. (1967) made wind tunnel studies of the flow through triangular towers. Booms for meteorological sensors were mounted in two configurations: 
- face configuration--boom protruding normal to face of tower

- corner configuration--boom protruding from corner of tower, parallel to one face,

Experiments were performed for boom lengths of $1 D$ and $2 D$ where $D$ is the width of the face of the tower. The arc lengths, centered around the upwind direction of the boom over which specified accuracies of wind speed and direction measurements are achieved, are presented in Table 10.4. From these results, Gill et al. make the following recommendations:

- Corner configuration of the boom is preferred.

- Boom should be downwind of direction of least frequent winds or winds of least concern.

- Boom should be located at height of minimum tower density, above or below horizontal cross members.

- If accuracies of $\pm 5 \%$ i $n$ wind speed and \pm 5 " in wind direction are required, two sets of sensors mounted on two corner configured booms point in opposite directions with lengths of at least 1-1/2 D are required.

TABLE 10.4. Arc Over Which Wind Speed and Wind Direction Measurements are Accurate (Gill et al. 1967)

\begin{tabular}{|c|c|c|c|c|}
\hline \multirow[b]{2}{*}{$\begin{array}{l}\text { Boom } \\
\text { Length }\end{array}$} & \multicolumn{4}{|c|}{ Accuracy of Speed $(\%)$ and Direction $\left({ }^{\circ}\right)$} \\
\hline & $\begin{array}{r} \pm 5 \%, \pm \\
\text { Corr } \\
\end{array}$ & $\frac{0 \%, \pm 10^{\circ}}{\text { ation }}$ & $\begin{array}{l} \pm 5 \%, \pm \\
\mathrm{Fac}\end{array}$ & $\begin{array}{l}0 \%, \pm 10^{\circ} \\
\text { tion }\end{array}$ \\
\hline $1 \mathrm{D}$ & 180 & 310 & 200 & 230 \\
\hline $2 \mathrm{D}$ & 240 & 330 & 260 & 330 \\
\hline
\end{tabular}

Towers may require a person to climb the tower to retrieve and service instruments or the instruments may be mounted on an elevator system so that no climbing is required. The latter system is safer and can be a lighter, less expensive tower.

Tall towers require long signal cables. For instruments that produce pulsed or digital information this is not a problem. However, there will be 
signal loss from anemometers that generate a voltage. The magnitude of this loss is easily calculated and may be checked along with an anemometer generator calibration check whereby the anemometer shaft is spun with an electric motor a t a known rate.

Erecting towers of height $\geq 200 \mathrm{ft}$ may require a careful assessment of cost-benefit trade-offs. The Federal Aviation Administration requires reliable aircraft beacon lights on those towers. Achieving reliability can be fairly expensive if ac power must be brought into a remote site. Of course, if a candidate site is found to be economically viable, power for construction will be brought in anyway. Also, where large clusters are being considered, the price of bringing power in may be negligible. However, one should keep i $n$ mind the option of using $199-\mathrm{ft}$ tall towers during the candidate site evaluation stage.

\subsection{QUALITY ASSURANCE}

The decision to install millions of dollars' worth of wind turbines at a site will be based, in part, upon the meteorological measurements made throughout the siting process. Quality data are crucial to a quality'decision. If meteorological consultants are used to gather data for a utility, it is ultimately the responsibility of the utility to see that the data collected conform to desired quality specifications. This is best achieved through a written quality assurance (QA) plan, probably prepared by the consultant but certainly agreed to by both the utility and the consultant.

A quality assurance plan documents the procedures to be followed that should result in maintaining specified levels of data accuracy and recovery rates. Those levels can be set to any mutually agreed upon standards matched appropriately to the problem. In the candidate site evaluation and site development stages of siting, the standards required by the Nuclear Regulatory Commission (NRC) for meteorological measurements at nuclear power plant sites (NRC Regulatory Guide 1.23) serve as stringent goals for a wind data collection program. (a) The NRC guidelines specify that measurement system (i.e., sensor,

(a) These are also the goals of the Department of Energy's Candidate Site meteorological measurement program. 
cables, signal conditioner, recorders, and data reduction) errors should not exceed $\pm 5^{\circ}$ in wind direction, $\pm 0.22 \mathrm{~m} / \mathrm{sec}(0.5 \mathrm{mph})$ in wind speed, and recorded time within $5 \mathrm{~min}$ of actual time. The data recovery rate of $90 \%$ is specified and redundant systems may be required to achieve this.

A strict QA plan would describe procedures used to initially calibrate each sensor, and how those calibrations may be traced to the National Bureau of Standards. The periodic (e.g., quarterly or semiannual) system calibration process should be described. This process might entail testing that the torque required to turn anemometer or vane shafts remains within specified limits, that anemometers driven at known speeds produce the expected signal, that known signals applied to the signal conditioning equipment and data loggers produce the expected recorded output, and that time is being kept properly. The documentation and correction procedures applied to components found to be out of tolerance should be described. Standard procedures or checklists of routine site inspection and tape changing activities help ensure that no problems go unnoticed. For example, without a checklist a technician might fail to properly reset the data logger clock, or fail to note potentially troublesome corrosion. In the same way, strict documentation of changes in data reduction and processing procedures provides a traceable trail should questions arise at some time about data quality.

\subsection{SUMMARY}

In this chapter measurement systems, sensors, and recorders are described. A summary of the instrumentation applicable at each stage of the siting process outlined in Chapter 2 is presented in Table 10.5. Typical problems and tips for deployment of these instrument systems are also summarized. Because the risk of making decisions on poor quality data belongs to the utility, it is recommended that the utility review and agree upon quality assurance procedures implemented by those individuals or consul tants responsible for making the measurements. 


\section{TABLE 10.5. Summary of Measurements and Instrumentation for Wind Prospecting Strategy}

\begin{tabular}{lll}
\multicolumn{1}{c}{ Stage } & \multicolumn{1}{c}{ Sensor } & Recording System \\
$\begin{array}{l}\text { Region of } \\
\text { Interest } \\
\text { Analysis }\end{array}$ & $\begin{array}{l}\text { Satelite or } \\
\text { aircraft camera }\end{array}$ & $\begin{array}{l}\text { Film or digital } \\
\text { imagery }\end{array}$ \\
$\begin{array}{l}\text { Candidate } \\
\text { Resource Area } \\
\text { Evaluation }\end{array}$ & $\begin{array}{l}\text { Satellite or } \\
\text { aircraft cameras }\end{array}$ & $\begin{array}{l}\text { Film or digital } \\
\text { imagery }\end{array}$ \\
$\begin{array}{l}\text { Wind } \\
\text { Analysis }\end{array}$ & Cup and vane & $\begin{array}{l}\text { Class IV; } \\
\text { preprocessed or } \\
\text { raw data }\end{array}$ \\
& & \\
& & \\
Wind & Cup and vane & Class III \\
Documen- & Wind-run anemometer & Class II \\
tation & &
\end{tabular}

$\overrightarrow{0}$

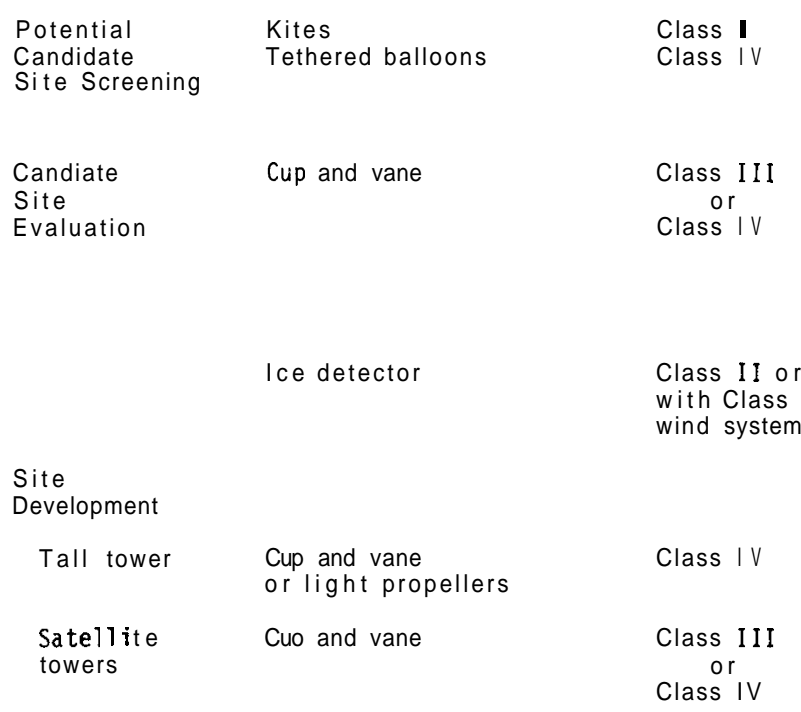

Use, Deployment Considerations, or Problems

Used for biological, geomorphological, and cultural indicator techniques.

Expertise in interpretation of Eolian landforms required.

Data are easily obtained, quickly analyzed, but have limited information content.

Same as Region of Interest analysis.

Measurements at 10 to $15 \mathrm{~m}$. Tubular aluminum poles with one detatchable guy wire (to lower pole for servicing instruments) is least expensive and most mobile. Telephone poles are more rugged and withstand icing, but are imnobile and canno be erected everywhere. Steel towers are probably more expensive than they are worth for this stage.

Site poles away from minor topographical features that are unrepresentative of deeper layers or surrounding region.

Problems include:

lcing of sensors (use spray silicone lubricants or spray non-stick coatings). lcing of poles (telephone poles are durable).

Electrical interference (avoid high voltage lines, lightning prone areas:

check shielding and grounds). Vandalism (avoid popular trails and roads; disguise or hide equipment). Extreme temperatures (change batteries frequently; evaluate temperature specifications on equipment).

Strip chart failures: pen clogging, inaccurate chart speed.

Use for detecting flow separation zones and for investigating the existence of significant horizontal wind structure at hub height (which makes site evaluation more difficult).

Check aerodynamic trim of kites and local FAA restrictions on balloons.

Tcwers with elevators for instrument booms is most convenient.

Consider tower shadowing of sensors.

If high rate sampling is to be used in the site development state, consider using data loggers capable of both low rate preprocessing and high rate raw data sampling.

Other problems are similar to candidate resource area evaluation stage.

Towers $200 \mathrm{ft}$ tall or more require beacons satisfying FAA standards. This will usually require reliable ac power at the site.

Same as in candidate site evaluation stage.

or 


\section{REFERENCES}

Barnett, K. M. and R. D. Reynolds. 1980. Assessing the Local Wind Field at Sierra Grande Mountain in New Mexico With Instrumentation. PNL-3623. Pacific Northwest Laboratory, Richland, Washington.

Beran, D. W. and F. F. Hall, Jr. 1974. "Remote Sensing for Air Pollution Meteorology." Bull. Am. Meteor. Soc. 55:1097-1105.

Cliff, W. C. 1977. The Effect of Generalized Wind Characteristics on Annual Power Estimates from Wind Turbine Generators. PNL-2436, Pacific Northwest Laboratory, Richland, Washington.

Derr, V. E. (Ed.) 1972. Remote Sensing in the Troposphere. Wave Propagation Laboratory and University of Colorado, Boulder, Colorado.

Doran, J. C. 1979. "Wind Direction Variations in Strong Winds." Proceedings of the Conference and Workshop on Wind Energy Characteristics and Wind Energy Siting 1979. American Meteorology Society, Boston, Massachusetts.

Gill, G. L., L. E. Olsson, J. Sela and M. Suda. 1967. "Accuracy of Wind Measurements on Towers or Stacks. Bull. Amer. Meteor. Soc. 48:665-674.

Heald, R. C. 1979. "Boundary Layer Wind Shear." Proceedings of the Conference and Workshop on Wind Energy Characteristics and Wind Energy Siting 1979. American Meteorological Society, Boston, Massachusetts.

Hooke, W. H. 1980. A Review of Remote-Sensor Potential for Wind Energy Studies. DOE/ET-23151-80-1. Available from National Technical Information Service, Springfield, Virginia.

Justus, C. G., W. R. Harqroves and A. Yalcin. 1976. "Nationwide Assessment of Potential output-from Wind Powered Generators." J. Appl. Meteor. $15: 673-678$.

Kaimal, J. C., H. W. Baynton and J. E. Gaynor. 1980. The Boulder Low-Level Intercomparison Experiment. National Oceanic and Atmospheric Administration Wave Propagation Laboratory, Boulder, Colorado, pp. 189.

Lawrence, R. S., G. R. Ochs and S. F. Clifford. 1972. "Use of Scintillations to Measure Average Wind Across a Light Beam." Appl. Optics 11:239-243.

Linscott, B. S. 1978. Ice Detector for MOD-OA Rotor Blades. Inforniation Request/Release No. 86, NASA Lewis Research Center, Cleveland, Ohio.

Lumley, J. L. and H. A. Panofsky. 1964. The Structure of Atmospheric Turbulence. John Wiley and Sons, Inc., New York, pp. 239. 
Nuclear Regulatory Commission (NRC). "Onsite Meteorological Programs." In Regulatory Guide 1.23 (Safety Guide 23), U.S. Nuclear Regulatory Commission, Washington, DC.

Ramsde11, J. V., S. Houston and H. L. Wegley. 1980. Measurement Strategies for Estimating Long-Term Average Wind Speeds. PNL-3448, Pacific Northwest Laboratory, Richland, Washington.

Rosenfield, C. L. and P. A. Maule. 1979. "Remote Sensor Applications to Wind Power Facility Siting." Proceedings of the Conference and Workshop on Wind Energy Characteristics and Wind Energy Siting. American Meteorological Society, Boston, Massachusetts,

Woodhouse, C. F. 1980. "Tethered Aerodynamically Lifting Anemometer (TALA). " In The Boulder Low-Level Intercomparison Experiment. J. C. Kaimal, H. W. Baynton and J. E. Gaynor, eds. National Oceanic and Atmospheric AdministrationWave Propagation Laboratory, Boulder, Colorado, pp. 33-37.

Wyngaard, J. C. 1973. On Surface-Layer Turbulence, Workshop on Micrometeorology. D. A. Haugen, ed. American Meteorological Society, Boston, Massachusetts.

Zambrano, T. G. 1980. Assessing the Local Wind Field With Instrumentation. PNL-3622, Pacific Northwest Laboratory, Richland, Washington. 
APPENDIX 1

REGIONAL WIND RESOURCE ASSESSMENT 


\section{APPENDIX 1}

\section{REGONAL WN RESOURCE ASSESSMENT}

Three national wind energy resource assessments were performed early in the Federal Wind Energy Program (Reed 1975; Coty 1976 and Garate 1977). Although a large portion of the data used in these assessments was common to a 11, the analysis techniques differed considerably. Not surprisingly, significant discrepancies in the magnitude and the distribution of wind power density can be found among the three national assessments. A re-analysis and synthesis were also completed (Elliott 1977) to determine the causes of the discrepancies and to include the most plausible results in a single assessment (see Figure 1.2).

As interest in wind energy increased, a need arose for resource assessments that were more refined and reliable than the national assessments so that energy planners, WECS manufacturers and public utilities could assess the wind power potential on a state and regional basis. Assessments for the twelve regions of the United States are being prepared by the Department of Energy (Figure A-1.1). (a) Each regional assessment consists of a regional wind energy atlas, a regional wind power data base and a narrative report describing the procedures used in producing the assessment. The regional assessments are the starting point for a utility considering the inclusion of WECS into its mix of generating equipment. The analyses provide preliminary information for assessing the potential for wind energy utilization within a utility's area. The assessments also indicate likely areas for finding sites.

\section{A.1.1 THE WND ENERGY ATLASES}

Each wind energy atlas contains wind resource information summarized by region and by state. The atlas also contains more detailed resource information at individual weather stations. Information that is provided in a regional format incl udes:

(a) Copies of the regional assessments will be available from the National Technical Information Service as they are completed. All assessments should be available by early 1981. A 1 ist of titles is provided at the end of this appendix . 


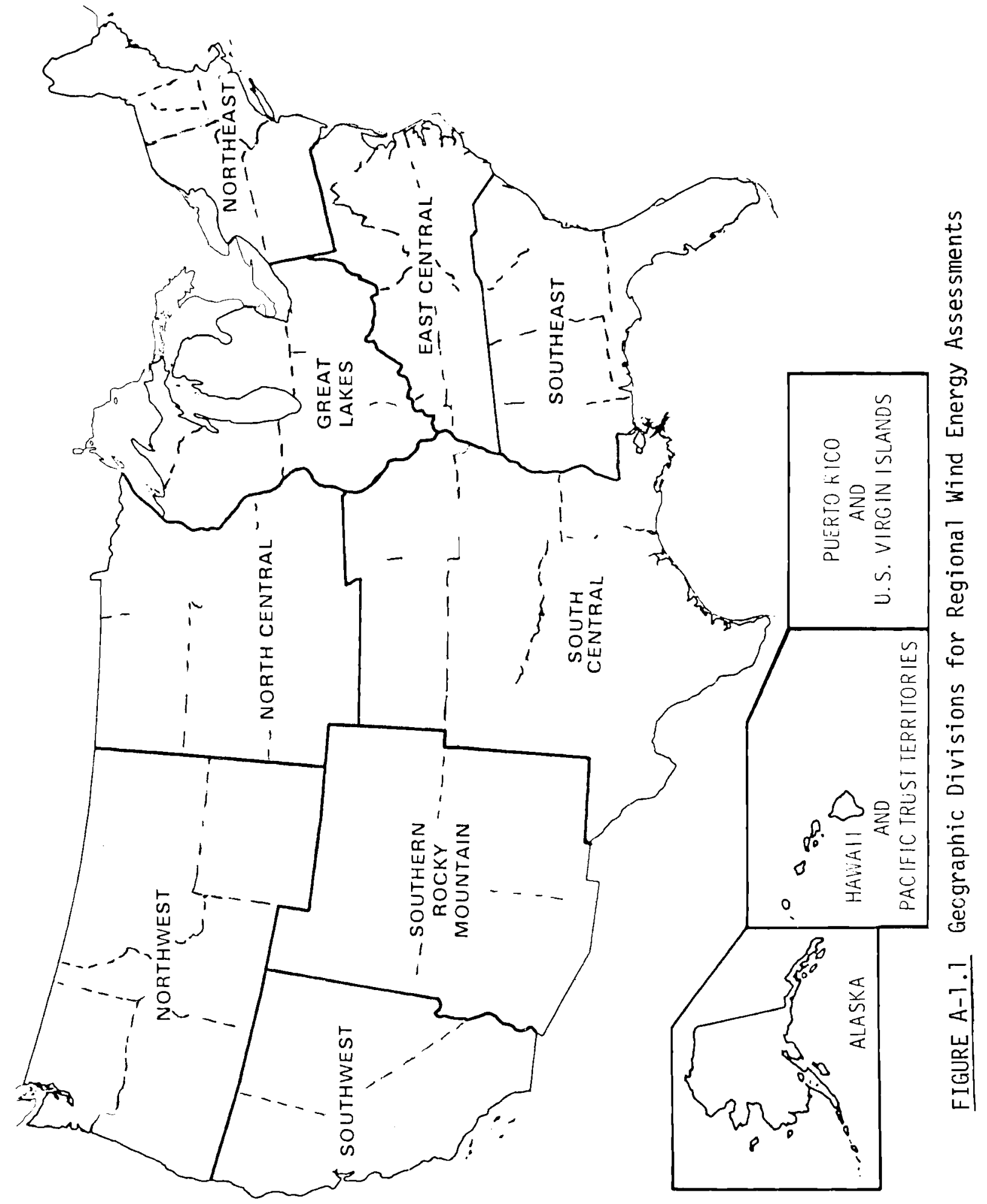

A. 1-2 
- maps of cultural geographic, topographic, and land-surface form classification

- a map of the annual available wind power

- a map showing the season of maximum wind power.

An example of the level of detail provided in the regional format is given in Figure A-1.2, which shows annual average wind power for the Northwest region. Considerably more detail is provided on a state-by-state basis. State features include:

- maps of cultural geographic, topographic, and land-surface form classification

- maps showing the location of data stations

- maps of annual and seasonal wind power

- aerial distribution of wind power

- maps of confidence level of resource estimates.

Figures A-1.3 and A-1.4 show average available wind power during summer and winter for the state of Washington. Maps of this type are useful for identifying areas where the seasonal variations in wind power best match the seasonal needs of the utility.

The numbers shown on the maps correspond to wind power categories defined in the figure legends. Each category reflects the best exposed sites within the overall area associated with that category. Mountainous areas are stippled on the map to indicate that the values shown on the map are estimates of the wind resource on exposed mountain ridge crests or summits. Most of the structure shown in the analysis is a result of interpreting meteorological and topographical indicators.

One of the principal problems in wind resource assessment is characterizing the spatial variability of the wind (see Appendix 3). Available wind power can change drastically over the distance of a few miles; therefore, one must account for the percentage of land that is not well exposed to prevailing winds in order to realistically assess the total wind resource. Each region was divided into a grid one-third of a degree in longitude by one-fourth of a 


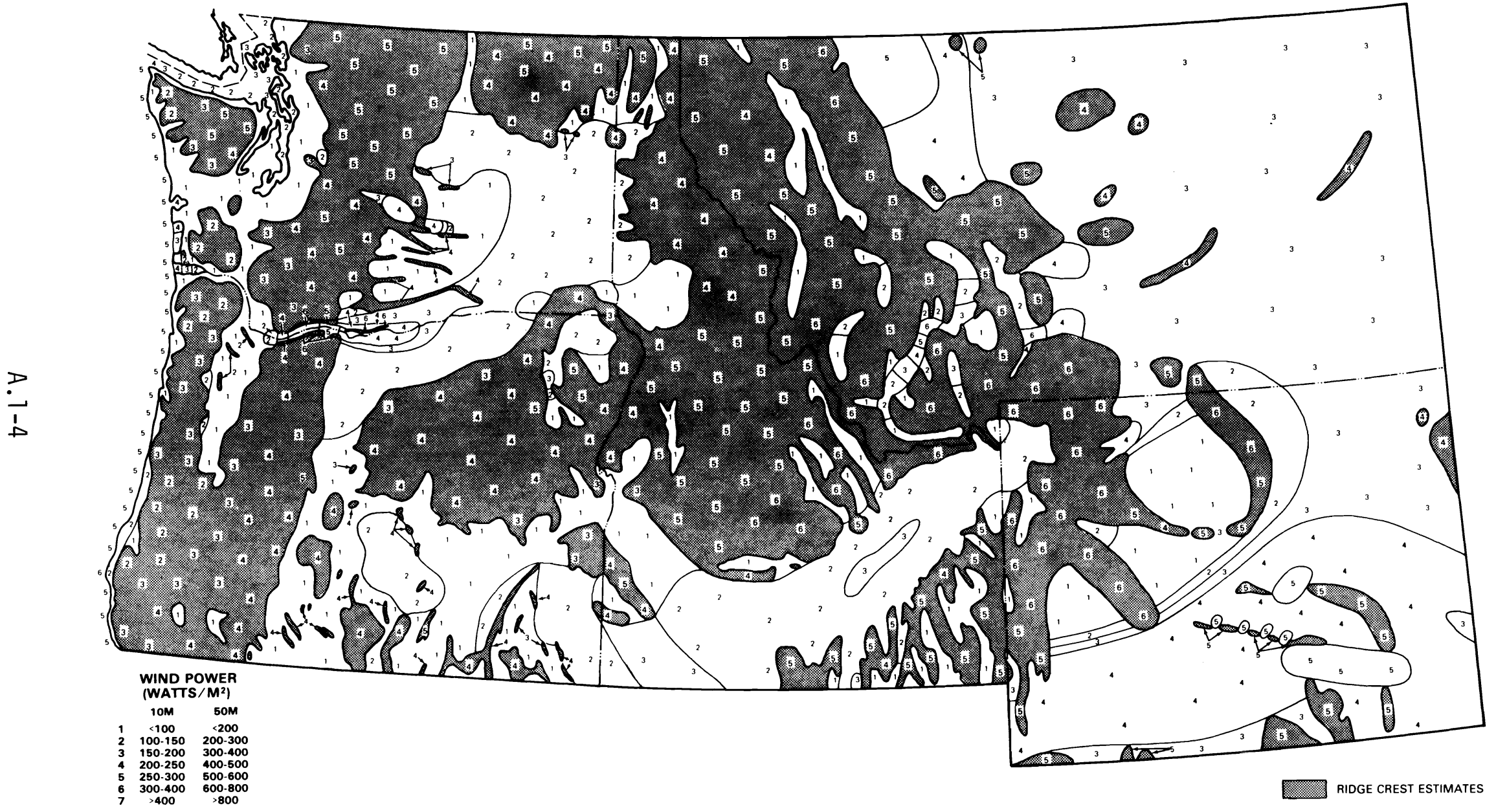

FIGURE A-1.2 Northwe: Region Annual Average Wind Power 
ज

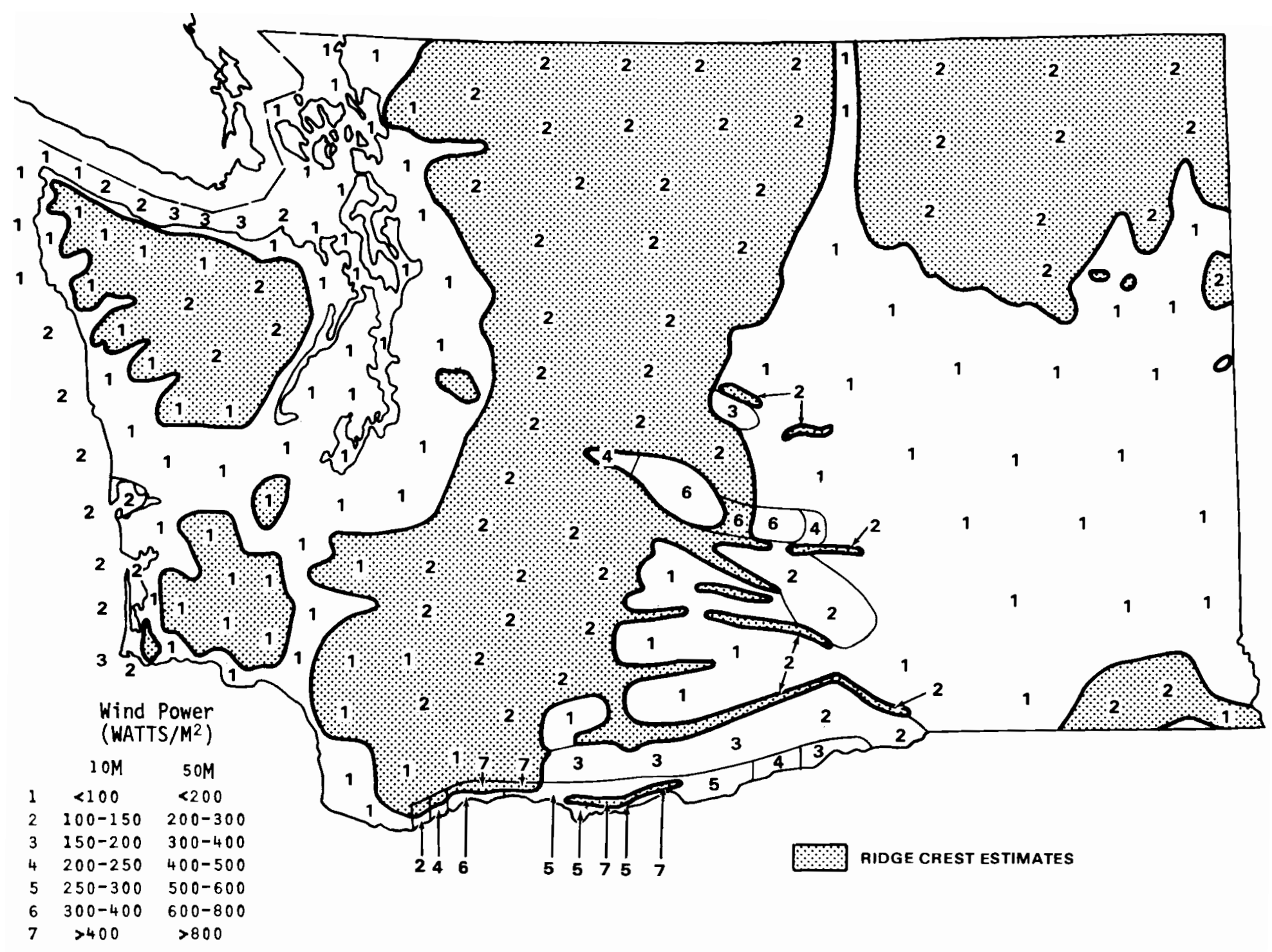

FIGURE A-1.3 Washington Summer Average Wind Power 


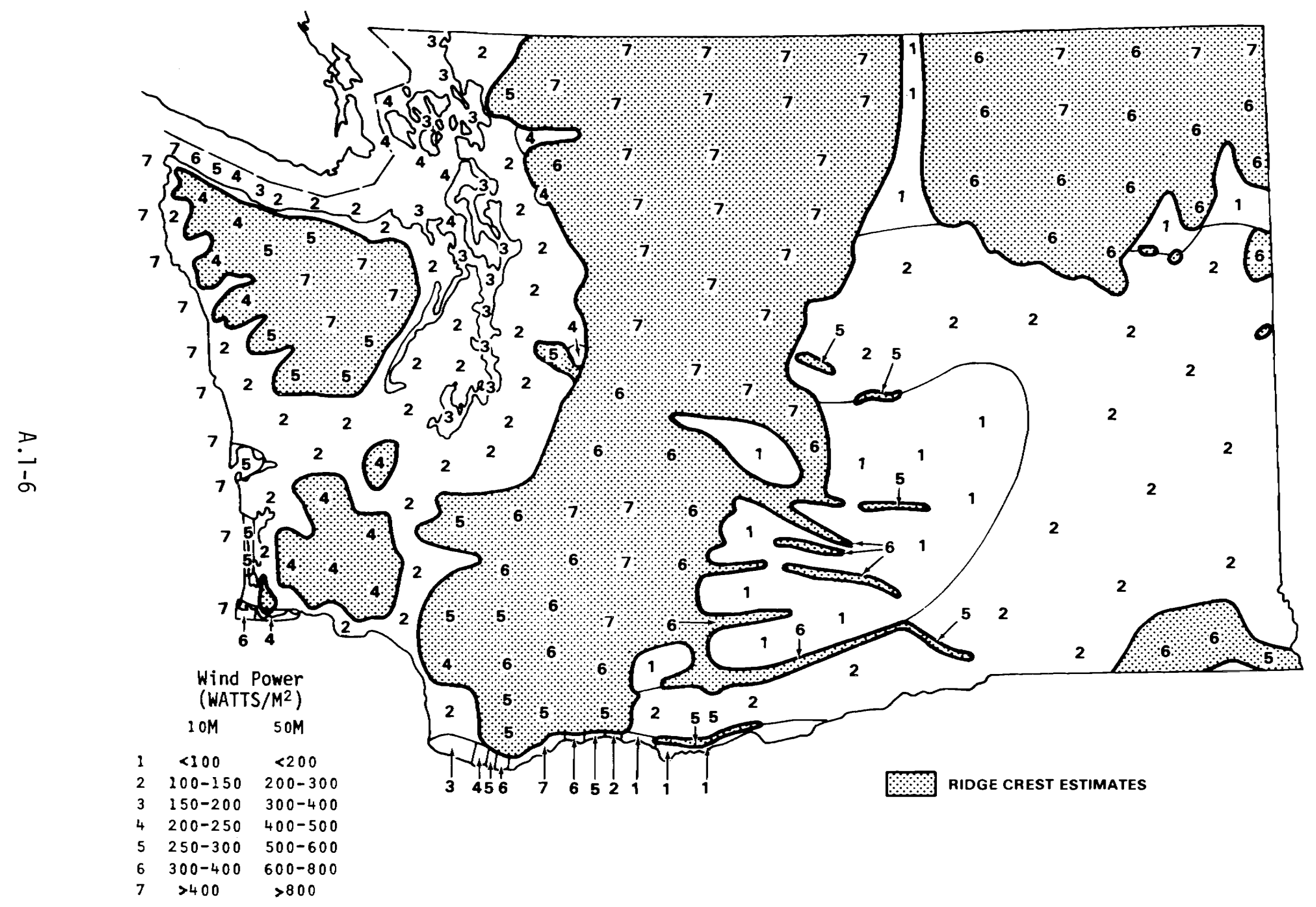

FIGURE A-1.4 Washington Winte Average Wind Powe 
degree in latitude, and the representative value of the wind power density at exposed sites within the grid box was determined. A percentage of the land area falling into several categories of wind exposure was estimated using the land-surface form classification scheme of Hammond (1964). From this information, an estimate was made of the percentage of the land area within a grid box experiencing available wind power densities greater than, equal to, slightly less than, or far less than the value for the grid box. The assignment of wind power categories to these percentage areas was based on the land-surface form classification and the amount of local terrain relief. The fractional values for each area in each grid box were summed to determine the areal distribution of the annual average wind power density. Figure A-1.5 shows the percentage of land area in each grid box where the wind resource exceeds class 3 for the state of Washington.

For the smallest space-scale treated in the atlas (a single weather station), the wind resource can be described in considerable detail. Stations selected for this portion of the atlas are those for which hourly or threehourly data are available on magnetic tape at the NCC. Information provided for individual stations includes:

- a map of station location

- tables of station location and wind power

- graphs of monthly speed and power

- graphs showing interannual variation

- plots of speed frequency

- plots of speed-direction frequency

- plots of speed duration

- graphs of power duration plots of diurnal variation by season.

Examples of weather station data presented in graphical form are provided in Figures A-1.6 to A-1.12.

\section{A.1.2 NARRATIVE REPORTS}

Summary reports of the methods used to evaluate the wind energy resource in each region are also being prepared. In addition to the methodology descriptions, lists of all data stations and some data are included. Titles of these reports are listed at the end of this appendix. 


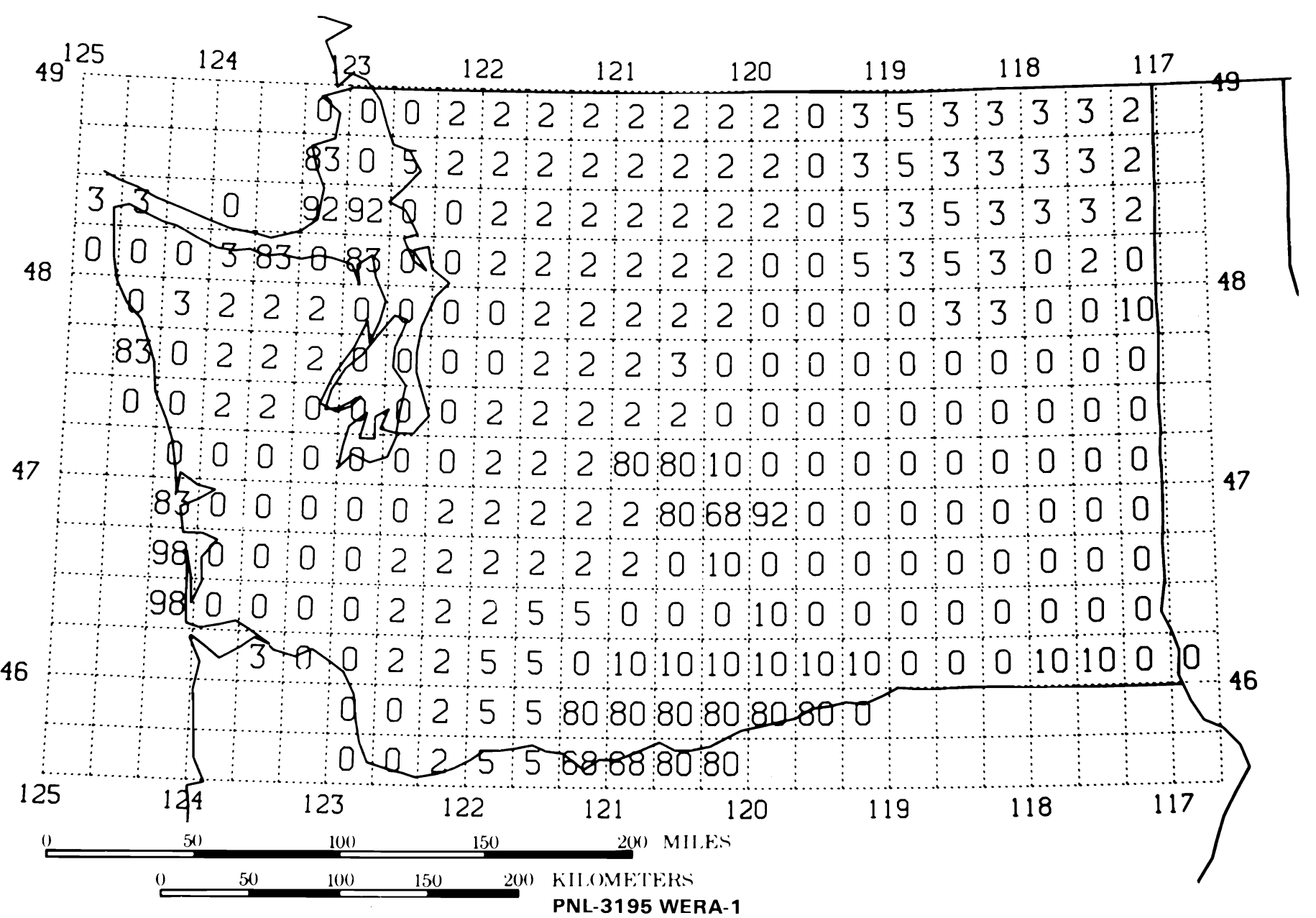

FIGURE A-1.5 Areal Distribution of Wind Resource in Washington.

Percent of land area with or exceeding power class 3. 


\section{MONTHLY AVERAGE WIND POWER AND SPEED \\ WIND POWER \\ WIND SPEED \\ LEFT ORDINATE - WATTS $/ \mathrm{M}^{2}$ \\ RIGHT ORDINATE - M/SEC \\ ABSCISSA - MONTH}
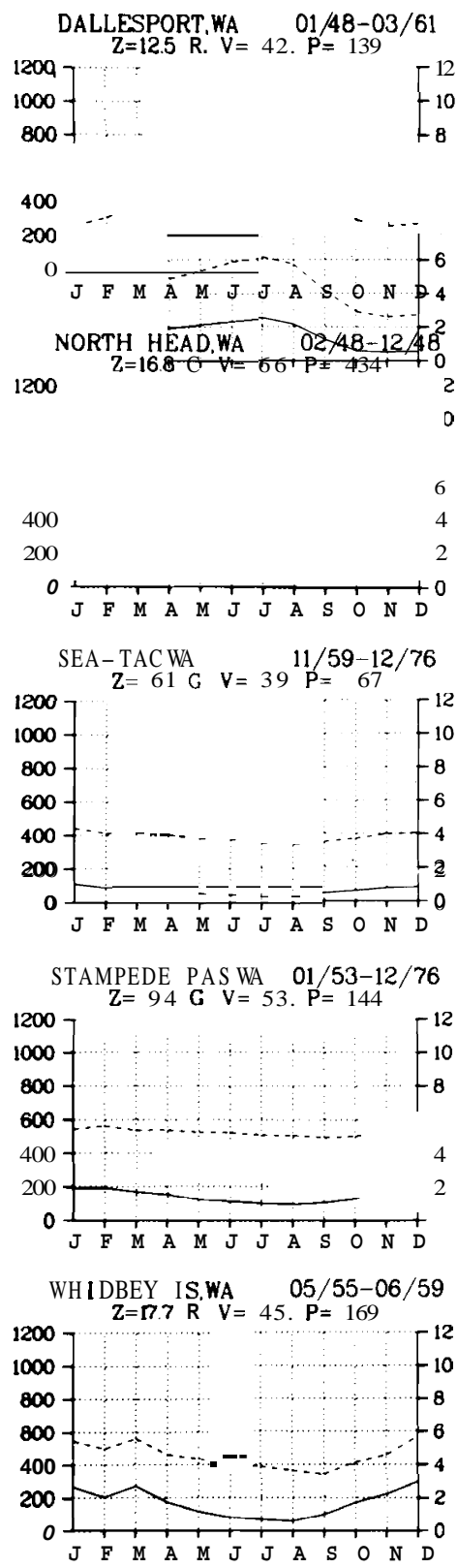
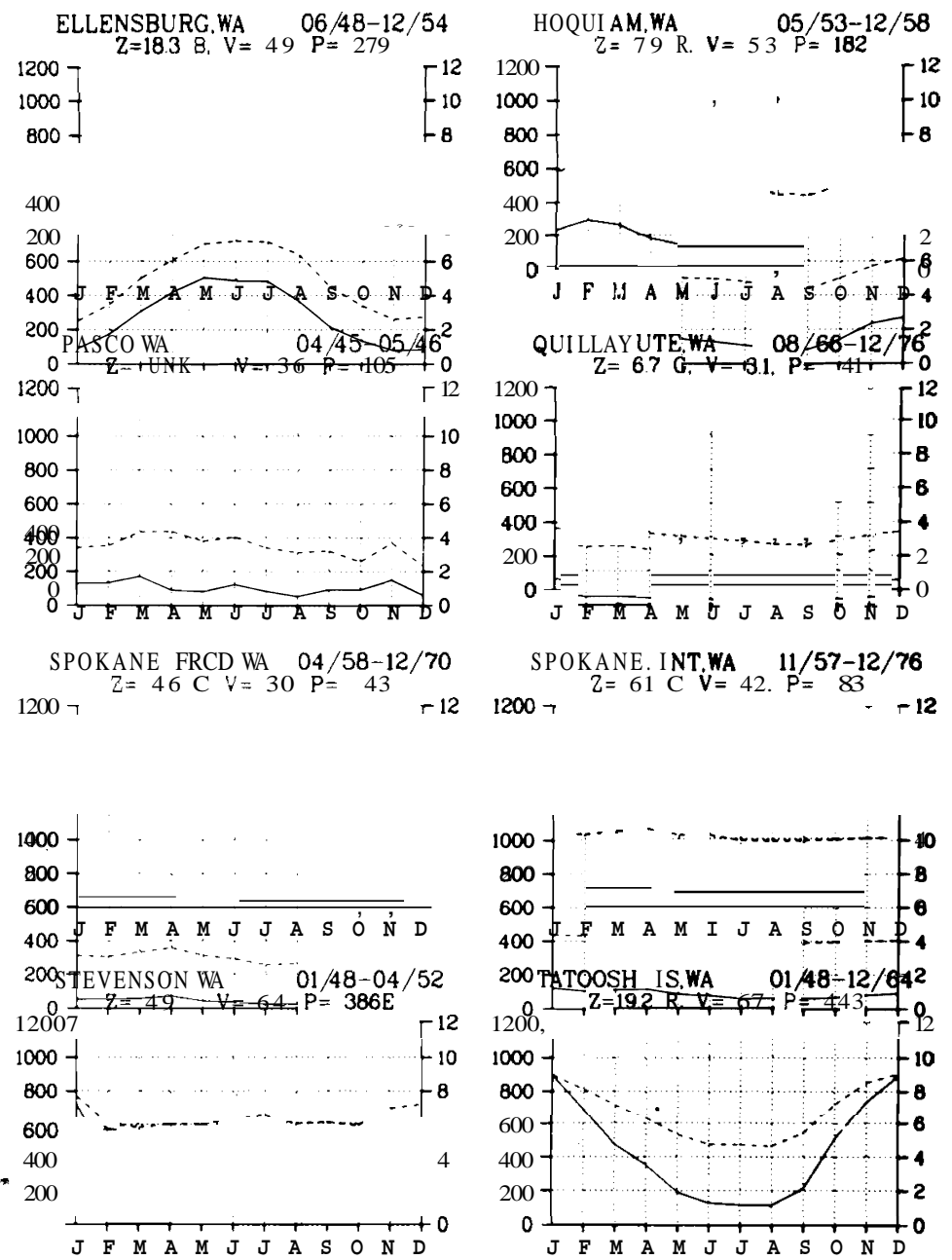

FIGURE A-1.6 Monthly Average Wind Power and Speed for Washington 


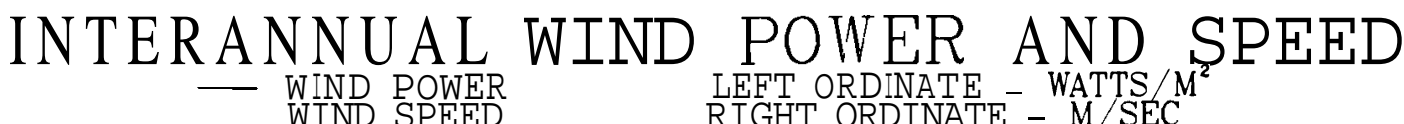 \\ LEFT ORDINATE - WATTS/M
RIGHT ORDINATE - $M /$ SEC \\ ABSCISSA - YEAR}

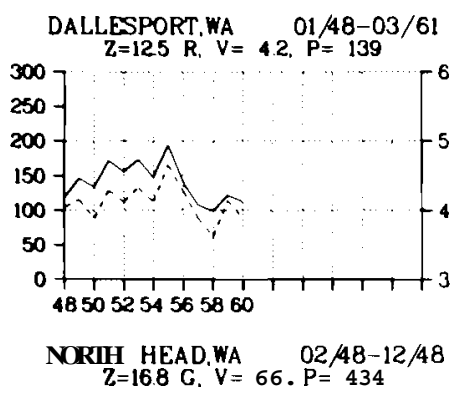

LESS THAN TWO YEARS OF DATA
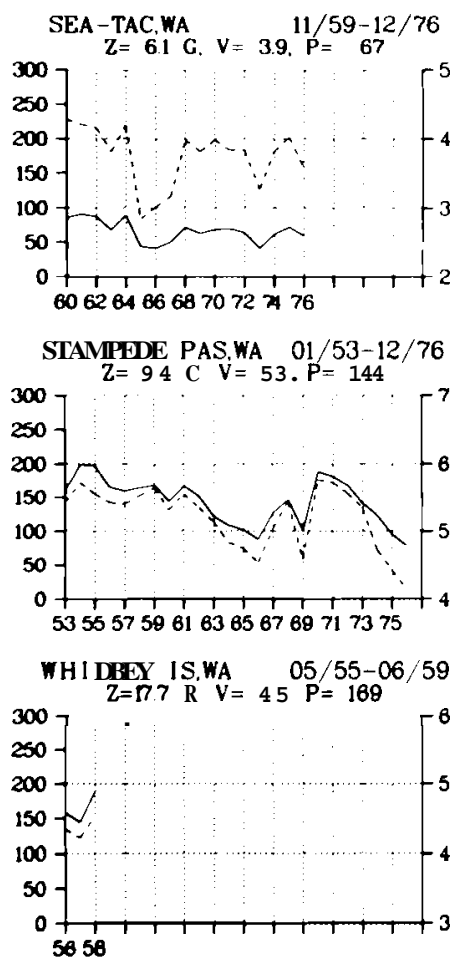

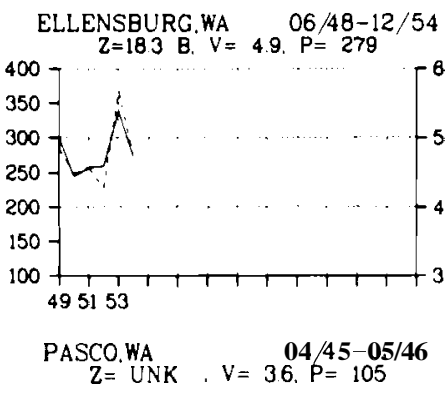

LESS THAN TWO YEARS OF DATA
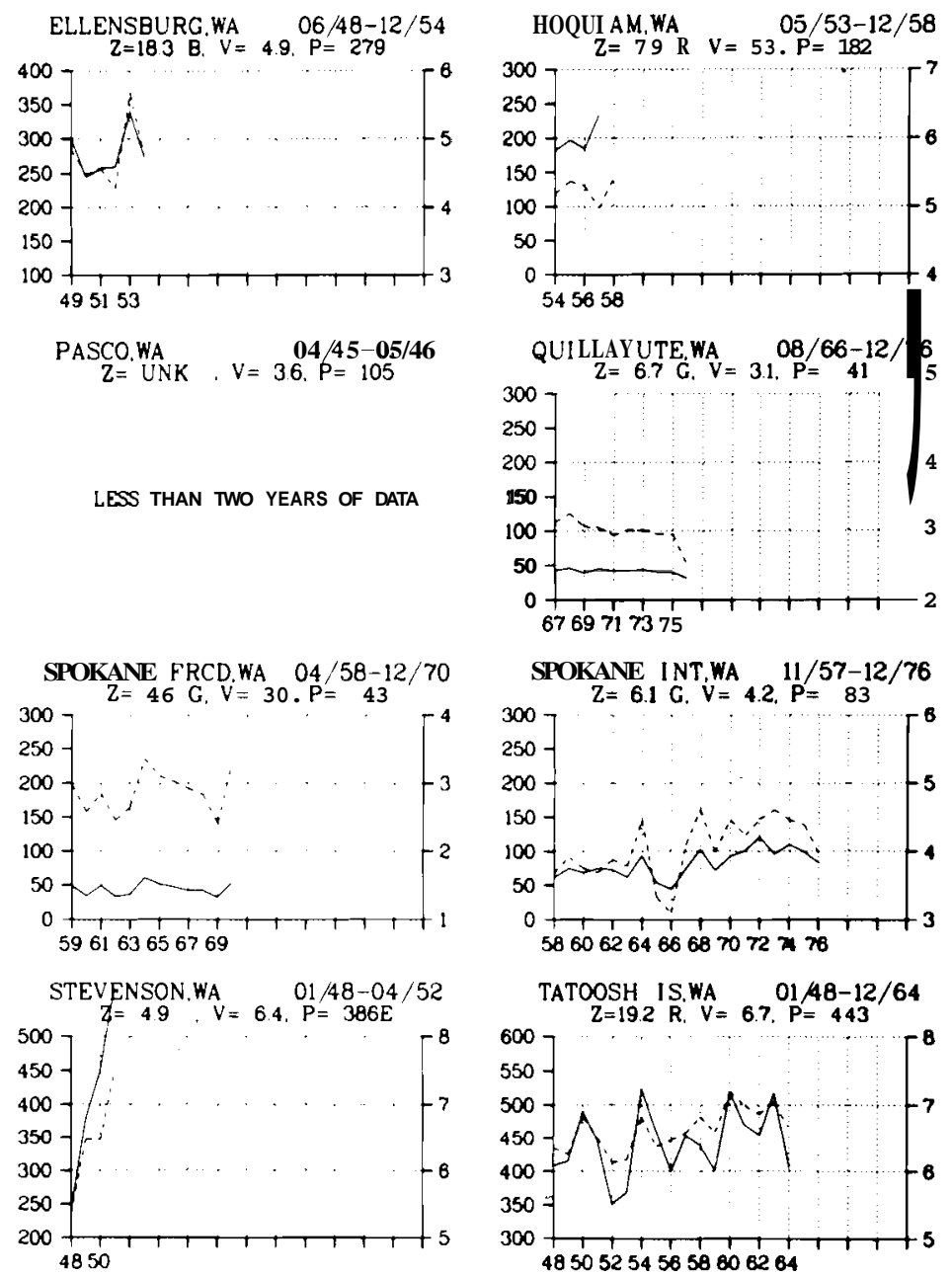

FIGURE A-1.7 Interannual Wind Power and Speed for Washington 


\section{DIURNAL WIND SPEED BY SEASON \begin{tabular}{ll} 
WINTER & $\cdots \cdot$...SPRINC \\
\hline- SUMMER & $-\cdots$-AUTUMN
\end{tabular} \\ ORDINATE - M/SEC \\ ABSCISSA - HOUR}
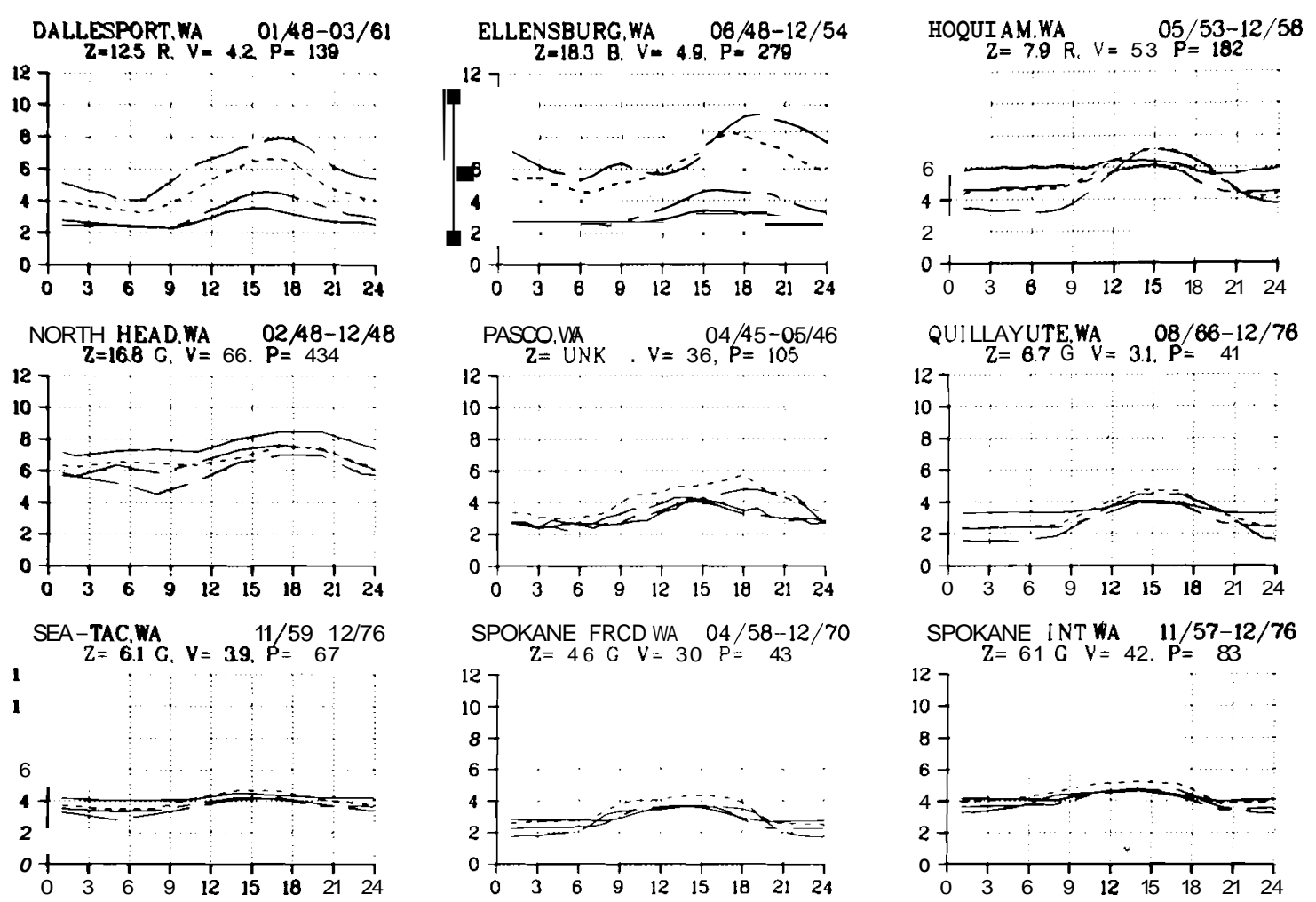

STAYPEDE PAS.WA $01 / 53-12 / 76$
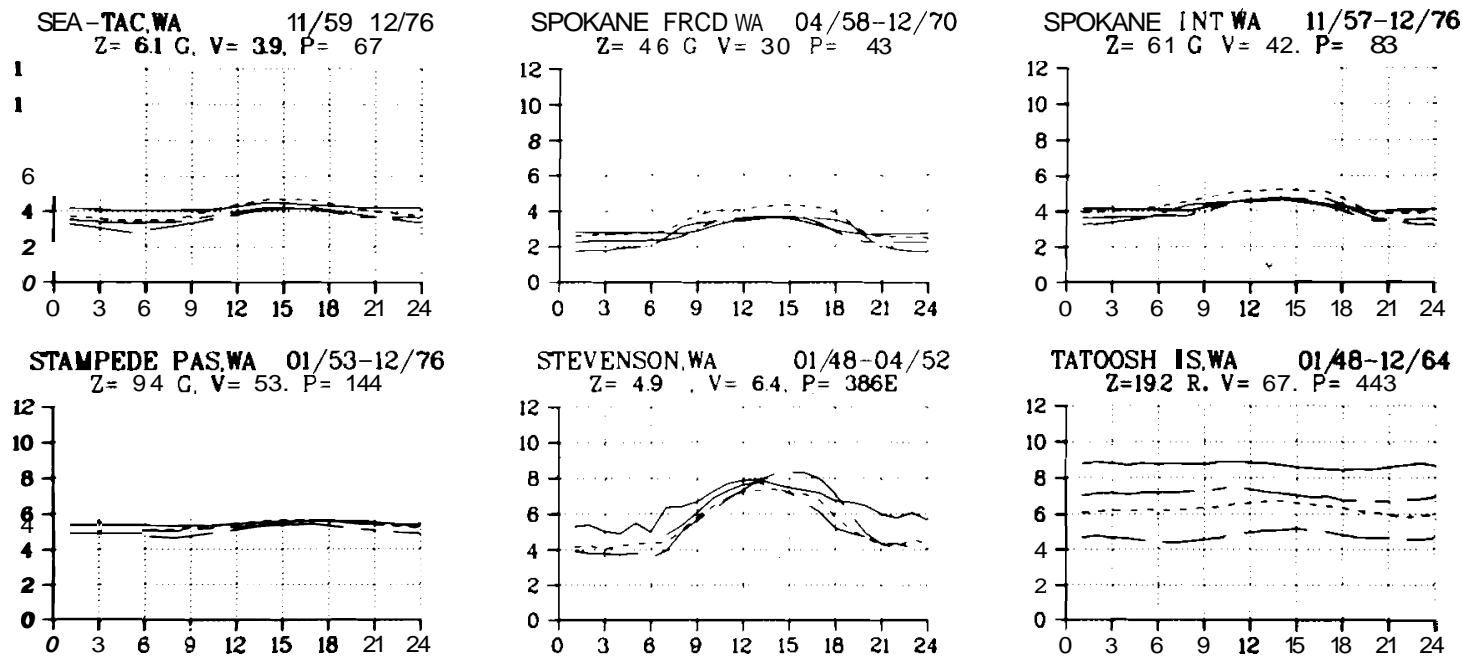

WHIDBEY IS.WA 05/55-06/59

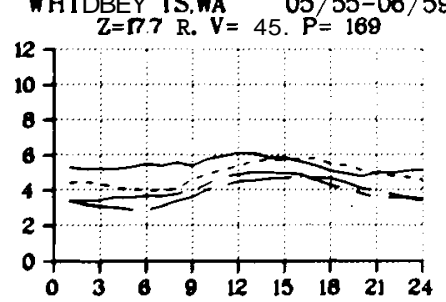

FIGURE A-1.8 Diurnal Wind Speed by Season for Washington 


\section{ANNUAL AVERAGE WIND SPEED FREQUENCY \\ — ACTUAL DISTRIBUTION ORDINATE - PERCENT \\ ...... RAYLEIGH DISTRIBUTION ABSCISSA - M/SEC}
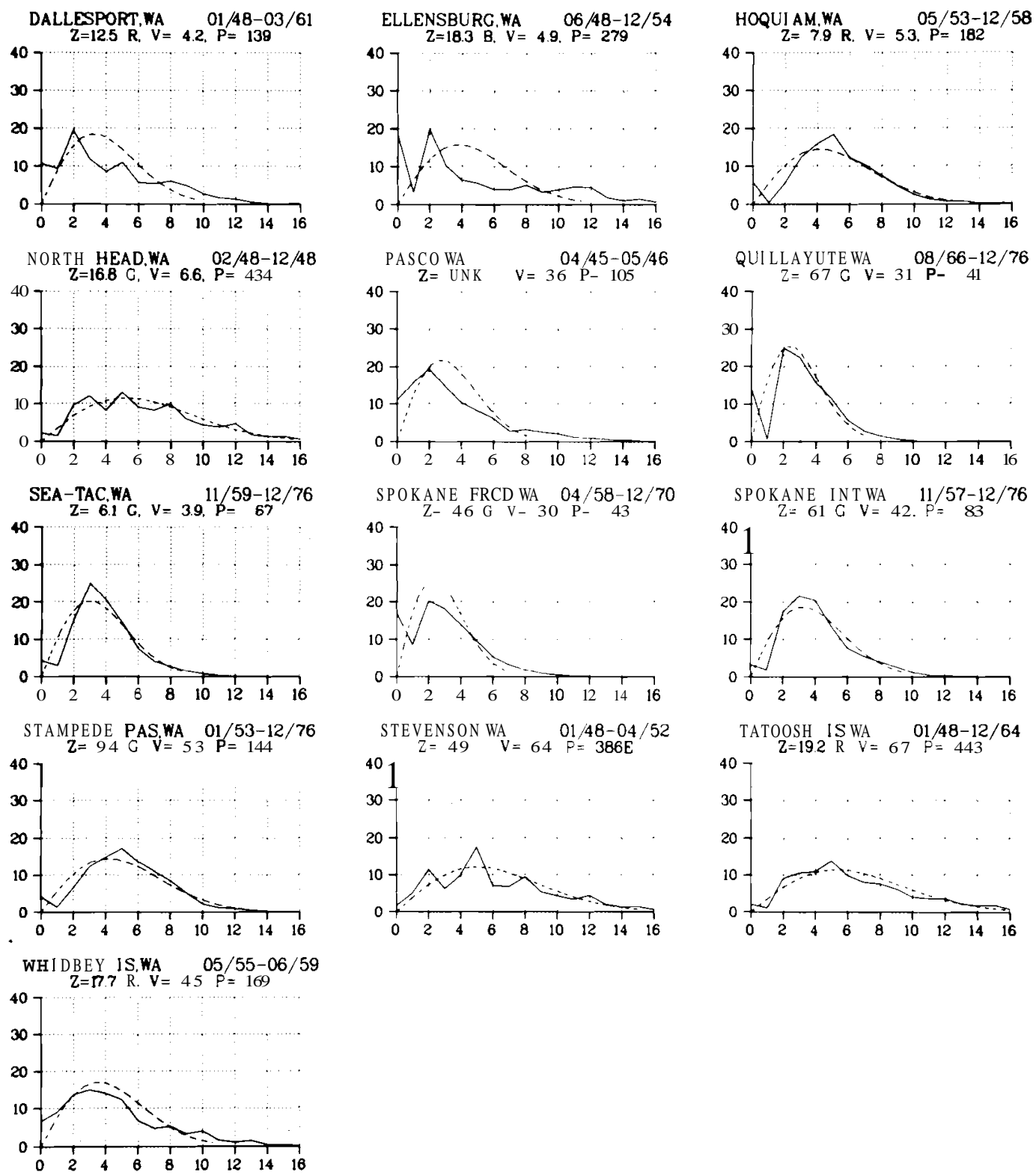

FIGURE A-1.9 Annual Average Wind Speed Frequency for Washington 


\section{DIRECTIONAL FREQUENCY AND AVERAGE SPEED PERCENT FREQUENCY LEFT ORDI NATE - . PERCENT \\ RIGHT ORDINATE - M/SEC \\ ABSCISSA - WIND DIRECTION}
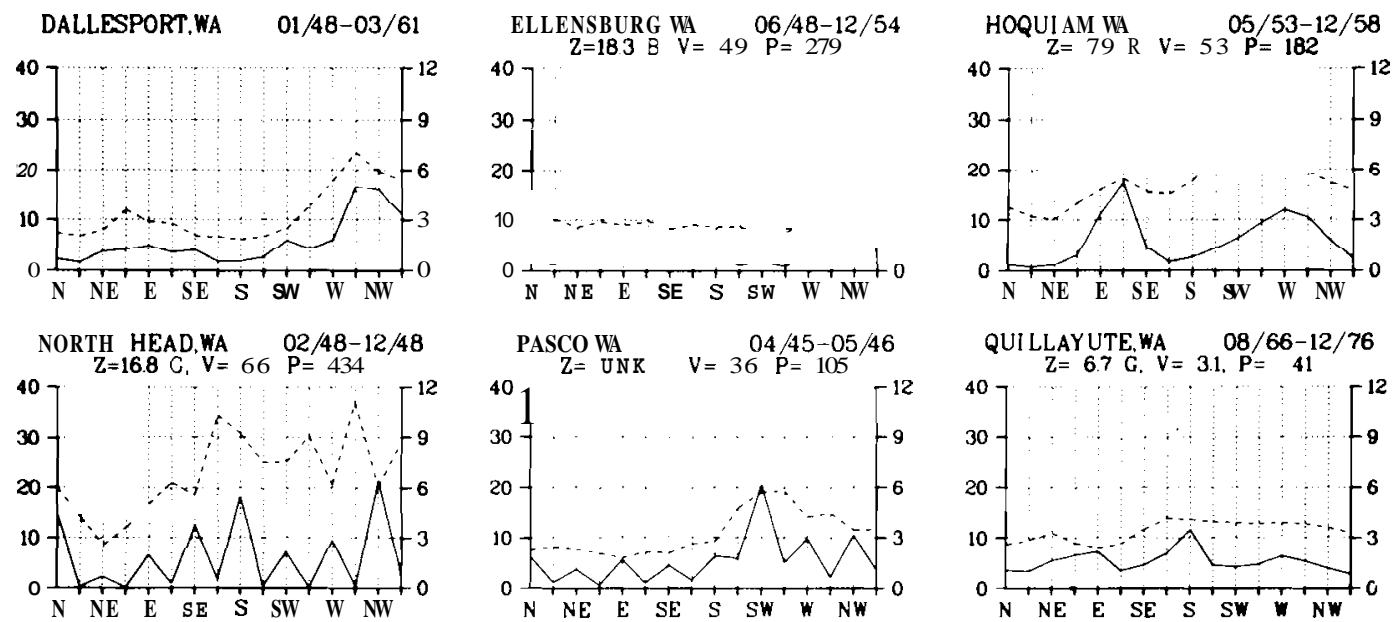

$\mathrm{Z}=\mathrm{UNK} \quad \mathrm{V}=36 \mathrm{P}=105$
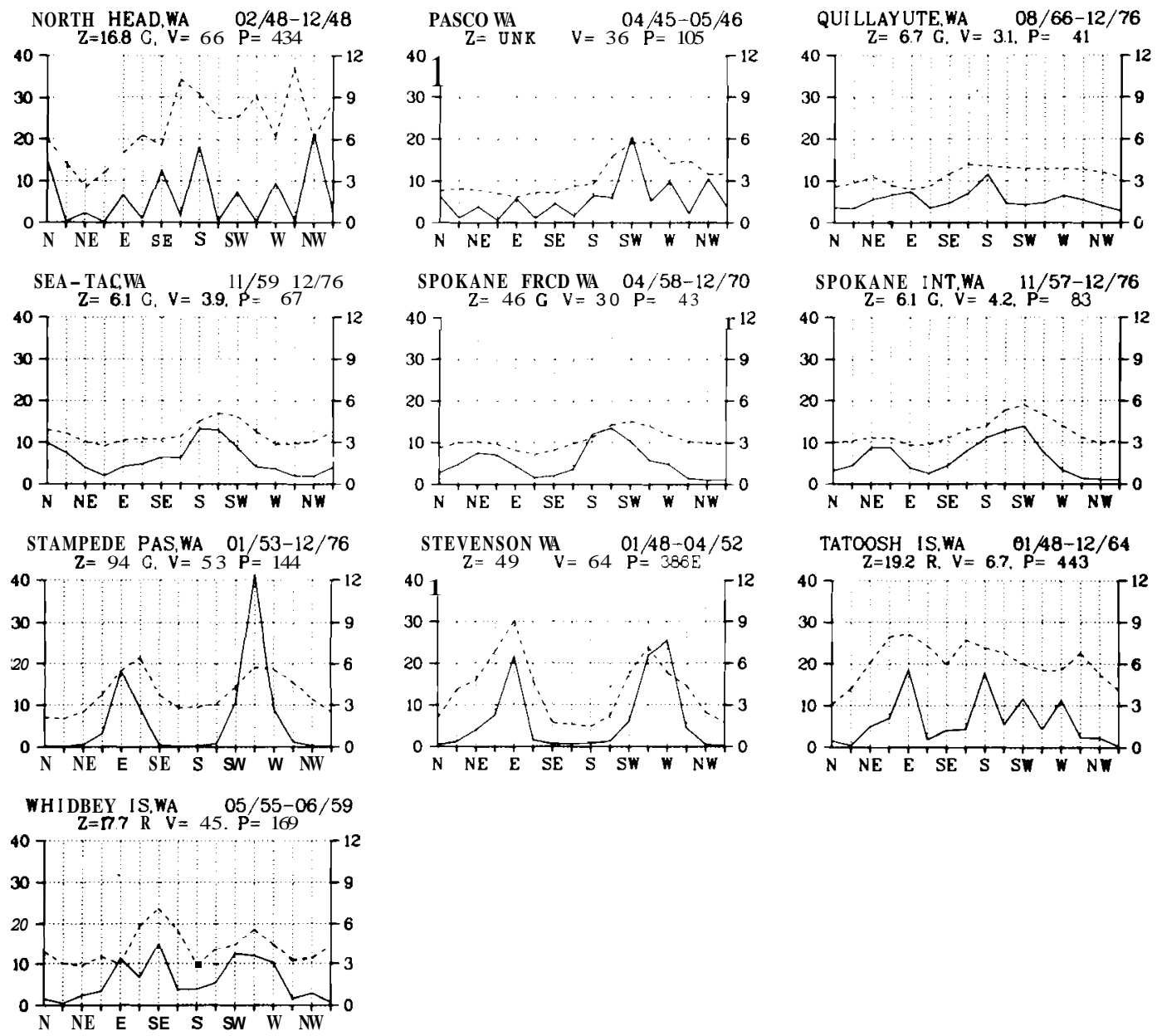

FIGURE A-1.10 Directional Frequency and Average Speed for Washington 


\section{WIND SPEED DURATION - ANNUAL AVERAGE ORDINATE - PERCENT ABSCISSA - M/SEC}
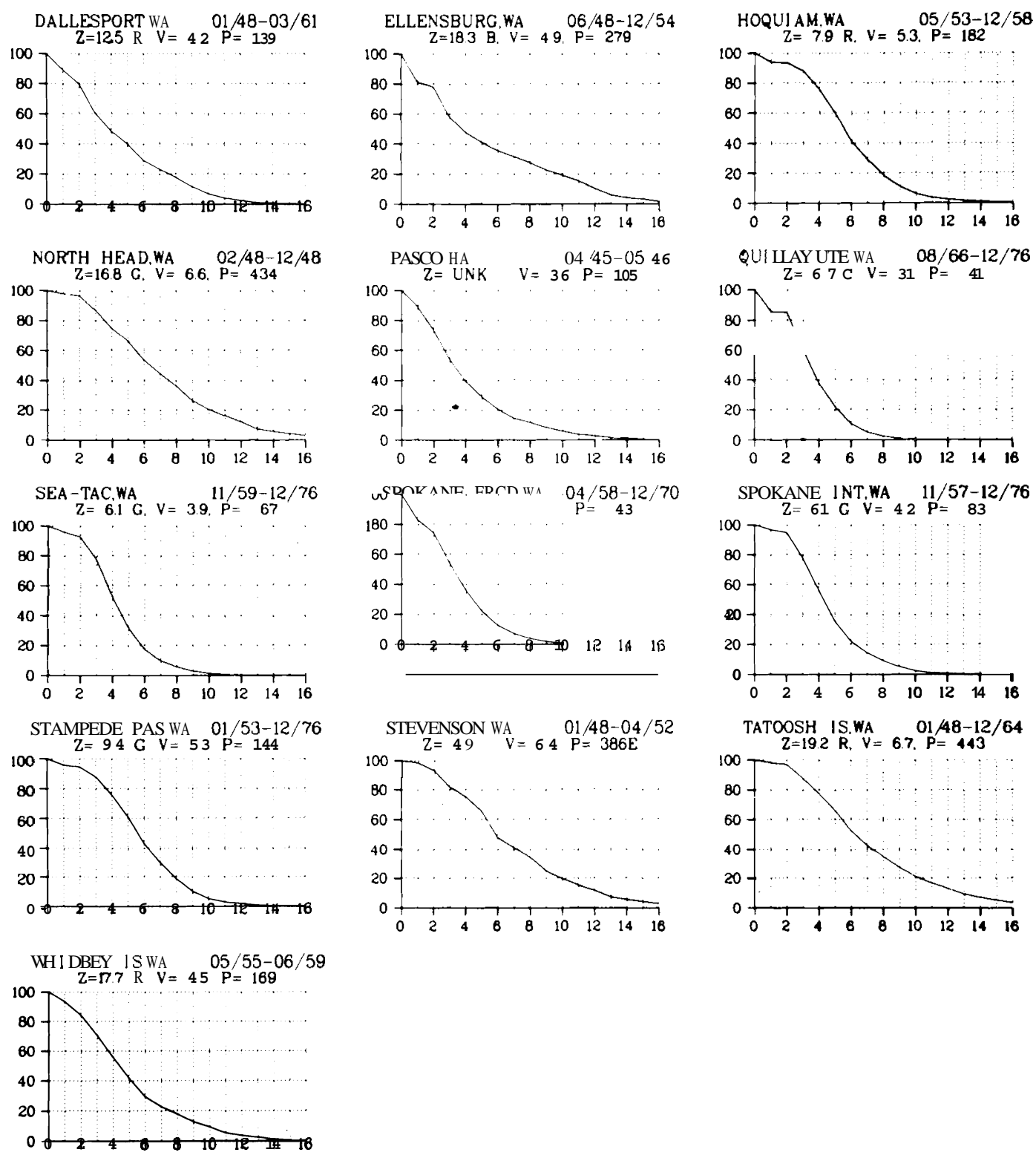

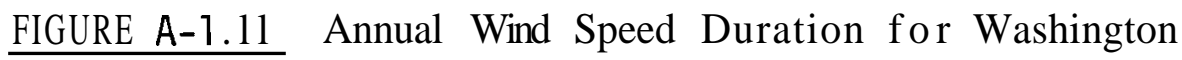




\section{WIND POWER DURATION - ANNUAL AVERAGE ORDINATE - PERCENT \\ ABSCISSA - WATTS $/ M^{2}$}
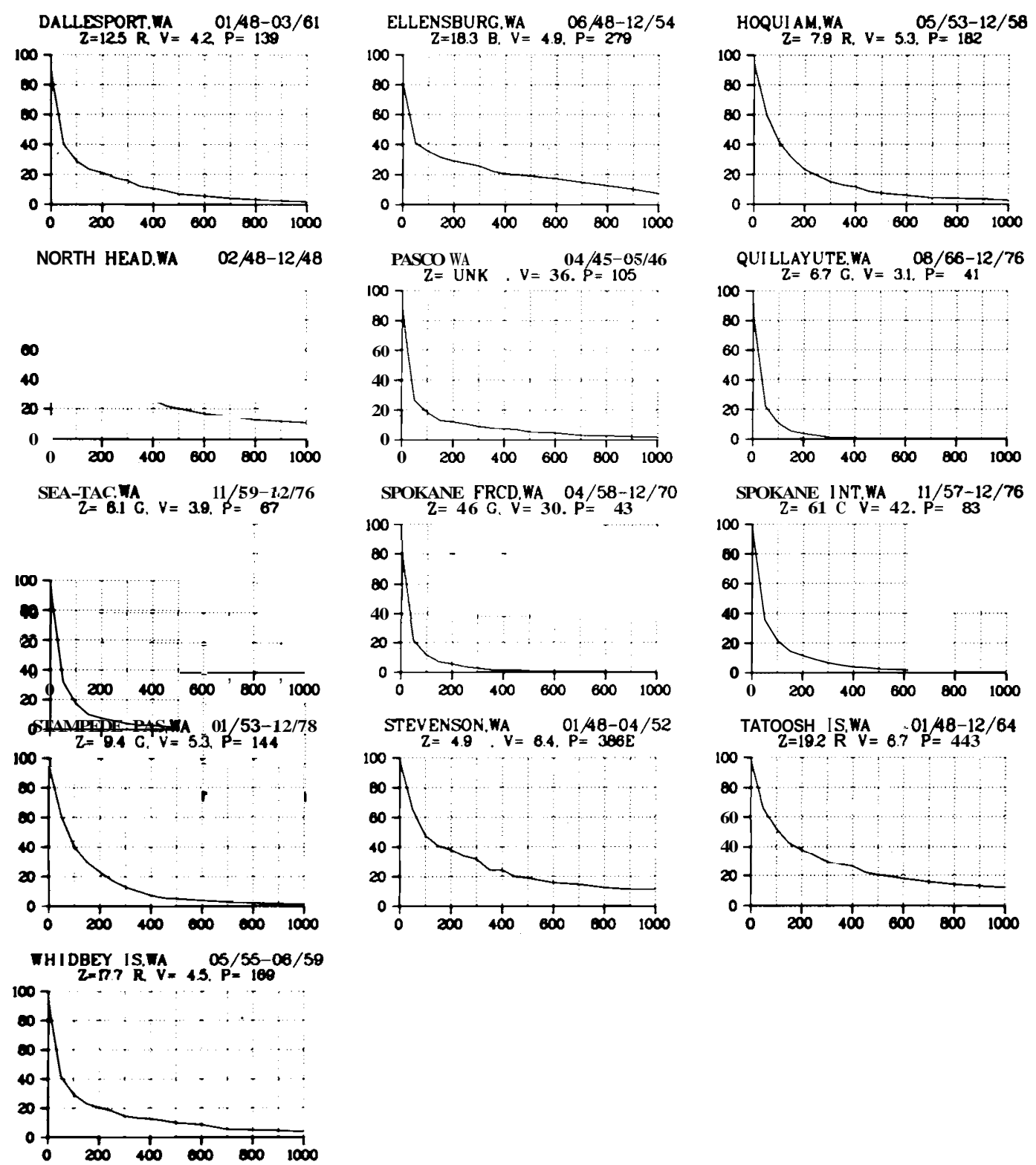

FIGURE A-1.12 Annual Wind Power Duration for Washington 


\section{A.1.3 THE WIND ENERGY DATA BASE}

In addition to the regional wind energy atlases, a regional wind energy data base will be maintained. This data base will be available from DOE's Pacific Northwest Laboratory. The data base will include all data acquired during the preparation of the regional atlases. The data base will also include gridded annual and seasonal wind power information and gridded information on the character of the surface topography. This information will be available on magnetic tape. Tabular data of the parameters presented in graphic form and other pertinent statistics will be available on microfiche.

A11 atlases are available from National Technical Information Service in Springfield, Virginia.

\section{A.1.4 LIST OF ATLAS TITLES}

D. L. Elliott et al. 1980. Wind Energy Resource Atlas: Volume 1 - The Northwest Region. PNL-3195 WERA-1.

D. L. Freeman et al. 1980. Wind Energy Resource Atlas: Volume 2 - The North Central Region. PNL-3195 WERA-2.

D. L. Paton et a1. 1980. Wind Energy Resource Atlas: Volume 3 - The Great Lakes Region. PNL-3195 WERA-3.

K. E. Pickering et al. 1980. Wind Energy Resource Atlas: Volume 4 - The Northeast Region. PNL-3195 WERA-4.

R. Brode et al. 1980. Wind Energy Resource Atlas: Volume 5-The East Central Region. PNL-3195 WERA-5.

J. M. Zabransky et al. 1980. Wind Energy Resource Atlas: Volume 6 - The Southeast Region. PNL-3195 WERA-6.

L. Graves et al. 1980. Wind Energy Resource Atlas: Volume 7 - The South Central Region.

S. P. Anderson et al. 1980. Wind Energy Resource Atlas: Volume 8 - The Southern Rocky Mountain Region. PNL-3195 WERA-8.

G. L. Norman et al. 1980. Wind Energy Resource Atlas: Volume 9 - The Southwest Reaion. PNL-3195 WERA-9. 
J. E. Wise et al. 1980. Wind Energy Resource Atlas: Volume 10 - Alaska. PNL-3195 WERA-10.

T. A. Schroeder et al. 1980. Wind Energy Resource Atlas: Volume 11 - Hawaii and Pacific Trust Territories. PNL-3195 WERA-11.

H. L. Wegley et a1. 1980. Wind Energy Resource Atlas: Volume 12 - Puerto Rico and U.S. Virgin Islands. PNL-3195 WERA-12.

D. L. Elliott et a1. 1980. Wind Energy Resource Atlas: Volume 13 - National Synthesis. PNL-3195 WERA-13.

The narrative reports describing the development of each of the wind energy atlases are:

D. L. Elliott et al. 1981. Techniques for Assessing the Wind Resource in the Northwest Region. PNL-3360.

D. L. Freeman et al. 1981. Techniques for Assessing the Wind Resource in the North Central Reaion. PNL-3667.

D. L. Paton et al. 1981. Techniques for Assessing the Wind Resource in the Great Lakes Region. PNL-3668.

K. E. Pickering et al. 1981. Techniques for Assessing the Wind Resource in the Northeast Region. PNL-3452.

R. Brode et al. 1981. Techniques for Assessing the Wind Resource in the East Central Region. PNL-3451.

J. M. Zabransky et al. 1981. Techniques for Assessing the Wind Resource in the Southeast Reaion. PNL-3669.

L. Graves et al. 1981. Techniques for Assessing the Wind Resource in the South Central Region. PNL-3670.

S. R. Anderson et al. 1981. Techniques for Assessing the Wind Resource in the Southern Rocky Mountain Region. PNL-3671.

G. T. Norman et al. 1981. Techniques for Assessing the Wind Resource in the Southwest Region. PNL-3672.

T. Wentink et al. 1981. Techniques for Assessing the Wind Resource in Alaska. PNL-3519.

T. A. Schroeder et al. 1981. Techniques for Assessing the Wind Resource in Hawaii and the Pacific Trust Territories. PNL-3673. 
H. L. Wegley et a1. 1981. Techniques for Assessing the Wind Resource in Puerto Rico and the U.S. Virgin Islands. PNL-3674. 


\section{REFERENCES}

Coty, U. A. 1976. Wind Energy Mission Analysis. SAN/1075-1/1,2,3; LockheedCalifornia Company, Burbank, California.

Elliott, D. L. 1977. Synthesis of National Wind Energy Assessments. BNWL-2220 WIND-5, Pacific Northwest Laboratory, Richland, Washington.

Garate, J. A. 1977. Wind Energy Mission Analysis. C00/2578-1/1,2,3; General Electric Company, Philadelphia, Pennsylvania.

Hammond, E. H. 1964. "Analysis of Properties in Landform Geography: An Application to Broadscale Landform Mapping." Annals, Association of American Geographers. $54: 11-19$, Map Supplement Number 4.

Reed, J. W. 1975. Wind Power Climatology of the U.S. SAND 74-0348, Sandia Laboratories, Albuquerque, New Mexico. 


\section{APPENDIX 2}

SOME BACKGROUND METEOROLOGY PERTINENT TO SITING LARGE WECS 


\section{APPENDIX 2 \\ SOME BACKGROUND METEOROLOGY PERIINENT TO SITING LARGE WBCS}

To properly site large wind energy conversion systems (WECS), the interaction of the air flow with the earth's surface must be considered. Terrainflow interaction is a continual process that is rarely simple to understand. This appendix provides a groundwork for understanding the fundamental principles of terrain-flow interaction that are relevant to the problem of siting large WECS.

Important characteristics of the earth's surface are the distribution of:

- surface elevations or topography

surface roughness

- surface temperature.

These characteristics force or modify the atmospheric flow, depending on the ambient characteristics of the flow. The most important flow characteristics are the distribution of:

- air temperature

- pressure

- wind speed and direction

- turbulence.

These flow characteristics are related to each other so that terrain modifications affecting one flow characteristic affect all of them. Terrain forcing of the atmosphere occurs on a range of scales from the planetary scale to the small scale near the earth's surface where molecular viscosity is a significant force.

Scales relevant to the siting of large wind turbines are considered here. The horizontal scale ranges from tens of kilometers in complex terrain to tens of meters near a mountain summit. The vertical scale ranges from $<2 \mathrm{~km}$, a value based on the structure of the atmospheric flow, to $10 \mathrm{~m}$, approximately the lowest point on the rotor disk of a large wind turbine.

Understanding basic flow features is necessary to understand more complicated terrain and flow interactions. Therefore, this introduction deals with 
the most basic of flows, which is flow over flat terrain. It begins with a look at the equations that govern atmospheric flows. The central discussion is a brief review of knowledge about flow over horizontally uniform terrain on which the surface temperature varies with the heating by the sun. Such a flow is a good approximation to flow characteristics over many flat sites. The problems of vertical extrapolations of winds and the turbulence characteristics over flat land are discussed. The characteristics of the boundary layer over flat terrain should be understood in order to attempt to understand the response of the atmosphere in flow over complex terrain.

\section{A.2.1 FUNDAMENTAL EQUATIONS AND DEFINITIONS}

A small number of equations describe the physical constraints that atmospheric flows obey. First, the continuity equation, based on the conservation of mass, is:

$$
-\frac{1}{\rho} \frac{d \rho}{d t}=\frac{\partial U}{\partial x}+\frac{\partial V}{\partial z}+\frac{\partial W}{\partial z}
$$

where

- $p=$ density

- $U=$ velocity component in $x$ direction

- $V=$ velocity component in y direction

- $W$ = velocity component in $\mathbf{z}$ (vertical) direction.

The derivative of density is the total derivative following the fluid motion. The total derivative operator, $\frac{d}{d t}$, may be separated into a local (fixed coordinates) time derivative and the terms describing the advection by the wind of the operand along the $x, y$, and $z$ directions, as in the following equation:

$$
\frac{d}{d t}=\frac{\partial}{\partial t}+1 \frac{\partial}{\partial x}+V \frac{\partial}{\partial y}+w \frac{\partial}{\partial z}
$$

Density variations as a result of pressure fluctuations caused by flow over small obstacles are negligible so the flow is treated as incompressible. Larger scale motions do involve density changes, however, and the full continuity equation must be considered. 
Secondly, the equations of motion express the conservation of momentum. For the horizontal wind components, this is:

$$
\begin{aligned}
& \frac{\partial \bar{U}}{\partial t}+\bar{U} \frac{\partial \bar{U}}{\partial x}+\bar{V} \frac{\partial \bar{U}}{\partial y}+W_{\frac{\partial}{\partial z}}=f \bar{V}-\frac{1}{\rho} \frac{\partial p}{\partial x}-F_{x} \\
& \frac{\partial \bar{V}}{\partial t}+\bar{U} \frac{\partial \bar{V}}{\partial x}+\bar{V} \frac{\partial \bar{V}}{\partial y}+\bar{W}_{\partial z} \frac{\partial \bar{V}}{\partial z}=-f \bar{U}-\frac{1}{\rho} \frac{\partial p}{\partial y}-F_{y}
\end{aligned}
$$

where

$$
\begin{aligned}
- & \text { an averaging operator, aueraging over a period that is long } \\
& \text { compared to the time scales of turbulent motions but short } \\
& \text { compared to time scales of significant changes in the mean } \\
& \text { flow, say about } 1 \text { hour. } \\
\mathrm{f}= & 2 \Omega \sin \phi \text { (the Coriolis parameter) with } \\
& \quad \begin{array}{l}
\text { angular velocity of earth } \\
\end{array} \\
& =\text { latitude } \\
\mathrm{P}= & \text { pressure }
\end{aligned}
$$

In a turbulent atmosphere, the friction terms represent the divergence of the turbulence transport of momentuni. Turbulence momentum transport is a result of the covariance of the turbulence wind components. The friction terms ma be written then as:

$$
\begin{aligned}
& F_{x}=\frac{\partial}{\partial x} \overline{u u}+\frac{\partial}{\partial y} \overline{u v}+\frac{\partial}{\partial z} \overline{u w} \\
& F_{y} \frac{\partial}{\partial x} \overline{u v}+\frac{\partial}{\partial y} \overline{v v}+\frac{\partial}{\partial z} \overline{v w}
\end{aligned}
$$

where the turbulence fluctuations from the mean are:

$$
\begin{aligned}
& \mathrm{u}=U-\bar{U} \\
& \mathrm{v}=\mathrm{V}-\overline{\mathrm{V}} \\
& \mathrm{w}=\mathrm{W}-\overline{\mathrm{W}} \\
& \overline{\mathrm{uW}}, \overline{\mathrm{VW}}, \text { and } \overline{\mathrm{uV}} \text { are called Reynolds stresses }
\end{aligned}
$$


the turbulence kinetic energy (TKE) is proportional to the sum of $\overline{\mathrm{UU}}, \overline{\mathrm{VV}}$, and $\overline{\mathrm{wW}}$.

(See Busch 1973 for a derivation of the mean momentum and turbulence equations).

For example, consider flow in the $x$ direction at some height $z$ above the ground. Since the wind at $z+\Delta z$ is usually stronger than at $z$, downward turbulent motions $(w<0)$ at $z$ are bringing higher momentum air down from above $(u>0)$ so that the average correlation $\overline{u w}<0$. Also, since the increase in windspeed with height is generally greater at $z$ than at $z+\Delta z$, we see that $\overline{u w}$ is less negative at $z+\Delta z$ so that $\frac{\partial u w}{\partial z}>0$ and there is a positive term in $F_{x}$ which in turn becomes a sink for horizontal momentum. This sink must be balanced by some other term in the equations of motion. A great deal of research goes into finding ways to express the friction terms as functions of known or easily obtained quantities.

When vertical accelerations are small, the vertical equation of motion reduces to a statement of hydrostatic balance:

$$
\frac{\partial p}{\partial z}=-\rho g
$$

where

$\mathrm{g}=$ acceleration of gravity.

Thirdly, the equation of state is:

$$
p=p r t
$$

where

$$
\begin{aligned}
& T=\text { absolute temperature in Kelvins }(K) \\
& R=\text { gas constant for air }=287 \text { joules } \mathrm{kg}^{-1} \mathrm{~K}^{-1}
\end{aligned}
$$

Fourthly, conservation of energy is expressed in the first law of thermodynamics as:

$$
\frac{d H}{d t}=C_{p} \frac{d T}{d t}-\frac{1}{p} \frac{d p}{d t}
$$


where

$\frac{d H}{d \mathbf{t}}=$ rate of external heat addition per unit mass of air

$C_{P}=1004$ joules $\mathrm{kg}^{-1} \mathrm{~K}^{-1}$, the specific heat at constant pressure for air.

When an air parcel is moved from one pressure level to another adiabatically, with no external heat addition, Equation (A-2.9) can be used to determine the change in temperature associated with the work done against (by) the lower (higher) pressure at the new level. It is convenient, especially where vertical motions are involved, to define a potential temperature, 8, as the temperature a parcel of air would have if it were brought adiabatically to the pressure level of $1000 \mathrm{mb}$. This relation is readily derived from Equations (A-2.9) and (A-2.8) to be:

$$
\theta=T\left(\frac{1000 \mathrm{mb}}{\mathrm{p}}\right)^{R / C_{p}}
$$

The static stability of a layer in the atmosphere is measured in terms of the vertical gradient of potential temperature. If $\partial \theta / \partial z$ is positive, then when an air parcel of potential temperature $\theta_{0}$ at height $\mathrm{z}$ is displaced adiabatically (its potential temperature is conserved) to height $\mathrm{z}+\Delta z$, the potential temperature $\theta$ and consequently the temperature $T$ of the parcel will be lower than the ambient temperature at height $z+\Delta z$. The now cooler parcel is forced back down. Such a layer is said to be stable. Gravity waves are oscillations of fluid in a stable layer. If $\partial \theta / \partial z<0$ the layer is unstable and a disturbed parcel continues its motions away from its origin. A neutral layer is one where $\partial \theta / \partial z=0$ and a disturbed parcel experiences no restoring or destabilizing forces.

Another form of the conservation of energy may be approximately expressed in the thermodynamic energy equation, which is:

$$
\begin{gathered}
\frac{a \theta}{\bar{a} t}+U \frac{a d}{a x}+V \frac{a e}{a \sim}+W \frac{\partial \theta}{\partial z}=-\frac{1}{P C P} \frac{a}{a x}\left(\rho C_{p} \bar{u} \theta^{\prime}+R x\right) \\
+\frac{a}{\partial y}\left(\rho C_{P} \overline{v \theta^{\prime}}+R y\right)+\frac{a}{\partial z}\left(\rho C_{P} w \theta r+R z\right)
\end{gathered}
$$


where

$\bar{\theta}=$ averaged potential temperature

$\theta^{\prime}=\theta-\bar{\theta}$ is the fluctuating potential temperature

$R x, R y, R z=$ the components of the radiant energy flux

$\rho C_{P} \overline{u \theta^{\top}}, \rho C_{P} \overline{v \theta^{\top}}$ and $\rho C_{P} \overline{w \theta^{\top}}$ are turbulentheat fluxes.

It is through this equation that turbulent heat fluxes above a hot surface add heat to the atmosphere.

If all terms in the horizontal momentum Equations $(A-2.3)$ and $(A-2.4)$ are small except the Coriolis and pressure gradient forces, the flow is in "geostrophic" balance and the wind vector $\left(U_{g}, V_{g}\right)$ is the geostrophic wind where:

and

$$
U_{g}=\frac{-1}{\rho f} \frac{\partial p}{\partial y}
$$

$$
V_{g}=\frac{1}{\rho f} \frac{\partial p}{\partial x}
$$

If the winds are not in geostrophic balance, one may still use the geostrophic wind as a shorthand way of describing the pressure gradient force or as an approximation to the actual winds.

The geostrophic wind may be a function of height. If so, the atmosphere is called baroclinic; if not, it is called barotropic. Differentiating Equations $(A-2.12)$ and $(A-2.13)$ with respect to height, using the equation of state (Equation $A-2.8$ ) and the hydrostatic equation (Equation A-2.7) results in:

$$
\begin{aligned}
& \frac{\partial U_{g}}{\partial z}=\frac{-g}{f T} \frac{\partial T}{\partial y}+\frac{U_{g}}{T} \frac{\partial T}{\partial z} \\
& \frac{\partial V_{g}}{\partial z}=\frac{g}{f T} \frac{\partial T}{\partial x}+\frac{V_{g}}{T} \frac{\partial T}{\partial z} .
\end{aligned}
$$

The difference between the geostrophic wind at $z_{1}\left(U_{g} z_{1}, V_{g} z_{1}\right)$ and at $z_{2}\left(U_{g} z_{2}\right.$, $\left.V_{g} z_{2}\right)$ is called the thermal wind. The components of the thermal wind $\left(U_{T}, V_{T}\right)$ are obtained by integrating Equations $(A-2.14)$ and $(A-2.15)$, [neglecting the small terms on the right sides of $(A-2.14)$ and $(A-2.15)]$. The result is: 


$$
\begin{aligned}
& U_{T}=U_{g} z_{1}-U_{g} z_{2}=\frac{-g}{f<T\rangle} \frac{\partial\langle T\rangle}{\partial y}\left(z_{2}-z_{1}\right) \\
& v_{T}=v_{g} z_{2}-v_{g} z_{1}=\frac{g}{f\langle T\rangle} \frac{\partial\langle T\rangle}{\partial x}\left(z_{2}-z_{1}\right)
\end{aligned}
$$

where $\langle T\rangle$ is the average temperature in the layer between $z_{2}$ and $z_{1}$. The thermal wind equations show the important relationship between horizontal temperature structure of a baroclinic atmosphere and the vertical structure of the winds.

The planetary boundary layer (PBL) is loosely defined as that layer of the atmosphere near the earth's surface where interaction of the flow and the earth's surface is achieved, usually through turbulent transport of momentum and heat. Therefore, in the PBL, $F_{X}$ and $F_{Y}$ are significant terms in the momentum balance. In the PBL, Equations (A-2.3) and (A-2.4) are used to describe the mean flow. Those equations may be rewritten as:

$$
\begin{aligned}
& \frac{d \bar{U}}{d t}+f\left(V_{g}-\bar{V}\right)=F_{x} \\
& \frac{d V}{d t}-f\left(U_{g}-\bar{U}\right)=F_{y} .
\end{aligned}
$$

The PBL depth may be quite variable. Over the Great Plains i t may grow from a few hundred meters in the morning to a few $\mathrm{km}$ in the afternoon. Over coastal areas it may remain a constant depth for days. The magnitudes of the components of the frictional force at a given height $z$ also varies tremendously. To understand the winds in the PBL one must understand the turbulence structure that determine $F_{X}$ and $F_{Y}$,

A number of mechanisms influence the structure of turbulence in the PBL. Turbulent kinetic energy (TKE) is mechanically produced by wind shear. It is also thermally produced by the buoyancy forces associated with an upward heat flux and unstable stratification. Turbulence generated near the earth's surface, where production by shear and an upward heat flux is greatest, may be transported upward to higher levels in the PBL. If horizontal gradients of mean quantities are not small, which certainly occurs in flow over uneven 
terrain, turbulence may accumulate at a point because of transport and diffusion of TKE from upstream sources. At a point, TKE is reduced by buoyancy forces associated with a downward heat flux in stable stratification, by molecular viscosity in the smallest turbulent eddies, and by transport of TKE out of the neighborhood. Turbulence also interacts with gravity waves in a stably stratified flow in ways that are poorly understood. The TKE can be radiated away by gravity waves, or gravity waves can "break" thereby adding TKE to the flow.

Describing turbulence involves scaling parameters that are assumed to characterize turbulent flows (Tennekes 1973b, Wyngaard 1973). There are two important scaling parameters that relate to the production of TKE. Mechanical production of TKE is parameterized by the friction velocity $u_{*}$, which is the square root of the kinematic surface stress and is related to the Reynolds stresses as:

$$
u_{*}=\sqrt{\frac{\tau_{0}}{\rho}}=\left[(\overline{u w})_{0}+(\overline{v w})_{0}\right]^{\frac{1}{2}}
$$

where

$\tau=$ horizontal shear stress

subscript 0 means the values are measured very near the surface.

Buoyancy production of turbulence is related to the near surface vertical heat flux $\left(H_{0}=C_{p} p\left(\overline{w \theta^{\top}}\right)_{0}\right)$.

The Obukhov length $(L)$ is a scale relating the strengths of shear produced and thermally produced turbulence, as:

$$
L=\frac{-\theta}{g k} \frac{u_{\star}^{3}}{(\overline{w \theta})_{0}}
$$

where $k=0.35$ (Businger et al. 1971) is the von Karman constant. In unstable conditions with an upward heat flux, $-L$ is about twice the distance above the surface where buoyancy production of turbulence becomes larger than shear production. In stable conditions with a downward heat flux, $L$ is about the size of the largest turbulent eddies. 
Boundary layer height (h) is another scaling parameter that characterizes the flow. A dimensionless stability parameter $(h / L)$ can be formed which appropriately represents stability of the PBL. Consider an unstable PBL where $h / L<0$. Increasing $h$ while keeping $L$ constant is equivalent to increasing the heat flux while keeping $h$ and $u_{\star}$ fixed, or decreasing $u_{\star}$ while keeping $h$ and $\left(\bar{w}^{\top}\right)_{0}$ fixed. In all cases, the final state results in the buoyancy production of turbulence dominating a larger fraction of the PBL, which is tantamount to a more unstable PBL.

The texture of the earth's surface is described by the roughness length scale $\left(z_{0}\right)$. The roughness length is empirically derived from the logarithmic wind profile described in Section A.2.2. Table A-2.1 compares roughnesses over several surfaces. For a given geostrophic wind, the stress near the ground is greater for a rougher surface than for a smooth one. Likewise, the near-surface turbulence intensity is greater and the near surface wind speed is less over a rougher surface.

The roughness length must sometimes be qualified. It can be a function of wind speed: strong winds create a rougher sea surface but bend wheat over resulting in a smaller $z_{0}$ for wheatlands. Or, when the surface is densely covered with trees, $z_{0}$ may be somewhat smaller than for a sparser forest, since the overlying flow responds only to the surface of the forest canopy, i.e., the top of the tree cover. However, a downward extrapolation of the logarithmic wind profile from above the canopy yields zero wind speed at some height above the forest floor and within the canopy. This height is the displacement height (D). The extrapolation to zero wind speed does not mean that no flow exists below $D$. Usually, a weak and complicated flow known as canopy flow exists below the displacement height. The most severe qualifications on the use of the roughness length arise when the land is not flat. The logarithmic wind profile has no validity in uneven terrain yet intuitively the texture of the earth's surface is important. Therefore, the roughness of uneven land is often described in analogy to $z_{0}$. It is not clear however how one should separate out the effects of, for example, trees and terrain in a description of the roughness of rolling forested hills. 
In this section, basic definitions and fundamental equations were introduced with which basic features of turbulent atmospheric flows may be discussed. The governing equations include the momentum equations, continuity equation, hydrostatic equation, equation of state, and the first law of thermodynamics. From these equations definitions were provided for the geostrophic wind, thermal wind, the force balance within the planetary boundary layer, and potential temperature and atmospheric stability. The generation of turbulence was briefly discussed and some of the scaling parameters necessary to describe turbulent flows were presented. These include the friction velocity $\left(u_{*}\right)$, the heat flux $\left(\rho C_{P} \overline{w \theta^{\top}}\right)$, the Obukhov length $(L)$, the PBL height $(h)$, and the roughness length $z_{0}$. With these definitions we can discuss flow over uniform flat terrain. TABLE A-2.1. Typical Values of Surface Roughness Length $\left(z_{0}\right)$, for
Various Types of Surfaces (Frost et al. 1978)

Type of Surface

Mud Flats, Ice

Smooth Sea

Sand

Snow Surface

Mown Grass (0.01 m)

Low Grass, Steppe

Fallow Field

High Grass

Palmetto

Forest and Woodland

Suburbia

City

\begin{tabular}{rl} 
Range of $z$ & $\mathrm{~m}$ \\
\hline $10^{-5}-3 \cdot 10^{-5}$ \\
$2 \cdot 10^{-4}-3 \cdot 10^{-4}$ \\
$10^{-4}-10^{-3}$ \\
$10^{-3}-6 \cdot 10^{-3}$ \\
$10^{-3}-10^{-2}$ \\
$10^{-2}-4 \cdot 10^{-2}$ \\
$2 \cdot 10^{-2}-3 \cdot 10^{-2}$ \\
$4 \cdot 10^{-2}-10^{-1}$ \\
$10^{-1}-3 \cdot 10^{-1}$ \\
$10^{-1}-1$ \\
$1-2$ \\
$1-4$
\end{tabular}

\section{A.2.2 FOW OVER UNIFORM FLAT TERRAIN}

Flow in the PBL over uniform flat terrain is the simplest PBL flow conceivable. Even so no exact solutions to the equations of motion are possible 
because of the nature of turbulence. However, combined theoretical and experimental progress has led to a good understanding of these most simple PBL flows. Many WECS will be sited in flat terrain where the concepts presented here will be suitable for quantitative application. Much of the basic structure presented here is also present in the more complicated flows discussed in Section A.2.3. In this section we present discussions on:

- diurnal cycle of PBL structure

- extrapolation of near surface winds to heights of $100 \mathrm{~m}$

- turbulence characteristics.

\section{A.2.2.1 Diurnal Cycle of PBL Structure}

During the course of daily heating by the sun and cooling of the earth by radiation at night, the temperature structure of the lower atmosphere changes. The temperature structure is important in defining the characteristics of the turbulence in the boundary layer. The turbulence structure controls the friction terms in the momentum balance which controls the wind structure. The wind structure feeds back into the turbulence structure to modify the temperature structure. These processes occur within the planetary boundary layer which may be changing in depth rapidly. Add in the effects of advection and baroclinicity that occur in realistic flows and it is no wonder that predictions of boundary layer structures or extrapolations of characteristics from one level to another are very uncertain.

Three broaa classes of PBL structure can be identified, based on the stability near the surface. These classes are neutral, unstable, and stable. The characteristics of these classes are significant to the siting of WECS in two ways. First, WECS operate in the lowest portion of the PBL where the wind characteristics are determined by the larger PBL structure; and second, the response of the PBL to non-uniform terrain depends upon ambient PBL structure.

Figure A-2.1 shows potential temperature profiles, wind profiles, and boundary layer heights during the course of a day. This hypothetical flow is unrealistically simple because there is no time variation of the upper level flow and the flow is barotropic. Figure A-2.1 also exaggerates some characteristics in order to demonstrate a number of important properties. 

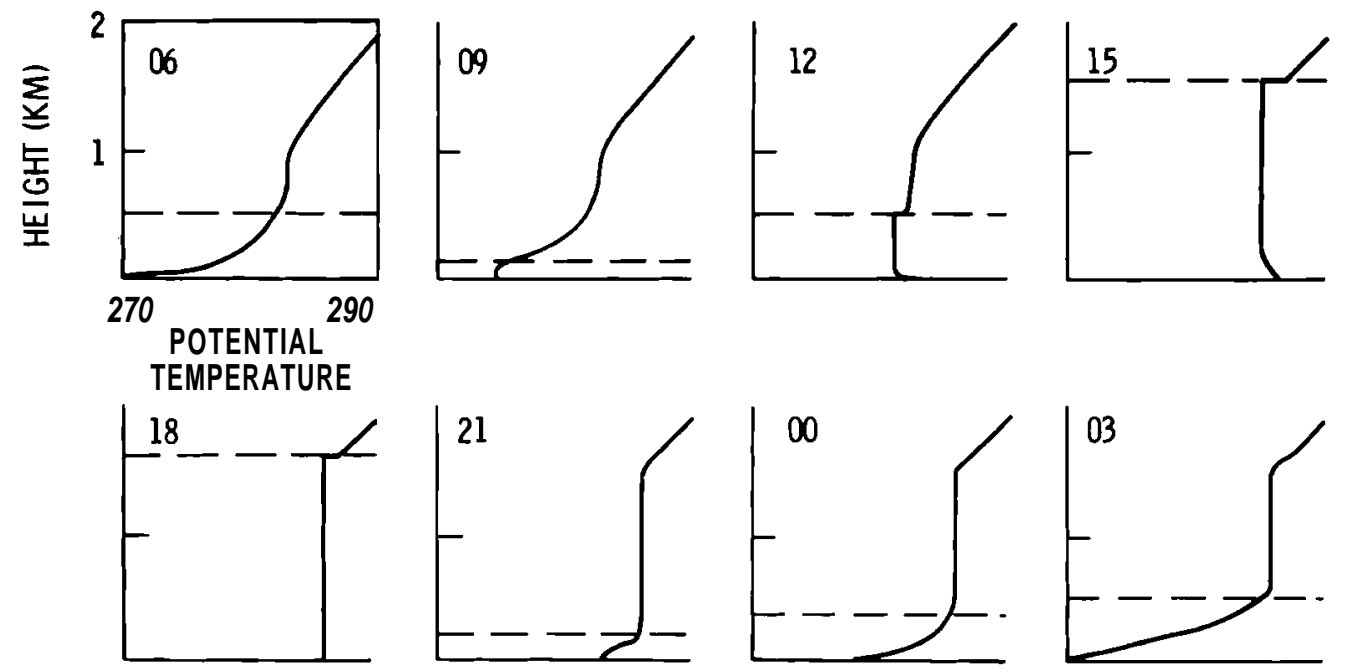

WIND DIRECTION

(a)

(DEGREES)
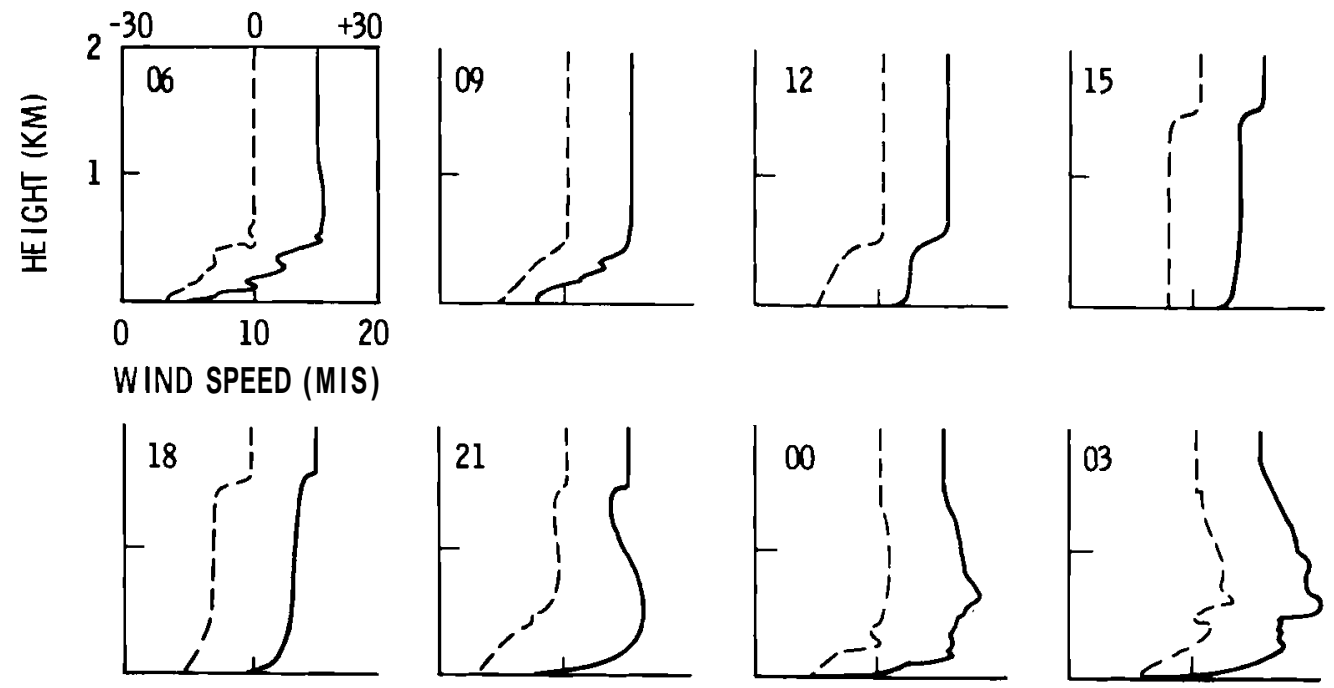

(b)

FIGURE A-2.1. Potential Temperature Profiles and Boundary Layer Heiyht (part a) and Wind Speed Profiles (solid) and Wind Direction Profiles (dashed, part b) Every Three Hours, for a Hypothetical Boundary Layer over Uniform Terrain 
By 0600 the nightime radiational cooling and downward heat flux has established a nocturnal temperature inversion and boundary layer height to about $500 \mathrm{~m}$. Turbulence in a nocturnal boundary layer achieves some mixing between different levels, thereby reducing vertical gradients of both wind and potential temperature. However, to maintain shear production of turbulent kinetic energy (TKE), the only source of TKE in the stable PBL, the wind shear must be large enough to overcome the effects of the stable stratification; or:

$$
R i \equiv \frac{g}{\theta} \frac{\partial \theta / \partial z}{(\partial \bar{U} / \partial z)^{2}} \leqslant 0.2
$$

where

$\mathrm{Ri}$ is the Richardson number stability parameter

$\mathrm{Ri} \approx 0.2$ is the critical Richardson number.

If $\mathrm{Ri} \gtrsim 0.2$ the flow becomes laminar and turbulent transfer of heat and momentum ceases. In this way layers in the atmosphere can become decoupled and behave independently. This is presented in the highly variable wind profile of 0600 . In turbulent layers above and below a laminar or quiescent region, downward momentum transport continues, which increases the wind shear enough to drop the Richardson number below its critical value and the layer once again becomes turbulent. Patchy intermittent turbulence also arises from the poorly understood interaction between turbulence and gravity waves which propagate through the stable PBL. At 0600, the wind direction changes fairly continuously through the boundary layer, except at the decoupled layers, from the frictional force balance at the surface to geostrophic balance at the top of the PBL.

By 0900 solar heating of the earth's surface has begun. In the surface layer the lowest 20-30 meters, the potential temperature profile is superadiabatic, i.e., $\partial \theta / \partial z<0$. From the surface layer, warm bubbles rise through an adiabatic layer $(\partial \theta / \partial z \simeq 0)$ called the mixed layer, until they encounter the stable potential temperature profile of the remnant nocturnal inversion. The base of the temperature inversion separates the turbulent mixed layer from the non-turbulent flow aloft. Therefore, the height of the unstable PBL is the height of the lowest potential temperature inversion. The warm bubbles penetrate a small distance into the inversion layer where they entrain some 
of the warm air of the inversion layer into the mixed layer. Hence, the mixed layer grows by entrainment. There is a downward heat flux in the upper portion of the boundary layer.

Figure A-2.1 shows that by noon the mixed layer has grown by entrainment to $500 \mathrm{~m}$. In the unstable PBL, vertical mixing is strong, thus all levels are strongly coupled. The rapid communication between levels makes gradients of potential temperature and wind become very small. Shortly after noon the mixed layer entrains the rest of the nocturnal inversion and encounters the remnant of the previous day's mixed layer (seen between 500 and $1000 \mathrm{~m}$ on the noon potential temperature profile). The remnant mixed layer presents no obstacle to PBL growth so the boundary layer quickly grows to the height of the previous day's mixing depth.

By 1500, when the mixing is at its strongest, the winds are nearly uniform in speed and direction throughout the mixed layer. The PBL is as deep as it is going to get. The transport of high momentum air entrained at the inversion down to near the surface results in the daily maximum wind speed near the surface. The momentum transport near the surface is higher than in the morning so that the surface stress is larger. This means that, although the mixed layer has little wind shear, the lowest ten of meters exhibits higher wind shear than early in the morning. Note that most of the adjustment of wind direction to the geostrophic wind direction occurs in the inversion layer atop the mixed layer.

After solar heating stops, the surface heat flux decreases to 0 . The 1800 potential temperature profile shows $\partial \theta / \partial z=0$ throughout the boundary layer. This is an example of a type of neutral boundary layer.

The neutral boundary layer in an atmosphere with a constant potential temperature has been theoretically studied extensively. It is from this assumption that the various forms of the classic "Ekman spiral" wind profile are derived. In such a boundary layer where thermally forced convection is nonexistent, the dominant length scale was presumed to be $u_{*} / f$. The neutral PBL then was determined to have a depth of $0.2 u_{\star} / f$ to $0.3 u_{\star} / f$. This boundary layer, however, is virtually never observed. 
A somewhat more likely case of a neutral PBL is like that shown at 1800 in Figure A-2.1. In this case $\partial \theta / \partial z=0$ up to the temperature inversion that clearly separates the turbulent PBL from the non-turbulent air aloft. Therefore, the height of the inversion base is still the appropriate height scale for the boundary layer. Entrainment at the inversion causes a downward heat flux which goes to 0 at the surface. Even this type of boundary layer is rarely observed, at least as a steady-state phenomenon. Instead, the neutral PBL is a very non-stationary transition state that occurs between the unstable daytime PBL and the stable nightime PBL. (a)

By 2100 the ground is cooling. The downward heat flux has cooled a thin layer near the surface that is the nocturnal boundary layer. Above, in the remnants of the day's mixed layer, there is no production of turbulence and so the turbulence dies out quickly. If the geostrophic winds are strong enough the PBL will remain continuously turbulent. The PBL then slowly grows. However, by midnight, Figure A-2.1 shows the boundary layer has reverted to being sporadically turbulent, and will remain so until morning starts the cycle anew.

Re-establishing the boundary layer height near the ground after sunset and the subsequent decay of turbulence in what was the daytime mixed layer cause the friction terms in the momentum budget to become negligibly small. The upper leveis are then suddenly decoupled from the PBL below. When the decoupling occurs the winds are sub-geostrophic, i.e., the wind speed is below the geostrophic wind speed and the wind direction is counterclockwise of the geostrophic wind direction by ten or more degrees. With friction removed from the force balance we can write the equations of motion for the ageostrophic wind as:

$$
\frac{d u^{\prime}}{d t}-f v^{\prime}=0
$$

(a) In Section A.2.2.2 wind profiles near the ground are discussed for stable, neutral, and unstable conditions. In that context, neutral conditions imply that the region being considered is sufficiently near the ground that shear production of turbulence is much larger than either buoyancy production or buoyancy destruction of turbulence. Viewed that way, a neutral wind profile can exist as a stationary state. 


$$
\frac{d v^{\prime}}{d t}+f u^{\prime}=0
$$

where

$$
\begin{aligned}
& u^{\prime}=\bar{U}-U_{g}, v^{\prime}=V-V_{g} \\
& \frac{d U_{g}}{d t}=\frac{d V_{g}}{d t}=0 .
\end{aligned}
$$

The solution to these equations is that of an inertia circle:

$$
\begin{aligned}
& u^{\prime}=u_{0}^{\prime} \cos (f t)+v_{0}^{\prime} \sin (f t) \\
& v^{\prime}=v_{0}^{\prime} \cos (f t)-u_{0}^{\prime} \sin (f t)
\end{aligned}
$$

where $u_{0}{ }^{\prime}$ and $v_{0}$ ' are the values of the ageostrophic wind components when the upper flow becomes decoupled. The winds above the nocturnal PBL then begin to oscillate about the geostrophic wind vector with a frequency of $2 \pi / f$. In fact, daytime heating will interrupt the inertia circle before it is complete, but the flow may reach its maximum winds, above the geostrophic wind speed in $1 / 2$ inertial period or $\pi / f$ after sunset. This is illustrated in the 0300 wind profile of Figure A-2.1. It is obvious from Equations (A-2.25) and $(A-2.26)$ that the more subgeostrophic the wind speed in the mixed layer is during the day, the larger will be the acceleration at night. If there is a substantial increase of the wind with height in the daytime mixed layer or a decrease in the geostrophic wind with height, the winds will accelerate most just above the top of the nocturnal PBL, leading to a low-level nocturnal jet (Blackadar 1957). (a)

The differences between the diurnal wind speeds near the surface and those one to two hundred meters above the surface deserve emphasis. The daytime coupling of all levels mixes high momentum air down to the surface causing a daytime wind maximum. The nighttime stability causes a decoupling of many layers from the effects of the surface friction resulting in a nighttime wind speed maximum, sometimes as low as 100 meters. This behavior is not always

(a) Terrain and baroclinicity of the flow can amplify the inertial oscillations. This is discussed in Chapter 5. 
the same from day to day, however, since it is very sensitive to the conditions of a specific day. This makes the use of near-surface winds to interpret the winds that a WECS will experience very uncertain. In spite of the difficulties of categorizing an infinite variety of flow regimes, some basic characteristics of unstable, stable but continuously turbulent, or stable and sporadically turbulent boundary layers are always observed.

\section{A.2.2.2 Vertical Extrapolation of Near-Surface Winds}

To estimate the nature of the wind resource that a large wind turbine will experience, it is necessary to estimate the wind characteristics everywhere between 10 and 100 meters above the surface. These approximations could be made by an arbitrary guess, installation of a plethora of wind sensors on a 100 meter tower, or ultimately by installing the wind turbine. The quality required of the estimates depends upon the end use of the data. How much is spent on obtaining the data involves cost and benefit tradeoffs.

Only direct measurements at least as high as the hub height of the proposed machine wi11 be accurate enough to make a responsible assessment of the value of the resource, because of the extreme complexity of even very simple flows such as that discussed in the preceding section. Nevertheless, preliminary estimates of winds at or above hub height may be desirable without the expense of making direct measurements. Extrapolations of winds from lower and more easily intrumented heights to higher levels are one possible approach.

Two approaches to extrapolating the winds to higher levels are commonly used. The first, the log law, has its origins in the forefront of fluid mechanics and atmospheric research. It stems from a combination of theoretical and semi-empirical research. The second approach, used extensively by the wind engineering community, is the power law. The log law has the better claim to accuracy and physical justification. The strictly empirical power law is popular because of its simplicity in calculations, although attempts to incorporate new parameters into it to describe more complicated flow features result in substantial complexity also. Both approaches are subject to uncertainty caused by the variable, complicated nature of turbulent flows.

In this section we will discuss the log law, the power law, and the relation between the two approaches. As we describe the uses of the power law for 
extrapolation, the fundamental reasons for the inadequacy of all schemes for extrapolation, even over the most uniform sites, will become apparent.

\section{Log Law}

In the atmosphere there are a number of ways to arrive at a prediction of a logarithmic wind profile; e.g., mixing length theory, eddy viscosity theory, and similarity theory (Businger 1973). These approaches usually begin with the definition (assumption) of the surface layer where the fluxes of heat and momentum are constant with height and the effects of the Coriolis force are negligible. The Monin-Obukhov similarity theory (see Monin and Yaglom 1971) for example, states that, under all the restrictive assumptions that define flow in the surface layer, the only parameters available to govern the flow are: $g / T, u_{*}, \overline{w \theta^{\top}}$, and $z$. The only dimensionless group of these parameters is $z / L$, therefore dimension1ess quantities are hypothesized to be functions of $z / L$ only. As discussed in Section A.2.1, $z / L$ may be interpreted as a stability parameter where:

- $z / L<0$ is unstable

- $z / L=0$ is neutral stability

- $z / L>$ is stable.

One can define a dimensionless wind shear ${ }^{(a)}$ which must be a function of $\mathrm{z} / \mathrm{L}$ as:

$$
\frac{k z}{u_{\star}} \frac{\partial \bar{U}}{\partial z} \equiv \Phi_{m}(z / L)
$$

where $k=0.35$ is the Von Karman constant. This functional form has been verified by measurements and found to be (Businger et al. 1971):

$$
\begin{array}{ll}
\Phi_{m}=\left(1-15 \frac{z}{L}\right)^{-\frac{1}{4}} & \text { for } z / L \leq 0 \\
\Phi m=1+4.7 \frac{z}{L} & \text { for } z / L \geq 0 .
\end{array}
$$

(a) It should be emphasized that we are speaking of average wind speeds and wind shears where the average represents an average over 15 minutes to an hour. 
One way to measure the stability in the surface layer (a simpler but less accurate method is discussed under the power law section below) is to measure the Richardson number (Equation A-2.22) using two wind speed and two very sensitive temperature sensors or a thermocouple. The Richardson number is related to $\mathrm{z} / \mathrm{L}$ by:

$$
\begin{array}{ll}
R i=\frac{0.74 \frac{Z}{L}\left(1-15 \frac{Z}{L}\right)^{\frac{1}{4}}}{\left(1-9 \frac{Z}{L}\right)^{\frac{1}{2}}} & \text { for } \frac{Z}{L}<0 \\
R i=\frac{0.74 \frac{Z}{L}+4.7\left(\frac{Z}{L}\right)^{2}}{\left(1+4.7 \frac{Z}{L}\right)^{2}} & \text { for } \frac{z}{L}>0 .
\end{array}
$$

If one knows the Richardson number one can use Equations (A-2.28), (A-2.29), and $(\mathrm{A}-2.27)$ to determine $u$

Another approximate method to obtain $u_{*}$ uses bulk transfer techniques. One such "bulk transfer" or "resistance" law is due to Ziltinkevich and Deardorff (1974):

$$
\frac{u_{\star}}{u_{h}}=k\left\{\left[\ln \frac{h}{z_{0}}-b\right]^{2}+a^{2}\right\}^{-\frac{1}{2}}
$$

where $U_{h}$ is the wind speed just above the top of the PBL, which presumably may be estimated from a nearby rawinsonde or from the $850 \mathrm{mb}$ geopotential field. Here, $a$ and $b$ are functions of the stability parameter $h / L$. Resistance laws may also be modified to account for baroclinicity (Arya 1978). Curves of $a$ and $b$ have been derived from experimental data by Melgarejo and Deardorff (1974). These are shown in Figure A-2.2. Figure A-2.3 shows three curves from Equation $(A-2.34)$ as functions of $h / z_{0}$; for $h / L=10, h / L=0$, and $h / L=-10$. The dependence of $u_{*} / u_{h}$ is only very sensitive to $h$ for unstable cases over very rough surfaces or for very low boundary layer heights. Equation (A-2.32) provides a convenient way to illustrate the changes in the wind profile as boundary layer height changes or as stability changes, as will be seen below. 

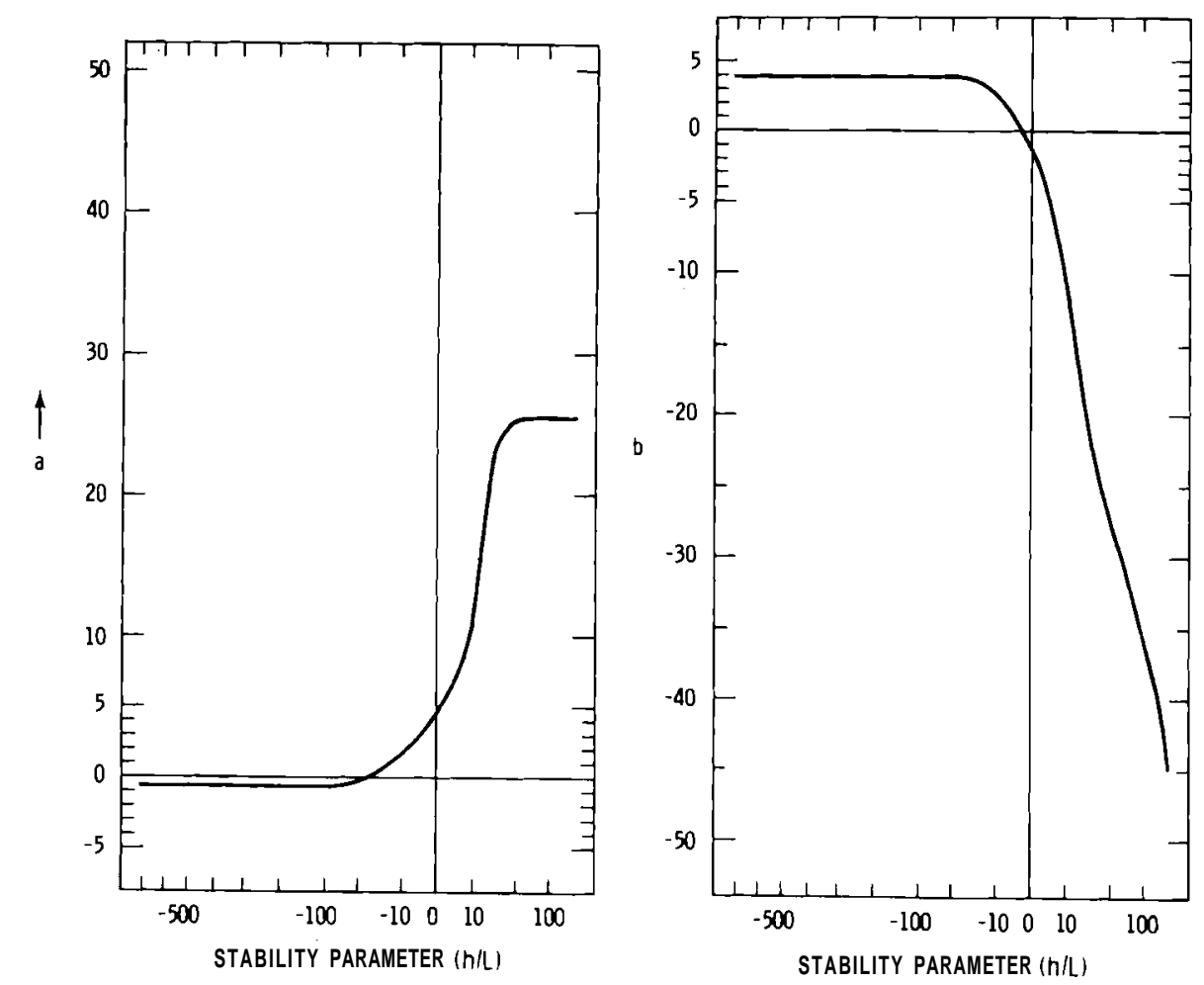

FIGURE A-2.3. Resistance Law Parameters $a$ and $b$ as Functions of the Stability Parameter ( $h / L)$

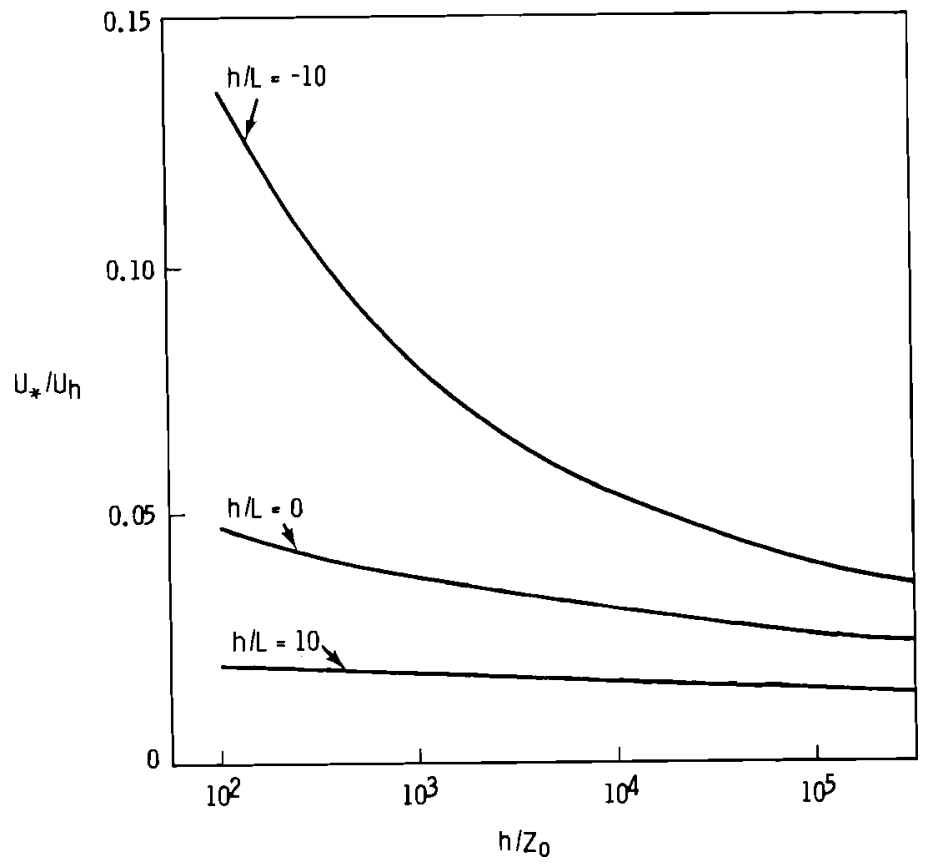

FIGURE A-2.4. Relation Between the Bulk Momentum Transfer Coefficient $\left(u_{*} / u_{h}\right)$ as a Function of the Stability Parameter $(h / L)$ and $h / z_{0}$. 
When $z / L=0$, Equation (A-2.27) is readily integrated to give the wind profile for neutral conditions as:

$$
\bar{U}=\frac{u_{x}}{k} \ln \left(\frac{z+z_{0}}{z_{0}}\right)
$$

where $z_{0}$ is the roughness length. Above the canopy of displacement height (D) :

$$
\bar{U}=\frac{u_{t}}{k} \ln \left(\frac{z-D+z_{0}}{z_{0}}\right) .
$$

If $z>z_{0}, z_{0}$ in the numerator of Equation $(A-2.33)$ and $(A-2.34)$ may be dropped.

Paul son (1970) integrated Equation (A-2.27) using Equations (A-2.28) and (A-2.29) to get the surface layer wind profiles over the entire range of stability. These profiles are:

$$
\bar{U}=\frac{u_{*}}{k}\left(\ln \frac{z}{z_{0}}-\Psi_{1}\right) \text { for } \frac{z}{L}<0
$$

where:

- $\psi_{1}=2 \ln [(1+x) / 2]+\ln \left[\left(1+x^{2}\right) / 2\right]-2 \tan ^{-1}(x)+\pi / 2$

- $x=\left(1-15 \frac{z}{L}\right)^{1 / 4}$

and

$$
U=\frac{u_{*}}{k}\left(\ln \frac{z}{z_{0}}+4.7 \frac{z}{L}\right) \text { for } \frac{z}{L}>0 .
$$

Equations (A-2.32) and (A-2.33) are theoretically only valid in the surface layer. While theoretically matching the surface-layer log law with outer PBL velocity profiles. Tennekes (1973a, b) showed that the matching layer must also obey a log law. As a result, the log law may be applied through about the first $150 \mathrm{~m}$ of the neutral PBL. Panofsky (1973) presents data that show that the Monin-Obukhov similarity theory is applicable to at least $150 \mathrm{~m}$ in the unstable PBL as well. However, Wyngaard (1973) points out the possibility that variations in the PBL height $h$ may influence $\Phi_{m}$. Businger 
and Arya (1974) argue that in a continuously turbulent stable boundary layer, Equation (A-2.33) will hold for the component of wind parallel to the surface wind (there will be clockwise turning of wind with height in the stable boundary layer) through at least $15 \%$ of the boundary layer.

Figure A-2.4 shows three wind profiles: one for a stable, a neutral, and an unstable boundary layer. Equations (A-2.33), (A-2.35) and (A-2.36) were used. The same roughness, PBL height, and wind speed at the top of the PBL have been assumed for each profile. The resistance law plotted in Figure A-2.3 was used to obtain the appropriate $u_{\star}$ for each profile. The PBL height used is probably too large for a stable PBL but Figure A-2.3 shows that $u$ is not very sensitive to $\mathrm{h}$ in the stable case. It is also unlikely that a case as windy as this could be as unstable as $h / L=-10$. From the Obukhov length and \. the heat flux can be estimated at 900 watts $/ \mathrm{m}^{2}$ or $65 \%$ of the solar constant, which is extremely large. Therefore the unstable profile of Figure A.2-4 is viewed as a limiting case.

Figure A-2.4 does demonstrate that in an unstable atmosphere high momentum air is mixed down near the surface. The strong coupling of all layers causes the wind shear over most of the WECS rotor disk to be least for an unstable boundary layer, although the wind shear is significant very near the ground. In the stable case the wind shear is almost constant over the WECS rotor disk, thereby causing near-surface winds to be substantially weaker than those higher up. In addition, in the stable case there is usually some turning of the wind with height.

Figure A-2.5 describes the effects of surface roughness on the neutral stability wind profile. Wind speeds at 15,65 , and $115 \mathrm{~m}$ are shown as a function of $z_{0}$, assuming $U_{h}=20 \mathrm{~m} / \mathrm{sec}$ and using $u_{*}$ obtained from Figure A-2.3. The advantage to siting wind turbines in smooth terrain is clear.

In summary, there are four main points to remember concerning the log law. First, Equations $(\mathrm{A}-2.33),(\mathrm{A}-2.35)$ and $(\mathrm{A}-2.36)$ are accepted as the best description of the wind profile in the surface layer over uniform flat terrain because of their theoretical foundation and empirical confirmation. 
Second, those equations, or any other equations describing a wind profile based on near surface measurements or theory, are suspect when applied above $30 \mathrm{~m}$. However, evidence exists to suggest that the log law equations may be used through 10 to $15 \%$ of the boundary layer under some restrictive conditions. Third, with measurements of the Richardson number, Equations (A-2.30) and (A-2.31) may be used, in principle, to measure $u_{\star}$ via Equations (A-2.27) and $(A-2.28)$, and then to obtain $z_{0}$ using Equations (A-2.33), (A-2.35) and (A-2.36). Finally, with the use of a bulk transfer or resistance law such as Equation (A-2.32), the response of the boundary layer to varying conditions beneath a constant geostrophic wind heuristically may be examined.

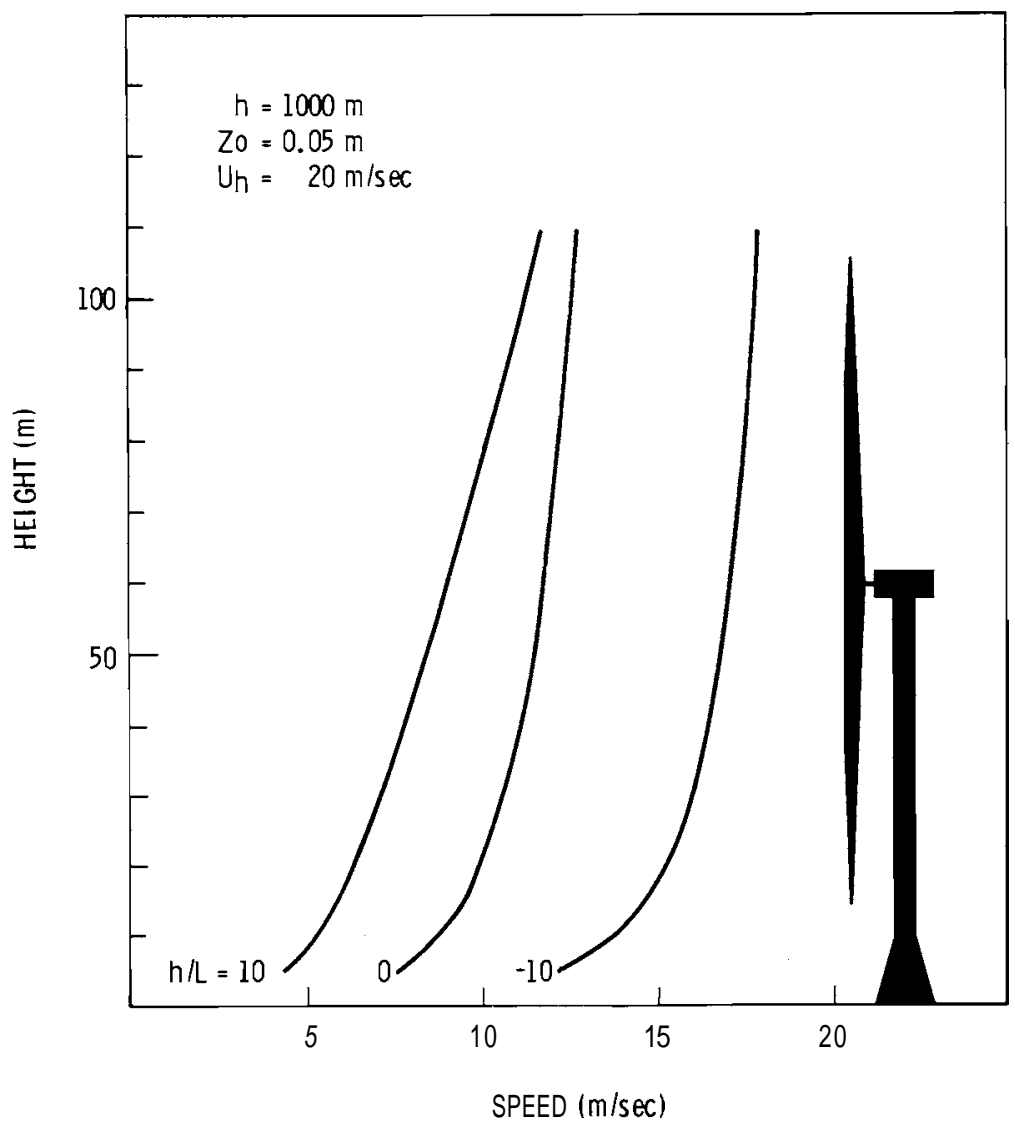

FIGURE A-2.4. Stable, Neutral and Unstable Wind Profiles for a Roughness Typical of High Grass and a Wind Speed at the Top of a $1-\mathrm{km}$ Deep Boundary Layer of $20 \mathrm{~m} / \mathrm{sec}$ 


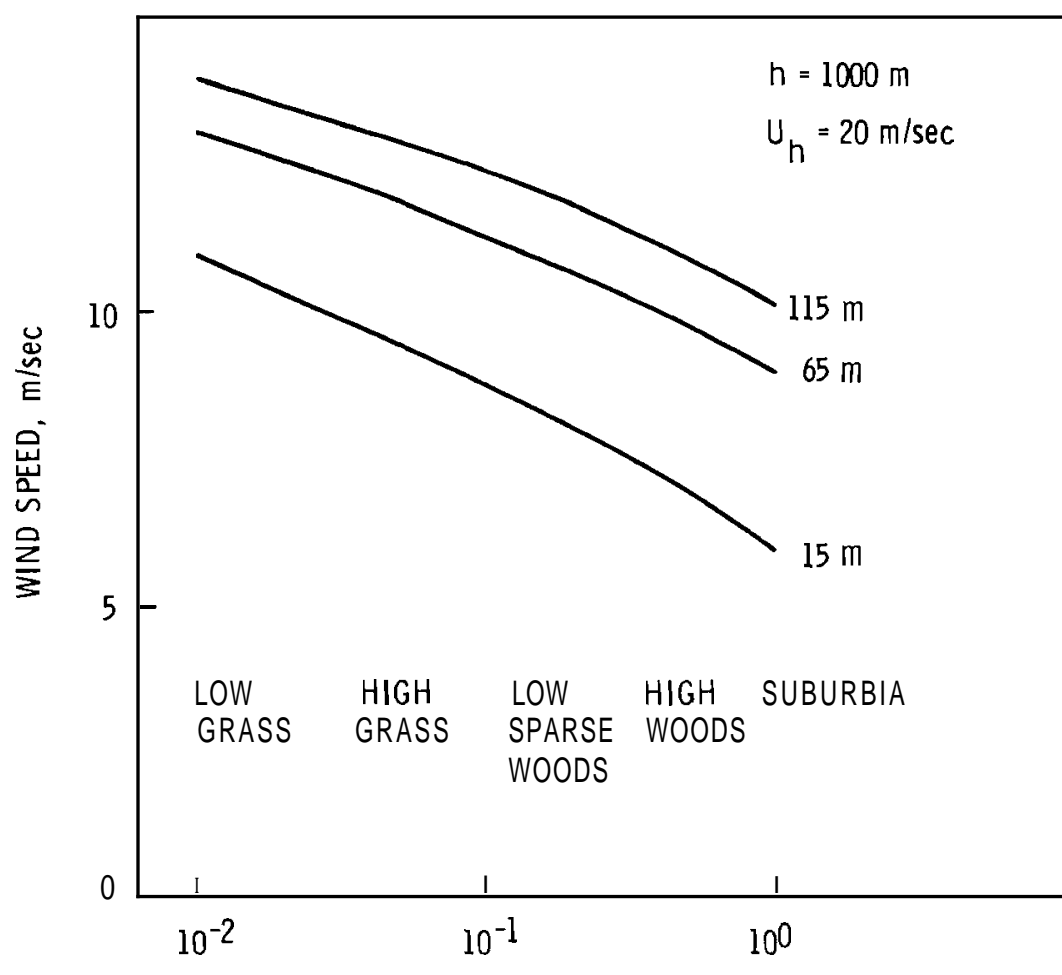

Zo. M

FIGURE A-2.5. Wind Speeds Near the Bottom and Top of a Large WECS Rotor Disk, and Near Hub Height Versus Roughness. A wind speed of $20 \mathrm{~m} / \mathrm{sec}$ above a $1-\mathrm{km}$ deep boundary layer was assumed.

\section{Power Law}

An alternative description of the wind profile is the power law. In its most general form it is:

$$
\frac{U_{2}}{U_{1}}=\left(\frac{z_{2}}{z_{1}}\right)^{\alpha}
$$

where

$$
\begin{aligned}
& \mathrm{U}_{2}=\text { wind speed at height } \mathrm{z}_{2} \\
& \mathrm{U}_{1}=\text { wind speed at height } \mathrm{z}_{1} \\
& \mathrm{a}=\text { power law exponent. }
\end{aligned}
$$


The exponent $a$ is the key parameter that determines the shape of the wind profile. One hopes that $a$ is a simple function so that the simplicity of Equation (A-2.37) is preserved.

The power law has not proven to be a simple parameter. Early assumptions or hopes that a is a constant for all sites have been discarded, unless the use of a more complicated function is not justified by the accuracy of the input data or the required accuracy of the extrapolated wind speed. Several investigators report or employ $a$ as a decreasing function of wind speed (Justus and M khail 1976, Fichtl and Smith 1977, American Society of Civil Engineers 1961 and Fales 1961). Spera and Richards (1979) have proposed a model of a that depends on roughness and wind speed.

There is no question that the log law expressed by Equations (A-2.33), $(A-2.35)$, and (A-2.36) are the most accurate description of the wind profile in the surface layer over uniform terrain and, with due caution, that those equations are usable through 10-15\% of the boundary layer. Beyond that height, even in the boundary layer over uniform terrain, there are too many parameters to describe the wind profile accurately in a tractable way with that or any other set of equations.

The log law and the power law may be equated to see how the power law exponent should vary over flat terrain. This is done by using the log law equations at 2 heights $z_{1}$, and $z_{2}$, equating their quotient to Equation (A-2.37), and solving for a. The results are:

$$
\begin{array}{ll}
\alpha=\ln \left\{\frac{\ln \left(z_{2} / z_{0}\right)}{\ln \left(z_{1} / z_{0}\right)}\right\} & \text { for } \frac{z}{L}=0 \\
\alpha=\ln \left(z_{2} / z_{1}\right) & \text { for } \frac{Z}{L}>0 \\
\alpha=\ln \left(\frac{\ln \left(z_{2} / z_{1}\right)+4.7 z_{2} / L}{\ln \left(z_{1} / z_{0}\right)+4.7 z_{1} / L}\right\} & \ln \left(z_{2} / z_{0}\right)-\psi\left(z_{2} / L\right) \\
\frac{\ln \left(z_{1} / z_{0}\right)-\psi\left(z_{1} / L\right)}{\ln \left(z_{2} / z_{1}\right)} & \text { for } \frac{z}{L}<0
\end{array}
$$


where

$$
\begin{aligned}
\psi\left(\frac{Z}{L}\right) & =2 \ln [(1+x) / 2]+\ln \left[\left(1+x^{2}\right) / 2\right]-2 \tan ^{-1}(x)+\pi / 2 \\
x & =\left(1-15 \frac{Z}{L}\right)^{-1 / 4}
\end{aligned}
$$

Therefore, a is a function of $z_{2}, z_{1}, z_{0}$, and $L$; it is not a function of wind speed. From this parameter $\mathrm{I}$ is t alone, it is apparent that the wind speed dependence reported is a reflection of the variation in stability. This will be discussed further after the sensitivity of a to other parameters is examined.

The sensitivity of a to the parameters affecting it determines, in turn, the sensitivity of the extrapolation to some greater height. Differentiate Equation (A-2.37) to determine the sensitivity of the extrapolation to variations in a. This results in:

$$
\frac{\delta u_{2}}{u_{2}} \sim \operatorname{In} \frac{z_{2}}{z_{1}} \delta \alpha .
$$

So if one wishes to extrapolate a $10 \mathrm{~m}$ wind speed measurement to $60 \mathrm{~m}$ within $\pm 10 \%$, a must be known to within \pm 0.06 .

Figure A-2.6 shows a as a function of $z_{2}$ for three stability groups, three values of the reference height $z_{,}$, and for $z_{0}=0.05 \mathrm{~m}$. For neutral and unstable conditions, extrapolations to hub heights above $30 \mathrm{~m}$ may be well approximated by an a based on $60 \mathrm{~m}\left(\alpha_{60}\right)$. There is somewhat more sensitivity to the choice of a reference height. For stable conditions, systematic errors of $5-10 \%$ could easily occur if the variation of a with $z_{2}$ and $z_{1}$ are ignored.

The variation of a to $z_{0}$ and $L$ will be considered next, but first consider the Pasquill stability category scheme (Pasquill 1974) which is frequently used as an easy way to estimate the stability with limited information.

(a) We will use $\alpha_{\left(z 2, z_{1}\right)}$ to indicate the levels at which the power law exponent applies. When $z_{1}$ is absent, $z_{1}=10 \mathrm{~m}$ is assumed. 
Pasquill categories are determined from estimates of:

- the wind speed at $10 \mathrm{~m}$

- daytime insolation and cloud cover

- nighttime radiation and cloud cover.

The Pasquil1 stability system neglects temperature advection. This is an omission of convenience but is serious for realistic flows. Figure A-2.7 summarizes the Pasquill categories: $A$ is the most unstable and $F$ the most stable (Turner 1970). Figure A-2.8 relates these stability groups and the roughness length to the Obukhov length (Golder 1972).

Figure A-2.9 shows the dependence of a on roughness length and the Obukhov length, for an extrapolation from $10 \mathrm{~m}$ to $60 \mathrm{~m}$. It is clear that a is a very weak function of stability when there is even a slight upward heat flux, but when conditions are stable, a becomes a very strong function of stability. The power law coefficient increases with roughness length, and increases with stability most quickly over the rougher surfaces.

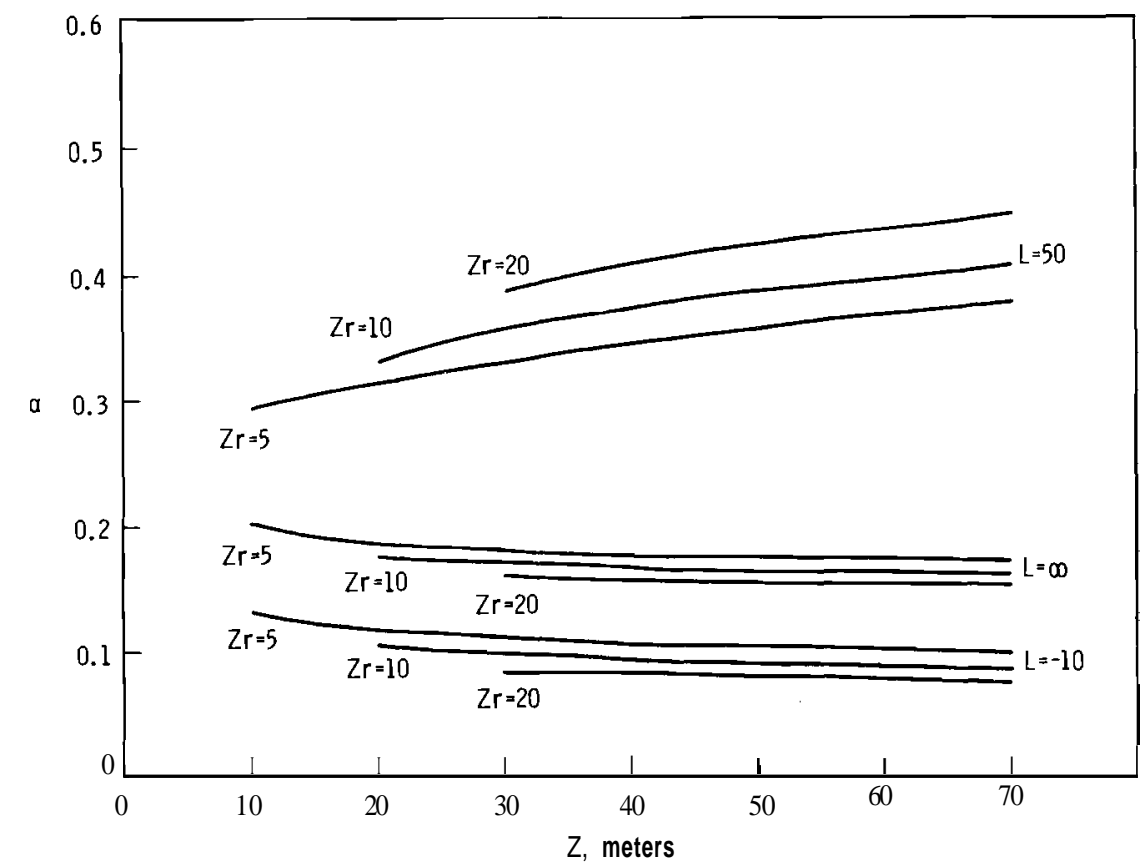

FIGURE A-2.6. The Power Law Coefficient as a Function of Height for Different Stability and Reference Height groups 


\begin{tabular}{|c|c|c|c|c|c|c|c|c|c|}
\hline \multirow{2}{*}{$\begin{array}{l}\text { SOLAR } \\
\text { ALTITUDE } \\
\text { (DEGREES) }\end{array}$} & \multirow{2}{*}{$\begin{array}{l}\text { CLOUD } \\
\text { COVER }\end{array}$} & \multirow{2}{*}{$\begin{array}{c}\text { CEILING } \\
\text { (KM) }\end{array}$} & \multicolumn{7}{|c|}{ 10M WIND SPEED (M/SEC) } \\
\hline & & & 1 & 2 & 3 & 4 & 5 & 6 & $>6$ \\
\hline \multirow{7}{*}{$>60$} & $\leq 0.5$ & & A) & $\bar{A}$ & $A$ & $B$ & $B$ & $\mathrm{c}$ & $c$ \\
\hline & & $>5$ & As & A) & $A$ & B & $B$ & $c:$ & $c$ \\
\hline & $>0.5$ & $<5$ & A) & 6 & B & $B$ & $c$ & $\mathrm{C}$ & $D$ \\
\hline & & $<2$ & $B^{\prime}$ & (a) & $c$ & $\mathrm{C}$ & $c$ & D & D \\
\hline & & $>5$ & $A$ & & $B$ & $B$ & 0 & 6 & D \\
\hline & 1.0 & $<5$ & B & 8 & $c$ & $c$ & $C$ & D & D \\
\hline & & $<2$ & D & D & D & D & $D$ & $D$ & D \\
\hline \multirow{7}{*}{$35-60$} & $\leq 0.5$ & & A) & $B^{\prime}$ & $B$ & B & $c$ & $c$ & D \\
\hline & & $>5$ & A & & $B$ & $B$ & $c$ & $\mathrm{c}$ & D \\
\hline & $>0.5$ & $<5$ & B & B & $c$ & $\mathrm{c}$ & $\mathrm{Cl}$ & D & D \\
\hline & & $<2$ & $c$ & $\mathrm{C}$ & D & D & D & D & D \\
\hline & & $>5$ & B) & $B$ & $C$ & $\mathrm{C}$ & 0 & D & D \\
\hline & 1.0 & $<5$ & $c$ & $c$ & D & D & D & D & D \\
\hline & & $<2$ & D & D & D & D & D & D & D \\
\hline \multirow{7}{*}{$15-35$} & $\leq 0.5$ & & $\mathrm{~B}^{\prime}$ & $B$ & $\mathrm{Cl}$ & $\mathrm{c}$ & $c$ & $D$ & D \\
\hline & & $>5$ & $B$ & $B$ & $c$ & $\mathrm{CO}$ & $\mathrm{c}$ & D & D \\
\hline & $>0.5$ & $<5$ & $c$ & $c$ & D & D & D & D & D \\
\hline & & $<2$ & $\mathrm{c}$ & $c$ & D & D & D & D & D \\
\hline & & $>5$ & $\mathrm{c}$ & $\mathrm{c}$ & D & D & D & D & D \\
\hline & 1.0 & $<5$ & $\mathrm{C}$ & $\mathrm{C}$ & D & D] & D & D & D \\
\hline & & $<2$ & D & D & D & D & $D$ & $D$ & D \\
\hline \multirow{7}{*}{$<15$} & $\leq 0.5$ & & $c$ & $c$ & $D$ & $D$ & D & $D$ & $D$ \\
\hline & & $>5$ & $\mathrm{c}$ & $\mathrm{C}$ & D & D & D & D & D \\
\hline & $>0.5$ & $<5$ & $\mathrm{C}$ & $\mathrm{C}$ & D & D & D & D & $D$ \\
\hline & & $<2$ & $\mathrm{C}$ & $\mathrm{C}$ & D & D & D & D & D \\
\hline & & $>5$ & $c$ & $c$ & D & $D$ & D & D & D \\
\hline & 1.0 & $<5$ & $\mathrm{c}$ & $c$ & $D$ & D & D & D & $D$ \\
\hline & & $<2$ & D & D & D & D & D & D & D \\
\hline \multirow{2}{*}{ NIGHT } & $>0.5$ & & $\vec{F}$ & $F$ & $E$ & E,D & $D$ & D & D \\
\hline & $<0.5$ & & $\mathrm{~F}$ & $F$ & $\mathrm{~F}$ & $F, E$ & & $E, D$ & D \\
\hline
\end{tabular}

FIGURE A-2.7. The Pasquil1 Stability Groups (Turner 1970) 


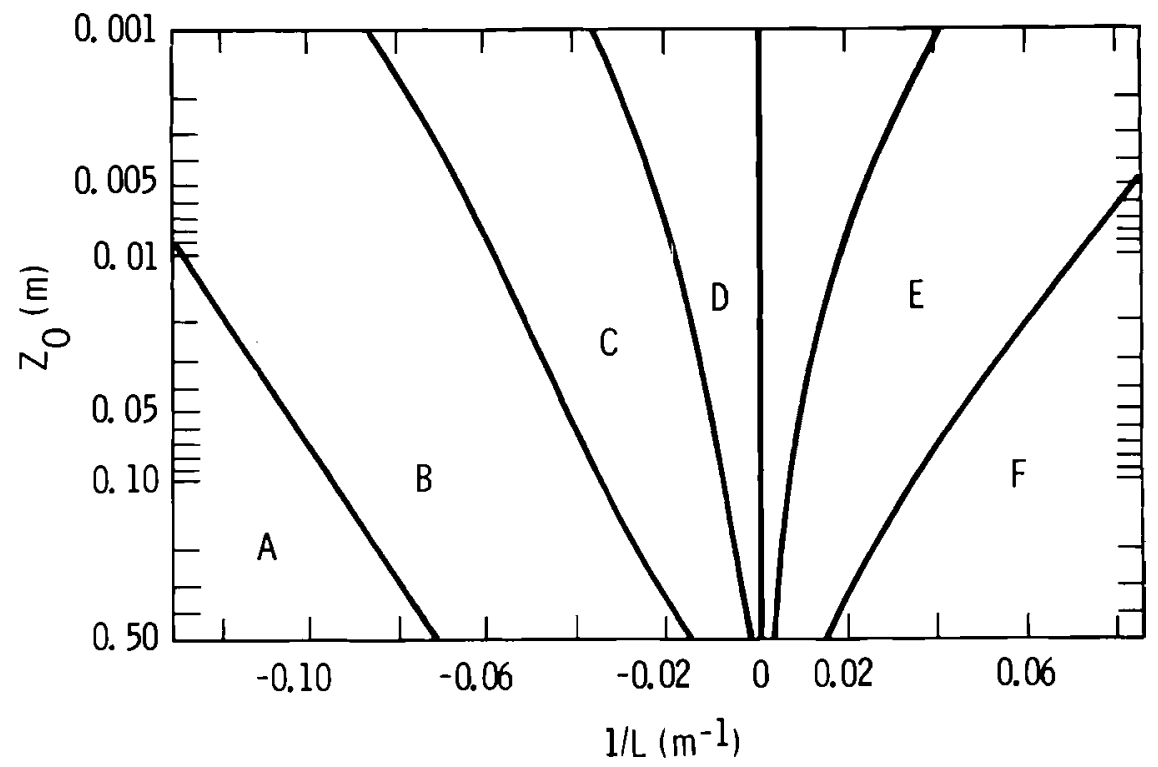

FIGURE A-2.8. Obukhov Length Related to Pasquil1 Stability Category and Roughness Length (Golder 1972)

It is evident why many investigators see a as a decreasing function of wind speed. Over most flat land surfaces, daytime heating causes unstable conditions, which mix high momentum air from higher levels in the PBL down to near the surface; the daytime surface winds are maximum when a is at a minimum. Conversely at night the near-surface wind speed minimum is associated with a larger a and stable stratification. This behavior may not hold true in complex terrain.

Figure A-2.9 provides a convenient way to look up the accuracy of a given extrapolation using Equation (A-2.41), or alternatively, to determine the accuracy with which the roughness length and Obukhov length must be known. For example, if it is believed that $0.05 \leq z_{0}(\mathrm{~m}) \leq 0.15$ and that $1000 \geq \mathrm{L}(\mathrm{m})$ $\geq 200$ then $0.18 \leq \alpha_{60} \leq 0.24$, which by Equation (A-2.41) implies that the extrapolation is good to about $\pm 5 \%$.

The Pasquill stability determination scheme is inadequate for determining the stability to use in extrapolations. The Pasquill groups are drawn into Figure A-2.9 using Figure A-2.8. The near neutral group encompasses a variation in a of nearly 0.2 for flow over high grass. Are there other ways to determine L, short of measuring the Richardson number? 


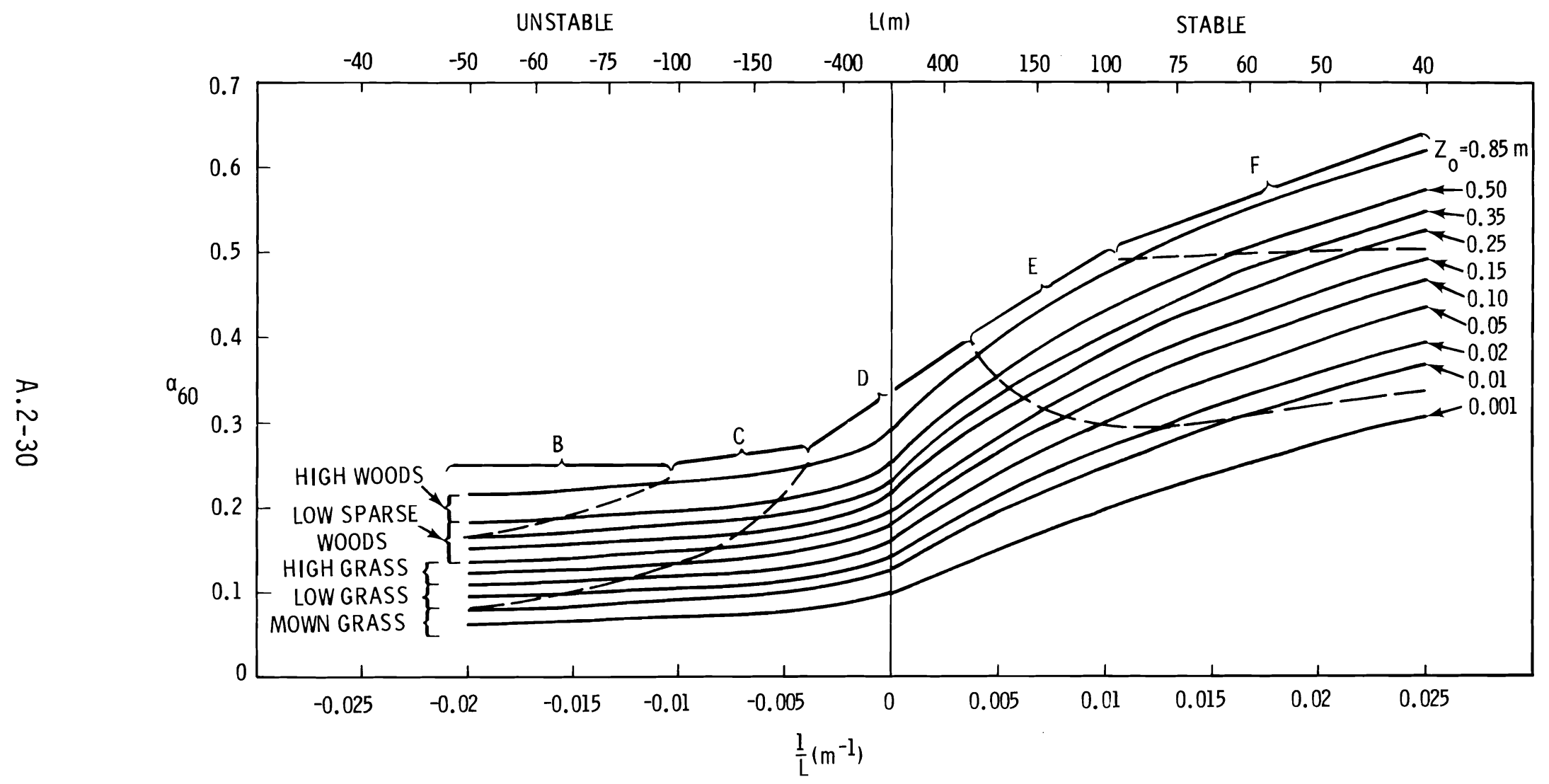

FIGURE A-2.9. The Power Low Costficient fior $z_{2}=50 \mathrm{~m}$ and $z_{1}=10 \mathrm{~m}$ Versus Obukhov Length $(L)$ and Rowghnsss $L \frac{1}{2}$ ngth $\left(z_{0} \mid\right.$. The Pasquill stability groups are shown as regions betwe in dashes curves. 
If wind speed measurements are available at two levels, e.g, 10 and $20 \mathrm{~m}$ or 5 and $10 \mathrm{~m}$, and if $z_{0}$ is known, the $\log$ law equations can be used in principle to relate $u_{2} / u_{1}$ to $L$. Figure A-2.10 is similar to Figure A-2.9 except the ordinate is in terms of $u_{2} / u_{1}$ instead of $a$, and $z_{2}=10$ or $20 \mathrm{~m}$ and $z_{1}=5$ or $10 \mathrm{~m}$. (a) Consider, though, a given anemometer system that is accurate to $\pm 2 \%$. Then $u_{2} / u_{1}$ is known to within $\pm 4 \%$. If, for example, $u_{20} / u_{10}=1.2$ over terrain where $z_{0}$ is known to be equal to $0.25 \mathrm{~m}$, then one can only estimate the Obukhov length to be $L>150 \mathrm{~m}$ or $\mathrm{L}<-150 \mathrm{~m}$. Compare this with Figure A-2.9 and note that this method proves no better than the Pasquil1 method.

Compounding these difficulties are uncertainties in the value of $z_{0}$. Figure A-2.10 shows that if $z_{0}$ is not known fairly closely, there are substantial systematic bias errors introduced in the estimate of the stability, which feed through to become errors in the extrapolation. The roughness length can be determined more accurately by using multiple levels of wind speed and temperature measurements, but this requires a level of sophistication beyond that assumed for routine siting programs.

These conclusions can be drawn about extrapolations of wind speeds from 5,10 , or $20 \mathrm{~m}$ to hub height in order to obtain a time series of hub height wind speeds sufficiently accurate for responsible approximations of wind turbine output. Extrapolations are possible if it is certain that the heat flux is upward. This is not determinable using either the Pasquill stability groups or two anemometers if conditions are unstable but near neutral. Accurate determination of stability is only possible using sensitive wind and temperature instrumentation. During stable conditions, no accurate time-series extrapolation is feasible, even over the simplest terrain. Extrapolations will be inaccurate over any terrain in which the power-law coefficient is a rapidly varying function of some parameter, e.g., stability or wind direction over a ridge. The implications of this inadequacy of short-term wind extrapolations extend also to short-term wind profile measurement programs. A series of wind profiles obtained with tethered balloon systems or kites cannot be

(a) Figure A-2.9 could also have been presented in terms of $u_{2} / u_{7}$ instead of $a$, but $u_{2} / u_{1}$ is a stronger function of $z_{2}$ and $z_{1}$ than is a; therefore a is more convenient. 


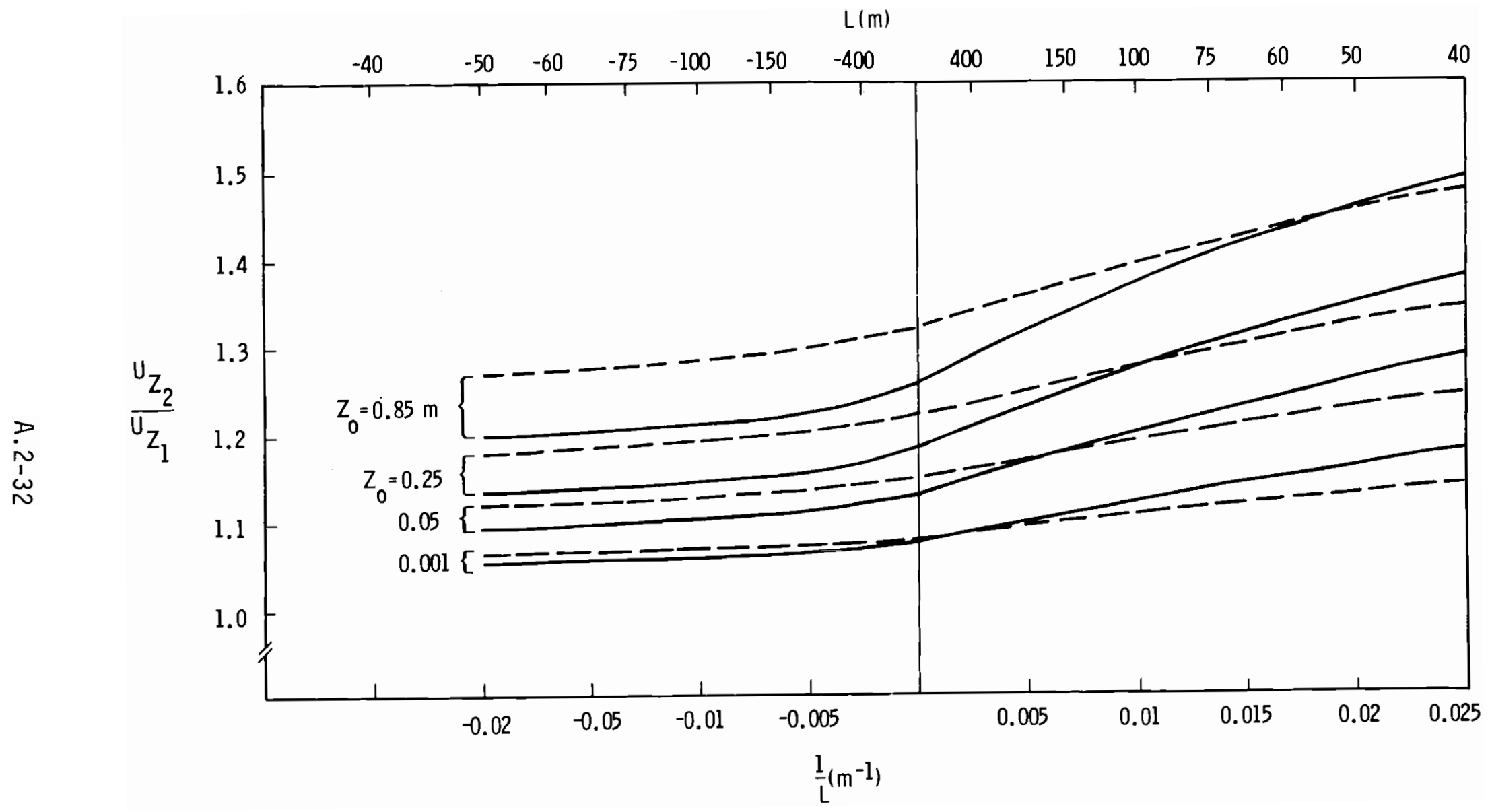

FIGURE A-2.10. Ratio of Winds at 2 Heights Versus

Obukhov Length $(L)$ and Roughness Length $\left(z_{0}\right)$ 
regarded as having any climatological significance, nor can a site-specific wind extrapolation scheme be devised from those measurements. Direct measurements of winds at hub height are the only possible method for obtaining reasonably accurate hub-height wind-speed time series.

We might also consider the problem of extrapolations on longer term wind averages such as annual average wind speeds or the average wind speed for a given hour of the day for a given season. An error analysis of these estimates will not be simple because many of the statistics that enter the problem will be non-Gaussian; in part due to the non-linear relation between a and $L$. There is at least a simple limiting answer to the question of whether such extrapolations are useful. If it takes a full season of wind speed measurements to come within $10 \%$ of the seasonal mean wind speed with $90 \%$ confidence (Corotis et al. 1977) then an extrapolation to the hub height annual average will certainly not improve upon that accuracy. Since the extrapolations are expected to be sensitive to the site-specific wind regime, it is also as yet very unclear whether empirical evidence gathered from existing tall towers will shed much light on the confidence with which long-term average wind speed extrapolations can be made at a new site.

\section{Summary}

In this section the logarithmic wind profile for neutral conditions and its variations for stable and unstable conditions were presented. These profiles given by Equations $(A-2.33),(A-2.35)$ and $(A-2.36)$ are the accepted descriptions of the wind structure in the lowest $30 \mathrm{~m}$ of the boundary layer. Under some conditions they may be applied through $10-15 \%$ of the boundary layer. A bulk transfer relation, Equation (A-2.32) was presented so that the reader can investigate for himself the variations of wind profile structure as various boundary layer parameters change underneath a constant geostrophic wind speed above the PBL. The power law, which is a convenient description of the wind profile used by the wind engineering community was compared to the log law. From this comparison, the problems of extrapolating near-surface wind speeds to hub height were considered. Even over the simplest terrain, a time series of hub-height winds sufficiently accurate for assessing the performance of wind turbines cannot be obtained. Direct measurements are necessary. 
Short term wind profile measurements are meaningless for determining a wind profile climatology. The accuracy of extrapolating long-term average wind speeds to hub height were not considered; however the accuracy of these estimates is certainly worse than the accuracy of the estimates of the near-surface measurements used in the extrapolation.

\section{A.2.2.3 Turbulence Characteristics}

Wind turbines operate in a turbulent environment; therefore the characteristics of the turbulence that they interact with are of interest. Most questions of wind turbine and turbulence interaction are of importance to design engineers only. However, people involved in site selection for WECS need to be cognizant of the behavior of turbulence behavior too. Excessive turbulence may exceed design specifications of a given wind turbine. The operating strategies of a large wind turbine work on time scales that coincide with time scales of large turbulence eddies or gusts. And finally, the nature of turbulence dictates in part the usefulness of short-term measurements.

This section provides a brief summary of the magnitude of turbulence wind fluctuations over uniform terrain and the spectral distribution of those fluctuations. The summary serves as a ballpark indication of the turbulence levels to expect in simple flows, and a benchmark to use in understanding the departures from ideal characteristics that occur in more complicated flows. Most of the information presented here is derived from surface-layer measurements and therefore provides only a rough guideline for the behavior from 30 to $100 \mathrm{~m}$.

Turbulence Intensity

The amount of turbulent kinetic energy per unit mass in a unit volume (e) is just one half the sum of the variances of the three wind components, or:

$$
\mathrm{e}=1 / 2\left(\sigma_{u}^{2}+\sigma_{v}^{2}+\sigma_{w}^{2}\right) \text {. }
$$

The variance of the vertical velocity is not very important for the site selection problem, except that as $\sigma_{u}$ becomes large, the vertical momentum transfer becomes greater. This implies that $\sigma_{u}$ normalized by the friction 
velocity should be a universal function of the governing parameters. In the unstable surface layer the observations fit the following form (Panofsky et al. 1977) :

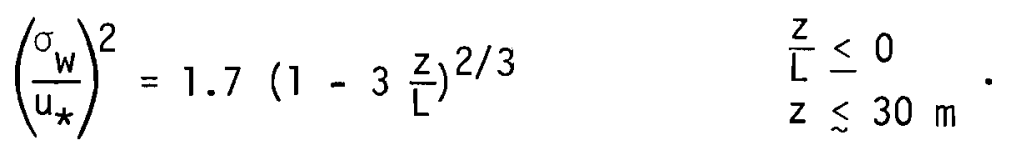

This is only good for the surface layer; it overestimates $\sigma_{\mathrm{w}}$ if $z \geq 0.1 \mathrm{~h}$. A form that is useful under unstable conditions above the surface layer is (Hiester 1977):

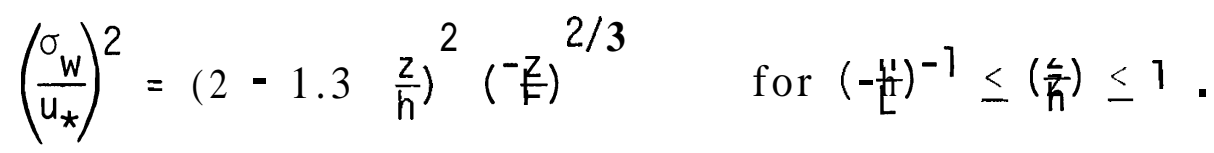

Measurements are sparse in the continuously stable PBL and very rare in the sporadically turbulent PBL. In the continuously turbulent PBL we can only crudely estimate that the near-surface values of $\sigma_{w}{ }^{2}$ are roughly equal to the neutral stability value and that $\sigma_{w}$ decreases to 0 at $z=h$, or:

$$
\left(\frac{\sigma_{w}}{u_{*}}\right)^{2} \approx 1.7\left(1-\frac{z}{h}\right) \quad \text { for } \frac{z}{L} \geq 0
$$

In the stable PBL for $z>L$ where the turbulence is sporadic, internal waves are responsible for variances of $\mathrm{u}, \mathrm{v}$, and $\mathrm{w}$, although very little turbulent transport of heat or momentum occurs. We can only indicate that under these conditions $\sigma_{\mathrm{W}}<\sigma_{\mathrm{V}}<\sigma_{\mathrm{U}}$.

Descriptions of the horizontal velocity variances are less universal than the descriptions of the vertical velocity variances. This is partly a result of the difficulty in handling the averaging and filtering of the data and because of the spectrum of the horizontal variances which is influenced by the boundary layer height, even in the unstable surface layer. For the unstable surface layer Panofsky et al. (1977) present data that shows both components of the horizontal velocity variances may be described by: 


$$
\left(\frac{\sigma_{u}}{u_{*}}\right)^{2} \approx\left(\frac{\sigma_{v}}{u_{*}}\right)^{2} \approx\left(12-0.5 \frac{h}{L}\right)^{2 / 3} \quad \text { for } \frac{h}{L}<0 .
$$

There is evidence to suggest however that the variances decrease with height in the lower mixed layer (Deardorff 1974). Some data show the constant, 12, decreases linearly with height to about 8.6 at $z=0.15 \mathrm{~h}$, then decreases more slowly so that $\sigma_{u}$ and $\sigma_{v}$ are approximately constant with height in the upper mixed layer (Hiester 1977).

Equation (A-2.46) shows $\sigma_{u} \approx \sigma_{v}$ which is true under very unstable conditions where these variances are produced primarily by transfer of energy from the buoyancy-produced $\sigma_{w}$. However, for near-neutral and stable conditions, shear production of TKE goes directly into the $\sigma_{u}$ (where the $x$ coordinate axis is parallel to the mean wind) and then some of this is transferred to the $v$ and w components. Therefore, near-neutral $\sigma_{u}$ is larger than $\sigma_{v}$. The best evidence for neutral conditions is summarized by Panofsky et al. (1979) which shows:

$$
\begin{array}{ll}
\left(\frac{\sigma_{u}}{u_{*}}\right)^{2}=5.8 & \text { for } \frac{z}{L}=0 \\
\left(\frac{\sigma_{v}}{u_{*}}\right)^{2}=3.6 & \text { for } \frac{z}{L}=0 .
\end{array}
$$

For stable conditions we can again only roughly say that the variances decrease with height as:

$$
\begin{array}{ll}
\left(\frac{\sigma_{u}}{u_{*}}\right)^{2} \approx 5.8\left(1-\frac{z}{L}\right) & \text { for } \frac{z}{L}>0 \\
\left(\frac{\sigma_{v}}{u_{*}}\right)^{2} \approx 3.6\left(1-\frac{z}{h}\right) & \text { for } \frac{z}{L}>0 .
\end{array}
$$

Any of these equations of the normalized variances may be related to mean wind speed using the log-law equations, Equations (A-2.33), (A-2.35), and (A-2.36) 
to relate $u_{\star}$ and $\bar{U}$. For example, Equations (A-2.47) and (A-2.48) for neutral conditions become:

$$
\begin{array}{ll}
\frac{\sigma_{u}}{\bar{U}}=\frac{0.84}{\ln z / z_{0}} & \text { for } \frac{Z}{L}=0 \\
\frac{\sigma_{v}}{U}=\frac{0.67}{\ln z / z_{0}} & \text { for } \frac{z}{L}=0 .
\end{array}
$$

\section{Turbulence Spectra}

The formulas listed above that quantify the magnitude of the wind component variances represent, for example, the variances of a l-hr time series of the fluctuations measured at a single point. The total variance represents a sum of the contributions to the variance by fluctuations of an entire spectrum of frequencies. When the velocities of turbulence fluctuations are small compared to the mean wind speed, the turbulence structure is advected along by the mean wind speed without substantial change. Then the Taylor hypothesis or "frozen turbulence approximation" may be used to view the turbulence spatially in terms of wavenumbers and wavelengths instead of frequencies and periods. The variances represent then a summation of contributions of different wavelengths. A spectrum is normally derived from the time series recorded at a single anemometer. The spectral energy density gives an estimate of the mean amplitude of fluctuations near a particular frequency or wavenumber.

Strictly speaking these are one-dimensional longitudinal spectra and one must be cautious when attempting to decipher the structure of the threedimensional flow from a one-dimensional spectrum. For example, waves of wavelength $\lambda$ approaching a beach appear to have a wavelength of $1.41 \lambda$ if one views the wave motion at a $45^{\circ}$ angle from the beach. The same aliasing occurs in the boundary layer; smaller eddies can show up at longer wavelengths in the spectrum. This is not a problem at the high wavenumber end of the spectrum. For wavelengths very much larger than the rotor diameter of the turbine we can assume that the disturbance is affecting the entire wind turbine; i.e., a low frequency gust or wind direction oscillation. One should keep in mind, however, 
that for large WECS, some of the energy that appears in the one-dimensional spectrum at wavelengths that appear to be larger than the rotor diameter may be associated with three-dimensional eddies with sizes that are about the same size as the rotor. Eddies that are the same size as the rotor will create the largest component of the fluctuating wind shear seen by the blade. (For a more detailed discussion of the spectral properties of turbulence see Tennekes and Lumley 1972).

It is not simple to obtain turbulence spectra in geophysical flows. The point of examining the spectrum is to study the fluctuations caused by turbulence and ignore the longer term fluctuations associated with the diurnal cycle or changing weather. In some turbulent flows over uniform terrain there is a spectral gap at periods of about an hour where there is very little energy. This is the fundamental reason for averaging over periods of about an hour; most of the turbulence is sampled but the longer term, externally forced variations are screened out. In most realistic flows, however, there is a significant amount of energy in and around the frequency where the spectral gap is supposed to be, especially for the u and v component variances. These low frequency oscillations arise from nonturbulent secondary circulations within the PBL, from remnants of distant mesoscale weather disturbances, or from horizontal shear production of $u$ and $v$ variance by interaction of the flow and the topography if the terrain is not completely flat. For example, Panofsky e t a1. (1979) suggest that $\sigma_{u}$ and $\sigma_{v}$ are $30 \%$ larger over rolling farmland and perhaps doubled in the lee of low wooded mountains. These contributions to the variances enter the spectrum at the low frequency end.

There has been some success in finding universal descriptions of spectra in the unstable boundary layer and stable suface layer over flat terrain. W will first discuss the spectra of the velocity components in the unstable boundary layer and conclude with a look at spectra in the continuously turbulent stable boundary layer.

Figure A-2.11 shows spectra for the w, u, and v fluctuating wind components (Kainial et al. 1976). The units of the abscissa and ordinate are chosen to provide universal curves at the high frequency end of the spectrum. The presentation of these normalized spectra is such that the area under a given 

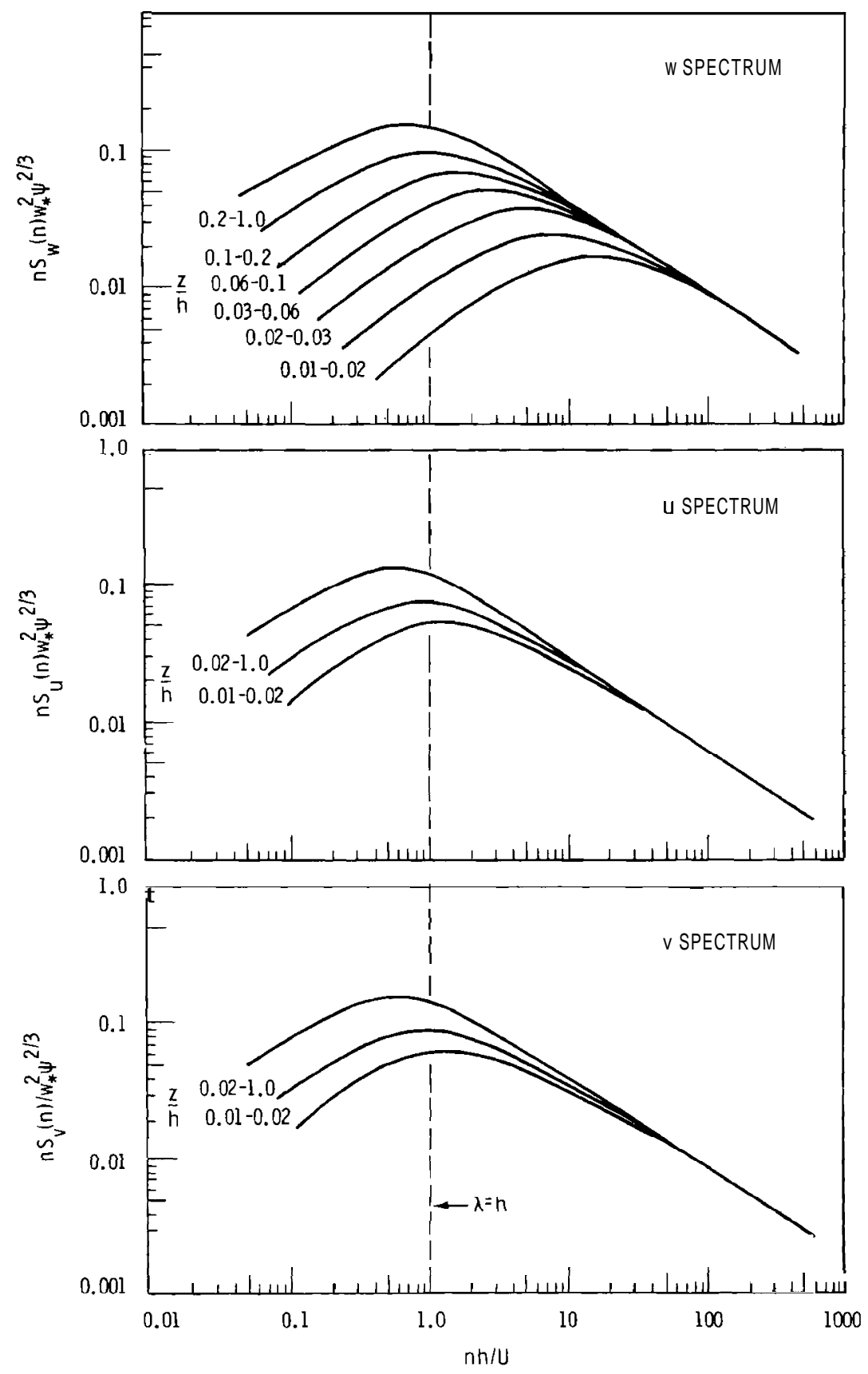

FIGURE A-2.11. One-Dimensional Longitudinal Velocity Spectra in the Unstable Boundary Layer. From Kaimal et a1. (1976). Average value of $\mathrm{h} / \mathrm{L}=-137$. w is a buoyancy scaling veiocity related to the friction scaling velocity by $\left(w_{*} / u_{*}\right)^{3}=-h / k L$. $\psi$ is the rate of dissipation of turbulence kinetic energy (TKE) divided by the rate of buoyancy production of TKE, and is of order unity. 
logarithmic increment of the abscissa is proportional to the fraction of the total variance that is contributed by frequencies (or wavenumbers) within that increment. Figure A-2.11 shows that in the unstable PBL the $u$ and $v$ spectra are not very height dependent, in contrast to the $w$ spectrum. The frequency of the peak of the $u$ and $v$ spectra at the hub height of a WECS occurs at a frequency of about $n=0.5 \bar{U} / \mathrm{h}$. In a $10 \mathrm{~m} / \mathrm{sec}$ wind in a $1 \mathrm{~km}$ deep boundary layer the waves with the most energy have a wavelength (one-dimensional) of about $2 \mathrm{~km}$ and one wavelength passes the wind turbine in about 3 minutes.

The large amount of variance at the low frequencies has significance for the design of control strategies for a wind turbine. If a wind turbine that is designed to adjust to changes in wind direction requires a similar time period to diagnose and make the adjustment, the wind turbine could oscillate around the true mean wind direction, never catching up to the changing wind. Similarly, a machine may cut in and cut out too often if there is substantial variance in periods that are close to the time scale of the control strategy's startup cycle. The designers can optimize the machine control strategies by adjusting the averaging intervals of the wind signals that are inputs to machine control algorithms, or by adjusting the out-of-bounds limits for direction error etc. to be more tolerant.

The site evaluation personnel need not worry about designing a control strategy that optimizes wind turbine performance; that is the machine designer's domain. However, the site evaluation personnel should be aware of the response characteristics of the machine and the spectral characteristics of the $u$ and $v$ variances at a site. A site with amplitudes in the low frequency wind components that are large enough to frequently activate machine adjustment procedures is less preferable than a site with more benign winds, even if a machine is optimized for the wind characteristics at the site.

Turbulence at a site that threatens the structural integrity of the machine is all-important. One does not want to select a site where either the fluctuating or mean wind shears are so large and so frequent that they exceed the fatigue-loading design specifications of the machine. Consider the fluctuating wind shear across a $50 \mathrm{~m}$ blade of a $100 \mathrm{~m}$ diameter rotor. The maximum contribution to the fluctuating wind shear across that blade will come 
from eddies that are about $50 \mathrm{~m}$ in diameter. Eddies of this size are not simple circular whorls but are elongated by the mean wind shear; nevertheless, we can crudely approximate that eddies of this size are roughly associated with wavelengths of the same magnitude. Then in a $20 \mathrm{~m} / \mathrm{sec}$ wind, these eddies contribute variance at a frequency of $0.4 \mathrm{~Hz}$. Near this frequency, then, one does not wish to see excessive energy in the spectrum of $u$.

This frequency also dictates the sampling rate that anemometers (placed at, say, hub height, bottom of rotor, and top of rotor) must sample at if measurements of the turbulence shears between those levels are required. In the above example, one needs to sample at least as frequently as every 2.5 seconds. Sampling at substantially higher frequencies only obtains information on the finer scale turbulence in the vicinity of each anemometer, which doesn't give useful information on the wind shear across the entire blade length. Sampling should be two or more times as fast if the spectrum is to be computed (see Lumley and Panofsky 1964). However, having made these arguments to determine data-sampling rates based on knowledge of the turbulence spectrum, simple statistics of the wind shears at those sampling rates should answer the questions about turbulence intensity without having to compute a spectrum.

In the continuously turbulent, stable surface layer, universal forms of the velocity component spectra have also been determined. Figure A-2.12 shows spectra for the $u, v$, and $w$ components. The boundary layer is generally continuously turbulent if $z<L$. For $\frac{z}{L}<0.2$, the Richardson number is roughly proportional to $\frac{Z}{L}$; therefore, the spectrum in a continuously turbulent stable PBL is approximately independent of height up to about hub height if the Obukhov length is large.

Spectra for the sporadically turbulent boundary layer have not been collapsed to any universal functions. The physical processes that provide energy to the spectrum include internal wave action and wave-turbulence interaction. Neither of these have yielded to parameterization efforts. 


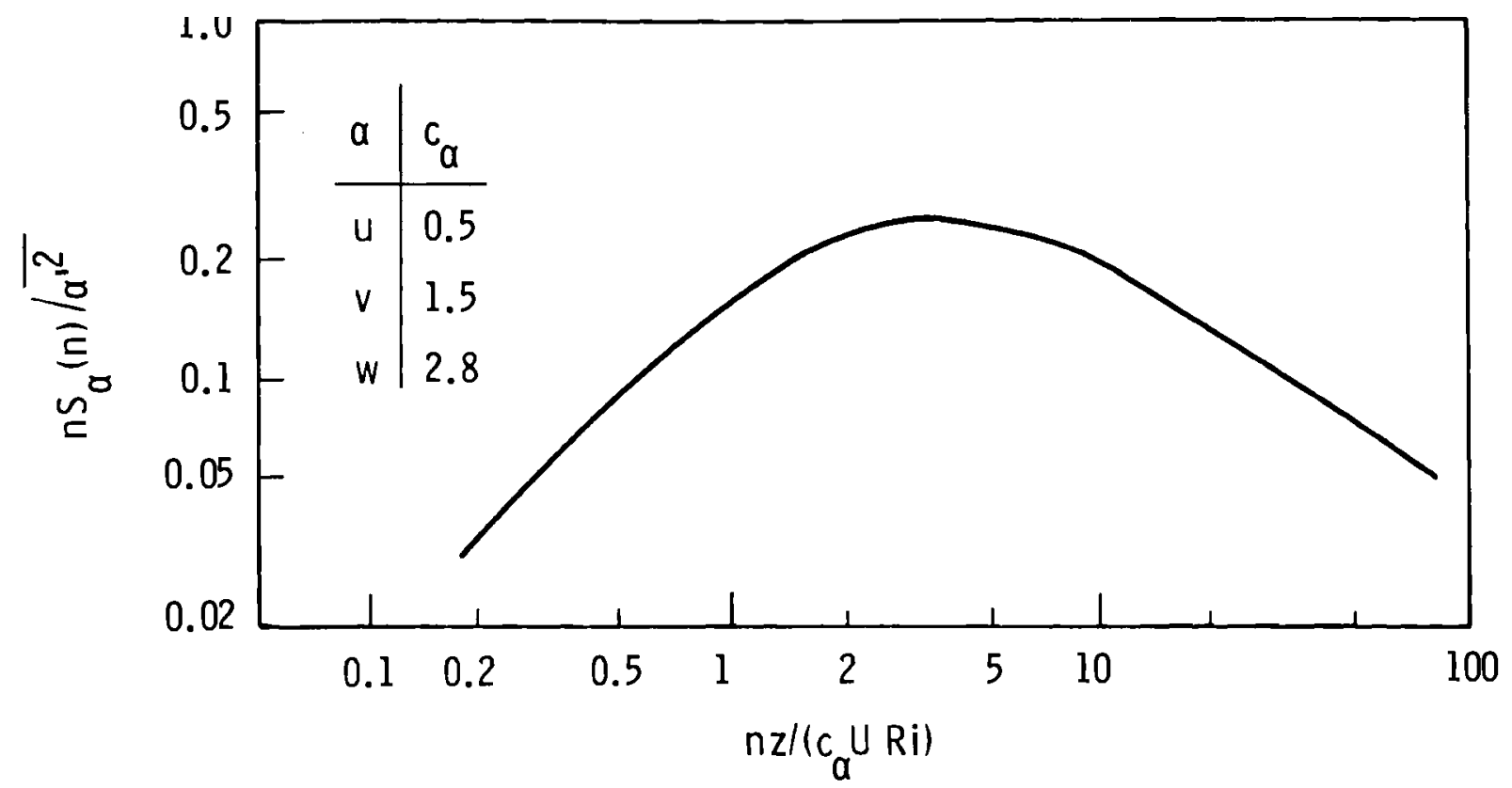

FIGURE A-2.12. Frequency-Weighted Spectra of $u, v$, and $w$, as a Function of $\mathrm{nz} /\left(\mathrm{c}_{\alpha} \cup \mathrm{Ri}\right)$, Where $\mathrm{n}=$ (period)-1, from Kaimal (1973). Respective values of $c_{\alpha}$ are given within the figure.

\section{A.2.3 SUMMARY}

In this section, a general guideline to the turbulence characteristics of boundary layers over flat terrain were presented. The magnitudes and the spatial distribution of the variances of the three wind components were discussed. The problem of turbulence interaction with a wind turbine is a design problem: It becomes a siting problem if turbulence characteristics at a site exceed design criteria. The numbers and figures presented here give a rough idea of the turbulence characteristics over flat terrain so that real site characteristics may be compared in perspective. It is not necessary for a site evaluation team to compute spectra if their data collection and processing reflects an understanding of the nature of turbulence. 


\section{REFERENCES}

American Society of Civil Engineers. 1961. Part 2 of "Wind Forces On Structures." Trans. 126:1124.

Arya, S. P. S. 1978. "Comparative Effects of Stability, Baroclinicity, and The Scale-Height Ratio on Drag Laws for the Atmospheric Boundary Layer." J. Atmos. Sci. 35:40-46.

Blackadar, A. K. 1957. "Boundary Layer Wind Maxima and Their Significance for the Growth of Nocturnal Inversions." Bull. Amer. Meteor. Soc. 38:283-290.

Busch, N. E. 1973. "On the Mechanics of Atmospheric Turbulence." In Workshop on Micrometeorology, D. A. Haugen, ed. American Meteorological Society, Boston, Massachusetts, pp. 1-65.

Businger, J. A., J. C. Wyngaard, Y. Izumi and E. F. Bradley. 1971. "FluxProfile Relationships in the Atmosphere Surface Layer." J. Atmos. Sci. $28: 181-189$.

Businger, J. A. 1973. "Turbulent Transfer in the Atmospheric Surface Layer." In Workshop on Micrometeorology, D. A. Haugen, ed. American Meteorological Society, Boston, Massachusetts, pp. 67-100.

Businger, J. A. and S. P. S. Arya. 1974. "Height of the Mixed Layer in the Stably Stratified Planetary Boundary Layer." Advances in Geophysics.

Academic Press, New York, pp. 73-92.

Corotis, R. B., A. B. Sigl and M. P. Cohen. 1977. "Variance Analysis of Wind Characteristics for Energy Conversion." J. Appl. Meteor. 16:1149-1157.

Deardorff, J. W. 1974. "Three-dimensional Study of Turbulence in an Entraining Mixed Layer." Boundary Layer Meteor. 7:199-226.

Fales, E. N. 1967. "Windmills." Standard Handbook for Mechanical Engineers, 7 th ed., Baumeister and Marks, eds., McGraw-Hill, New York, pp. 9-13.

Fichtl. G. H. and 0. E. Smith. 1977. "Wind." In Terrestrial Environment (Climate) Criteria Guidelines for Use in Aerospace Vehicle Development. 1977 revision. J. W. Kaufman (ed). NASA-TM-78118, National Aeronautics and Space Administration, Washington, DC, pp. 8.15-8.17.

Frost, W., B. H. Long and R. E. Turner. 1978. Engineering Handbook on the Atmospheric Environmental Guidelines For Use in Wind Turbine Generator Development. NASA-TP-1359, National Aeronautics and Space Administration, Washington, DC, pp. 3.15.

Golder, D. 1972. "Relations Among Stability Parameters in the Surface Layer." Boundary Layer Meteor. 3:47-58. 
Hiester, T. R. 1977. "Parameterization of the Unstably Stratified Planetary Boundary Layer Using Aircraft Data." M.S. thesis. University of Washington, Seattle, Washington.

Justus, C. G. and A. Mikhail. 1976. "Height Variation of Wind Speed and Wind Distribution Statistics." Geophys. Res. Letters 3:261-264.

Kaimal, J. C. 1973. "Turbulence Spectra, Length Scales, and Structure Parameters in the Stable Surface Layer." Boundary Layer Meteor. 4:289-309.

Kaimal, J. C., J. C. Wyngaard, D. A. Haugen, O. R. Coté, Y. Izumi, J. S. Caughey and C. J. Readings. 1976. "Turbulence Structure in the Convective Boundary Layer." J. Atmos. Sci. 33:2152-2169.

Lumley, J. L. and H. A. Panofsky. 1964. The Structure of Atmospheric Turbulence. John Wiley and Sons, Inc., New York, pp. 239.

Melgarejo, J. W. and J. W. Deardorff. 1974. "Stability Functions for the Boundary Layer Resistance Laws Based Upon Observed Boundary Layer Heights." J. Atmos. Sci. $31: 1324-1333$.

Monin, A. S. and A. M. Yaglom. 1971. Vol. 1 of Statistical Fluid Mechanics: Mechanics of Turbulence. M.I.T. Press, Cambridge, Massachusetts, pp. 769.

Panofsky, H. A. 1973. "Tower Micrometeorology." In Workshop on Micrometeorology. D. A. Haugen, ed. American Meteorological Society, Boston, Massachusetts, pp. 151-176.

Panofsky, H. A., H. Tennekes, D. H. Lenschow and J. C. Wyngaard. 1977. "The Characteristics of Turbulent Velocity Components in the Surface Layer Under Convective Conditions." Boundary Layer Meteor. 11:355-361.

Panofsky, H. A., J. M. Vilardo, H. N. Shirer, R. C. Lipschutz and D. E. Larko. "Effect of Complex Terrain on Wind Fluctuations." In Proceedings of Conference and Workshop on Wind Energy Characteristics and Wind Energy Siting 1979. American Meteorological Society, Boston, Massachusetts.

Pasquill, F. 1974. Atmospheric Diffusion. 2nd ed. John Wiley and Sons, New York.

Paulson, C. A. 1970. "The Mathematical Representation of Wind Speed and Temperature Profiles in the Unstable Atmospheric Surface Layer." J. Appl. Meteor. 9:857-861.

Siera, D. A. and T. R. Richards. 1979. Modified Power Law Eauations for vertical Wind Prof iles. DOE/NASA/1059-79-4, NASA-TM-79275: National Aeronautics and Space Administration, Washington, DC.

Tennekes, H. 1973a. "The Logarithmic Wind Profile." J. Atmos. Sci. 30:234-238. 
Tennekes, H. 1973b. "Similarity Laws and Scale Relations in Planetary Boundary Layers." In Workshop on Micrometeorology. D. A. Haugen, ed. American Meteorological Society, Boston, Massachusetts, pp. 177-216.

Tennekes, H. and J. L. Lumley. 1972. A First Course in Turbulence.

M. I.T. Press, Cambridge, Massachusetts, pp. 300.

Turner, D. B. 1970. Workbook of Atmospheric Dispersion Estimates. Office of A ir Progress Bulletin No. Ap-25, Environmental Protection Agency, Research Triangle Park, North Carolina.

Wyngaard, J. C. 1973. "On Surface-Layer Turbulence." In Workshop on Micrometeorology. D. A. Haugen, ed. American Meteorological Society, Boston, Massachusetts, pp. 101-149.

Ziltinkevich, S. S. and J. W. Deardorff. 1974. "Similarity Theory for the Planetary Boundary Layer of Time-Dependent Height." J. Atmos. Sci. $31: 1449-1452$. 


\section{APPENDIX 3}

VARIABILITY OF THE WIND RESOURCE 
APPENDIX 3

\section{VARIABILITY OF THE WIND RESOURCE}

The spatial and temporal variability of the wind resource is the principal problem in wind turbine siting. Wind characteristics such as wind speed, turbulence levels, and seasonal and diurnal modulations can change drastically over relatively short distances. Therefore, the usefulness of existing wind measurements in evaluating the wind energy potential of nearby sites is limited. This appendix illustrates the degree of spatial and temporal variability that can be expected in key wind characteristics and examines limitations to the use of the existing data base in determining the economic value of windgenerated electricity. Examples of the spatial and temporal variability of critical wind characteristics have been included because a sound appreciation for the complexities of wind climatology is necessary before realistic siting strategies can be derived.

Spatial variability of the wind depends on topography, the complexity of the meteorology within a region, the season of the year, and the time of day. Some indication of spatial variability will be illustrated in this section by actual wind data. As the data show, large differences in wind behavior can be found in places a short distance apart (i.e., with little horizontal separation). Differences in observed wind characteristics can also result from differences in elevation. Very significant vertical differences can occur over the scale of large wind turbines.

Temporal variations, such as the year-to-year variability of the wind, are also very important issues in siting. Year-to-year variability governs the length of time that wind measurements at a site must be made. A few examples are given of the year-to-year variability of some important wind characteristics.

\section{A.3.1 EFFECTS OF HORIZONTAL SEPARATION}

To illustrate how certain wind characteristics may vary with horizontal separation, eight stations in the state of Montana have been chosen. Table A-3.1 lists the station names and locations, heights of the stations above sea level 
TABLE A-3.1 Montana Stations Selected for Detailed Wind Characteristics

\begin{tabular}{|c|c|c|c|c|c|c|}
\hline Station ID & Station Name ${ }^{(a)}$ & Latitude & Longi tude & $\begin{array}{l}\text { Elevation } \\
\text { of Station }\end{array}$ & $\begin{array}{l}\text { Period of Record, } \\
\text { Month and Year }\end{array}$ & $\begin{array}{l}\text { Anemometer } \\
\text { Height, } \mathrm{m}\end{array}$ \\
\hline $\begin{array}{l}\text { Billings, } \\
\text { Montana }\end{array}$ & $\begin{array}{l}\text { Billings Logan } \\
\text { International Airport }\end{array}$ & 45.80 & 108.53 & 1092 & $06 / 58-12 / 76$ & 7.6 \\
\hline $\begin{array}{l}\text { Butte, } \\
\text { Montana }\end{array}$ & $\begin{array}{l}\text { Silver Bow County } \\
\text { Airport }\end{array}$ & 45.95 & 112.50 & 1689 & $01 / 48-12 / 59$ & 18.0 \\
\hline $\begin{array}{l}\text { Glasgow, } \\
\text { Montana }\end{array}$ & Glasgow AFB & 48.40 & 106.52 & 853 & $06 / 61-06 / 68$ & 4.0 \\
\hline $\begin{array}{l}\text { Glasgow, } \\
\text { Montana }\end{array}$ & $\begin{array}{l}\text { Glasgow } \\
\text { International Airport }\end{array}$ & 48.22 & 106.62 & 695 & $08 / 62-12 / 76$ & 6.1 \\
\hline $\begin{array}{l}\text { Great Falls, } \\
\text { Montana }\end{array}$ & Ma $1 \mathrm{~ms}$ trom AFB & 47.52 & 111.17 & 1056 & $03 / 58-11 / 65$ & 4.6 \\
\hline $\begin{array}{l}\text { Great Falis, } \\
\text { Montana }\end{array}$ & $\begin{array}{l}\text { Great Falls } \\
\text { International Airport }\end{array}$ & 47.48 & 111.37 & 1124 & $08 / 59-12 / 76$ & 6.7 \\
\hline $\begin{array}{l}\text { Miles City, } \\
\text { Montana }\end{array}$ & $\begin{array}{l}\text { Miles City } \\
\text { Airport }\end{array}$ & 46.43 & 105.87 & 802 & $01 / 53-12 / 76$ & 12.2 \\
\hline $\begin{array}{l}\text { Whitehal1, } \\
\text { Montana }\end{array}$ & Whitehall CAA & 45.82 & 112.20 & 1403 & $01 / 48-12 / 54$ & 9.1 \\
\hline
\end{tabular}

(a) CAA - Civil Aeronautics Administration Facility; AFB - Air-Force Base 
and the heights of the anemometers above the ground. Figure A-3.1 is a map of the station locations. As seen from the map, several pairs of stations can be selected that would show how wind characteristics vary over fairly short distances.

Figure A-3.2 is a time-series plot of monthly average wind speeds for two Montana stations. Only 13 miles apart, both stations are located in tablelands, with local relief of less than $500 \mathrm{ft}$. The $5-\mathrm{yr}$ averaged wind speeds at the two sites differ by about $0.5 \mathrm{~m} / \mathrm{sec}$ and the figure shows a very high correlation between the two sites. Although a high correlation between monthly averages of surface wind speed is not surprising for two nearby stations in fairly simple terrain, monthly averages of wind speed can also show a high degree of correlation over considerable distances. For example, Figure A-3.3 shows a 14-yr time series of monthly averaged wind speeds for two stations in eastern Montana separated by 130 miles: Miles City and Glasgow International Airport (Int.). For the 9-yr period from January 1968 to December 1976, both the phase and the amplitude of the two time series are nearly identical. In fact, the average wind speeds at the two stations for this 9 -yr period show them to be equal.

Figure A-3.3 also illustrates one of the more maddening aspects of wind behavior. The behavior of the wind at Miles City during the 5 -yr period from January 1963 to December 1967 differs substantially from the behavior recorded at Glasgow Int. for the same period. For example, the average wind speed at Miles City was $0.8 \mathrm{~m} / \mathrm{sec}$ lower than the average over the same period for Glasgow Int. At Glasgow, however, there is no indication that this 5-yr period was unusual. In fact, the 5-yr average wind at Glasgow is the same as the 74-yr mean. A possible reason for the discrepancy between these two stations could be a change in the way wind data were recorded at Miles City, for example: a change in anemometer location, a change in the surroundings of the anemometer, a change in the type of instrument used, a change in calibration procedures, or a change in the way data were sampled. However, changes in anemometer locations are usually well documented. A check of weather service records for Miles City shows no change in anemometer location between 1963 and 1977. Other possible reasons for the discrepancy are extremely 


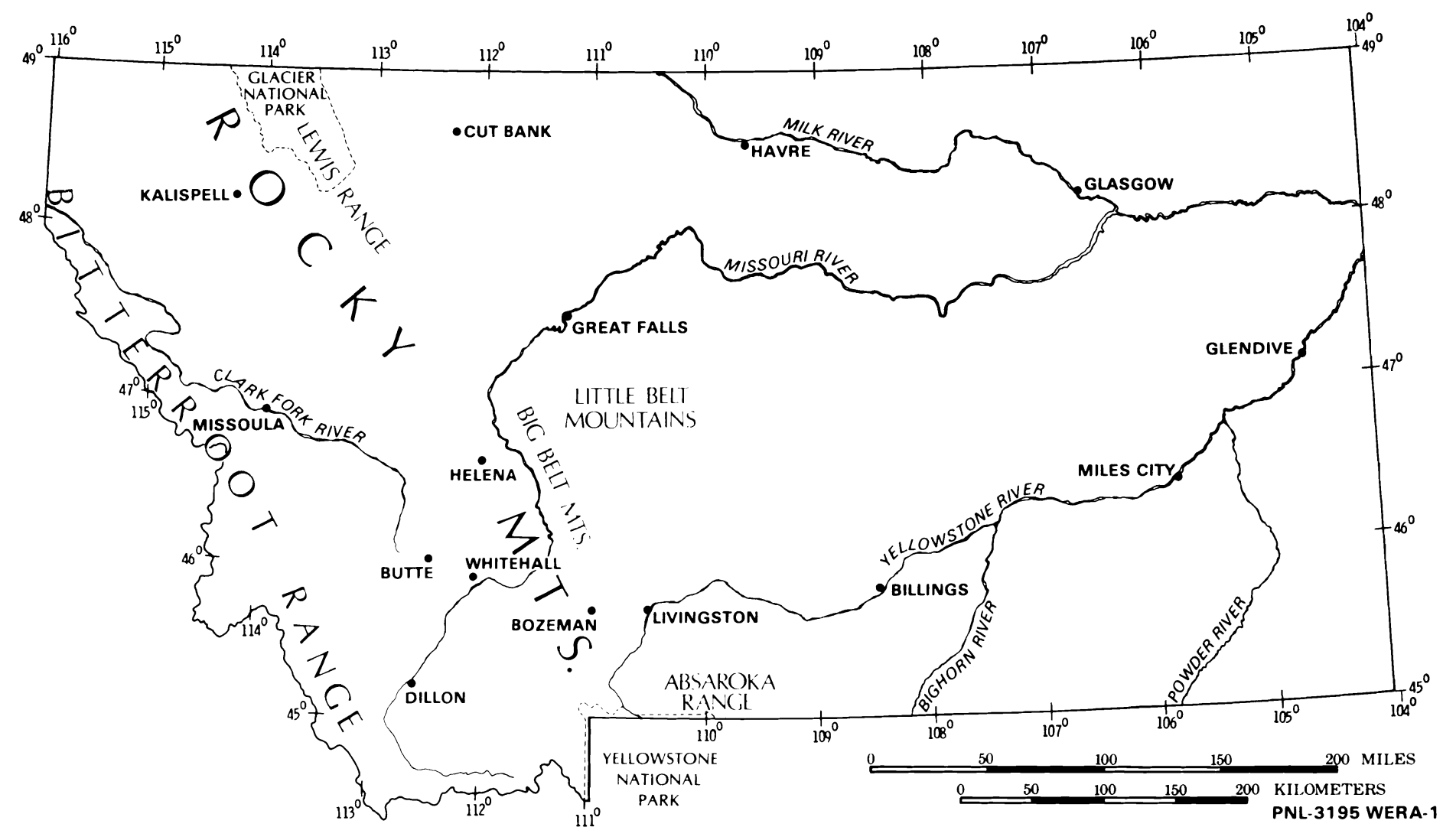

FIGURE A-3.1. Geographic Map of Montana 


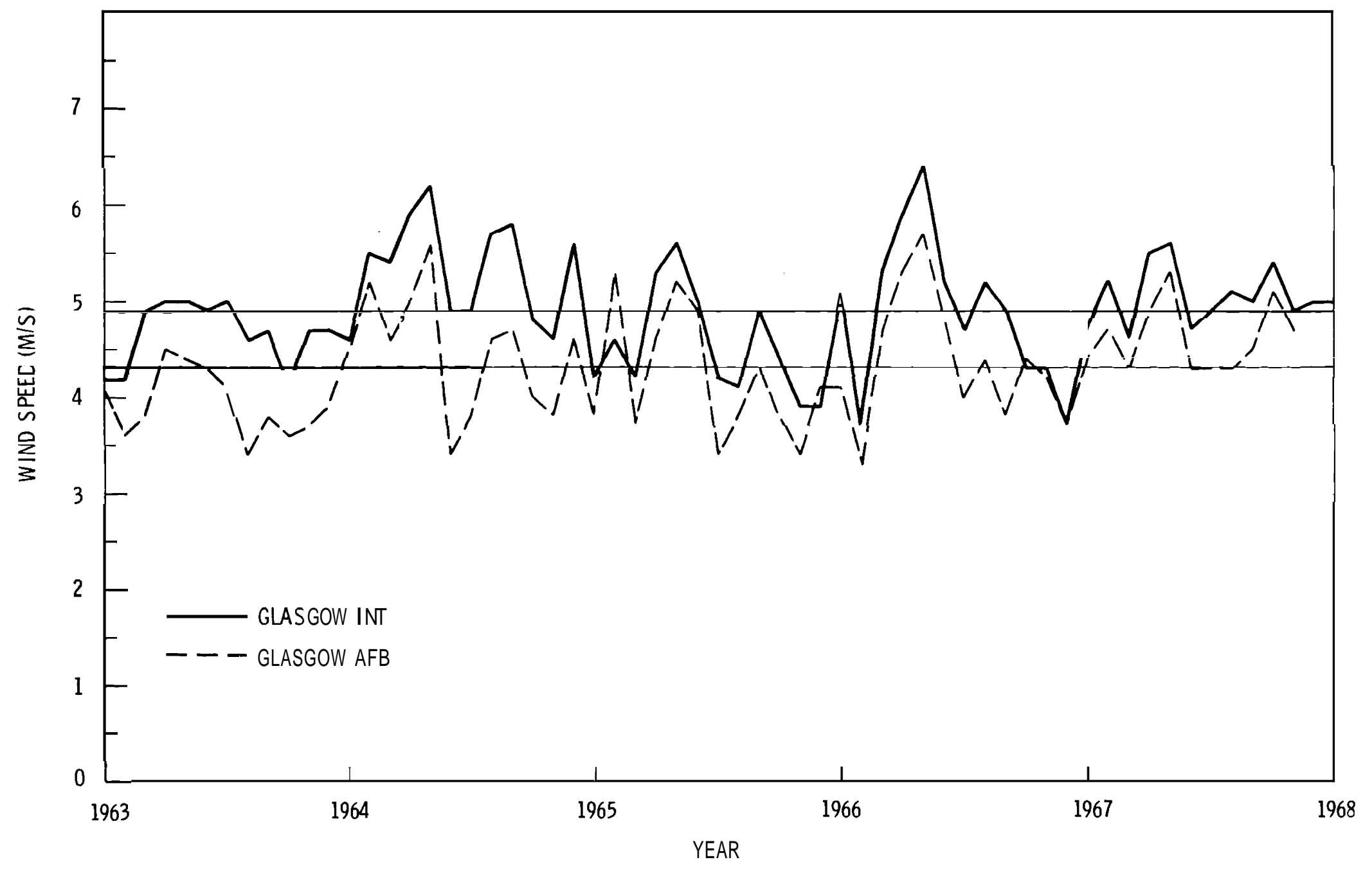

FIGURE A-3.2. Time Series of Monthly Averaged Wind Speed for Glasgow (Montana) International Airport and Glasgow (Montana) A ir Force Base. The stations are 13 miles apart. Horizontal lines represent 5 -yr average wind speed. 


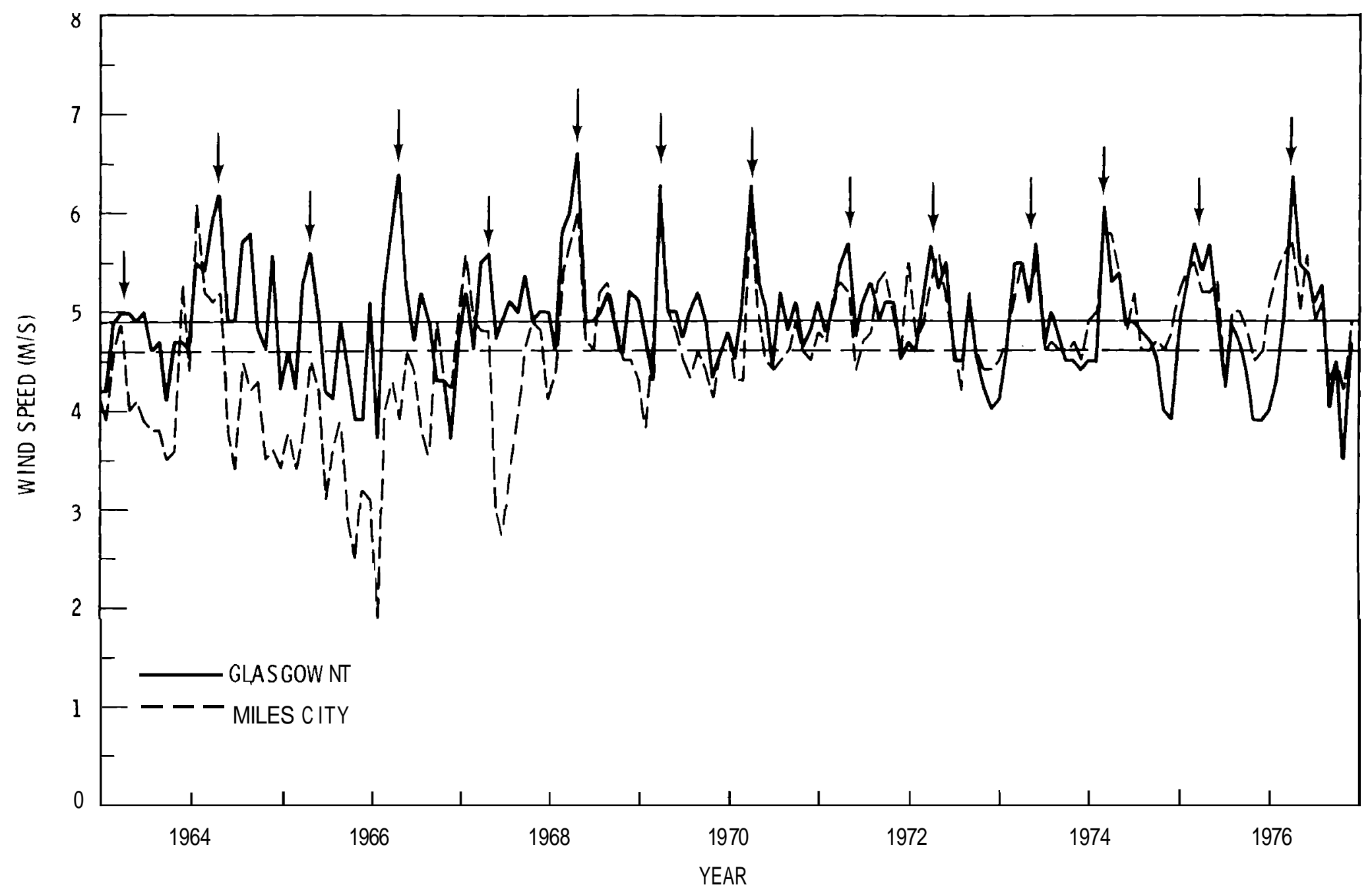

FIGURE A-3.3. Time Series of Monthly Averaged Wind Speed for Glasgow (Montana) International Airport and Miles City, Montana. The two stations are 130 miles apart. Horizontal lines represent $14-y r$ average wind speed. 
difficult, if not impossible, to eliminate. Much of the information on exposure, calibration and sampling is not recorded. Thus, if wind speed records show anomalous behavior, it may be impossible to tell whether the anomaly is real or caused by instrumentation and the way this instrumentation was sampled.

Separation distance is not an infallible predictor of wind speed correlations. If time series of monthly averaged wind speeds for a 14-yr period are compared for Billings and Miles City (about 135 miles apart), the correlation is not nearly as good as for Glasgow Int. and Miles City. Billings shows a very strong early winter wind maximum (see Figure A-3.4). At Miles City, however, the seasonal wind maximum is less pronounced, broader, and occurs 2 to 3 no later--in the spring. These phase differences are even more evident if Bill ings and $\mathbf{G}$ asgow Int. (190 miles apart) are compared (see Figure A-3.5)

Figures A-3.3 through A-3.5 indicate that the wind climatology in Billings is different from that of Glasgow and Miles City. The latter two stations are roughly the same distance from the Rocky Mountains and are situated in the plains of eastern Montana. In the eastern plains, the average winds are strongest in the springtime and are caused by strong pressure gradients associated with spring storms. Billings, on the other hand, is mudh closer to the mountains and is located in a region of greater local terrain relief. The strong winter winds at Billings are caused by strong pressure gradients that form just to the east of the mountains. The pressure pattern is a prevalent mid-winter feature along the eastern slopes of the northern Rockies. The patterns result from interactions between both large- and small-scale features of the topography and certain characteristics of the wintertime meteorology (Barchet and Eliott 1979).

Very significant changes in wind climatology can occur even over short distances. Such behavior is common in regions with large topographic relief or in areas of significant changes in terrain type (perpendicular to coast lines, for example). Figure A-3.6 illustrates the behavior of monthly averaged wind speeds at Butte and Whitehall, 17 miles apart. Butte is located in a sheltered valley in the mountains of southwestern Montana. Whitehall is on the other side of a ridge and is near the mouth of a long, northeasterly sloping valley. In Montana, winds are usually strong in large valleys having 


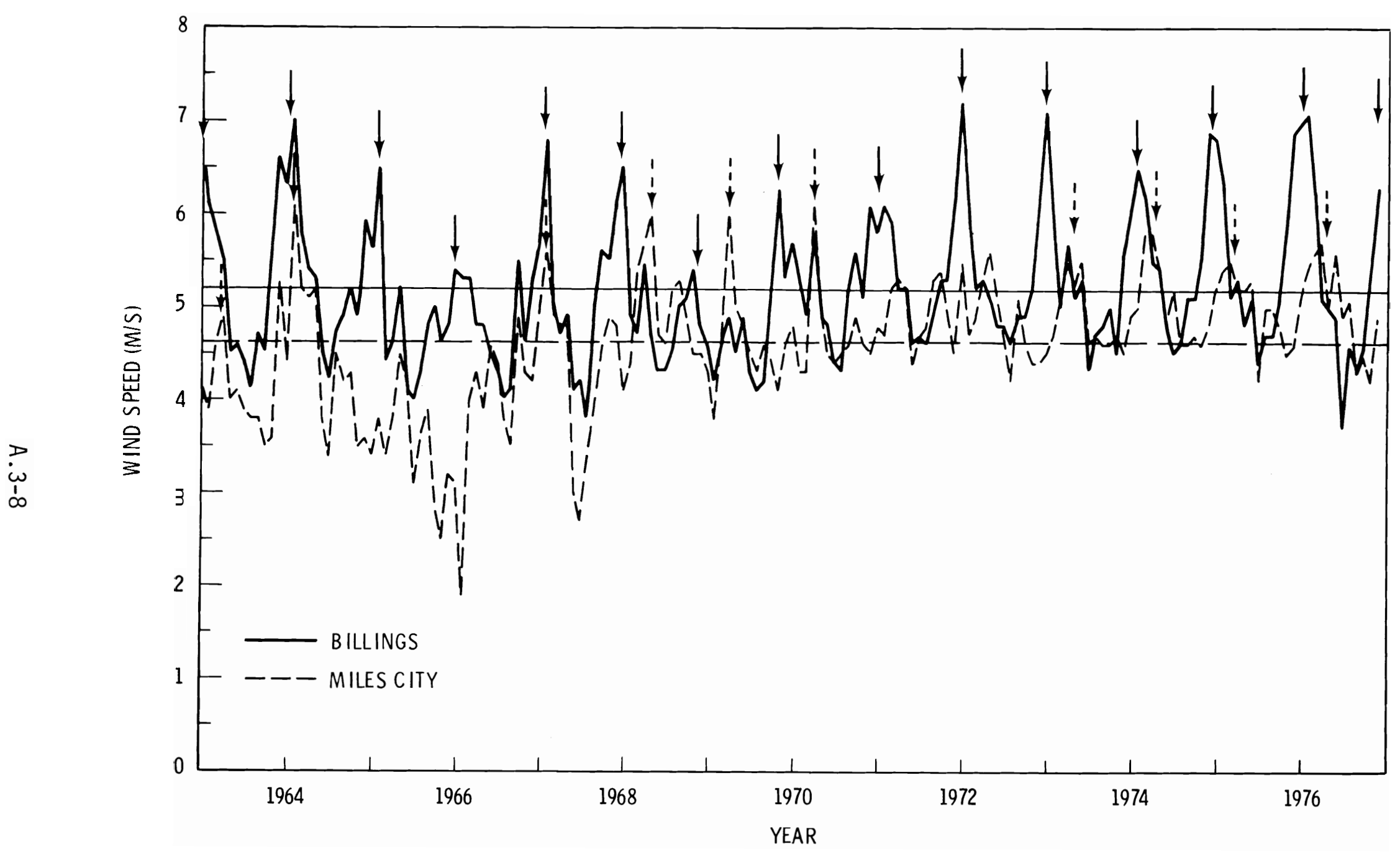

FIGURE A-3.4. Time Series of Monthly Averaged Wind Speed for Billings, Montana and Miles City, Montana. The two stations are 135 miles apart. Horizontal lines represent the $14-y r$ average wind speed. 


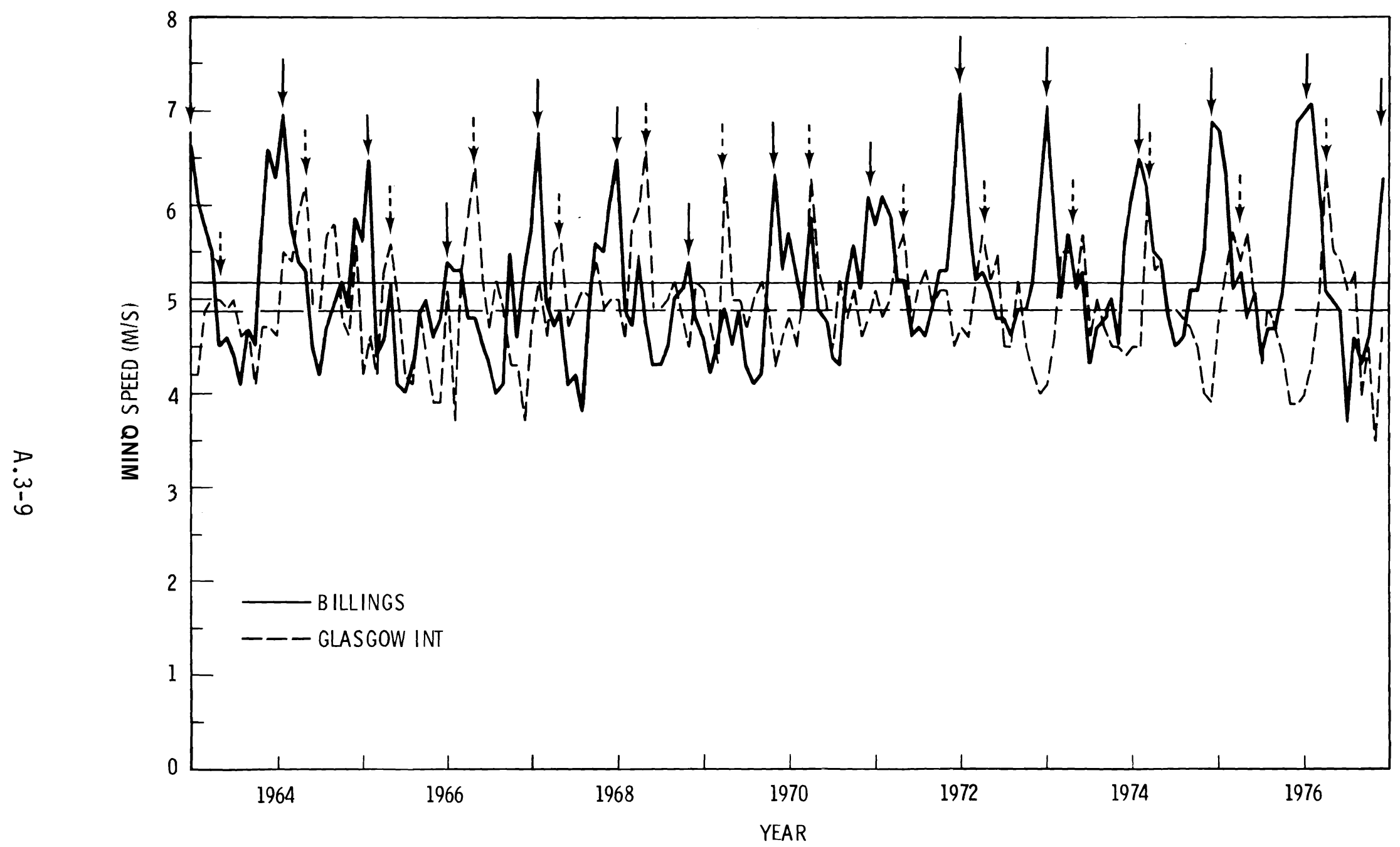

FIGURE A-3.5. Tims Jarips of Monthly Aversged Wind 3pzed for Billings, Montana and Glasgow (Montema) Internotional Airport. The stitions are 190 milss apart. Horizontal linzs repwesent the 14-yr avaweg wind speed. 


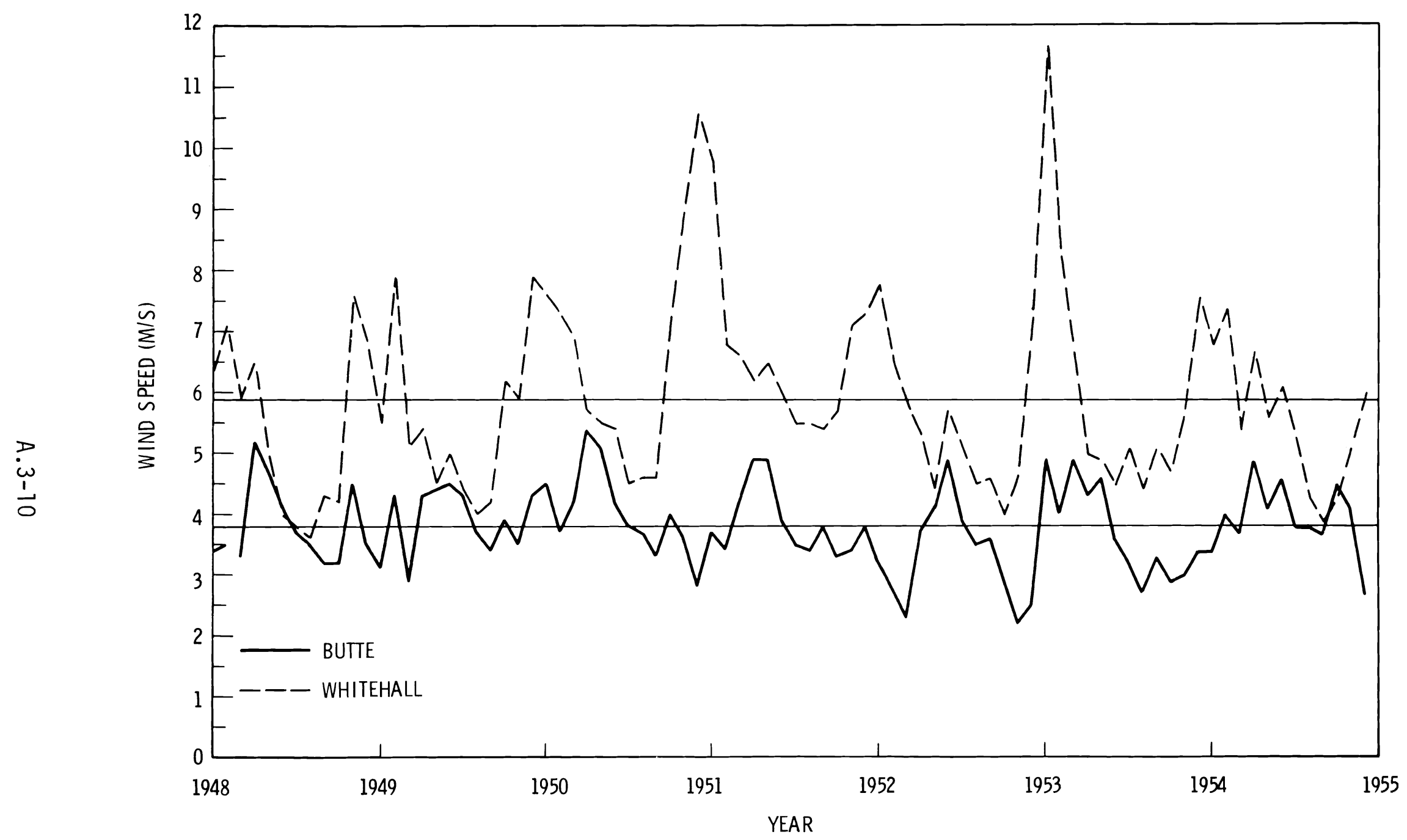

FIGURE A-3.6. Time Series of Monthly Averaged Wind Speed for Butte, Montana and Whitehal1, Montana. The two stations are 17 miles apart. Horizontal lines represent the 7-yr average wind speed. 
this orientation. The highest winds are observed in the wintertime when strong winds at higher elevations in the atmosphere are from the southwest and along the valley axis. Wind speeds at Whitehall are a good illustration of this phenomenon.

\section{A.3.2 EFFECTS OF ELEVATION DIFFERENCES}

Differences in elevation can also lead to differences in the wind climatology of adjacent sites. The effects elevation changes can have on the wind's diurnal bahavior are particularly important (see Figures A-3.7 and A-3.8). Figure A-3.7 shows the average diurnal variation of the wind speed at Dallesport, Washington (see Figure A-3.9) for 4 individual months. These curves represent $12 \mathrm{yr}$ of data, so the $4 \mathrm{mo}$ should represent average conditions for each of the four seasons. The data show summer and spring as the windiest times of the year with winter and fall winds as very light. The seasonal modulation illustrated at Dallesport is typical of the seasonal characteristics of wind speed in the eastern portion of the Columbia River gorge (Elliott and Barchet 1980).

Figure A-3.8 shows the average diurnal variation in wind speed for 4 individual months for an anemometer located on Goodnoe Hills (Figure A-3.9). (a) Goodnoe Hills is about 30 miles east of Dallesport, but the most significant difference is that the Goodnoe Hills anemometer is located on a ridge, $2200 \mathrm{ft}$ above the Dallesport site.

Although only 1 yr of data was analyzed, the results show a wind climatology at Goodnoe Hills different from locations near the floor of the gorge. At Goodnoe Hills, seasonal variations in wind speed appear much less pronounced (at least for the year during which data were collected). Diurnal behavior is also different. Although the magnitude of the diurnal modulation varies with season, higher winds consistently occur at night at Goodnoe Hills. This is a phenomenon not uncommon on isolated mountains and ridges.

(a) December 1978 is used rather than January 1979 because wind speed data are available for only a portion of the month of January. 


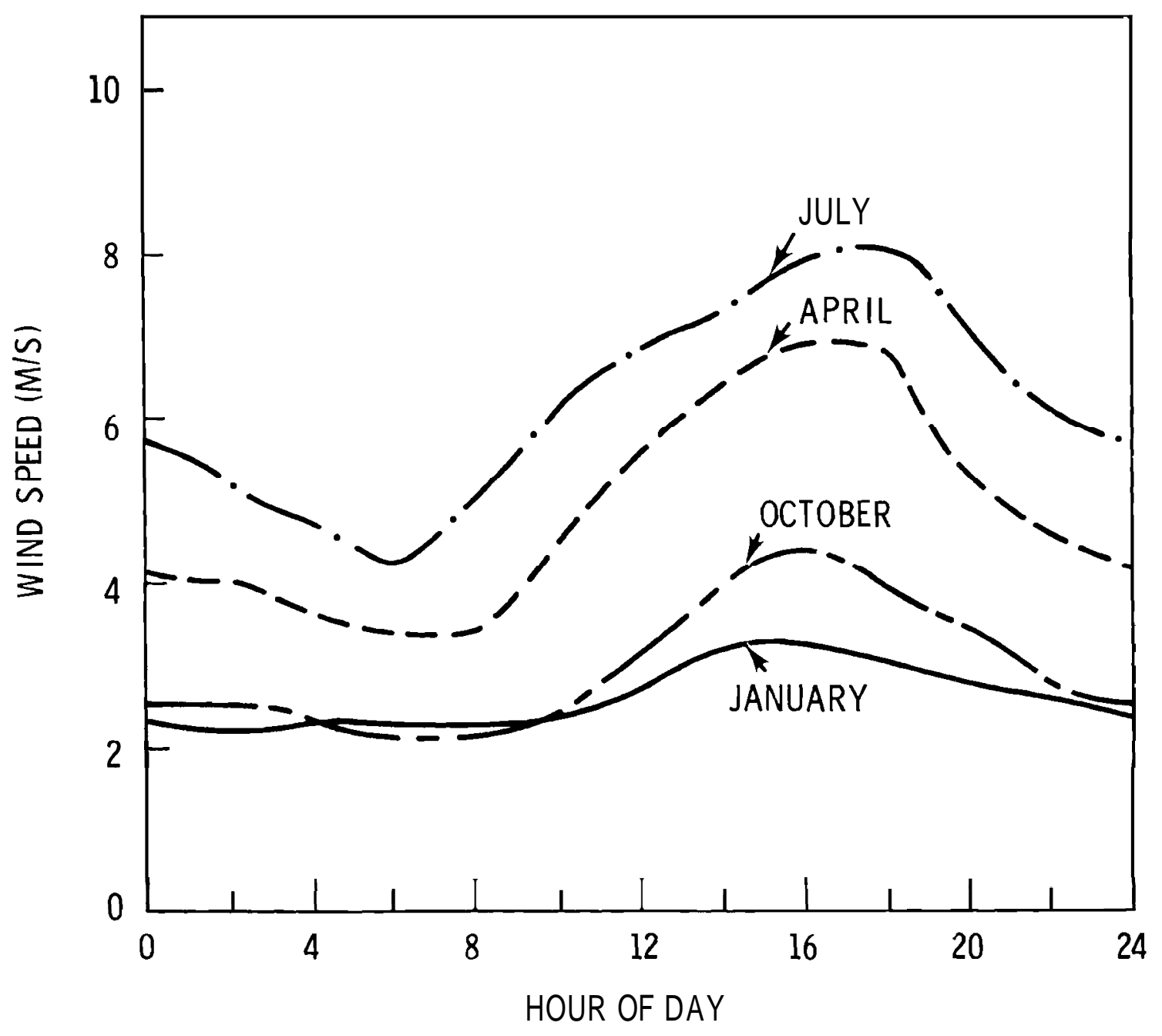

FIGURE A-3.7. Monthly Average Diurnal Variation in Wind Speed at Dallesport, Washington- Curves are based on $12 \mathrm{yr}$ of data. 


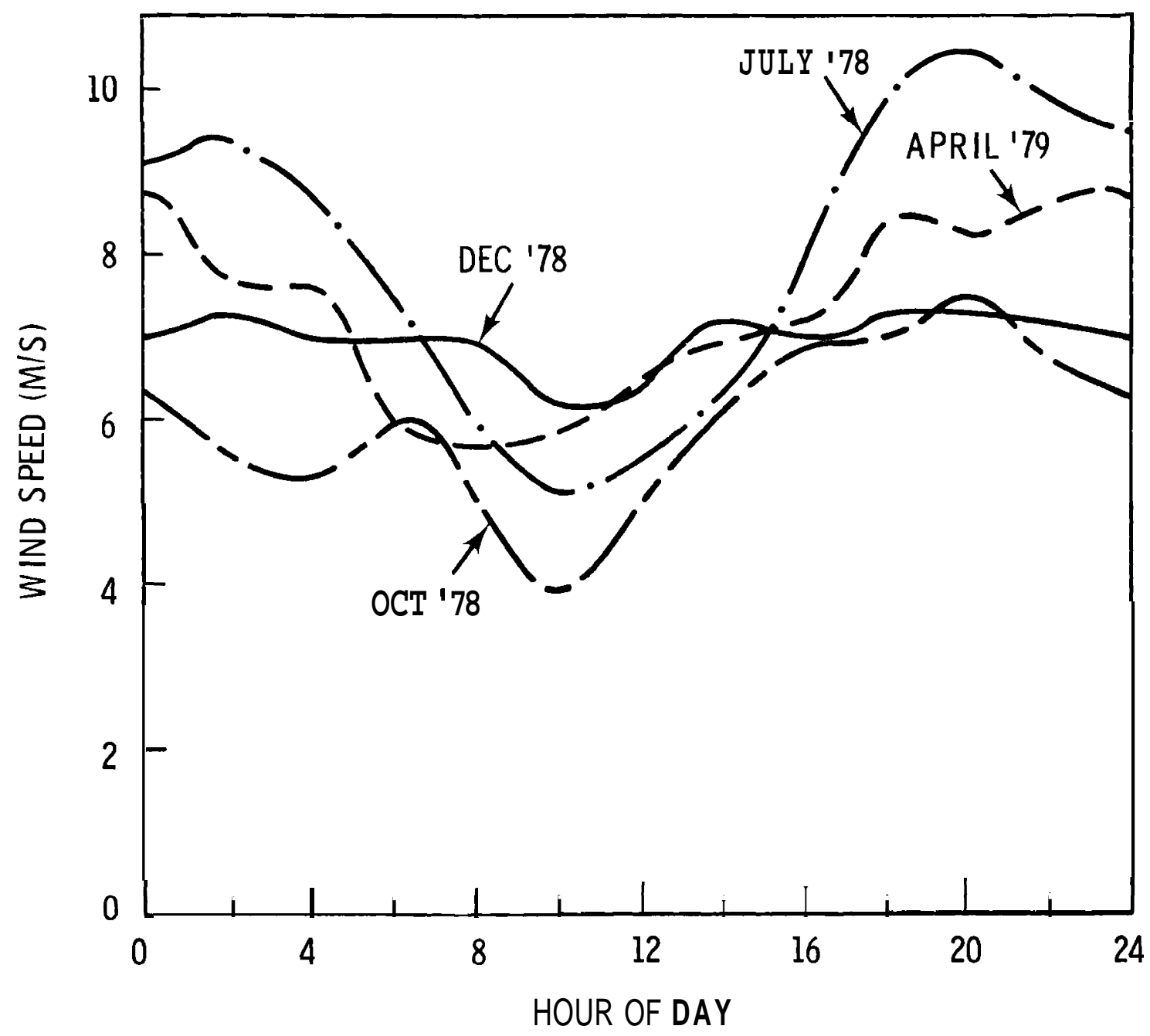

FIGURE A-3.8. Monthly Average Diurnal Variation in Wind Speed a t Goodnoe Hills, Washington. Curves are based on a single year of measurement. 


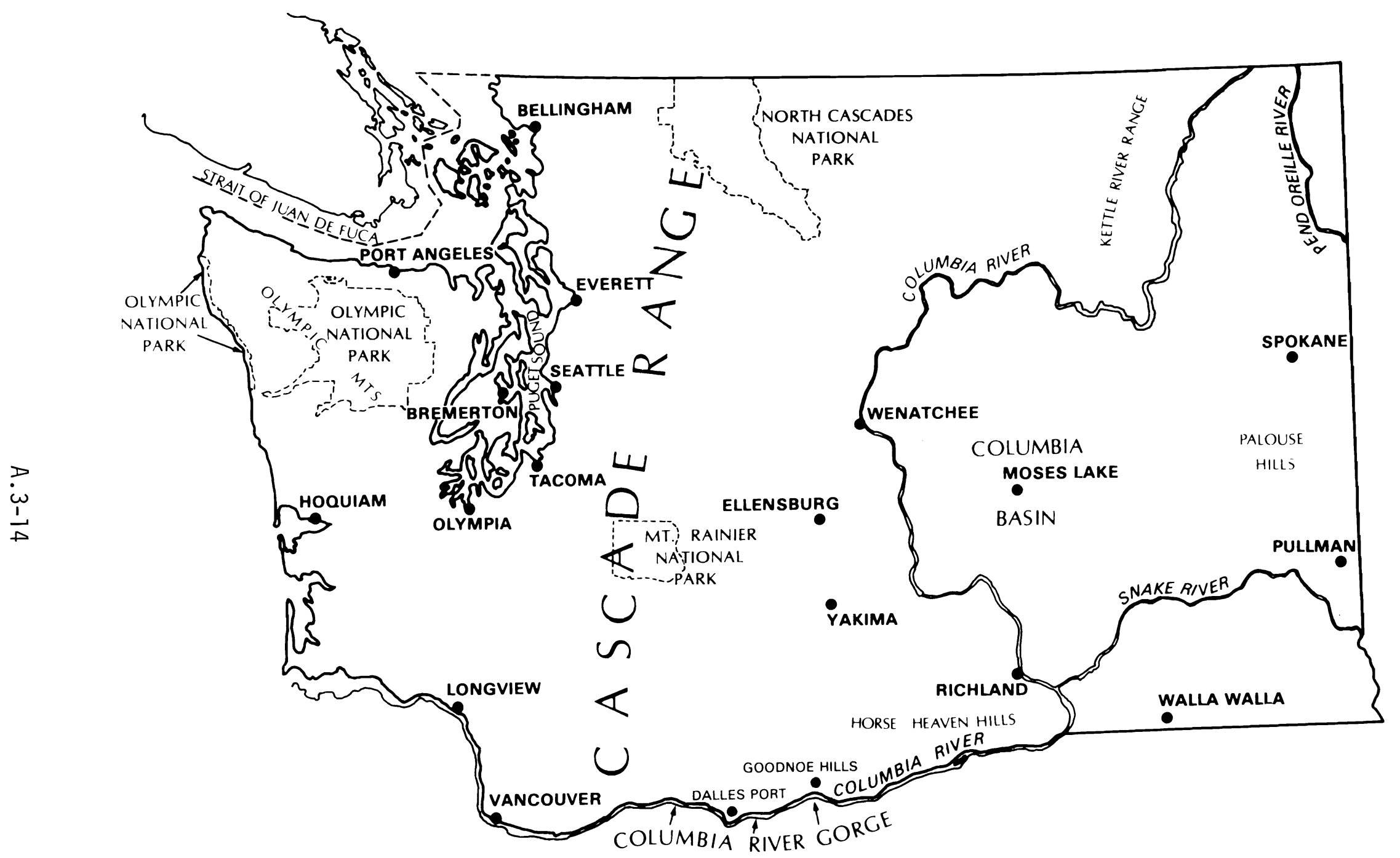

FIGURE A-3.9 Geographic Map of Washington Showing Dallesport in the Columbia River Gorge and Goodnoe Hills, Which is 30 Miles East of Dallesport and $2200 \mathrm{Ft}$ Above Dallesport Along the Rim of the Gorge 
Significant changes in the diurnal behavior of the wind can occur, however, over small vertical distances. Figure A-3.10 shows the annual average diurnal wind variation at three levels observed at a site in central Oklahoma. The curves were created from the analysis of a single year's data at a very tall meteorological tower (Crawford and Hudson 1973). The levels shown correspond to: the height at which surface wind measurements are typically taken, the hub height for a multi-megawatt horizontal-axis wind turbine, and the top of the rotor disk for such a turbine.

Figure A-3.10 shows how greatly diurnal behavior can change over vertical distances comparable to the size of large wind turbines. A wind turbine located at a site with wind behavior as depicted in the figure would, on an annual average, produce more energy at night than during the day. (a) This is contrary to what would have been expected from the surface data.

As was mentioned in Chapter 1, the temporal match between energy generation and a utility's load characteristics has an important bearing on the economic value of wind turbines (JFB Scientific Corp. 1979, Marsh 1979). Thus, a thorough understanding of the diurnal behavior of the wind flowing through the rotor of a wind turbine is important in determining the diurnal characteristics of energy production. Very few locations exist where wind measurements have been made for long periods at elevations comparable to the heights of large wind turbines. The wind behavior illustrated in Figure A-3.10 is thought to be typical of the Great Plains, although details of this behavior are very site specific. The height at which the diurnal modulation of wind speed changes from a daytime maximum to a nighttime maximum varies from day to day, depending on the time of year, local topography, and local meteorology. The shape, phase and magnitude of the diurnal modulation also show similar changes. Since the behavior of the nighttime wind at heights comparable to the heights of typical large wind turbines is so complicated, it is not possible to deduce its behavior by vertically extrapolating surface measurements. The diurnal behavior must be defined by actual measurements. (b)

(a) Changes in diurnal behavior with height can be very dependent on the season. Unfortunately, Crawford and Hudson (1973) do not present seasonal information.

(b) See Appendix 2 for a more thorough discussion of this subject. 


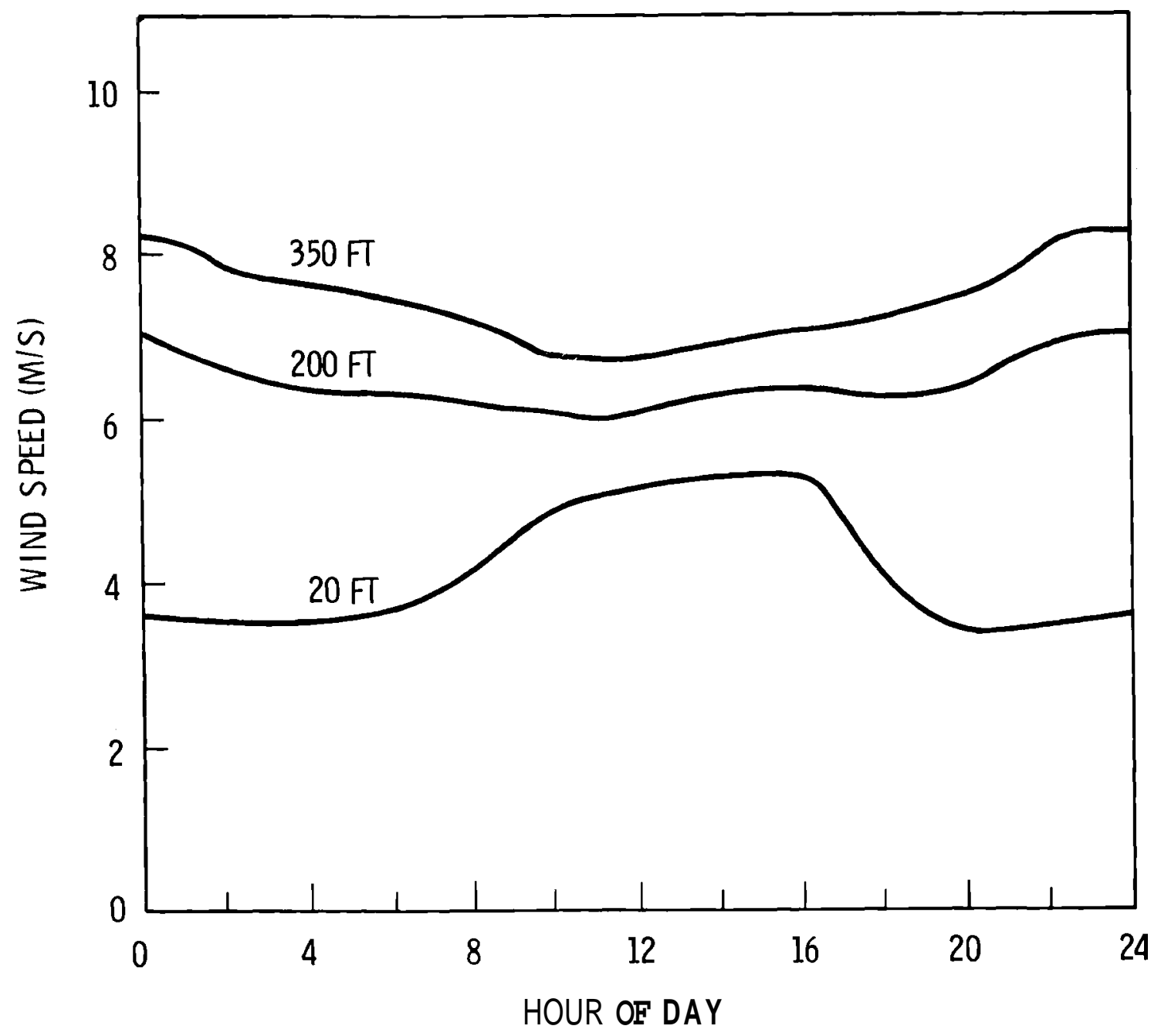

EIGURE A-3.10. Annual-Average Diurnal Variation at Three Elevations. Results are based on a single year of measurement at a site near Oklahoma City, Oklahoma. Elevations correspond to a typical near-surface measurement height; hub height for a large, horizontal-axis wind turbine; and the top of the rotor disk of such a turbine. 


\section{A.3.3 NIERANNUAL VARIABILITY}

The variability of key wind characteristics from year to year is important in determining the value of wind energy conversion to a utility and in determining the length of time data should be collected at a potential site. However, the magnitude of this variability depends on the particular characteristics examined. In this discussion, the interannual variability of the mean wind speed, the seasonal variation in wind speed, and the diurnal modulation of wind strength are examined.

The annual mean wind speed for any given year is a good estimate of the 1ong-term average (Corotis e t a1. 1977, Ramsdel1 1979). Figure A-3.11 shows the variation in the relative uncertainty of a wind speed measurement as a function of the sample length (Ramsdell 1979). The figure is based on up to $30 \mathrm{yr}$ of data from 40 stations throughout the United States. A long-term mean was defined at each station by averaging all monthly means. At each location, the standard deviation of the monthly means about the long-term average was computed. This standard deviation was defined as the base standard deviation $\sigma_{0}$. Next, the standard deviation of estimates of the 30-yr mean based on 2 consecutive months was computed. The same thing was done for 3 consecutive months, $4 \mathrm{mo}$, and so forth. Relative uncertainty was defined as the ratio of these standard deviations to $\sigma_{0}$.

Figure A-3.11 shows the relative uncertainty at these stations dropping very quickly for the first 12 mo of measurements. As sampling time increases beyond $12 \mathrm{mo}$, the change in relative uncertainty is slow. This slow decrease in relative uncertainty indicates that year-to-year variations in annual mean wind speed should be small.

Year-to-year variations in annual mean wind speed are shown more explicitly in Figure A-3.12, which presents time series of annual means at eight Montana locations. Rarely, at any of these stations, did the annual mean change by more than $1 \mathrm{~m} / \mathrm{sec}$ in consecutive years. In general, year-to-year variations are small (less than $0.5 \mathrm{~m} / \mathrm{sec}$ ). However, the results of these eight stations suggest that the more complicated the topography and meteorology, the larger the interannual variability will be. 


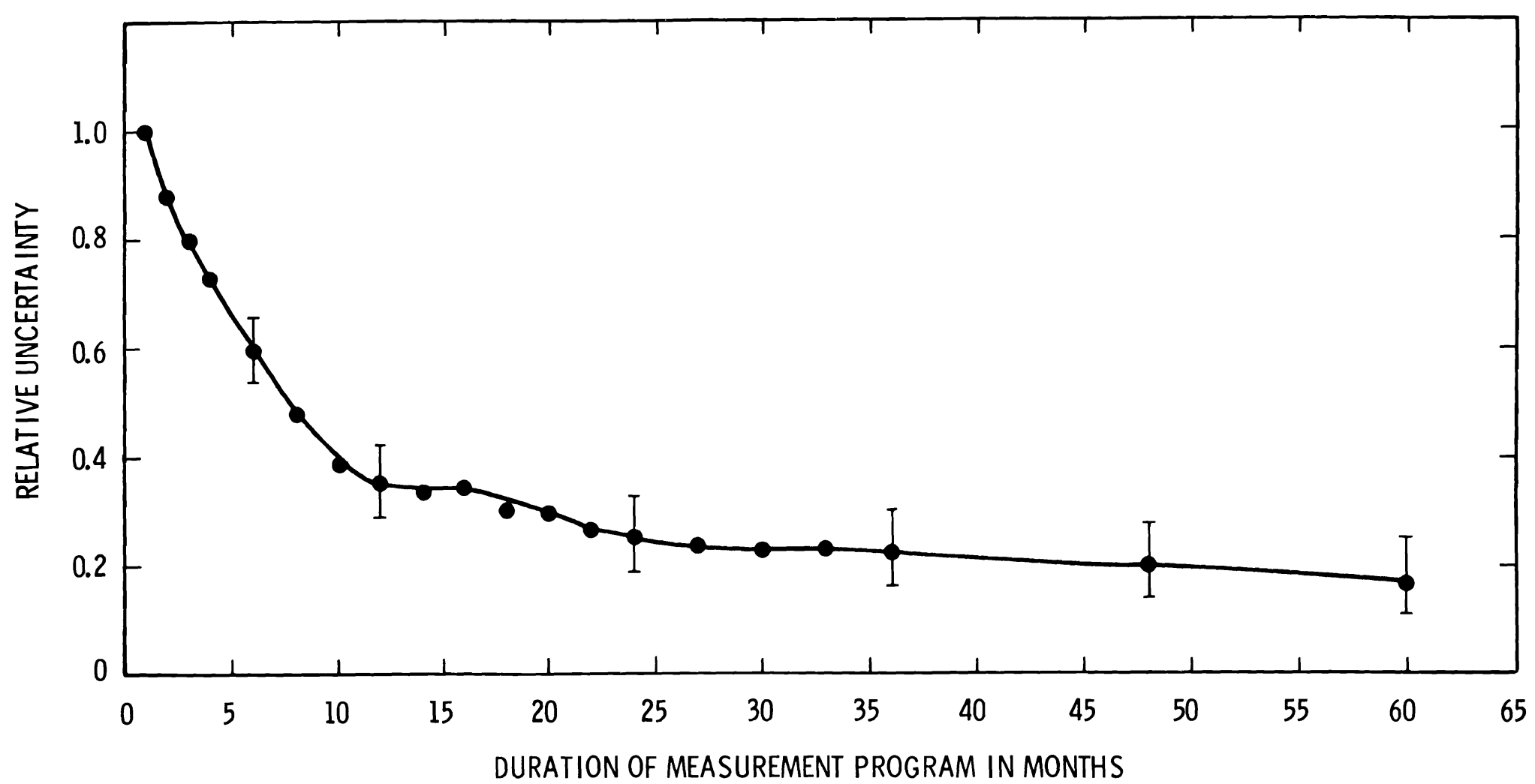

FIGURE A-3.11. Relative Uncertainty of the Measurement of the Climatological Mean Wind as a Function of the Duration of a Measurement Program (Ramsdel1 1979) 

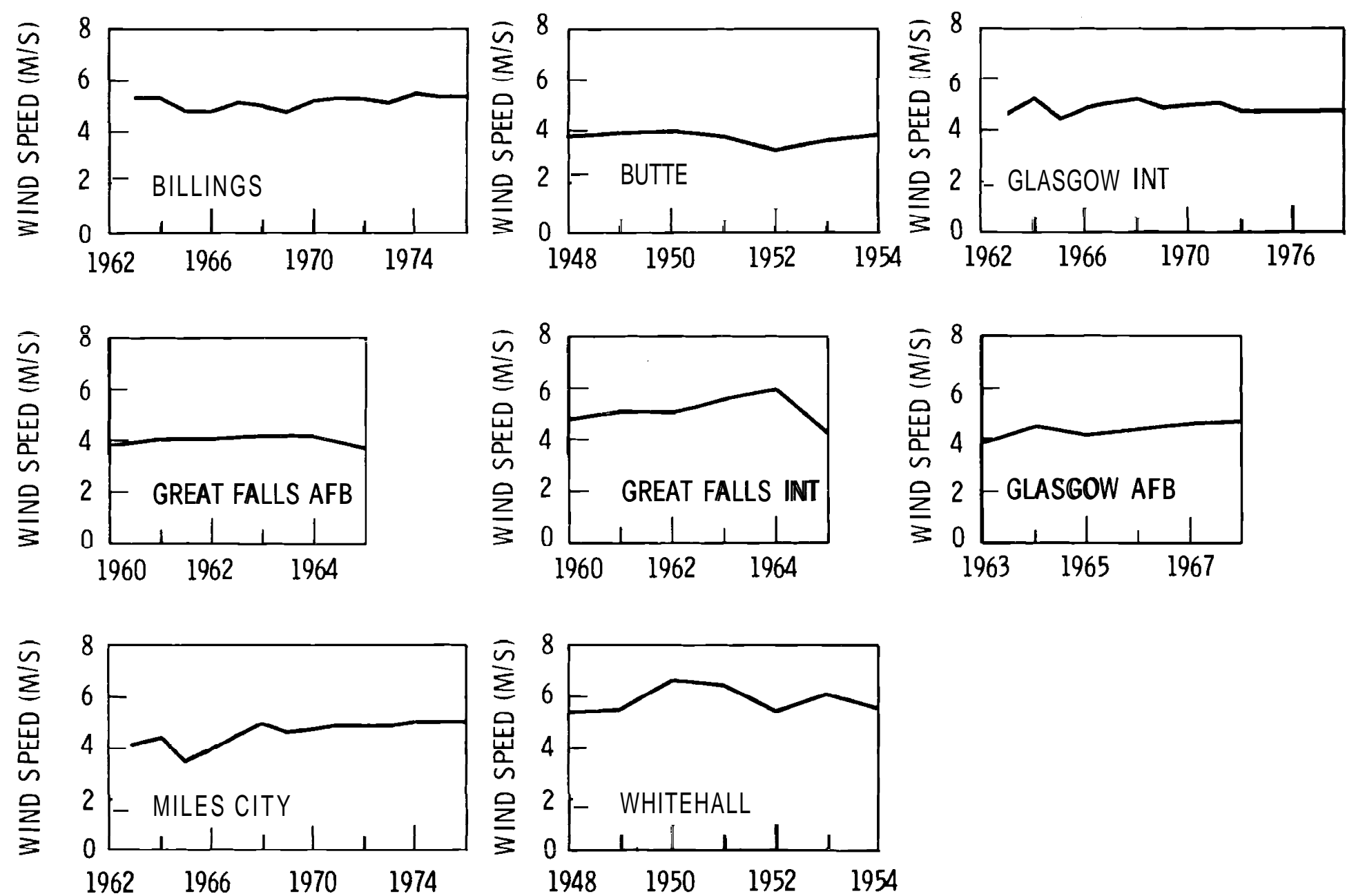

FIGURE A-3.12. Time Series of Yearly Averaged Wind Speed at Eight Locations in Montana 
The lack of correlation in the annual mean between adjacent sites is particularly interesting. At Great Falls International Airport (Int.), for example, 1964 appears to have been an exceptionally windy year; yet at Great Fal1s Air Force Base (AFB), only 10 mi les away, 1964 was not very unusual. Similar behavior is observed when Whitehall is compared with Butte (separation distance of 17 miles). At Whitehal1, 1950 and 1951 appear to have been windier than normal, whereas these same years were fairly normal for Butte. Thus, an abnormal year at one site may not correspond to an abnormal year at a nearby site, particularly in regions where the topography significantly affects the meteorology .

Seasonal changes in wind energy potential have an important influence on the economic value of wind-generated electricity. A close examination of long-term time-series data, however, shows that seasonal modulation can change significantly from year to year (see Figures A-3.2 through A-3.6). Year-to-year changes in the seasonal modulation of wind speed are shown more explicitly in Figures A-3.13 to A-3.15 for Billings, Montana.

Figure A-3.13 compares the seasonal variation of monthly averaged wind speeds for 3 consecutive years with the 18-yr mean. This figure, as well as Figures A-3.2 through A-3.6, shows that the mean behavior of the seasonal variation is not well defined by a single year of data. A better estimate of the cliniatological mean is obtained by examining wind data from more than 1 yr. Figure A-3.14 compares 2-, 3-, and 5-yr averages with the 18-yr mean. Several years of data are needed to define seasonal wind characteristics. Even with $3 \mathrm{yr}$ of data, the windiest month at Billings would have been incorrectly identified as February. The 3-yr mean also shows secondary wind speed maxima in May and October, which do not show up in the 18-yr average.

Figures A-3.14 and A-3.15 indicate that a 5-yr average defines the general nature of the seasonal modulation. However, the data for Billings show that for a given month, the 5-yr average can differ from the 18-yr mean by as much as $10 \%$. At locations where the year-to-year variability is greater (e.g., Whitehall), this difference will probably be greater.

A wind climatologist would consider the year-to-year differences in the seasonal behavior of the wind as described above to be significant. Whether 


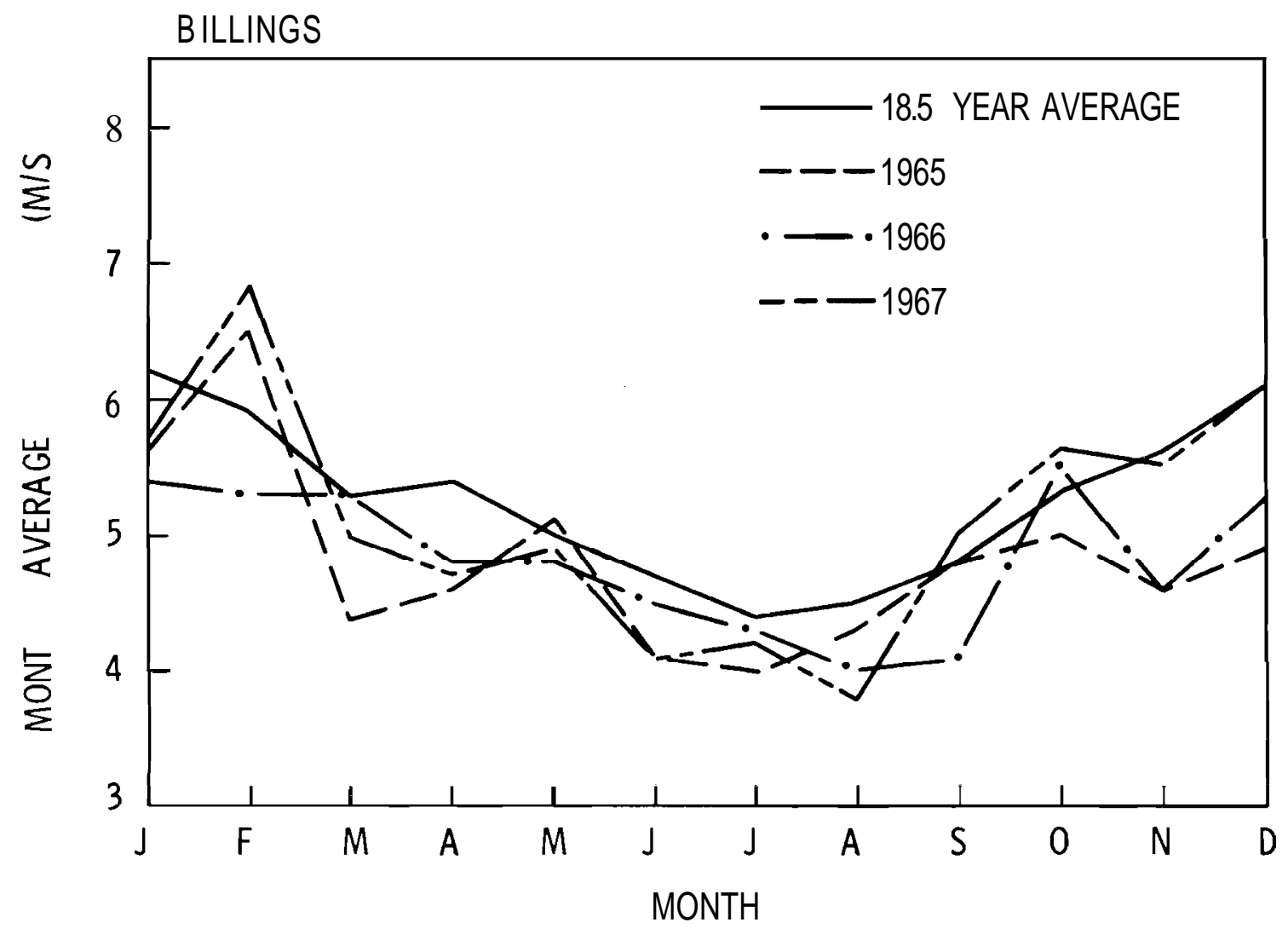

FIGURE A-3.13. Seasonal Variation of Monthly Averaged Wind Speeds at Billings, Montana. Three consecutive years are compared with an 18.5-yr mean. 


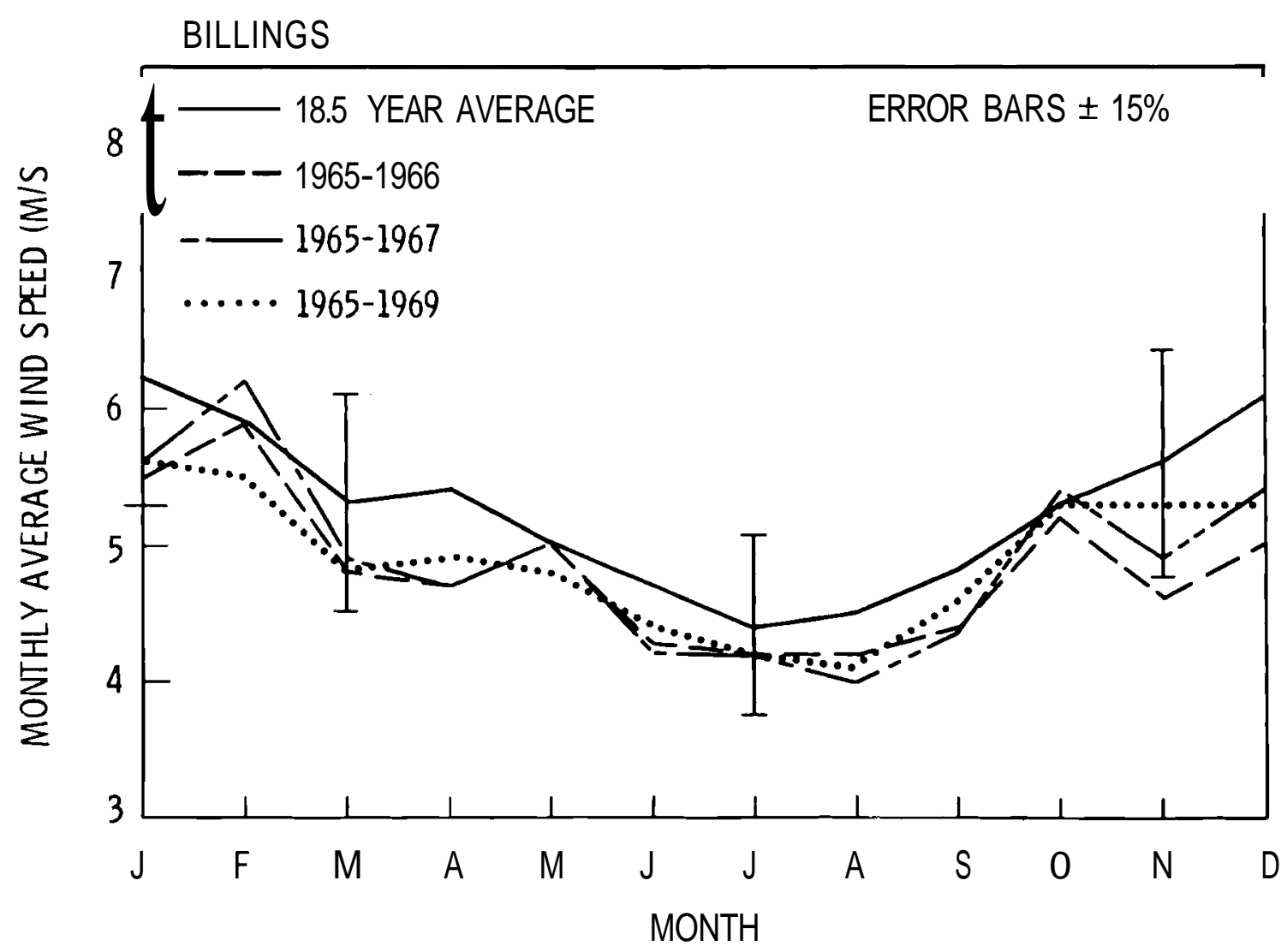

FIGURE A-3.14. Seasonal Variation of Monthly Averaged Wind Speeds at Billings, Montana. The 18.5-yr mean is compared with 2-, 3-, and 5-yr averages. 


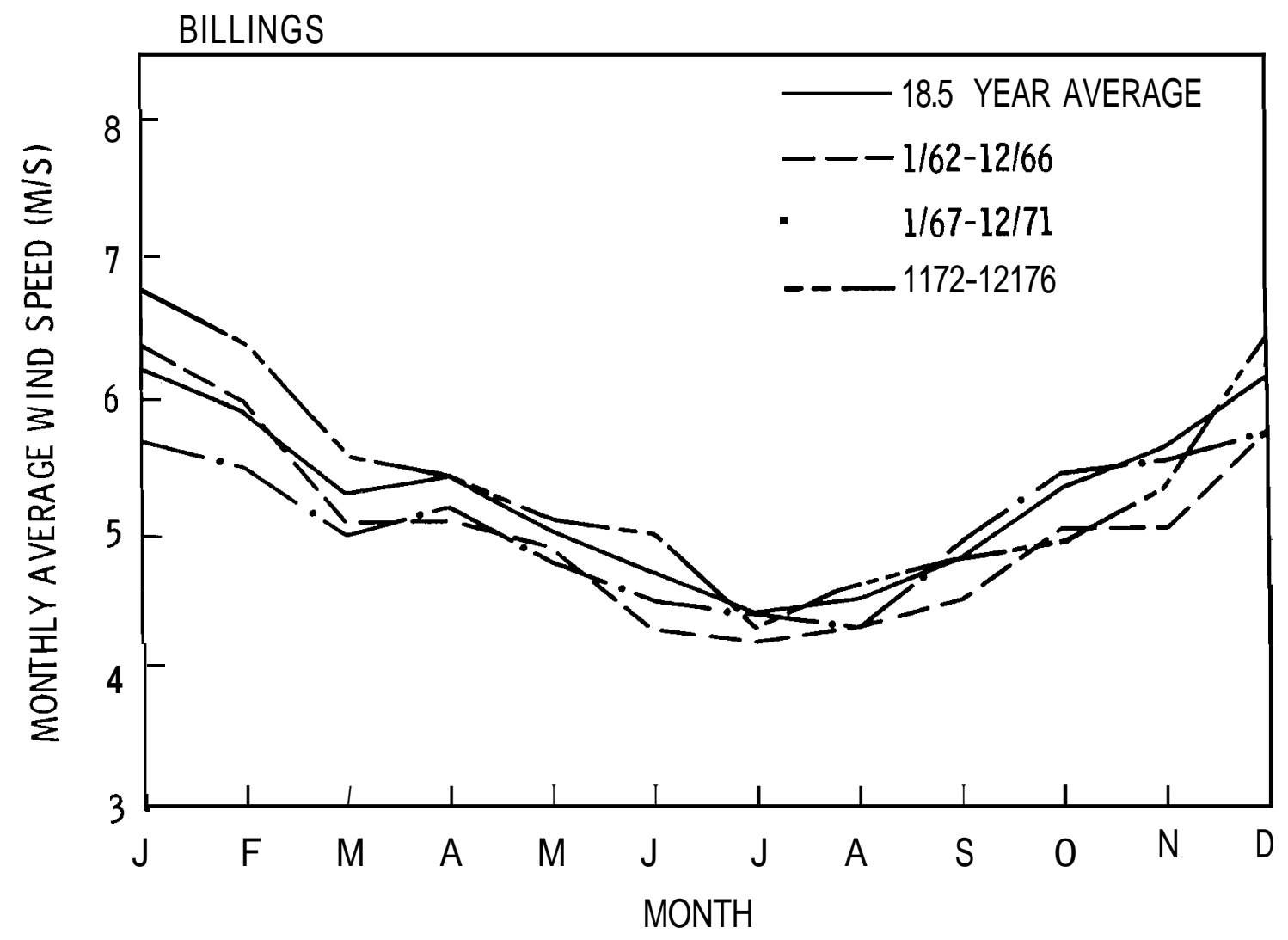

FIGURE A-3.15. Seasonal Variation of Monthly Averaged Wind Speeds at Billings, Montana. The 18.5-yr mean is compared with successive $5-y r$ averages. 
such differences would be significant to the economic value of wind-generated electricity for a particular utility depends on the nature of the utility and it s load.

Examples of year-to-year differences in diurnal behavior at two fairly windy locations are given in Figures A-3.16 and A-3.17. In these figures, the monthly average diurnal cycles for 3 consecutive years are compared to the 1ong-term means. The figures show that year-to-year differences can be significant. Although gross features of the diurnal cycle (such as whether the strongest winds come at night or during the day) can be established with a single year of data, more detailed characteristics (such as the amplitude of the diurnal oscillation and the time-of-day of maximum winds) cannot.

\section{A.3.4 CLIMATOLOGICAL ADJUSTMENT OF SHORT-TERM RECORDS}

Because of the length of time required to establish the climatological average of many wind characteristics, methods have been proposed for adjusting short-term estimates of the characteristics to the climatological mean. All of these techniques require simultaneous wind measurements at the site in question and at a nearby site with a long history of wind measurements.

Comparisons are made between the measurements at the two sites and between the measurements at the historical site and its long-term mean. The measurement at the site with the short-term record is then adjusted accordingly.

To use such a method requires that the wind at the two sites be highly correlated (Corotis 1977). (a) High correlation can be found over large distances (e.g., Figure A-3.3) or may not be found over very short distances (e.g., Figure A-3.6). However, if a high correlation exists, adjusting shortterm measurements can be very accurate. For example, Table A-3.2 shows the results of estimating the 5 -yr mean wind speed at Glasgow AFB from a 3-mo measurement period. In adjusting the short-term estimates of the 5 -yr mean at Glasgow AFB (see Figure A-3.2), Glasgow Int. was used as the historical site.

(a) High correlation must exist for the time scales of interest. For example, the monthly averages of wind speed between two sites could be highly correlated even iftheir diurnal behavior, for example, were completely different. Likewise, hourly data for two sites having similar diurnal behavior could be more highly correlated than their monthly averages. 
DIURNAL WIND BEHAVIOR @ GREAT FALLS INT

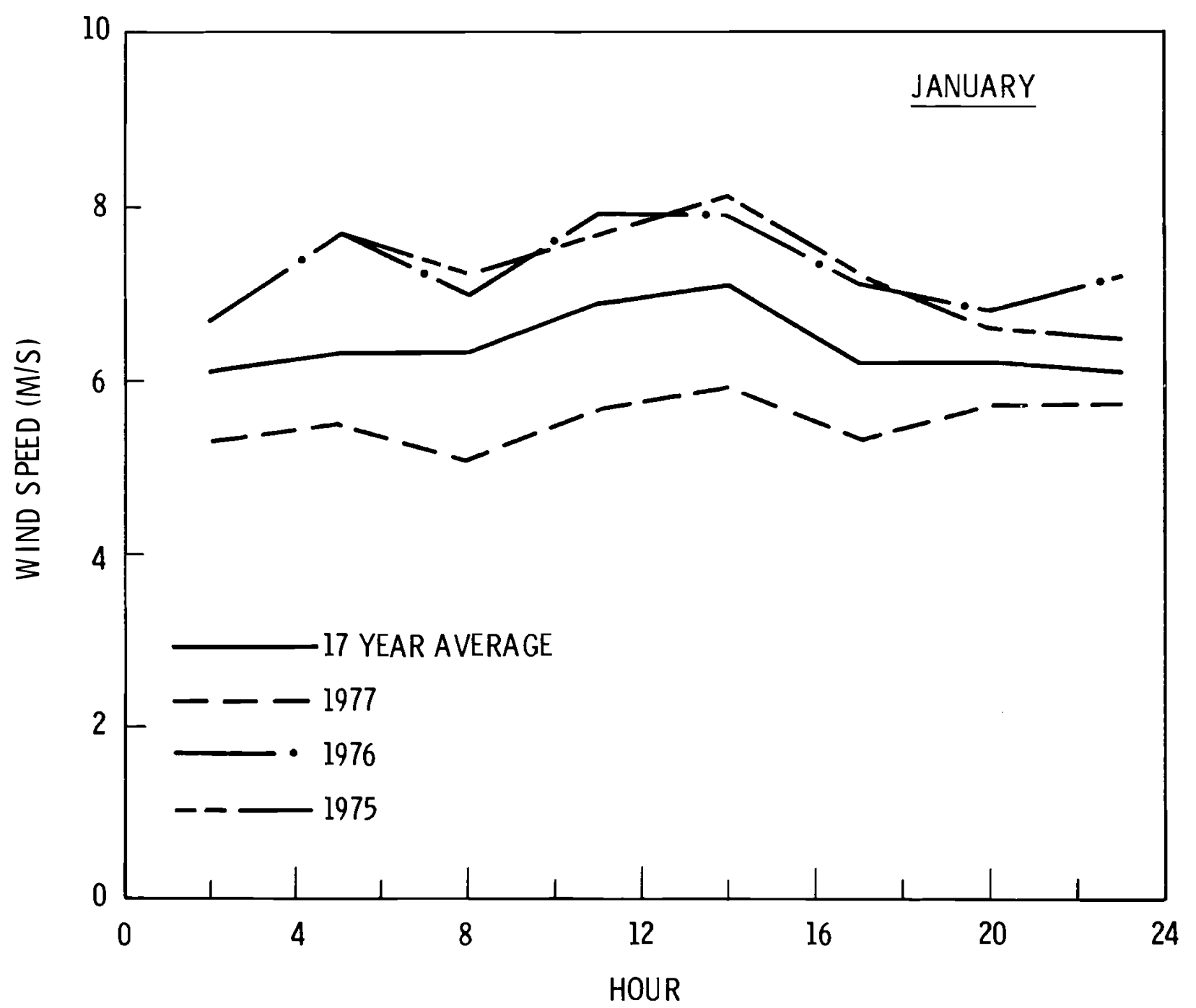

FIGURE A-3.16. Monthly Averaged Diurnal Wind Speed Cycle for Great Falls, Montana. Average diurnal cycle for 1 mo in each season for 3 consecutive years are compared with a $17-y r$ mean. 


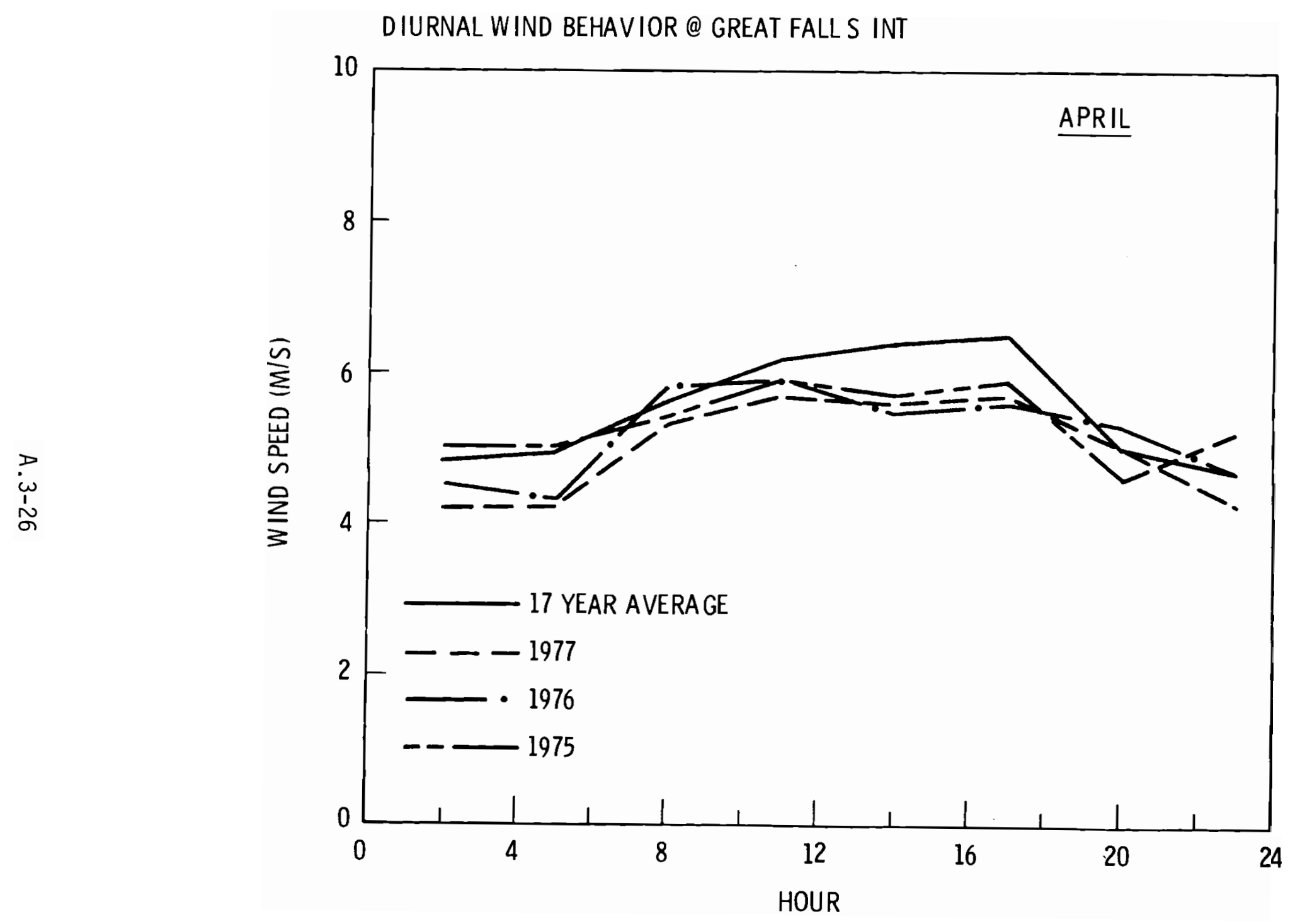

FIGURE A-3.16 (contd.) 


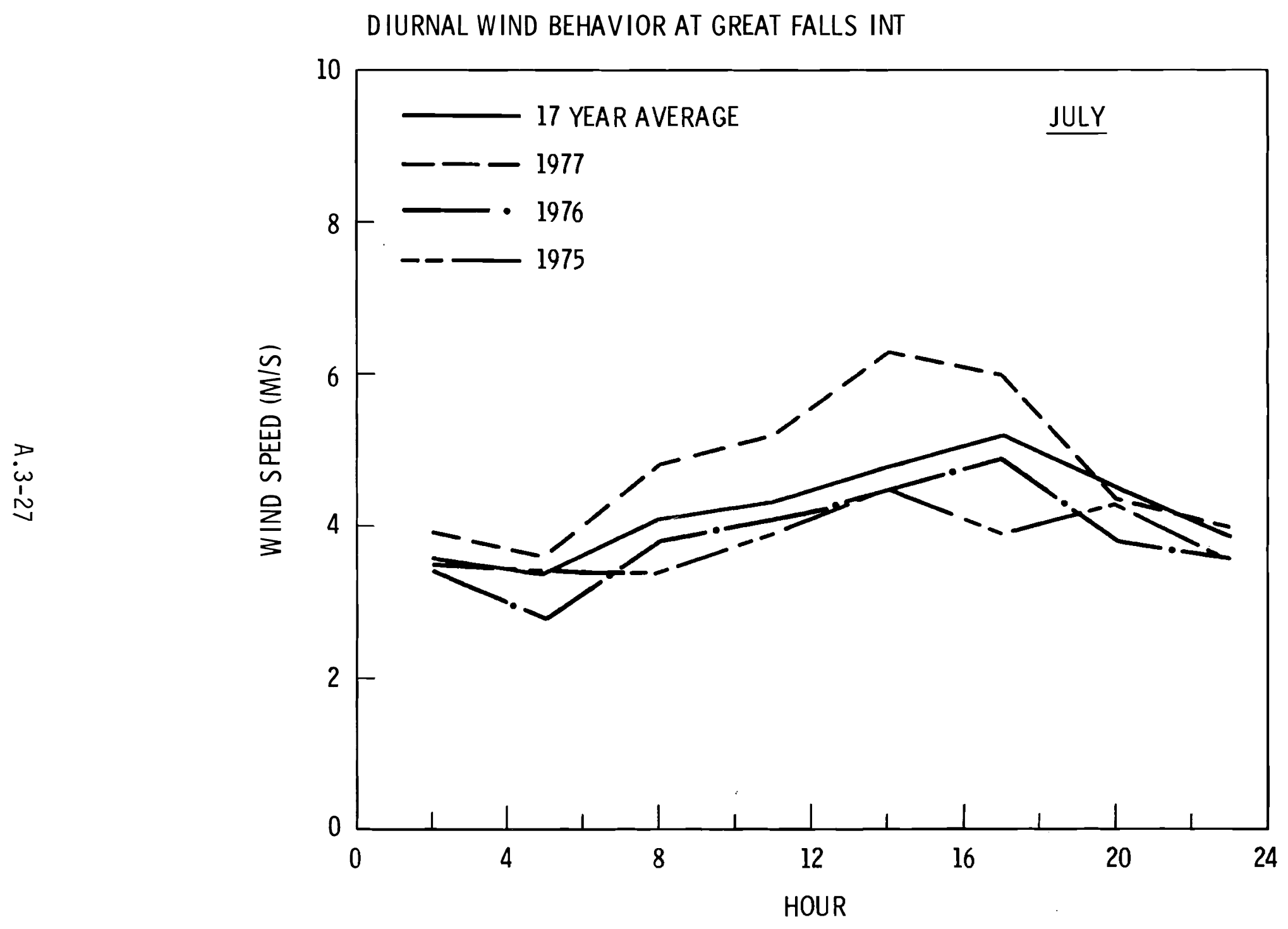

FIGURE A-3.16. (contd.) 


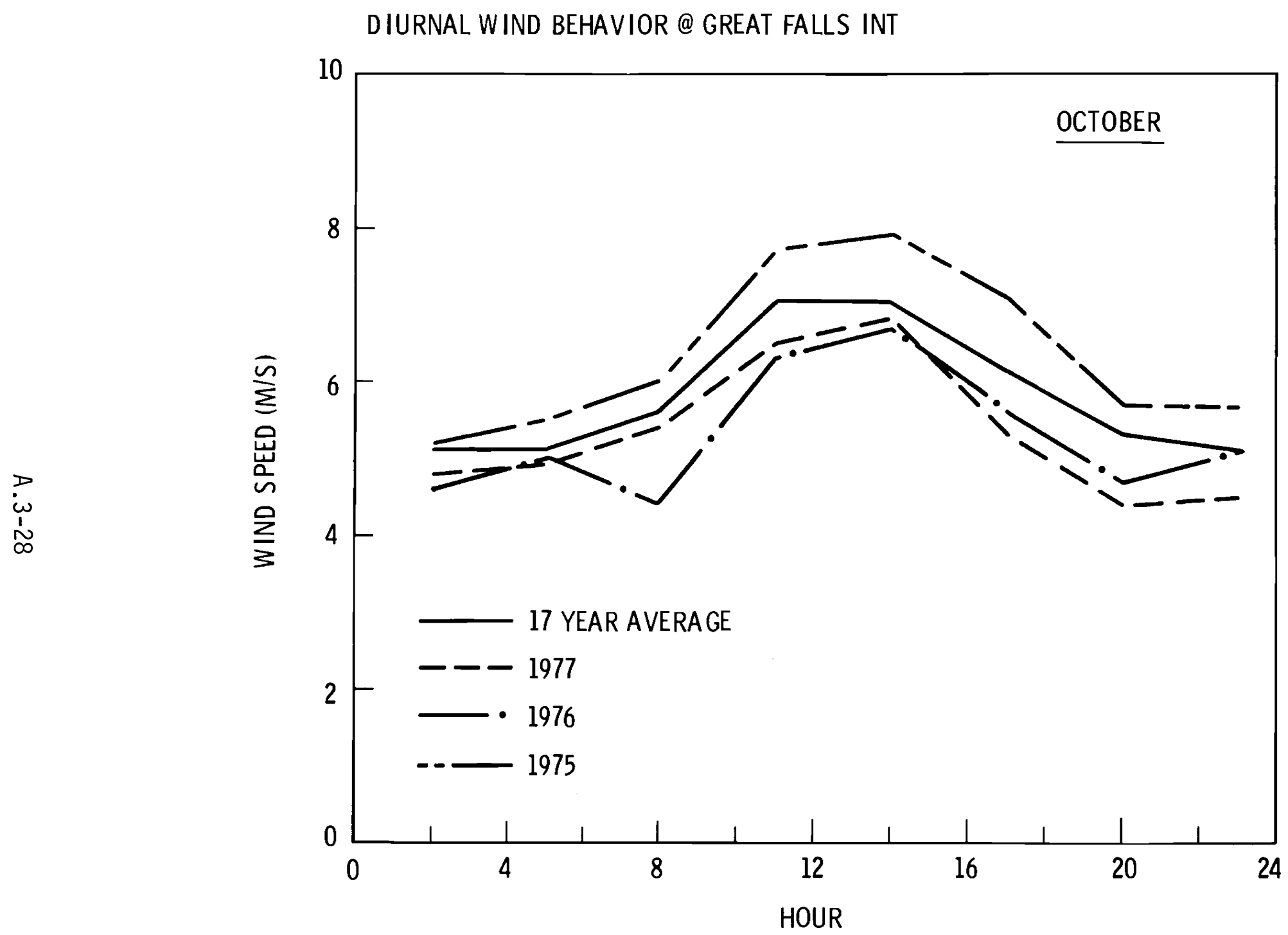

FIGURE A-3.16. contd.) 


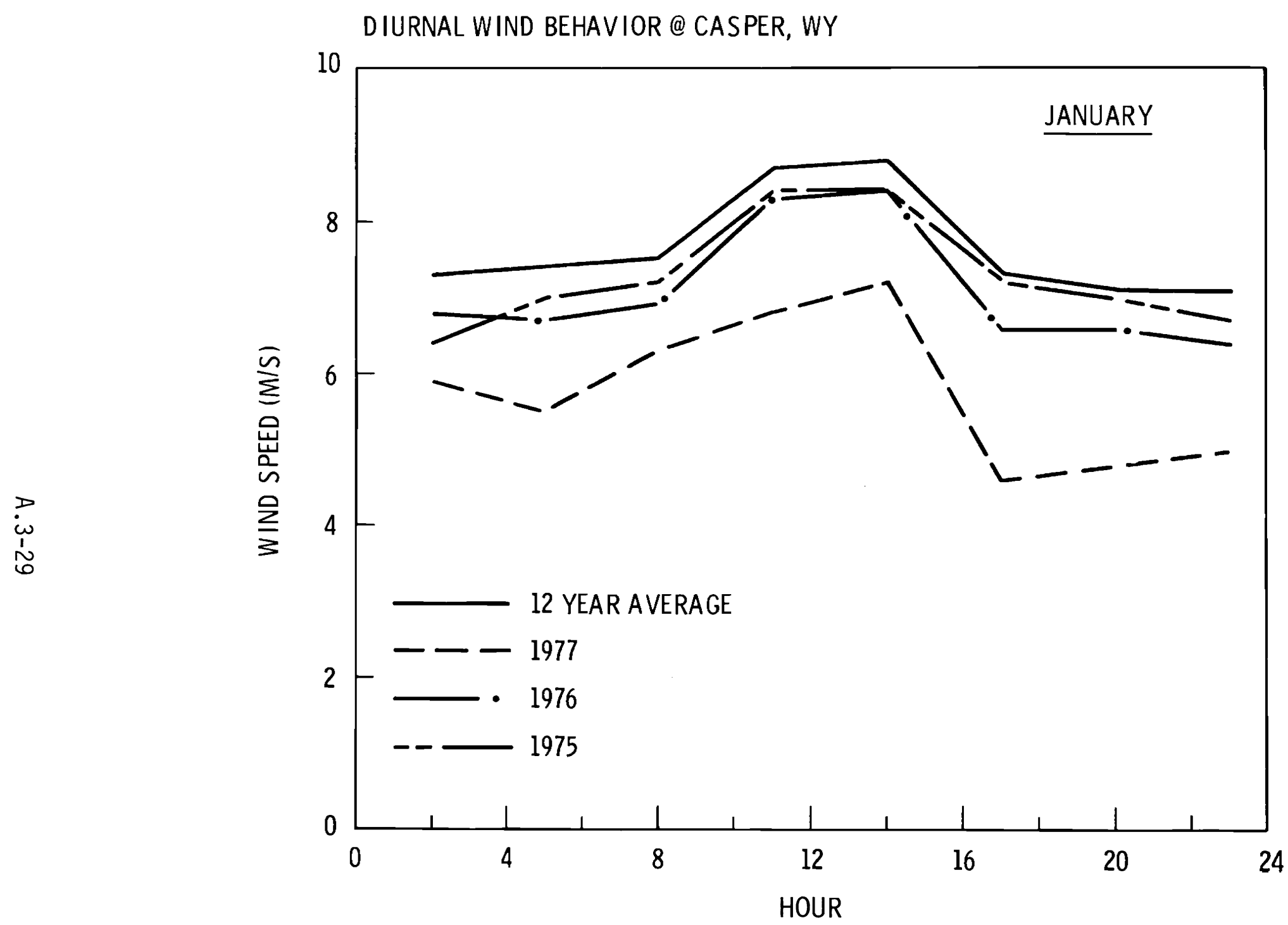

FIGURE A-3.17 Monthly Averaged Diurnal Wind Speed Cycle for Casper, Wyoming. Average diurnal cycle for 1 mo in each season for 3 consecutive years are compared to a 12-yr mear 


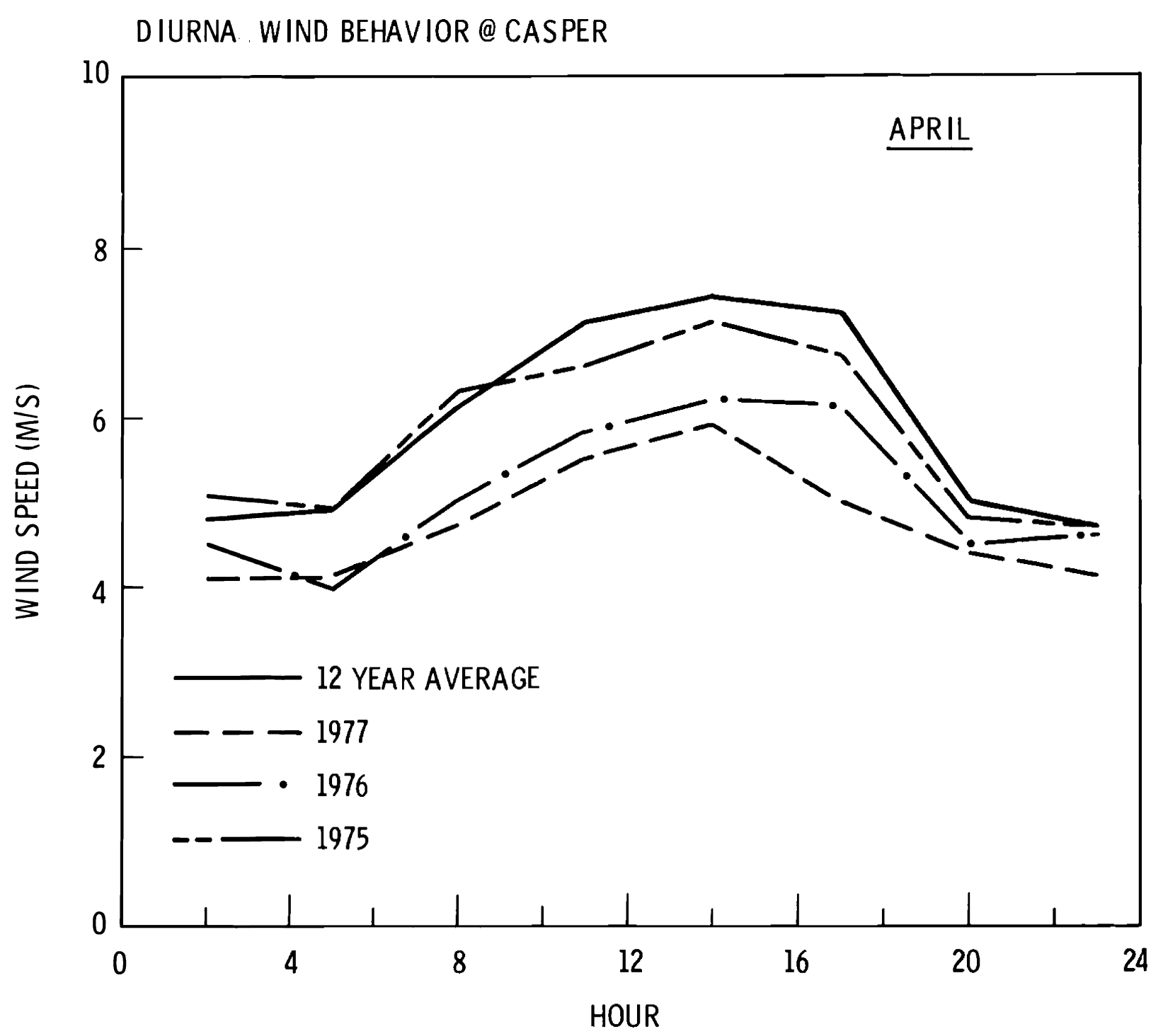

FIGURE A-3.17 contd.) 


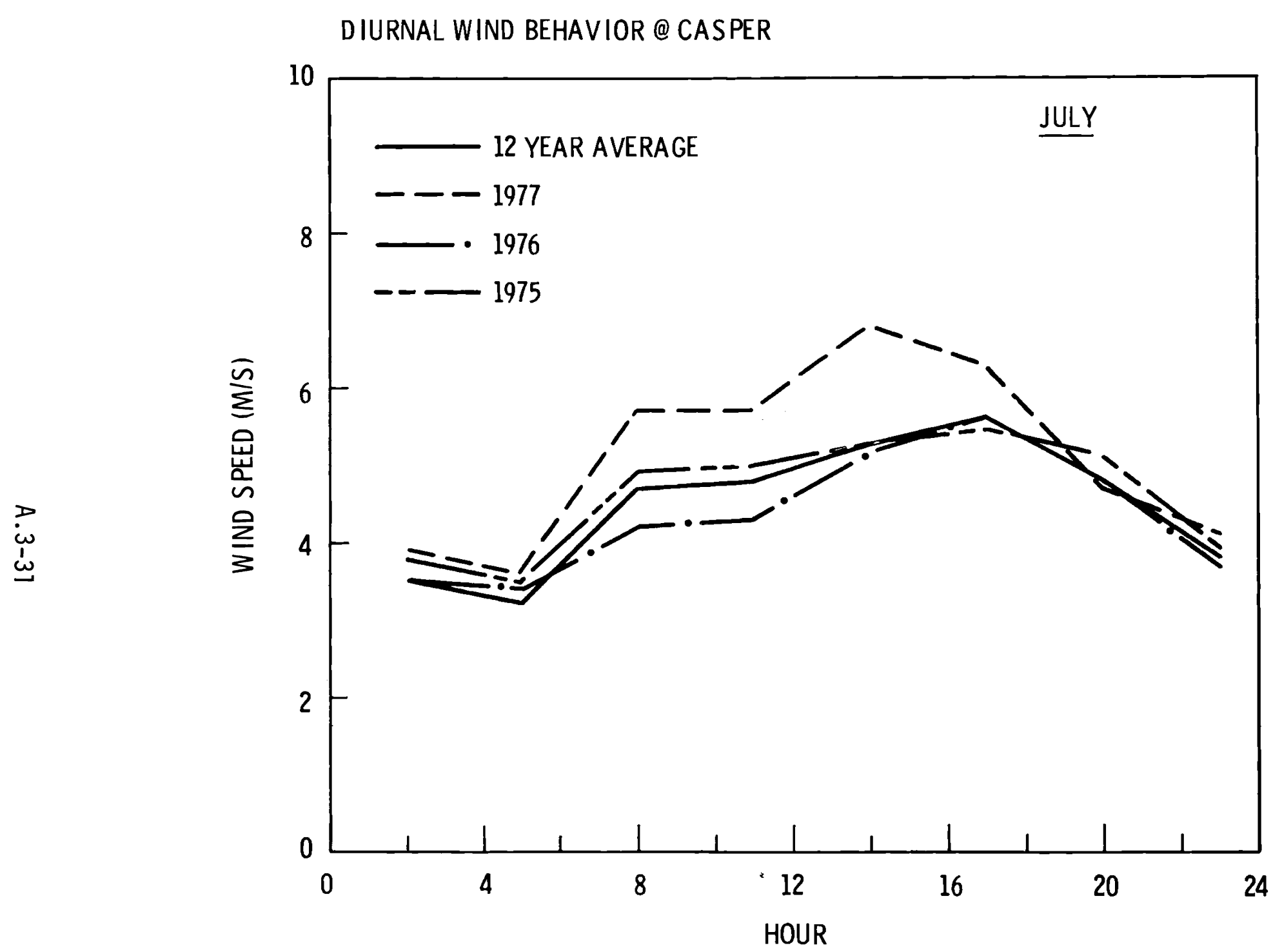

FIGURE A-3.17 (contd.) 
DIURNALWINDBEHAVIOR @ CASPER

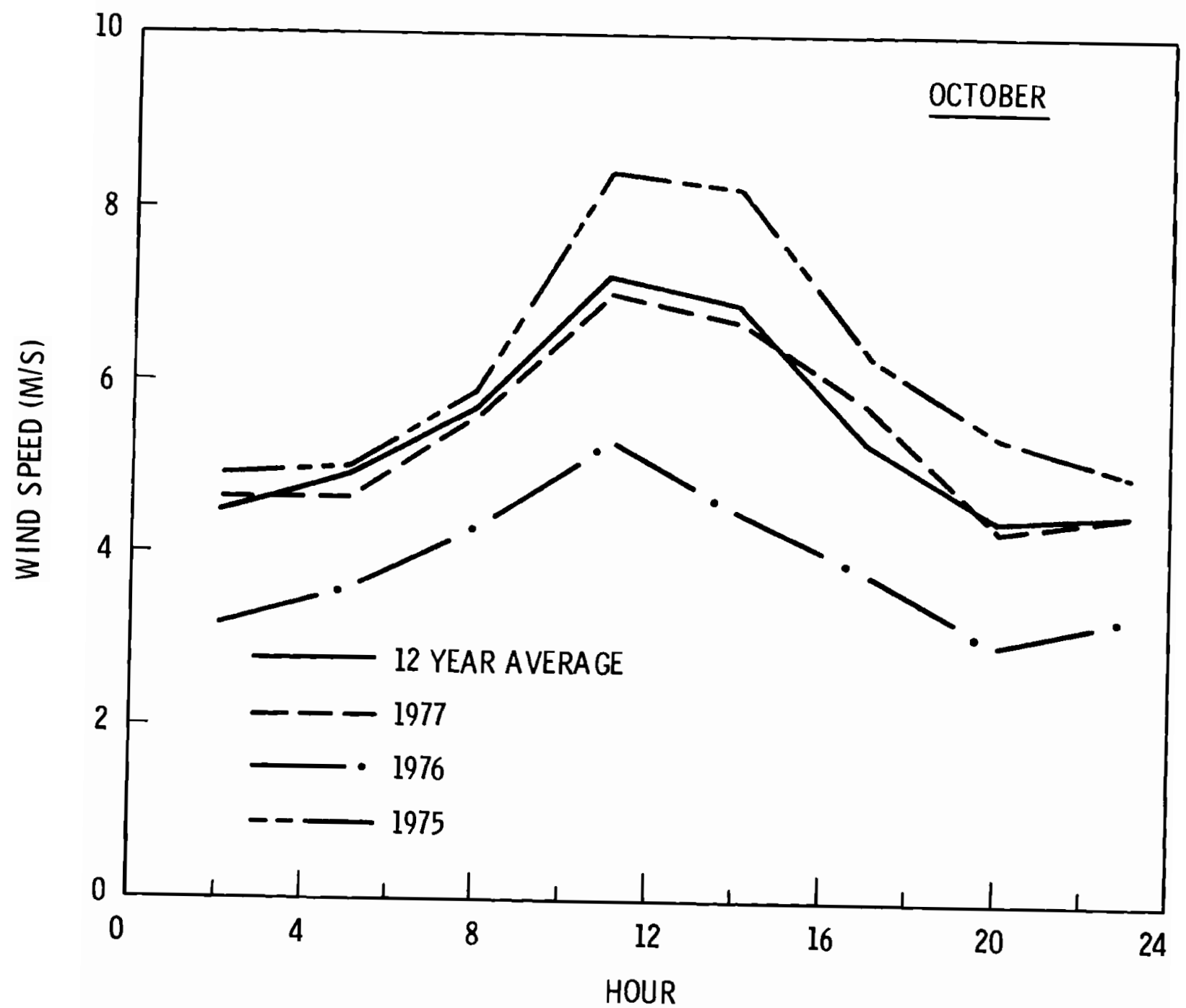

FIGURE A-3.17. (contd) 
Wind measurements were assumed to have been made during the windiest season (March, April, and May) and the method of adjustment was:

$$
\begin{aligned}
& d=\frac{x^{\prime}-y^{\prime}}{y^{\prime}} \\
& y=\frac{x}{1+d}
\end{aligned}
$$

where $x=$ short-term average at Glasgow AFB (March, April, May)

$X^{\prime}=$ simultaneous short-term average at Glasgow Int. (the historical site)

$y=$ estimated $5-y r$ mean at Glasgow AFB

$y^{\prime}=$ actual $5-y r$ mean at Glasgow Int.

$d=$ correction factor.

\begin{tabular}{|c|c|c|c|c|}
\hline Year & $\begin{array}{l}\text { Average Wind } \\
\text { Speed, } \mathrm{m} / \mathrm{sec}\end{array}$ & $\begin{array}{c}\text { Difference From } \\
5-Y r \text { Mean } \\
\end{array}$ & $\begin{array}{l}\text { Adjusted Wind } \\
\text { Speed, m/sec }\end{array}$ & $\begin{array}{c}\text { Difference From } \\
5-Y r \text { Mean } \\
\end{array}$ \\
\hline 1963 & 4.2 & -0.1 & 4.2 & -0.1 \\
\hline 1964 & 5.1 & +0.8 & 4.3 & 0 \\
\hline 1965 & 4.5 & +0.2 & 4.4 & +0.1 \\
\hline 1966 & 5.2 & +0.9 & 4.3 & 0 \\
\hline 1967 & 4.8 & +0.5 & 4.5 & +0.2 \\
\hline
\end{tabular}

TABLE A-3.2. Adjusting Short-Term Records at Glasgow AFB(a)

(a) Months used were March, April, and May. The 5-yr average (1963-1967) was $4.3 \mathrm{~m} / \mathrm{sec}$ for Glasgow AFB and $4.9 \mathrm{~m} / \mathrm{sec}$ for Glasgow Int., the historical site.

If this same technique is applied to a pair of stations having poorer correlation, the results are not as good (see Table A-3.3). In this example the 7-yr average wind speed at Whitehall was estimated using Butte as the historical station and assuming measurements were made during January, February and March. 
TABLE A-3.3. Adjusting Short-Term Records at Whitehall (a)

\begin{tabular}{lccccc}
$\underline{\text { Year }}$ & $\begin{array}{c}\text { Average Wind } \\
\text { Speed, m/sec }\end{array}$ & $\begin{array}{c}\text { Difference From } \\
\text { 7-Yr Mean }\end{array}$ & $\begin{array}{c}\text { Adjusted Wind } \\
\text { Speed, m/sec }\end{array}$ & $\begin{array}{c}\text { Difference From } \\
\text { 7-Yr Mean }\end{array}$ \\
\hline 1948 & 5.4 & -0.5 & & 7.2 & \\
1949 & 5.5 & -0.4 & & 6.8 & 1.3 \\
1950 & 6.6 & +0.7 & 6.7 & 0.9 \\
1951 & 6.5 & +0.6 & 7.8 & 0.8 \\
1952 & 5.5 & -0.4 & 9.2 & 1.9 \\
1953 & 6.1 & +0.2 & & 7.4 & 3.3 \\
1954 & 5.6 & -0.3 & 6.7 & 1.5
\end{tabular}

(a) Months used were January, February, and March. The 7-yr average (1948-1954) was $5.9 \mathrm{~m} / \mathrm{sec}$ at Whitehall and $3.8 \mathrm{~m} / \mathrm{sec}$ at Butte, the historical site.

There are several problems with climatological adjustment techniques such as the one described above. First, it is difficult to accurately estimate long-term averages with less than $10 \mathrm{yr}$ of data at the historical site. Few stations have wind records exceeding $10 \mathrm{yr}$, particularly in the western United States. Secondly, the correlation between two sites cannot be estimated accurately by short-term comparisons.

Wind speed correlations can be affected by seasons. For example, at temperate latitudes winter winds are driven by large-scale pressure patterns associated with large storms. Therefore, the correlation between a coastal site and a weather station in the interior could be substantially higher in winter than in summer when winds on the coast are greatly affected by the sea breeze.

The interannual variability of the correlation between sites can also be large. Table A-3.4 summarizes year-to-year variations in the correlation of monthly mean wind speeds for two sets of sites in Montana--one for which the correlation is higher and one for which it is lower. For the two stations 
near Gl asgow, year-to-year changes in the correlation coefficient ( $r$ ) were small (with the exception of 1963). For the uncorrelated pair, $\mathbf{r}$ varied from -0.38 to +0.54 reflecting the large variance in monthly means at the Whitehall site.

TABLE A-3.4. Variation of Yearly Estimates of the Correlation Coefficient of Monthly Mean Wind Speed Between Two Stations

$$
\text { Glasgow AFB and Glasgow Int. }
$$

Correlation Coefficient Over 60 Months: $\quad r=0.79$

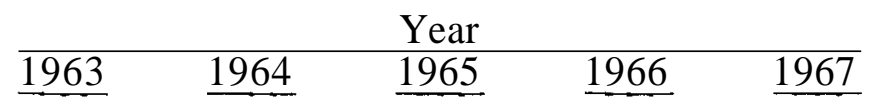

Correlation Coefficient

$\begin{array}{lllll}0.59 & 0.81 & 0.78 & 0.89 & 0.85\end{array}$

$\underline{\text { Whitehall and Butte }}$

Correlation Coefficient Over 84 Months: $\quad r=0.12$

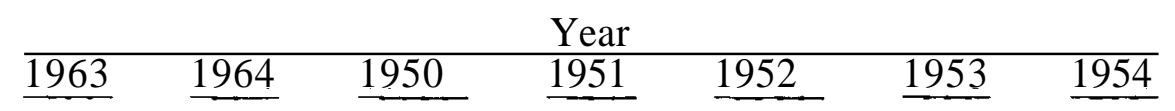

Correlation

Coefficient

$\begin{array}{lllllll}0.27 & 0.23 & -0.38 & 0.01 & -0.20 & 0.54 & 0.03\end{array}$

\section{A.3.5 CONCLUSIONS}

Wind characteristics critical to the performance of wind energy conversion systems can show considerable spatial and temporal variation. Spatial variability in the horizontal is most important when a large area is screened for viable wind energy conversion sites. The spatial variability of the wind has both positive and negative aspects. When wind turbines or wind turbine clusters are placed at neighboring sites with low or even negative correlation, the reliability of the wind generation system increases. This, of course, is a positive aspect of spatial variability. The negative aspect is that existing wind data cannot be used reliably to predict wind behavior at adjacent sites. 
This aspect magnifies the site selection problem. Temporal variability is most important once potential sites are chosen. Since it governs the temporal characteristics of energy production and the length of time, site-specific measurements may have to be made.

The vertical variation of wind characteristics is more important than horizontal variability because of the problems it presents in wind turbine siting. Since wind behavior is observed to change significantly over vertical distances comparable to the size of large wind turbines, such critical characteristics as the diurnal modulation of wind speed cannot be inferred from surface wind measurements. Information on diurnal load matching will require wind data representative of the average wind speed across the rotor disk of the machine. For large, horizontal-axis machines, wind data from at least as high as hub height will be required.

Interannual variability (and the sensitivity of economic value to this variability) dictates how long wind data must be sampled at a particular site. The amount of interannual variability varies with the particular wind characteristic of interest. The cost of energy, for example, depends on the annual average of the energy produced by a machine at a particular site. Both a study by Doran et al. (1977) and experience in the DOE candidate site evaluation program have shown estimates of annual average energy production to correlate very well with average annual wind speed. Since the annual mean wind speed is reasonably well defined by a single year of measurements, estimates of average annual energy production will be reasonably well known with a year of on-site data.

The value of energy, however, is determined by load matching, which is governed by the seasonal and diurnal characteristics of the wind flowing through the rotor disk. If wind-generated electricity is to be of the greatest value, it must be consistently available during the times of peak demand. However, a single year's data is not sufficient to establish the seasonal and diurnal characteristics of the wind. How accurately these characteristics must be known will undoubtedly depend on the load characteristics and generating mix of the utility. 


\section{REFERENCES}

Barchet, W. R. and D. L. Elliott. 1979. "Pacific Northwest Regional Assessment." In Proceedings of the Conference and Workshop on Wind Energy Characteristics and Wind Energy Siting 1979. American Meteorological Society, Boston, Massachusetts.

Corotis, R. B., A. B. Sigl and M. P. Cohen. 1977. "Variance Analysis of Wind Characteristics for Energy Conversion." J. Appl. Meteor. 16:1149-1157.

Crawford, K. C. and H. R. Hudson. 1973. "The Diurnal Wind Variation in the Lowest $1500 \mathrm{Ft}$ i n Central Oklahoma: June 1966 - May 1967." J. Appl. Meteor. 12:127-132.

Doran, J. C., J. A. Bates, P. J. Liddell and T. D. Fox. 1977. Accuracy of Wind Power Estimates. PNL-2442, Pacific Northwest Laboratory, Richland, Washington.

Elliott, D. L. and W. R. Barchet. 1980. Wind Energy Resource Atlas: The Northwest Region. PNL-3195, WERA-1, Pacific Northwest Laboratory, Richland, Washington.

Glasgow, J. C. and W. H. Robbins. 1979. "Utility Operational Experience on the NASA/DOE MOD-CA 200-kW Wind Turbine." In Proceedings of the Workshop on Economic and Operational Requirements and Status of Large Wind Systems. EPRI ER-1110-SR (DOE Conf. \#790352), ATtas Corp., Santa Cruz, California.

JBF Scientific Corp. 1979. Vol. 1 of Wind Energy Systems Application to Regional Utilities. JBF Scientific Corp., Wilmington, Massachusetts.

Marsh, W. D. 1979. Vols. 1, 2, 3 of Requirements Assessment of Wind Power Plants in Electric Utility Systems. EPRI ER-978-SY, General Electric Company, Schenectady, New York.

Neustadter, H. E. 1979. "The Use of Wind Data With an Operation Wind Turbine in a Research and Development Environment." In Proceedings of the Conference and Workshop on Wind Energy Characteristics and Wind Energy Siting 1979. American Meteorological Society, Boston, Massachusetts.

Ramsdell, J. V., S. Houston and H. L. Wegley. 1979. Mleasurement Strategies for Estimating Long-Term Average Wind Speeds. PNL-3348, Pacific Northwest Laboratory, Richland, Washington. 


\section{APPENDIX 4}

\section{ESTIMATING AVERAGE WIND SPEEDS FROM AVERAGE SAND TRANSPORT RATES}




\section{APPENDIX 4 \\ ESTIMATING AVERAGE WIND SPEEDS RROM AVERAGE SAND TRANSPORT RATES}

Bagnold's theory of sand transport suggests that the mass transport rate, q, per unit width of path in $\mathrm{kg} \mathrm{m}^{-1} \mathrm{sec}^{-1}$ is given by:

$$
\begin{aligned}
q & =\frac{C \rho}{g}\left(\frac{d}{D}\right)^{\frac{1}{2}} u_{*}^{3} & & u_{*} \geq u_{*} \\
& =0 & & u_{*}<u_{*} .
\end{aligned}
$$

The average rate of sand transport, $\bar{q}$, may be computed from the following convolution:

$$
\bar{q}=\int_{u_{\star} T}^{\infty} q\left(u_{\star}\right) p\left(u_{\star}\right) d u_{\star}
$$

where $p\left(u_{*}\right)$ is the probability density function of $u$ If a probability density function is assumed then $\bar{q}$ may be determined as a function of the probability density function parameters. As a reasonable approximation, we will assume that $p\left(u_{*}\right)$ is given by a Rayleigh distribution. This may not be the best assumption, as discussed in Section 7.2.2; however, only efforts to obtain approximate results are warranted due to the uncertainties in the theory of sand transport. Since the Rayleigh distribution depends on just one parameter, the mean of the distribution $\bar{u}_{*}$, we look to discover a functional relationship of the form

$$
\bar{q}=f\left(\bar{u}_{\star}, u_{\star}\right)
$$

Plugging the Rayleigh distribution into Equation (A-4.2) (we shall drop the asterisk for convenience but recall that we are speaking of friction velocities) one obtains:

$$
\bar{q}=A \int_{u_{T}}^{\infty} u^{4} e^{-\alpha u^{2}} d u
$$


where

$$
\begin{aligned}
& \alpha=\frac{\pi}{4 \vec{u}^{2}} \\
& A=\frac{C \rho}{g}\left(\frac{d}{D}\right)^{\frac{1}{2}} 2 \alpha .
\end{aligned}
$$

The terms in Equation $(\mathrm{A}-4.6)$ are defined in Chapter 7.

To evaluate the integral in $(A-4.4)$ we will perform the integration in terms of easily solved definite integrals, i.e.,

$$
I=\int_{u_{T}}^{\infty} u^{4} e^{-\alpha u^{2}} d u=\int_{0}^{\infty} u^{4} e^{-\alpha u^{2}} d u-\int_{0}^{u_{T}} u^{4} e^{-\alpha u^{2}} d u .
$$

Consider the integral

$$
I(n)=\int_{0}^{x} u^{n} e^{-r \cdot u^{2}} d u
$$

Note that by differentiating with respect to a we obtain the following recursion relation

$$
I(n)=-\frac{\partial}{\partial \alpha} \int_{0}^{x} u^{n-2} e^{-\alpha u^{2}} d u=-\frac{\partial I(n-2)}{\partial \alpha}
$$

Let

$$
\begin{aligned}
u & =\alpha^{-\frac{1}{2}} y \\
d u & =\alpha^{-\frac{1}{2}} d y \\
z & =\alpha^{\frac{1}{2}} x=\alpha^{\frac{1}{2}} u_{T}
\end{aligned}
$$

so that Equation $(\mathrm{A}-4.8)$ becomes

$$
I(n)=\alpha\left\{\frac{-(n+1)}{2}\right\} \int_{0}^{z} y^{n} e^{-y^{2}} d y .
$$


Then we can see that

$$
I(0)=\alpha^{-\frac{1}{2}} \int_{0}^{z} e^{-y^{2}} d y=\frac{\sqrt{\pi}}{2} \alpha^{-\frac{1}{2}} \operatorname{erf} z
$$

where the error function is defined as

$$
\operatorname{erf} Z \equiv \frac{2}{\sqrt{\pi}} \int_{0}^{Z} e^{-y^{2}} d y
$$

The error function is computed numerically and tabulated in handbooks of mathematical functions. In the 7 imit as $Z$ approaches infinity the error function approaches 1 , so that

$$
\int_{0}^{\infty} e^{-y^{2}} d y=\frac{\sqrt{\pi}}{2}
$$

It is straightforward to determine $1(1)$ as

$$
\begin{aligned}
I(1) & =\alpha^{-1} \int_{0}^{z} y e^{-y^{2}} d y \\
& =\frac{1}{2} \alpha^{-1}\left[1-e^{-z^{2}}\right] .
\end{aligned}
$$

To evaluate Equation (A-4.7) we need I(4), which by Equation (A-4.9) may be determined as

$$
\begin{aligned}
I(4) & =\frac{\partial^{2} I(0)}{\partial \alpha^{2}} \\
& =\frac{3}{8}^{\sqrt{\pi}} \alpha-5 / 2 \text { erf } z-\frac{3 u_{T}}{4} \alpha^{-2} e^{-z^{2}}-\frac{u_{T}{ }^{3}}{2} \alpha^{-1} e^{-z^{2}} .
\end{aligned}
$$


Since

$$
\int_{0}^{\infty} e^{-y^{2}} d y=\frac{\sqrt{\pi}}{2}
$$

it is also easily shown that

$$
\int_{0}^{\infty} u^{4} e^{-\alpha u^{2}} d u=\frac{3}{8} \sqrt{\pi} \alpha^{-5 / 2}
$$

We can now write Equation (A-4.4) in its simplest form, using Equations (A-4.5) through $(A-4.7),(A-4.12),(A-4.18)$ and $(A-4.20)$, as:

$$
\bar{q}=\frac{C \rho}{g}\left(\frac{d}{D}\right)^{\frac{1}{2} u_{\star}}{ }^{3}\left\{\frac{6}{\pi} \beta^{3}\left[1-\operatorname{erf}\left(\frac{\sqrt{\pi}}{2 \beta}\right)\right]+\left(1+\frac{6}{\pi} \beta^{2}\right) \exp \left(-\frac{\pi}{4 \beta^{2}}\right)\right\}(A-4.21)
$$

where

$$
\beta=\bar{u}_{\star} / u_{\star} T
$$

The quantity in braces can be defined as:

$$
f(\beta)=\frac{6}{\pi} \beta^{3}\left[1-\operatorname{erf}\left(\frac{\sqrt{\pi}}{2 \beta}\right)\right]+\left(1+\frac{6}{\pi} \beta^{2}\right) \exp \left(-\frac{\pi}{4 \beta^{2}}\right) .
$$

This function is plotted in Figure 7.6; the use of this figure is discussed in Chapter 7 .

In a similar way a function could be generated to describe the average sand transport rate according to the theory of Lettau et al. (1978). We have chosen to use Bagnold's theory because of its simplicity and completeness, and in consideration of the very gross approximations and assumptions in the use of sand transport rate measurements of climatological wind speed. 


\section{REFERENCES}

Lettau, H. H. and K. Lettau, eds. 1978. Exploring the World's Driest Climate. IES Report 101, Center for Climatic Research, Institute for Environmental Studies, University of Wisconsin, Madison, Wisconsin. 
GLOSSARY 


\section{GLOSSARY}

ADIABATIC LAPSE RATE - The rate of decrease of temperature with height of a parcel of air lifted adiabatically (without external heat addition; temperature changes as internal energy is converted into work done against the environment) through an atmosphere in hydrostatic equilibrium. For dry air this is equal to $g / C_{P}$ where $g$ is the acceleration of gravity and $C_{P}$ is the specific heat of air.

ADVECTION - The process of transport of an atmospheric property solely by the mass motion of the atmosphere; e.g., the advection of temperature $(T)$ by the mean wind $(\vec{V})$ is described by $-\vec{V} \cdot \nabla T$.

ANABATIC WIND - An upslope wind; usually applied only when the wind is blowing up a hill or mountain as the result of local surface heating, and apart from the effects of the larger-scale circulation.

ANALYSIS - Used in this work to signify a detailed examination of the state of the atmosphere based on actual observations, usually including a separation of the field being analyzed into component patterns and involving the drawing of families of isopleths for various elements, and usually subject to constraints required by the physical laws governing the atmosphere (e.g., conservation of mass).

ANEMOMETER, - An instrument to measure wind speed.

BAROCLINIC ATMOSPHERE - An atmosphere in which the density depends on both the temperature and the pressure. In a baroclinic atmosphere the geostrophic wind generally has vertical shear, and this shear is related to the horizontal temperature gradient by the thermal wind equation.

BETZ LIMIT - The limiting efficiency of an ideal windmill extracting energy from air flowing through the windmill. The Betz limit is $16 / 27=59 \%$.

BLOCKING - The process whereby a barrier to stratified flow prevents the dense fluid layers nearest the surface from crossing the barrier because of insufficient kinetic energy. 
BOUNDARY LAYER - The layer of the atmosphere where the direct effect of turbulent transfer of heat and momentum is observable. Above the boundary layer the free atniosphere is in approximate geostrophic or gradient wind balance.

CAPACITY FACTOR - The ratio of the average power load of an electric power plant to its rated capacity. Also known as plant factor.

CIRCULATION - The line integral of the tangential component of the velocity field about a closed path. The circulation (C) is also equal to the average normal component of the vorticity $(\zeta)$ times the area enclosed by the loop; i.e.,

$$
c=\oint \vec{V} \cdot d 1=\zeta A .
$$

CLUSTER - A group of any number of wind turbines sited within a sufficiently close proximity that they may be considered, for most purposes, as a single power producing unit; also called a WECS farm.

COEFFICIENT OF PERFORMANCE - See Power Coefficient

CONIROL STRATEGY - The algorithm of the wind turbine microprocessor that takes wind data and wind turbine status or performance data to determine what, if any, changes in machine operation should be initiated (e.g., startup, shutdown, yaw maneuver, pitch control).

CONVECTION - Atmospheric motions initiated or perpetuated by buoyancy forces.

CORIOLIS EFFECT - The deflection relative to the earth's surface of any object moving above the earth; an object moving horizontally is deflected to the right in the northern hemisphere, to left in the southern. Therefore, air accelerating toward low pressure in the northern hemisphere is eventually deflected toward the right so that the air moves along lines of constant pressure with low pressure on the left. This is geostrophic balance.

CORIOLIS PARAMETER - Denoted by the symbol $\mathbf{f}$.

$f=2 \Omega \sin \phi$ where $\Omega=$ the angular velocity of the earth, $\phi$ is the latitude. The magnitude of the horizontal component of the Coriolis force per unit mass on a horizontally moving fluid parcel is equal to the product of the Coriolis parameter and the speed of the parcel. 
COST OF ENERGY - A minimum levelized price that must be charged for energy consumed to recover expenses for purchasing, installing, owning, operating, and maintaining an energy producing facility. Distinct from value of energy.

CUT-IN - The wind speed at which the wind turbine control system initiates startup procedures.

CUT-OUT - Cut-out is the wind speed at which the wind turbine control system initiates shutdown procedures.

DIAGNOSTIC EQUATION - Any equation governing a system which contains no time derivative and therefore specifies a balance of quantities in space at a moment of time.

DRY ATMOSPHERE - An atmosphere in which condensation of water vapor has not or is not taking place and therefore where the thermodynamics of transition between vapor, liquid and ice phases of water may be ignored.

DYNAMICAL SIMILARITY - A relation between two systems (one of which may be a model) such that by proportional alterations of the units of length, mass, time, etc., measured quantities in one system go identically (or with a constant multiple for each) into those in the other; in particular, this implies constant ratios of forces in the two systems.

EINSTEIN SUMMATION CONVENTION - A notational convenience used in vector and tensor analysis whereupon it is agreed that any term in which an index appears twice will stand for the sum of all such terms as the index assumes all of a preassigned range of values.

EULERIAN EQUATION - A mathematical representation of the motions of a fluid in which the behavior and the properties of the fluid are described at fixed points in a coordinate system. The properties of a fluid are assigned to points in space at each given time, without attempt to identify individual fluid parcels from one time to the next; as opposed to a Lagrangian description of flow. For example, an anemometer at a fixed point makes an Eulerian measurement of wind speed. 
FLAGGED TREE - A tree, deformed by the wind, with characteristic crown asymmetry.

FLAT - In the micrometeorological sense terrain may be considered flat only if topographical protrusions or slopes have no significant influence on the statistics of interest (e.g., wind speed or wind shear) throughout the region of interest (e.g., the height of a wind turbine or the dimensions of a cluster). The criteria for flatness may be a function of stability.

ROW SEPARATION - Flow is said to separate from an object when upstream of the separation point the streamline near the surface follows the surface whereas at the separation point the streamline departs from the surface. Downstream of the separation point and beneath the separation streamline a reverse flow eddy forms. At some distance further downstream the separation streamline comes back to the surface, follows the surface, and the flow is said to be reattached.

Flow separation may be caused when fluid momentum, depleted by downward momentum transport, is insufficient to allow the flow to proceed into regions of increasing pressure, or when the inertia of the flow passing over a sharp edge does not enable the flow to negotiate the sudden change in surface contour.

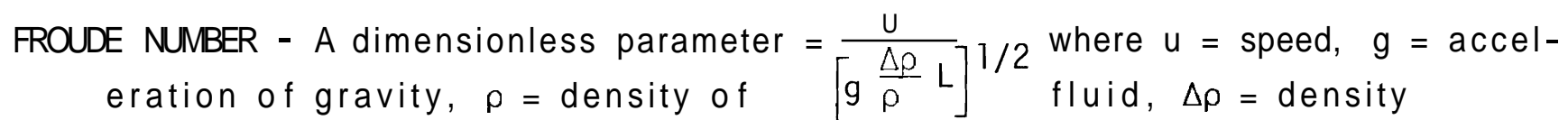
difference between two layers of fluid, and $L$ is a characteristic length scale. This parameter is a ratio of inertia to buoyancy forces, or equivalently a ratio of fluid speed to gravity wave speed.

GEOMETRICAL SIMILARITY - Property of two fluid flows for which a simple alteration of scales and length and velocity transforms one into the other.

GEOSTROPHIC WIND - The horizontal wind velocity for which the Coriolis acceleration exactly balances the horizontal pressure gradient force; i.e.,

$$
\vec{v}_{g}=\hat{k} \times \frac{1}{\rho f} \nabla_{H} P
$$

where $\vec{V}_{g}$ is the geostrophic wind vector, $\nabla_{H} P$ is the horizontal pressure gradient, $k$ is a vertical unit vector, $\rho$ is density, and $f$ is the Corio1 is parameter. 
GRAVITY WAVE - A wave in a fluid medium in which restoring forces are primarily due to buoyancy (that is, gravity) rather than by conipression.

HEAT FLUX - In the context used here this usually means turbulent transfer of heat either up or down within the planetary boundary layer.

HISTOGRAM - A graphical representation of a distribution function by means of rectangles whose widths represent intervals into which a range of observed values is divided and whose heights represent the number of observations occurring in each interval.

HORIZONTAL AXIS WIND TURBINE - A wind turbine in which the rotor rotates about an axis that is horizontal and parallel to the wind. The axis is at hub height above ground.

HUB HEIGHT - The height above ground of the axis of a horizontal axis wind turbine. It is frequently assumed that hub height winds may be used to construct an adequate representation of the winds affecting the entire wind turbine. For a vertical axis wind turbine such a height would be midway between the top and bottom of the rotor.

HYDRAULIC JUMP - A steady state, finite-amplitude disturbance in a channel, in which water passes turbulently from a region of (uniform) low depth and high velocity to a region of (uniform) high depth and low velocity. The atmosphere analog is the pressure jump where dense air beneath the inversion is the fluid, and the inversion height changes.

HYDROSTATIC APPROXIMATION - The assumption that the atmosphere is in hydrostatic equilibrium, i.e., that the pressure at a point is solely due to the weight of the air mass above that point. This assumption holds if vertical accelerations are small compared to the acceleration of gravity.

INERTIAL FORCE - The fictitious force acting on a body as a result of using a noninertial frame of reference; examples are centrifugal and Coriolis forces that appear in the rotating earth coordinate system. 
INERTIAL OSCILLATION - When the force balance on a fluid parcel changes quickly there is a component of the flow that is not in equilibrium with the forces upon that parcel, i.e., due to the inertia of the fluid equilibrium is not achieved instantaneously. This component, with no real forces on it in an inertial frame of reference, experiences only the fictitious Coriolis and centrifugal forces in the rotating frame of reference. The unbalanced component oscillates at a point with a period of $2 \pi \div$ Coriolis parameter.

INTERNAL BOUNDARY LAYER - Within the planetary boundary layer when discrete changes in the surface occur (e.g., roughness, surface temperature) the effects of the change are observed within an internal boundary layer which grows downstream of the change.

INVERSION - See Temperature Inversion.

ISOPLETH - A line of constant value of a given quantity with respect to either space or time.

KATABATIC WIND - A wind (or component thereof) directed down the slope of an incline and caused by greater air density near the slope than at the same levels some distance horizontally from the slope. Also known as drainage wind.

KELVIN-HELMHOLTZ WAVE - An unstable were forming in a system of two layers of fluid with a velocity discontinuity at the interface. The unstable wave gains kinetic energy at the expense of the kinetic energy of the winds in each layer. Ultimately the wave breaks, like an ocean wave, and becomes turbulent. Kelvin-Helmholtz waves occur in the atmosphere at inversion surfaces where there is high wind shear.

KINEMATIC SIMILARITY - A relationship between fluid flow systems in which corresponding fluid velocities and velocity gradients are in the same ratios at corresponding locations.

L - See Obukhov Length. 
LAGRANGIAN DESCRIPTION - A description of fluid motion in which one considers volume elements which are carried along with the fluid, and across whose boundaries material does not flow; in contrast to the Eulerian description. For example, following the motion of a free balloon provides a Lagrangian measurement of the wind field.

LAPSE RATE - The rate of change with height of temperature or potential temperature.

LARGE SCALE TERRAIN - Defined for this document to be terrain in which the vertical relief is a significant fraction of the boundary layer depth or exceeds the boundary layer depth.

LEE WAVE - A gravity wave train which is excited by a mountain or ridge and extends downwind, in the lee, of the mountain. Wave clouds frequently form at the crest of each wave.

LEVELIZATION - The process by which a series of nonuniform future payments is converted to a uniform (level) series of payments whose present value is equal to that of the original nonuniform series.

LOAD MATCH - The phase relationship between the load on the utility network and the wind generated power; i.e., wind generated power peaking in the afternoon with maximum load occurring in the afternoon is a good load match. Load matching also applies to seasonal wind and wind variations.

LOG LAW - In this document log law refers to a wind profile in which the wind speed increases with the logarithm of height, or to wind profile descriptions that modify the logarithmic description to account for stability effects.

MEAN WIND - The definition varies with context. If no averaging interval is specified such as annual mean wind the reference is probably indicative of an averaging interval just long enough to filter out the turbulence in the wind, e.g., 15 min to $1 \mathrm{hr}$. 
MESOSCALE - A scale of atmospheric motions that are smaller than synoptic scale but larger than microscale; i.e., horizontal scales between $1 \mathrm{~km}$ and hundreds of $\mathrm{km}$. Mesoscale meteorology is especially difficult because the mesoscale flow interacts with the synoptic scale and the microscale in ways that are poorly understood.

MICROSCALE - The scale of motions in the atmosphere associated with turbulence; i _.e., scales smaller than about $1 \mathrm{~km}$.

MIXED LAYER - The portion of the planetary boundary layer in unstable conditions above the surface layer and below the inversion where convection couples (mixes) all layers resulting in small gradients of potential temperature, wind speed, and wind direction.

MOMENTUM FLUX - In the context used here this means turbulent transfer of momentum within the planetary boundary layer. However, momentum may also be transported by gravity waves in a nonturbulent flow.

NEUTRAL STABILITY - Locally, neutral stability exists when the vertical gradient of potential temperature equals zero so that perturbed parcels will not be accelerated or retarded by buoyancy forces. However, in a mixed layer the lapse rate of potential temperature may equal zero over much of the mixed layer but the boundary layer would not be considered neutrally stable since there is an upward heat flux originating from the unstable surface layer.

NOCTURNAL JET - A low-level wind speed maximum that forms at the top of the nocturnal boundary layer.

NONDIMENSIONAL PARAMETER - A ratio of various physical properties (such as density, heat capacity) and conditions (wind speed, temperature) of such nature that the resulting number has no defining units of length, time, etc. Study of fluid flows, where no exact solutions are possible, may be simplified by transforming measured solutions for one flow into solutions for another flow, if the two flows have identical values of the nondimensional parameters which govern the flow, such as the Reynolds number, Froude number, and $z / L$ where $z$ is height and $L$ is the Obukhov length. See Similarity, Dynamical Similarity, Kinematic Similarity, Geometrical Similarity. 
NUMERICAL MODELING - The forecasting or diagnosis of the behavior of atniospheric disturbances by the numerical solution of the governing fundamental equations of hydrodynamics, subject to observed initial and/or boundary conditions. See Diagnostic Equation, Prognostic Equation, and Primitive Equations.

OBJECTIVE ANALYSIS - Analysis of the flow field from a few observations where the relation of flow variables at separate points in space or time is constrained to satisfy governing equations, which may or may not include all relevant physics. This is opposed to a subjective analysis where the individual performing the analysis relies, as best he can, on his understanding of all of the governing physics to guide his interpretation of flow features between observations.

OBUKHOV LENGTH - A length scale which relates the rate of shear production of turbulence kinetic energy to buoyancy production of turbulence kinetic energy. $z / L$ and $h / L$ where $z$ is the height above ground and $h$ is the height of the boundary layer are fundamental nondimensional parameters of turbulent boundary layer flow.

PHYSICAL MODELING - The attempt to measure solutions to a small scale-flow, e.g., in a wind tunnel or a water towing tank, so that the measured solutions may be applied to full-scale flow features. Physical modeling requires strict attention to preserving dynamical, kinematic and geometrical siniilarity between model and full scale-flow.

PITCH CONTROL - The mechanisms by which the change of the orientation of the wind turbine blade relative to the wind is achieved, thereby altering the aerodynamic efficiency of the blade.

PLANETARY BOUNDARY LAYER (PBL) - See Boundary Layer.

PLANT FACTOR - Same as Capacity Factor.

POTENTIAL TEMPERATURE - The temperature that a parcel of a i $r$ would have i $\mathbf{f}$ it were brought adiabatically (i.e., without external heat addition) to a pressure of $1000 \mathrm{mb}$. Potential temperature is a conservative property (i.e., does not change) for vertical motion of short-time scales where condensation has not taken place. 
POWER COEFFICIENT - The ratio of the energy converted to the wind energy intercepted. This can be a power coefficient of a rotor, specifying the efficiency of the rotor, or a power coefficient of the entire system, including all losses from aerodynamic to mechanical to electrical.

POWE CURVE - The curve specifying the power produced by a wind turbine when the turbine is synchronized with the grid as a function of wind speed (measured at hub height, for example).

POWER LAW - A description of the variation of wind speed with height used extensively in the engineering community where the ratio of wind speeds at two heights is equal to the ratio of the two heights raised to some power. This is opposed to the log law used extensively by the meteorological research community.

PRESENT VALUE - The principal of a sum of money payable at a future date that upon drawing interest at a given rate will equal the sum at the date on which this sum is to be paid. Present value (PV) calculations compare investment options against each other and against investing the money at the highest available interest rate. For example, at $8 \%$ interest the PV of an investment option that yields $\$ 200$ at the end of 1 year and $\$ 100$ at the end of the second year is:

$$
\mathrm{PV}=\left(\frac{1}{1.08}\right) 200+\left(\frac{1}{1.08}\right)^{2} 100=\$ 271 .
$$

The PV of a second option yielding $\$ 40$ at the end of 1 year and $\$ 270$ at the end of 2 years is $\$ 269$. Therefore, the first option is the superior of the two investments and if it can be purchased for less than $\$ 271$, is also superior to investing at $8 \%$ per year.

PRIMITIVE EQUATIONS - The Eulerian equations of motion of a fluid in which the primary dependent variables are the fluid's velocity components. In meteorology these equations are frequently specialized to apply directly to large-scale motions by the introduction of filtering approximations. PROBABILITY DENSITY FUNCTION (PDF) - A function whose integral over any set gives the probability that a random variable has values in this set. Also known as density function, frequency distribution. 
PROFILE - A graphic representation of the variation of one property, such as wind speed or temperature, with respect to another property such as distance or position.

PROGNOSTIC EQUATION - Any equation governing a system which contains a time derivative of a quantity and therefore can be used to determine the value of that quantity at a later time when other terms in the equation are known.

RADIOSONDE - A balloon-borne instrument for the siniultaneous measurement and transmission of atmospheric pressure, temperature and humidity.

RATED WIND SPEED - The wind speed at which the power output of a wind turbine reaches its rated value.

RAWINSONDE - A radiosonde system with the additional capability of evaluating wind speed and direction aloft by means of tracking by radar or radio direction finder.

RAYLEIGH DISTRIBUTION - A unimodal one parameter probability density function described by:

$$
p(x)=\frac{\pi x}{2 x^{2}} \exp \left[-\pi x^{2} / 4 \bar{x}^{2}\right]
$$

where $\bar{x}$ is the average value of the variate $x$. The Rayleigh distribution is frequently used to describe the distribution of wind speed.

REGIONAL RESOURCE ASSESSMENT - In this document this refers to detailed wind resource assessments carried out by U.S. Department of Energy subcontractors in 12 regions of the United States and its territories.

RESOURCE ASSESSMENT - In this document this refers to interpretations of the spatial and temporal distributions of the wind climatology and wind resource interpolated between permanent or long-term data stations using historical data from those stations. The resolution of these assessments is as fine as can be responsibly made from sparse data, but is too coarse to be used in WECS site selection except to provide a preliminary indication of the magnitude and distribution of the wind resource for an area. 
REYNOLS NUMBER - A dimensionless number which is significant in the design of a model of any system in which the effect of viscosity is important in controlling the velocities or the flow pattern of a fluid:

$$
\operatorname{Re}=\frac{\mathrm{uL} \rho}{\mu}
$$

where $u$ is a characteristic velocity

$L$ is a characteristic length

$p$ is the density

and $\mu$ is the dynamic viscosity of the fluid.

At high Reynolds numbers in the atmosphere $\left(\mu=0.181 \mathrm{~kg} / \mathrm{m} \mathrm{sec}\right.$ at $20^{\circ} \mathrm{C}$ ) the large scale structure of turbulence becomes independent of Reynolds number; however, fine structure turbulence is very dependent on Reynolds number since fine structure turbulence is dissipated by viscous forces.

REYNOLS STRESS - The net transfer of momentum across a surface in a turbulent fluid because of fluctuations in fluid velocity; also known as eddy stress. The eddy stress, due to the àverage correlation of fluctuating velocity coniponents is

$$
\tau=-\rho \overline{U_{i} U_{j}}
$$

where $\tau=$ Reynolds stress

$p=$ density

$U_{j}$ and $U_{j}$ are fluctuating wind components and the Einstein sumniation convention is used, and an overbar denotes an averaging operator.

ROSSBY NUMBER - The nondimensional ratio of the inertial force to the Coriolis force for a given flow of a rotating fluid given as:

$$
\mathrm{R}_{\mathrm{O}}=\mathrm{U} / \mathrm{fL}
$$

where $U$ is a characteristic velocity, $f$ is the Coriolis parameter, and $L$ a characteristic length. The smallness of the Rossby number is an indication of the goodness of the geostrophic approximation to the winds. 
ROTOR - 1 - The rotating device of a wind turbine that converts wind power into torque on a rotating shaft.

2 - A turbulent eddy that forms beneath the crest of some lee wave disturbances.

ROUGHNESS LENGTH - A parameter $\left(z_{0}\right)$ used to describe the texture of the earth's surface and its effects upon the logarithmic wind profile. Since the logarithmic wind profile is valid only over flat terrain, the roughness length is not really an applicable parameter for nonflat terrain, however, the texture of nonflat terrain is, for comparison, often described in terms of a roughness length.

SAMPLING ERROR - The error or uncertainty resulting from the attempt to infer the characteristics of the true population from the statistics of a limited sample or subset of the population. For example, when one wishes to measure the wind speed corresponding to a given set of external conditions the sampling error is determined by the nature of the turbulence; if one averages over only a portion of or a few large turbulent eddies the estimate of the true average wind speed can be in considerable error.

SAMPLING RATE - The rate at which measurements of physical quantities are made. This rate is determined by the temporal resolution required of the data and sampling error considerations.

SCALE - A characteristic value of a property or feature of a flow or an object or a domain of consideration, such as a characteristic length, frequency, time, velocity, etc.

SECONDARY CIRCULATION - A circulation superimposed upon the mean flow so that iflong average or large spatial averages are taken the circulation is averaged out. These circulations are, however, distinct from turbulence in that their dynamics are not characteristic of turbulence. Nevertheless, secondary circulations can exist in the planetary boundary layer causing periodic variations of wind speed and direction at a given location with time scales of an hour or more, thereby compounding sampling error problems. An example of secondary circulations is horizontal roll vortices, long 
vortices with diameters of the same order as boundary layer height.

These vortices are not quite, but close to parallel to the mean wind, so that it takes a substantial time for one wavelength to pass a fixed point on the ground.

SEPARATION STREAMLINE - The streamline separating the separated flow region beneath the streamline from smoother flow above the streamline. See Flow Separation.

SHEAR - In this document used synonymously with wind shear.

SHEAR STRESS - A stress in which the material on one side of a surface (or in the case of fluids, an imaginary plane between layers of fluid, or fluid above a solid boundary) pushes on the other side of the surface with a force which is parallel to the surface.

In a turbulent flow this is the momentum transport.

SHUTDOWN - The procedure whereby a wind turbine returns to parked standby status. Shutdown procedures may be initiated by wind speeds exceeding cut-out, wind speeds dropping below cut-in before start-up is complete, by power output falling below a prescribed value, or by excessive changes in wind speed or wind direction.

SMAL SCALE TERRAIN - Defined for this document to mean nonflat terrain in which the vertical relief is a small fraction $(<10 \%)$ of the boundary layer dep.th.

SPECTRUM - A plot of the distribution of variance (spectral density) of a given signal as a function of frequency or wave number (1/wavelength). When the plot is displayed with the logarithm of the product of the frequency and the spectral density on the ordinate and the logarithm of the frequency on the abscissa, the area under the curve between a given set of points represents the fraction of the variance contained between those frequencies. 
SPEEDUP - Relates the wind speed at height $z$ above the crest of a hill to the height $z$ above flat terrain upstream of the hill:

$$
S(z)=\frac{U_{C}(z)}{U_{0}(z)}
$$

where $U_{C}=$ wind speed over the crest

$U_{0}=$ upstream wind speed.

STABILITY or STATIC STABILITY - The property of a fluid in which any departure from an equilibrium state gives rise to forces which tend to return the fluid to an equilibrium state. In a dry atmosphere, a positive vertical gradient of the potential temperature causes buoyancy restoring forces to act on a vertical disturbance; this is a stable atmosphere. If the gradient of potential temperature is negative, disturbed parcels experience forces that drive the parcel further from their origin; this is an unstable atmosphere. If the potential temperature is constant with height, disturbed parcels experience no restoring or destablizing forces and conditions are said to be neutrally stable.

STABLE - See Stability

STARTUP - The procedure whereby a wind turbine starts from parked standby status, is made operationally ready, achieves synchronization with the grid and begins to satisfy load. Startup requires several minutes.

STREAM FUNCTION - A scalar function of position used to describe steady, incompressible two-dimensional flow. The stream function $\psi$ is related to velocity components $U$ and $W$ for flow in a vertical plane as:

$$
\begin{aligned}
& U=-\frac{\partial \psi}{\partial \zeta} \\
& W=\frac{\partial \psi}{\partial x}
\end{aligned}
$$

STREAMLINE - A line which is everywhere parallel to the direction of fluid flow at a given instant. When flow is two-dimensional, streamlines represent lines of constant values of the stream function, and hence the more closely spaced the streamlines are the higher the velocity of the fluid is. 
SYNOPTIC SCALE - The scale of migratory high and low-pressure systems of the lower troposphere, with wavelengths of 1000-2500 kni. A reference to synoptic scale winds refers to winds due to the pressure gradient of the synoptic scale waves. There may be mesoscale wind disturbances superimposed upon the synoptic scale windfield.

TEMPERATURE INVERSION - A layer of the atmosphere where the temperature increases with height. In this document temperature inversion or inversion is also used to imply a layer where the potential temperature increases with height forming a stable layer, however, the reader should realize that the potential temperature can be increasing with height while the temperature is decreasing with height. The stable boundary layer is a potential temperature inversion whereas the base of the lowest potential temperature inversion marks the top of an unstable or nearly neutral boundary layer.

THERMAL WIND - The wind shear vector between the geostrophic winds at two pressure levels and related to the mean temperature gradient between the two constant pressure surfaces.

THRESHOD VELOCITY - The wind speed at which sand first begins to move (fluid threshold velocity) or the minimum speed necessary to maintain sand motion once it has been set into motion (impact threshold velocity).

TURBULENCE - Motion of fluids in which local velocities and pressures fluctuate irregularly in a random manner. Additionally, three-dimensional turbulence, usually known just as turbulence, is distinguished from other random flows in the atmosphere by the cascade of kinetic energy of the disturbance to smaller and smaller scales caused by the stretching and amplification of vorticity.

TURBULENCE INTENSITY - A statistic to convey the niagnitude of turbulence kinetic energy of a component of a flow. Turbulence intensity of the longitudinal wind component as expressed by micrometeorologists is

$$
\frac{\sigma_{u}}{u_{\star}}
$$


where $\sigma_{u}$ is the standard deviation of the velocity fluctuations of the wind component parallel to the mean wind, and $u_{*}$ is the friction velocity. Another expression of turbulence intensity used more by engineers is

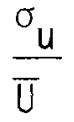

where $\bar{U}$ is the mean wind speed.

TWO-DIMENSIONAL ROW - Flow in which is independent of one space coordinate and hence all cross sections of the flow normal to that coordinate axis are identical.

$u_{*}$ - A reference wind velocity, known also as the friction velocity, defined by the relation

$$
u_{*}=\sqrt{\tau / \rho}
$$

where $\tau$ is the Reynolds stress

$\rho$ is the density

$u_{*}$ is the friction velocity.

UNSTABLE - See Stability.

VALUE OF ENERGY - for WECS, this is the present value of the savings obtained by the addition of WECS to the current or planned generation mix of the utility. The value of energy represents the amounts of money that a utility could afford to spend on a WECS or WECS cluster, over the expected lifetime of the cluster. This is distinct from the cost of energy.

VERTICAL AXIS WIND TURBINE - A wind turbine for which the rotor rotates about a vertical axis. The concept of hub height does not exist for a vertical axis machine so references to measurements at hub height should be substituted by nieasurements at the midpoint of the rotor.

VORTEX - A flow with closed streamlines; any flow possessing vorticity, e.g., an eddy, whirlpool. 
VORTEX LINE - A line drawn through a fluid such that it is everywhere tangent to the vorticity.

VORTEX SHEDDING - In flow of fluid past objects, the shedding of fluid vortices periodically downstream from the restricting object.

VORTICITY - A vector equal to the curl of the velocity arid used as a measure of the local angular velocity of the fluid;

$$
\vec{\zeta}=\nabla \times \vec{V} \text {. }
$$

WAKE - The region downstream of a body immersed in a fluid in which the effects of the body on the fluid's motion are concentrated.

WECS - Wind Energy Conversion System.

WEIBULL DISTRIBUTION - A unimodal probability density function depending on two parameters, a scale factor and a shape factor. The Weibull distribution is given by

$$
p(x)=\left(\frac{k}{C}\right)\left(\frac{x}{c}\right)^{k-1} \exp \left[-(x / C)^{k}\right]
$$

where $C$ is the scale factor, related to the mean value of $x(\bar{x})$ by

$$
\bar{x}=C \Gamma(1+1 / k)
$$

where $\Gamma$ is the gamma function and $k$ is the shape factor which is related to the variance of $x\left(a,{ }^{2}\right)$ by

$$
\sigma_{x}^{2}=c^{2}\left\{\Gamma(1+2 / k)-[\Gamma(1+1 / k)]^{2}\right\} \text {. }
$$

The Rayleigh distribution is a s'pecial case of the Weibull distribution where $k=2$.

WIND PROFILE - See Profile.

WIND SHEAR - The local variation or gradient of the wind vector or any of its components in a given direction. Unless otherwise stated wind shear refers to the vertical shear of the horizontal winds.

YAW - The rotation or movement of a horizontal axis about a vertical axis. 


\section{DISTRIBUTION}

No. of

Copies

\section{OFFSITE}

A. A. Churm

DOE Chicago Patent Group

9800 S. Cass Avenue

Argonne, IL 60439

5 D. F. Ancona/C. I . Aspliden

Department of Energy

600 E. Street, N.W.

Washington, DC 20545

5 G. P. Tennyson

Department of Energy

Albuquerque Operations Office

4501 Indian School Road N.E.

Suite 151

Albuquerque, M 87110

G. T. Ankrum

U.S. Department of Energy

600 E Street N.W.

Washington, DC 20461

J. Rumbaugh

Division of Electrical Energy Systems

U.S. Department of Energy

20 Massachusetts Avenue

Washington, DC 20585

F. R. Summers, P.E.

U.S. Department of Energy

Alaska Power Administration

P.O. Box 50

Juneau, AK 99802

27 DOE Technical Information Center

Peter Lissaman

Aerovironment, Inc.

145 Vista Avenue

Pasadena, CA 91107
No. of

Copies
W. A. Vachon

Arthur D. Little, Inc.

20 Acorn Park

Cambridge, MA 02140

Peter Smeallie

AWEA Windletter

1609 Connecticut Avenue N.W.

Washington, DC 20008

Ben Wolf $f$

American Wind Energy Association

1621 Connecticut Avenue, N.W.

Fifth Floor

Washington, D 20009

Richard Katzenberg

American Wind Energy Association

Natural Power, Inc.

New Boston, NH 03070

A. B. Van Rennes

Corporate Director

External Research and Development

The Bendix Corporation

Bendix Center

Southfield, MI 48037

John Lowe

Boeing Engineering and Construction Company

P.O. Box 3707

Seattle, WA 98214

Wayne Wiesner

Wind Turbine Group

Boeing Engineering and Construction

P.O. Box 3707

Seattle, WA 98124 
Nicholas Butler

Bonneville Power Administration

P.O. Box 3261-EIC

Portland, OR 97208

M. Ginosar/J. Lerner

California Solar Energy Commission

Energy Resources, Conservation and Development Commission

1111 Howe Avenue, Mail Stop 15

Sacramento, CA 95825

R. N. Meroney

Department of Civil Engineering

Colorado State University

Fort Collins, CO 80523

D. E. Smith

Public Service Company of Colorado

P.O. Box 840

Denver, CO 80201

E. H. Gi1more

Department of Agriculture

Agriculture Research Service

Bushland, TX 79012

L. A. Liljedahl

Department of Agriculture

Agriculture Wind Energy Program

Building 001, Room 126 BARC-West

Beltsville, MD 20705

Jay Tappan

Department of Energy

State of Oregon

Labor and Industries Building

Room 111

Salem, OR 98310

Edgar Demeo

Electric Power Research Institute 3412 Hillview Avenue

Palo Alto, CA 94303
4 Frank Goodman, Jr.

Electric Power Research Institute

P.O. Box 10412

Palo Alto, CA 94025

Wilson Prichett

Energy R\&D, NRECA

1800 Massachusetts Avenue NW

Washington, DC 20036

W. H. Snyder

Fluid Model ing Section (MD-81)

Environmental Protection Agency

Research Triangle Park, NC 27711

3. J. Riley

Senior Research Scientist

Flow Industries, Inc.

21414-68th Avenue S.

Kent, WA 98031

W. D. Marsh

General Electric Company

Electrical Utility Systems Engineering Department

Schenectady, NY 12345

R. H. Poor

General Electric Company

Philadelphia, PA 19101

E. E. Johanson

JBF Scientific Corporation

2 Jewel Drive

Wilmington, MA 01887

J. J. Walt on

L 262

Lawrence Livermore Laboratory

Livermore, CA 94596

Gerald Park

Department of Electrical Engineering

Michigan State University

East Lansing, MI 48824 
No. of

Copies

Ms. Abbey Page

Maine Office of Energy Resources

55 Capital

Augusta, ME 04330

J. Konigsberg

Montana Energy Office

Capital Station

Helena, MT 59601

2 W. H. Robbins

NASA/Lewis Research Center

21000 Brookpark Road

Cleveland, $\mathrm{OH} 44135$

Phillip French

NASA Scientific and Technical Information Facility

P.O. Box 8757

Baltimore/Washington International Airport

Baltimore, MD 21240

D. H. Lenschow

National Center for Atmospheric Research

P.O. Box 3000

Boulder, CO 80309

M. J. Changery

National Oceanic and Atmospheric Administration

National Climatic Center

Federal Building

Asheville, NC 28801

William Hooke

Wave Propagation Laboratory

National Oceanic and Atmospheric Administration

Boulder, CO 80302

J. W. Deardorff

Department of Atmospheric Sciences

Oregon State University

Corvallis, OR 97331
No. of

Copies

E. W. Hewson/J. E. Wade Oregon State University

Corvallis, OR 97331

2 R. L. Simon

Pacific Gas and Electric

215 Market Street

San Francisco, CA 94106

E. L. Luther

PRC Eng. Analysis Co.

01d Springhouse Road

McLean, VA 22102

P. M. Sforza

Department of Mechanical and Aerospace Engineering

Polytechnic Institute of New York

Long Island Center

Route 110

Farmingdale, NY 11735

Terry J. Healy/C. Hansen

Rockwe11 International

Rocky Flats Plant

P.O. Box 464

Golden, CO 80401

E. Kadlec

Sandia Laboratories

Division 5443

P.O. Box 5800

Albuquerque, NM 87115

R. M. Traci

Science Applications, Inc.

1200 Prospect Street

P.O. Box 2351

La Jolla, CA 92038

I. Vas/Roger Taylor

Solar Energy Research Institute 1536 Cole Blvd.

Golden, CO 80401 
No. of

Copies

C. M. Bhumralkar

SRI International

333 Ravenswood Avenue

Menlo Park, CA 94205

C. A. Mazzola

Stone \& Webster Engineering Corporation

245 Summer Street

P.0. Box 2325

Boston, MA 02107

W. Owens

Tennessee Val ley Authority

426 United Bank Building

Chattanooga, TN 37401

Thomas A. Schroeder

University of Hawaii at Monoa

2525 Correa Road

Honolulu, HI 96822

R. A. Pielke

University of Virginia

Department of Environmental Sciences

Clark Hall

Charlottesville, VA 22903

Ms. Susan Hosch

Washington State Energy Office $400 \mathrm{E}$. Union Avenue, 1s t Floor

Olympia, WA 98504

John Young

Washington Public Power Supply System

P.O. Box 968

Richland, WA 99352

L. L. Nelson

U.S. Water \& Power Resource Service

Denver Federal Center, Bldg. 20

Denver, CO 80225
No. of

Copies

G. D. Thomann

Wichita State University

P.O. Box 44

Wichita, KS 67208

Farrell Smith Sei Ter

Wind Energy Report

Box 14 - 104 X. Village Avenue

Rockville Centre, NY 11571

R. W. Marrs

University of Wyoming

Department of Geology

Laramie, WY 82071

V. Barros

28 De Julio 28

3120 Puerto Madryn

Chulr e t

R. ARGENTINA

P. A. Taylor (ARQL)

Atmospheric Environment Service 4905 Dufferin Street

Downsview, Ontario

M3H 5T4

CANADA

S. Venkatesh (ARMS)

Atmospheric Environment Service 4905 Dufferin Street

Downsview, Ontario

M3H 5T4

CANADA

R. J. Templin

National Research Counci 1

Montreal Road

Ottawa, Ontario

KIA OR6

CANADA 
No. of

Copies

D. J. Mi1borrow

Central Electricity Research Laboratories

Kelvin Avenue

Leatherhead, Surrey

KT227SE

ENGLAND

K. M. Carpenter

Meteorological Office

London Road

Bracknell, Berkshire

RG12 2SZ

ENGLAND

D. Lindley

Taylor Woodrow Construction, Limited

Taywood House

345 Ruislip Road

Southhall

Middl esex

UBI 2QX

ENGLAND

Peter Builtjes

Department of Fluid Flow Technology

TNO

P.O. Box 342

7300 Apeldoorn

NETHERLANDS

Paul Vermeu Ten

Department of Fluid Flow Technology

TNO

P.O. Box 342

7300 Apeldoorn

NETHERLANDS

Anders Gustafsson

Coordinator Wind Energy

P.O. Box 11021

S-161 11 Bromnia 11

SWEDEN
No. of

Copies

Olle Ljungstrom

FFA, The Aeronautical Research Institute

Forskningsstationen i Stockholm

Drottning Kristinas Vag 47

S-114 29 Stockholm

SWEDEN

\section{ONS ITE}

2 DOE Richland Operations Office

P.0. Box 550

Richland, WA 99352

H. E. Ransom

R. K. Stewart

44 Pacific Northwest Laboratory Battelle Boulevard

Richland, WA 99352

W. R. Barchet

J. C. Barnard

J. W. Buck

J. R. Connell

J. C. Doran

K. Drumheller

C. E. Elderkin

D. L. Elliott

R. L. George

D. L. Hadley

T. R. Hiester

A. H. Miller

E. L. Owzarski

W. T. Pennell

E. H. Phinney

D. C. Powell

J. M. Proud (15)

J. W. Ramsde11

D. S. Renné

H. L. Wegley

L. L. Wendell

R. K. Woodruff

R. Zengerle

Technical Information - Library (5)

Publishing Coordination (2) 